\title{
Sanitary Landfill Groundwater Monitoring Report - Fourth Quarter 1998 and 1998 Summary
}

by

J. Chase

RECORDS ADMINISTRATION

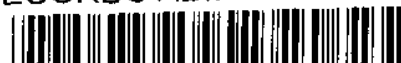

Westinghouse Savannah River Company

Savannah River Site

Aiken, South Carolina 29808

DOE Contract No. DE-AC09-96SR18500

This paper was prepared in connection with work done under the above contract number with the U.S.

Department of Energy. By acceptance of this paper, the publisher and/or recipient acknowledges the U.S. Government's right to retain a nonexclusive, royalty-free license in and to any copyright covering this paper, along with the right to reproduce and to authorize others to reproduce all or part of the copyrighted paper. 
WSRC-TR-99-00011

Unclassified

\section{SANITARY LANDFILL GROUNDWATER MONITORING REPORT (U)}

\section{FOURTH QUARTER 1998 \\ AND 1998 SUMMARY}

Publication Date: March 1999

Key Words

Chloroethene (vinyl chloride)

LFW wells

Tetrachloroethylene

Trichloroethylene

Authorized Derivative Classifier / Reviewing Official

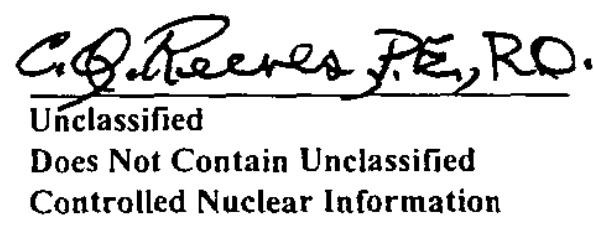

Westinghouse Savannah River Company Savannah River Site

Aiken, SC 29808 


\section{DISCLAMER}

This report was prepared by Westinghouse Savannah River Company (WSRC) for the United States Department of Energy under Contract No. DE-AC09-96SR 18500 and is an account of work performed under that contract. Reference herein to any specific commercial product, process, or service by trademark, name, manufacturer, or otherwise does not necessarily constitute or imply endorsement, recommendation, or favoring of same by WSRC or by the United States Government or any agency thereof. 


\section{SANITARY LANDFILL GROUNDWATER MONITORING REPORT (U)}

\section{FOURTH QUARTER 1998}

AND 1998 SUMMARY

Publication Date: March 1999

Key Words

Chloroethene (vinyl chloride)

LFW wells

Tetrachloroethylene

Trichloroethylene

Authorized Derivative Classifier I

Reviewing Official

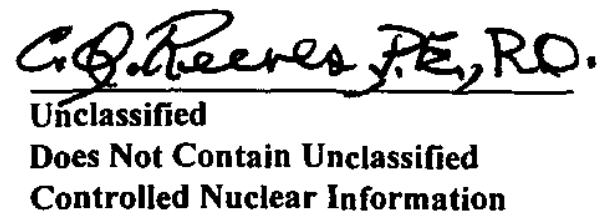

Westinghouse Savannah River Company

Savannah River Site

Aiken, SC 29808

Prepared for the U.S. Department of Energy under Control Contract No. DE-AC09-96SR18500 


\section{DISCLAIMER}

This report was prepared as an account of work sponsored by an agency of the United States Government. Neither the United States Government nor any agency thereof, nor any of their employees, makes any warranty, express or implied, or assumes any legal liability or responsibility for the accuracy, completeness, or usefulness of any information, apparatus, product, or process disclosed, or represents that its use would not infringe privately owned rights. Reference herein to any specific commercial product, process, or service by trade name, trademark, manufacturer, or otherwise does not necessarily constitute or imply its endorsement, recommendation, or favoring by the United States Government or any agency thereof. The views and opinions of authors expressed herein do not necessarily state or reflect those of the United States Government or any agency thereof.

This report has been reproduced directly from the best available copy.

Available to DOE and DOE contractors from the Office of Scientific and Technical Information, P.O. Box 62, Oak Ridge, TN 37831; prices available from (615) 576-8401.

Available to the public from the National Technical Information Service, U.S. Department of Commerce, 5285 Port Royal Road, Springfield, VA 22161. 


\section{SANITARY LANDFILL GROUNDWATER MONITORING REPORT (U)}

\section{FOURTH QUARTER 1998}

Publication Date: March 1999

Key Words

Chloroethene (vinyl chloride)

LFW wells

Tetrachloroethylene

Trichloroethylene

Authorized Derivative Classifier/

Reviewing Official

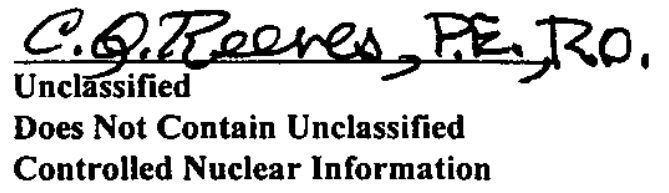

Westinghouse Savannah River Company

Savannah River Site

Aiken, SC 29808

Prepared for the U.S. Department of Energy under Control Contract No. DE-AC09-96SR18500 
THIS PAGE LEFT BLANK INTENTIONALLY. 


\title{
Abstract
}

\begin{abstract}
A maximum of fifty-three wells of the LFW series monitor groundwater quality in the Steed Pond Aquifer (Water Table) beneath the Sanitary Landfill at the Savannah River Site (SRS). These wells are sampled quarterly to comply with the South Carolina Department of Health and Environmental Control Domestic Water Permit DWP-087A and as part of the SRS Groundwater Monitoring Program.
\end{abstract}

Iron (total recoverable), chloroethene (vinyl chloride) and 1,1-dichloroethane were the most widespread constituents exceeding the Final Primary Drinking Water Standards during 1998. Trichloroethylene, 1,4-dichlorobenzene, 1,1-dichloroethelene, 1,4-dichlorobenzene, sulfide, $\mathrm{pH}$, trichlorofluoromethane, benzene, dichloromethane (methylene chloride), a common laboratory contaminant, tetrachloroethylene, tritium, aluminum (total recoverable), dichlorodifluoromethane, cis-1,2-dichloroethylene, and arsenic (total recoverable) also exceeded standards in one or more wells.

The groundwater flow direction in the Steed Pond Aquifer (Water Table) beneath the Sanitary Landfill was to the southeast (universal transverse Mercator coordinates). The flow rate in this unit was approximately $153 \mathrm{ft} /$ year during first quarter 1998 and $164 \mathrm{ft} /$ year during fourth quarter 1998. 
THIS PAGE LEFT BLANK INTENTIONALLY. 


\section{Contents}

\section{Page}

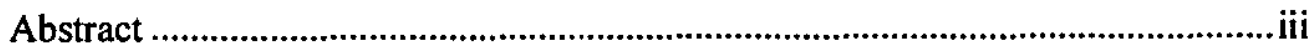

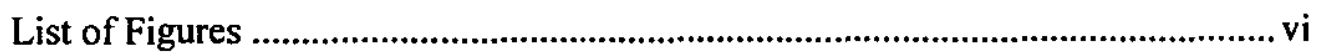

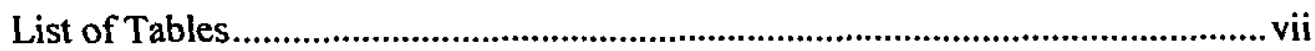

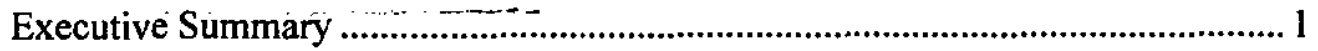

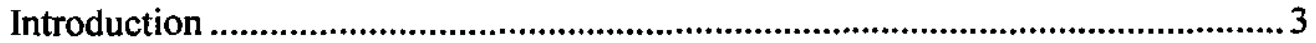

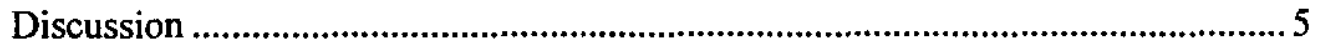



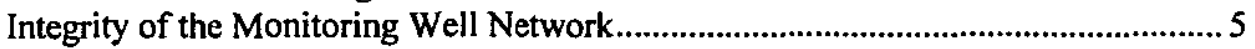

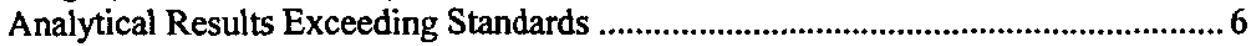

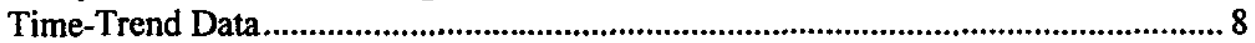

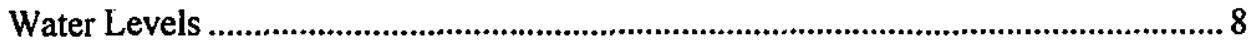

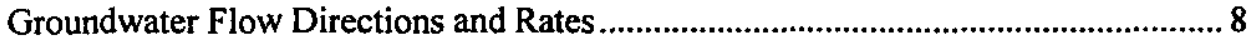

Upgradient Versus Downgradient Results .................................................................... 9

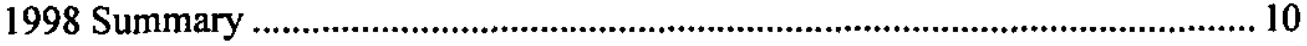

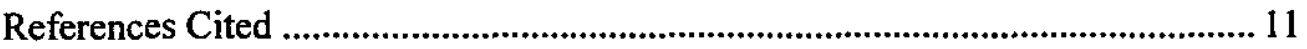

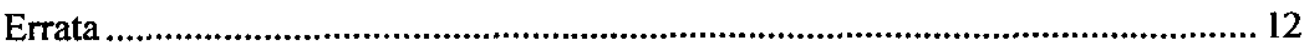

Appendix A - Final Primary Drinking Water Standards ..................................A-1

Appendix B - Flagging Criteria ...................................................................... B-1

Appendix C - Figures .......................................................................................

Appendix D - Groundwater Monitoring Results Tables ................................... D-1

Appendix E - Data Quality/Usability Assessment.............................................. E-1

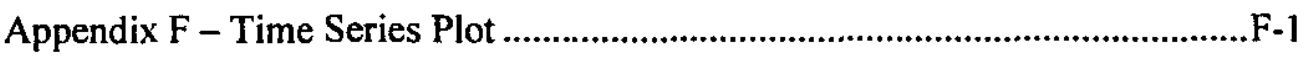

Appendix G - Hydrographs .............................................................................. 


\section{List of Figures}

\section{Page}

1. Location of the Sanitary Landfill at the Savannah River Site

C-3

2. Boundaries of Original Sanitary Landfill and Expansions and Locations of Monitoring Wells at the Sanitary Landfill

3. Chloroethene Concentrations at the Sanitary Landfill, First Quarter 1998.

4. Chloroethene Concentrations at the Sanitary Landfill, Fourth Quarter 1998

5. Trichloroethylene Concentrations at the Sanitary Landfill, First Quarter 1998.

6. Trichloroethylene Concentrations at the Sanitary Landfill, Fourth Quarter 1998.

7 Tritium Concentrations at the Sanitary Landfill, First Quarter 1998

8. Tritium Concentrations at the Sanitary Landfill, Fourth Quarter 1998

9. Piezometric Surface Map of the Steed Pond Aquifer (Water Table) at the Sanitary Landfill, First Quarter 1998

10. Piezometric Surface Map of the Steed Pond Aquifer (Water Table) at the Sanitary Landfill, Fourth Quarter 1998.

11. Regional Correlation of Hydrostratigraphic and Lithostratigraphic Nomenclature 


\section{List of Tables}

1. Maximum Page Water Standards D-3

2. Maximum Levels of Constituents Exceeding Other Flag 2 Criteria D-7

3. Groundwater Monitoring Results for Individual Wells D-13 
THIS PAGE LEFT BLANK INTENTIONALLY. 


\section{Fourth Quarter 1998 Data Review}

This report contains analytical data for samples taken during Fourth Quarter 1998 from wells of the LFW series located at the Sanitary Landfill at the Savannah River Site (SRS). The data are submitted in reference to the Sanitary Landfill Operating Permit (DWP-087A). The report presents monitoring results that equaled or exceeded the Safe Drinking Water Act final Primary Drinking Water Standards (PDWS) or screening levels established by the U.S. Environmental Protection Agency (Appendix A), the South Carolina final Primary Drinking Water Standard for lead (Appendix A), or the SRS flagging criteria (Appendix B).

Mechanical failure prevented sample collection and analysis of the groundwater at well LFW10A.

\section{Key to Reading the Tables}

The following abbreviations may appear in the data tables:

\section{Constituents}

\section{$1,2,3,4,6,7,8-\mathrm{HPCDD}$ \\ $1,2,3,4,6,7,8-\mathrm{HPCDF}$ \\ $1,2,3,4,7,8-\mathrm{HXCDD}$ \\ $1,2,3,4,7,8-\mathrm{HXCDF}$ \\ Lindane \\ PCB \\ 1,2,3,7,8-PCDD \\ 1,2,3,7,8-PCDF \\ Sp. Conductance \\ TCDD \\ TCDF}

Laboratories
$\mathrm{CN}$
EM

GE and GP

$\mathrm{SC}$

SP

TM

WA and WS

Sampling Codes
B
C
D
E
I

L.

Sampling Codes (cont'd) 1,2,3,4,6,7,8-heptachlorodibenzo-p-dioxin $1,2,3,4,6,7,8$-heptachlorodibenzo-p-furan 1,2,3,4,7,8-hexachlorodibenzo-p-dioxin 1,2,3,4,7,8-hexachlorodibenzo-p-furan gamma-benzene hexachloride poiychlorinated bipheny! 1,2,3,7,8-pentachlorodibenzo-p-dioxin 1,2,3,7,8-pentachlorodibenzo-p-furan specific conductance tetrachlorodibenzo-p-dioxin tetrachlorodibenzo-p-furan

Clemson Technical Center, Inc.

Environmental Protection Department/ Environmental Monitoring Section (EPD/EMS) Laboratory

General Engineering Laboratories

Savannah River Technology Center Spencer Testing Services, Inc.

TMA/Eberline

Roy F. Weston, Inc.

blank sample was collected

well was pumping continuously

well was dry

equipment blank was collected

well went dry during sampling; insufficient water to collect all samples

well went dry before sampling began; only depth to water can be determined 
$\mathbf{P}$

S

$\mathrm{X}$ inaccessibility or mechanical failure prevented sample collection and field analysis of the water no water in standpipe; for water level events only well went dry during purging; samples collected after well recovered

Sampling Methods

\begin{tabular}{|c|c|c|}
\hline B & & sample collected using an open-bucket bailer \\
\hline & & sample collected using a bladder pump \\
\hline & " & $\begin{array}{l}\text { sample collected using a single-speed centrifugal } \\
\text { downhole pump }\end{array}$ \\
\hline & & sample collected using a variable-speed pump \\
\hline
\end{tabular}

Units

E

$\mathrm{mg} / \mathrm{L}$

$\mathrm{msl}$

MSL

NTU

$\mathrm{pCi} / \mathrm{L}$

$\mathrm{pCi} / \mathrm{mL}$

$\mathrm{pH}$

$\mu \mathrm{g} / \mathrm{L}$

$\mu \mathrm{S} / \mathrm{cm}$ exponential notation (e.g., $1.1 \mathrm{E}-09=1.1 \times 10^{-9}=$ 0.0000000011 )

milligrams per liter

mean sea level

million structures per liter

turbidity unit

picocuries per liter

picocuries per milliliter

pH unit

micrograms per liter

microsiemens per centimeter

Other

$\begin{array}{ll}\text { CS } & \begin{array}{l}\text { carbon steel } \\ \text { primary drinking water standard (PDWS) column in data } \\ \text { tables }\end{array} \\ \text { GS } & \begin{array}{l}\text { groundwater protection standard column in data tables } \\ \text { holding time column in data tables } \\ \text { modifier column in data tables }\end{array} \\ \text { H } & \text { primary drinking water standard } \\ \text { Mod } & \text { polyvinyl chloride } \\ \text { PDWS } & \text { top of casing } \\ \text { TOC } & \end{array}$

\section{Holding Times}

Standard analytical methods include a limit, called holding time, on the maximum elapsed time between sample collection and extraction or analysis by the laboratory. In the data tables, a large bultet ( $)$ in the $H$ (holding time) column indicates that holding time was exceeded. Analyses performed beyond holding times may not yield valid results.

The South Carolina Department of Health and Environmental Control allows only 15 minutes to elapse between sampling and analysis for $\mathrm{pH}$. Thus, only field $\mathrm{pH}$ measurements can meet the holding time criterion; laboratory $\mathrm{pH}$ analyses always will exceed it. 
WSRC-TR-99-00011

Unclassified

The laboratory procedure used for the determination of specific conductance allows one day to elapse between sampling and analysis. Thus, laboratory specific conductance measurements may exceed the holding time criterion. 


\section{Data Rounding}

Constituent results in analytical results tables that appear to equal the final PDWS but are not marked in the $D$ (exceeded the final PDWS or screening level) column are below the final PDWS in the database. Values stored in the database contain more significant digits than the reported results. Apparent discrepancies in the tables are due to the rounding of reported results.

\section{Data Qualification}

The contract laboratories continually assess their own accuracy and precision according to U.S. Environmental Protection Agency-(EPA) guidelines. They submit sample- or batch-specific quality assurance/quality control information either at the same time as analytical results or in a quarterly summary. Properly defined and used result modifiers (also referted to as qualifiers) can be a key component in assessing data usability. Result modifiers designed by the Environmental Protection Department/Environmental Monitoring Section and provided to the primary laboratories are defined below. These modifiers appear in the data tables under the column Mod. The lettered modifiers are based on EPA's STORET codes.

\section{Result Modifier}

(Blank)

A

I

J

L

M

$\mathrm{R}$

$\mathrm{T}$

$\mathrm{U}$

V

Result Modifier (cont'd)
Data are not qualified. Numbers should be interpreted exactly as reported.

Value reported is the mean of two or more determinations.

The value in the result field is the instrument reading, not the sample quantification limit. Always used with the result qualifier $U$.

Value is estimated because quantitation in the sample or in associated quality control samples did not meet specifications.

Value is off-scale high. The actual value is not known but is known to be greater than the value shown.

Presence of the analyte is verified but not quantified.

Result was rejected because performance requirements in the sample analysis or associated quality control analyses were not met.

Analyte was not detected; if present, it was below the criteria for detection.

Material analyzed for but not detected. Analytical result reported is less than the sample quantitation limit.

Analyte was detected in an associated method blank. 
Resuit was obtained from an unpreserved or improperly preserved sample. Data may not be accurate.

Result may be an underestimation of the true value due to analytical bias.

Result may be an overestimation of the true value due to analytical bias.

The associated result may be of poor precision (high variability) due to analytical bias.

Result is associated with QA results indicating matrix interference.

The associated result is from a reanalysis performed out of holding time due to problems with an earlier analysis. 
THIS PAGE LEFT BLANK INTENTIONALLY. 


\section{Executive Summary}

The Sanitary Landfill at the Savannah River Site (SRS) is composed of the original 32-acre landfill, plus expansion areas to the north and south that add 16 and 22 acres, respectively, to the facility. The landfill is subject to the requirements of the Resource Conservation and Recovery Act and is subject to the South Carolina Department of Health and Environmental Control (SCDHEC) Domestic Waste Permit DWP-087A and the Sanitary Landfill groundwater quality assessment plan.

A maximum of fifty-three wells of the LFW series monitored the groundwater quality in the Steed Pond Aquifer (Water Table) beneath the Sanitary Landfill during 1998. These wells sampled quarterly for selected indicator parameters, inorganics, radionuciides, volatile organics, and other constituents to comply with the Sanitary Landfill groundwater quality assessment plan and as part of the SRS Groundwater Monitoring Program. No new wells were abandoned or installed in 1998.

During 1998, samples analyzed from 32 of the 53 monitoring welts (60 percent) exhibited levels of benzene, chloroethene (vinyl chloride), dichloromethane (methylene chloride), 1,2-dichloroethane, 1,4-dichlorobenzene, aluminum (total recoverable), lead (total recoverable), mercury (total recoverable), tetrachloroethylene, trichloroethylene, or tritium that exceeded their final Primary Drinking Water Standards (PDWS).

Aluminum (total recoverable), Iron, (total recoverable), Chloroethene, and 1,1-Dichloroethane were the most widespread constituents exceeding standards during 1998. Aluminum and iron, occurred in concentrations that exceeded its final PDWS in 17 wells. Chloroethene occurred in concentrations that exceeded its final PDWS in 13 wells; 1,1 -Dichloroethane exceeded its final PDWS in 12 wells during the year. Trichlorofluoromethane exceeded its final PDWS in 10 wells during the year. Dichloromethane (Methylene chloride) was elevated in $8 \mathrm{LFW}$ wells; Trichloroethylene was elevated in 7 wells, Dichlorodifluoromethane was elevated in 6 wells, Benzene was elevated in 4 wells, 1,2-Dichloroethane, $\mathrm{pH}$, Tetrachloroethylene, and Tritium was elevated in 3 wells. The remaining elevated constituents occurred with less frequency during the year. These results are similar to the results for 1997 .

The groundwater flow direction in the Steed Pond Aquifer (Water Table) beneath the Sanitary Landfill during first and fourth quarters was to the southeast (universal transverse Mercator coordinates) toward Upper Three Runs Creek. Estimated flow rates in this aquifer during the same periods were $153 \mathrm{ft} /$ year during first quarter 1998 and $164 \mathrm{ft} /$ year during fourth quarter 1998 . These directions and flow rates are very similar to directions and flow rates during 1997. 
THIS PAGE LEFT BLANK INTENTIONALLY. 


\section{Introduction}

The Sanitary Landfill (740-G) (Figures 1 and 2, Appendix C) is located southwest of Road C, about midway down the slope from the Aiken Plateau to Upper Three Runs Creek. The following description outlines important events in the history of the landfill:

- The original 32-acre landfill began receiving sanitary waste, including paper and paper products, plastics, scrap metal, rubber, food waste, glass, packing material, miscellaneous construction debris, empty paint cans, and other nonradioactive waste materials in 1974.

- On June 12,1978; the South-Garolina Department of Health and Environmental Control (SCDHEC) issued permit DWP-087 to the U.S. Department of Energy (DOE) for the operation of the Sanitary Landfill for inert and domestic solid waste disposal.

- On September 9, 1986, SCDHEC issued permit DWP-087A which allowed expansion areas to the north and south of the original 32-acre landfill, adding 16 and 22 acres, respectively (WSRC, 1993).

- The original landfill was filled to capacity in early 1987 (WSRC, 1990).

- In 1988, the Sanitary Landfill was placed on the Resource Conservation and Recovery Act (RCRA) Facility Investigation (RFI) list because elevated levels of hazardous constituents were detected in the groundwater beneath the facility (WSRC, 1990).

- A groundwater quality assessment plan for the Sanitary Landfill was submitted to SCDHEC in 1990 (WSRC, 1990).

- SCDHEC renewed permit DWP-087A on July 24, 1991 (SCDHEC, 1991).

- As a result of Settlement Agreement 91-51-SW, effective August 24, 1991, the Sanitary Landfill became subject to RCRA requirements because wipes and rags contaminated with RCRA-listed solvents were disposed there.

- As a result of RCRA permit investigation, the U.S. Environmental Protection Agency (EPA) removed the Sanitary Landfill from the RFI/RI unit list in August 1991.

- The Northern Expansion began operations in mid-1992 when the Southern Expansion was filled (WSRC, 1993).

- $\quad$ SRS submitted a closure plan (rev. 0) for the Sanitary Landfill to SCDHEC on February 28, 1993. Based on comments received from the SCDHEC, a revised closure plan (rev. 1) was submitted in January 1994. Additional comments were received from SCDHEC in August 1995; the closure plan was revised in response to these comments and the Sanitary Landfill Closure Plan (rev. 2) was submitted in October 1995. This revision was released for public comment on November 6, 1995. The public comment period ended on December 6, 1995. There were no comments received during the public comment period. SCDHEC granted conditional approval of the Sanitary Landfill Closure Plan (rev. 2) in December 1995. In order to meet the conditions of the approval, SRS submitted revised pages to the Sanitary Landfill Closure Plan in February 1996. In addition, installation of the geosynthetic closure cap, as specified in the approved Closure Plan, was initiated in February 1996. The Sanitary Landfill was certified closed on October 26, 1997.

- A RCRA Part B post-closure care permit application was submitted to SCDHEC on March 31, 1993. 
- A modification to the Sanitary Landfill groundwater quality assessment plan was approved by SCDHEC in November 1993. The modified plan included further characterization studies and proposed the installation of additional monitoring wells. Thirty-two new monitoring wells were sampled for the first time during second quarter 1994.

- The Northern Expansion, the last open portion of the Sanitary Landfill, ceased actual operations on November 1, 1994.

- A RCRA style closure was completed over the central and southern portions of the landfill in April 1997.

- Two horizontal remediation wells were completed on the southern and western sides of the landfill in Febriary 1998.

The SRS Environmental Protection Department/Environmental Monitoring Section (EPD/EMS) samples the monitoring wells at the Sanitary Landfill quarterly for selected parameters. The Environmental Restoration Department provides quarterly reports of the monitoring results to SCDHEC to meet regulatory requirements. 


\section{Discussion}

\section{Groundwater Monitoring Data}

Groundwater samples were collected from the LFW wells during December 1997 for first quarter 1998, during March for second quarter 1998, during June for third quarter 1998 and during September for fourth quarter 1998.

The WSRC sampling procedure requires evacuation of a minimum of two well volumes and stabilization of $\mathrm{pH}$, specific conductance, and turbidity prior to sample collection. Stability is established when a minimum of three successive measurements, taken for each stability parameter within a given time period, are within a specified tolerance range. If a well pumps dry before two well volumes are purged or before stabilization is achieved, it must be revisited within 24 hours for the data to be considered from a single sampling event. On the second visit within 24 hours, samples are taken without purging or stability measurements; thus, these samples may not be representative of groundwater quality.

Throughout 1998, samples from wells at the Sanitary Landfill were analyzed for selected indicator parameters, inorganics, radionuclides, volatile organics, and other constituents. For simplicity, results that equaled or exceeded standards are described as exceeding standards, above standards, or as elevated. These following standards were used in this report:

- The Safe Drinking Water Act final Primary Drinking Water Standards (PDWS) or drinking water screening levels, as established by the U. S. EPA (Appendix A)

- The South Carolina final PDWS for lead (Appendix A)

- SRS flagging criteria based on PDWS, Secondary Drinking Water Standards, or method detection limits (Appendix B)

The final PDWS for individual analytes provided in Appendix A may not always match the SRS flagging criteria provided in Appendix B. The final PDWS are used as guidelines in this compliance report to meet regulatory requirements; the flagging criteria are used by EPD/EMS to identify relative levels of constituents in the groundwater and as guides for scheduling groundwater sampling.

\section{Integrity of the Monitoring Well Network}

A maximum of fifty-three wells of the LFW series monitored groundwater quality in the Steed Pond Aquifer (Water Table) beneath the Sanitary Landfill (Figure 2, Appendix C) during 1998. Fourteen wells were installed between 1981 and 1984, 17 wells were installed around the southern and northern expansions in late 1986, and 26 additional wells were installed in 1991. Between December 1993 and February 1994, 32 additional LFW wells were installed southeast of the southern expansion. During January 1996, fifty-five wells were deleted from analyses according to the Groundwater Quality Assessment Plan Amendment. Of these fifty-five wells, four are still being monitored in order to comply with SCDHEC Subtitle D regulations for the interim Sanitary Landfill. The designated background wells at the Sanitary Landfill are wells LFW29, 30, and 31, and well cluster LFW43.

SRS rehabilitates and replaces wells that do not produce representative groundwater samples. A complete record of well installations, replacements, and abandonments at the Sanitary Landfill is provided in the EPD/EMS well inventory. During 1998, no wells were abandoned or replaced. 
- Table 3-(Appendix D) lists the number of well volumes purged from each of the LFW series wells during each quarter 1998 and provides samples codes (I, P, X, etc. [see Appendix D front matter]) that describes unusual sampling events. Although not all wells were sampled during every quarter, there were no unusual sampling events recorded in 1998. Mechanical failure was the reason for missing. Sampling events.

\section{Analytical Results Exceeding Standards}

Results for analytes that exceeded the final PDWS (Appendix A) during each quarter 1998 are summarized in Table 1 (Appendix D) and described in the following text. The text includes the maximum level for each constituent in parentheses following the well in which it was detected.

During first quarter 1998, 19 of the $53 \mathrm{LFW}$ wells contained elevated constituents.

- 1,1-Dichloroethane was elevated in 15 wells: LFW10A (maximum concentration at $172 \mu \mathrm{g} / \mathrm{L}), 21$, 58D, 60C, 60D, 61D, 62C, 62D, 65D, 67C, 67D, and 69D.

- 1,2-Dichloroethane was elevated in 3 wells: LFW43B (maximum concentration at $7.5 \mu \mathrm{g} / \mathrm{L}), 62 \mathrm{C}, 62 \mathrm{D}$.

- 1,4-Dichlorobenzene was elevated in one well: LFW62D maximum concentration of $86 \mu \mathrm{g} / \mathrm{L}$

- Benzene was elevated in 3 wells: LFW62D (maximum concentration at $25.6 \mu \mathrm{g} / \mathrm{L}$ ), $10 \mathrm{~A}$ and $67 \mathrm{C}$.

- Chloroethene was elevated in 12 wells: LFW10A (maximum concentration at 34.8 $\mu \mathrm{g} / \mathrm{L}$ ), 6R, 8R, 18, 21, 36R, 58D, 60C, 62D, 63D, 64D, and 69D.

- Dichlorofluoromethane was elevated in 6 wells: LFW 58D, 61D LFW59D (maximum concentration at $38.5 \mu \mathrm{g} / \mathrm{L}$ ), 62D, 63D, 67C, and 67D.

- Dichloromethane (Methylene Chloride) was elevated in 3 wells: LFW10A (maximum concentration at $100 \mu \mathrm{g} / \mathrm{L}$ ), 61D and 63D.

- Iron (total recoverable) was elevated in one well: LFW62D maximum concentration of $34.5 \mu \mathrm{g} / \mathrm{L}$

- Mercury (total recoverable) was elevated at two wells: LFW60D, and 62C (maximum concentration of $3.21 \mu \mathrm{g} / \mathrm{L}$ ).

- Tetrachloroethylene was elevated in 2 wells: LFW58D, and 61D (maximum concentration of $8.31 \mu \mathrm{g} / \mathrm{L}$

- Trichloroethylene was elevated in 7 wells: LFW58D, 61D, 62C, 62D (maximum concentration at $34.5 \mu \mathrm{g} / \mathrm{L}), 65 \mathrm{D} ; 67 \mathrm{C}$, and $67 \mathrm{D} . \cdots$

- Trichlorofluoromethane was elevated in 7 wells: LFW10A, 58D, 61D, 62D, 63D, $65 \mathrm{D}$, and $67 \mathrm{D}$ (maximum concentration at $40.1 \mu \mathrm{g} / \mathrm{L}$ ).

- Tritium was elevated in 2 wells: LFW10A (maximum concentration of 27.71 $\mathrm{pCi} / \mathrm{mL}$ ), and $67 \mathrm{C}$. 
During second quarter 1998, 15 of the 53 LFW wells contained elevated constituents.

- 1,1-Dichloroethane was elevated in 6 wells: LFW10A, (maximum concentration at $130 \mu \mathrm{g} / \mathrm{L}), 21,61 \mathrm{D}, 65 \mathrm{D}, 67 \mathrm{C}$, and 67D.

- Benzene was elevated in 2 wells: LFW 10A and $67 \mathrm{C}$ (maximum concentration at 8.9 $\mu \mathrm{g} / \mathrm{L})$.

- Chloroethene was elevated in 10 wells: LFW6R, 10A (maximum concentration at 24 $\mu \mathrm{g} / \mathrm{L}), 18,21,36 \mathrm{R}, 60 \mathrm{C}, 63 \mathrm{D}, 64 \mathrm{D}, 67 \mathrm{C}$ and $69 \mathrm{D}$.

- Dichlorodifluoromethane was elevated in 4 wells: LFW61D, 63D (maximum concentration at $190 \mu \mathrm{g} / \mathrm{L}), 67 \mathrm{c}$, and 67D.

- Dichloromethane (methylene chloride) was elevated in 5 wells: LFW8R, 10A (maximum concentration at $190 \mu \mathrm{g} / \mathrm{L}$ ), 21, 63D, and 67D.

- Trichloroethylene was elevated in 4 wells: LFW61D, 65D, 67C, and 67D (maximum concentration at $17 \mu \mathrm{g} / \mathrm{L}$ ).

- Trichlorofluoromethane was elevated in 7 wells: LFW6R (maximum concentration of $34 \mu \mathrm{g} / \mathrm{L}), 10 \mathrm{~A}, 31,63 \mathrm{D}, 65 \mathrm{D}, 67 \mathrm{C}$, and 67D.

- Tritium was elevated in well LFW67C with maximum activity level of $25.4 \mathrm{pCi} / \mathrm{mL}$.

During third quarter 1998,30 of the 53 LFW wells contained elevated constituents.

- 1,1-Dichloroethane was elevated in 9 wells: LFW10A (maximum concentration at $200 \mu \mathrm{g} / \mathrm{L}), 21,58 \mathrm{D}, 60 \mathrm{C}, 61 \mathrm{D}, 62 \mathrm{D}, 65 \mathrm{D}, 67 \mathrm{C}$, and 67D.

- 1,4-Dichlorobenzene was elevated in well LFW58D, with a concentration of 170 $\mu \mathrm{g} / \mathrm{L}$.

- Aluminum (total recoverable) was elevated in 16 wells: LFW56D, 58D, 59D, 60D, $61 \mathrm{D}, 62 \mathrm{D}, 63 \mathrm{~B}, 63 \mathrm{C}, 64 \mathrm{C}$ (maximum concentration at $731 \mu \mathrm{g} / \mathrm{L}$ ), 65B, 67B, 67D, $69 \mathrm{C}, 69 \mathrm{D}, 71 \mathrm{~B}$, and $71 \mathrm{C}$.

- Benzene was elevated in 4 wells: LFW10A, 58D (maximum concentration at 24 $\mu \mathrm{g} / \mathrm{L}) 62 \mathrm{D}$ and $67 \mathrm{C}$.

- Chloroethene (vinyl chloride) was elevated in 12 wells: LFW6R, 8R, 10A, 21, 36R, 58D (maximum concentration at $40 \mu \mathrm{g} / \mathrm{L}$ ), 60C, 62D, 63D, 64D, 67C, and 69D.

- Dichlorodifluoromethane was elevated in 6 wells: LFW58D, 61D, 62D, 63D (maximum concentration at $20 \mu \mathrm{g} /$ ), $67 \mathrm{C}$, and $67 \mathrm{D}$.

- Dichloromethane (methylene chloride) was elevated at 3 wells: LFW10A (maximum concentration at $59.6 \mu \mathrm{g} / \mathrm{L}), 58 \mathrm{D}$, and $62 \mathrm{D}$.

- Iron (total recoverable) was elevated at 15 wells: LFW6R, 8R, 10A, 18, 21, 36R, $56 \mathrm{D}, 58 \mathrm{D}, 60 \mathrm{C}, 60 \mathrm{D}, 61 \mathrm{D}, 62 \mathrm{D}, 67 \mathrm{C}$ (maximum concentration at $65300 \mu \mathrm{g} /$ ), $68 \mathrm{D}$, and 69D.

- Trichloroethylene was elevated in 5 wells: LFW58D, (maximum concentration at 16 $\mu \mathrm{g} / \mathrm{L}), 61 \mathrm{D}, 62 \mathrm{D}, 67 \mathrm{C}$, and 67D. 
- Trichlorofluoromethane was elevated in 8 wells: LFW6R (maximum concentration at $37 \mu \mathrm{g} / \mathrm{L}), 31,58 \mathrm{D}, 62 \mathrm{D}, 63 \mathrm{D}, 65 \mathrm{D}, 67 \mathrm{C}$ and $67 \mathrm{C}$.

- Tritium was elevated in 3 wells: LFW 10A, 61D, and 67C (maximum activity level of $25.56 \mathrm{pCi} / \mathrm{mL}$ ).

During fourth quarter 1998, 15 of the 53 LFW wells contained elevated constituents.

- 1,1-dichloroethane was elevated in 8 wells: LFW 10A (maximum concentration of $151 \mu \mathrm{g} / \mathrm{L}), 21,58 \mathrm{D}, 61 \mathrm{D}, 62 \mathrm{D}, 65 \mathrm{D}, 67 \mathrm{C}$, and 67D.

- 1,1-Dichloroethylene was elevated at well LFW67D with a concentration of 1.69 $\mu \mathrm{g} / \mathrm{L}$.

- 1,4-Dichlorobenzene was elevated at well LFW58D, with a concentration of 173 $\mu \mathrm{g} / \mathrm{L}$.

- Aluminum (total recoverable) was elevated at 3 wells: LFW32C, 59D, and 62D (maximum concentration of $861 \mu \mathrm{g} / \mathrm{L}$ ).

- Arsenic was elevated at well LFW67C with a concentration of $68.8 \mu \mathrm{g} / \mathrm{L}$.

- Benzene was elevated in 4 wells: LFW10A, 58D (maximum concentration of 13.3 $\mu \mathrm{g} / \mathrm{L}), 62 \mathrm{~d}$, and $67 \mathrm{C}$.

- Chloroethene ( vinyl chloride) was elevated in 6 wells: LFW8R, 10A, 21, 58D, 67C (maximum concentration at $41.3 \mu \mathrm{g} / \mathrm{L}$ ), and 69D.

- Cis-1,2-Dichloroethylene was elevated in 2 wells: LFW62D, and 67C (maximum concentration of $201 \mu \mathrm{g} / \mathrm{L}$ ).

- Dichlorodifluoromethane was elevated in 5 wells: LFW58D (maximum concentrations of $27.7 \mu \mathrm{g} / \mathrm{L}), 61 \mathrm{D}, 63 \mathrm{D}, 67 \mathrm{C}$, and $67 \mathrm{D}$.

- Dichloromethane (methylene chloride) was elevated in well LFW62D with a concentration of $10.3 \mu \mathrm{g} / \mathrm{L}$.

- Iron (total recoverable) was elevated in 11 wells: LFW8R, 10A, 18, 21, 58D, 59D, 61D, 62D, 67C (maximum concentration of $60200 \mu \mathrm{g} / \mathrm{L}$ ), 67D, and 69D.

- Tetrachloroethylene was elevated in well LFW62D with a concentration of $7.05 \mu \mathrm{g} / \mathrm{L}$.

- Trichloroethylene was elevated in 6 wells: LFW58D, 61D, 62D (maximum concentration at $19.2 \mu \mathrm{g} / \mathrm{L}), 65 \mathrm{D}, 67 \mathrm{C}$, and 67D.

$39.9 \mu \mathrm{g} / \mathrm{L}), 58 \mathrm{D}, 61 \mathrm{D}, 62 \mathrm{D}, 63 \mathrm{D}, 65 \mathrm{D}$, and $67 \mathrm{D}$.

- Tritium was elevated in 2 wells: LFWIOA and 67C (maximum activity level of 34.53 $\mathrm{pCi} / \mathrm{mL})$.

Results for analytes that exceeded other SRS Flag 2 criteria (appendix B) during fourth quarter 1998 are summarized in Table 2 (Appendix D). 
WSRC-TR-99-00011

Unclassified

Table 3 (Appendix D) shows the results during each quarter of 1998 for all of the constituents. The analytical laboratories that conducted the analyses, the dilution factors used in the analyses, and the analyses that received modifiers (which help define the laboratory accuracy and precision) or that exceeded the EPA-approved holding times listed in Table 3 are for fourth quarter 1998 data only. Constituents results in Table 3 that appear to equal the final PDWS but are not marked in the $S T$ column (exceeded the final PDWS or screening level) are below the final PDWS in the database. Database results," the results that are compared to the final PDWS, contain more significant digits than the results given in this report. Apparent discrepancies are the result of the rounding of reported results.

In addition to the results tables, Appendix $\mathrm{D}$ provides definitions of the abbreviations and modifiers used in the results tables as well as descriptions of holding times, data rounding, and data qualification practices. Appendix E provides a general assessment of the quality and usability of EPD/EMS data.

Isoconcentration maps of chloroethene, trichloroethylene, and tritium for first and fourth quarters 1998 are presented in Figures 3 through 8 (Appendix C).

\section{Time-Trend Data}

Appendix $\mathrm{F}$ presents time series plots for benzene in wells LFW8R, 21, 22, 36, 48D, 58D, 59D, 61C, and 61D; 1,4-dichlorobenzene in wells LFW21,22,36,48D, 61C, and 61D; dichloromethane in wells LFW 8R, 21, 22, 36, 39, 48D, 58D, 59D, 61C, and 61D; ethylbenzene in wells LFW21, 22, 36, 39, 48D, 58D, 59D, 61C, and 61D; tetrachloroethylene in wells LFW8R, 21, 22, 36, 39, 48D, 58D, 59D, 61C, and 61D; trichloroethane in wells LFW8R, 21, 22, 39, 48D, 58D, 59D, 61C, and 61D; trichlorofluoromethane in wells LFW8R, 21, 22, 36, 39, 58D,59D, 61C, and 61D; and xylenes in wells LFW8R, 21, 22, 36, 39, 48D, 58D, 59D, 61C, and 61D in the Sanitary Landfill through fourth quarter 1997.

\section{Water Levels}

Hydrographs showing the water elevations through time for wells at the Sanitary Landfill are provided in Appendix G. The average water elevations in the Steed Pond Aquifer (Water Table) for each quarter of 1997 and 1998 are presented below.

Average Water Elevations at the Sanitary Landfill

\begin{tabular}{|c|c|c|}
\hline Quarter & $\begin{array}{c}1997 \\
\text { Average Water Elevation } \\
(\mathrm{ft} \mathrm{msl})\end{array}$ & $\begin{array}{c}1998 \\
\text { Average Water Elevation } \\
\text { (ft msl) }\end{array}$ \\
\hline First & 141.4 & 143.4 \\
\hline Second & 145.3 & 150.3 \\
\hline Third & 142.1 & 143.4 \\
\hline Fourth & 143.0 & 145.5 \\
\hline
\end{tabular}




\section{Groundwater Flow Directions and Rates}

Historically, the groundwater flow direction in the Steed Pond Aquifer (Water Table) hydrostratigraphic unit beneath the Sanitary Landfill has been to the southeast (Universal Transverse Mercator [UTM] coordinates) toward Upper Three Runs Creek. During the first and fourth quarters 1998, the flow direction was to the southeast (Figures 9 and 10, Appendix C). Figure 11 presents the regional correlation of hydrostratigraphic and lithostratigraphic nomenclature.

Horizontal flow rate calculations provide estimates of the transport rate of constituents originating from the Sanitary landfill. Flow rates are estimated using the following equation:

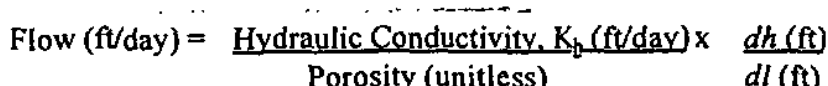

where the hydraulic conductivity $\left(K_{h}\right)$ constant is $10 \mathrm{ft} /$ day, the effective porosity value is 20 percent, the change in head is $d h$, and the horizontal distance along each flow direction arrow is $d l$.

Flow path length is calculated to the nearest $50 \mathrm{ft}$. Flow rate per day is calculated to two significant figures, then multiplied by 365 and rounded to two significant figures for the flow rate per year. Flow rate estimated vary depending upon the vertical gradient between wells, the size of the area under consideration, the number of data points, and the length and location of the flow path. Because these are inferred or estimated parameters, flow rate estimates should be considered accurate to an order of magnitude only.

The approximate groundwater flow rate in the Steed Pond Aquifer (Water Table) during first quarter 1998 was estimated as follows (Figure 9, Appendix C):

$$
\begin{aligned}
& \frac{10}{0.20} \times \frac{16}{1908}=0.42 \mathrm{ft} / \text { day } \\
& 0.42 \mathrm{ft} / \text { day } \times 365 \text { days } \approx 153 \mathrm{ft} / \text { year }
\end{aligned}
$$

The approximate groundwater flow rate in the Steed Pond Aquifer (Water table) during fourth quarter 1998 is estimated as follows (Figure 10, Appendix C):

$$
\begin{aligned}
& \frac{10}{0.20} \times \frac{12}{1337}=0.45 \mathrm{ft} / \text { day } \\
& 0.45 \mathrm{ft} / \text { day } \times 365 \text { days } \approx 164 \mathrm{ft} / \text { year }
\end{aligned}
$$

\section{Upgradient versus Downgradient Results}

Wells LFW 43B, 43C, and 43D, at the northern end of the Sanitary Landfill, are designated upgradient wells for the Steed Pond Aquifer (Water Table). All remaining Steed Pond Aquifer

(Wäter Table) wells monitor downgradient or sidegradient water quality. (Figures 2, 09, and 10, Appendix C). 1,2-Dichloroethane, and $\mathrm{pH}$ constituents exceeded their final PDWS during the first quarter period in LFW 43B well.

1,1-Dichloroethane, 1,1-Dichloroethylene, 1,2-Dichloroethane, 1,4-Dichlorobenzene, Aluminum (total recoverable), Arsenic (total recoverable), Benzene. Chloroethene (Vinyl chloride), Cis-1,2Dichloroethylene, Dichlorodifluoromethane, Dichloromethane (Methylene chloride), Iron (tota! recoverable), Mercury (total recoverable), $\mathrm{Ph}$, Sulfide, Tetrachloroethylene, Trichloroethylene, Trichlorofluoromethane, and Tritiumwere elevated in one or more of the downgradient or sidegradient wells during the year. 
recoverable), Mercury (total recoverable), $\mathrm{Ph}$, Sulfide, Tetrachloroethylene, Trichloroethylene, Trichlorofluoromethane, and Tritium were elevated in one or more of the downgradient or sidegradient wells during the year.

Wells LFW 32, 74C, 74D, 75C, and 75D are upgradient wells, which are monitored to meet the requirements of Subtitle D of the SCDHEC permit (nonhazardous solid waste) for the Interim Sanitary Landfill. These wells are monitored for the Solid Waste Program and are not part of the 48 well monitoring network. However, information provided by these wells is germane to the groundwater monitoring scheme for the Sanitary Landfill. None of the constituents exceeded their final PDWS in wells LFW32, 74C, 74D, 75C, and 75D. 
During first quarter 1998, 19 of the 53 LFW wells contained levels of 1,1-Dichloroethane, 1,2Dichloroethane, 1,4-Dichlorobenzene, Benzene, Chloroethene (Vinyl chloride), Dichlorodifluoromethane, Dichloromethane (Methylene chloride), Iron and (total recoverable), Mercury (total recoverable), Tetrachloroethylene, Trichloroethylene, Trichlorofluoromethane, and Tritium that exceeded the final PDWS in the groundwater beneath the Sanitary Landfill. Elevated leveis of 1,1-Dichloroethane "and Chloröethene (Vinyl chloride) occurred most frequently: 1,1Dichloroethane exceeded its PDWS in 13 wells, with the highest concentration in well LFW10A at 172 $\mathrm{ug} / \mathrm{L}$; chloroethene exceeded the final PDWS in 12 wells, with the maximum concentration detected in well LFW $10 \mathrm{~A}$ at $34.8 \mathrm{ug} / \mathrm{L}$. The remaining constituents were elevated in 7 or fewer wells during first quarter.

Elevated levels of 1,1-Dichloroethane, Benzene, Chloroethene (Vinyi chloride), Dichlorodifluoromethane, Dichloromethane (Methylene chloride), Trichloroethylene, Trichlorofluoromethane, and Tritium exceeded their final PDWS in one or more of 15 wells at the Sanitary Landfill during second quarter 1997. Chloroethene and trichlorofluoromethane exceeded its PDWS in 7 wells, with the highest concentration in well LFW6R at $34 \mathrm{ug} / \mathrm{L}$. The remaining constituents were elevated in 6 or fewer wells during second quarter.

During third quarter 1998, 30 of the 53 LFW wells contained levels of chloroethene, trichloroethylene, benzene, 1,4-dichlorobenzene, tetrachloroethylene, tritium, and mercury that exceeded the final PDWS in the groundwater beneath the Sanitary Landfill. Elevated levels of chloroethene and trichloroethylene occurred most frequently: chloroethene exceeded the final PDWS in 11 wells, with the maximum concentration detected in well LFW58D at $45.5 \mathrm{ug} / \mathrm{L}$; trichloroethylene exceeded its PDWS in 8 wells, with the highest concentration in well LFW59D at $26.4 \mathrm{ug} / \mathrm{L}$. The remaining constituents were elevated in 3 or fewer wells during third quarter.

Elevated levels of Iron (total recoverable), 1,1-Dichloroethane, Trichlorofluoromethane, Chloroethene (Vinyl chloride), Dichlorodifluoromethane, Trichloroethylene, Benzene, Aluminum (total recoverable), Cis-1,2-Dichloroethylene, Tritium, 1,4-Dichlorobenzene, Arsenic (total recoverable), Dichloromethane (Methylene chloride), and Tetrachloroethylene exceeded the final PDWS in 9 wells, with the highest concentration in well LFW10A at $151 \mathrm{ug} / \mathrm{L}$. The remaining constituents were elevated in 6 or fewer wells during fourth quarter.

The analytical results for 1998 are similar to the results for 1997 . One of the constituents ( $\mathrm{pH}$ for well LFW43B)exceeded their final PDWS in the upgradient wells LFW43B, 43C, and 43D. And none of the constituents exceeded their_final PDWS in the Interim Sanitary Landfill wells LFW32, 74C, 74D, $75 \mathrm{C}$, and $75 \mathrm{D}$.

The groundwater flow direction in the Steed Pond Aquifer (Water Table) beneath the Sanitary Landfill is to the southeast (UTM coordinates) toward Upper Three Runs Creek. The flow rate in this aquifer during first quarter 1998 was estimated to be approximately $124 \mathrm{ft} /$ year and during fourth quarter 1998 , it was estimated at approximately $200 \mathrm{ft} / \mathrm{year}$. Flow directions during 1998 were very similar to 1997 findings. However, the rate of flow increased approximately $44 \%$ during the fourth quarter of 1998 over 1997. 
WSRC-TR-99-00011

Unclassined

\section{References Cited}

Heffiner, J.D., and Exploration Resources, Inc., 1991. Technical Summary of Groundwater Quality Protection Program at the Savannah River Site (1952-1986), Volume I - Site Geohydrology and Waste Sites, DPSP-88-1002. Westinghouse Savannah River Company, Savannah River Site, Aiken, SC.

SCDHEC (South Carolina Department of Health and Environmental Control), 1991. Sanitary Landfill Domestic Waste Permit 087A, April 1991. Columbia, SC.

WSRC (Westinghouse Savannah River Company), 1990. Sanitary Landfill Groundwater Quality Assessment Plan, WSRC-TR-90-167. Savannah River Site, Aiken, SC. 


\section{Errata}

Result values for earlier quarters presented in this report may differ from the values for those same quarters presented in earlier reports because some reanalysis may have been performed by the laboratories after the reports were printed.

Fourth Quarter 1998:

- No errata have been reported. 
WSRC-TR-99-00011

Unclassified

\section{Appendix A}

Final Primary Drinking Water Standards 
WSRC-TR-99-00011

Unclassified

THIS PAGE LEFT BLANK INTENTIONALLY. 
WSRC-TR-99-00011

Unclassified

Final Primary Drinking Water Standards

\begin{tabular}{|c|c|c|c|c|}
\hline Analyte & Unit & Level & $\underline{\text { Status }}$ & Source \\
\hline Alachlor & $\mu \mathrm{g} / \mathrm{L}$ & 2 & Final & EPA, 1993 \\
\hline Aldicarb a & $\mu g / L$ & 3 & Final & EPA, 1993 \\
\hline Aldicarb sulfone & $\mu g / L$ & 2 & Final & EPA, 1993 \\
\hline Aldicarb sulfoxide " & $\mu g / L$ & 4 & Final & EPA, 1993 \\
\hline Antimony & $\mu \mathrm{g} / \mathrm{L}$ & 6 & Final & EPA, 1993 \\
\hline Arsenic & $\mu \mathrm{g} / \mathrm{L}$ & 50 & Final & EPA, 1993 \\
\hline Asbestos & Fibers/L & $7,000,000$ & Final & EPA, 1993 \\
\hline Atrazine & $\mu \mathrm{g} / \mathrm{L}$ & 3 & Final & EPA, 1993 \\
\hline$\cdot \quad \cdot \quad \ldots \ldots \cdot-\cdots$ & $\mu g / L$ & 2,000 & Final & EPA, 1993 \\
\hline Benzene & $\mu \mathrm{g} / \mathrm{L}$ & 5 & Final & EPA, 1993 \\
\hline Benzo $[a]$ pyrene & $\mu \mathrm{g} / \mathrm{L}$ & 0.2 & Final & EPA, 1993 \\
\hline Beryllium & $\mu \mathrm{g} / \mathrm{L}$ & 4 & Final & EPA, 1993 \\
\hline Bis(2-ethylhexyl) phthalate & $\mu \mathrm{g} / \mathrm{L}$ & 6 & Final & EPA, 1993 \\
\hline Bromodichloromethane & $\mu \mathrm{g} / \mathrm{L}$ & 100 & Final & EPA, 1993 \\
\hline Bromoform & $\mu \mathrm{g} / \mathrm{L}$ & 100 & Final & EPA, 1993 \\
\hline 2-sec-Butyl-4,6-dinitrophenol & $\mu \mathrm{g} / \mathrm{L}$ & 7 & Final & EPA, 1993 \\
\hline Cadmium & $\mu g / L$ & 5 & Final & EPA, 1993 \\
\hline Carbofuran & $\mu \mathrm{g} / \mathrm{L}$ & 40 & Final & EPA, 1993 \\
\hline Carbon tetrachloride & $\mu g / L$ & 5 & Final & EPA, 1993 \\
\hline Chlordane & $\mu \mathrm{g} / \mathrm{L}$ & 2 & Final & EPA, 1993 \\
\hline Chlorobenzene & $\mu \mathrm{g} / \mathrm{L}$ & 100 & Final & EPA, 1993 \\
\hline Chloroethene (Vinyl chloride) & $\mu \mathrm{g} / \mathrm{L}$ & 2 & Final & EPA, 1993 \\
\hline Chloroform & $\mu g / L$ & 100 & Final & EPA, 1993 \\
\hline Chromium & $\mu g / L$ & 100 & Final & EPA, 1993 \\
\hline Copper & $\mu \mathrm{g} / \mathrm{L}$ & 1,300 & Final & EPA, 1993 \\
\hline Cyanide & $\mu \mathrm{g} / \mathrm{L}$ & 200 & Final & EPA, 1993 \\
\hline Dalapon * & $\mu \mathrm{g} / \mathrm{L}$ & 200 & Final & EPA, 1993 \\
\hline Dibromochloromethane & $\mu \mathrm{g} / \mathrm{L}$ & 100 & Final & EPA, 1993 \\
\hline 1,2-Dibromo-3-chloropropane & $\mu \mathrm{g} / \mathrm{L}$ & 0.2 & Final & EPA, 1993 \\
\hline 1,2-Dibromoethane & $\mu \mathrm{g} / \mathrm{L}$ & 0.05 & Final & EPA, 1993 \\
\hline 1,2-Dichlorobenzene & $\mu \mathrm{g} / \mathrm{L}$ & 600 & Final & EPA, 1993 \\
\hline 1,4-Dichlorobenzene & $\mu \mathrm{g} / \mathrm{L}$ & 75 & Final & EPA, 1993 \\
\hline 1,2-Dichloroethane & $\mu g / L$ & 5 & Final & EPA, 1993 \\
\hline 1,1-Dichloroethylene & $\mu \mathrm{g} / \mathrm{L}$ & 7 & Final & EPA, 1993 \\
\hline 1,2-Dichloroethylene & $\mu \mathrm{g} / \mathrm{L}$ & 50 & Final & EPA, 1993 \\
\hline cis-1,2-Dichioroethylene & $\mu \mathrm{g} / \mathrm{L}$ & 70 & Final & EPA, 1993 \\
\hline trans-1,2-Dichloroethylene & $\mu \mathrm{g} / \mathrm{L}$ & 100 & Final & EPA, 1993 \\
\hline Dichloromethane (Methylene chloride) & $\mu g / L$ & 5 & Final & EPA, 1993 \\
\hline 2,4-Dichlorophenoxyacetic acid & $\mu \mathrm{g} / \mathrm{L}$ & 70 & Final & EPA, 1993 \\
\hline 1,2-Dichloropropane & $\mu \mathrm{g} / \mathrm{L}$ & 5 & Final & EPA, 1993 \\
\hline Di(2-ethylhexyl) adipate " & $\mu \mathrm{g} / \mathrm{L}$ & 400 & Final & EPA, 1993 \\
\hline Diquat dibromide " & $\mu \mathrm{g} / \mathrm{L}$ & 20 & Final & EPA, 1993 \\
\hline Endothall : & $\mu \mathrm{g} / \mathrm{L}$ & 100 & Final & EPA, 1993 \\
\hline Endrin & $\mu g / L$ & 2 & Final & EPA, 1993 \\
\hline Ethylbenzene & $\mu g / L$ & 700 & Final & EPA, 1993 \\
\hline Fluoride & $\mu \mathrm{g} / \mathrm{L}$ & 4,000 & Final & EPA, 1993 \\
\hline Glyphosate " & $\mu \mathrm{g} / \mathrm{L}$ & 700 & Final & EPA, 1993 \\
\hline Gross alpha ${ }^{b}$ & $\mathrm{pCi} / \mathrm{L}$ & $1.5 \mathrm{E}+01$ & Final & EPA, 1993 \\
\hline Heptachlor & $\mu \mathrm{g} / \mathrm{L}$ & 0.4 & Final & EPA, 1993 \\
\hline Heptachlor epoxide & $\mu \mathrm{g} / \mathrm{L}$ & 0.2 & Final & EPA, 1993 \\
\hline
\end{tabular}




\begin{tabular}{|c|c|c|c|c|}
\hline Analyte $^{-} \quad-\quad \cdots \cdots$ & Unit & Levèl & Status - & Source \\
\hline Hexachlorobenzene & $\mu \mathrm{g} / \mathrm{L}$ & 1 & Final & EPA, 1993 \\
\hline Hexachlorocyclopentadiene & $\mu \mathrm{g} / \mathrm{L}$ & 50 & Final & EPA, 1993 \\
\hline Lead & $\mu g / L$ & 50 & Final & SCDHEC, 198 \\
\hline Lindane & $\mu g / L$ & 0.2 & Final & EPA, 1993 \\
\hline Mercury & $\mu \mathrm{g} / \mathrm{L}$ & 2 & Final & EPA, 1993 \\
\hline Methoxychlor & $\mu \mathrm{g} / \mathrm{L}$ & 40 & Final & EPA, 1993 \\
\hline Nickel & $\mu \mathrm{g} / \mathrm{L}$ & 100 & Final & EPA, 1993 \\
\hline Nitrate as nitrogen & $\mu \mathrm{g} / \mathrm{L}$ & 10,000 & Final & EPA, 1993 \\
\hline Nitrate-nitrite as nitrogen & $\mu \mathrm{g} / \mathrm{L}$ & 10,000 & Final & EPA, 1993 \\
\hline Nitrite as nitrogen & $\mu \mathrm{g} / \mathrm{L}$ & 1,000 & Final & EPA, 1993 \\
\hline Nonvolatile beta & $\mathrm{pCi} / \mathrm{L}$ & $5 \mathrm{E}+01$ & Interim Final & EPA, 1977 \\
\hline Oxamyl & $\mu \mathrm{g} / \mathrm{L}$ & 200 & Final & EPA, 1993 \\
\hline PCB 1016 & $\mu g / L$ & 0.5 & Final & EPA, 1993 \\
\hline PCB 1221 & $\mu \mathrm{g} / \mathrm{L}$ & 0.5 & Final & EPA, 1993 \\
\hline PCB 1232 & $\mathrm{Hg} / \mathrm{L}$ & 0.5 & Final & EPA, 1993 \\
\hline PCB 1242 & $\mu \mathrm{g} / \mathrm{L}$ & 0.5 & Final & EPA, 1993 \\
\hline PCB 1248 & $\mu \mathrm{g} / \mathrm{L}$ & 0.5 & Final & EPA, 1993 \\
\hline PCB 1254 & $\mu \mathrm{g} / \mathrm{L}$ & 0.5 & Final & EPA, 1993 \\
\hline РCB 1260 & $\mu \mathrm{g} / \mathrm{L}$ & 0.5 & Final & EPA, 1993 \\
\hline PCB 1262 & $\mu \mathrm{g} / \mathrm{L}$ & 0.5 & Final & EPA, 1993 \\
\hline Pentachlorophenol & $\mu \mathrm{g} / \mathrm{L}$ & 1 & Final & EPA, 1993 \\
\hline Picloram ${ }^{2}$ & $\mu \mathrm{g} / \mathrm{L}$ & 500 & Final & EPA, 1993 \\
\hline Selenium & $\mu \mathrm{g} / \mathrm{L}$ & 50 & Final & EPA, 1993 \\
\hline Simazine & $\mu \mathrm{g} / \mathrm{L}$ & 4 & Final & EPA, 1993 \\
\hline Strontium $-89 / 90^{c}$ & $\mathrm{pCi} / \mathrm{L}$ & $8 \mathrm{E}+00$ & Final & EPA, 1993 \\
\hline Strontium-90 & $\mathrm{pCi} / \mathrm{L}$ & $8 \mathrm{E}+00$ & Final & EPA, 1993 \\
\hline Styrene & $\mu \mathrm{g} / \mathrm{L}$ & 100 & Final & EPA, 1993 \\
\hline $2,3,7,8-\mathrm{TCDD}$ & $\mu \mathrm{g} / \mathrm{L}$ & 0.00003 & Final & EPA, 1993 \\
\hline Tetrachloroethylene & $\mu \mathrm{g} / \mathrm{L}$ & 5 & Final & EPA, 1993 \\
\hline Thallium & $\mu g / L$ & 2 & Final & EPA, 1993 \\
\hline Toluene & $\mu \mathrm{g} / \mathrm{L}$ & 1,000 & Final & EPA, 1993 \\
\hline Toxaphene & $\mu \mathrm{g} / \mathrm{L}$ & 3 & Final & EPA, 1993 \\
\hline 2,4,5-TP (Silvex) & $\mu g / L$ & 50 & Final & EPA, 1993 \\
\hline 1,2,4-Trichlorobenzene & $\mu \mathrm{g} / \mathrm{L}$ & 70 & Final & EPA, 1993 \\
\hline 1,1,1-Trichloroethane & $\mu \mathrm{g} / \mathrm{L}$ & 200 & Final & EPA, 1993 \\
\hline 1,1,2-Trichloroethane & $\mu \mathrm{g} / \mathrm{L}$ & 5 & Final & EPA, 1993 \\
\hline Trichloroethylene & $\mu \mathrm{g} / \mathrm{L}$ & 5 & Final & EPA, 1993 \\
\hline Tritium & $\mathrm{pCi} / \mathrm{mL}$ & $2 \mathrm{E}+01$ & Final & EPA, 1993 \\
\hline Xylenes & $\mu \mathrm{g} / \mathrm{L}$ & 10,000 & Final & EPA, 1993 \\
\hline
\end{tabular}

Note: Final PDWS were assigned to alachlor, aldicarb, aldicarb sulfone, aldicarb sulfoxide, atrazine, carbofuran, dalapon, di(2-ethylhexyl) adipate, diquat dibromide, endothall, glyphosate, oxamyl, picloram, and simazine in the SRS Groundwater Monitoring Program for the first time beginning first quarter 1994.

- At present, EMS does not perform this analysis because the constituent is not in the current contract.

- The standard given is for gross aipha including radium-226 but excluding radon and uranium.

c For double radionuclide analyses where each separate radionuclide has its own standard, the more stringent standard is used. 
WSRC-TR-99-0001 1

Unclassified

\section{References}

EPA (U.S. Environmental Protection Agency), 1977. National Interim Primary Drinking Water Regulations, EPA-570/9-76-003. Washington, DC

EPA (U.S. Environmental Protection Agency), 1993. National Primary Drinking Water Regulations, Code of Federal Regulations, Section 40, Part 141, pp. 592-732. Washington, DC.

SCDHEC (South Carolina Department of Health and Environmental Control), 1981. State Primary Drinking Water Regulations, R.61-58.5. Columbia, SC. 
THIS PAGE LEFT BLANK INTENTIONALLY. 
WSRC-TR-99-00011

Unclassified

\section{Appendix B}

\section{Flagging Criteria}




\section{Flagging Criteria}

The Savannah River Site Environmental Protection Department/Environmental Monitoring Section (EPD/EMS) flagging criteria are as follows:

- Flag 2 criteria for constituents equal the Safe Drinking Water Act (SDWA) final Primary Drinking Water Standards (PDWS), the SDWA proposed PDWS, or the SDWA Secondary Drinking Water Standards (SDWS). If a constituent does not have a drinking water standard, the Flag 2 criterion equals 10 times the method detection limit (MDL) calculated as the 90th percentile detection limit obtained recently by one of the primary analytical laboratories.

- Flag 1. criteria for constituents_equal one-half of the final PDWS, one-half the proposed PDWS, or one-half the SDWS. If a constituent does not have a drinking water standard, the Flag 1 criterion equals 5 times the MDL calculated as the 90th percentile detection limit obtained recently by one of the primary analytical laboratories.

- Flag 0 criteria are assigned to constituent levels below Flag 1 criteria, constituent levels below the sample detection limits, or constituents having no flagging criteria.

The following parameters are exceptions to the flagging rules:

- EPD/EMS sets flagging criteria for specific conductance and $\mathrm{pH}$. No flags are set for alkalinity, calcium, carbonate, magnesium, potassium, silica, sodium, total dissolved solids, total phosphates (as P), and total phosphorus. Analyses for these parameters are conducted as part of the biennial comprehensive analyses or by special request.

- Aesthetic parameters such as color, corrosivity, Eh, odor, surfactants, and turbidity are not assigned flagging criteria but are analyzed by special request.

- Common laboratory contaminants and cieaners such as dichloromethane (methylene chloride), ketones, phthalates, and toluene are not assigned flagging criteria unless they have primary drinking water standards. These constituents are analyzed by special request.

Anglyte
Acenaphthene
Acenaphthylene
Acetone
Acetonitrile (Methyl cyanide)
Acetophenone
2-Acetylaminofluorene
Acrolein
Acrylonitrile
Actinium-228
Alachlor
Aldicarb
Aldicarb sulfone
Aldicarb sulfoxide
Aldrin
Alkainity (as CaCO,
Allyl chloride
Aluminum
Aluminum, dissolved
Aluminum, total recoverable

\begin{tabular}{lll} 
Unit & Flag 1 & Flag 2 \\
\cline { 3 - 3 }$\mu \mathrm{g} / \mathrm{L}$ & 50 & 100 \\
$\mu \mathrm{g} / \mathrm{L}$ & 50 & 100 \\
$\mu \mathrm{g} / \mathrm{L}$ & 500 & 1,000 \\
$\mu \mathrm{g} / \mathrm{L}$ & 500 & 1,000 \\
$\mu \mathrm{g} / \mathrm{L}$ & 50 & 100 \\
$\mu \mathrm{g} / \mathrm{L}$ & 50 & 100 \\
$\mu \mathrm{g} / \mathrm{L}$ & 100 & 200 \\
$\mu \mathrm{g} / \mathrm{L}$ & 100 & 200 \\
$\mathrm{pCi} / \mathrm{L}$ & $1.64 \mathrm{E}+03$ & $3.27 \mathrm{E}+03$ \\
$\mu \mathrm{g} / \mathrm{L}$ & 1 & 2 \\
$\mu \mathrm{g} / \mathrm{L}$ & 1.5 & 3 \\
$\mu \mathrm{g} / \mathrm{L}$ & 1 & 2 \\
$\mu \mathrm{g} / \mathrm{L}$ & 2 & 4 \\
$\mu \mathrm{g} / \mathrm{L}$ & 0.25 & 0.5 \\
& No flag & No flag \\
$\mu \mathrm{g} / \mathrm{L}$ & 250 & 500 \\
$\mu \mathrm{g} / \mathrm{L}$ & 25 & 50 \\
$\mu \mathrm{g} / \mathrm{L}$ & 25 & 50 \\
$\mu \mathrm{g} / \mathrm{L}$ & 25 & 50
\end{tabular}

\author{
Source * \\ EPA Method 8270 \\ EPA Method 8270 \\ EPA Method 8240 \\ EPA Method 8240 \\ EPA Method 8270 \\ EPA Method 8270 \\ EPA Method 8240 \\ EPA Method 8240 \\ Proposed PDWS (EPA, 1991) \\ Final PDWS (EPA, 1993a) \\ Final PDWS (EPA, 1993a) \\ Final PDWS (EPA, 1993a) \\ Final PDWS (EPA, 1993a) \\ EPA Method 8080 \\ Set by EPD/EMS \\ EPA Method 8240 \\ SDWS (EPA, 1993b) \\ SDWS (EPA, 1993b) \\ SDWS (EPA, 1993b)
}


WSRC-TR-99-00011

Unclassified

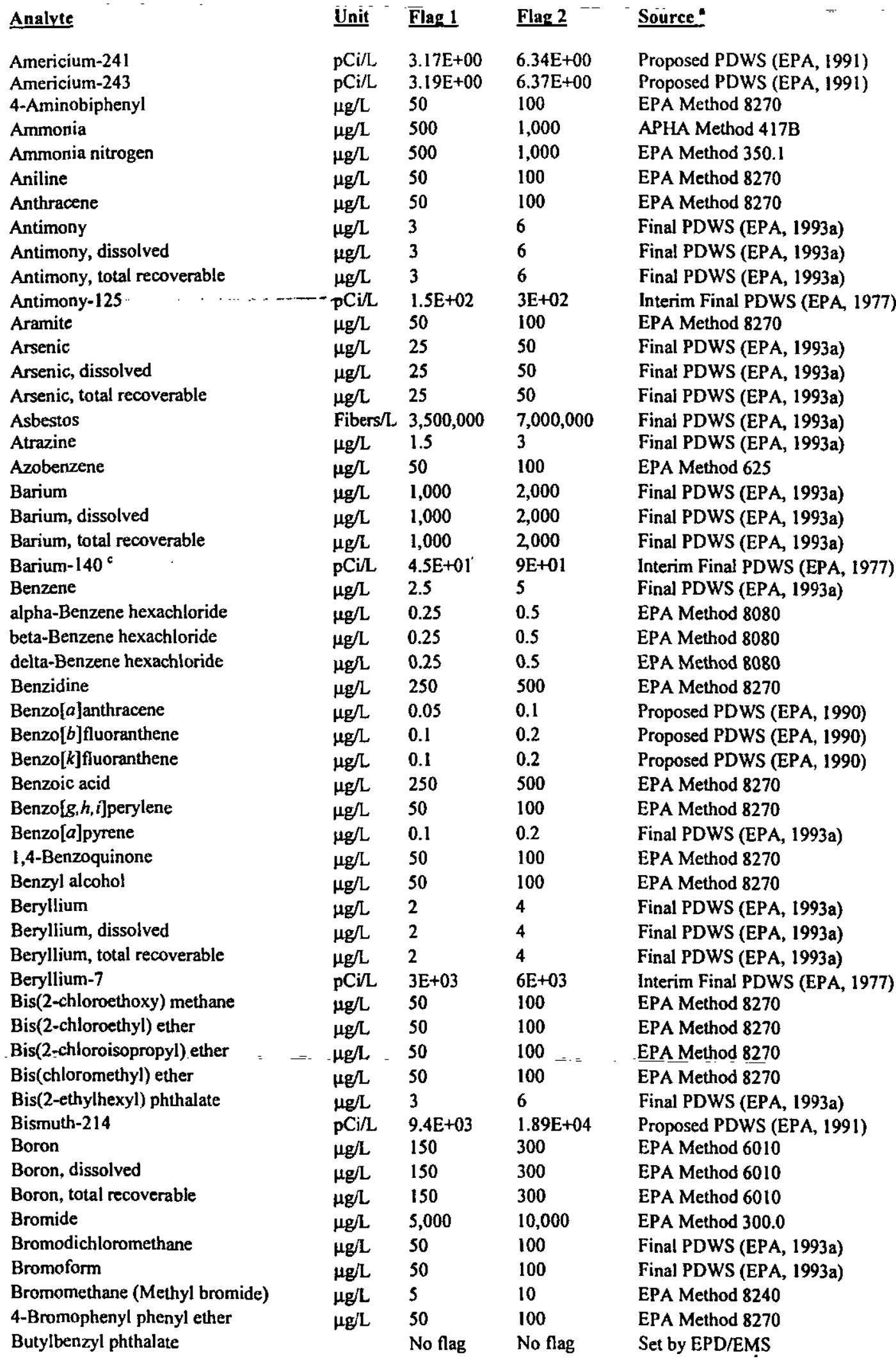


WSRC-TR-99-00011

Unclassified

\begin{tabular}{|c|c|c|c|c|}
\hline 2-sec-Butyl-4,6-dinitrophenol & $\mu g / L$ & 3.5 & 7 & Final PDWS (EPA, 1993a) \\
\hline Cadmium & $\mu \mathrm{g} / \mathrm{L}$ & 2.5 & 5 & Final PDWS (EPA, 1993a) \\
\hline & Unit & Flag 1 & Flag 2 & Source" \\
\hline Analyte & & & & \\
\hline Cadmium, dissolved & $\mu \mathrm{g} / \mathrm{L}$ & 2.5 & 5 & Final PDWS (EPA, 1993a) \\
\hline Cadmium, total recoverable & $\mu \mathrm{g} / \mathrm{L}$ & 2.5 & 5 & Final PDWS (EPA, 1993a) \\
\hline Calcium & & No flag & No flag & Set by EPD/EMS \\
\hline Calcium, dissolved & & No flag & No flag & Set by EPD/EMS \\
\hline Caicium, total recoverable & & No flag & No flag & Set by EPD/EMS \\
\hline Carbofuran & $\mu g / L$ & 20 & 40 & Final PDWS (EPA, 1993a) \\
\hline Carbon-14 & $\mathrm{pCi} / \mathrm{L}$ & $1 \mathrm{E}+03$ & $2 \mathrm{E}+03$ & Interim Final PDWS (EPA, 1977) \\
\hline Carbonate & & No flag & No flag & Set by EPD/EMS \\
\hline Carbon disulfide & $\mu \mathrm{g} / \mathrm{L}$ & 5 & 10 & EPA Method 8240 \\
\hline Carbon tetrachloride & $\mu \mathrm{g} / \mathrm{L}$ & 2.5 & 5 & Final PDWS (EPA, 1993a) \\
\hline Cerium-141 ${ }^{c}$ & $\mathrm{pCi} / \mathrm{L}$ & $1.5 \mathrm{E}+02$ & $3 E+02$ & Interim Final PDWS (EPA, 1977) \\
\hline Cerium-144 & $\mathrm{pCiL}$ & $1.31 \mathrm{E}+02$ & $2.61 \mathrm{E}+02$ & Proposed PDWS (EPA, 1991) \\
\hline Cesium-134 & $\mathrm{pCi} / \mathrm{L}$ & $4.07 \mathrm{E}+01$ & $8.13 \mathrm{E}+01$ & Proposed PDWS (EPA, 1991) \\
\hline Cesium-137 & $\mathrm{pCi} / \mathrm{L}$ & $1 E+02$ & $2 \mathrm{E}+02$ & Interim Final PDWS (EPA, 1977) \\
\hline Chlordane & $\mu \mathrm{g} / \mathrm{L}$ & 1 & 2 & Final PDWS (EPA, 1993a) \\
\hline Chloride & $\mu g / L$ & 125,000 & 250,000 & SDWS (EPA, 1993b) \\
\hline 4-Chloroaniline & $\mu \mathrm{g} / \mathrm{L}$ & 50 & 100 & EPA Method 8270 \\
\hline Chlorobenzene & $\mu \mathrm{g} / \mathrm{L}$ & 50 & 100 & Final PDWS (EPA, 1993a) \\
\hline Chlorobenzilate & $\mu g / L$ & 50 & 100 & EPA Method 8270 \\
\hline 4-Chloro-m-cresol & $\mu g / L$ & 50 & 100 & EPA Method 8270 \\
\hline Chloroethane & $\mu \mathrm{g} / \mathrm{L}$ & 5 & 10 & EPA Method 8240 \\
\hline Chloroethene (Vinyl chloride) & $\mu g / L$ & 1 & 2 & Final PDWS (EPA, 1993a) \\
\hline Chloroethyl vinyl ether & $\mu \mathrm{g} / \mathrm{L}$ & 5 & 10 & EPA Method 8240 \\
\hline 2-Chloroethyl vinyl ether & $\mu \mathrm{g} / \mathrm{L}$ & 5 & 10 & EPA Method 8240 \\
\hline Chloroform & $\mu \mathrm{g} / \mathrm{L}$ & 50 & 100 & Final PDWS (EPA, 1993a) \\
\hline Chloromethane (Methyl chloride) & $\mu \mathrm{g} / \mathrm{L}$ & 5 & 10 & EPA Method 8240 \\
\hline 2-Chloronaphthalene & $\mu g / L$ & 50 & 100 & EPA Method 8240 \\
\hline 2-Chlorophenol & $\mu g / L$ & 50 & 100 & EPA Method 8270 \\
\hline 4-Chlorophenyl phenyl ether & $\mu \mathrm{g} / \mathrm{L}$ & 50 & 100 & EPA Method 8270 \\
\hline Chloroprene & $\mu g / L$ & 1,000 & 2,000 & EPA Method 8240 \\
\hline Chromium & $\mu \mathrm{g} / \mathrm{L}$ & 50 & 100 & Final PDWS (EPA, 1993a) \\
\hline Chromium, dissolved & $\mu \mathrm{g} / \mathrm{L}$ & 50 & 100 & Final PDWS (EPA, 1993a) \\
\hline Chromium, total recoverable & $\mu \mathrm{g} / \mathrm{L}$ & 50 & 100 & Final PDWS (EPA, 1993a) \\
\hline Chromium-51 ${ }^{c}$ & $\mathrm{pCi} / \mathrm{L}$ & $3 \mathrm{E}+03$ & $6 \mathrm{E}+03$ & Interim Final PDWS (EPA, 1977) \\
\hline Chrysene & $\mu \mathrm{g} / \mathrm{L}$ & 0.1 & 0.2 & Proposed PDWS (EPA, 1990) \\
\hline Cobalt & $\mu \mathrm{g} / \mathrm{L}$ & 20 & 40 & EPA Method 6010 \\
\hline Cobalt, dissolved & $\mu \mathrm{g} / \mathrm{L}$ & 20 & 40 & EPA Method 6010 \\
\hline Cobalt, total recoverable & $\mu \mathrm{g} / \mathrm{L}$ & 20 & 40 & EPA Method 6010 \\
\hline Cobalt-57 & $\mathrm{pCi} / \mathrm{L}$ & $5 E+02$ & $1 E+03$ & Interim Final PDWS (EPA, 1977) \\
\hline Cobalt- $58^{d}$ & $\mathrm{pCi} / \mathrm{L}$ & $4.5 \mathrm{E}+03$ & $9 \mathrm{E}+03$ & Interim Final PDWS (EPA, 1977) \\
\hline Cobalt-60 & $\mathrm{pCi} / \mathrm{L}$ & $5 \mathrm{E}+01$ & $1 E+02$ & Interim Final PDWS (EPA, 1977) \\
\hline Color & & No flag & No flag & Set by EPD/EMS \\
\hline Copper & $\mu g / L$ & 500 & 1,000 & Final PDWS (SCDHEC, 1981) \\
\hline Copper, dissolved & $\mu g / L$ & 500 & 1,000 & Final PDWS (SCDHEC, 1981) \\
\hline Copper, total recoverable & $\mu g / L$ & 500 & 1,000 & Final PDWS (SCDHEC, 1981) \\
\hline Corrosivity & & No flag & No flag & Set by EPD/EMS \\
\hline m-Cresol (3-Methylphenol) & $\mu \mathrm{g} / \mathrm{L}$ & & 100 & EPA Method 8270 \\
\hline o-Cresol (2-Methylphenol) & $\mu \mathrm{g} / \mathrm{L}$ & 50 & 100 & EPA Method 8270 \\
\hline p-Cresol (4-Methylphenol) & $\mu \mathrm{g} / \mathrm{L}$ & 50 & 100 & EPA Method 8270 \\
\hline Curium-242 & $\mathrm{pCi} / \mathrm{L}$ & $6.65 \mathrm{E}+01$ & $1.33 \mathrm{E}+02$ & Proposed PDWS (EPA, 1991) \\
\hline Curium-243 & $\mathrm{pCi} / \mathrm{L}$ & $4.15 E+00$ & $8.3 E+00$ & Proposed PDWS (EPA, 1991) \\
\hline Curium-243/244 ${ }^{\circ}$ & $\mathrm{pCi} / \mathrm{L}$ & $4.15 \mathrm{E}+00$ & $8.3 \mathrm{E}+00$ & Proposed PDWS (EPA, 1991) \\
\hline
\end{tabular}


WSRC-TR-99-00011

Unclassified

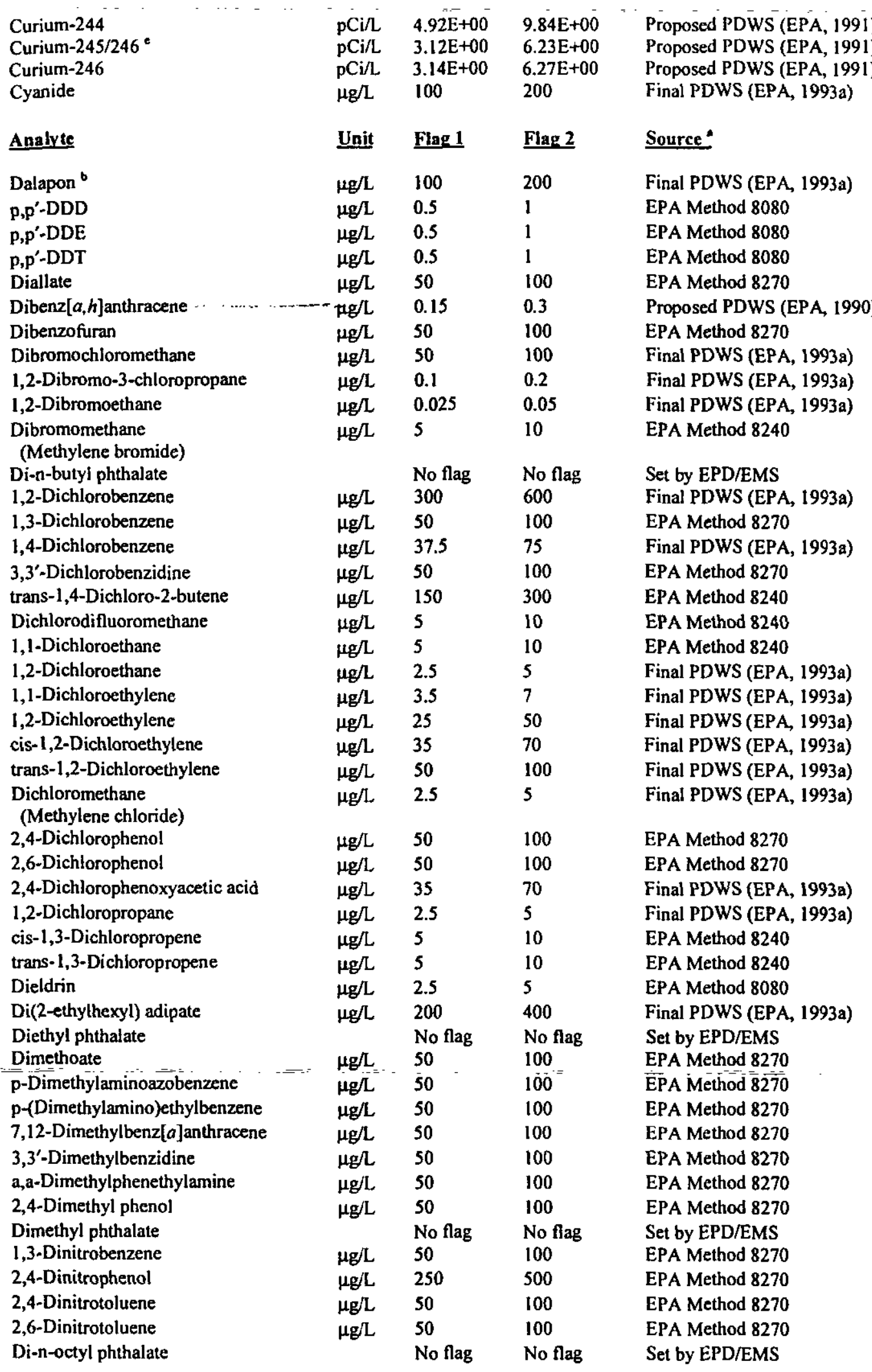


1,4-Dioxane

Diphenylamine

1,2-Diphenylhydrazine

Diquat dibromide ${ }^{b}$

Dissolved organic carbon

Disulfoton

Eh

\section{Analyte}

Endosulfan I

Endosulfan II

Endosulfan sulfate

Endothall $^{\mathrm{b}}$

Endrin

Endrin aldehyde

Endrin ketone

Ethylbenzene

Ethyl methacrylate

Ethyl methanesulfonate

Europium-152

Europium-154

Europium-155

Famphur

Fluoranthene

Fluorene

Fluoride

Glyphosate b

Gross alpha

Heptachlor

Heptachlor epoxide

Heptachlorodibenzo-p-dioxin

isomers

1,2,3,4,6,7,8-HPCDD

Heptachlorodibenzo-p-furan

isomers

$1,2,3,4,6,7,8$-HPCDF

Hexachlorobenzene

Hexachlorobutadiene

Hexachlorocyclopentadiene

Hexachlorodibenzo-p-dioxin

isomers

1,2,3,4,7,8-HXCDD

Hexachlorodibenzo-p-furan

isomers

$1,2,3,4,7,8-\mathrm{HXCDF}$

Hexachloroethane

Hexachlorophene

Hexachloropropene

2-Hexanone

Indeno $[1,2,3-c, d]$ pyrene

Iodine

lodine-129

Iodine-131 ${ }^{\mathrm{c}}$

Iodomethane (Methyl iodide)

Iron
WSRC-TR-99-00011

Unclassified

$\begin{array}{lll}\mu \mathrm{g} / \mathrm{L} & 50 & 100 \\ \mu \mathrm{g} / \mathrm{L} & 50 & 100 \\ \mu \mathrm{g} / \mathrm{L} & 50 & 100 \\ \mu \mathrm{g} / \mathrm{L} & 10 & 20 \\ \mu \mathrm{g} / \mathrm{L} & 5,000 & 10,000 \\ \mu \mathrm{g} / \mathrm{L} & 50 & 100 \\ & \text { No flag } & \text { No flag }\end{array}$

EPA Method 8270

EPA Method 8270

EPA Method 8270

Final PDWS (EPA, 1993a)

EPA Method 9060

EPA Method 8270

Set by EPD/EMS

\section{Unit Flag 1 $\underline{\text { Flag } 2}$ Source:}

$\mu \mathrm{g} / \mathrm{L} \quad 0.5 \quad 1$

$\mu \mathrm{g} / \mathrm{L} \quad 0.5 \quad 1$

$\mu \mathrm{g} / \mathrm{L} \quad 0.5$

$\mu \mathrm{g} / \mathrm{L} \quad 50 \quad 100$

$\mu \mathrm{g} / \mathrm{L} \quad 1 \quad 2$

$\mu \mathrm{g} / \mathrm{L} \quad 0.5 \quad 1$

$\mu \mathrm{g} / \mathrm{L} \quad 350 \quad \begin{array}{ll}\text { No flag } & \text { No f }\end{array}$

$\mu \mathrm{g} / \mathrm{L} \quad 50 \quad 100$

$\mu \mathrm{g} / \mathrm{L} \quad 50 \quad 100$

$\mathrm{pCi} / \mathrm{L} \quad 3 \mathrm{E}+01 \quad 6 \mathrm{E}+01$

$\mathrm{pCi} / \mathrm{L} \quad \mathrm{IE}+02 \quad 2 \mathrm{E}+02$

$\mathrm{pCi} / \mathrm{L} \quad 3 \mathrm{E}+02 \quad 6 \mathrm{E}+02$

$\mu \mathrm{g} / \mathrm{L} \quad 50 \quad 100$

$\mu \mathrm{g} / \mathrm{L} \quad 50 \quad 100$

$\mu \mathrm{g} / \mathrm{L} \quad 50 \quad 100$

$\mu \mathrm{g} / \mathrm{L} \quad 2,000 \quad 4,000$

$\mu \mathrm{g} / \mathrm{L} \quad 350 \quad 700$

$\mathrm{pCi} / \mathrm{L} \quad 7.5 \mathrm{E}+00 \quad 1.5 \mathrm{E}+01$

$\mu \mathrm{g} / \mathrm{L} \quad 0.2 \quad 0.4$

$\mu \mathrm{g} / \mathrm{L} \quad 0.1 \quad 0.2$

$\mu \mathrm{g} / \mathrm{L} \quad 0.00325 \quad 0.0065$

$\mu \mathrm{g} / \mathrm{L} \quad 0.00325 \quad 0.0065$

$\mu \mathrm{g} / \mathrm{L} \quad 0.00225 \quad 0.0045$

$\mu \mathrm{g} / \mathrm{L} \quad 0.00225 \quad 0.0045$

$\mu \mathrm{g} / \mathrm{L} \quad 0.5 \quad 1$

$\mu \mathrm{g} / \mathrm{L} \quad 50 \quad 100$

$\mu \mathrm{g} / \mathrm{L} \quad 25 \quad 50$

$\mu \mathrm{g} / \mathrm{L} \quad 0.00225 \quad 0.0045$

$\mu \mathrm{g} / \mathrm{L} \quad 0.00225 \quad 0.0045$

$\mu \mathrm{g} / \mathrm{L} \quad 0.002 \quad 0.004$

EPA Method 8080

EPA Method 8080

EPA Method 8080

Final PDWS (EPA, 1993a)

Final PDWS (EPA, 1993a)

EPA Method 8080

Set by EPD/EMS

Final PDWS (EPA, 1993a)

EPA Method 8270

EPA Method 8270

Interim Final PDWS (EPA, 1977)

Interim Final PDWS (EPA, 1977)

Interim Final PDWS (EPA, 1977)

EPA Method 8270

EPA Method 8270

EPA Method 8270

Final PDWS (EPA, 1993a)

Final PDWS (EPA, 1993a)

Final PDWS (EPA, 1993a)

Final PDWS (EPA, 1993a)

Final PDWS (EPA, 1993a)

EPA Method 8280

EPA Method 8280

EPA Method 8280

EPA Method 8280

Final PDWS (EPA, 1993a)

EPA Method 8270

Final PDWS (EPA, 1993a)

EPA Method 8280

EPA Method 8280

EPA Method 8280

$\mu \mathrm{g} / \mathrm{L} \quad 0.002 \quad 0.004$

$\mu \mathrm{g} / \mathrm{L} \quad 50 \quad 100$

$\mu \mathrm{g} / \mathrm{L} \quad 250 \quad 500$

$\mu \mathrm{g} / \mathrm{L} \quad 50 \quad 100$

$\mu \mathrm{g} / \mathrm{L} \quad 50 \quad 100$

$\mu \mathrm{g} / \mathrm{L} \quad 50 \quad 100$

$\mu \mathrm{g} / \mathrm{L} \quad 250 \quad 500$

$\mathrm{pCi} / \mathrm{L} \quad 5 \mathrm{E}-01 \quad 1 \mathrm{E}+00$

$\mathrm{pCi} / \mathrm{L} \quad 1.5 \mathrm{E}+00 \quad 3 \mathrm{E}+00$

$\mu \mathrm{g} / \mathrm{L} \quad 75 \quad 150$

$\mu \mathrm{g} / \mathrm{L} \quad 150 \quad 300$
EPA Method 8280

EPA Method 8270

EPA Method 8270

EPA Method 8270

EPA Method 8240

EPA Method 8270

APHA Method 415A

Interim Final PDWS (EPA, 1977)

EPA Method 8240

SDWS (EPA, 1993b)
Interim Final PDWS (EPA, 1977) 
Iron, dissolved

Iron, total recoverable

Iron- $55^{\mathrm{c}}$

Iron-59

Isobutyl alcohol

Isodrin

Isophorone

Isosafrole

Kepone

Lanthanum- $140^{\circ}$

Lead

Analyte

Lead, dissolved

Lead, total recoverable

Lead-212

Lindane

Lithium

Lithium, dissolved

Lithium, total recoverable

Magnesium

Magnesium, dissolved

Magnesium, total recoverable

Manganese

Manganese, dissolved

Manganese, total recoverable

Manganese-54

Mercury

Mercury, dissolved

Mercury, total recoverable

Methacrylonitrile

Methapyrilene

Methoxychlor

3-Methylcholanthrene

2-Methyl-4,6-dinitrophenol

Methyl ethyl ketone

Methyl isobutyl ketone

Methyl methacrylate

Methyl methanesulfonate

2-Methylnaphthalene

Molybdenum

Molybdenum, dissolved

Molybdenum, total recoverable

Naphthalene

$=\mathrm{l}$,4-Naphthoquinone $=\quad:-=-$

1-Naphthylamine

2-Naphthylamine

Neptunium-237

Nickel

Nickel, dissolved

Nickel, total recoverable

Nickel-59 ${ }^{\circ}$

Nickel-63 ${ }^{\mathrm{c}}$

Niobium-95

Nitrate as nitrogen

Nitrate-nitrite as nitrogen

\begin{tabular}{|c|c|c|c|}
\hline $\begin{array}{l}\mu \mathrm{g} / \mathrm{L} \\
\mu \mathrm{g} / \mathrm{L}\end{array}$ & 150 & $\begin{array}{l}300 \\
300\end{array}$ & SDWS (EPA, 1993b) \\
\hline $\mathrm{pCi} / \mathrm{L}$ & $1 \mathrm{E}+03$ & $2 \mathrm{E}+03$ & Interim Final PDWS (EPA, 1977) \\
\hline $\mathrm{pCi} / \mathrm{L}$ & $1 E+02$ & $2 \mathrm{E}+02$ & Interim Final PDWS (EPA, 1977) \\
\hline$\mu \mathrm{g} / \mathrm{L}$ & 500 & 1,000 & EPA Method 8240 \\
\hline$\mu \mathrm{g} / \mathrm{L}$ & 50 & 100 & EPA Method 8270 \\
\hline$\mu \mathrm{g} / \mathrm{L}$ & 50 & 100 & EPA Method 8270 \\
\hline$\mu \mathrm{g} / \mathrm{L}$ & 50 & 100 & EPA Method 8270 \\
\hline$\mu \mathrm{g} / \mathrm{L}$ & 50 & 100 & EPA Method 8270 \\
\hline $\mathrm{pCi} / \mathrm{L}$ & $3 E+01$ & $6 \mathrm{E}+01$ & Interim Final PDWS (EPA, 1977) \\
\hline$\mu g / L$ & 25 & 50 & Final PDWS (SCDHEC, 1981) \\
\hline Unit & Flag 1 & Flag 2 & Source " \\
\hline
\end{tabular}

$\mu \mathrm{g} / \mathrm{L} \quad 25 \quad 50$

$\mu \mathrm{g} / \mathrm{L} \quad 25 \quad 50$

$\mathrm{pCi} / \mathrm{L} \quad 6.2 \mathrm{E}+01 \quad 1.23 \mathrm{E}+02$

$\mu \mathrm{g} / \mathrm{L} \quad 0.1 \quad 0.2$

$\mu \mathrm{g} / \mathrm{L}, \quad 25 \quad 50$

$\mu \mathrm{g} / \mathrm{L} \quad 25 \quad 50$

$\mu \mathrm{g} / \mathrm{L} \quad 25 \quad 50$

No flag No flag

No flag No flag

No flag No flag

$\mu \mathrm{g} / \mathrm{L} \quad 25 \quad 50$

$\mu g / \mathrm{L} \quad 25 \quad 50$

$\mu \mathrm{g} / \mathrm{L} \quad 25 \quad 50$

$\mathrm{pCi} / \mathrm{L} \quad 1.5 \mathrm{E}+02 \quad 3 \mathrm{E}+02$

$\mu \mathrm{g} / \mathrm{L} \quad 1 \quad 2$

$\mu \mathrm{g} / \mathrm{L} \quad 1 \quad 2$

$\mu \mathrm{g} / \mathrm{L} \quad 1 \quad 2$

$\mu \mathrm{g} / \mathrm{L} \quad 250 \quad 500$

$\mu \mathrm{g} / \mathrm{L} \quad 50 \quad 100$

$\mu \mathrm{g} / \mathrm{L} \quad 20 \quad 40$

$\mu \mathrm{g} / \mathrm{L} \quad 50 \quad 100$

$\mu \mathrm{g} / \mathrm{L} \quad 250 \quad 500$

No flag No flag

No flag No flag

$\mu \mathrm{g} / \mathrm{L} \quad 50 \quad 100$

$\mu \mathrm{g} / \mathrm{L} \quad 50 \quad 100$

$\mu g / L \quad 50 \quad 100$

$\mu \mathrm{g} / \mathrm{L} \quad 250 \quad 500$

$\mu \mathrm{g} / \mathrm{L} \quad 250 \quad 500$

$\mu \mathrm{g} / \mathrm{L} \quad 250 \quad 500$

$\mu \mathrm{g} / \mathrm{L} \quad 50 \quad 100$

$=100$.

$\mu \mathrm{g} / \mathrm{L} \quad 50 \quad 100$

$\mu \mathrm{g} / \mathrm{L} \quad 50 \quad 100$

$\mathrm{pCi} / \mathrm{L} \quad 3.53 \mathrm{E}+00 \quad 7.06 \mathrm{E}+00$

$\mu \mathrm{g} / \mathrm{L} \quad 50 \quad 100$

$\mu \mathrm{g} / \mathrm{L} \quad 50 \quad 100$

$\mu \mathrm{g} / \mathrm{L} \quad 50 \quad 100$

$\mathrm{pCi} / \mathrm{L} \quad 1.5 \mathrm{E}+02 \quad 3 \mathrm{E}+02$

$\mathrm{pCi} / \mathrm{L} \quad 2.5 \mathrm{E}+01 \quad 5 \mathrm{E}+01$

$\mathrm{pCi} / \mathrm{L} \quad 1.5 \mathrm{E}+02 \quad 3 . \mathrm{E}+02$

$\mu \mathrm{g} / \mathrm{L} \quad 5,000 \quad 10,000$

$\mu \mathrm{g} / \mathrm{L} \quad 5,000 \quad 10,000$
Final PDẂS (SCDHEC, 1981)

Final PDWS (SCDHEC, 1981)

Proposed PDWS (EPA, 1991)

Final PDWS (EPA, 1993a)

EPA Method 6010

EPA Method 6010

EPA Method 6010

Set by EPD/EMS

Set by EPD/EMS

Set by EPD/EMS

SDWS (EPA, 1993b)

SDWS (EPA, 1993b)

SDWS (EPA, 1993b)

Interim Final PDWS (EPA, 1977)

Final PDWS (EPA, 1993a)

Final PDWS (EPA, 1993a)

Final PDWS (EPA, 1993a)

EPA Method 8240

EPA Method 8270

Final PDWS (EPA, 1993a)

EPA Method 8270

EPA Method 8270

Set by EPD/EMS

Set by EPD/EMS

EPA Method 8270

EPA Method 8270

EPA Method 8270

EPA Method 6010

EPA Method 6010

EPA Method 6010

EPA Method 8270

EPA Method 8270

EPA Method 8270

EPA Method 8270

Proposed PDWS (EPA, 1991)

Final PDWS (EPA, 1993a)

Final PDWS (EPA, 1993a)

Final PDWS (EPA, 1993a)

Interim Final PDWS (EPA, 1977)

Interim Final PDWS (EPA, 1977)

Interim Final PDWS (EPA, 1977)

Final PDWS (EPA, 1993a)

Final PDWS (EPA, 1993a) 
WSRC-TR-99-00011

Unclassified

Nitrite as nitrogen

m-Nitroaniline

o-Nitroaniline

p-Nitroaniline

Nitrobenzene

Nitrogen by Kjeldahl method

2-Nitrophenol

4-Nitrophenol

4-Nitroquinoline-1-oxide

N-Nitrosodi-n-butylamine

N-Nitrosodiethylamine

$\mathrm{N}$-Nitrosodimethylamine

N-Nitrosodiphenylamine

Analyte

N-Nitrosodipropylamine $\mathrm{N}$-Nitrosomethylethylamine $\mathrm{N}$-Nitrosomorpholine

$\mathrm{N}$-Nitrosopiperidine

$\mathrm{N}-\mathrm{Nitrosopyrrolidine}$

5-Nitro-o-toluidine

Nonvolatile beta

Octachlorodibenzo-p-dioxin isomers

Octachlorodibenzo-p-furan

isomers

Odor

Oil \& Grease

Oxamyl ${ }^{b}$

Parathion

Parathion methy?

PCB 1016

PCB 1221

PCB 1232

PCB 1242

PCB 1248

PCB 1254

PCB 1260

PCB 1262

Pentachlorobenzene

Pentachlorodibenzo-p-dioxin isomers

1,2,3,7,8-PCDD

Pentachlorodibenzo-p-furan isomers

1,2,3,7,8-PCDF

Pentachloroethane

Pentachloronitrobenzene

Pentachlorophenol

$\mathrm{pH}$

$\mathrm{pH}$

Phenacetin

Phenanthrene

Phenol

$\begin{array}{lll}\mu \mathrm{g} / \mathrm{L} & 500 & 1,000 \\ \mu \mathrm{g} / \mathrm{L} & 50 & 100 \\ \mu \mathrm{g} / \mathrm{L} & 50 & 100 \\ \mu \mathrm{g} / \mathrm{L} & 50 & 100 \\ \mu \mathrm{g} / \mathrm{L} & 50 & 100 \\ \mu \mathrm{g} / \mathrm{L} & 500 & 1,000 \\ \mu \mathrm{g} / \mathrm{L} & 50 & 100 \\ \mu \mathrm{g} / \mathrm{L} & 50 & 100 \\ \mu \mathrm{g} / \mathrm{L} & 50 & 100 \\ \mu \mathrm{g} / \mathrm{L} & 50 & 100 \\ \mu \mathrm{g} / \mathrm{L} & 50 & 100 \\ \mu \mathrm{g} / \mathrm{L} & 50 & 100 \\ \mu \mathrm{g} / \mathrm{L} & 50 & 100\end{array}$

Final PDWS (EPA, 1993a)

EPA Method 8270

EPA Method 8270

EPA Method 8270

EPA Method 8270

EPA Method 351.2

EPA Method 8270

EPA Method 8270

EPA Method 8270

EPA Method 8270

EPA Method 8270

EPA Method 8270

EPA Method 8270

\section{Unit Flag 1 Flag 2 Source*}

$\begin{array}{llll}\mu \mathrm{g} / \mathrm{L} & 50 & 100 & \text { EPA Method 8270 } \\ \mu \mathrm{g} / \mathrm{L} & 50 & 100 & \text { EPA Method 8270 } \\ \mu \mathrm{g} / \mathrm{L} & 50 & 100 & \text { EPA Method 8270 } \\ \mu \mathrm{g} / \mathrm{L} & 50 & 100 & \text { EPA Method 8270 } \\ \mu \mathrm{g} / \mathrm{L} & 50 & 100 & \text { EPA Method 8270 } \\ \mu \mathrm{g} / \mathrm{L} & 50 & 100 & \text { EPA Method 8270 } \\ \mathrm{pC} / \mathrm{L} & 2.5 \mathrm{E}+01 & 5 \mathrm{E}+01 & \text { Interim Final PDWS (EPA, 1977) } \\ \mu \mathrm{g} / \mathrm{L} & 0.005 & 0.01 & \text { EPA Method 8280 }\end{array}$

$\mu \mathrm{g} / \mathrm{L} \quad 0.005 \quad 0.01 \quad$ EPA Method 8280

\begin{tabular}{llll} 
& No flag & No flag & Set by EPD/EMS \\
\hline $\mathrm{g} / \mathrm{L}$ & 5,000 & 10,000 & EPA Method 413.1
\end{tabular}

$\mu \mathrm{g} / \mathrm{L} \quad 100 \quad 200$

$\mu \mathrm{g} / \mathrm{L} \quad 0.25 \quad 0.5$

$\mu \mathrm{g} / \mathrm{L} \quad 0.25 \quad 0.5$

$\mu \mathrm{g} / \mathrm{L} \quad 0.25 \quad 0.5$

$\mu \mathrm{g} / \mathrm{L} \quad 0.25 \quad 0.5$

$\mu \mathrm{g} / \mathrm{L} \quad 0.25 \quad 0.5$

$\mu g / \quad 0.25 \quad 0.5$

$\mu \mathrm{g} / \mathrm{L} \quad 0.25 \quad 0.5$

$\mu \mathrm{g} / \mathrm{L} \quad 0.25 \quad 0.5$

$\mu \mathrm{g} / \mathrm{L} \quad 0.25 \quad 0.5$

$\mu \mathrm{g} / \mathrm{L} \quad 0.25 \quad 0.5$

$\mu \mathrm{g} / \mathrm{L} \quad 50 \quad 100$

$\mu \mathrm{g} / \mathrm{L} \quad 0.00275 \quad 0.0055$

$\mu \mathrm{g} / \mathrm{L} \quad 0.00275 \quad 0.0055$

$\mu g / L \quad 0.00275 \quad 0.0055$

$\mu \mathrm{g} / \mathrm{L} \quad 0.00275 \quad 0.0055 \quad$ EPA Method 8280

$\mu \mathrm{g} / \mathrm{L} \quad 50 \quad 100 \quad$ EPA Method 8270

$\mu \mathrm{g} / \mathrm{L} \quad 50 \quad 100$

$\mu \mathrm{g} / \mathrm{L} \quad 0.5 \quad 1$

$\mathrm{pH} \quad 8 \quad 10$

$\mathrm{pH} \quad 4 \quad 3$

$\mu \mathrm{g} / \mathrm{L}, \quad 50 \quad 100$

$\mu \mathrm{g} / \mathrm{L} \quad 50 \quad 100$

$\mu \mathrm{g} / \mathrm{L} \quad 50 \quad 100$
Final PDWS (EPA, 1993a)

EPA Method 8080

Final PDWS (EPA, 1993a)

Final PDWS (EPA, 1993a)

Final PDWS (EPA, 1993a)

Final PDWS (EPA, 1993a)

Final PDWS (EPA, 1993a)

Final PDWS (EPA, 1993a)

Final PDWS (EPA, 1993a)

Final PDWS (EPA, 1993a)

EPA Method 8270

EPA Method 8280

EPA Method 8280

EPA Method 8280

EPA Method 8270

Final PDWS (EPA, 1993a)

Set by EPD/EMS

Set by EPD/EMS

EPA Method 8270

EPA Method 8270
EPA Method 8080

EPA Method 8270 


Phenols
p-Phenylenediamine
Phorate
Picloram
2-Picoline
Plutonium-238
Plutonium-239
Plutonium-239/240
Plutonium-240
Plutonium-241
Plutonium-242
Potassium
Potassium, dissolved
Potassium, total recoverable
Potassium-40
Promethium-144
Promethium-146
Promethium-147

Analyte
Pronamid
Propionitrile
Pyrene
Pyridine
Radium (alpha-emitting)
Radium-226
Radium-228
Radon-222
Ruthenium-103
Ruthenium-106
Safrole
Selenium
Selenium, dissolved
Selenium, total recoverable
Silica
Silica, dissolved
Silica, total recoverable
Silver
Silver, dissolved
Silver, total recoverable
Simazine
Sodium
Sodium, dissolved
Sodium,total recoverable
Sodium-22
Specific conductance
Strontium-89
Strontium-89/90 e
Strontium-90
Styrene
Sulfate
Sulfide
Sulfotepp
Surfactants
$2,3,7,8-T C D D$
2,3,7,8-TCDF
Sil

\begin{tabular}{|c|c|c|}
\hline$\mu \mathrm{g} / \mathrm{L}$ & $25^{\circ}$ & $50^{-}$ \\
\hline $\mathrm{Hg} / \mathrm{L}$ & 50 & 100 \\
\hline$\mu \mathrm{g} / \mathrm{L}$ & 0.5 & 1 \\
\hline$\mu \mathrm{g} / \mathrm{L}$ & 250 & 500 \\
\hline$\mu \mathrm{g} / \mathrm{L}$ & 50 & 100 \\
\hline $\mathrm{pCi} / \mathrm{L}$ & $3.51 \mathrm{E}+00$ & $7.02 \mathrm{E}+00$ \\
\hline $\mathrm{pCi} / \mathrm{L}$ & $3.11 E+01$ & $6.21 E+01$ \\
\hline $\mathrm{pCi} / \mathrm{L}$ & $3.11 \mathrm{E}+01$ & $6.21 \mathrm{E}+01$ \\
\hline $\mathrm{pCi} / \mathrm{L}$ & $3.11 \mathrm{E}+01$ & $6.22 E+01$ \\
\hline $\mathrm{pCi} / \mathrm{L}$ & $3.13 \mathrm{E}+01$ & $6.26 \mathrm{E}+01$ \\
\hline $\mathrm{pCi} / \mathrm{L}$ & $3.27 \mathrm{E}+01$ & $6.54 \mathrm{E}+01$ \\
\hline & No flag & No flag \\
\hline & No flag & No flag \\
\hline & No flag & No flag \\
\hline$i / L$ & $1.5 \mathrm{E}+02$ & $3 E+02$ \\
\hline $\mathrm{i} / \mathrm{L}$ & $5 E+01$ & $1 \mathrm{E}+02$ \\
\hline $\mathrm{pCi} / \mathrm{L}$ & $5 E+01$ & $1 E+02$ \\
\hline $\mathrm{pCi} / \mathrm{L}$ & $2.62 \mathrm{E}+03$ & $5.24 E+03$ \\
\hline
\end{tabular}

\begin{tabular}{|c|c|c|c|}
\hline Unit & Flag 1 & Flag 2 & Source" \\
\hline$\mu \mathrm{g} / \mathrm{L}$ & 50 & 100 & EPA Method 8270 \\
\hline$\mu \mathrm{g} / \mathrm{L}$ & 1,000 & 2,000 & EPA Method 8240 \\
\hline$\mu \mathrm{g} / \mathrm{L}$ & 50 & 100 & EPA Method 8270 \\
\hline$\mu \mathrm{g} / \mathrm{L}$ & 50 & 100 & EPA Method 8270 \\
\hline $\mathrm{pCi} / \mathrm{L}$ & $1 E+01$ & $2 E+01$ & Proposed PDWS (EPA, 1991) \\
\hline $\mathrm{pCi} / \mathrm{L}$ & $1 \mathrm{E}+01$ & $2 \mathrm{E}+01$ & Proposed PDWS (EPA, 1991) \\
\hline $\mathrm{pCi} / \mathrm{L}$ & $1 \mathrm{E}+01$ & $2 \mathrm{E}+01$ & Proposed PDWS (EPA, 1991) \\
\hline $\mathrm{pCi} / \mathrm{L}$ & $1.5 \mathrm{E}+02$ & $3 E+02$ & Proposed PDWS (EPA, 1991) \\
\hline $\mathrm{pCi} / \mathrm{L}$ & $1 \mathrm{E}+02$ & $2 \mathrm{E}+02$ & Interim Final PDWS (EPA, 1977) \\
\hline $\mathrm{pCi} / \mathrm{L}$ & $1.5 \mathrm{E}+01$ & $3 \mathrm{E}+01$ & Interim Final PDWS (EPA, 1977) \\
\hline$\mu \mathrm{g} / \mathrm{L}$ & 50 & 100 & EPA Method 8270 \\
\hline$\mu \mathrm{g} / \mathrm{L}$ & 25 & 50 & Final PDWS (EPA, 1993a) \\
\hline$\mu \mathrm{g} / \mathrm{L}$ & 25 & 50 & Final PDWS (EPA, 1993a) \\
\hline \multirow[t]{4}{*}{$\mu \mathrm{g} / \mathrm{L}$} & 25 & 50 & Final PDWS (EPA, 1993a) \\
\hline & No flag & No flag & Set by EPD/EMS \\
\hline & No flag & No flag & Set by EPD/EMS \\
\hline & No flag & No flag & Set by EPD/EMS \\
\hline$\mu \mathrm{g} / \mathrm{L}$ & 50 & 100 & SDWS (EPA, 1993b) \\
\hline$\mu \mathrm{g} / \mathrm{L}$ & 50 & 100 & SDWS (EPA, 1993b) \\
\hline$\mu \mathrm{g} / \mathrm{L}$ & 50 & 100 & SDWS (EPA, 1993b) \\
\hline \multirow[t]{4}{*}{$\mu g / L$} & 2 & 4 & Final PDWS (EPA, 1993a) \\
\hline & No flag & No flag & Set by EPD/EMS \\
\hline & No flag & No flag & Set by EPD/EMS \\
\hline & No flag & No flag & Set by EPD/EMS \\
\hline $\mathrm{p} \overline{\mathrm{C}} / \mathrm{L}$ & $2.33 \mathrm{E}+02$ & $4.66 \mathrm{E}+02$ & Proposed PDWS $(E P A, 1991)$ \\
\hline$\mu \mathrm{S} / \mathrm{cm}$ & 250 & 500 & Set by EPD/EMS \\
\hline $\mathrm{pCi} / \mathrm{L}$ & $|E+0|$ & $2 \mathrm{E}+01$ & Interim Final PDWS (EPA, 1977) \\
\hline $\mathrm{pCi} / \mathrm{L}$ & $4 \mathrm{E}+00$ & $8 \mathrm{E}+00$ & Final PDWS (EPA, 1993a) \\
\hline $\mathrm{pCi} / \mathrm{L}$ & $4 \mathrm{E}+00$ & $8 \mathrm{E}+00$ & Final PDWS (EPA, 1993a) \\
\hline$\mu \mathrm{g} / \mathrm{L}$ & 50 & 100 & Final PDWS (EPA, 1993a) \\
\hline$\mu \mathrm{g} / \mathrm{L}$ & 200,000 & 400,000 & Proposed PDWS (EPA, 1990) \\
\hline$\mu \mathrm{g} / \mathrm{L}$ & 5,000 & 10,000 & EPA Method 9030 \\
\hline$\mu \mathrm{g} / \mathrm{L}$ & 50 & 100 & EPA Method 8270 \\
\hline & No flag & No flag & Set by EPD/EMS \\
\hline $\mathrm{g} / \mathrm{L}$ & 0.000015 & 0.00003 & Final PDWS (EPA, 1993a) \\
\hline $\mathrm{L}$ & 0.002 & 0.004 & EPA Method 8280 \\
\hline
\end{tabular}

EPA Method 420.1'

EPA Method 8080

Final PDWS (EPA, 1993a)

EPA Method 8270

Proposed PDWS (EPA, 1991)

Proposed PDWS (EPA, 1991)

Proposed PDWS (EPA, 1991)

Proposed PDWS (EPA, 1991)

Proposed PDWS (EPA, 1991)

Proposed PDWS (EPA, 1991)

Set by EPD/EMS

Set by EPD/EMS

Set by EPD/EMS

Proposed PDWS (EPA, 1986)

EPA Method 901.1

EPA Method 901.1

Proposed PDWS (EPA, 1991)
EPA Method 8270

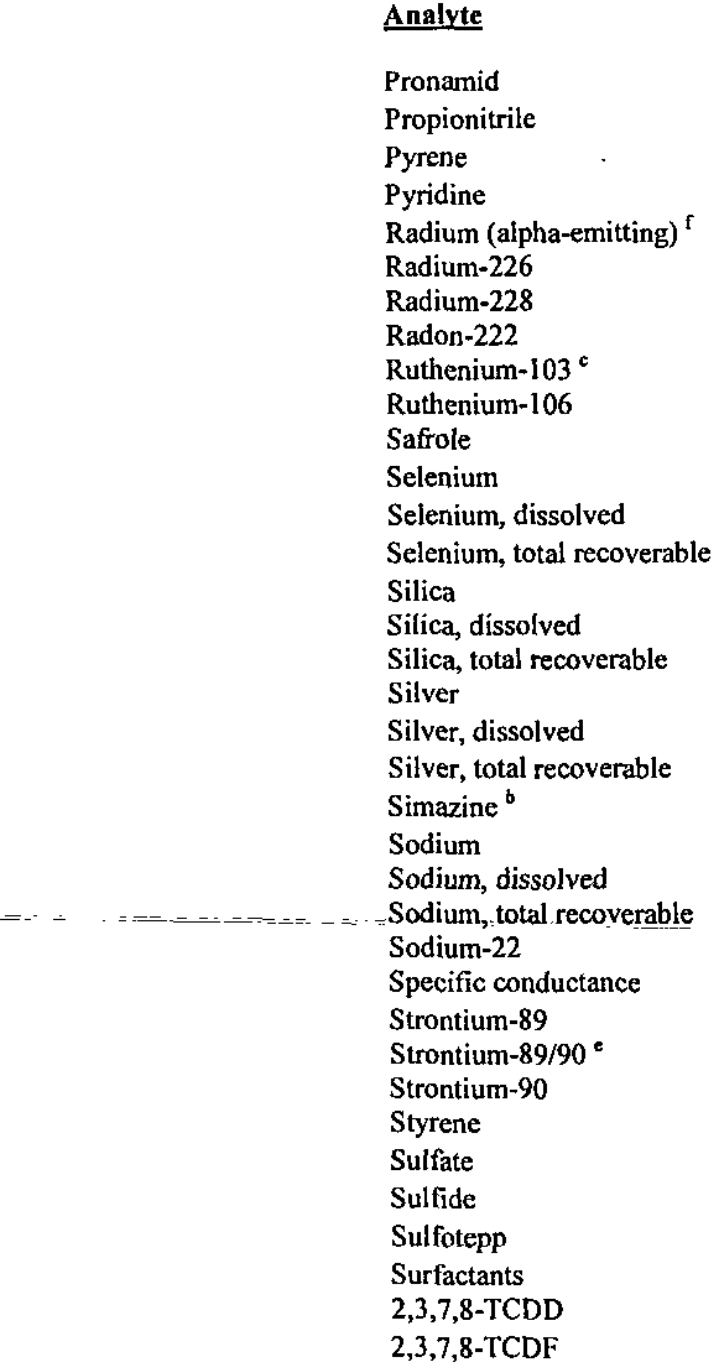

Sanitary Landfill 


Uranium, dissolved
Uranium, total recoverable
Uranium alpha activity
Uranium-233/234
Uranium-234
Uranium-235
Uranium-238
Vanadium
Vanadium, dissolved
Vanadium, total recoverable
Vinyl acetate
Xylenes
Yttrium-88
Zinc
Zinc, dissolved
Zinc, total recoverable
Zinc-65
Zirconium-95
Zirconium/Niobium-95

$\begin{array}{lll}\mu \mathrm{g} / \mathrm{L} & 10 & -\cdots \\ \mu \mathrm{g} / \mathrm{L} & 10 & 20 \\ \mathrm{pCi} / \mathrm{L} & 1.5 \mathrm{E}+01 & 3 \mathrm{E}+01 \\ \mathrm{pCi} / \mathrm{L} & 6.9 \mathrm{E}+00 & 1.38 \mathrm{E}+01 \\ \mathrm{pCi} / \mathrm{L} & 6.95 \mathrm{E}+00 & 1.39 \mathrm{E}+01 \\ \mathrm{pCi} / \mathrm{L} & 7.25 \mathrm{E}+00 & 1.45 \mathrm{E}+01 \\ \mathrm{pCi} / \mathrm{L} & 7.3 \mathrm{E}+00 & 1.46 \mathrm{E}+01 \\ \mu \mathrm{g} / \mathrm{L} & 40 & 80 \\ \mu \mathrm{g} / \mathrm{L} & 40 & 80 \\ \mu \mathrm{g} / \mathrm{L} & 40 & 80 \\ \mu \mathrm{g} / \mathrm{L} & 5 & 10 \\ \mu \mathrm{g} / \mathrm{L} & 5,000 & 10,000 \\ \mathrm{pCi} / \mathrm{L} & 5 \mathrm{E}+01 & 1 \mathrm{E}+02 \\ \mu \mathrm{g} / \mathrm{L} & 2,500 & 5,000 \\ \mu \mathrm{g} / \mathrm{L} & 2,500 & 5,000 \\ \mu \mathrm{g} / \mathrm{L} & 2,500 & 5,000 \\ \mathrm{pCi} / \mathrm{L} & 1.5 \mathrm{E}+02 & 3 \mathrm{E}+02 \\ \mathrm{pCi} / \mathrm{L} & 1 \mathrm{E}+02 & 2 \mathrm{E}+02 \\ \mathrm{pCi} / \mathrm{L} & 1 \mathrm{E}+02 & 2 \mathrm{E}+02\end{array}$

Proposed-PDWS (EPA, 1991)

Proposed PDWS (EPA, 1991)

Proposed PDWS (EPA, 1991)

Proposed PDWS (EPA, 1991)

Proposed PDWS (EPA, 1991)

Proposed PDWS (EPA, 1991)

Proposed PDWS (EPA, 1991)

EPA Method 6010

EPA Method 6010

EPA Method 6010

EPA Method 8240

Final PDWS (EPA, 1993a)

EPA Method 901.1

SDWS (EPA, 1993b)

SDWS (EPA, 1993b)

SDWS (EPA, 1993b)

Interim Final PDWS (EPA, 1977)

Interim Final PDWS (EPA, 1977)

Interim Final PDWS (EPA, 1977)

- References for methods are in Appendix E; references for dated sources are at the end of this appendix.

b EMS is currently unable to perform this analysis.

c EMS discontinued monitoring this radionuclide because it is inappropriate for the SRS Groundwater Monitoring Program.

d EPD/EMS set this flagging criterion using the 1991 proposed PDWS because the final PDWS in 1977 may have been in error.

- For double radionuclide analyses where each separate radionuclide has its own standard, the more stringent standard is used.

f The applied standard is for radium-226.

g The primary maximum contaminant level range for turbidity is 1-5 NTU, which is inappropriate for the SRS Groundwater Monitoring Program.

\section{References}

EPA (U.S. Environmental Protection Agency), 1977. National Interim Primary Drinking Water Regulations, EPA-570/9-76-003. Washington, DC.

EPA (U.S. Environmental Protection Agency), 1986. Water Pollution Control; National Primary Drinking Water Regulations, Radionuclides (Proposed). Federal Register, September 30, 1986, pp. 34835-34862. Washington, DC.

=EPA_(U.S.=Environmental Protection Agency), 1990. National Primary and Secondary Drinking Water Regulations; Synthetic Organic Chemicals and Inorganic Chèmicals (Proposed Rule): Federal-Register, July:25, 1990, pp. 30369-30448. Washington, DC.

EPA (U.S. Environmental Protection Agency), 1991. National Primary Drinking Water Regulations; Radionuclides; Proposed Rule. Federal Register, July 18, 1991, pp. 33052-33127. Washington, DC.

EPA (U.S. Environmental Protection Agency), 1993a. National Primary Drinking Water Regulations. Code of Federal Regulations, Section 40, Part 141, pp. 592-732. Washington, DC.

EPA (U.S. Environmental Protection Agency), 1993b. National Secondary Drinking Water Regulations. Code of Federal Regulations, Section 40, Part 143, pp. 774-777. Washington, DC. 
WSRC-TR-99-00011

Unclassified

SCD $\overline{H E} \bar{C}$ (South Carolina Department of Health and Environmental Control), 1981. State Primary Drinking Water Regulations, R.61-58.5. Columbia, SC. 


\section{Appendix C}

Figures 


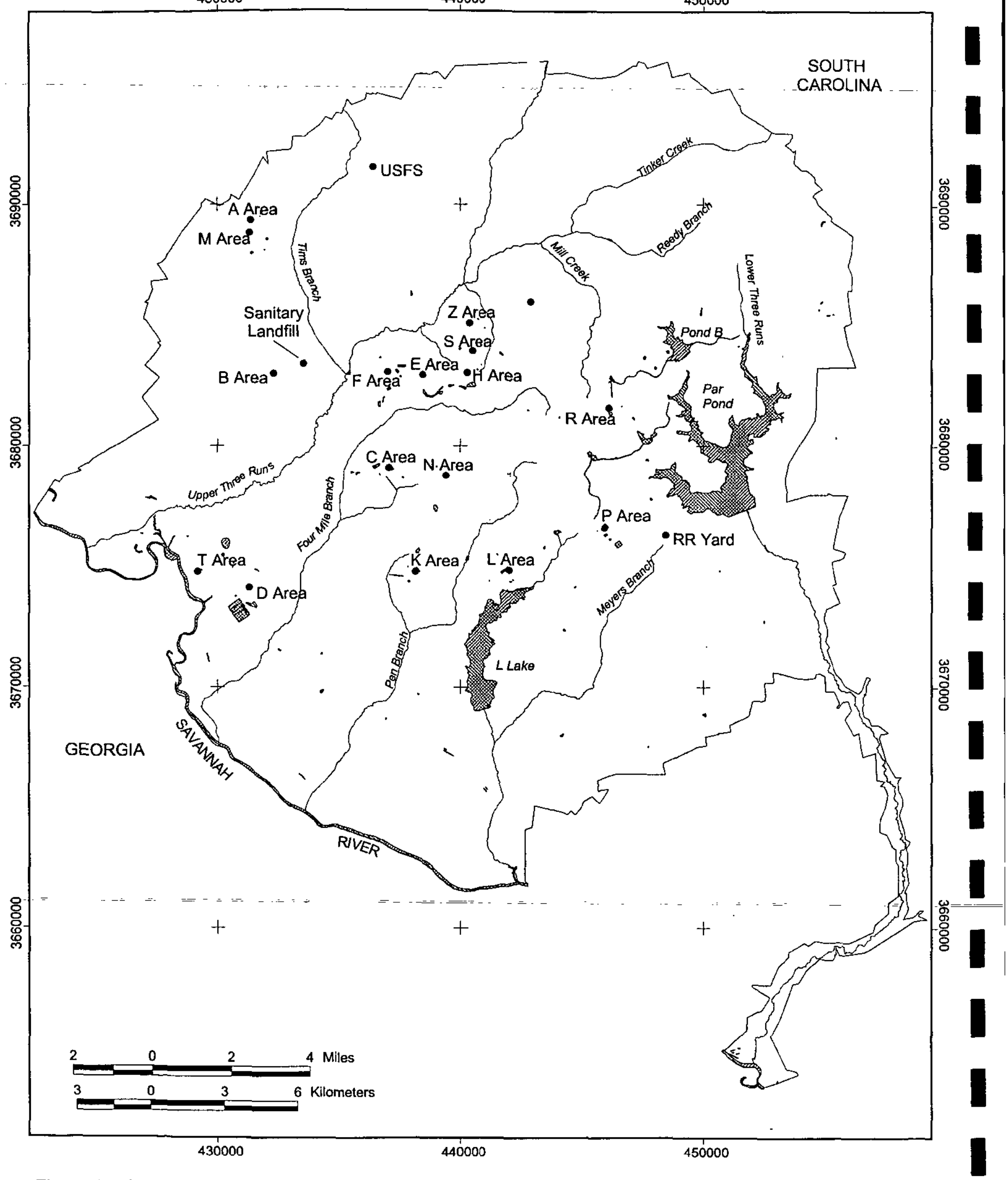

Figure 1. Location of the Sanitary Landfill at the Savannah River Site. 




Figure 2. Boundaries of the Original Sanitary Landfill Expansion and Location of the Monitoring Wells at the Sanitary Landfll 


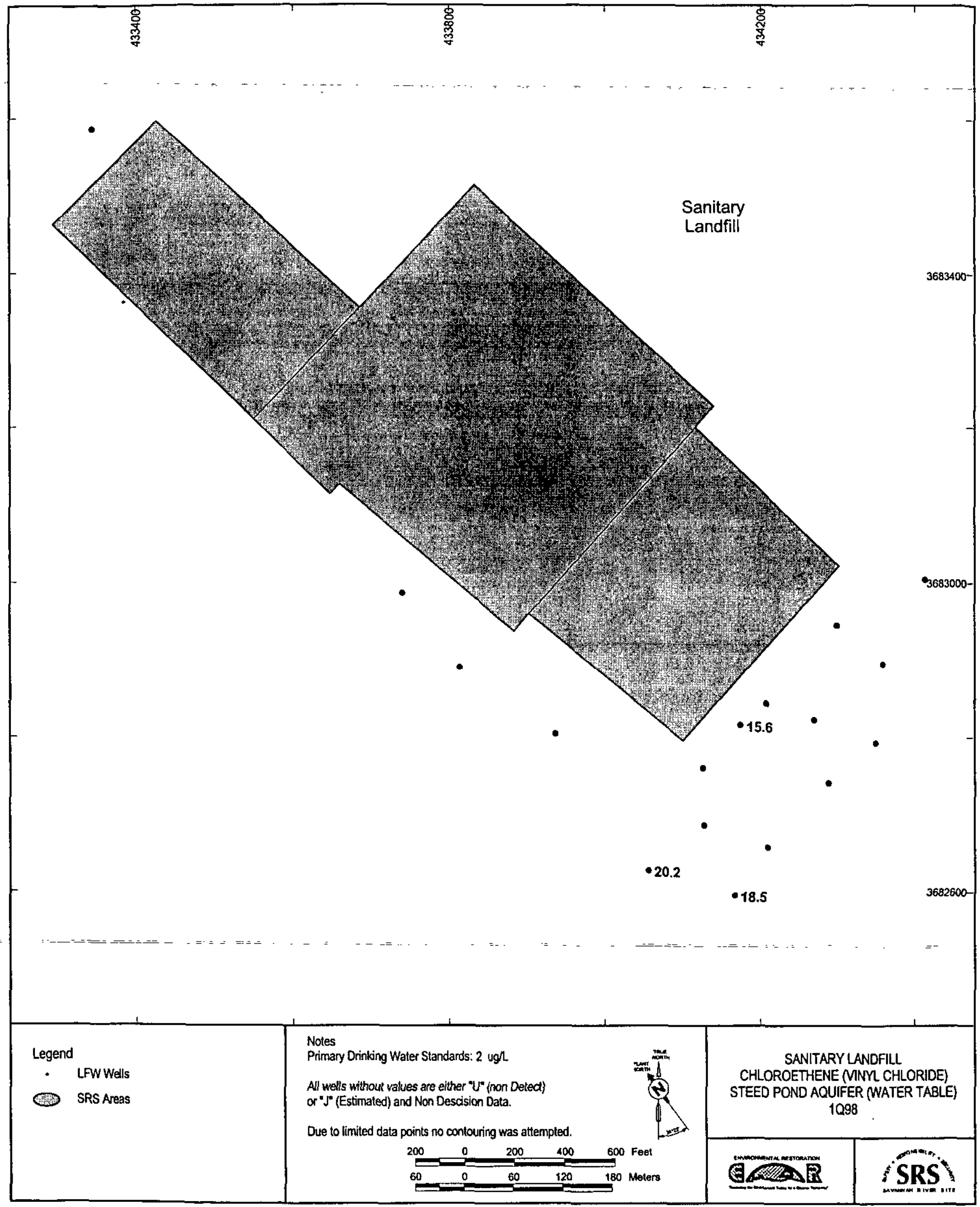

Figure 3. Chloroethene (Vinyl Chloride) Concentrations at the Sanitary Landfill, First Quarter 1998. 



3683000

$9.14^{\bullet}$

$\bullet 7.99$

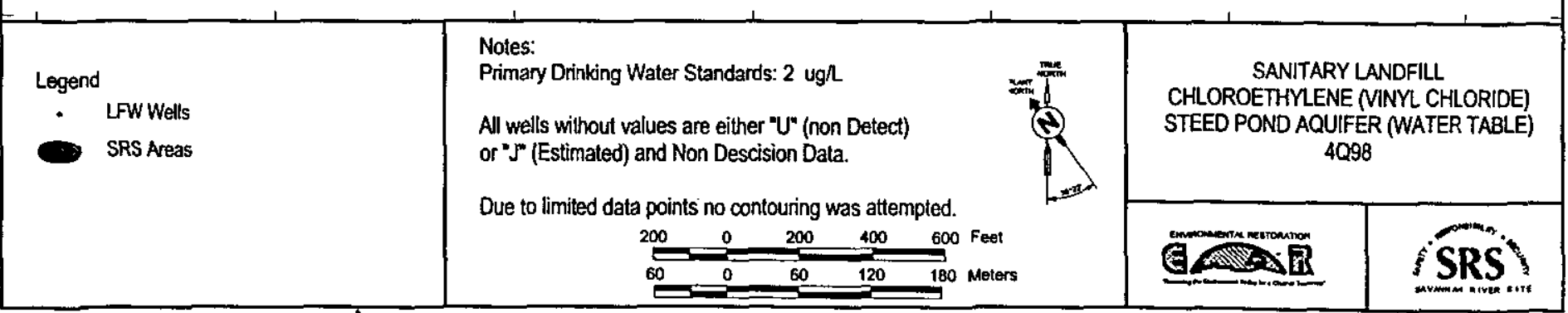

Figure 4. Chloroethene (Vinyl Chloride) Concentrations at the Sanitary Landfill, Fourth Quarter 1998. 


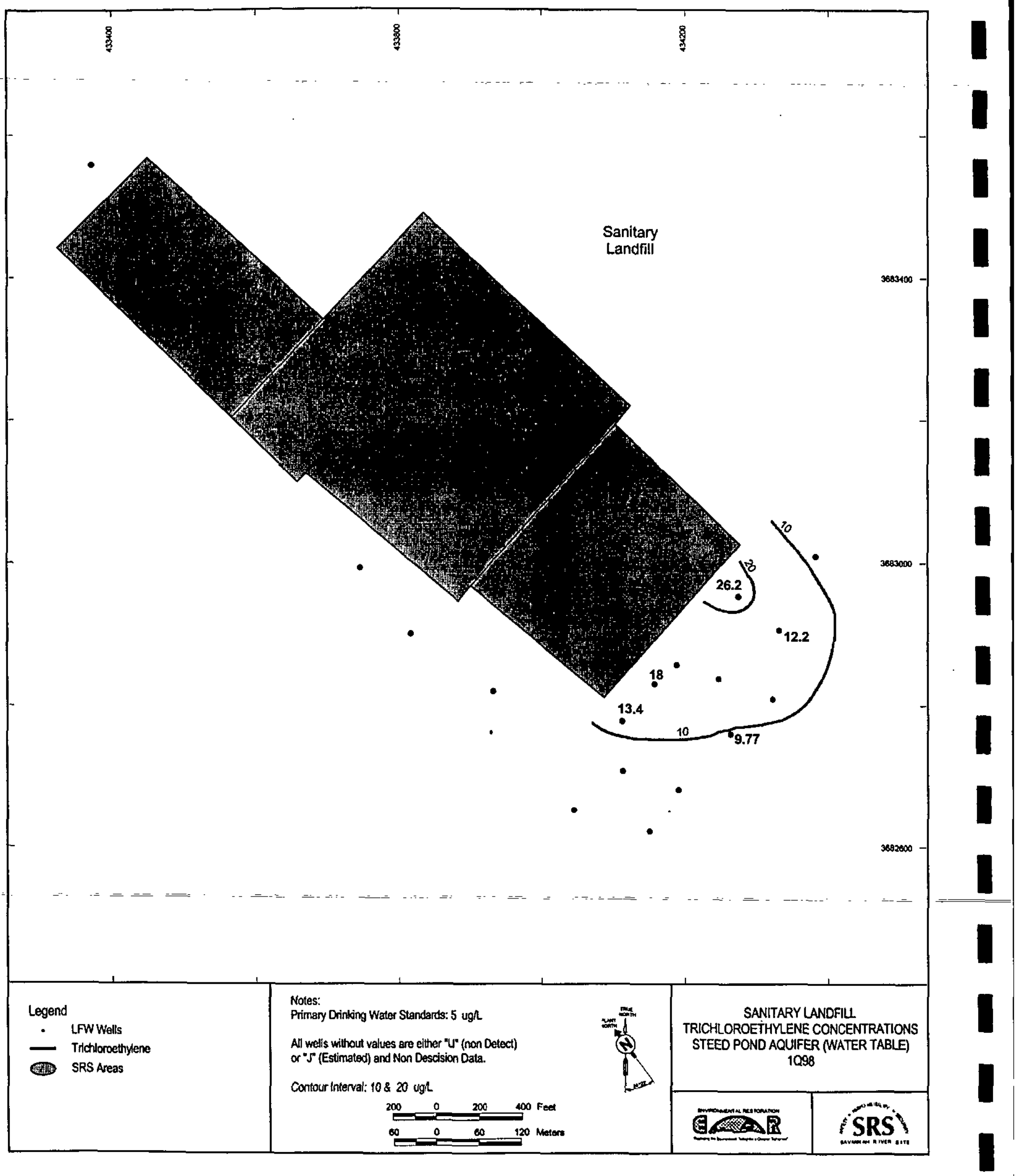

Figure 5. Trichloroethylene Concentrations at the Sanitary Landfill, First Quarter 1998. 

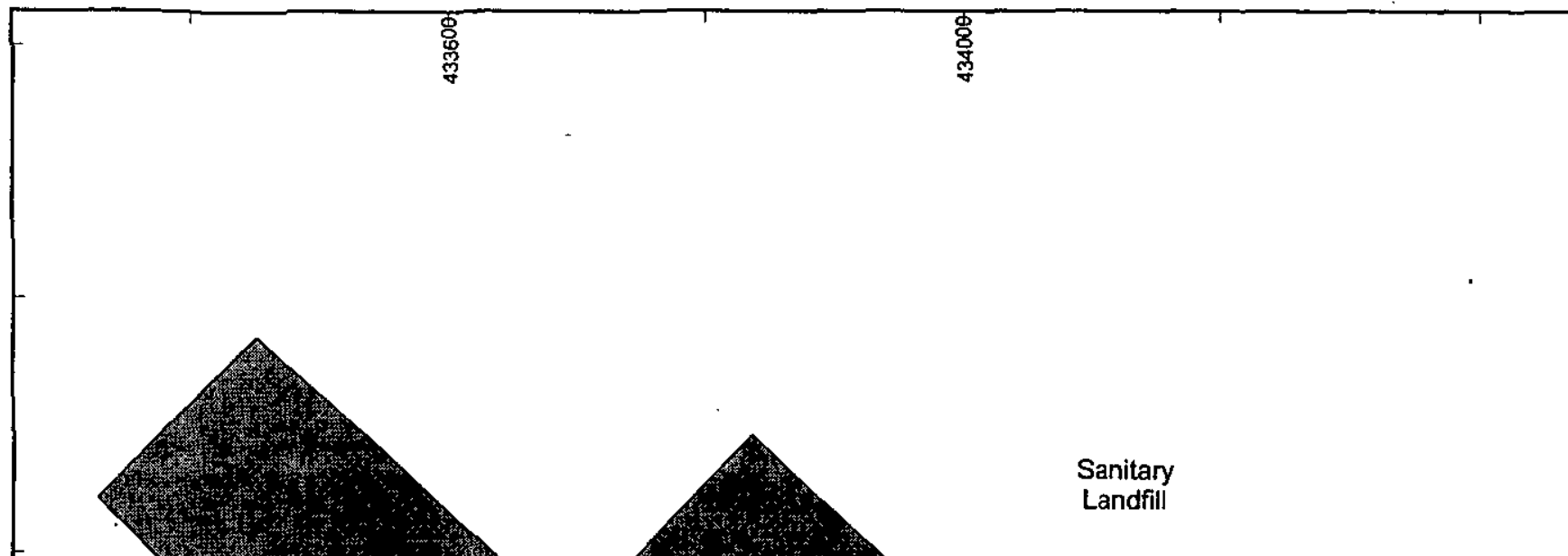


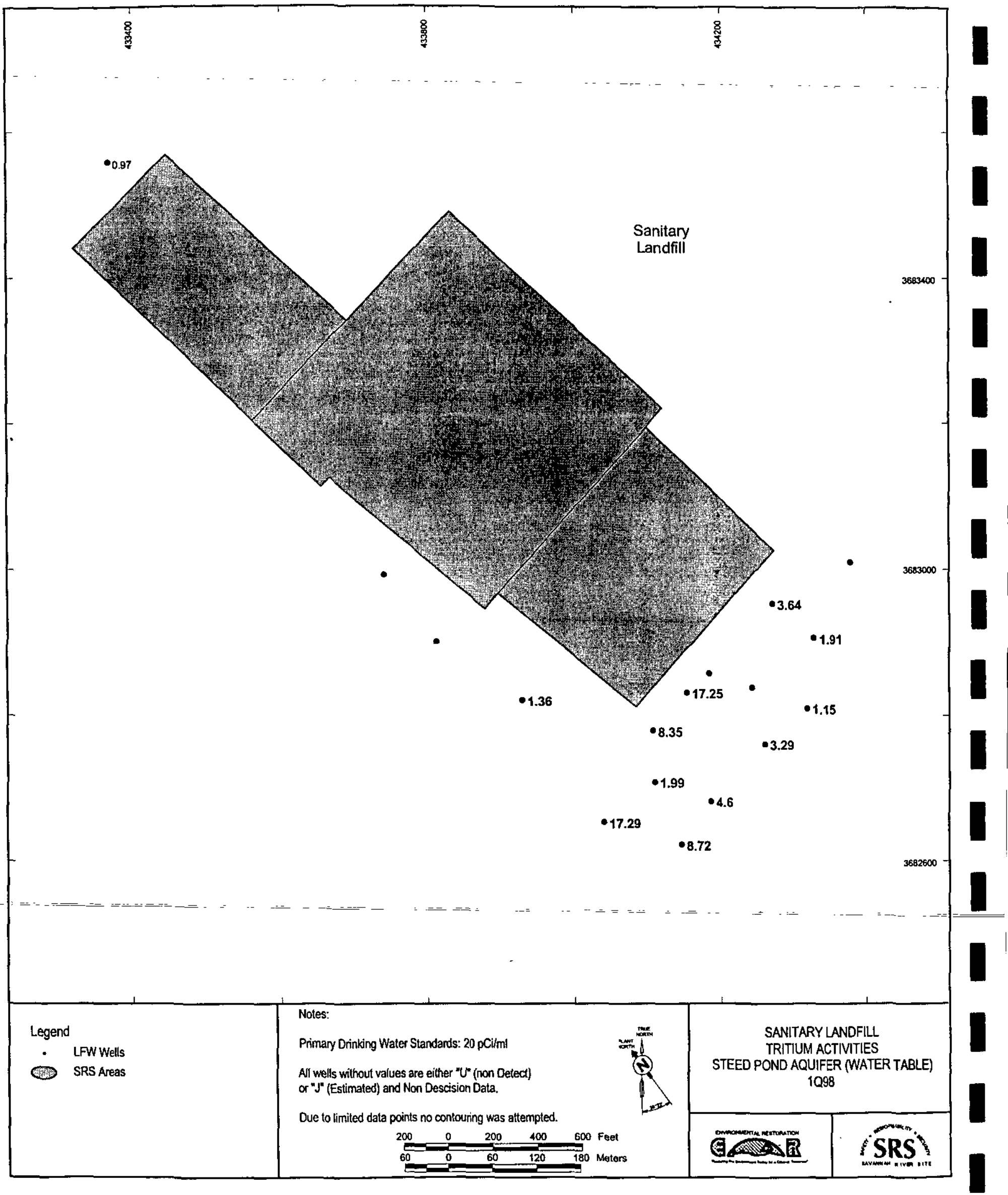

Figure 7. Tritium Activities at the Sanitary Landfill, First Quarter 1998. 


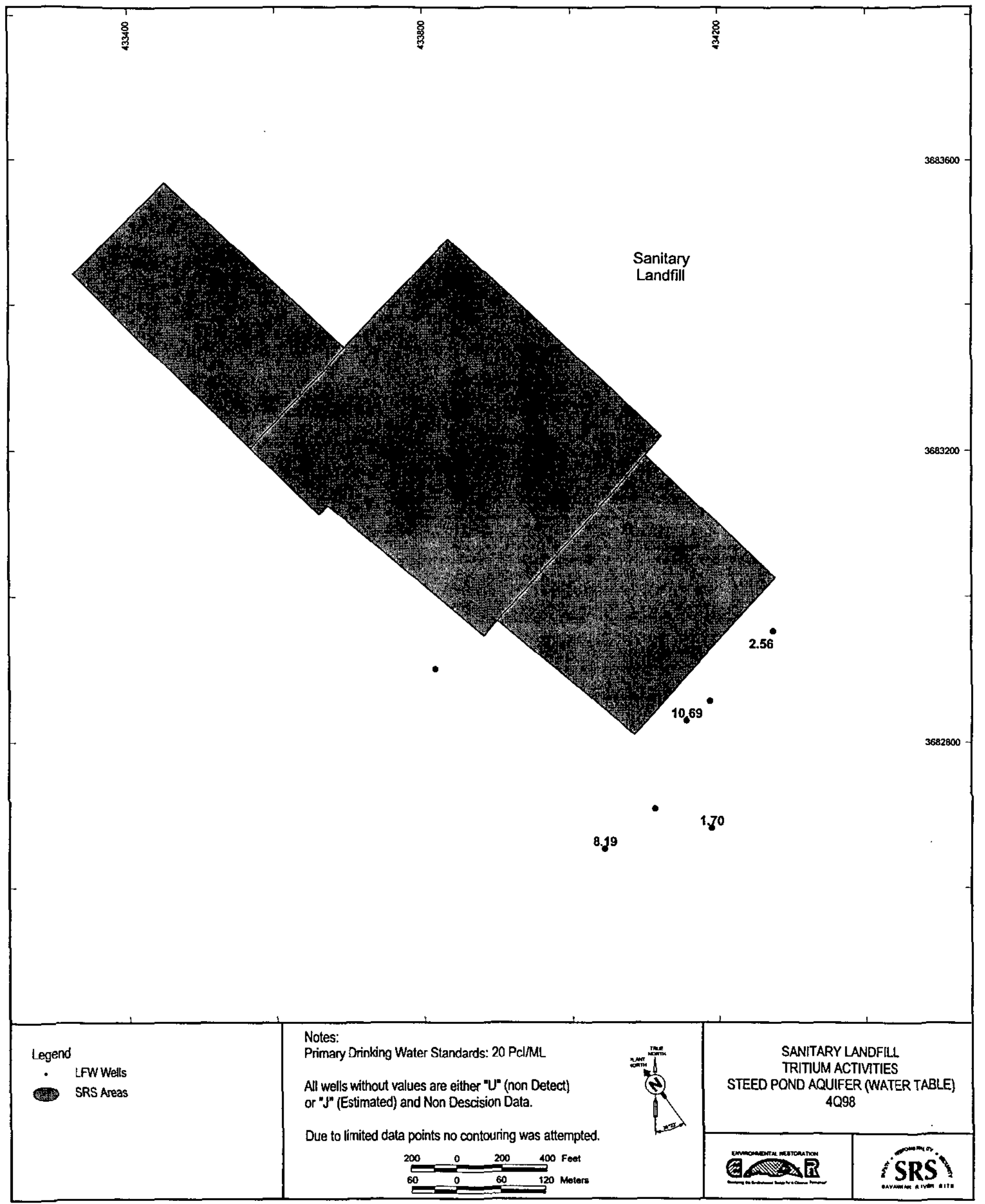

Figure 8. Tritium Activities at the Sanitary Landfill, Fourth Quarter 1998. 




Figure 9. Pieziometric Surface of the Steed Pond (Water Table) at the Sanitary Landfill, First Quarter 1998. 




Figure 10. Pieziometric Surface of the Steed Pond (Water Table) at the Sanitary Landfill, Fourth Quarter 1998. 
WSRC-TR-99-00011

Unclassified

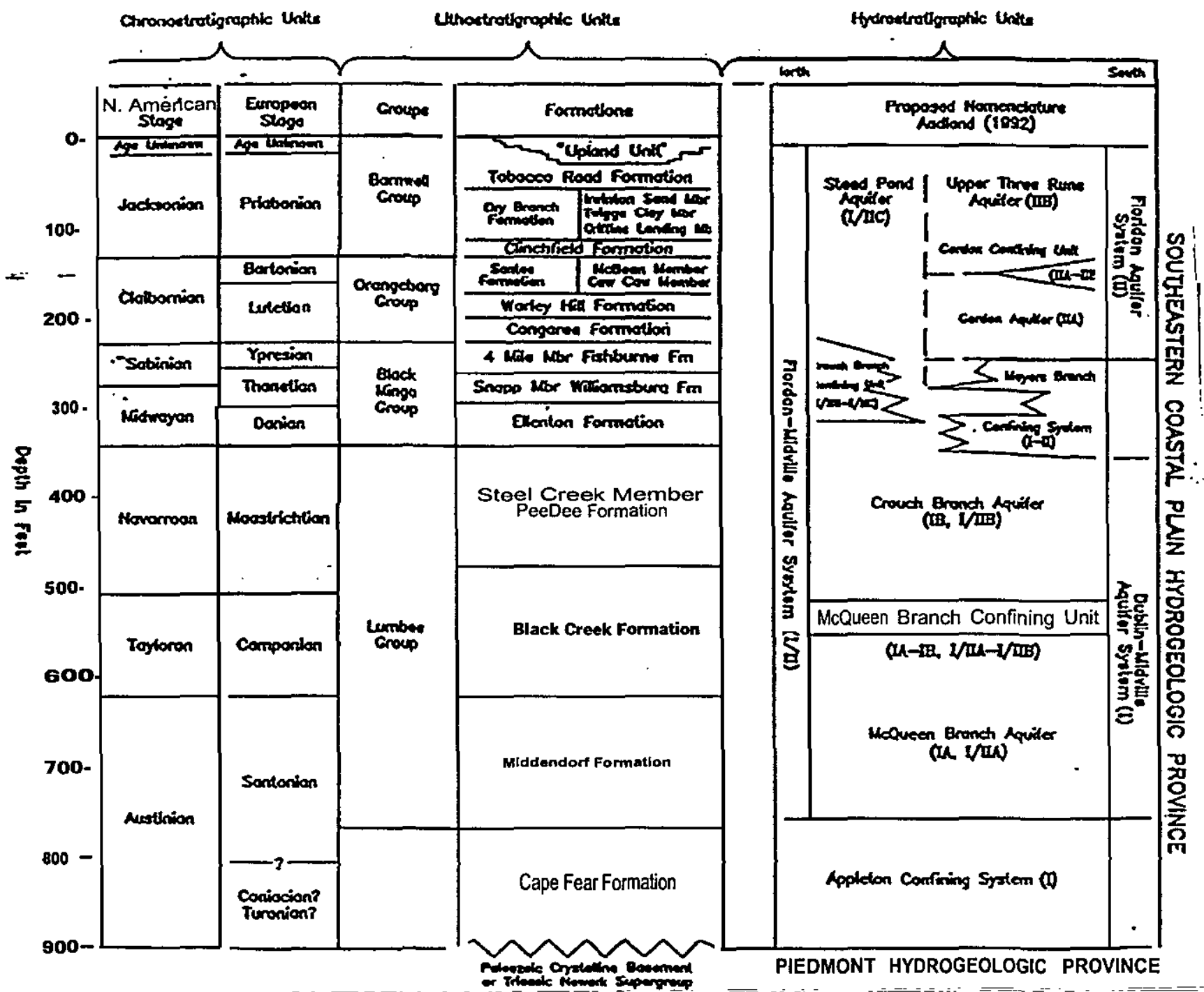

Figure 11. Regional Correlation of Hydrostratigraphic and Lithographic 
WSRC-TR-99-00011

Unclassified

\section{Appendix D}

Tables 
WSRC-TR-99-00011

Unclassified

THIS PAGE LEFT BLANK INTENTIONALLY. 


\section{Fourth Quarter 1998 Data Review}

This report contains analytical data for samples taken during Fourth Quarter 1998 from wells of the LFW series located at the Sanitary Landfill at the Savannah River Site (SRS). The data are submitted in reference to the Sanitary Landfill Operating Permit (DWP-087A). The report presents monitoring results that equaled or exceeded the Safe Drinking Water Act final Primary Drinking Water Standards (PDWS) or screening levels established by the U.S. Environmental Protection Agency (Appendix A), the South Carolina final Primary Drinking Water Standard for lead (Appendix A), or the SRS flagging criteria (Appendix B).

Mechanical failure prevented sample collection and analysis of the groundwater at well LFW10A.

\section{Key to Reading the Tables}

The following abbreviations may appear in the data tables:

\section{Constituents}

\section{1,2,3,4,6,7,8-HPCDD \\ $1,2,3,4,6,7,8$-HPCDF \\ $1,2,3,4,7,8-\mathrm{HXCDD}$ \\ $1,2,3,4,7,8-\mathrm{HXCDF}$ \\ Lindane \\ PCB}

1,2,3,7,8-PCDD

$1,2,3,7,8-\mathrm{PCDF}$

Sp. Conductance

TCDD

TCDF

\section{Laboratories}

\section{$\mathrm{CN}$}

EM

GE and GP

$\mathrm{SC}$

SP

TM

WA and WS

Sampling Codes

\section{B}

C

D

E

I

L

$\mathrm{P}$

S $1,2,3,4,6,7,8$-heptachlorodibenzo-p-dioxin

$1,2,3,4,6,7,8$-heptachlorodibenzo-p-furan

$1,2,3,4,7,8$-hexachlorodibenzo-p-dioxin

$1,2,3,4,7,8$-hexachlorodibenzo-p-furan

gamma-benzene hexachloride

polychlorinated biphenyl

$1,2,3,7,8$-pentachlorodibenzo-p-dioxin

$1,2,3,7,8$-pentachlorodibenzo-p-furan

specific conductance

tetrachlorodibenzo-p-dioxin

tetrachlorodibenzo-p-furan

Clemson Technical Center, Inc.

Environmental Protection Department/ Environmental Monitoring Section (EPD/EMS) Laboratory

General Engineering Laboratories

Savannah River Technology Center

Spencer Testing Services, Inc.

TMA/Eberline

Roy F. Weston, Inc.

blank sample was collected

well was pumping continuously

well was dry

equipment blank was collected

well went dry during sampling; insufficient water to collect all samples

well went dry before sampling began; only depth to water can be determined

inaccessibility or mechanical failure prevented sample

collection and field analysis of the water

no water in standpipe; for water level events only 
Sampling Codes (cont'd)

Sampling Methods

B

S

V

Units

$\mathrm{E}$

$\mathrm{mg} / \mathrm{L}$

$\mathrm{ms} !$

MSL

NTU

$\mathrm{pCi} / \mathrm{L}$

$\mathrm{pCi} / \mathrm{mL}$

$\mathrm{pH}$

$\mu \mathrm{g} / \mathrm{L}$

$\mu \mathrm{S} / \mathrm{cm}$

Other

CS
D
GS
H
Mod
PDWS
PVC
TOC

well went dry during purging; samples collected after well recovered sample collected using an open-bucket bailer sample collected using a bladder pump sample collected using a single-speed centrifugal downhole pump sample collected using a variable-speed pump exponential notation (e.g., $1.1 \mathrm{E}-09=1.1 \times 10^{-9}=$ 0.0000000011 )

milligrams per liter

mean sea level

million structures per liter

turbidity unit

picocuries per liter

picocuries per milliliter

$\mathrm{pH}$ unit

micrograms per liter

microsiemens per centimeter carbon steel

primary drinking water standard (PDWS) column in data tables

groundwater protection standard column in data tables holding time column in data tables modifier column in data tables primary drinking water standard polyvinyl chloride top of casing

\section{Holding Times}

Standard analytical methods include a limit, called holding time, on the maximum elapsed time between sample collection and extraction or analysis by the laboratory. In the data tables, a large

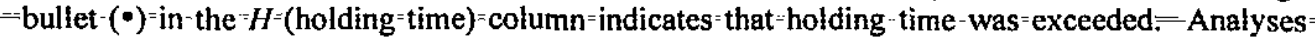
performed beyond holding times may not yield valid results.

The South Carolina Department of Health and Environmental Control allows only 15 minutes to elapse between sampling and analysis for $\mathrm{pH}$. Thus, only field $\mathrm{pH}$ measurements can meet the holding time criterion; laboratory $\mathrm{pH}$ analyses always will exceed it.

The laboratory procedure used for the determination of specific conductance allows one day to elapse between sampling and analysis. Thus, laboratory specific conductance measurements may exceed the holding time criterion. 


\section{Data Rounding}

Constituent results in analytical resuits tables that appear to equal the final PDWS but are not marked in the $D$ (exceeded the final PDWS or screening level) column are below the final PDWS in the database. Values stored in the database contain more significant digits than the reported results. Apparent discrepancies in the tables are due to the rounding of reported results.

\section{Data Qualification}

The contract laboratories continually assess their own accuracy and precision according to U.S. Environmental Protection Agency (EPA) guidelines. They submit sample- or batch-specific quality assurance/quality control information either at the same time as analytical results or in a quarterly summary. Properly defined and used result modifiers (also referred to as qualifiers) can be a key component in assessing data usability. Result modifiers designed by the Environmental Protection Department/Environmental Monitoring Section and provided to the primary laboratories are defined below. These modifiers appear in the data tables under the column Mod. The lettered modifiers are based on EPA's STORET codes.

\section{Result Modifier}

(Blank)

Data are not qualified. Numbers should be interpreted exactly as reported.

A

I

J

L

M

R

$\mathrm{T}$

U

V

$\mathbf{Y}$

Value reported is the mean of two or more determinations.

The value in the result field is the instrument reading, not the sample quantification limit. Always used with the result qualifier $U$.

Value is estimated because quantitation in the sample or in associated quality control samples did not meet specifications.

Value is off-scale high. The actual value is not known but is known to be greater than the value shown.

Presence of the analyte is verified but not quantified.

Result was rejected because performance requirements in the sample analysis or associated quality control analyses were not met.

Analyte was not detected; if present, it was below the criteria for detection.

Material analyzed for but not detected. Analytical result reported is less

than the sample quantitation limit.

Analyte was detected in an associated method blank.

Result was obtained from an unpreserved or improperly preserved sample. Data may not be accurate. 


\section{Result Modifier (cont'd)}

2

3

4

6
Result may be an underestimation of the true value due to analytical bias.

Result may be an overestimation of the true value due to analytical bias.

The associated result may be of poor precision (high variability) due to analytical bias.

Result is associated with QA results indicating matrix interference.

The associated result is from a reanalysis performed out of holding time due to problems with an earlier analysis.

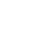


WSRC-TR-99-00011

Unclassified

THIS PAGE LEFT BLANK INTENTIONALLY. 
Table 1. Maximum Levels of Constituents Exceeding the Final Primary Drinking Water Standards Steed Pond Aquifer (Water Table)

\begin{tabular}{|c|c|c|c|c|c|c|c|}
\hline Well & Constituent & $\underline{\text { Units }}$ & $\underline{1098}$ & $\underline{2098}$ & $\underline{3098}$ & $\underline{4098}$ & $\underline{\text { Modifiers }}$ \\
\hline \multirow[t]{3}{*}{ LFW6R } & Chloroethene (Vinyl chloride) & $\mu \mathrm{g} / \mathrm{L}$ & 2.54 & 2.4 & 4 & $N A^{b}$ & $\mathbf{J}$ \\
\hline & Iron, (total recoverable) & $\mu \mathrm{g} / \mathrm{L}$ & $N A^{b}$ & $N A^{b}$ & 488 & $N A^{b}$ & \\
\hline & Trichlorofluoromethane & $\mu \mathrm{g} / \mathrm{L}$ & $--^{2}$ & 34 & 37 & $\ldots$ & J \\
\hline \multirow[t]{3}{*}{ LFW8R } & Chloroethene (Vinyl chloride) & $\mu \mathrm{g} / \mathrm{L}$ & 10.4 & $--{ }^{8}$ & 7.2 & 13.3 & \\
\hline & $\begin{array}{l}\text { Dichloromethane } \\
\text { (methylene chloride) }\end{array}$ & $\mu \mathrm{g} / \mathrm{L}$ & $\ldots$ & --- & 8.3 & $--^{\mathbf{a}}$ & $\mathrm{U}$ \\
\hline & Iron, (total recoverable) & $\mu \mathrm{g} / \mathrm{L}$ & $\mathrm{NA}^{\mathrm{b}}$ & $N A^{b}$ & 24000 & 43900 & \\
\hline \multirow[t]{7}{*}{ LFW10A } & 1,1-Dichloroethane & $\mu \mathrm{g} / \mathrm{L}$ & 172 & 130 & 200 & 151 & \\
\hline & Benzene & $\mu \mathrm{g} / \mathrm{L}$ & 7.44 & 7 & 11 & 9.53 & \\
\hline & Chloroethene (Vinyl chloride) & $\mu \mathrm{g} / \mathrm{L}$ & 34.8 & 24 & 36 & 27.1 & \\
\hline & $\begin{array}{l}\text { Dichloromethane } \\
\text { (Methylene chloride) }\end{array}$ & $\mu \mathrm{g} / \mathrm{L}$ & $--{ }^{*}$ & 100 & 190 & 59.6 & \\
\hline & Iron (total recoverable) & $\mu \mathrm{g} / \mathrm{L}$ & $N_{A}^{b}$ & $N A^{b}$ & 36600 & 37000 & \\
\hline & Trichlorofluoromethane & $\mu \mathrm{g} / \mathrm{L}$ & 23.6 & 19 & $---^{2}$ & $\ldots$ & \\
\hline & Tritium & $\mathrm{pCi} / \mathrm{mL}$ & 27.71 & $-m^{a}$ & 20.33 & 23.87 & \\
\hline \multirow[t]{2}{*}{ LFW 18} & Chloroethene (Vinyl chloride) & $\mu \mathrm{g} / \mathrm{L}$ & 3.24 & 2.8 & $--{ }^{*}$ & $--x^{\prime}$ & \\
\hline & Iron (total recoverable) & $\mu \mathrm{g} / \mathrm{L}$ & $N^{b}$ & $\mathrm{NA}^{\mathrm{b}}$ & 11500 & 15600 & \\
\hline \multirow[t]{4}{*}{ LFW21 } & 1,1-Dichloroethane & $\mu \mathrm{g} / \mathrm{L}$ & 79.2 & 40 & 62 & 55 & \\
\hline & Chloroethene (Vinyl chloride) & $\mu \mathrm{g} / \mathrm{L}$ & 3.24 & 5.1 & 6.8 & 18.8 & \\
\hline & $\begin{array}{l}\text { Dichloromethane } \\
\text { (Methylene chloride) }\end{array}$ & $\mu \mathrm{g} / \mathrm{L}$ & $---{ }^{\prime}$ & $\ldots$ & 5.4 & $--\mathbf{a}^{\mathbf{a}}$ & \\
\hline & Iron (total recoverable) & $\mu \mathrm{g} / \mathrm{L}$ & $N A^{b}$ & $N^{b}$ & 15600 & 19900 & \\
\hline LFW31 & Trichlorofluoromethane & $\mu \mathrm{g} / \mathrm{L}$ & --- & 12 & 28 & 39.9 & \\
\hline LFW32C & Aluminum (total recoverable) & $\mu \mathrm{g} / \mathrm{L}$ & $N A^{b}$ & $N A^{b}$ & $N A^{b}$ & 52.7 & J \\
\hline \multirow[t]{2}{*}{ LFW36R } & Chloroethene (Vinyl chloride) & $\mu \mathrm{g} / \mathrm{L}$ & 19.6 & 22 & 13 & $N A^{b}$ & \\
\hline & Iron (total recoverable) & $\mu \mathrm{g} / \mathrm{L}$ & $N A^{b}$ & $N A^{b}$ & 29700 & $N^{b}$ & \\
\hline LFW43B & 1,2-Dichloroethane & $\mu \mathrm{g} / \mathrm{L}$ & 7.5 & $\cdots a^{n}$ & $--x^{*}$ & $\mathrm{NA}^{\mathbf{b}}$ & \\
\hline \multirow[t]{2}{*}{ LFW56D } & Aluminum (total recoverable) & $\mu \mathrm{g} / \mathrm{L}$ & $N^{b}$ & $\mathrm{NA}^{\mathrm{b}}$ & 83.1 & $\mathrm{NA}^{\mathrm{b}}$ & \\
\hline & Iron (total recoverable) & $\mu \mathrm{g} / \mathrm{L}$ & $N A^{b}$ & $N^{b}$ & 330 & $\mathrm{NA}^{\mathrm{b}}$ & \\
\hline \multirow[t]{2}{*}{ LFW59D } & Aluminum (total recoverable) & $\mu \mathrm{g} / \mathrm{L}$ & $N A^{b}$ & $N A^{b}$ & 145 & 119 & $\mathrm{~J}$ \\
\hline & Iron (total recoverable) & $\mu \mathrm{g} / \mathrm{L}$ & $N A^{b}$ & $N A^{b}$ & --- & 358 & \\
\hline
\end{tabular}


WSRC-TR-99-00011

Unclassified

$\begin{array}{cl}\text { Well } & \text { Constituent } \\ \text { LFW58D } & \text { 1,1-Dichloroethane } \\ & \text { 1,4-Dichlorobenzene } \\ \text { Aluminum (total recoverable) } & \\ \text { Benzene } & \text { Chloroethene (Vinyl chloride) } \\ \text { Dichlorodifluoromethane } \\ \text { Dichloromethane } \\ \text { (Methylene chloride) } \\ \text { Iron (total recoverable) } \\ \text { Tetrachloroethylene } \\ \text { Trichloroethylene } \\ \text { Trichlorofluoromethane }\end{array}$

\begin{tabular}{|c|c|c|c|c|c|}
\hline Units & $\underline{1098}$ & $\underline{2098}$ & $\underline{3098}$ & $\underline{4098}$ & Modifiers \\
\hline$\mu \mathrm{g} / \mathrm{L}$ & 67.7 & $N A^{b}$ & 140 & 92.3 & \\
\hline$\mu \mathrm{g} / \mathrm{L}$ & $\cdots$ & $N A^{b}$ & 170 & 173 & \\
\hline$\mu \mathrm{g} / \mathrm{L}$ & $N A^{b}$ & $N A^{b}$ & 630 & $\ldots$ & \\
\hline$\mu \mathrm{g} / \mathrm{L}$ & $--{ }^{n}$ & $N A^{b}$ & 24 & 13.3 & \\
\hline$\mu \mathrm{g} / \mathrm{L}$ & 7.95 & $N_{A}^{b}$ & 40 & 15.1 & \\
\hline$\mu \mathrm{g} / \mathrm{L}$ & 24.6 & $\cdot N A^{b}$ & 15 & 27.7 & \\
\hline$\mu \mathrm{g} / \mathrm{L}$ & $---^{2}$ & $\mathrm{NA}^{\mathrm{b}}$ & $--{ }^{*}$ & 8.6 & $\mathbf{J}$ \\
\hline$\mu \mathrm{g} / \mathrm{L}$ & $N^{b}$ & $N A^{b}$ & 7980 & 17900 & \\
\hline$\mu \mathrm{g} / \mathrm{L}$ & 5.25 & $N A^{b}$ & $--{ }^{\prime}$ & $--{ }^{\star}$ & \\
\hline$\mu \mathrm{g} / \mathrm{L}$ & 13.4 & $N A^{b}$ & 16 & 16.2 & \\
\hline$\mu \mathrm{g} / \mathrm{L}$ & 17.5 & $N^{b}{ }^{b}$ & 34 & 33.9 & \\
\hline
\end{tabular}

LFW60C 1,1-Dichloroethane Chloroethene (Vinyl chloride) Iron (total recoverable)

$\mu \mathrm{g} / \mathrm{L}$

$\mu \mathrm{g} / \mathrm{L}$

12.7

$13 \quad \mathrm{NA}^{\mathrm{b}}$

$\mu \mathrm{g} / \mathrm{L}$

2.87

4.

$\mathrm{NA}^{\mathrm{b}}$

$\mathrm{NA}^{\mathrm{b}}$

5.7

$N A^{b}$

LFW60D 1,1-Dichloroethane

Aluminum (total recoverable)

Iron (total recoverable)

Mercury (total recoverable)

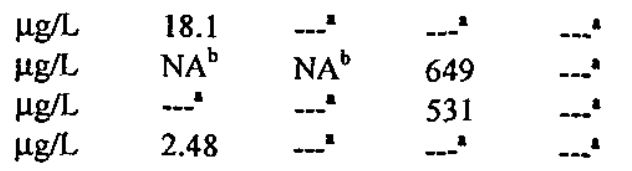

LFW61D 1,1-Dichloroethane

Aluminum (total recoverable)

Dichlorodifluoromethane

Dichloromethane

(Methylene chloride)

Iron (total recoverable)

Tetrachloroethylene

Trichloroethylene

Trichlorofluoromethane

Tritium

LFW62C 1,1-Dichloroethane

1,2-Dichloroethane

Mercury (total recoverable)

Trichloroethylene

$\mu \mathrm{g} / \mathrm{L} \quad 75.9$

$\mu \mathrm{g} / \mathrm{L} \quad \mathrm{NA}^{\mathrm{b}}$

$46 \quad 49$

$\mu \mathrm{g} / \mathrm{L} \quad 38.5$

$\mathrm{NA}^{\mathrm{b}}$

203

59.1

$\mu \mathrm{g} / \mathrm{L}$

$--{ }^{2}$

17

17

…

$\mu \mathrm{g} / \mathrm{L} \quad \mathrm{NA}^{\mathrm{b}}$

14

18.7

$\mu \mathrm{g} / \mathrm{L} \quad 8.31$

\begin{tabular}{ccc} 
NA $^{b}$ & 23000 & 19200 \\
\hdashline$-n^{*}$ & $-n^{*}$
\end{tabular}

$\mu \mathrm{g} / \mathrm{L} \quad 26.2$

$\mu \mathrm{g} / \mathrm{L} \quad 14.8$

12

14.4

$\mathrm{pCi} / \mathrm{mL} \quad$-.-

-..2 $=1$

$\mu \mathrm{g} / \mathrm{L} \quad 45.7$

$--^{a}$

2.39

16

$\mu \mathrm{g} / \mathrm{L} \quad 5.22$

$N A^{b}$

$N A^{b}$

$\mathrm{NA}^{\mathrm{b}}$

$\mu \mathrm{g} / \mathrm{L} \quad 3.21$

$N A^{b}$

$N A^{b}$

$\mathrm{NA}^{\mathrm{b}}$

$\mu \mathrm{g} / \mathrm{L}$

13.2

$N A^{b}$

$N A^{b}$

$N A^{b}$

$\mu \mathrm{g} / \mathrm{L} \quad 119$

$\mathrm{NA}^{\mathrm{b}}$

$N A^{b}$

$N A^{b}$

LFW62D 1,1-Dichloroethane

1,2-Dichloroethane

1,4-Dichlorobenzene

$\mu \mathrm{g} / \mathrm{L}$

$N A^{b}$

82

$\mu \mathrm{g} / \mathrm{L}$

5.38

Aluminum (total recoverable)

$\mu \mathrm{g} / \mathrm{L}$

86

$N A^{b}$

79.1

Benzene

$\mu \mathrm{g} / \mathrm{L} \quad 25.6$

$N A^{b}$

$-^{2} \quad-a^{2}$

$\mu \mathrm{g} / \mathrm{L} \quad 15.6$

$N A^{b}$

369

$N A^{b}$

369

Chloroethene (Vinyl chloride)

Dichlorodifluoromethane

$\mu \mathrm{g} / \mathrm{L}$

$N A^{b}$

12

19.9

$\mathrm{NA}^{\mathrm{b}}$

5.2

Dichloromethane(Methylene chloride)

$\mu \mathrm{g} / \mathrm{L} \quad \mathrm{NA}^{\mathrm{b}}$

$N A^{b}$

12

$\mu \mathrm{g} / \mathrm{L}$

Iron (total recoverable)

Tetrachloroethylene

$\mu \mathrm{g} / \mathrm{L}$

$\begin{array}{llll}34.5 & \mathrm{NA}^{\mathrm{b}} & 660 & 1970 \\ \cdots-\mathrm{N}^{2} & \cdots & \cdots^{2} & 7.05\end{array}$




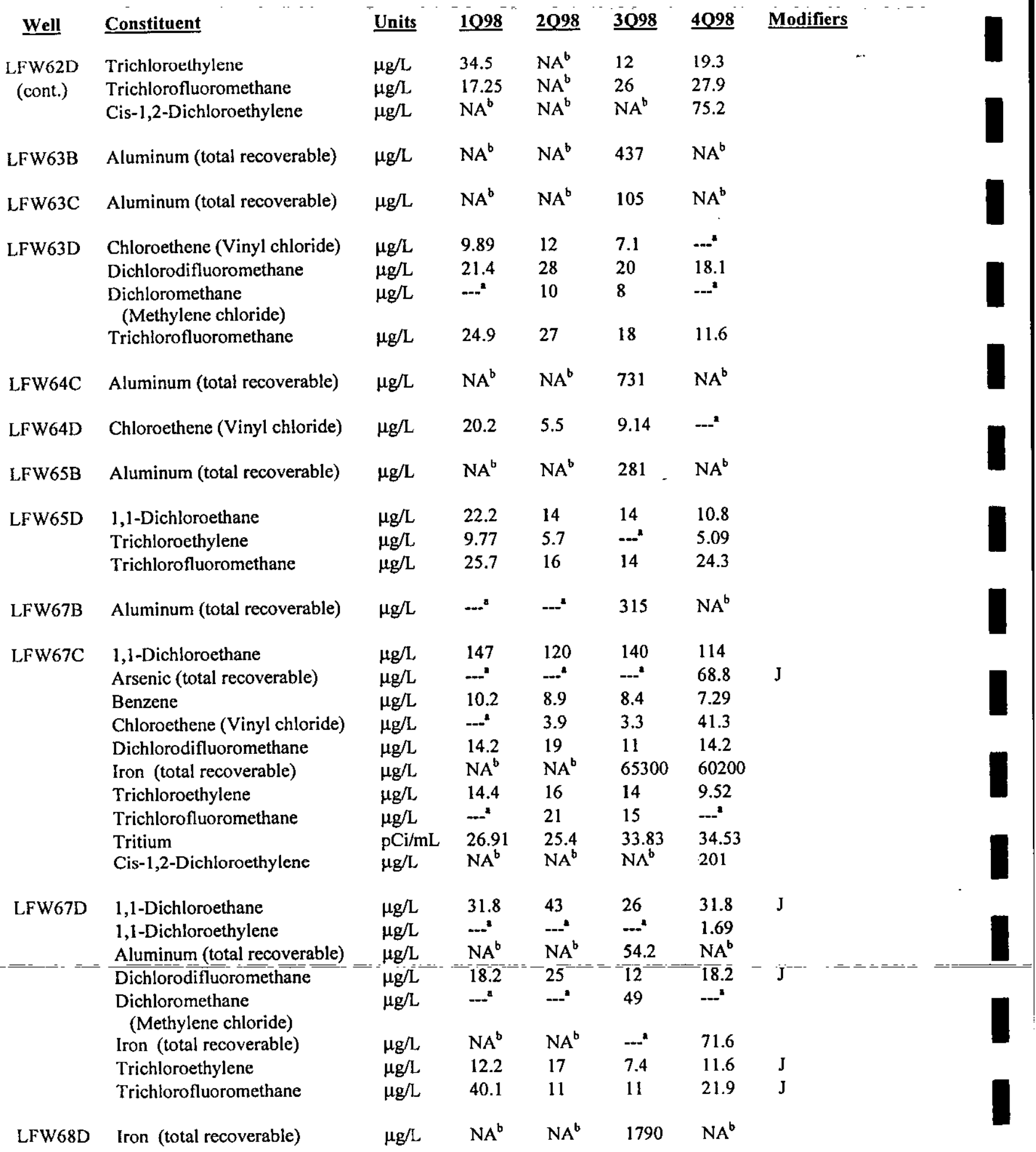


WSRC-TR-99-00011

Unclassified

\begin{tabular}{|c|c|c|c|c|c|c|c|}
\hline Well & Constituent & Units & 1098 & $\underline{2098}$ & $\underline{3098}$ & $\underline{4098}$ & Modifiers \\
\hline LFW69C & Aluminum (total recoverable) & $\mu \mathrm{g} / \mathrm{L}$ & $N A^{b}$ & $N A^{b}$ & 321 & $N_{A}^{b}$ & \\
\hline \multirow[t]{4}{*}{ LFW69D } & 1,1-Dichloroethane & $\mu \mathrm{g} / \mathrm{L}$ & 10.2 & $-m^{2}$ & $\ldots$ & $--^{2}$ & \\
\hline & Aluminum (total recoverable) & $\mu \mathrm{g} / \mathrm{L}$ & $N^{b}$ & $N A^{b}$ & 110 & $--{ }^{\star}$ & \\
\hline & Chloroethene (Vinyl chloride) & $\mu \mathrm{g} / \mathrm{L}$ & 18.5 & 7.8 & 17 & 18.5 & \\
\hline & Iron (total recoverable) & $\mu \mathrm{g} / \mathrm{L}$ & $N A^{b}$ & $N A^{b}$ & 3140 & 629 & \\
\hline LFW71B & Aluminum (total recoverable) & $\mu \mathrm{g} / \mathrm{L}$ & $N A^{b}$ & $N A^{b}$ & 309 & $N A^{b}$ & \\
\hline LFW71C & Aluminum (total recoverable) & $\mu \mathrm{g} / \mathrm{L}$ & $N A^{b}$ & $N A^{b}$ & 90.1 & $N A^{b}$ & \\
\hline
\end{tabular}


WSRC-TR-99-00011

Unclassified

Table 2. Maximum Levels of Constituents Exceeding Other Flag 2 Criteria

\begin{tabular}{|c|c|c|c|c|c|c|c|}
\hline Well & Constituent & Units & $\underline{1098}$ & 2098 & $\underline{3098}$ & $\underline{4098}$ & Modifiers \\
\hline LFW31 & $\mathrm{pH}$ & PH & $N A^{b}$ & -.-' & $N A^{b}$ & 5.37 & \\
\hline LFW32C & $\mathrm{PH}$ & $\mathrm{PH}$ & $N A^{b}$ & $\ldots$ & $N A^{b}$ & 5.33 & $J$ \\
\hline LFW43B & pH & $p H$ & 5.27 & $N A^{b}$ & $N A^{b}$ & $N A^{b}$ & \\
\hline
\end{tabular}

Notes: The groundwater samples are unfiltered. Therefore, the results for metals are for total recoverable metals. The modifier column applies to fourth quarter 1997 data only.

$--^{2}=$ analyzed but not above final PDWS

$\mathrm{NA}^{\mathrm{b}} \quad=$ not analyzed 
Table 3. Groundwater Monitoring Results for Individual Wells

WELL: LFW 6R

\begin{tabular}{|c|c|c|c|c|c|c|}
\hline SRS Coord. & Lat/Longitude & Screen Zone Elevation & Top of Casing & Casing & Pump & Formation \\
\hline $\begin{array}{l}\text { N } 84413.9 \\
\text { E } 45194.0\end{array}$ & $\begin{array}{l}33.286 \operatorname{Deg} N \\
81.712 \operatorname{Deg} W\end{array}$ & $1=2$ & 1 & & $\mathbf{V}$ & ond \\
\hline
\end{tabular}
E 45194.0 81.712 Deg W

$\begin{array}{llll}\text { SAMPLE DATE } & 12 / 01 / 97 & 03 / 12 / 98 & 06 / 12 / 98\end{array}$

FIELD DATA

Analyte

Water Elevation

Depth to water

pH

Sp. Conductance

Water temperature

Alkalinity as $\mathrm{CaCO}$

Turbidity

Volumes purged

Sampling code

\begin{tabular}{|c|c|c|c|c|}
\hline 101998 & 201998 & 301998 & 401998 & Unit \\
\hline 150.65 & 153.46 & 152.35 & & ft msl \\
\hline 17.35 & 14.54 & 15.65 & & ft \\
\hline 4.6 & 4.9 & 4.6 & & pH \\
\hline 42 & 85 & 34 & & $\mathbf{u S} / \mathbf{c m}$ \\
\hline 20 & 17 & 16 & & deg. C \\
\hline $\mathbf{0}$ & 7 & 0 & & $\mathbf{m g} / \mathbf{L}$ \\
\hline 3.3 & 1.6 & 1.1 & & NTU \\
\hline 3.61580 & 4.85293 & 3.61337 & & well volumes \\
\hline
\end{tabular}

\section{ANALYTICAL DATA}

\section{H ST Analyte}

1,1,1,2-Tetrachloroethane

1,1,1-Trichloroethane

1,1,2,2-Tetrachloroethane

1,1,2-Trichloroethane

1,1-Dichloroethane

1,1-Dichloroethylene

1,2,3-Trichloropropane

1,2-Dibromo-3-chloropropane

1,2-Dibromoethane

1,2-Dichloroethane

1,2-Dichloropropane

1,4-Dichlorobenzene

2-Hexanone

Acetone

Acetonitrile (Methyl cyanide)

Acrolein

Acrylonitrile

Allyl chloride

Aluminum, total recoverable

Arsenic, total recoverable

Barium, total recoverable

Benzene

Bromodichloromethane

Bromoform

Bromomethane (Methyl bromide)

Cadmium, total recoverable well volumes

Compliance

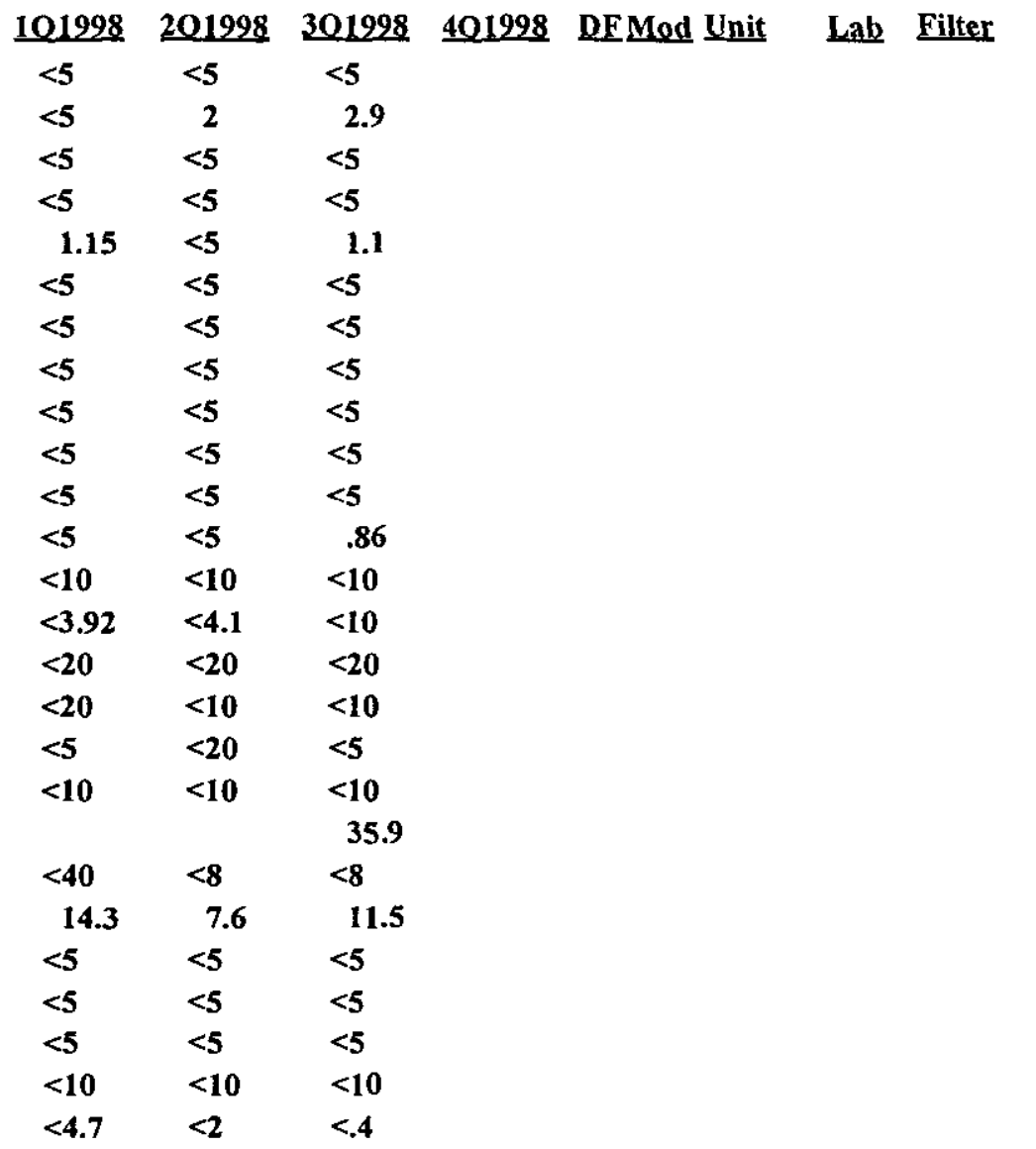

Note: Flagging, dilution factors, modifiers, and laboratories are for fourth quartei998 data only. See Appendix B for flagging criteria. * a exceeded holding time for fourth quarter 1998.

$+=$ exceeded screening level or final prima ry d rinking water standard for fourth quartert 998 .

Sanitary Landfill

D-13

Fourth Quarter 1998 and Annual Summary 
WELL: LFW 6R

\section{ANALYTICAL DATA}

\section{H ST Analyte}

Carbon disulfide

Carbon tetrachloride

Chlorobenzene

Chloroethane

Chloroethene (Vinyl chloride)

Chloroform

Chloromethane (Methyl chloride)

Chloroprene

Chromium, total recoverable

Dibromochloromethane

Dibromomethane (Methylene bromide)

Dichlorodifluoromethane

Dichloromethane (Methylene chloride)

Ethylbenzene

Gross alpha

Iodomethane (Methyl iodide)

Iron, total recoverable

Isobutyl alcohol

Lead, total recoverable

Mercury, total recoverable

Methacrylonitrile

Methyl ethyl ketone

Methyl isobutyl ketone

Methyl methacrylate

Propionitrile

Selenium, total recoverable

Silver, total recoverable

Styrene

Tetrachloroethylene

Toluene

Trichloroethylene

Trichlorofluoromethane

Tritium

Unknown

Vinyl acetate

Xylenes

cis-1,3-Dichloropropene

trans-1,2-Dichloroethylene

trans-1,3-Dichloropropene

trans-1,4-Dichloro-2-butene
101998

$\begin{array}{ccccccc}<5 & <5 & <5 & & & \\ <5 & <5 & <5 & & & \\ <5 & .83 & 1.5 & \end{array}$

$<10 \quad 4.3 \quad 5.3$

$\begin{array}{lll}2.56 & 2.4 & 4\end{array}$

$<5 \quad<5 \quad<5$

$<10<10<10$

$<5 \quad<5 \quad<5$

$4.8<3<3$

$<5<5 \quad<5$

$<5 \quad<5 \quad<5$

$<10<5 \quad 1.4$

$<4.75<2.5 \quad<2.8$

$<5<5 \quad<5$

$\begin{array}{lll}5.79 & 3.83 & 7.04\end{array}$

$<5 \quad<5 \quad<5$

488

$<100<100<100$

$21.9<5 \quad 6$

$.08 \quad .13<.2$

$<10<5<5$

$<10<10<10$

$<10<12<12$

$<5<5$

$<50<5 \quad<5$

$<66<5<5$

$<5<2<2$

$<5<5<5$

$3.31<5<5$

$<5<5<5$

$<5 \quad<5 \quad .86$

$\begin{array}{lll}1.46 & 34 & 37\end{array}$

$\leq 1.11=1950+2.1$

$<11.62$

$<10<5<5$

$4.35<5<5$

$<5<5 \quad<5$

$<5$

$<5<5<5$

$<20<5 \quad<5$

Note: Flagging, dilution factors, modifiers, and laboratories are for fourth quartei998 data only. See Appendix B for flagging criteria.

* = exceeded holding time for fourth quarter 1998 .

$+=$ exceeded screening level or final primary drinking water standard for fourth quarten 998 .

Sanitary Landfill

D-14

Fourth Quarter 1998 and Annual Summary 
WELL: LFW $8 R$

\begin{tabular}{|c|c|c|c|c|c|c|}
\hline$\frac{\text { SRS Coord }}{\text { N } 83949.0}$ & $\frac{\text { Lat/Longitude }}{33.286 \text { Deg N }}$ & $\frac{\text { Screen Zone Elevation }}{154.9-134.9 \text { ft msI }}$ & $\frac{\text { Ton of Casing }}{170.6 \mathrm{ft} \mathrm{msl}}$ & $\frac{\text { Casing }}{2^{1 " P V C}}$ & $\frac{\text { Pump }}{V}$ & $\begin{array}{l}\text { Formation } \\
\text { U Steed Pond }\end{array}$ \\
\hline
\end{tabular}

SAMPLE DATE

FIELD DATA

Analyte
Water Elevation
Depth to water
pH
Sp. Conductance
Water temperature
Alkalinity as CaCO3
Turbidity
Volumes purged
Sampling code

ANALYTICAL DATA

\section{H ST Analyte}

1,1,1,2-Tetrachloroethane

1,1,1-Trichloroeth ane

1,1,2,2-Tetrachloroethane

1,1,2-Trichloroethane

1,1-Dichloroethane

1,1-Dichloroethylene

1,2,3-Trichloropropane

1,2-Dibromo-3-chloropropane

1,2-Dibromoethane

1,2-Dichlorobenzene

1,2-Dichloroethane

1,2-Dichloropropane

1,3-Dichlorobenzene

1,4-Dichlorobenzene

1,4-Dioxane

2-Hexanone

Acetone

Acetonitrile (Methyl cyanide)

Acrolein

Acrylonitrile

Altyl chloride

Aluminum, total recoverable

Arsenic, total recoverable

Barium, total recoverable

Benzene

Bromodichloromethane

\section{$\begin{array}{llll}12 / 02 / 97 & 03 / 16 / 98 & 06 / 10 / 98 & 09 / 15 / 98\end{array}$}

$\begin{array}{lllll}101998 & 201998 & 301998 & 401998 & \text { Unit } \\ 147.08 & 149.07 & 148.13 & 148.23 & \text { ft msl } \\ 21.4 & 19.41 & 20.35 & 20.25 & \text { ft } \\ 6.4 & 6.1 & 6.1 & 7.2 & \text { pH } \\ 240 & 110 & 120 & 200 & \text { uS/cm } \\ 21 & 22.4 & 21.3 & 22 & \text { deg. C } \\ 19 & 29 & 46 & 75 & \text { mg/L } \\ .8 & .9 & 1.6 & .2 & \text { NTU } \\ 5.12400 & 8.61979 & 11.1371 & 8.69387 & \text { well volumes } \\ \text { tV } & & & & \end{array}$

\begin{tabular}{|c|c|c|c|c|c|c|c|c|}
\hline & & & & & & & & npli \\
\hline & 201998 & 301998 & 401998 & DF & Mod & Unit & $\mathbf{L a b}$ & Eilter \\
\hline$<5$ & $<5$ & $<5$ & & & & & & \\
\hline$<5$ & $<5$ & $<5$ & $<5$ & 1 & $\mathbf{U}$ & $\mathrm{ug} / \mathrm{L}$ & EX & $<$ EQL \\
\hline$<5$ & $<5$ & $<5$ & $<5$ & 1 & $\mathbf{U}$ & $\mathbf{u g} / \mathbf{L}$ & EX & $<$ EQL \\
\hline$<5$ & $<5$ & $<5$ & $<5$ & 1 & $\mathbf{U}$ & $\mathrm{ug} / \mathrm{L}$ & EX & $<\mathrm{EQL}$ \\
\hline 1.93 & $<5$ & 2.9 & 3.06 & 1 & $\boldsymbol{J}$ & $\mathbf{u g} / \mathbf{L}$ & $\mathbf{E X}$ & NDD \\
\hline$<5$ & $<5$ & $<5$ & $<5$ & 1 & $\mathbf{U}$ & ug/L & $\mathbf{E X}$ & $<$ EQL \\
\hline$<5$ & $<5$ & $<5$ & & & & & & \\
\hline$<5$ & $<5$ & $<5$ & $<5$ & 1 & $\mathbf{U}$ & $\mathbf{u g} / \mathbf{L}$ & EX & $<$ EQL \\
\hline$<5$ & $<5$ & $<5$ & $<5$ & 1 & $\mathbf{U}$ & 1g/L & EX & $<$ EQL \\
\hline & & & $<5$ & 1 & $\mathbf{U}$ & $\mathbf{u g} / \mathbf{L}$ & EX & $<$ EQL \\
\hline$<5$ & $<5$ & $<5$ & $<5$ & 1 & $\mathbf{U}$ & $\mathbf{u g} / \mathbf{L}$ & EX & $<$ EQL \\
\hline$<5$ & $<5$ & $<5$ & $<5$ & 1 & $\mathbf{U}$ & $\mathbf{u g} / \mathbf{L}$ & EX & $<$ EQL \\
\hline & & & $<5$ & 1 & $\mathbf{U}$ & $\mathbf{u g} / \mathbf{L}$ & EX & $<\mathrm{EQL}$ \\
\hline 3.78 & $<5$ & 2 & 6.28 & 1 & & $\mathbf{u g} / \mathrm{L}$ & $\mathbf{E X}$ & 6.28 \\
\hline & & & $<1000$ & 1 & $\mathbf{U}$ & $\mu \mathrm{g} / \mathrm{L}$ & EX & $<$ EQL \\
\hline$<10$ & $<10$ & $<10$ & $<5$ & 1 & $\mathbf{U}$ & $\mathbf{u g} / \mathbf{L}$ & $\mathbf{E X}$ & $<$ EQL \\
\hline$<7.84$ & $<10$ & $<5$ & $<20$ & 1 & $\mathbf{U}$ & ug/L & $\mathbf{E X}$ & < EQL \\
\hline$<\mathbf{2 0}$ & $<20$ & $<20$ & $<500$ & 1 & $\mathbf{U}$ & $u g / L$ & EX & $<$ EQL \\
\hline$<20$ & $<10$ & $<10$ & $<\mathbf{5 0}$ & 1 & $\mathbf{U}$ & $\mathbf{u g} / \mathbf{L}$ & $\mathbf{E X}$ & $<$ EQL \\
\hline$<5$ & $<20$ & $<5$ & $<50$ & 1 & $\mathbf{U}$ & $\mathbf{u g} / \mathrm{L}$ & EX & $<$ EQL \\
\hline$<10$ & $<10$ & $<10$ & $<10$ & 1 & $\mathbf{U}$ & ug/L & $\mathbf{E X}$ & $<\mathbf{E Q L}$ \\
\hline & & 11.6 & $<200$ & 1 & $\mathbf{U}$ & $\mathbf{u g} / \mathbf{L}$ & EX & $<\mathrm{EQL}$ \\
\hline 20.4 & 10.1 & 10 & $<37.1$ & 1 & $\mathbf{U}$ & $\mathbf{u g} / \mathbf{L}$ & $\mathbf{E X}$ & $<\mathbf{E Q L}$ \\
\hline 12.9 & 7.4 & 15.9 & 11 & 1 & & ug/L & EX & 11 \\
\hline$<5$ & $<5$ & $<5$ & $<5$ & 1 & $\mathbf{U}$ & $u g / L$ & EX & $<\mathrm{EQL}$ \\
\hline$<5$ & $<5$ & $<5$ & $<5$ & 1 & $\mathbf{U}$ & $\mathbf{u g} / \mathbf{L}$ & EX & $<$ EQL \\
\hline
\end{tabular}

Note: Flagging, ditution factors, modifiers, and laboratories are for fourth quartet 998 data only. See Appendix B for flagging criteria.

$\star$ = exceeded holding time for fourth quarter 1998 .

$+=$ exceeded screening level or final primary drinking water standard for fourth quarter 998 .

Sanitary Landfill

D-15

Fourth Quarter 1998 and Annual Summary 
WSRC-TR-99-00011

Unclassified

WELL: LFW 8R

ANALYTICAL DATA

H ST Analyte

Bromoform

Bromomethane (Methyl bromide)

Cadmium, total recoverable

Carbon disulfide

Carbon tetrachloride

Chlorobenzene

Chloroethane

+ Chloroethene (Vinyl chloride)

Chloroform

Chloromethane (Methyl chloride)

Chloroprene

Chromium, total recoverable

Dibromochloromethane

Dibromomethane (Methylene bromide)

Dichlorodifluoromethane

Dichloromethane (Methylene chloride)

Ethyl methacrylate

Ethylbenzene

Gross alpha

Iodomethane (Methyl iodide)

+ Iron, total recoverable

Isobutyl alcohol

Lead, total recoverable

Mercury, total recoverable

Methacrylonitrile

Methyl ethyl ketone

Methyl isobutyl ketone

Methyl methacrylate

Pentachloroethane

Propionitrile

Selenium, total recoverable

Silver, total recoverable

Styrene

Tetrachloroethylene

Toluene

Trichloroethylene

Trichlorofluoromethane

Tritium

Vinyl acetate

Xylenes

cis-1,2-Dichloroethylene

cis-1,3-Dichloropropene
101998

$<5$

$<10<10$

$<4.7<2$

$<5<5$

$<5<5$

$2.21<5$

$<10<10$

$10.4<10$

$<5<5$

$<10<10$

$<5 \quad<5$

$<7<3$

$<5<5$

$<5<5$

$1.58<5$

$<7.89<2.4$

$<5<5 \quad<5$

6.39

$<5$

$<100$

$<1.81$

$<5$

$<47$

$<.7$

$<10$

$<10$

$<10$

$$
24000
$$

$<100<100$

14.8

24.6

$<.2$

$<5$

$<10$

$<12$

$<5$

Compliance

Lab Filter

$<5 \quad<5 \quad 1 \quad \mathrm{U} \quad \mathrm{ug} / \mathrm{L} \quad \mathrm{EX}<\mathrm{EQL}$

$\begin{array}{lllllll}1.6 & <5 & 1 & \mathrm{U} & \mathrm{ug} / \mathrm{L} & \mathrm{EX} & <\mathrm{EQL}\end{array}$

$<.9<10 \quad 1 \quad \mathrm{U} \quad \mathrm{ug} / \mathrm{L} \quad \mathrm{EX}<\mathrm{EQL}$

$<5 \quad<5 \quad 1 \quad$ U $\quad$ ug/L $\quad$ EX $<$ EQL

$<5 \quad<5 \quad 1 \quad$ U $\quad$ ug/L $\quad$ EX $<$ EQL

$\begin{array}{llllllll}1.4 & 3.75 & 1 & J & u g / L & \text { EX } & \text { NDD }\end{array}$

$6.7<10 \quad 1 \quad \mathrm{U} \quad \mathrm{ug} / \mathrm{L} \quad \mathrm{EX} \quad<$ EQL

$\begin{array}{lllllll}7.2 & 13.3 & 1 & \mathrm{ug} / \mathrm{L} & \mathrm{EX} & 13.3\end{array}$

\begin{tabular}{lllllll}
\hline & 1 & $\mathrm{U}$ & $\mathrm{ug} / \mathrm{L}$ & $\mathrm{EX}$ & $<\mathrm{EQL}$
\end{tabular}

$\begin{array}{lllllll}1.1 & <5 & 1 & \mathrm{U} & \mathrm{ug} / \mathrm{L} & \mathrm{EX} & <\mathrm{EQL}\end{array}$

$\begin{array}{lllll}<50 & \text { U } & \text { ug/L } & \text { EX } & <\text { EQL }\end{array}$

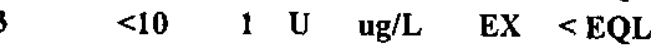

$5 \quad<5 \quad 1 \quad U \quad$ ug/L $/$ EX $<E Q L$

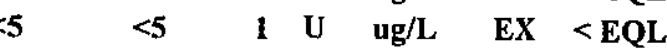

$\begin{array}{lllllll}2.1 & 2.5 & 1 & \mathrm{~J} & \mathrm{ug} / \mathrm{L} & \mathrm{EX} & \mathrm{NDD}\end{array}$

$8.3<10 \quad 1 \quad \mathrm{U} \quad \mathrm{ug} / \mathrm{L} \quad \mathrm{EX}<\mathrm{EQL}$

$<5 \quad 1 \quad \mathrm{U} \quad \mathrm{ug} / \mathrm{L} \quad \mathrm{EX} \quad<\mathrm{EQL}$

$<5 \quad 1$ U $\quad$ ug/L $\quad$ EX $<$ EQL

$\begin{array}{lllll}11.04 & 1 & \mathrm{pCi} / \mathrm{L} & \mathrm{TM} & 11.04\end{array}$

$<5 \quad 1 \quad$ U $\quad$ ug/L $\quad$ EX $<$ EQL

$\begin{array}{lllll}43900 & 1 & \mathrm{ug} / \mathrm{L} & \mathrm{EX} & 43900\end{array}$

$<100 \quad$ I $U$ ug/L $\quad$ EX $<$ EQL

$<.5 \quad 1 \quad \mathrm{U} \quad \mathrm{ug} / \mathrm{L} \quad \mathrm{EX} \quad<\mathrm{EQL}$

$<500 \quad 1$ U ug/L EX $<$ EQL

$<10$ I U ug/L EX $<$ EQL

$<5 \quad 1$ U ug/L EX $<$ EQL

$<50 \quad 1 \quad$ U $\quad$ ug/L $\quad$ EX $<$ EQL

$<200 \quad 1$ U $\quad$ ug/L $\quad$ EX $<$ EQL

$\begin{array}{lllllllll}<50 & <5 & <5 & & & & & & \\ <66 & <5 & <5 & <200 & 1 & \text { U } & \text { ug/L } & \text { EX } & <\text { EQL }\end{array}$

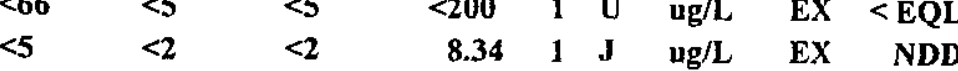

$\leq 5_{-} \ldots \leq 5 \ldots \leq 5 \ldots$

$\begin{array}{lllllllll}<5 & <5 & <5 & <5 & 1 & \mathrm{U} & \mathrm{ug} / \mathrm{L} & \mathrm{EX} & <\mathrm{EQL}\end{array}$

$<5 \quad<5 \quad<5 \quad<5 \quad 1 \quad \mathrm{U} \quad \mathrm{ug} / \mathrm{L} \quad \mathrm{EX} \quad<\mathrm{EQL}$

$<5 \quad<5 \quad<5 \quad<5 \quad 1 \quad \mathrm{U}, \mathrm{ug} / \mathrm{L} \quad \mathrm{EX} \quad<\mathrm{EQL}$

$<5 \quad<5 \quad<5 \quad<5 \quad 1 \quad$ U $\quad u g / L \quad$ EX $<\mathrm{EQL}$

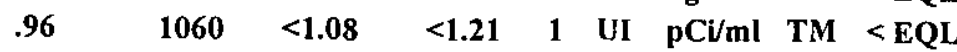

$<10<<50 \quad<20 \quad 1 \quad \mathrm{U} \quad \mathrm{ug} / \mathrm{L} \quad \mathrm{EX}<\mathrm{EQL}$

$<5 \quad<5 \quad<5 \quad<10 \quad 1 \quad$ U $\quad$ ug/L $\quad$ EX $\quad<$ EQL

$4.39 \quad 1 \quad \mathrm{~J} \quad \mathrm{ug} / \mathrm{L} \quad \mathrm{EX} \quad$ NDD

$\begin{array}{lllllllll}< & <5 & <5 & <5 & 1 & \mathrm{U} & \mathrm{ug} / \mathrm{L} & \mathrm{EX} & <\mathrm{EQL}\end{array}$

Note: Flagging, dilution factors, modifiers, and laboratories are for fourth quartet 998 data only. See Appendix B for flagging criteria.

* = exceeded holding time for fourth quarter 1998.

$+=$ exceeded screening level or final primary drinking water standard for fourth quarten 998 .

Sanitary Landfill 
WELL: LFW 8R

\section{ANALYTICAL DATA}

\section{H ST Analyte}

trans-1,2-Dichloroethylene trans-1,3-Dichloropropene trans-1,4-Dichloro-2-butene

$\begin{array}{ccc}101998 & 201998 & \text { 301998 } \\ <5 & & \\ <5 & <5 & <5 \\ <20 & <5 & <5\end{array}$

401998
$<5$
$<5$
$<20$

\section{Compliance}

Lab Filter

EX $<$ EQL

EX $<$ EQL

EX $<$ EQL

Note: Flagging, dilution factors, modifiers, and laboratories are for fourth quarte1998 data only. See Appendix B for flagging criteria. * $=$ exceeded holding time for fourth quarter 1998 .

$t=$ exceeded screening level or final primary drinking water standard for fourth quarten 998 . 
WELL: LFW IOA

\begin{tabular}{|c|c|c|c|c|c|c|}
\hline SRS Coord. & Lat/Longitude & Screen Zone Elevation & Top of Casing & Casing & Pump & Formation \\
\hline $\begin{array}{l}\text { N } 84369.6 \\
\text { E } 45935.6\end{array}$ & $\begin{array}{l}\text { 33.287 Deg } N \\
\text { 81.710 Deg W }\end{array}$ & $164.4-134.4$ ft msl & $181.6 \mathrm{ft} \mathrm{msl}$ & VC & $\mathbf{S}$ & ed Pond \\
\hline
\end{tabular}

$\begin{array}{lllll}\text { SAMPLE DATE } & 12 / 01 / 97 & 03 / 16 / 98 & 06 / 12 / 98 & 09 / 16 / 98\end{array}$

FIELD DATA

\begin{tabular}{|c|c|c|c|c|c|}
\hline Analyte & 101998 & 201998 & $\underline{301998}$ & 401998 & Unit \\
\hline Water Elevation & 149.14 & 151.69 & 151.66 & 150.96 & $\overrightarrow{f t} \mathrm{~ms}$ \\
\hline Depth to water & 30.3 & 27.75 & 27.78 & 28.48 & ft \\
\hline pH & 6.2 & 6.2 & 6.4 & 6.4 & pH \\
\hline Sp. Conductance & 420 & 340 & 400 & 320 & uS $/ \mathrm{cm}$ \\
\hline Water temperature & 18 & 21 & 22 & 21.5 & deg. $C$ \\
\hline Alkalinity as $\mathrm{CaCO}$ & 140 & 16 & 97 & 99 & $\mathrm{mg} / \mathrm{L}$ \\
\hline Turbidity & 20.2 & 2.2 & 5.1 & 5.3 & NTU \\
\hline $\begin{array}{l}\text { Volumes purged } \\
\text { Sampling code }\end{array}$ & 3.70480 & 5.41622 & 5.73907 & 4.48590 & well volumes \\
\hline
\end{tabular}

\section{ANALYTICAL DATA}

H ST Analyte

1,1,1,2-Tetrachloroethane

1,1,1-Trichloroethane

1,1,2,2-Tetrachloroethane

1,1,2-Trichloroethane

+ 1,1-Dichloroethane

1,1-Dichloroethylene

1,2,3-Trichloropropane

1,2-Dibromo-3-chloropropane

1,2-Dibromoethane

1,2-Dichlorobenzene

1,2-Dichloroethane

1,2-Dichloropropane

1,3-Dichlorobenzene

1,4-Dichlorobenzene

1,4-Dioxane

2-Hexanone

Acetone

Acetonitrile (Methyl cyanide)

Acrolein

Acrylonitrile

Allyl chloride

Aluminum, total recoverable

Arsenic, total recoverable

Barium, total recoverable

+ Benzene

Bromodichloromethane

\begin{tabular}{|c|c|c|c|c|c|c|c|c|}
\hline \multirow{3}{*}{$\begin{array}{c}101998 \\
<5 \\
<5\end{array}$} & \multirow{2}{*}{$\frac{201998}{<5}$} & \multirow{2}{*}{$\frac{301998}{<5}$} & \multirow[t]{2}{*}{$\underline{401998}$} & \multicolumn{3}{|c|}{ DEMod Unit } & Lab & Eilter \\
\hline & & & & & & & & \\
\hline & $<5$ & $<5$ & $<5$ & 1 & $\mathbf{U}$ & $\mathbf{u g} / \mathrm{L}$ & EX & $<\mathrm{EQL}$ \\
\hline$<5$ & $<5$ & $<5$ & $<5$ & 1 & $\boldsymbol{U}$ & $\mathbf{u g} / \mathrm{L}$ & EX & $<$ EQL \\
\hline$<5$ & $<5$ & $<5$ & $<5$ & 1 & $\mathbf{U}$ & $\mathbf{u g} / \mathbf{L}$ & EX & < EQL \\
\hline 172 & 130 & 200 & 151 & 1 & & $\mathbf{u g} / \mathbf{L}$ & $\mathbf{E X}$ & 151 \\
\hline$<5$ & $<5$ & $<5$ & $<5$ & 1 & $\mathbf{U}$ & $\mathbf{u g} / \mathbf{L}$ & EX & $<$ EQL \\
\hline$<5$ & $<5$ & $<5$ & & & & & & \\
\hline$<5$ & $<5$ & $<5$ & $<5$ & 1 & $\mathbf{U}$ & $\mathbf{u g} / \mathbf{L}$ & EX & $<$ EQL \\
\hline \multirow[t]{2}{*}{$<5$} & $<5$ & $<5$ & $<5$ & 1 & $\mathbf{U}$ & $\mathbf{u g} / \mathbf{L}$ & $\mathbf{E X}$ & $<$ EQL \\
\hline & & & $<5$ & 1 & $\mathbf{U}$ & $\mathbf{u g} / \mathrm{L}$ & EX & $<\mathbf{E Q L}$ \\
\hline$<5$ & $<5$ & 1.6 & $<5$ & 1 & $U$ & $\mathbf{u g} / \mathrm{L}$ & EX & $<$ EQL \\
\hline \multirow[t]{2}{*}{3.62} & $<5$ & 4 & 3.73 & 1 & $\mathbf{J}$ & $\mathbf{u g} / \mathbf{L}$ & $\mathbf{E X}$ & NDD \\
\hline & & & $<5$ & $\mathbf{I}$ & $\mathbf{U}$ & ug/L & EX & $<\mathbf{E Q L}$ \\
\hline \multirow[t]{2}{*}{28} & 26 & 38 & 43.5 & 1 & & $\mathbf{u g} / \mathrm{L}$ & EX & 43.5 \\
\hline & & & $<1000$ & 1 & $\mathbf{U}$ & $\mathbf{u g} / \mathrm{L}$ & EX & < EQL \\
\hline 3.13 & $<10$ & 5.7 & 5.98 & 1 & & $u g / L$ & EX & 5.98 \\
\hline 129 & 110 & $<32$ & 41.3 & 1 & & $\mathrm{ug} / \mathrm{L}$ & $\mathbf{E X}$ & 41.3 \\
\hline$<20$ & $<20$ & $<20$ & $<500$ & 1 & $\overline{\mathbf{U}}$ & $\mathrm{ug} / \mathrm{L}$ & $\mathbf{E X}$ & $<\mathrm{EQL}$ \\
\hline$<20$ & $<10$ & $<10$ & $<50$ & 1 & $\mathbf{U}$ & $\mathrm{ug} / \mathrm{L}$ & $\mathbf{E X}$ & $<$ EQL \\
\hline$<5$ & $<20$ & $<5$ & $<50$ & 1 & $\mathbf{U}$ & ug/L & EX & $<\mathrm{EQL}$ \\
\hline \multirow[t]{2}{*}{$<10$} & $<10$ & $<10$ & $<10$ & 1 & $\mathbf{U}$ & $\mathrm{ug} / \mathrm{L}$ & EX & $<\mathbf{E Q L}$ \\
\hline & & 13.5 & $<200$ & 1 & $\mathbf{U}$ & ug/L & $\mathbf{E X}$ & $<\mathrm{EQL}$ \\
\hline 17.7 & 14.9 & 16.9 & $<100$ & 1 & $\mathbf{U}$ & ug/L & $\mathbf{E X}$ & $<\mathbf{E Q L}$ \\
\hline 4.7 & 2.7 & 4.1 & $<10$ & 1 & $\mathbf{U}$ & ug/L & EX & < EQL \\
\hline 7.44 & 7 & 11 & 9.53 & 1 & & ug/L & EX & 9.53 \\
\hline$<5$ & $<5$ & $<5$ & $<5$ & 1 & $\mathbf{U}$ & ug/L & EX & $<$ EQL \\
\hline
\end{tabular}

Note: Flagging, dilution factors, modifiers, and laboratories are for fourth quartet 998 data only. See Appendix B for flagging criteria.

* = exceeded holding time for fourth quarter 1998 .

+ = exceded screening level or final primary drinking water standard for fourth quarter 998 . 
WELL: LFW 10A

\section{ANALYTICAL DATA}

\section{H ST Analyte}

Bromoform

Bromomethane (Methyl bromide)

Cadmium, total recoverable

Carbon disulfide

Carbon tetrachloride

Chlorobenzene

Chloroethane

+ Chloroethene (Vinyl chloride)

Chloroform

Chloromethane (Methyl chloride)

Chloroprene

Chromium, total recoverable

Dibromochloromethane

Dibromomethane (Methylene bromide)

Dichlorodiftuoromethane

+ Dichloromethane (Methylene chloride)

Ethyl methacrylate

Ethylbenzene

Gross alpha

Iodomethane (Methyl iodide)

+ Iron, total recoverable

Isobutyl alcohol

Lead, total recoverable

Mercury, total recoverable

Methacrylonitrile

Methyl ethyl ketone

Methyl isobutyl ketone

Methyl methacrylate

Pentachloroethane

Propionitrile

Selenium, total recoverable

Silver, total recoverable

Styrene

Tetrachloroethylene

Toluene

Trichloroethylene

Trichlorofluoromethane

+ Tritium

Vinyl acetate

Xylenes

cis-1,2-Dichloroethylene

cis-1,3-Dichloropropene

$\begin{array}{cccc}101998 & 201998 & 301998 & 40 \\ <5 & <5 & <5 & 1.8 \\ <10 & <10 & <2 \\ <4.7 & <2 & <5 \\ <5 & <5 & <5 \\ <5 & <5 & 1.1 \\ 1.31 & <5 & 5 \\ <10 & 4.5 & 36 \\ 34.8 & 24 & <5 \\ <5 & <5 & <10 \\ <10 & <10 & <5 \\ <5 & <5 & <3 \\ 17.3 & <3 & <5 \\ <5 & <5 & <5 \\ <5 & <5 & 2.6 \\ 7.24 & 2.9 & 190 \\ <9.93 & 100 & \end{array}$

\begin{tabular}{|c|c|c|c|c|c|c|c|c|}
\hline 43.5 & 56 & 69 & 65.9 & 1 & & $u g / L$ & EX & 65.9 \\
\hline$<1.92$ & $<-1.48$ & 3.07 & $<.99$ & 1 & UI & $\mathrm{pCi} / \mathrm{L}$ & TM & $<$ EQL \\
\hline \multirow[t]{2}{*}{$<5$} & $<5$ & $<5$ & $<5$ & 1 & $\mathbf{U}$ & $\mathbf{u g} / \mathbf{L}$ & EX & $<\mathrm{EQL}$ \\
\hline & & 36600 & 37000 & I & & $\mathbf{u g} / \mathbf{L}$ & EX & 37000 \\
\hline$<100$ & $<100$ & $<100$ & & & & & & \\
\hline$<47$ & $<5$ & 3.3 & $<100$ & 1 & $\mathbf{U}$ & $\mathrm{ug} / \mathrm{L}$ & EX & $<\mathrm{EQL}$ \\
\hline$<.7$ & $<.2$ & $<.2$ & $<.5$ & 1 & $\mathbf{U}$ & ug/L & EX & $<$ EQL \\
\hline$<10$ & $<5$ & $<5$ & $<500$ & 1 & $\mathbf{U}$ & $\mathbf{u g} / \mathbf{L}$ & $\mathbf{E X}$ & $<$ EQL \\
\hline$<237$ & $<150$ & $<41$ & $<36.3$ & 1 & & ug $/ \mathbf{L}$ & $\mathbf{E X}$ & 36.3 \\
\hline \multirow[t]{3}{*}{$<152$} & $<110$ & $<170$ & $<133$ & 1 & & $\mathbf{u g} / \mathbf{L}$ & $\mathbf{E X}$ & 133 \\
\hline & $<5$ & $<5$ & $<50$ & 1 & $\mathbf{U}$ & $\mathrm{ug} / \mathrm{L}$ & EX & $<$ EQL \\
\hline & & & $<200$ & 1 & $\mathbf{U}$ & ug/L & EX & $<$ EQL \\
\hline
\end{tabular}

$<50<5<5$

$<66<5$

$<5<2$

$<5<5$

$4.34 \quad 2.7 \quad 3.6$

$\begin{array}{llllllll}53.3 & 88 & 130 & 133 & 1 & \text { ug/L } & \text { EX } & 133\end{array}$

$\begin{array}{lllllllll}1.78 & 2.2 & 4.2 & <5 & 1 & \mathrm{U} & \mathrm{ug} / \mathrm{L} & \mathrm{EX} & <\mathrm{EQL}\end{array}$

$\begin{array}{lllllllll}23.6 & 19 & <5 & <5 & 1 & \mathrm{U} & \mathrm{ug} / \mathrm{L} & \mathrm{EX} & <\mathrm{EQL}\end{array}$

$\begin{array}{lllllllll}27.71 & 13400 & 20.33 & 23.87 & 1 & \mathrm{pC} / \mathrm{ml} & \mathrm{TM} & >20\end{array}$

$<10 \quad<5 \quad<5 \quad<20 \quad 1 \quad \mathrm{U} \quad \mathrm{ug} / \mathrm{L} \quad \mathrm{EX}<\mathrm{EQL}$

$\begin{array}{llllllll}138 & 200 & 250 & 209 & 1 & \text { ug/L } & \text { EX } & 209\end{array}$

$\begin{array}{lllllllll} & & & <5 & 1 & \mathrm{U} & \mathrm{ug} / \mathrm{L} & \mathrm{EX} & <\mathrm{EQL} \\ & <5 & <5 & <5 & 1 & \mathrm{U} & \text { ug/L } & \text { EX } & <\mathrm{EQL}\end{array}$

Note: Flagging, dilution factors, modifiers, and laboratories are for fourth quartet 998 data only. See Appendix B for fagging criteria. * = exceded holding time for fourth quarter 1998.

$+=$ exceeded screening level or final primary drinking water standard for fourth quarten 998 . 
WSRC-TR-99-00011 Unclassified

WELL: LFW 10A

\section{ANALYTICAL DATA}

H ST Analyte

trans-1,2-Dichloroethylene trans-1,3-Dichloropropene trans-1,4-Dichloro-2-butene

\section{$101998 \quad \underline{201998} \quad 301998 \quad 401998 \quad$ DFMod Unit}

$<5$

$<5$

$<20$

$<5$
$<5$

$<5$

$<20$
Compliance

Lab Filter
$<5$

$<5$

\section{$1 U$ ug/L}

$1 \mathrm{U} \quad \mathrm{ug} / \mathrm{L}$

$1 \mathrm{U}$ ug/L
EX $<$ EQL

EX $<$ EQL

Note: Flagging, dilution factors, modifiers, and laboratories are for fourth quarte1998 data only. See Appendix B for flagging criteria.

* = exceeded holding time for fourth quarter 1998.

$+=$ exceeded screening level or final primary drinking water standard for fourth quarteri 998 . 
WSRC-TR-99-00011

Unclassified

WELL: LFW 18

\begin{tabular}{|c|c|c|c|c|c|c|}
\hline $\begin{array}{l}\text { SRS Coord } \\
\text { N } 84577.3 \\
\text { E } 45459.4\end{array}$ & $\begin{array}{l}\text { Lat/Longitude } \\
\text { 33.287 Deg N } \\
\text { 81.711 Deg W }\end{array}$ & $\frac{\text { Screen Zone Elevation }}{167.7-137.7 \mathrm{ft} \mathrm{msl}}$ & $\frac{\text { Top of Casing }}{183.9 \mathrm{ft} \mathrm{msl}}$ & $\frac{\text { Casing }}{4^{\text {"PVC }}}$ & $\frac{\text { Pump }}{S}$ & $\frac{\text { Formation }}{\text { U Steed Pond }}$ \\
\hline
\end{tabular}

SAMPLE DATE

$12 / 01 / 97$

$03 / 16 / 98$

$06 / 12 / 98$

$09 / 16 / 98$

FIELD DATA

Analyte

Water Elevation

Depth to water

pH

Sp. Conductance

Water temperature

Alkalinity as $\mathrm{CaCO} 3$

Turbidity

Volumes purged

Sampling code

\begin{tabular}{|c|c|c|c|c|}
\hline 101998 & 201998 & 301998 & 401998 & Unit \\
\hline 151.3 & 154.1 & 153.75 & 153.8 & ft msl \\
\hline 30.35 & 27.55 & 27.9 & 27.85 & ft \\
\hline 5.8 & 5.7 & 6 & 5.2 & pH \\
\hline 120 & 100 & 110 & 100 & $\mathrm{uS} / \mathrm{cm}$ \\
\hline 17 & 19.7 & 22.6 & 20.9 & deg. $C$ \\
\hline 33 & 16 & 29 & 37 & $\mathrm{mg} / \mathrm{L}$ \\
\hline .6 & 2.3 & .9 & 1 & NTU \\
\hline 3.35136 & 3.01295 & 5.80878 & 2.06893 & well volumes \\
\hline
\end{tabular}

\section{ANALYTICAL DATA}

H ST Analyte

1,1,1,2-Tetrachloroethane

1,1,1-Trichloroethane

1,1,2,2-Tetrachloroethane

1,1,2-Trichloroethane

1,1-Dichloroethane

1,1-Dichloroethylene

1,2,3-Trichloropropane

1,2-Dibromo-3-chloropropane

1,2-Dibromoethane

1,2-Dichlorobenzene

1,2-Dichloroethane

1,2-Dichloropropane

1,3-Dichlorobenzene

1,4-Dichlorobenzene

1,4-Dioxane

2-Hexanone

Acetone

Acetonitrile (Methyl cyanide)

Acrolein

Acrylonitrile

Allyl chloride

Aluminum, total recoverable

Arsenic, total recoverable

Barium, total recoverable

Benzene

Bromodichloromethane

\begin{tabular}{|c|c|c|}
\hline 101998 & $\underline{201998}$ & 301998 \\
\hline$<5$ & $<5$ & $<5$ \\
\hline$<5$ & $<5$ & $<5$ \\
\hline$<5$ & $<5$ & $<5$ \\
\hline$<5$ & $<5$ & $<5$ \\
\hline 1.02 & $<5$ & $<5$ \\
\hline$<5$ & $<5$ & $<5$ \\
\hline$<5$ & $<5$ & $<5$ \\
\hline$<5$ & $<5$ & $<5$ \\
\hline$<5$ & $<5$ & $<5$ \\
\hline$<5$ & $<5$ & $<5$ \\
\hline$<5$ & $<5$ & $<5$ \\
\hline 6.31 & 5.8 & 2.7 \\
\hline$<10$ & $<10$ & $<10$ \\
\hline$<5.38$ & $<10$ & $<3.6$ \\
\hline$<\mathbf{2 0}$ & $<20$ & $<20$ \\
\hline$<20$ & $<10$ & $<10$ \\
\hline$<5$ & $<20$ & $<5$ \\
\hline$<10$ & $<10$ & $\begin{array}{c}<10 \\
14.9\end{array}$ \\
\hline 18.1 & 17 & 12.8 \\
\hline 3.4 & 2.5 & 3.1 \\
\hline$<5$ & $<5$ & $<5$ \\
\hline$<5$ & $<5$ & $<5$ \\
\hline
\end{tabular}

Compliance

Lab Filter

$\begin{array}{llllll}< & 1 & \mathbf{U} & \text { ug/L } & \text { EX } & <\text { EQL }\end{array}$

$<5 \quad 1 \quad U \quad$ ug/L $\quad$ EX $<$ EQL

$\begin{array}{llllll}<5 & \text { I } & \text { U } & \text { ug/L } & \text { EX } & <\text { EQL }\end{array}$

$<5 \quad 1 \quad$ U $\quad$ ug/L $\quad$ EX $<$ EQL

$<5 \quad 1 \quad \mathrm{U} \quad$ ug/L $\quad$ EX $<$ EQL

$\begin{array}{lllllll}<5 & & 1 & \mathrm{U} & \mathrm{ug} / \mathrm{L} & \mathrm{EX} & <\mathrm{EQL}\end{array}$

$<5 \quad 1 \quad U \quad \mathrm{ug} / \mathrm{L} \quad \mathrm{EX} \quad<$ EQL

$<5 \quad 1 \quad \mathrm{U} \quad \mathrm{ug} / \mathrm{L} \quad \mathrm{EX}<\mathrm{EQL}$

$\begin{array}{lllllll}<5 & 1 & \mathrm{U} & \mathrm{ug} / \mathrm{L} & \mathrm{EX} & <\text { EQL }\end{array}$

$\begin{array}{lllllll}<5 & 1 & \mathrm{U} & \mathrm{ug} / \mathrm{L} & \mathrm{EX} & <\mathrm{EQL}\end{array}$

$<5 \quad 1 \quad \mathrm{U} \quad$ ug/L $\mathrm{EX} \quad \mathrm{EQL}$

$\begin{array}{llllll}4.94 & 1 & \mathrm{~J} & \mathrm{ug} / \mathrm{L} & \mathrm{EX} & \text { NDD }\end{array}$

$<1000 \quad 1 \quad \mathrm{U} \quad \mathrm{ug} / \mathrm{L} \quad \mathrm{EX}<\mathrm{EQL}$

$<5 \quad 1 \quad \mathrm{U} \quad \mathrm{ug} / \mathrm{L} \quad \mathrm{EX} \quad<\mathrm{EQL}$

$<20 \quad 1 \quad$ U $\quad$ ug/L $\quad$ EX $<$ EQL

$<500 \quad 1 \quad$ U $\quad$ ug/L $\quad$ EX $\quad<$ EQL

$<50 \quad 1 \quad$ U $\quad$ ug/L $\quad$ EX $<$ EQL

$<50 \quad 1 \quad$ U $\quad$ ug/L $\quad$ EX $<$ EQL

$<10 \quad 1 \quad U \quad$ ug/L $\quad$ EX $<$ EQL

$<200 \quad 1 \quad U \quad$ ug/L $\quad$ EX $\quad<$ EQL

$<100 \quad 1 \quad \mathrm{U}$ ug/L EX $<$ EQL

$<10 \quad 1 \quad \mathrm{U} \quad \mathrm{ug} / \mathrm{L} \quad$ EX $<$ EQL

$<5 \quad 1 \quad$ U $\quad$ ug/L $\quad$ EX $\quad<$ EQL

$<5 \quad 1 \quad \mathrm{U}$ ug/L EX $<$ EQL

Note: Flagging, dilution factors, modifiers, and laboratories are for fourth quartei 998 data only. See Appendix B for flagging criteria.

* = exceeded holding time for fourth quarter 1998.

+ = exceeded screening level or final primary drinking water standard for fourth quarteri 998 .

Sanitary Landfill

Fourth Quarter 1998 and Annual Summary 
WELL: LFW 18

\section{ANALYTICAL DATA}

H ST Analyte

Bromoform

Bromomethane (Methyl bromide)

Cadmium, total recoverable

Carbon disulfide

Carbon tetrachloride

Chlorobenzene

Chloroethane

Chloroethene (Vinyl chloride)

Chloroform

Chloromethane (Methyl chloride)

Chloroprene

Chromium, total recoverable

Dibromochloromethane

Dibromomethane (Methylene bromide)

Dichlorodifluoromethane

Dichloromethane (Methylene chloride)

Ethyl methacrylate

Ethylbenzene

Gross alpha

Iodomethane (Methyl iodide)

+ Iron, total recoverable

Isobutyl alcohol

Lead, total recoverable

Mercury, total recoverable

Methacrylonitrile

Methyl ethyl ketone

Methyl isobutyl ketone

Methyl methacrylate

Pentachloroethane

Propionitrile

Selenium, total recoverable

Silver, total recoverable

Styrene

Tetrachloroethylene

Toluene

Trichloroethylene

Trichlorofluoromethane

Tritium

Vinyl acetate

Xylenes

cis-1,2-Dichloroethylene

cis-1,3-Dichloropropene

$\begin{array}{lllllllll}<5 & <5 & <5 & <5 & 1 & \mathrm{U} & \mathrm{ug} / \mathrm{L} & \mathrm{EX} & <\mathrm{EQL}\end{array}$

$<10 \quad<10 \quad<10 \quad<5 \quad 1 \quad \mathrm{U} \quad \mathrm{ug} / \mathrm{L} \quad \mathrm{EX} \quad<\mathrm{EQL}$

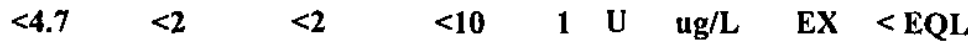

$\begin{array}{lllllllll}< & <5 & <5 & <5 & 1 & \mathrm{U} & \mathrm{ug} / \mathrm{L} & \mathrm{EX} & <\mathrm{EQL}\end{array}$

$\begin{array}{lllllllll}<5 & <5 & <5 & <5 & 1 & \mathrm{U} & \mathrm{ug} / \mathrm{L} & \mathrm{EX} & <\mathrm{EQL}\end{array}$

$\begin{array}{lllllllll}1.79 & 1.7 & .8 & 1.54 & 1 & \mathrm{~J} & \mathrm{ug} / \mathrm{L} & \mathrm{EX} & \mathrm{NDD}\end{array}$

$\begin{array}{lllllllll}10 & <10 & <10 & <10 & \text { l } & \text { U } & \text { ug/L } & \text { EX } & <\text { EQL }\end{array}$

$\begin{array}{lllllllll}3.24 & 2.8 & 1.1 & <5 & 1 & \text { U } & \text { ug/L } & \text { EX } & <\text { EQL }\end{array}$

$\begin{array}{lllllllll}<5 & <5 & <5 & <5 & 1 & \text { U } & \text { ug/L } & \text { EX } & <\text { EQL }\end{array}$

$<10<10 \quad<10 \quad<5 \quad 110$ ug/L $\quad$ EX $<$ EQL

$\begin{array}{lllllllll}< & <5 & <5 & <50 & 1 & \text { U } & \text { ug/L } & \text { EX } & <\text { EQL }\end{array}$

$\begin{array}{lllllllll}<1.1 & <3 & <3 & <10 & 1 & \mathrm{U} & \mathrm{ug} / \mathrm{L} & \mathrm{EX} & <\mathrm{EQL}\end{array}$

$\begin{array}{lllllllll}<5 & <5 & <5 & <5 & 1 & \text { U } & \text { ug/L } & \text { EX } & <\text { EQL }\end{array}$

$\begin{array}{llllllllll}<5 & <5 & <5 & <5 & 1 & \mathrm{U} & \mathrm{ug} / \mathrm{L} & \mathrm{EX} & <\mathrm{EQL}\end{array}$

$\begin{array}{llllllllll}<10 & <5 & <5 & <5 & 1 & \mathrm{U} & \mathrm{ug} / \mathrm{L} & \mathrm{EX} & <\mathrm{EQL}\end{array}$

$\begin{array}{lllllllll}<.14 & <2.7 & <2.2 & <10 & 1 & \mathrm{U} & \mathrm{ug} / \mathrm{L} & \mathrm{EX} & <\mathrm{EQL}\end{array}$

$<5 \quad 1 \quad \mathrm{U} \quad$ ug/L $\quad$ EX $<$ EQL

$\begin{array}{lllllllll}<5 & <5 & <5 & <5 & 1 & \mathrm{U} & \mathrm{ug} / \mathrm{L} & \mathrm{EX} & <\mathrm{EQL}\end{array}$

$\begin{array}{llllllll}1.43 & <.36 & 2.14 & 1.1 & 1 & \mathrm{pCi} / \mathrm{L} & \mathrm{TM} & 1.1\end{array}$

$\begin{array}{lllllllll}<5 & <5 & <5 & <5 & 1 & \mathrm{U} & \mathrm{ug} / \mathrm{L} & \mathrm{EX} & <\mathrm{EQL}\end{array}$

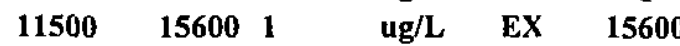

$\begin{array}{ccccccccc}<100 & <100 & <100 & & & & & \\ <47 & 9.6 & 4.2 & <100 & 1 & \mathrm{U} & \mathrm{ug} / \mathrm{L} & \mathrm{EX} & <\mathrm{EOL}\end{array}$

$\begin{array}{lllllllll}<.7 & <.2 & <.2 & <.5 & 1 & \mathrm{U} & \mathrm{ug} / \mathrm{L} & \mathrm{EX} & <\mathrm{EQL}\end{array}$

$\begin{array}{lllllllll}<10 & <5 & <5 & <500 & 1 & \mathrm{U} & \mathrm{ug} / \mathrm{L} & \mathrm{EX} & <\mathrm{EQL}\end{array}$

$<3.28<10 \quad<10 \quad<10 \quad 11 \quad$ U $\quad$ ug/L $\quad$ EX $<$ EQL

$\begin{array}{lllllllll}<.44 & <12 & <12 & <5 & 1 & \mathrm{U} & \mathrm{ug} / \mathrm{L} & \mathrm{EX} & <\mathrm{EQL}\end{array}$

$\begin{array}{llllllll}<5 & <5 & <50 & 1 & \mathrm{U} & \mathrm{ug} / \mathrm{L} & \mathrm{EX} & <\mathrm{EQL}\end{array}$

$<200 \quad 1 \quad$ U $\quad$ ug/L $\quad$ EX $\quad<$ EQL

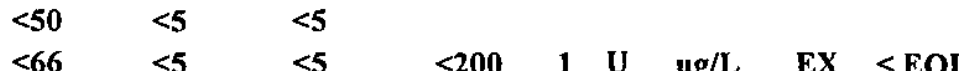

$\begin{array}{lllllllll}<66 & <5 & <5 & <200 & 1 & \text { U } & \text { ug/L } & \text { EX } & <\text { EQL }\end{array}$

$<5<2<2 \quad<20 \quad 1 \quad$ U $\quad$ ug/L $\quad$ EX $\quad<$ EQL

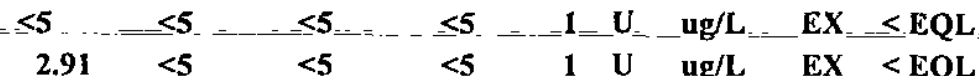

$\begin{array}{lllllllll}<5 & <5 & <5 & <5 & 1 & \mathrm{U} & \mathrm{ug} / \mathrm{L} & \mathrm{EX} & <\mathrm{EQL}\end{array}$

$\begin{array}{lllllllll}1.14 & <5 & <5 & <5 & 1 & \mathrm{U} & \mathrm{ug} / \mathrm{L} & \mathrm{EX} & <\mathrm{EQL}\end{array}$

$\begin{array}{lllllllll}<5 & <5 & <5 & <5 & 1 & \mathrm{U} & \mathrm{ug} / \mathrm{L} & \mathrm{EX} & <\mathrm{EQL}\end{array}$

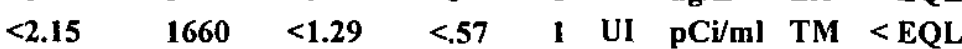

$<10<5 \quad<5 \quad<20 \quad 1 \quad$ U $\quad$ ug/L $\quad$ EX $<$ EQL

$<5<5 \quad<5 \quad<10 \quad 1 \quad$ U $\quad$ ug/L $\quad$ EX $\quad<$ EQL

$<5 \quad 1 \quad$ U $\quad$ ug/L $\quad$ EX $\quad<$ EQL

$\begin{array}{llllllllll}< & <5 & <5 & <5 & 1 & \mathrm{U} & \mathrm{ug} / \mathrm{L} & \mathrm{EX} & <\mathrm{EQL}\end{array}$

Note: Flagging, dilution factors, modifiers, and laboratories are for fourth quartei 998 data only. See Appendix B for fagging criteria. * = exceeded holding time for fourth quarter 1998.

$+=$ exceeded screening level or final primary drinking water standard for fourth quarten 998 
WSRC-TR-99-00011

Unclassified

WELL: LFW 18

ANALYTICAL DATA

\section{H ST Analyte}

trans-1,2-Dichloroethylene trans-1,3-Dichloropropene trans-1,4-Dichloro-2-butene $\begin{array}{lllll}101998 & 201998 \quad 301998 \quad 401998 \quad \text { DFMOd Unit }\end{array}$

$<5$

$<5$

$<\mathbf{2 0}$
$<5$

$<5$
$<5$

$<5$

$<20$
Compliance

Lab Filter

EX $<$ EQL

EX $<$ EQL

EX $<$ EQL

Note: Flagging, dilution factors, modifiers, and laboratories are for fourth quartet 998 data only. See Appendix B for fagging criteria. * = exceeded holding time for fourth quarter 1998 .

$+=$ exceeded screening level or final primary drinking water standard for fourth quarten 998 . 
WSRC-TR-99-0001 1

Unclassified

WELL: LFW 21

\begin{tabular}{|c|c|c|c|c|c|c|}
\hline RS Coord. & Lat/Lengitude & Screen Zone Elevation & Top of Casing & Casing & Pump & Forn \\
\hline $\begin{array}{l}\text { N } 84178.3 \\
\text { E } 46149.4\end{array}$ & $\begin{array}{l}\text { 33.287 Deg N } \\
\text { 81.709 Deg W }\end{array}$ & $37.9 \mathrm{ft} \mathrm{msl}$ & & $4 " \mathrm{PVC}$ & & ond \\
\hline
\end{tabular}

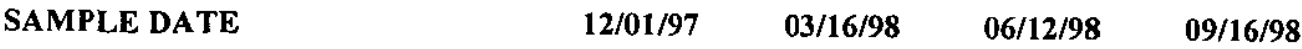

FIELD DATA

Analyte
Water Elevation
Depth to water
pH
Sp. Conductance
Water temperature
Alkalinity as CaCO3
Turbidity
Volumes purged
Sampling code

ANALYTICAL DATA

\section{H ST Analyte}

1,1,1,2-Tetrachloroethane

1,1,1-Trichloroethane

1,1,2,2-Tetrachloroethane

1,1,2-Trichloroethane

+ 1,1-Dichloroethane

1,1-Dichloroethylene

1,2,3-Trichloropropane

1,2-Dibromo-3-chloropropane

1,2-Dibromoethane

1,2-Dichlorobenzene

1,2-Dichloroethane

1,2-Dichloropropane

1,3-Dichlorobenzene

1,4-Dichlorobenzene

1,4-Dioxane

2-Hexanone

Acetone

$\frac{101998}{147.46}$
35.45
6.2
300
19
130
4.2
6.38420

201998
150.16
32.75
6
200
20
115
1.5
8.57470

301998
149.63
33.28
6.3
220
22
83
3.5
7.69339

\begin{tabular}{l}
401998 \\
\hline 149.04 \\
33.87 \\
6.6 \\
200 \\
20.7 \\
98 \\
4.6 \\
3.32886
\end{tabular}

101998

$$
\begin{aligned}
& <5 \\
& <5 \\
& <5 \\
& <5 \\
& 7 \\
& <5 \\
& <5 \\
& <5 \\
& <5 \\
&
\end{aligned}
$$

$\begin{array}{lll}<5 & <5 & <5 \\ <5 & <5 & <5 \\ <5 & <5 & <5 \\ <5 & <5 & <5 \\ 79.2 & 40 & \\ <5 & <5 & \\ <5 & <5 & <5 \\ <5 & <5 & <5 \\ <5 & <5 & <5 \\ & & \\ <5 & <5 & <5 \\ 1.92 & <5 & <5\end{array}$

Unit
ft msl
ft
pH
uS/cm
deg. C
mg/L
NTU
well volumes

Acetonitrile (Methyl cyanide)

Acrolein

Acrylonitrile

Allyl chloride

Aluminum, total recoverable

Arsenic, total recoverable

Barium, total recoverable

Benzene

Bromodichloromethane 
WSRC-TR-99-00011

Unclassified

WELL: LFW 21

\section{ANALYTICAL DATA}

H ST Analyte

\section{Bromoform \\ Bromomethane (Methyl bromide) \\ Cadmium, total recoverable \\ Carbon disulfide \\ Carbon tetrachloride \\ Chlorobenzene \\ Chloroethane \\ + Chloroethene (Vinyl chloride) \\ Chloroform \\ Chloromethane (Methyl chloride) \\ Chloroprene}

Chromium, total recoverable

Dibromochloromethane

Dibromomethane (Methylene bromide)

Dichlorodifuoromethane

Dichloromethane (Methylene chloride)

Ethyl methacrylate

Ethylbenzene

Gross alpha

Iodomethane (Methyl iodide)

+ Iron, total recoverable

Isobutyl alcohol

Lead, total recoverable

Mercury, total recoverable

Methacrylonitrile

Methyl ethyl ketone

Methyl isobutyl ketone

Methyl methacrylate

Pentachloroethane

Propionitrile

Selenium, total recoverable

Silver, total recoverable

Styrene

Tetrachloroethylene

Toluene

Trichloroethylene

Trichlorofluoromethane

Tritium

Vinyl acetate

Xylenes

cis-1,2-Dichloroethylene

cis-1,3-Dichloropropene

\section{8}

$$
<5
$$$$
<
$$$$
<4
$$$$
<5
$$$$
<5
$$

2.15

$<10$

3.24

$<5$$$
<1
$$$$
<
$$

$<1.7<5$

$<5<5$

$<5<5<5$

$<10 \quad<5 \quad 1.3$

$<5.18<4<5.4$$$
3
$$

$34 \quad 5.4$

$4.25<.19$

$<5$

$\begin{array}{rll} & 5.6 & <5 \\ & & 15600 \\ <100 & <100 & <100\end{array}$

\begin{tabular}{ccc}
8.4 & 4.2 & \multicolumn{1}{c}{9.1} \\
$<.7$ & .05 & .05 \\
$<10$ & $<5$ & $<5$ \\
$<10$ & $<10$ & $<10$ \\
$<10$ & $<12$ & $<12$ \\
& $<5$ & $<5$
\end{tabular}

$<50<5<$

$<66<5<5$

$<5<2 \quad<2$

$<5<5<5$

$<5<5<5$

13.922 .6

$\begin{array}{lll}1.02 & .75 & 1.2\end{array}$

$\begin{array}{lll}7.08 & 3.9 & <5\end{array}$

$<10.2 \quad 4120$

$<10$

98.1

$<5$

14

2.56

$<5$

29

$<5 \quad<5 \quad<5$
Compliance

401998 DFMod Unit

$\begin{array}{llllll}<5 & 1 & \mathrm{U} & \mathrm{ug} / \mathrm{L} & \mathrm{EX} & <\mathrm{EQL}\end{array}$

$<5 \quad 1 \quad U \quad$ ug/L $\quad$ EX $<$ EQL

$<10 \quad 1$ U ug/L EX $<$ EQL

$<5$ I U ug/L EX $<$ EQL

$<5 \quad 1 \quad \mathrm{U} \quad \mathrm{ug} / \mathrm{L} \quad \mathrm{EX} \quad<\mathrm{EQL}$

$\begin{array}{llllll}2.25 & 1 & J & \mathrm{ug} / \mathrm{L} & \mathrm{EX} & \text { NDD }\end{array}$

$<10 \quad$ I U ug/L EX $<$ EQL

$\begin{array}{llllll}18.8 & 1 & \text { ug/L } & \text { EX } & 18.8\end{array}$

$\begin{array}{llllll}<5 & 1 & \mathrm{U} & \mathbf{u g} / \mathrm{L} & \mathrm{EX} & <\mathrm{EQL}\end{array}$

$<5 \quad 1 \quad$ U $\quad$ ug/L $\quad$ EX $<$ EQL

$<50$ I U ug/L EX $<$ EQL

$<10$ I U ug/L EX $<$ EQL

$\begin{array}{llllll}<5 & 1 & \mathrm{U} & \mathrm{ug} / \mathrm{L} & \mathrm{EX} & <\text { EQL }\end{array}$

$\begin{array}{llllll}<5 & 1 & \mathbf{U} & \mathrm{ug} / \mathrm{L} & \mathrm{EX} & <\mathrm{EQL}\end{array}$

$<5 \quad 1 \quad \mathrm{U} \quad \mathrm{ug} / \mathrm{L} \quad \mathrm{EX}<\mathrm{EQL}$

$\begin{array}{llllll}4.07 & 1 & J & u g / L & E X & \text { NDD }\end{array}$

$\begin{array}{llllll}<5 & 1 & \mathrm{U} & \mathrm{ug} / \mathrm{L} & \mathrm{EX} & <\mathrm{EQL}\end{array}$

$\begin{array}{lllll}9.63 & 1 & \mathrm{ug} / \mathrm{L} & \mathrm{EX} & 9.63\end{array}$

$\begin{array}{lllll}2.3 & 1 & \mathrm{pCi} / \mathrm{L} & \mathrm{TM} & 2.3\end{array}$

$\begin{array}{llllll}<5 & 1 & \mathrm{U} & \mathrm{ug} / \mathrm{L} & \mathrm{EX} & <\mathrm{EQL}\end{array}$

$\begin{array}{llll}199001 & \mathrm{ug} / \mathrm{L} & \mathrm{EX} & 19900\end{array}$

$<100 \quad 1 \quad U \quad u g / L \quad$ EX $<$ EQL

$<.5 \quad 1 \quad \mathrm{U} \quad \mathrm{ug} / \mathrm{L} \quad \mathrm{EX}<\mathrm{EQL}$

$<500 \quad 1 \quad$ U $\quad$ ug/L $\quad$ EX $<$ EQL

$<10 \quad 1 \quad \mathrm{U} \quad \mathrm{ug} / \mathrm{L} \quad \mathrm{EX} \quad<$ EQL

$<5 \quad 1 \quad$ U $\quad$ ug/L $\quad$ EX $\quad<$ EQL

$<501 \mathrm{U} \quad \mathrm{ug} / \mathrm{L} \quad \mathrm{EX} \quad<$ EQL

$<2001 \mathrm{U}$ ug/L EX $<$ EQL

$<200 \quad$ I U U U / L EX $\quad$ EQL

$<20 \quad 1 \quad \mathrm{U} \quad \mathrm{ug} / \mathrm{L} \quad \mathrm{EX} \quad<\mathrm{EQL}$

$<5 \quad 1 \quad \mathrm{U} \quad \mathrm{ug} / \mathrm{L} \quad$ EX $\quad<$ EQL

$<5 \quad 1 \quad \mathrm{U} \quad \mathrm{ug} / \mathrm{L} \quad \mathrm{EX} \quad<\mathrm{EQL}$

$\begin{array}{llllll}2.9 & 1 & J & \text { ug/L } & \text { EX } & \text { NDD }\end{array}$

$<5 \quad 1 \quad \mathrm{U} \quad \mathrm{ug} / \mathrm{L} \quad \mathrm{EX} \quad<$ EQL

$<5$ I U ug/L EX $<$ EQL

$\begin{array}{lllll}3.14 & \mathrm{pCi} / \mathrm{ml} & \text { TM } & 3.14\end{array}$

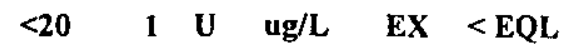

$\begin{array}{lllll}29.6 & 1 & \mathrm{ug} / \mathrm{L} & \mathrm{EX} & 29.6\end{array}$

$\begin{array}{lllll}52.5 & 1 & \mathrm{ug} / \mathrm{L} & \mathrm{EX} & 52.5\end{array}$

$<5 \quad 1 \quad$ U $\quad$ ug/L EX $\quad$ EQL

Note: Flagging, dilution factors, modifiers, and laboratories are for fourth quarteit 998 data only. See Appendix B for flagging criteria. * = exceeded holding time for fourth quarter 1998.

$+=$ exceeded screening level or final primary drinking water standard for fourth quarten 998 . 
WSRC-TR-99-00011

Unclassified

WELL: LFW 21

ANALYTICAL DATA

H ST Analyte

\begin{tabular}{ccccccccc}
101998 & 201998 & 301998 & & 401998 & \multicolumn{2}{c}{ DFMod Unit } & Lab & Filter \\
.941 & & & $<5$ & 1 & U & ug/L & EX & $<$ EQL \\
$<5$ & $<5$ & $<5$ & $<5$ & 1 & U & ug/L & EX & $<$ EQL \\
$<20$ & $<5$ & $<5$ & $<20$ & 1 & U & ug/L & EX & $<$ EQL
\end{tabular}

trans-1,2-Dichloroethylene trans-1,3-Dichloropropene

trans-1,4-Dichloro-2-butene

Compliance

Lab Filter

$$
\text { EX }<\text { EQL }
$$

Note: Flagging, dilution factors, modifiers, and laboratories are for fourth quartel 998 data only. See Appendix B for flagging criteria.

* = exceeded holding time for fourth quarter 1998.

$+=$ exceeded screening level or final primary drinking water standard for fourth quarterf 998 . 
WSRC-TR-99-00011

Unclassified

WELL: LFW 23R

\begin{tabular}{|c|c|c|c|c|c|c|}
\hline SRS Coord. & Lat/Longitude & Screen Zone Elevation & Top of Casing & Casing & Pump & Eormation \\
\hline $\begin{array}{l}\text { N } 84206.1 \\
\text { E } 46512.9\end{array}$ & $\begin{array}{ll}\text { 33.288 } & \text { Deg } N \\
\text { 81.708 } & \text { Deg W }\end{array}$ & $138.2-118.2$ ft msl & $1703 \mathrm{ft} \mathrm{mcl}$ & VC & $\mathbf{V}$ & ond \\
\hline
\end{tabular}

SAMPLE DATE

$12 / 05 / 97 \quad 03 / 16 / 98 \quad 06 / 12 / 98$

\section{FIELD DATA}

Analyte
Water Elevation
Depth to water
pH
Sp. Conductance
Water temperature
Alkalinity as CaCO3
Turbidity
Volumes purged
Sampling code

ANALYTICAL DATA

H ST Analyte

1,1,1,2-Tetrachloroethane
1,1,1-Trichloroethane
1,1,2,2-Tetrachloroethane
1,1,2-Trichloroethane
1,1-Dichloroethane
1,1-Dichloroethylene
1,2,3-Trichloropropane
1,2-Dibromo-3-chloropropane
1,2-Dibromoethane
1,2-Dichloroethane
1,2-Dichloropropane
1,4-Dichlorobenzene
2-Hexanone
Acetone
Acetonitrile (Methyl cyanide)
Acrolein
Acrylonitrile
Allyl chloride
Aluminum, total recoverable
Arsenic, total recoverable
Barium, total recoverable
Benzene
Bromodichloromethane
Bromoform
Bromomethane (Methyl bromide)
Cadmium, total recoverable

\begin{tabular}{lllll}
101998 & $\underline{201998}$ & $\underline{301998}$ & 401998 & Unit \\
\hline 147.37 & 150.02 & 149.22 & & ft msl \\
20.85 & 18.2 & 19 & ft \\
4.2 & 4.8 & 4.6 & $\mathrm{pH}$ \\
30 & 28 & 24 & uS/cm \\
22 & 16 & 16 & deg. C \\
0 & 0 & 0 & $\mathrm{mg} / \mathrm{L}$ \\
1 & .4 & .3 & NTU \\
12.6910 & 2.69963 & 2.76492 & well volumes \\
tV & & &
\end{tabular}

\begin{tabular}{|c|c|c|}
\hline 101998 & $\underline{201998}$ & 301998 \\
\hline$<5$ & $<5$ & $<5$ \\
\hline$<5$ & $<5$ & $<5$ \\
\hline$<5$ & $<5$ & $<5$ \\
\hline$<5$ & $<5$ & $<5$ \\
\hline$<5$ & $<5$ & $<5$ \\
\hline$<5$ & $<5$ & $<5$ \\
\hline$<5$ & $<5$ & $<5$ \\
\hline$<5$ & $<5$ & $<5$ \\
\hline$<5$ & $<5$ & $<5$ \\
\hline$<5$ & $<5$ & $<5$ \\
\hline$<5$ & $<5$ & $<5$ \\
\hline$<5$ & $<5$ & $<5$ \\
\hline$<10$ & $<10$ & $<10$ \\
\hline$<10$ & $<10$ & $<10$ \\
\hline$<20$ & $<20$ & $<20$ \\
\hline$<20$ & $<10$ & $<10$ \\
\hline$<5$ & $<20$ & $<5$ \\
\hline$<10$ & $<10$ & $\begin{array}{r}<10 \\
\quad 24.2\end{array}$ \\
\hline$<40$ & $<8$ & $<8$ \\
\hline 9 & 6.3 & 6.4 \\
\hline$<5$ & $<5$ & $<5$ \\
\hline$<5$ & $<5$ & $<5$ \\
\hline$<5$ & $<5$ & $<5$ \\
\hline$<10$ & $<10$ & $<10$ \\
\hline$<4.7$ & $<2$ & $<2$ \\
\hline
\end{tabular}

Note: Flagging, dilution factors, modifiers, and laboratories are for fourth quarte1998 data only. See Appendix B for flagging criteria. * $=$ exceeded holding time for fourth quarter 1998.

$+=$ exceeded screening level or final primary drinking water standard for fourth quarten 998 . 
WELL: LFW 23R

\section{ANALYTICAL DATA}

H ST Analyte

Carbon disulfide

Carbon tetrachloride

Chlorobenzene

Chloroethane

Chloroethene (Vinyl chloride)

Chloroform

Chloromethane (Methyl chloride)

Chloroprene

Chromium, total recoverable

Dibromochloromethane

Dibromomethane (Methylene bromide)

Dichlorodifluoromethane

Dichloromethane (Methylene chloride)

Ethylbenzene

Gross alpha

Iodomethane (Methyl iodide)

Iron, total recoverable

Isobutyl alcohol

Lead, total recoverable

Mercury, total recoverable

Methacrylonitrile

Methyl ethyl ketone

Methyl isobutyl ketone

Methyl methacrylate

Propionitrile

Selenium, total recoverable

Silver, total recoverable

Styrene

Tetrachloroethylene

Toluene

Trichloroethylene

Trichlorofluoromethane

Tritium

Vinyl acetate

Xylenes

cis-1,3-Dichloropropene

trans-1,2-Dichloroethylene

trans-1,3-Dichloropropene

trans-1,4-Dichloro-2-butene
101998

201998

301998

401998

DFMod Unit

Compliance

$<5<5<5$

$<5 \quad<5 \quad<5$

$<5<5<5$

$<10<10<10$

$<10<10<10$

$<5<5<5$

$<10<10<10$

$<5 \quad<5 \quad<5$

$.88 \quad .6 \quad<3$

$<5<5<5$

$<5 \quad<5 \quad<5$

$<10<5<5$

$<4.74<2.7<2.3$

$<5<5<5$

$\begin{array}{lll}8.89 & 4.94 & 9.45\end{array}$

$<5<5 \quad<5$

28.2

$<100<100<100$

$<47 \quad<5 \quad 3.7$

$<.7<.2<.2$

$<10<5 \quad<5$

$<10<10<10$

$<10<12<12$

$<5<5$

$<50<5<5$

$<66<5<5$

$.88<2<2$

$<5<5 \quad<5$

$<5<5 \quad<5$

$<5<5 \quad<5$

$<5<5<5$

$<5<5<5$

$1.7 \quad 1700<.78$

$<10<<<5$

$<5 \quad<5 \quad<5$

$<5$

$<5<5<5$

$<20<5 \quad<5$

Note: Flagging, dilution factors, modifiers, and laboratories are for fourth quarted 998 data only. See Appendix B for flagging criteria. * = exceeded holding time for fourth quarter 1998.

$+=$ exceeded screening level or final primary drinking water standard for fourth quarten 998 . 
WELL: LFW 28

\section{SRS Coord. \\ N 86079.6 \\ E 45555.3

$$
\begin{aligned}
& \text { Lat/Longitude } \\
& \text { 33.290 Deg N } \\
& \text { 81.714 Deg W }
\end{aligned}
$$ 33.290 Deg N}

SAMPLE DATE

\section{FIELD DATA}

Analyte

Water Elevation

Depth to water

pII

Sp. Conductance

Water temperature

Alkalinity as $\mathrm{CaCO} 3$

Turbidity

Volumes purged

Sampling code

\section{ANALYTICAL DATA}

\section{H ST Analyte}

1,1,1,2-Tetrachloroethane

1,1,1-Trichloroethane

1,1,2,2-Tetrachloroethane

1,1,2-Trichloroethane

1,1-Dichloroethane

1,1-Dichloroethylene

1,2,3-Trichloropropane

1,2-Dibromo-3-chloropropane

1,2-Dibromoethane

1,2-Dichlorobenzene

1,2-Dichloroethane

1,2-Dichloropropane

1,4-Dichlorobenzene

2-Hexanone

101998

201998
162.2
27.9
5.2
56
19.4
11
1
2.21493

Top of Casing

1621 - $141 . \mathrm{ft}$.

$192.4 \mathrm{ft} \mathrm{msl}$

$\frac{\text { Casing }}{4 \text { "PVC }}$

Pump Formation

$02 / 02 / 98$

.21493
301998

401998

Unit

ft msl

ft

pH

uS/cm

deg. C

mg/L

NTU

well volumes

Acetone

Acetonitrile (Methyl cyanide)

$<10$

$<20$

Acrylonitrile

$<10$

$<5$

$<8$

Arsenic, total recoverable

Barium, total recoverable

Benzene

Beryllium, total recoverable

$<1$

Bromochloromethane

$<10$

Bromodichloromethane

$<5$

Note: Flagging, dilution factors, modifiers, and laboratories are for fourth quartel998 data only. See Appendix B for flagging criteria.

* = exceeded holding time for fourth quarter 1998.

$+=$ exceeded screening level or final primary drinking water standard for fourth quarter 998. 
WELL: LFW 28

\section{ANALYTICAL DATA}

H ST Analyte

Bromoform

Bromomethane (Methyl bromide)

Cadmium, total recoverable

Carbon disulfide

Carbon tetrachloride

Chlorobenzene

Chloroethane

Chloroethene (Vinyl chloride)

Chloroform

Chloromethane (Methyl chloride)

Chloroprene

Chromium, total recoverable

Cobalt, total recoverable

Copper, total recoverable

Di-n-butyl phthalate

Dibromochloromethane

Dibromomethane (Methylene bromide)

Dichlorodifluoromethane

Dichloromethane (Methylene chloride)

Ethylbenzene

Iodomethane (Methyl iodide)

Isobutyl alcohol

Lead, total recoverable

Methacrylonitrile

Methyl ethyl ketone

Methyl isobutyl ketone

Methyl methacrylate

Nickel, total recoverable

Propionitrile

Selenium, total recoverable

Silver, total recoverable

Specific conductance

Styrene

Tetrachloroethylene

Thallium, total recoverable

Toluene

Trichloroethylene

Trichlorofluoromethane

Tritium

Unknown

Vanadium, total recoverable

Vinyl acetate
101998

201998

301998

401998 DEMod Unit

Compliance

$<5$

$<10$

$<2$

$<5$

$<5$

$<5$

$<10$

$<10$

$<5$

$<10$

$<5$

$<3$

$<5$

1.9

$<.77$

$<5$

$<5$

$<5$

$<2.7$

$<5$

$<5$

$<100$

$<5$

$<5$

$<10$

$<12$

$<5$

$<5$

$<5$

$<5$

$<2$

62.2
$<5$

$<5$

$<5$

$<5$

$<5$

$<5$

915

$<5.04$

$<2$

$<5$

Note: Flagging, dilution factors, modifiers, and laboratories are for fourth quarte 998 data only. See Appendix B for flagging criteria. * = exceeded holding time for fourth quarter 1998 .

$+=$ exceeded sereening level or final primary drinking water standard for fourth quarteri 998 . 
WSRC-TR-99-00011

Unclassified

WELL: LFW 28

\section{ANALYTICAL DATA}

\section{H ST Analyte}

Xylenes

Zinc, total recoverable

beta-Benzene hexachloride

cis-1,2-Dichloroethylene

cis-1,3-Dichloropropene

pH

trans-1,3-Dichloropropene

trans-1,4-Dichloro-2-butene

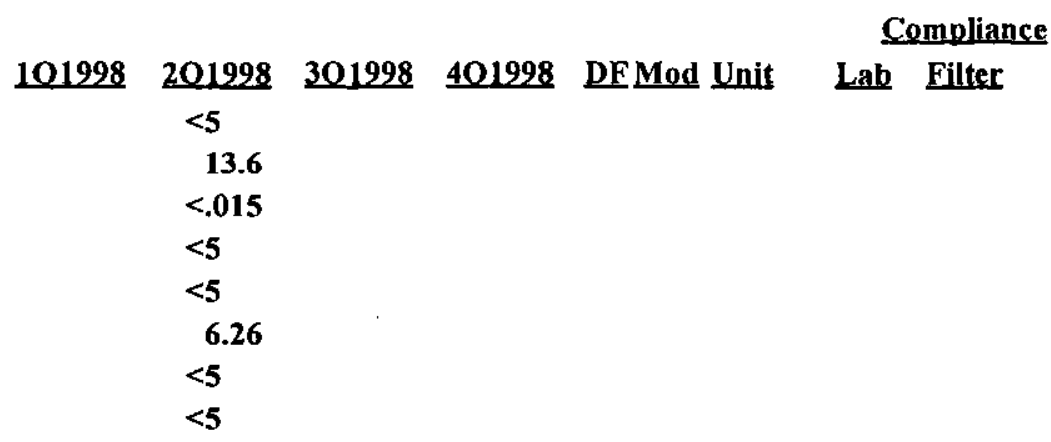

Note: Flagging, dilution factors, modifiers, and laboratories are for fourth quartei 998 data only. See Appendix B for fagging criteria. * = exceeded holding time for fourth quarter 1998.

$+=$ exceeded screening level or final primary drinking water standard for fourth quarter1 998 . 
WSRC-TR-99-00011

Unclassified

WELL: LFW 30

SRS Coord. N 86318.4

E 45170.9

\section{SAMPLE DATE}

\section{FIELD DATA}

Analyte

Water Elevation

Depth to water

pH

Sp. Conductance

Water temperature

Alkalinity as $\mathrm{CaCO} 3$

Turbidity

Volumes purged

Sampling code

\section{ANALYTICAL DATA}

\section{H ST Analyte}

1,1,1,2-Tetrachloroethane

1,1,1-Trichloroethane

1,1,2,2-Tetrachloroethane

1,1,2-Trichloroethane

1,1-Dichloroethane

1,1-Dichloroethylene

1,2,3-Trichloropropane

1,2-Dibromo-3-chloropropane

1,2-Dibromoethane

1,2-Dichlorobenxene

1,2-Dichloroethane

1,2-Dichloropropane

1,4-Dichlorobenzene

2-Hexanone

Acetone

-Acetonitrile (Methyl cyanide)

Acrolein

Acrylonitrile

Allyl chloride

Antimony, total recoverable

Arsenic, total recoverable

Barium, total recoverable

Benzene

Beryllium, total recoverable

Bromochloromethane

Bromodichloromethane

\section{Screen Zone Elevation $162.7-141.7$ ft msl}

$02 / 06 / 98$

$09 / 26 / 98$

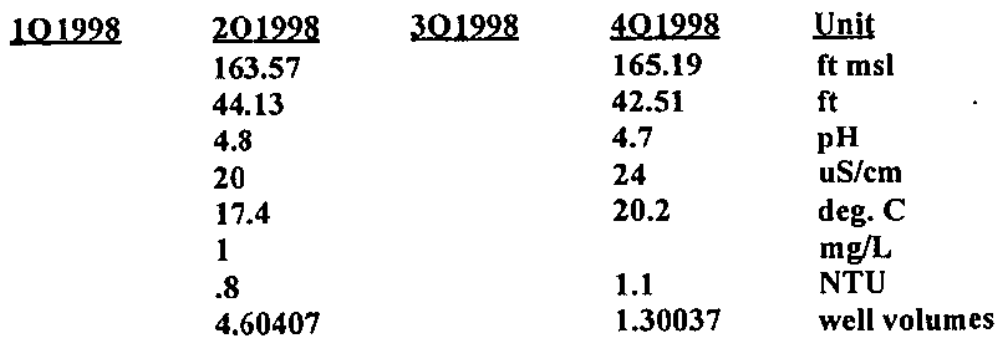

Compliance $101998 \underline{201998} 301998$ 401998 DFMod Unit Lab Filter $<5$

2.6

$<5$

$<5$

1

$<5$

$<5$

$<5$

$<5$

$<5$

$<5$

$<5$

$<5$

$<10$

$<5.6$

$<20$

$<10$

$-\overline{20}$

$<10$

$<5$

$<8$

5.4

$<5$

$<1$

$<10$

$<5$

Note: Flagging, dilution factors, modifiers, and laboratories are for fourth quarte1998 data only. See Appendix B for flagging criteria. * = cxceeded holding time for fourth quarter 1998 .

+- exceeded screening level or final primary drinking water standard for fourth quarten 998 . 
WSRC-TR-99-00011

Unclassified

WELL: LFW 30

\section{ANALYTICAL DATA}

\section{H ST Analyte}

Bromoform

Bromomethane (Methyl bromide)

Cadmium, total recoverable

Carbon disulfide

Carbon tetrachloride

Chlorobenzene

Chloroethane

Chloroethene (Vinyl chloride)

Chloroform

Chloromethane (Methyl chloride)

Chloroprene

Chromium, total recoverable

Cobalt, total recoverable

Copper, total recoverable

Di-n-butyl phthalate

Dibromochloromethane

Dibromomethane (Methylene bromide)

Dichlorodifluoromethane

Dichloromethane (Methylene chloride)

Ethylbenzene

Iodomethane (Methyl iodide)

Isobutyl alcohol

Lead, total recoverable

Methacrylonitrile

Methyl ethyl ketone

Methyl isobutyl ketone

Methyl methacrylate

Nickel, total recoverable

Propionitrile

Selenium, total recoverable

Silver, total recoverable

Specific conductance

Styrene

Tetrachloroethylene

Thallium, total recoverable

Toluene

Trichloroethylene

Trichlorofluoromethane

Tritium

Vanadium, total recoverable

Vinyl acetate

Xylenes
101998

20

$<5$

$<10$

$<2$

$<5$

$<5$

$<5$

$<10$

$<10$

$<5$

$<10$

$<5$

$<3$

$<5$

5.9

$<10$

$<5$

$<5$

$<5$

$<2.5$

$<5$

$<5$

$<100$

$<5$

$<5$

$<10$

$<12$

$<5$

$<5$

$<5$

$<5$

$<2$

20.4

$<5$

$<5$

$<5$

$<5$

.54

15

814

$<2$

$<5$

$<5$

Note: Flagging, dilution factors, modifiers, and laboratories are for fourth quartel998 data only. See Appendix B for flagging criteria. * = exceeded holding time for fourth quarter 1998 .

$+=$ exceeded screening level or final primary drinking water standard for fourth quarten 998 . 
WELL: LFW 30

\section{ANALYTICAL DATA}

H ST Analyte

Zinc, total recoverable beta-Benzene hexachloride cis-1,2-Dichloroethylene cis-1,3-Dichloropropene pH trans-1,3-Dichloropropene trans-1,4-Dichloro-2-butene
101998

201998

10.9

$<.015$

$<5$

$<5$

5.12

$<5$

$<5$

$<5$

\section{Compliance}

\section{DEMod Unit Lab Filter}

at

Note: Flagging, dilution factors, modifiers, and laboratories a re for fourth quartel 998 data only. See Appendix B for flagging criteria. * = exceeded holding time for fourth quarter 1998.

+ exceded screening level or final primary drinking water standard for fourth quarter1998. 
WELL: LFW 31

\begin{tabular}{|c|c|c|c|c|c|c|}
\hline SRS Coord. & Lat/Longitude & Screen Zone Elevation & Top of Casing & Casing & Pump & tion \\
\hline $\begin{array}{l}\text { N } 86262.2 \\
\text { E } 44869.0\end{array}$ & $\begin{array}{l}\text { 33.290 Deg } N \\
\text { 81.716 }\end{array}$ & $166.0-145.0 \mathrm{ft} \mathrm{msl}$ & 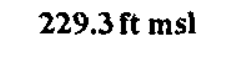 & $\mathbf{V C}$ & $\mathbf{S}$ & \\
\hline
\end{tabular}

\section{FIELD DATA}

Analyte
Water Elevation
Depth to water
pH
Sp. Conductance
Water temperature
Alkalinity as CaCO3
Turbidity
Volumes purged
Sampling code

$\begin{array}{lllll}101998 & 201998 & \frac{301998}{40.7} & 401998 & \text { Unit } \\ 160.7 & 163.55 & 165.41 & & \text { ft msl } \\ 66.3 & 63.45 & 61.59 & & \text { ft } \\ 4.8 & 5.2 & 4.5 & 4.8 & \text { pH } \\ 18 & 26 & 29 & 31 & \text { uS/cm } \\ 20 & 20 & 20.1 & 19.6 & \text { deg. C } \\ 4 & 0 & 0 & & \text { mg/L } \\ 3.2 & 1.4 & 1.4 & 1.2 & \text { NTU } \\ 16.5989 & 3.21694 & 3.96033 & & \text { well volumes } \\ \text { tS } & & & \mathrm{S} & \end{array}$

\section{ANALYTICAL DATA}

\section{H ST Analyte}

1,1,1,2-Tetrachloroethane

1,1,1-Trichloroethane

1,1,2,2-Tetrachloroethane

1,1,2-Trichloroethane

1,1-Dichloroethane

1,1-Dichloroethylene

1,2,3-Trichloropropane

1,2,4,5-Tetrachlorobenzene

1,2,4-Trichlorobenzene

1,2-Dibromo-3-chloropropane

1,2-Dibromoethane

1,2-Dichlorobenzene

1,2-Dichloroethane

1,2-Dichloropropane

1,3,5-Trinitrobenzene

1,3-Dichlorobenzene

1,3-Dinitrobenzene

1,4-Dichlorobenzene

1,4-Dioxane

1,4-Naphthoquinone

1-Naphthylamine

2,3,4,6-Tetrachlorophenol

2,4,5-Trichlorophenol

2,4,6-Trichlorophenol

2,4-Dichlorophenol

2,4-Dimethyl phenol

\section{$101998 \quad 201998$}

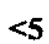

$<5$

$<5$

$<5$

$<5<5$

$<5<5$

$<5 \quad<5$

$<5<5$

$<5$

$<5$

$<5$

$<5$

$<5$

$<5$

$<5$

$<5$

$<5 \quad<5$

$<5$

$<5$

301998

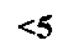

2.8

$<5$

$<5$

$<5$

$<5$

$<5$

$<$

$<5$

$$
<5
$$$$
<5
$$

$$
40
$$

401998 DEMod Unit

$\begin{array}{llll}<5 & 1 & \mathrm{U} & \mathrm{ug} / \mathrm{L}\end{array}$

$3.091 \mathrm{~J} \quad \mathrm{ug} / \mathrm{L}$

$<5 \quad 1 \quad \mathrm{U} \quad \mathrm{ug} / \mathrm{L}$

$<5 \quad 1 \quad \mathrm{U} \quad \mathrm{ug} / \mathrm{L}$

$<5 \quad 1 \quad U \quad \mathrm{ug} / \mathrm{L}$

$<5 \quad 1 \quad U \quad u g / L$

$<5 \quad 1 \quad \mathrm{U} \quad \mathrm{ug} / \mathrm{L}$

$<10.2 \quad 1 \quad \mathrm{U} \quad \mathrm{ug} / \mathrm{L}$

$<10.2 \quad 1 \quad \mathrm{UJ} \quad \mathrm{ug} / \mathrm{L}$

$<5 \quad 1 \quad U \quad u g / L$

$<5 \quad 1 \quad \mathrm{U} \quad \mathrm{ug} / \mathrm{L}$

$<10.2 \quad 1 \quad \mathrm{U} \quad \mathrm{ug} / \mathrm{L}$

$<5 \quad 1 \quad \mathrm{U} \quad \mathrm{ug} / \mathrm{L}$

$\begin{array}{lllll}<5 & 1 & \mathrm{U} & \mathrm{ug} / \mathrm{L}\end{array}$

$<10.2 \quad 1 \quad \mathrm{u} \quad \mathrm{ug} / \mathrm{L}$

$<10.2 \quad 1 \quad \mathrm{U} \quad \mathrm{ug} / \mathrm{L}$

$<10.2 \quad 1 \quad U \quad \mathrm{ug} / \mathrm{L}$

$<10.2 \quad 1$ UJ $\quad$ ug/L

$<10.2 \quad 1$ U ug/L

$<10.2 \quad 1 \quad \mathrm{U} \quad \mathrm{ug} / \mathrm{L}$

$<10.2 \quad 1 \quad \mathrm{U} \quad \mathrm{ug} / \mathrm{L}$

$<10.2 \quad 1 \quad \mathrm{U} \quad \mathrm{ug} / \mathrm{L}$

$<25.5 \quad 1 \quad \mathrm{U} \quad \mathrm{ug} / \mathrm{L}$

$<10.2 \quad 1 \quad \mathrm{U} \quad \mathrm{ug} / \mathrm{L}$

$<10.2 \quad 1 \quad \mathrm{U} \quad \mathrm{ug} / \mathrm{L}$

$<10.2 \quad 1 \quad \mathrm{U} \quad \mathrm{ug} / \mathrm{L}$
Compliance

Lab Filter

WA $<$ EQL

EX NDD

EX < EQL

EX < EQL

WA < EQL

WA <EQL

WA <EQL

WA $<$ EQL

WA < EQL

WA < EQL

WA $<$ EQL

WA $<$ EQL

WA $<$ EQL

WA < EQL

WA $<$ EQL

WA $<$ EQL

WA < EQL

WA < EQL

WA $<$ EQL

WA < EQL

WA < EQL

WA < EQL

WA < EQL

WA $<$ EQL

WA $<$ EQL

WA $<$ EQL

Note: Flagging, dilution factors, modifiers, and laboratories are for fourth quartet998 data only. See Appendix B for flagging criteria.

* = exceeded holding time for fourth quarter 1998 .

$+=$ exceeded screening level or final primary drinking water standard for fourth quarter 998 . 
WELL: LFW 31

\section{ANALYTICAL DATA}

H ST Analyte

2,4-Dinitrophenol

2,4-Dinitrotoluene

2,6-Dichlorophenol

2,6-Dinitrotoluene

2-Acetylaminofluorene

2-Chloronaphthalene

2-Chlorophenol

2-Hexanone

2-Methyl-4,6-dinitrophenol

2-Methylnaphthalene

2-Naphthylamine

2-Nitrophenol

2-Picoline

2-sec-Butyl-4,6-dinitrophenol

3,3"-Dichlorobenzidine

3,3'-Dimethylbenzidine

3-Methylcholanthrene

4-Aminobiphenyl

4-Bromophenyl phenyl ether

4-Chloro-m-cresol

4-Chloroaniline

4-Chlorophenyl phenyl ether

4-Nitrophenol

4-Nitroquinoline-1-oxide

5-Nitro-0-toluidine

7,12-Dimethylbenz(a)anthracene

Acenaphthene

Acenaphthylene

Acetone

Acetonitrile (Methyl cyanide)

Acetophenone

Acrolein

Acrylonitrile

Allyl chloride

Aluminum, total recoverable

Aniline

Anthracene

Antimony, total recoverable

Aramite

Arsenic, total recoverable

Barium, total recoverable

Benzene $\underline{301998}$

401998

$<10.2$ i $\quad$ U $\quad$ ug/ $/ \mathrm{L}$

$<10.2 \quad 1 \quad \mathrm{U} \quad \mathrm{ug} / \mathrm{L}$

$<10.2 \quad 1 \quad \mathrm{U} \quad \mathrm{ug} / \mathrm{L}$

$<10.2 \quad 1 \quad \mathrm{U} \quad \mathrm{ug} / \mathrm{L}$

$<10.2 \quad 1 \quad U \quad \mathrm{ug} / \mathrm{L}$

$<10.2 \quad 1 \quad U \quad$ ug/L

$<10$

$<10<10$

$<10$

$<25.5$

$<10.2$

$<10.2$

$<10.2$

$<10.2$

$<51$

$<10.2$

$<10.2$

$<10.2$

$<10.2$

$<10.2$

$<10.2$

$<10.2$

$<10.2$

$<25.5$

$<20.4$

$<10.2$

$<10.2$

$<10.2$

$<10.2$

$<4.88$

$<20$

$<10.2$

$<20$

$<5$

$--, \ldots+<$

$<20$

$<10$

$<5$

$<10$

30.6

1 U

$\begin{array}{llll}<10 & 1 & U \\ <200 & 1 & U\end{array}$

$<10.2 \quad 1 \quad \mathrm{U}$

$<10.2$ I U

$<35 \quad 1 \quad \mathrm{U}$

$<20.4 \quad 1 \quad \mathrm{U}$

$\begin{array}{lll}<28 & 1 & U\end{array}$

$5.6 \quad 1$

$<5$
Compliance

Lab Filter

WA $<$ EQL

WA $<$ EQL

WA $<$ EQL

WA $<$ EQL

WA $<$ EQL

WA $<$ EQL

WA $<$ EQL

WA $<$ EQL

WA $<$ EQL

WA $<$ EQL

$W_{A}<$ EQL

WA $<$ EQL

WA $<$ EQL

WA $<$ EQL

ug/L

WA $<$ EQL

ug/L

$\mathrm{ug} / \mathrm{L}$

ug/L

WA $<$ EQL

WA $<$ EQL

WA $<$ EQL

WA $<$ EQL

WA $<$ EQL

WA $<$ EQL

WA $<$ EQL

WA $<$ EQL

WA $<$ EQL

WA $<$ EQL

WA $<$ EQL

WA $<$ EQL

WA $<$ EQL

WA $<$ EQL

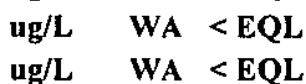

ug/L WA $<$ EQL ug/L WA $<$ EQL $\overline{\mathrm{ug} / \mathrm{L}} \mathrm{WA}<\mathrm{EQ} L$ ug/L WA $<$ EQL ug/L WA $<$ EQL ug/L WA $<$ EQL ug/L $\quad$ WA $<$ EQL ug/L WA $<$ EQL ug/L WA $<$ EQL

ug/L WA 5.6

$\mathrm{ug} / \mathbf{L} \quad$ WA $<$ EQL

Note: Flagging, dilution factors, modifiers, and laboratories are for fourth quartet 998 data only. See Appendix B for flagging criteria.

* $\approx$ exceeded holding time for fourth quarter 1998.

$+=$ exceded screening level or final primary drinking water standard for fourth quarter 998 . 


\section{H ST Analyte}

Benzo(a)anthracene

Benzo(a)pyrene

Benzo(b)fluoranthene

Benzo(g,h,i)perylene

Benzo(k)fluoranthene

Benzoic acid

Benzyl alcohol

Beryllium, total recoverable

Bis(2-chloroethoxy) methane

Bis(2-chloroethyl) ether

Bis(2-chloroisopropyl) ether

Bis(2-ethylhexyl) phthalate

Bromochloromethane

Bromodichloromethane

Bromoform

Bromomethane (Methyl bromide)

Butylbenzyl phthalate

Cadmium, total recoverable

Carbon disulfide

Carbon tetrachloride

Chlorobenzene

Chlorobenzilate

Chloroethane

Chloroethene (Vinyl chloride)

Chloroform

Chloromethane (Methyl chloride)

Chloroprene

Chromium, total recoverable

Chrysene

Cobalt, total recoverable

Copper, total recoverable

Di-n-butyl phthalate

Di-n-octyl phthalate

Diallate

Dibenz(a,h)anthracene

Dibenzofuran

Dibromochloromethane

Dibromomethane (Methylene bromide)

Dichlorodifluoromethane

Dichloromethane (Methylene chloride)

Diethyl phthalate

Dimethyl phthalate

\section{$101998201998 \quad 301998 \quad \underline{401998}$ DFMod Unit}

$<10.2 \quad 1 \quad \mathrm{U} \quad \mathrm{ug} / \mathrm{L}$

$<10.2 \quad 1 \quad \mathrm{U} \quad \mathrm{ug} / \mathrm{L}$

$<10.2 \quad 1 \quad \mathrm{U} \quad \mathrm{ug} / \mathrm{L}$

$<10.2 \quad 1 \quad \mathrm{U} \quad \mathrm{ug} / \mathrm{L}$

$<10.2 \quad 1 \quad \mathrm{U} \quad \mathrm{ug} / \mathrm{L}$

$<25.5 \quad 1 \quad \mathrm{U} \quad \mathrm{ug} / \mathrm{L}$

$<10.2 \quad 1 \quad \mathrm{U} \quad \mathrm{ug} / \mathrm{L}$

.2

$<$

$<10.2 \quad 1 \quad \mathrm{U}$

$<10.21 \mathrm{U}$

$<10.2$ I U

$\begin{array}{lll}1.22 & \mathrm{~J}\end{array}$

$<5 \quad 1 \mathrm{U}$

$<5 \quad 1 \quad$

$<5 \quad 1 \quad$ U

$<10 \quad 1 \mathrm{U}$

$<10.2 \quad 1 \mathrm{U}$

$<4 \quad 1 \quad \mathrm{U}$

$<5 \quad 1 \quad \mathrm{U}$

$<5 \quad 1 \quad \mathrm{U}$

$<5 \quad 1 \quad \mathrm{U}$

$<10.2 \quad 1 \quad \mathrm{U}$

$<10 \quad 1 \quad U$

$<10 \quad 1 \quad U$

$<5 \quad 1 \quad U$

$<10 \quad 1$

$<5 \quad 1 \quad$

$4.1 \quad 1 \quad J$

$<10.2 \quad 1 \quad$

$<7 \quad 1 \quad$ U

40.91

$<10.2 \quad 1 \quad \mathrm{U}$

$<10.2 \quad 1 \quad \mathrm{U}$

$<10.2 \quad 1 \quad U$

$<10.2 \quad 1 \quad U$

$<10.2 \quad 1 \quad U$

$<5 \quad 1 \quad U$

$<5 \quad 1 \quad$ U

$<10 \quad 1 \quad \mathrm{U}$

$<2.97$ I U

$<10.2 \quad 1 \mathrm{U}$

$<10.2 \quad 1 \quad U$
Compliance

Lab Eilter

WA < EQL

WA < EQL

WA < EQL

WA < EQL

WA < EQL

WA < EQL

WA < EQL

WA <EQL

WA < EQL

$\mathbf{u g} / \mathrm{L}$

ug/L

WA < EQL

$\mathrm{ug} / \mathrm{L}$

ug/L

ug/L.

ug/L

$\mathbf{u g} / \mathbf{L}$

ug/L

ug/L

ug/L

ug/L

ug/L

ug/L

ug/L

ug/L

ug/L

ug/L

ug/L

ug/L

ug/L

ug/L

ug/L

ug/L

WA $<$ EQL

WA NDD

WA $<$ EQL

WA <EQL

WA < EQL

WA < EQL

WA < EQL

WA $<$ EQL

WA $<$ EQL

WA $<$ EQL

WA < EQL

WA $<$ EQL

WA <EQL

WA $<$ EQL

WA < EQL

WA < EQL

WA $<$ EQL

WA NDD

WA < EQL

WA $<$ EQL

ug/L WA $<$ EQL

ug/L WA $<$ EQL

ug/L WA $<$ EQL

ug/L WA $<$ EQL

ug/L

ug/L

ug/L

WA $<$ EQL

WA $<$ EQL

WA $<$ EQL

ug/L WA $<$ EQL

ug/L WA $<$ EQL

ug/L WA $<$ EQL

ug/L WA $\quad$ EQL

Note: Flagging, dilution factors, modiliers, and laboratories are for fourth quartel 998 data only. See Appendix B for flagging criteria.

* = exceeded holding time for fourth quarter 1998.

$+=$ exceeded screening level or final primary drinking water standard for fourth quarter 998 .

Sanitary Landfill

D-37

Fourth Quarter 1998 and Annual Summary 
WELL: LFW 31

\section{ANALYTICAL DATA}

H ST Analyte

\section{Diphenylamine}

Ethyl methacrylate

Ethyl methanesulfonate

Ethylbenzene

Fluoranthene

Fluorene

Gross alpha

Hexachlorobenzene

Hexachlorobutadiene

Hexachlorocyclopentadiene

Hexachloroethane

Hexachlorophene

Hexachloropropene

Indeno(1,2,3-c,d)pyrene

Iodomethane (Methyl iodide)

Iron, total recoverable

Isobutyl alcohol

Isophorone

Isosafrole

Lead, total recoverable

Mercury, total recoverable

Methacrylonitrile

Methapyrilene

Methyl ethyl ketone

Methyl isobutyl ketone

Methyl methacrylate

Methyl methanesulfonate

N-Nitrosodi-n-butylamine

N-Nitrosodiethylamine

N-Nitrosodimethylamine

N-Nitrosodiphenylamine

N-Nitrosodipropylamine

N-Nitrosomethylethylamine

N-Nitrosomorpholine

N-Nitrosopiperidine

N-Nitrosopyrrolidine

Naphthalene

Nickel, total recoverable

Nitrobenzene

Pentachlorobenzene

Pentachloroethane

Pentachloronitrobenzene

\section{$101998 \quad 201998 \quad \underline{301998}$}

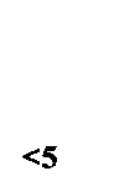

2.6

1.7

7.14

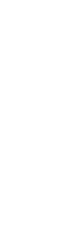

$\begin{array}{lll}<47 & <19.3 & 3.4 \\ <.7 & <.2 & <.2 \\ <10 & <5 & <5\end{array}$

$\begin{array}{lll}<10 & <5 & <5 \\ <10 & <10 & <10 \\ <10 & <12 & <12 \\ & <5 & <5\end{array}$

401998 DFMod Unit

$<10.2 \quad 1 \quad U \quad u g / L$

$<10.2 \quad 1 \quad \mathrm{U} \quad \mathrm{ug} / \mathrm{L}$

$<10.2$ I U ug/L

$<5 \quad 1 \quad U \quad u g / L$

$<10.2 \quad 1 \quad \mathrm{U}$ ug/L

$<10.2 \quad I \quad U \quad u g / L$

5.991

$<10.2$ I U

$<10.2 \quad \mathrm{U}$

$<10.2 \quad 1 \quad U$

$<10.2 \quad 1 \mathrm{U}$

$<102 \quad 1 \quad U$

$<10.2 \quad 1 \quad \mathrm{U}$

$<10.2 \quad 1 \quad \mathrm{U}$

$<5$

$214 \quad 1 \quad J$

$<100 \quad 1 \quad U$

$<10.2 \quad 1 \quad \mathrm{U}$

$<10.2 \quad 1 \quad \mathrm{U}$

$<17 \quad 1 \quad \mathrm{U}$

$<.5 \quad 1 \quad \mathrm{U}$

$<10 \quad 1 \mathrm{U}$

$<10.2 \quad 1 \mathrm{U}$

$<2.27 \quad 1 \mathrm{~J}$

$<10 \quad 1 \mathrm{U}$

$<10.2 \quad 1 \quad \mathrm{U}$

$<10.21 \mathrm{U}$

$<10.2 \quad 1 \quad U$

$<10.2 \quad 1 \quad U$

$<10.21 \mathrm{U} \mathrm{ug} / \mathrm{L}$

$<10.2 \quad 1 \quad \mathrm{U} \quad \mathrm{ug} / \mathrm{L}$

$<10.2 \quad 1$ UJ ug/L

$\mathrm{pCi} / \mathrm{L}$

ug/L

ug/L

ug/L

ug/L

ug/L

ug/L

ug/L

ug/L

ug/L

ug/L

ug/L

ug/L

ug/L

ug/L

ug/L

ug/L

ug/L

ug/L

ug/L

ug/L

$\mathrm{ug} / \mathrm{L}$

$<10.2 \quad 1$

ug/L

ug/L
ug/L

$\begin{array}{llll}<51 & 1 & \mathrm{U} & \mathrm{ug} / \mathrm{L} \\ <10.2 & 1 & \mathrm{U} & \mathrm{ug} / \mathrm{L}\end{array}$

ug/L

ug/L

ug/L

ug/L

ug/L

ug/L
Compliance

Lab Filter

WA $<$ EQL

WA < EQL

WA $<$ EQL

WA $<$ EQL

WA $<$ EQL

WA $<$ EQL

TM 5.99

WA $<$ EQL

WA $<$ EQL

WA < EQL

WA $<$ EQL

WA < EQL

WA < EQL

WA $<$ EQL

WA < EQL

WA NDD

WA $<$ EQL

WA $<$ EQL

WA $<$ EQL

WA $<$ EQL

EX < EQL

WA $<$ EQL

WA $<$ EQL

WA NDD

WA $<$ EQL

WA $<$ EQL

WA $<$ EQL

WA $<$ EQL

WA $<$ EQL

WA $<$ EQL

WA < EQL

WA $<$ EQL

WA $<$ EQL

WA $<$ EQL

WA < EQL

WA $<$ EQL

WA < EQL

WA NDD

WA $<$ EQL

WA $<$ EQL

WA < EQL

WA $<$ EQL

Note: Flagging, dilution factors, modifiers, and laboratories are for fourth quarte1998 data only. Sce Appendix B for flagging criteria.

* = exceeded holding time for fourth quarter 1998 .

$+=$ exceeded screening level or final primary drinking water standard for fourth quarter1998.

Sanitary Landfill

D-38

Fourth Quarter 1998 and Annual Summary 
WELL: LFW 31

\section{ANALYTICAL DATA}

\section{H ST Analyte}

Pentachlorophenol

Phenacetin

Phenanthrene

Phenol

Pronamid

Propionitrile

Pyrene

Pyridine

Safrole

Selenium, total recoverable

Silver, total recoverable

Specific conductance

Styrene

Tetrachloroethylene

Thallium, total recoverable

Toluene

Trichloroethylene

+ Trichlorofluoromethane

Tritium

Unknown

Unknown 12

Unknown 13

Unknown 14

Vanadium, total recoverable

Vinyl acetate

Xylenes

Zinc, total recoverable

a,a-Dimethylphenethylamine

beta-Benzene hexachloride

cis-1,2-Dichloroethylene

cis-1,3-Dichloropropene

m-Cresol (3-Methylphenol)

m-Nitroaniline

o-Cresol (2-Methylphenol)

o-Nitroaniline

o-Toluidine

p-Cresol (4-Methylphenol)

p-Dimethylaminoazobenzene

p-Nitroaniline

p-Phenylenediamine

pH

trans-1,2-Dichloroethylene

\section{$101998 \quad \underline{201998} \quad \underline{301998} \quad 40$}

$<25.51 \mathrm{U} \mathrm{ug} / \mathrm{L}$

$<10.2 \quad 1 \quad U \quad u g / L$

$<10.21 \mathrm{U} \quad \mathrm{ug} / \mathrm{L}$

$<10.2 \quad 1 \quad U \quad$ ug/L

$<10.21 \mathrm{U} \mathrm{ug} / \mathrm{L}$

$<50 \quad 1 \quad U$ ug/L

$<10.21 \mathrm{U} \quad \mathrm{ug} / \mathrm{L}$

$<10.2 \quad 1 \quad \mathrm{U} \quad \mathrm{ug} / \mathrm{L}$

$<10.2 \quad 1 \quad \mathrm{U} \quad \mathrm{ug} / \mathrm{L}$

$<36 \quad 1 \quad U \quad u g / L$

$\begin{array}{llll}<5 & 1 & \mathrm{U} & \mathrm{ug} / \mathrm{L}\end{array}$

uS/cm

17.51

$<5 \quad 1 \quad 0$

$<5 \quad 1 \quad$

ug/L

ug/L

$<28 \quad 1 \quad U \quad \mathrm{ug} / \mathrm{L}$

$\begin{array}{llll}< & 1 & \mathrm{U} & \mathbf{u g} / \mathrm{L}\end{array}$

$<5 \quad 1 \quad \mathrm{U} \quad \mathrm{ug} / \mathrm{L}$

$39.91 \quad$ ug/L

$<.54 \quad 1$

UI

1.72

$<1330$

$<.87$

$<5.02$

$<5.91$

$<4.42$

$<10.72$

$\begin{array}{ll}<10 & <5 \\ <5 & <5\end{array}$

.5
$<5$
$<5$

$<5$

$\begin{array}{lll}<6 & 1 & U \\ <20 & 1 & U \\ <10 & 1 & U \\ 25.9 & 1 & \\ <10.2 & 1 & U \\ <.052 & 1 & U \\ <5 & 1 & U \\ <5 & 1 & U \\ <10.2 & 1 & U \\ <25.5 & 1 & U \\ <10.2 & 1 & U \\ <25.5 & 1 & U \\ <10.2 & 1 & U \\ <10.2 & 1 & U \\ <10.2 & 1 & U \\ <25.5 & 1 & U \\ <10.2 & 1 & U \\ 5.37 & 1 & J \\ <5 & 1 & U\end{array}$

\section{ug/L}

ug/L

ug/L

ug/L

ug/L

ug/L

ug/L

ug/L

ug/L

ug/L

ug/L

ug/L

ug/L

ug/L

ug/L

ug/L

ug/L

pH

WA < EQL

EX < EQL

EX $<$ EQL

WA 25.9

WA $<$ EQL

GC $<$ EQL

WA $<$ EQL

WA $<$ EQL

WA < EQL

WA < EQL

WA < EQL

WA $<$ EQL

WA < EQL

WA $<$ EQL

WA <EQL

WA $<$ EQL

5.65

$<5$

WA NDD

Note: Flagging, dilution factors, modifiers, and laboratories are for fourth quartet 998 data only. See Appendix B for flagging criteria.

* = exceeded holding time for fourth quarter 1998.

$+=$ exceeded screening level or final primary drinking water standard for fourth quartert 998 .

Sanitary Landfill

D-39

Fourth Quarter 1998 and Annual Summary 
WELL: LFW 31

\section{ANALYTICAL DATA}

\section{H ST Anglyte}

trans-1,3-Dichloropropene

trans-1,4-Dichloro-2-butene
101998

$<5$

$<20$
201998

$<5$

$<5$
301998

$<5$

$<5$
Compliance

Lab Filter

$<5 \quad 1 \quad \mathrm{U} \quad \mathrm{ug} / \mathrm{L} \quad \mathrm{WA}<\mathrm{EQL}$

$<20 \quad 1 \quad U \quad$ ug/L $\quad$ EX $<$ EQL

Note: Flagging, dilution factors, modifiers, and laboratories are for fourth quartet 998 data only. See Appendix B for flagging criteria. * = exceeded holding time for fourth quarter 1998 .

$+=$ exceeded screening level or final primary drinking water standard for fourth quartern 998 . 
WELL: LFW 32

\begin{tabular}{|c|c|c|c|c|c|c|}
\hline SRS Coord, & Lat/Longitude & Screen Zone Elevation & Top of Casing & Casing & Pumn & Eormation \\
\hline $\begin{array}{l}\text { N } 85836.8 \\
\text { E } 44935.9\end{array}$ & $\begin{array}{l}\text { 33.289 Deg } N \\
81.715 \text { Deg W }\end{array}$ & - $144.3 \mathrm{ft}$ msl & $2237 \mathrm{ft} \mathrm{msl}$ & $4 "$ & $\mathbf{S}$ & \\
\hline
\end{tabular}

\section{SAMPLE DATE}

\section{FIELD DATA}

Analyte
Water Elevation
Depth to water
pH
Sp. Conductance
Water temperature
Alkalinity as CaCO3
Turbidity
Volumes purged
Sampling code

ANALYTICAL DATA

\section{H ST Analyte}

1,1,1,2-Tetrachloroethane

1,1,1-Trichloroethane

1,1,2,2-Tetrachloroethane

1,1,2-Trichloroethane

1,1-Dichloroethane

1,1-Dichloroethylene

1,2,3-Trichloropropane

1,2-Dibromo-3-chloropropane

1,2-Dibromoethane

1,2-Dichlorobenzene

1,2-Dichloroethane

1,2-Dichloropropane

1,4-Dichlorobenzene

2-Hexanone

Acetone

Acetonitrile (Methyl cyanide)

Acrolein

Acrylonitrile

Allyl chloride

Antimony, total recoverable

Arsenic, total recoverable

Barium, total recoverable

Benzene

Beryllium, total recoverable

Bromochloromethane

Bromodichloromethane
$02 / 02 / 98$

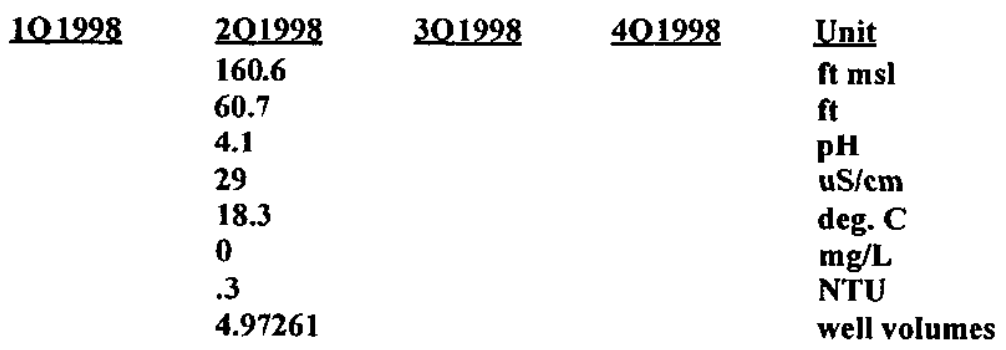

Compliance

101998201998 301998 401998 DFMod Unit Lab Filter

$<5$

12

$<5$

$<5$

4.5

1.2

$<5$

$<5$

$<5$

$<5$

$<5$

$<5$

$<5$

$<10$

$<10$

$<20$

$<10$

$<20$

$<10$

$<5$

$<8$

10.6

$<5$

$<1$

$<10$

$<5$

Note: Flagging, dilution factors, modifiers, and laboratories are for fourth quartei 998 data only. See Appendix B for flagging criteria. * a exceeded holding time for fourth quarter 1998 .

$+=$ exceeded screening level or final primary drinking water standard for fourth quarter 998 .

Sanitary Landfill 
WELL: LFW 32

\section{ANALYTICAL DATA}

H ST Analyte

Bromoform

Bromomethane (Methyl bromide)

Cadmium, total recoverable

Carbon disulfide

Carbon tetrachloride

Chlorobenzene

Chloroethane

Chloroethene (Vinyl chloride)

Chloroform

Chloromethane (Methyl chloride)

Chloroprene

Chromium, total recoverable

Cobalt, total recoverable

Copper, total recoverable

Di-n-butyl phthalate

Dibromochloromethane

Dibromomethane (Methylene bromide)

Dichlorodifluoromethane

Dichloromethane (Methylene chloride)

Ethylbenzene

Indomethane (Methyl iodide)

Isobutyl alcohol

Lead, total recoverable

Methacrylonitrile

Methyl ethyl ketone

Methyl isobutyl ketone

Methyl methacrylate

Nickel, total recoverable

Propionitrile

Selenium, total recoverable

Silver, total recoverable

Specific conductance

Styrene

Tetrachloroethylene

Thallium, total recoverable

Toluene

Trichloroethylene

Trichlorofluoromethane

Tritium

Unknown

Unknown 4

Vanadium, total recoverable
101998

201998

301998

$<5$

$<10$

$<2$

$<5$

$<5$

$<5$

$<10$

$<10$

$<5$

$<10$

$<5$

$<3$

$<5$

4.5

$<.79$

$<5$

$<5$

1.2

$<6.2$

$<5$

$<5$

$<100$

3.4

$<5$

$<10$

$<12$

$<5$

$<5$

$<5$

$<5$

$<2$

25.9

$<5$

$<5$
$<5$
$<5$
1.6
250
1950
$<42.7$
$<30.16$
.5

Comoliance

Lab Filter 
WSRC-TR-99-00011

Unclassified

WELL: LFW 32

\section{ANALYTICAL DATA}

\section{H ST Analyte}

Vinyl acetate

Xylenes

Zinc, total recoverable

beta-Benzene hexachloride

cis-1,2-Dichloroethylene

cis-1,3-Dichloropropene

pH

trans-1,3-Dichloropropene

trans-1,4-Dichloro-2-butene

$\begin{array}{rl}101998 & 201998 \\ <5 \\ <5 \\ 11.4 \\ <.015 \\ <5 \\ <5 \\ 5.02 \\ <5 \\ <5\end{array}$

11.4

015

5.02

$<5$
Compliance

Lab Eilter

Note: Flagging, dilution factors, modifiers, and laboratories are for fourth quartei998 data oniy. See Appendix B for flagging criteria. * = exceeded holding time for fourth quarter1998.

$+=$ exceeded screening level or final primary drinking water standard for fourth quarter1998. 
WELL: LFW 32C

\begin{tabular}{|c|c|c|c|c|c|c|}
\hline SRS Coord. & Lat/Longitude & Screen Zone Elevation & Top of Casing & Casing & Pump & Formation \\
\hline $\begin{array}{l}\text { N } 85837.8 \\
\text { E } 44923.0\end{array}$ & $\begin{array}{ll}33.289 & \text { Deg N } \\
81.715 & \text { Deg W }\end{array}$ & $113.6-98.6 \mathrm{ft} \mathrm{msl}$ & $223.6 \mathrm{ft} \mathrm{msl}$ & $2 " \mathrm{PVC}$ & $\mathbf{v}$ & M Steed Pond \\
\hline SAMPLE D & & & $6 / 98$ & & $6 / 98$ & \\
\hline
\end{tabular}

\section{FIELD DATA}

Analyte
Water Elevation
Depth to water
pH
Sp. Conductance
Water temperature
Alkalinity as CaCO3
Turbidity
Volumes purged
Sampling code

ANALYTICAL DATA

$101998 \quad 2019$

1,1,1,2-Tetrachloroethane 1,1,1-Trichloroethane 1,1,2,2-Tetrachloroethane 1,1,2-Trichloroethane 1,1-Dichloroethane 1,1-Dichloroethylene 1,2,3-Trichloropropane 1,2,4,5-Tetrachlorobenzene 1,2,4-Trichlorobenzene 1,2-Dibromo-3-chloropropane 1,2-Dibromoethane 1,2-Dichlorobenzene 1,2-Dichloroethane 1,2-Dichloropropane 1,3,5-Trinitrobenzene 1,3-Dichiorobenzene 1,3-Dinitrobenzene 1,4-Dichlorobenzene 1,4-Dioxane 1,4-Naphthoquinone 1-Naphthylamine

2,3,4,6-Tetrachlorophenol 2,4,5-Trichlorophenol 2,4,6-Trichlorophenol 2,4-Dichlorophenol 2,4-Dimethyl phenol

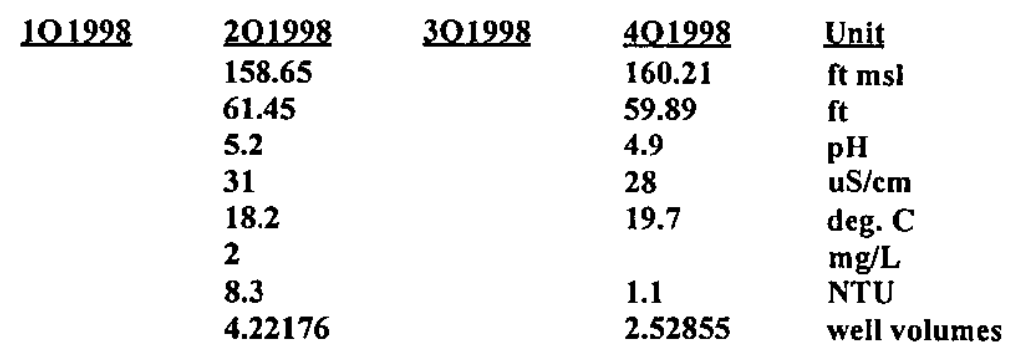

Compliance Lab Filter
$<5$

$<5$

$<5$

$<5$

$<5$

$<5$

$<5$

$<10.2 \quad I \quad U \quad$ ug/L $\quad$ WA $<$ EQL

$<10.211 \quad \mathrm{U} \quad$ ug/L $\quad$ WA $<$ EQL

$<5$

$<5$

$<5$

$<5$

$<5$

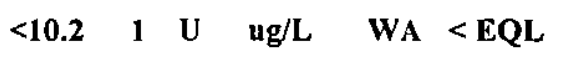

$<10.2 \quad 1 \quad$ U $\quad$ ug/L $\quad$ WA $<$ EQL

$<10.2 \quad 1 \quad \mathrm{U} \quad \mathrm{ug} / \mathrm{L} \quad \mathrm{WA}<\mathrm{EQL}$

$<10.2 \quad 1 \quad \mathrm{U} \quad \mathrm{ug} / \mathrm{L} \quad \mathrm{WA}<\mathrm{EQL}$

$-<10: 2--1=-\mathrm{U}=-\mathrm{ug} / \mathrm{L}=\mathrm{WA}=<\mathrm{EQL}$

$<10.2 \quad 1 \quad \mathrm{U} \quad$ ug/L $\quad$ WA $<$ EQL

$<10.2 \quad 1 \quad \mathrm{U} \quad \mathrm{ug} / \mathrm{L} \quad$ WA $<$ EQL

$<10.2 \quad 1 \quad \mathrm{U} \quad \mathrm{ug} / \mathrm{L} \quad \mathrm{WA}<\mathrm{EQL}$

$<10.2 \quad 1 \quad \mathrm{U} \quad \mathrm{ug} / \mathrm{L} \quad$ WA $<$ EQL

$<25.5 \quad 1 \quad U \quad$ ug/L $\quad$ WA $<$ EQL

$<10.2 \quad 1 \quad \mathrm{U} \quad \mathrm{ug} / \mathrm{L} \quad \mathrm{WA}<\mathrm{EQL}$

$<10.2 \quad 1 \quad \mathrm{U} \quad \mathrm{ug} / \mathrm{L} \quad$ WA $<$ EQL

$<10.2 \quad 1 \quad \mathrm{U} \quad \mathrm{ug} / \mathrm{L} \quad \mathrm{WA}<\mathrm{EQL}$

Note: Flagging, dilution factors, modifiers, and laboratories are for fourth quarte1998 data only. See Appendix B for nagging criteria. * = exceeded holding time for fourth quarter 1998.

$+=$ exceded screening level or final primary drinking water standard for fourth quarter1 998 . 
WELL: LFW 32C

\section{ANALYTICAL DATA}

\section{H ST Analyte}

2,4-Dinitrophenol

2,4-Dinitrotoluene

2,6-Dichlorophenol

2,6-Dinitrotoluene

2-Acetylaminofluorene

2-Chloronaphthalene

2-Chlorophenol

2-Hexanone

2-Methyl-4,6-dinitrophenol

2-Methylnaphthalene

2-Naphthylamine

2-Nitrophenol

2-Picoline

2-sec-Butyl-4,6-dinitrophenol

3,3"-Dichlorobenzidine

3,3"-Dimethylbenzidine

3-Methylcholanthrene

4-Aminobiphenyl

4-Bromophenyl phenyl ether

4-Chloro-m-cresol

4-Chloroaniline

4-Chlorophenyl phenyl ether

4-Nitrophenol

4-Nitroquinoline-1-oxide

5-Nitro-o-toluidine

7,12-Dimethylbenz(a)anthracene

Acenaphthene

Acenaphthylene

Acetone

Acetonitrile (Methyl cyanide)

Acetophenone

Acrolein

Acrylonitrile

Allyl chloride

+ Aluminum, total recoverable

Aniline

Anthracene

Antimony, total recoverable

Aramite

Arsenic, total recoverable

Barium, total recoverable

Benzene

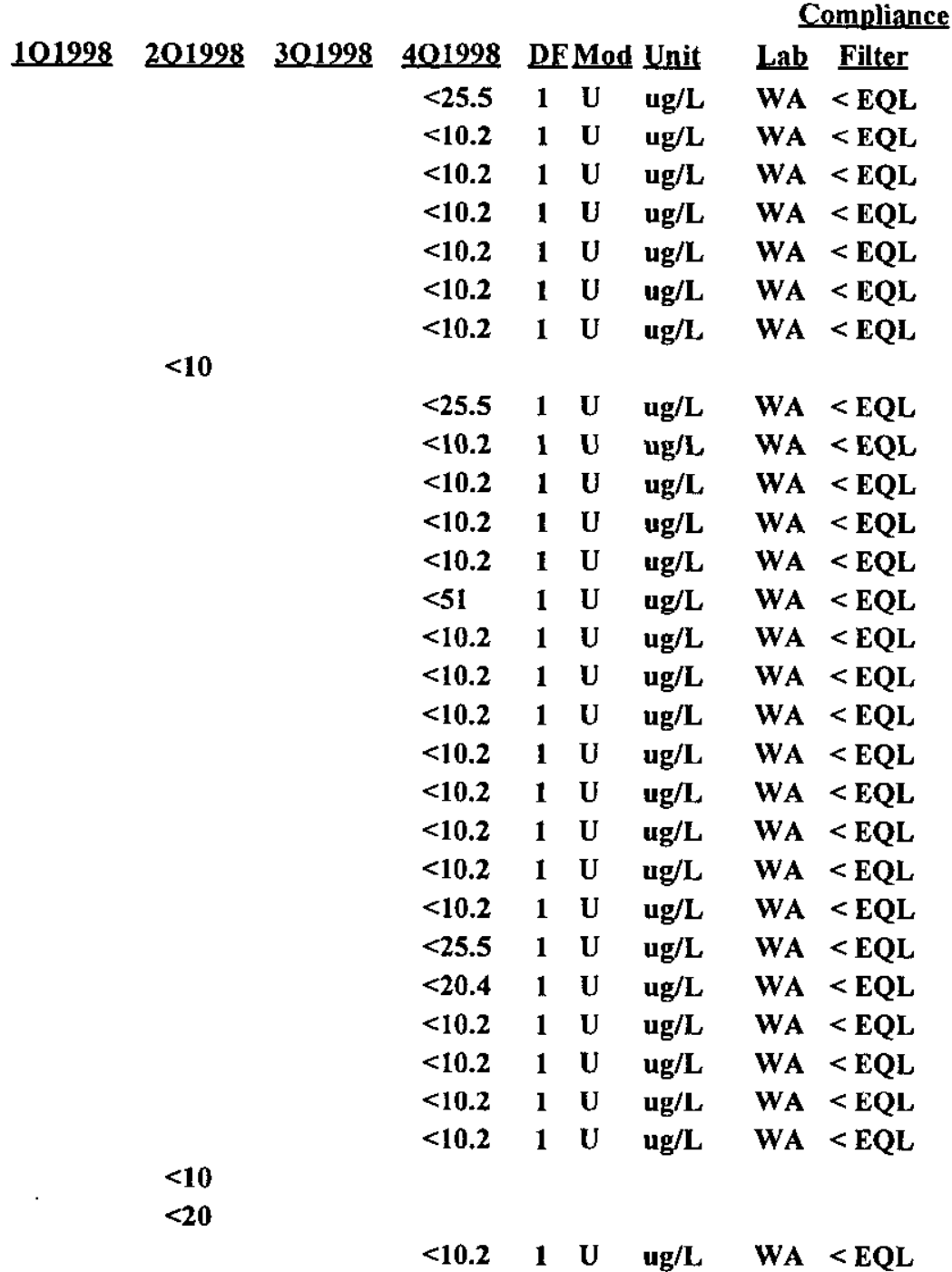

$<10$

$<20$

$<10$

$\begin{array}{ccccccc} & 52.7 & 1 & \mathrm{~J} & \mathrm{ug} / \mathrm{L} & \text { WA } & \text { NDD } \\ & <10.2 & 1 & \mathrm{U} & \mathrm{ug} / \mathrm{L} & \text { WA } & <\mathrm{EQL} \\ & <10.2 & 1 & \mathrm{U} & \mathrm{ug} / \mathrm{L} & \text { WA } & <\mathrm{EQL} \\ <5 & <35 & 1 & \mathrm{U} & \mathrm{ug} / \mathrm{L} & \text { WA } & <\mathrm{EQL} \\ & <20.4 & 1 & \mathrm{U} & \mathrm{ug} / \mathrm{L} & \text { WA } & <\mathrm{EQL} \\ <8 & <28 & 1 & \mathrm{U} & \mathrm{ug} / \mathrm{L} & \text { WA } & <\mathrm{EQL} \\ 7.5 & 5.5 & 1 & & \mathrm{ug} / \mathrm{L} & \text { WA } & 5.5 \\ <5 & & & & & & \end{array}$

Note: Flagging, dilution factors, modifiers, and laboratories are for fourth quartel998 data only. See Appendix B for fagging criteria. * = exceeded holding time for fourth quarter 1998.

$+=$ exceeded screening level or final primary drinking water standard for fourth quarten 998 . 
WELL: LFW 32C

\section{ANALYTICAL DATA}

\section{H ST Analyte}

Benzo(a)anthracene

Benzo(a)pyrene

Benzo(b)fluoranthene

Benzo(g,h,i)perylene

Benzo(k)fluoranthene

Benzoic acid

Benzyl alcohol

Beryllium, total recoverable

Bis(2-chloroethoxy) methane

Bis(2-chloroethyl) ether

Bis(2-chloroisopropyl) ether

Bis(2-ethylhexyl) phthalate

Bromochloromethane

Bromodichloromethane

Bromoform

Bromomethane (Methyl bromide)

Butylbenzyl phthalate

Cadmium, total recoverable

Carbon disulfide

Carbon tetrachloride

Chlorobenzene

Chlorobenzilate

Chloroethane

Chloroethene (Vinyl chloride)

Chloroform

Chloromethane (Methyl chloride)

Chloroprene

Chromium, total recoverable

Chrysene

Cobalt, total recoverable

Copper, total recoverable

Cyanide

Di-n-butyl phthalate

Di-n-octyl phthalate

Diallate

Dibenz(a,h)anthracene

Dibenzofuran

Dibromochloromethane

Dibromomethane (Methylene bromide)

Dichlorodifluoromethane

Dichloromethane (Methylene chloride)

Diethyl phthalate

\section{$101998201998301998 \quad 401998$ DFMod Unit Lab Filter}

$<10.2 \quad 1 \quad$ U $\quad$ ug/L $\quad$ WA $<$ EQL

$<10.2 \quad 1 \quad$ U $\quad$ ug/L $\quad$ WA $<$ EQL

$<10.2 \quad 1 \quad \mathrm{U} \quad$ ug/L $\quad$ WA $<$ EQL

$<10.2 \quad 1 \quad$ U $\quad$ ug/L $\quad$ WA $<$ EQL

$<10.2 \quad 1 \quad$ U $\quad$ ug/L $\quad$ WA $<$ EQL

$<25.5 \quad 1 \quad \mathrm{U} \quad \mathrm{ug} / \mathrm{L} \quad$ WA $<$ EQL

$<10.2 \quad 1 \quad \mathrm{U} \quad$ ug/L $\quad$ WA $<$ EQL

$<1$

$$
<1
$$

$<10.2 \quad 1 \quad \mathrm{U} \quad \mathrm{ug} / \mathrm{L}$

$<10.2 \quad 1 \quad \mathrm{U} \quad \mathrm{ug} / \mathrm{L}$

$<10.2 \quad 1 \quad \mathrm{U} \quad \mathrm{ug} / \mathrm{L}$

$<.805 \quad 1 \quad \mathrm{U} \quad \mathrm{ug} / \mathrm{L}$

$<10$

$<5$

$<5$

$<10$

$<2$

$<5$

$<5$

$<5$

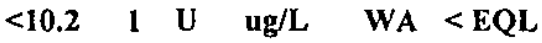

$<10$

$<10$

$<5$

$<10$

$<5$

$\begin{array}{lllllll}.6 & 2.6 & 1 & \mathrm{~J} & \mathrm{ug} / \mathrm{L} & \text { WA } & \text { NDD }\end{array}$

$<10.21 \mathrm{U}$ ug/L WA $<$ EQL

$<5 \quad<7 \quad 11 \quad \mathrm{U} \quad \mathrm{ug} / \mathrm{L} \quad$ WA $<$ EQL

$<3 \quad<3.4 \quad 1 \quad \mathrm{U} \quad u g / \mathrm{L} \quad$ WA $<$ EQL

$<15.21$ UJ ug/L WA $<$ EQL

$<10$

$<10.2 \quad 1 \quad \mathrm{U}$ ug/L WA $<$ EQL

$<10.21-\mathrm{U} / \mathrm{L}$ WA $<\mathrm{EQL}$

$<10.2 \quad 1 \quad \mathrm{U} \quad \mathrm{ug} / \mathrm{L} \quad \mathrm{WA}<\mathrm{EQL}$

$<10.2 \quad 1 \quad \mathrm{U} \quad \mathrm{ug} / \mathrm{L} \quad$ WA $<$ EQL

$<10.2 \quad 1 \quad U \quad$ ug/L $\quad$ WA $<$ EQL

$<5$

$<5$

$<5$

$<3.9$

$<10.2 \quad 1 \quad \mathrm{U} \quad \mathrm{ug} / \mathrm{L} \quad$ WA $<$ EQL

Note: Flagging, dilution factors, modifiers, and laboratories are for fourth quartet 998 data only. See Appendix B for flagging criteria.

* = exceeded holding time for fourth quarter 1998 .

+ - exceeded sereening level or final primary drinking water standard for fourth quarten 1998 .

Sanitary Landfill

D-46

Fourth Quarter 1998 and Annual Summary 
WELL: LFW 32C

\section{ANALYTICAL DATA}

H ST Analyte

Dimethoate

Dimethyl phthalate

Diphenylamine

Disulfoton

Ethyl methacrylate

Ethyl methanesulfonate

Ethylbenzene

Famphur

Fluoranthene

Fluorene

Hexachlorobenzene

Hexachlorobutadiene

Hexachlorocyclopentadiene

Hexachloroethane

Hexachlorophene

Hexachloropropene

Indeno(1,2,3-c,d)pyrene

Iodomethane (Methyl iodide)

Iron, total recoverable

Isobutyl alcohol

Isophorone

Isosafrole

Lead, total recoverable

Mercury, total recoverable

Methacrylonitrile

Methapyrilene

Methyl ethyl ketone

Methyl isobutyl ketone

Methyl methacrylate

Methyl methanesulfonate

N-Nitrosodi-n-butylamine

$\mathrm{N}$-Nitrosodiethylamine

$\mathrm{N}$-Nitrosodimethylamine

$\mathrm{N}$-Nitrosodiphenylamine

$\mathrm{N}$-Nitrosodipropylamine

$\mathrm{N}$-Nitrosomethylethylamine

$\mathrm{N}$-Nitrosomorpholine

$\mathrm{N}$-Nitrosopiperidine

N-Nitrosopyrrolidine

Naphthalene

Nickel, total recoverable

Nitrobenzene

\section{$101998 \quad 201998 \quad 301998$}

$<5$

$<5$

$<100$

$<5$

$<5$

$<10$

$<12$

$<5$

$<5$
Compliance

$\underline{\text { Lab }}$ Filter

$<1.02 \quad 1 \quad U$ ug/L WA $<$ EQL

$<10.2 \quad 1 \quad$ U $\quad$ ug/L $\quad$ WA $<$ EQL

$<10.2 \quad 1 \quad \mathrm{U}$ ug/L WA $<$ EQL

$<1.02$ I U ug/L WA $<$ EQL

$<10.2 \quad 1 \quad \mathrm{U}$ ug/L WA $<$ EQL

$<10.2$ I U ug/L WA $<$ EQL

$<2.65 \quad 1 \quad$ U $\quad$ ug/L $\quad$ WA $<$ EQL

$<10.2 \quad 1 \quad$ U $\quad$ ug/L $\quad$ WA $<$ EQL

$<10.2 \quad 1 \quad$ U $\quad$ ug/L $\quad$ WA $<$ EQL

$<10.2 \quad 1 \quad \mathrm{U} \quad$ ug/L $\quad$ WA $<$ EQL

$<10.2 \quad 1 \quad \mathrm{U} \quad$ ug/L $\quad$ WA $<$ EQL

$<10.2 \quad 1 \quad$ U $\quad$ ug/L $\quad$ WA $<$ EQL

$<10.2 \quad 1 \quad \mathrm{U} \quad$ ug/L $\quad$ WA $<$ EQL

$<102 \quad 1$ U ug/L WA $<$ EQL

$<10.2$ I U Ug/L $\quad$ WA $<$ EQL

$<10.2 \quad 1 \quad \mathrm{U} \quad$ ug/L $\quad$ WA $<$ EQL

$\begin{array}{llllll}152 & 1 & J & u g / L & \text { WA } & \text { NDD }\end{array}$

$<10.2 \quad 1 \quad$ U $\quad$ ug/L $\quad$ WA $<$ EQL

$<10.2 \quad 1$ U $\quad$ ug/L $\quad$ WA $<$ EQL

$<17 \quad 1 \quad$ U $\quad$ ug/L $\quad$ WA $<$ EQL

$\begin{array}{llllll}<1 & 1 & \text { U } & \text { ug/L } & \text { WA } & <\text { EQL }\end{array}$

$<10.2 \quad 1 \quad$ U $\quad$ ug/L $\quad$ WA $<$ EQL

$<10.2 \quad 1 \quad$ U $\quad$ ug/L $\quad$ WA $<$ EQL

$<10.2 \quad 1 \quad$ U $\quad$ ug/L $\quad$ WA $<$ EQL

$<10.2$ I $U$ ug/L WA $<$ EQL

$<10.2 \quad 1$ U ug/L WA $<$ EQL

$<10.2 \quad 1$ U ug/L WA $<$ EQL

$<10.2 \quad 1 \quad$ U $\quad$ ug/L $\quad$ WA $<$ EQL

$<10.2 \quad 1 \quad U \quad$ ug/L WA $<$ EQL

$<10.2 \quad 1 \quad$ U ug/L WA $\quad$ WQL

$<10.2 \quad 1 \quad$ U $\quad$ ug/L $\quad$ WA $<$ EQL

$\begin{array}{llllll}<1 & 1 & \text { U } & \text { ug/L } & \text { WA } & <\text { EQL }\end{array}$

$<10.2 \quad 1 \quad$ U $\quad$ ug/L $\quad$ WA $<$ EQL

$<10.2 \quad 1 \quad$ U $\quad$ ug/L $\quad$ WA $<$ EQL

$<15 \quad$ I U ug/L WA $<$ EQL

$<10.2 \quad 1 \quad \mathrm{U} \quad$ ug/L $\quad$ WA $<$ EQL

Note: Flagging, dilution factors, modifiers, and laboratories are for fourth quarte1998 data only. See Appendix B for flagging criteria.

* = exceeded holding time for fourth quarter 1998 .

$+=$ exceeded screening level or final primary drinking water standard for fourth quarten 998 . 
WELL: LFW 32C

\section{ANALYTICAL DATA}

H ST Analyte

0,0,0-Triethyl phosphorothioate

Parathion ethyl

Parathion methyl

Pentachlorobenzene

Pentachloroethane

Pentachloronitrobenzene

Pentachlorophenol

Phenacetin

Phenanthrene

Phenol

Phorate

Pronamid

Propionitrile

Pyrene

Pyridine

Safrole

Selenium, total recoverable

Silver, total recoverable

Specific conductance

Styrene

* Sulfide

Sulfotepp

Tetrachloroethylene

Thallium, total recoverable

Thionazin

Tin, total recoverable

Toluene

Trichloroethylene

Trichlorofluoromethane

Tritium

Vanadium, total recoverable

Vinyl acetate

Xylenes

Zinc, total recoverable

a,a-Dimethylphencthylamine

beta-Benzene hexachloride

cis-1,2-Dichloroethylene

cis-1,3-Dichloropropene

m-Cresol (3-Methylphenol)

m-Nitroaniline

o-Cresol (2-Methylphenol)

o-Nitroaniline

\section{Compliance}

$101998201998 \quad 301998$ 401998 DFMod Unit

$<1.02 \quad 1 \quad \mathrm{U} \quad \mathbf{u g} / \mathrm{L}$

$<1.02 \quad 1 \quad \mathrm{U} \quad \mathrm{ug} / \mathrm{L}$

$<1.021 \mathrm{U}$ ug/L

$<10.2 \quad 1 \quad \mathrm{U}$ ug/h

$<10.21 \mathrm{U} \quad \mathrm{ug} / \mathrm{L}$

$<51 \quad 1 \quad \mathrm{U} \quad \mathrm{ug} / \mathrm{L}$

$<25.5 \quad 1 \quad \mathrm{U} \quad \mathrm{ug} / \mathrm{L}$

$<10.2 \quad 1 \quad \mathrm{U} \quad \mathrm{ug} / \mathrm{L}$

$<10.2 \quad 1 \quad \mathrm{U} \quad \mathrm{ug} / \mathrm{L}$

$<10.2 \quad 1 \quad \mathrm{U} \quad \mathrm{ug} / \mathrm{L}$

$<2.04 \quad 1 \quad \mathrm{U} \quad \mathrm{ug} / \mathrm{L}$

$<10.2 \quad 1 \quad U \quad \mathrm{ug} / \mathrm{L}$

Lab Filter

WA $<$ EQL

WA $<$ EQL

WA $<$ EQL

WA $<$ EQL

WA $<$ EQL

WA $<$ EQL

WA $<$ EQL

WA $<$ EQL

WA $<$ EQL

WA $<$ EQL

WA $<$ EQL

WA $<$ EQL

$<5$

$<5$

$<2$

31.6

$<5$

$<10.2 \quad 1 \quad \mathrm{U} \quad \mathrm{ug} / \mathrm{L} \quad$ WA $<$ EQL

$<10.21 \mathrm{U} \quad \mathrm{ug} / \mathrm{L} \quad \mathrm{WA}<\mathrm{EQL}$

$<10.211$ U $\quad$ ug/L $\quad$ WA $<$ EQL

$<36 \quad 1 \quad$ U $\quad$ ug/L $\quad$ WA $<$ EQL

$<5 \quad 1 \quad \mathrm{U} \quad \mathrm{ug} / \mathrm{L} \quad$ WA $<$ EQL

$\begin{array}{lllll}18.4 & 1 & \text { uS } / \mathrm{cm} & \text { WA } & 18.4\end{array}$

$<10000 \quad 1 \quad$ UJ $\quad$ ug/L WA $\quad$ EQL

$<2.04 \quad 1 \quad \mathrm{U} \quad \mathrm{ug} / \mathrm{L} \quad \mathrm{WA}<$ EQL

$<5$

$<5$

$<28 \quad 1 \quad$ U $\quad$ ug/L $\quad$ WA $<$ EQL

$<1.02 \quad 1 \quad \mathrm{U} \quad$ ug/L $\quad$ WA $<$ EQL

$<31 \quad 1 \quad \mathrm{U} \quad \mathrm{ug} / \mathrm{L} \quad \mathrm{WA}<\mathrm{EQL}$

$<5$

$<5$

$<5$

$<690$

$1.1<6 \quad 1 \quad$ U $\quad$ ug/L $\quad$ WA $<$ EQL

$<5$

$<5$

$\begin{array}{rlllll}10.8 & = & \text { ug/L } & \text { WA } & 26.4 \\ & <10.2 & 1 & \text { U } & \text { ug/L } & \text { WA }<E Q L\end{array}$

$<.015$

$<5$

$<5$

\begin{tabular}{|c|c|c|c|c|c|}
\hline$<10.2$ & 1 & $\mathbf{U}$ & $\mathbf{u g} / \mathrm{L}$ & WA & $<$ EQL \\
\hline$<25$ & 1 & $\mathbf{U}$ & $\mathrm{ug} / \mathrm{L}$ & WA & $<$ EQL \\
\hline 10 & & 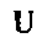 & $\mathbf{u g} / \mathrm{L}$ & WA & $<$ EQL \\
\hline 5 & & & $\mathbf{u g} / \mathbf{L}$ & WA & \\
\hline
\end{tabular}

Note: Flagging, dilution factors, modifiers, and laboratories are for fourth quartet 998 data only. See Appendix B for flagging criteria. * = exceeded holding time for fourth quarter 1998 .

$+=$ exceeded screening level or final primary drinking water standard for fourth quarter1998 . 
WELL: LFW 32C

\section{ANALYTICAL DATA}

\section{H ST Analyte}

o-Toluidine

p-Cresol (4-Methylphenol)

p-Dimethylaminoazobenzene

p-Nitroaniline

p-Phenylenediamine

* pH

trans-1,3-Dichloropropene

trans-1,4-Dichloro-2-butene

\section{$\underline{101998 \quad 201998 \quad \underline{301998}}$}

\begin{tabular}{|c|c|c|c|c|c|}
\hline 401998 & DF & Mo & Unit & Lab & Filter \\
\hline$<10.2$ & 1 & $\mathbf{U}$ & $\mathrm{ug} / \mathrm{L}$ & WA & $<\mathrm{EQL}$ \\
\hline$<10.2$ & 1 & $\mathrm{U}$ & ug/L & WA & $<\mathrm{EQL}$ \\
\hline$<10.2$ & 1 & $\mathbf{U}$ & $\mathbf{u g} / \mathrm{L}$ & WA & $<\mathrm{EQL}$ \\
\hline$<25.5$ & 1 & $\mathbf{U}$ & $\mathbf{u g} / \mathbf{L}$ & WA & $<\mathbf{E Q L}$ \\
\hline$<10.2$ & 1 & $\mathbf{U}$ & ug/L & WA & $<$ EQL \\
\hline 5.33 & 1 & $\mathbf{J}$ & pH & WA & NDD \\
\hline
\end{tabular}

\section{Compliance}

$<5$

$<5$

Note: Flagging, dilution factors, modifiers, and laboratories are for fourth quartei 998 data only. See Appendix B for flagging criteria. * = exceeded holding time for fourth quarter 1998 .

$+=$ exceeded screening levet or final primary drinking water standard for fourth quarter 998 . 
WELL: LFW 34

\section{SRS Coord. \\ N 85409.5 \\ E 45016.9 81.714 Deg W}

$\frac{\text { Screen Zone Elevation }}{164.7-143.7 \mathrm{ft} \mathrm{msl}}$

\section{Top of Casing}

201.0 ft msl $\frac{\text { Casing }}{4 \text { "PVC }}$

Punn

S

Eormation

\section{SAMPLE DATE}

FIELD DATA

Analyte
Water Elevation
Depth to water
pH
Sp. Conductance
Water temperature
Alkalinity as CaCO3
Turbidity
Volumes purged
Sampling code

ANALYTICAL DATA

1,1,1,2-Tetrachloroethane 1,1,1-Trichloroethane 1,1,2,2-Tetrachloroethane 1,1,2-Trichloroethane 1,1-Dichloroethane 1,1-Dichloroethylene 1,2,3-Trichloropropane 1,2-Dibromo-3-chloropropane 1,2-Dibromoethane 1,2-Dichlorobenzene 1,2-Dichloroethane 1,2-Dichloropropane 1,4-Dichlorobenzene 2-Hexanone 101998

$\begin{array}{ll}201998 & 301998 \\ 158.94 & \\ 39.76 & \\ 4.3 & \\ 29 & \\ 16.1 & \\ 0 & \\ .4 & \\ 6.34438 & \end{array}$

401998

Unit

ft msl ft pH uS/cm deg. C $\mathrm{mg} / \mathrm{L}$ NTU well volumes

\section{Acetone}

Acetonitrile (Methyl cyanide)

Acrolein $<5$ $<5$

$<5$

$<5$

2

$<5$

$<5$

$<5$

$<5$

$<5$

$<5$

$<5$

$<10$

$<5.2$

$<20$

$<10$

Acrylonitrile

$<20$

Allyl chloride

$<10$

Antimony, total recoverable

$<5$

Arsenic, total recoverable

Barium, total recoverable

$<8$

Benzene

Beryllium, total recoverable

Bromochloromethane

$<5$

$<1$

Bromodichloromethane

$<10$

$<5$

Compliance

Note: Flagging, dilution factors, modifiers, and laboratories are for fourth quartei 998 data only. See Appendix B for flagging criteria. * = excecded holding time for fourth quarter 1998 .

$+=$ excecded screening level or final primary drinking water standard for fourth quarteng98. 
WSRC-TR-99-00011

Unclassified

WELL: LFW 34

\section{ANALYTICAL DATA}

\section{H ST Analyte}

Bromoform

Bromomethane (Methyl bromide)

Cadmium, total recoverable

Carbon disulfide

Carbon tetrachloride

Chlorobenzene

Chloroethane

Chloroethene (Vinyl chloride)

Chloroform

Chloromethane (Methyl chloride)

Chloroprene

Chromium, total recoverable

Cobalt, total recoverable

Copper, total recoverable

Di-n-butyl phthalate

Dibromochloromethane

Dibromomethane (Methylene bromide)

Dichlorodifluoromethane

Dichloromethane (Methylene chloride)

Ethylbenzene

Iodomethane (Methyl iodide)

Isobutyl alcohol

Lead, total recoverable

Methacrylonitrile

Methyl ethyl ketone

Methyl isobutyl ketone

Methyl methacrylate

Nickel, total recoverable

Propionitrile

Selenium, total recoverable

Silver, total recoverable

Specific conductance

Styrene

Tetrachloroethylene

Thallium, total recoverable

Toluene

Trichloroethylene

Trichlorofluoromethane

Tritium

Unknown

Unknown 6

Unknown 7

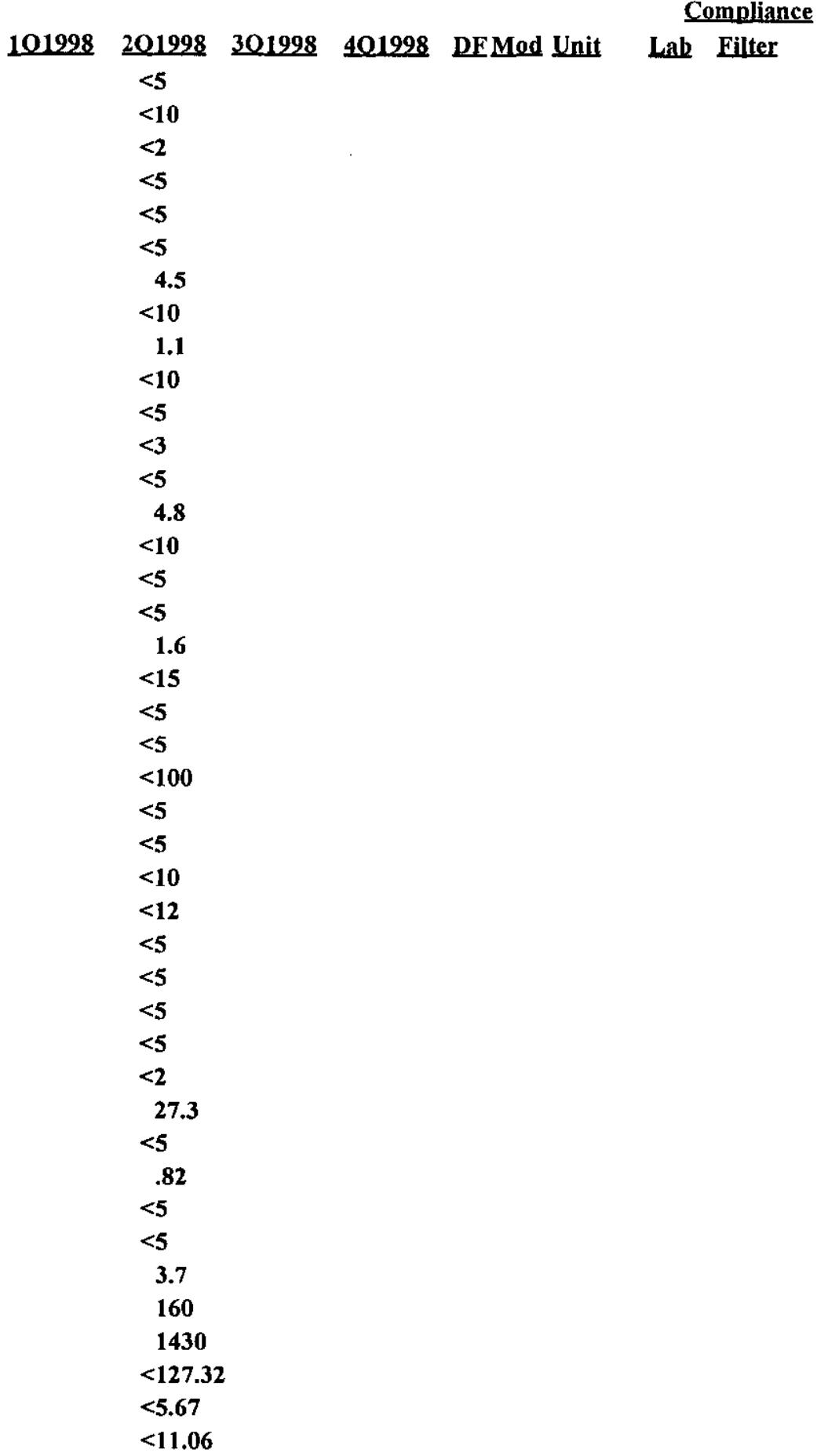

Note: Flagging, dilution factors, modifiers, and laboratories are for fourth quartei998 data only. See Appendix B for flagging criteria.

* = exceeded holding time for fourth quarter 1998 .

$+=$ exceeded screening level or final primary drinking water standard for fourth quarten 998 .

Sanitary Landfill

D-51

Fourth Quarter 1998 and Annual Summary 
WELL: LFW 34

ANALYTICAL DATA

H ST Analyte

Unknown 8

Vanadium, total recoverable

Vinyl acetate

Xylenes

Zinc, total recoverable

beta-Benzene hexachloride

cis-1,2-Dichloroethylene

cis-1,3-Dichloropropene

pH

trans-1,3-Dichloropropene

trans-1,4-Dichloro-2-butene

101998 \begin{aligned} & 201998 \\ &$<38.77 \\ &<2 \\ &<5 \\ &<5 \\ & 11.2 \multicolumn{2}{c}{$ Compliance } \\ &$<.015 \\ &<5 \\ &<5 \\ & 4.87$ Lab Filter \\ &$<5 \\ &<5\end{aligned}$

Note: Flagging, dilution factors, modifiers, and laboratories are for fourth quartei 998 data only. See Appendix B for flagging criteria. * exceeded holding time for fourth quarter 1998.

$+=$ exceeded screening level or final primary drinking water standard for fourth quarter 998 . 
WSRC-TR-99-00011

Unclassified

WELL: LFW 36R

\begin{tabular}{|c|c|c|c|c|c|}
\hline E. & $\frac{\text { Lat/Longitude }}{33.285 \text { Deg N }}$ & $\frac{\text { Screen Zone Elevation }}{141.8-121.8 \mathrm{ft} \mathrm{msl}}$ & $\frac{\text { Top of Casing }}{168.2 \mathrm{ft} \mathrm{msl}}$ & $\frac{\text { Casing }}{2 " P V C}$ & $\frac{\text { Pump }}{\mathrm{V}}$ \\
\hline
\end{tabular}

E 45519.1 81.709 Deg W

SAMPLE DATE

FIELD DATA

Analyte

Water Elevation

Depth to water

pH

Sp. Conductance

Water temperature

Alkalinity as $\mathrm{CaCO3}$

Turbidity

Volumes purged

Sampling code

$\begin{array}{llll}12 / 01 / 97 & 03 / 16 / 98 & 06 / 10 / 98 & 09 / 15 / 98\end{array}$

$\begin{array}{lllll}101998 & \frac{201998}{109} & \frac{301998}{401998} & \text { Unit } \\ 143.61 & 145.26 & 144.41 & 144.46 & \text { ft msi } \\ 22.55 & 20.9 & 21.75 & 21.7 & \text { ft } \\ 6.6 & 6.4 & 6 & & \text { pH } \\ 160 & 200 & 170 & & \text { uS/cm } \\ 19 & 19 & 20.8 & & \text { deg. C } \\ 70 & 70 & 73 & & \text { mg/L } \\ 3 & 1.6 & 1.4 & & \text { NTU } \\ 7.90897 & 5.01169 & 7.40594 & & \text { well volumes }\end{array}$

\section{ANALYTICAL DATA}

\section{H ST Analyte}

1,1,1,2-Tetrachloroethane

1,1,1-Trichloroethane

1,1,2,2-Tetrachloroethane

1,1,2-Trichloroethane

1,1-Dichloroethane

1,1-Dichloroethylene

1,2,3-Trichloropropane

1,2-Dibromo-3-chloropropane

1,2-Dibromoethane

1,2-Dichloroethane

1,2-Dichloropropane

1,4-Dichlorobenzene

2-Hexanone

Acetone

Acetonitrile (Methyl cyanide)

Acrolein

Acrylonitrile

Allyl chloride

Aluminum, total recoverable

Arsenic, total recoverable

Barium, total recoverable

Benzene

Bromodichloromethane

Bromoform

Bromomethane (Methyl bromide)

Cadmium, total recoverable

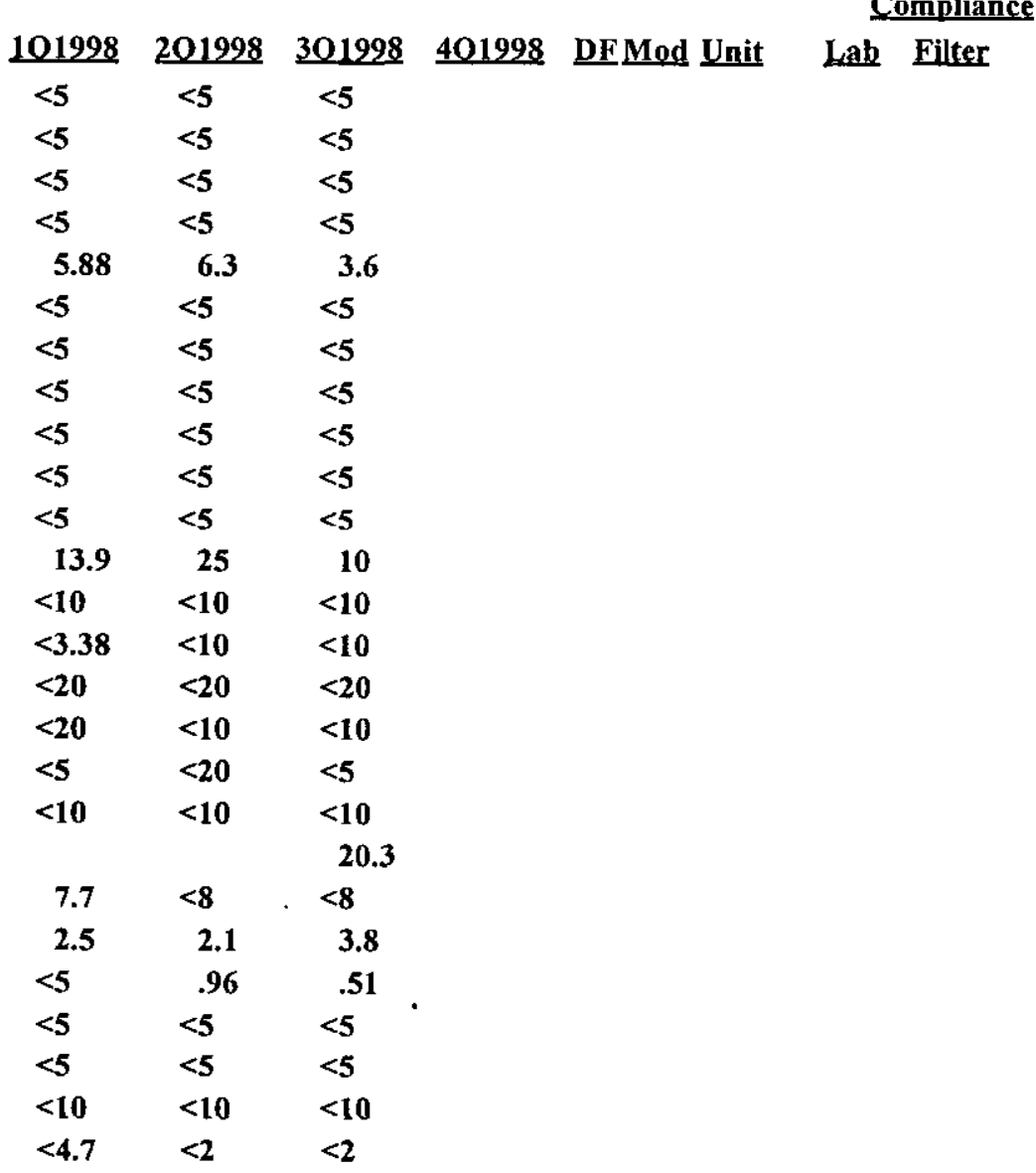

Note: Flagging, dilution factors, modifiers, and laboratories are for fourth quartel 998 data only. See Appendix B for nagging criteria.

* = exceeded holding time for fourth quarter1998.

$+=$ exceeded screening level or final primary drinking water standard for fourth quarter 1998 .

Sanitary Landfill

D-53

Fourth Quarter 1998 and Annual Summary 
WSRC-TR-99-00011

Unclassified

WELL: LFW 36R

ANALYTICAL DATA

H ST Analyte

Carbon disulfide

Carbon tetrachloride

Chlorobenzene

Chloroethane

Chloroethene (Vinyl chloride)

Chloroform

Chloromethane (Methyl chloride)

Chloroprene

Chromium, total recoverable

Dibromochloromethane

Dibromomethane (Methylene bromide)

Dichlorodifluoromethane

Dichloromethane (Methylene chloride)

Ethylbenzene

Gross alpha

Iodomethane (Methyl iodide)

Iron, total recoverable

Isobutyl alcohol

Lead, total recoverable

Mercury, total recoverable

Methacrylonitrile

Methyl ethyl ketone

Methyl isobutyl ketone

Methyl methacrylate

Propionitrile

Selenium, total recoverable

Silver, total recoverable

Styrene

Tetrachloroethylene

Toluene

Trichloroethylene

Trichlorofluoromethane

Tritium

VinyI acetate

Xylenes

cis-1,3-Dichloropropene

trans-1,2-Dichloroethylene

trans-1,3-Dichloropropene

trans-1,4-Dichloro-2-butene
$<5<5<5$

$<5<5 \quad<5$

$\begin{array}{lll}8.64 & 11 & 4.7\end{array}$

$<10<10<10$

$\begin{array}{lll}19.6 & 22 & 13\end{array}$

$<5<5 \quad<5$

$<10<10<10$

$<5<5 \quad<5$

$<7<3<3$

$<5<5 \quad<5$

$<5<5 \quad<5$

$\begin{array}{lll}<10 & 1.2 & .87\end{array}$

$<3.29<2<2.4$

$<5<5<5$

$2.99<-.61 \quad 2.73$

$<5<5 \quad<5$

29700

$<100<100<100$

$8.2<5 \quad 4.3$

$<.7 \quad<.2 \quad .06$

$<10<5 \quad<5$

$<10<10<10$

$<10<12 \quad<12$

$<5<5$

$<50<5 \quad<5$

$<66<5<5$

$<5<2 \quad<2$

$<5<5 \quad<5$

$<5<5<5$

$<5 \quad 1.1<5$

$<5<5<5$

$1.92<5<5$

$<3.89 \quad 3520 \quad 2.79$

$<10<5=-<5$

$<5 \quad 1.5 \quad<5$

$<5<5<5$

$<5$

$<5<5 \quad<5$

$<20<5 \quad<5$

3

06

10

5

5$$
5
$$

$\quad 2.79$
$<5$
$<5$
$<5$
$<5$
$<5$

Compliance

401998 DFMod Unit Lab Filter

7

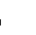

0

(2)

7

\footnotetext{
Note: Flagging, dilution factors, modifiers, and laboratories are for fourth quarte 998 data only. See Appendix B for flagging criteria. * = exceeded holding time for fourth quarter 1998 .

$+=$ exceded screening level or final primary drinking water standard for fourth quarten 998 .
} 
WELL: LFW 41R

\begin{tabular}{|c|c|c|c|c|c|c|}
\hline SRS Coord, & Lat/Longitude & Screen Zone Elevation & Ten of Casing & Casing & & 1001 \\
\hline $\begin{array}{l}\text { N } 83238.3 \\
\text { E } 46635.3\end{array}$ & $\begin{array}{ll}\text { 33.286 } & \text { Deg N } \\
\text { 81.706 } & \text { Deg W }\end{array}$ & $140.2-120.2 \mathrm{ft} \mathrm{msl}$ & $1607 \mathrm{ft}^{\mathrm{m}}$ & & V & \\
\hline
\end{tabular}
SAMPLE DATE
$12 / 04 / 97$
$03 / 12 / 98$
06/08/98

FIELD DATA

Analyte
Water Elevation
Depth to water
pH
Sp. Conductance
Water temperature
Alkalinity as CaCO3
Turbidity
Volumes purged
Sampling code

ANALYTICAL DATA

H ST Analyte

1,1,1,2-Tetrachloroethane

1,1,1-Trichloroethane

1,1,2,2-Tetrachloroethane

1,1,2-Trichloroethane

1,1-Dichloroethane

1,1-Dichloroethylene

1,2,3-Trichloropropane

1,2-Dibromo-3-chloropropane

1,2-Dibromoethane

1,2-Dichloroethane

1,2-Dichloropropane

1,4-Dichlorobenzene

2-Hexanone

Acetone

Acetonitrile (Methyl cyanide)

Acrolein

Acrylonitrile

Allyl chloride

Aluminum, total recoverable Arsenic, total recoverable

Barium, total recoverable

Benzene

Bromodichloromethane

Bromoform

Bromomethane (Methyl bromide)

Cadmium, total recoverable

\begin{tabular}{|c|c|c|}
\hline 101998 & 201998 & 301998 \\
\hline 141.6 & 143.95 & 142.25 \\
\hline 26 & 23.65 & 25.35 \\
\hline 4.6 & 4.4 & 5 \\
\hline 17 & 17 & 20 \\
\hline 18 & 19.9 & 19.3 \\
\hline 0 & 18 & 0 \\
\hline .2 & .3 & .2 \\
\hline 3.63259 & 2.83059 & 3.02984 \\
\hline
\end{tabular}

$\underline{401998}$

Unit

ft msl

ft

pH

uS/cm

deg. C

$\mathbf{m g} / \mathbf{L}$

NTU

well volumes

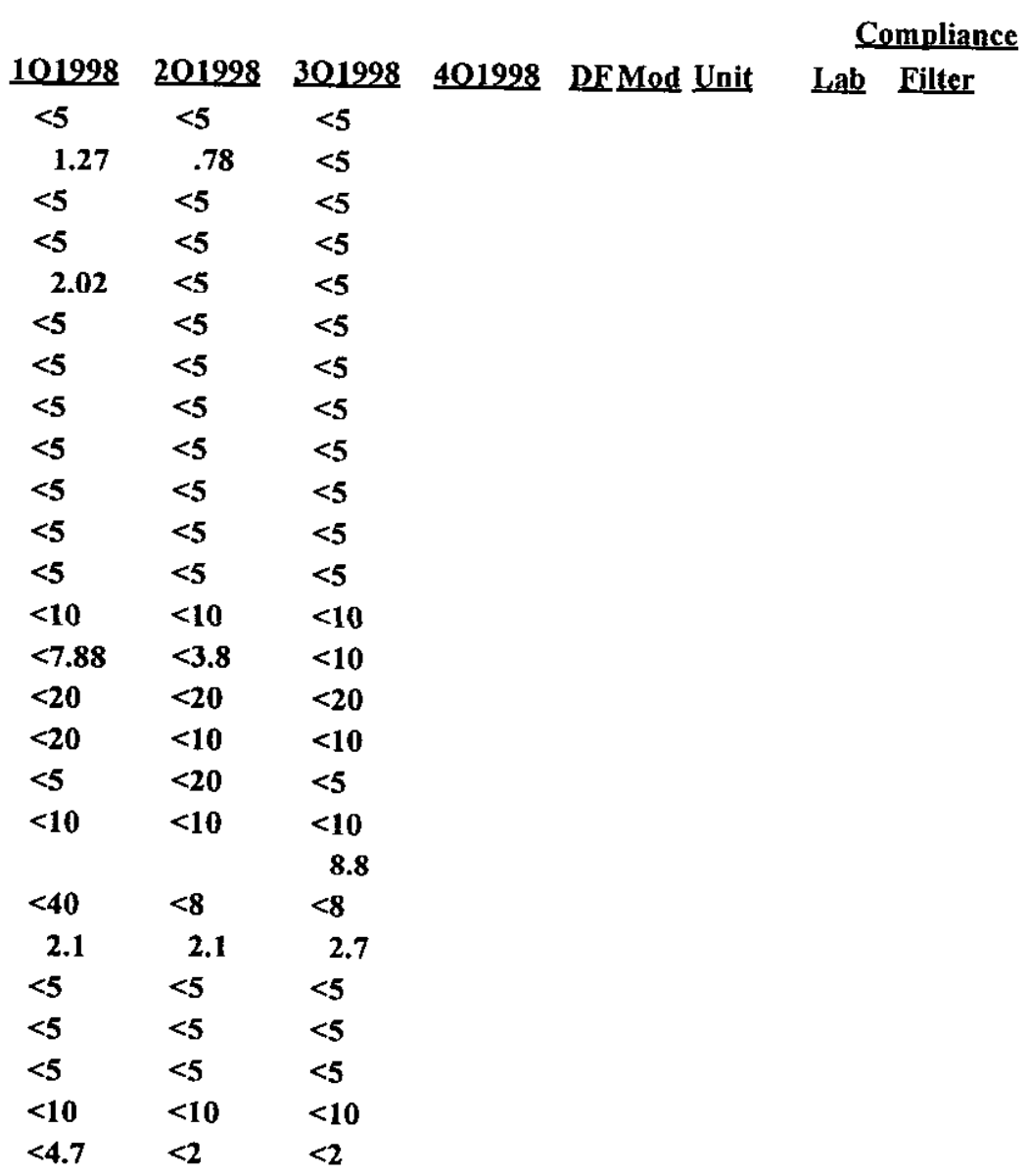

Note: Flagging, dilution factors, modifiers, and laboratories are for fourth quarte1998 data only. See Appendix B for fagging criteria.
$* \Rightarrow$ exceeded holding time for fourth quarter1998.

+ exceeded screening level or final primary drinking water standard for fourth quarter1 998 .

Sanitary Landfill

D-55

Fourth Quarter 1998 and Annual Summary 
WELL: LFW 41R

\section{ANALYTICAL DATA}

\section{H ST Analyte}

Carbon disulfide

Carbon tetrachloride

Chlorobenzene

Chloroethane

Chloroethene (Vinyl chloride)

Chloroform

Chloromethane (Methyl chloride)

Chloroprene

Chromium, total recoverable

Dibromochloromethane

Dibromomethane (Methylene bromide)

Dichlorodifluoromethane

Dichloromethane (Methylene chloride)

Ethylbenzene

Gross alpha

Iodomethane (Methyl iodide)

Iron, total recoverable

Isobutyl alcohol

Lead, total recoverable

Mercury, total recoverable

Methacrylonitrile

Methyl ethyl ketone

Methyl isobutyl ketone

Methyl methacrylate

Propionitrile

Selenium, total recoverable

Silver, total recoverable

Styrene

Tetrachloroethylene

Toluene

Trichloroethylene

Trichlorofluoromethane

Tritium

Unknown

Vinyl acetate

Xylenes

cis-1,3-Dichloropropene

trans-1,2-Dichloroethylene

trans-1,3-Dichloropropene

trans-1,4-Dichloro-2-butene
101998

$<5$

$<5$

$<5$

$<10$

$<10$

$<5$

$<10$

$<5$

$1.2<3$

$<5<5$

$<5<5$

$<24.6<2.2<2.1$

$<5<5 \quad<5$

$2.46<-.78 \quad 1.89$

$<5<5 \quad<5$

$<100<100<100$

$<47 \quad<5 \quad 7.7$

$<.7<.2<.2$

$<10<5<5$

$<10<10 \quad<10$

$<10<12<12$

$<5<5$

$<50<5<5$

$<66<5<5$

$<5<2<2$

$<5<5<5$

$<5<5<5$

$<5<5<5$

$1.1<5<5$

$6.88<5 \quad 3.9$

$<2.14 \quad 1730<82$

$\begin{array}{lll}<10 & <6.89 & \\ <5 & <5 & <5 \\ <5 & <5 & <5 \\ <5 & & \\ <5 & <5 & <5 \\ <20 & <5 & <5\end{array}$

5

5

2

5

5

5

5

5

$<5$
$<5$

Compliance

Lab Filter
39.6

$<5$

5

10

5

$<10$

3

5

4

89

7

.9

82

Note: Flagging, dilution factors, modifiers, and laboratories are for fourth quartei998 data only. See Appendix B for flagging criteria. * exceeded holding time for fourth quarter 1998.

$+=$ exceeded screening level or final primary drinking water standard for fourth quarter1998 . 
WSRC-TR-99-00011

Unclassified

WELL: LFW 43B

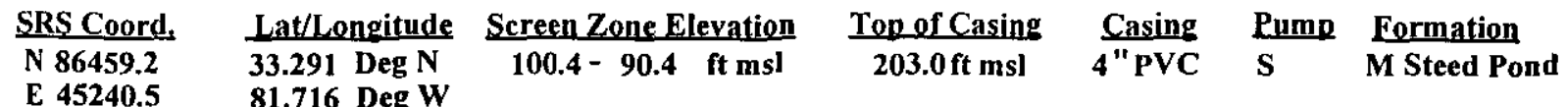

$\begin{array}{llll}\text { SAMPLE DATE } & 12 / 08 / 97 & 03 / 16 / 98 & 06 / 08 / 98\end{array}$

\section{FIELD DATA}

Analyte

Water Elevation

Depth to water

pH

Sp. Conductance

Water temperature

Alkalinity as $\mathrm{CaCO3}$

Turbidity

Volumes purged

$\begin{array}{lll}12 / 08 / 97 & 03 / 16 / 98 & 06 / 08 / 98\end{array}$

Sampling code

\begin{tabular}{|c|c|c|c|c|}
\hline 101998 & $\underline{201998}$ & 301998 & 401998 & Unit \\
\hline 161.75 & 165.54 & 165.8 & & $\overrightarrow{\mathrm{ft}} \mathrm{msl}$ \\
\hline 39.25 & 35.46 & 35.2 & & ft \\
\hline 5 & 5.4 & 5.2 & & pH \\
\hline 18 & 20 & 19 & & $\mathrm{uS} / \mathrm{cm}$ \\
\hline 20 & 16 & 18.7 & & deg. C \\
\hline 3 & 1 & 1 & & $\mathrm{mg} / \mathrm{L}$ \\
\hline .9 & .5 & .9 & & NTU \\
\hline 2.82641 & 3.77442 & 3.99808 & & well volumes \\
\hline
\end{tabular}

\section{ANALYTICAL DATA}

H ST Analyte

1,1,1,2-Tetrachloroethane

\section{1,1,1-Trichloroethane}

1,1,2,2-Tetrachloroethane

1,1,2-Trichloroethane

1,1-Dichloroethane

1,1-Dichloroethylene

1,2,3-Trichloropropane

1,2-Dibromo-3-chloropropane

1,2-Dibromoethane

1,2-Dichlorobenzene

1,2-Dichloroethane

1,2-Dichloropropane

1,3-Dichlorobenzene

1,4-Dichlorobenzene

1,4-Dioxane

2-Hexanone

2-Picoline

Acetone

Acetonitrile (Methyl cyanide)

Acrolein

Acrylonitrile

Allyl chloride

Aluminum, total recoverable

Antimony, total recoverable

Arsenic, total recoverable

Barium, total recoverable
10199

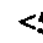

$<5$

$<5$

$<5$

$<5$

$<5$

$<5$

$<5$

$<5$

$<1$

$<5$

$<5$

$<1$

$<5$

$<1$

$<10$

$<1$

$<10$

$<20$

$<20$

$<5$

$<10$

$<10$

$<10 \quad<10$

$<20<20$

$<10<10$

$<20<5$

$<10<10$

13.2

$<5$

$<40 \quad<8$

3.8

3.7

$<8$

Note: Flagging, dilution factors, modifers, and laboratories are for fourth quarte1998 data only. See Appendix B for nagging criteria.

* = exceeded holding time for fourth quarter 1998 .

$+=$ exceeded screening tevel or final primary drinking water standard for fourth quarten 998 .

Sanitary Landfisl

D-57

Fourth Quarter 1998 and Annual Summary 


\section{ANALYTICAL DATA}

\section{H ST Analyte}

Benzene

Beryllium, total recoverable

Bromochloromethane

Bromodichloromethane

Bromoform

Bromomethane (Methyl bromide)

Cadmium, total recoverable

Carbon disulfide

Carbon tetrachloride

Chlorobenzene

Chloroethane

Chloroethene (Vinyl chloride)

Chloroform

Chloromethane (Methyl chloride)

Chloroprene

Chromium, total recoverable

Cobalt, total recoverable

Copper, total recoverable

Di-n-butyl phthalate

Dibromochloromethane

Dibromomethane (Methylene bromide)

Dichlorodifluoromethane

Dichloromethane (Methylene chloride)

Ethyl methacrylate

Ethylbenzene

Gross alpha

Iodomethane (Methyl iodide)

Iron, total recoverable

Isobutyl alcohol

Lead, total recoverable

Mercury, total recoverable

Methacrylonitrile

Methyl ethyl ketone

Methyl isobutyl ketone

Methyl methacrylate

Nickel, total recoverable

Pentachloroethane

Propionitrile

Selenium, total recoverable

Silver, total recoverable

Specific conductance

Styrene
10199

$<5$

$<1$

$<10$

$<5 \quad<5$

$<5 \quad<5$

$<10 \quad<10$

$<4.7<2$

$<5<5$

$<5 \quad<5$

$<5 \quad<5$

$<10<10$

$<10<10$

$<5<5$

$<10<10$

$<5<5$

$<7<3$

$<5$

$<3$

$<10$

$<5 \quad<5 \quad<5$

$<5<5<5$

$<10<5 \quad<5$

$<5.48<3.4<1.9$

$<1$

$<5 \quad<5 \quad<5$

$2.73<-.36 \quad 2.73$

$<5 \quad<5 \quad<5$

18.7

$<100<100<100$

$<47 \quad<5 \quad<5$

$<.7<.2<.2$

$<10<5<5$

$\leq 10 \ldots<10 \quad \leq 10$

$<10<12<12$

$<1<5 \quad<5$

$<5$

$<1$

$<50 \quad<5 \quad<5$

$<66<5<5$

$<5 \quad<2 \quad<2$

17.4

$<5<5<5$
Compliance

Lab Eilter

Note: Fagging, dilution factors, modifiers, and laboratories are for fourth quarte1998 data only. Sce Appendix B for flagging criteria.

* = exceeded holding time for fourth quarter 1998 .

+ - exceeded screening level or final primary drinking water standard for fourth quarter 998 . 
WSRC-TR-99-00011

Unclassified

WELL: LFW 43B

\section{ANALYTICAL DATA}

H ST Analyte

Tetrachloroethylene

Thallium, total recoverable

Toluene

Trichloroethylene

Trichlorofluoromethane

Tritium

Vanadium, total recoverable

Vinyl acetate

Xylenes

Zinc, total recoverable beta-Benzene hexachloride cis-1,2-Dichloroethylene cis-1,3-Dichloropropene pH

trans-1,2-Dichloroethylene trans-1,3-Dichloropropene trans-1,4-Dichloro-2-butene

\begin{tabular}{clllll}
101998 & $\frac{201998}{201998}$ & 401998 & DFMod Unit & \multicolumn{2}{c}{ Compliance } \\
$<5$ & $<5$ & $<5$ & & Filter \\
& $<5$ & & & \\
$<5$ & $<5$ & $<5$ & & \\
$<5$ & $<5$ & $<5$ & & \\
$<5$ & $<5$ & $<5$ & \\
.99 & $<1160$ & $<.27$ \\
& $<2$ & & \\
$<10$ & $<5$ & $<5$ \\
$<5$ & $<5$ & $<5$ \\
& $<10$ & \\
& $<.015$ & \\
$<5$ & $<5$ & $<5$ \\
& 5.27 & \\
$<5$ & $<5$ & $<5$ \\
$<5$ & $<5$ & $<5$ \\
$<20$ & $<5$ & $<5$
\end{tabular}

Note: Flagging, dilution factors, modifiers, and laboratories are for fourth quarted998 data only. See Appendix B for flagging criteria. * = exceeded holding time for fourth quarter 1998 .

$+=$ exceeded screening level or final primary drinking water standard for fourth quarten 998 .

Sanitary Landfill

D-59

Fourth Quarter 1998 and Annual Summary 
WSRC-TR-99-00011

Unclassified

WELL: LFW 43C

$\frac{\text { SRS Coord. }}{\text { N 86480.6 }} \quad \frac{\text { Lat/longitude }}{33.291 \text { Deg N }} \frac{\text { Screen ZoneElevation }}{138.5-128.5 \mathrm{ft} \mathrm{msl}} \quad \frac{\text { Ton of Casing }}{202.6 \mathrm{ft} \mathrm{msl}} \quad \frac{\text { Casing }}{4 " \mathrm{PVC}} \quad \frac{\text { Pump }}{\text { Formation }}$ U Steed Pond

E 45234.9 81.716 Deg W

SAMPLE DATE $\quad 12 / 05 / 97 \quad 03 / 16 / 98 \quad 06 / 08 / 98$

FIELD DATA

Analyte

Water Elevation

Depth to water

pH

Sp. Conductance

Water temperature

Alkalinity as $\mathrm{CaCO3}$

Turbidity

Volumes purged

Sampling code

\begin{tabular}{lllll}
101998 & $\underline{201998}$ & $\underline{301998}$ & $\underline{401998}$ & Unit \\
\hline 161.8 & 165.7 & 166.05 & & ft msl \\
38.7 & 34.8 & 34.45 & & ft \\
4.8 & 5 & 20 & 16 & pH \\
20 & 16 & 18.6 & uS/cm \\
21 & 1 & 0 & deg. $C$ \\
0 & .5 & .2 & mg/L \\
1 & 6.86557 & 3.61394 & NTU \\
5.21049 & & & well volumes
\end{tabular}

\section{ANALYTICAL DATA}

\section{H ST Analyte}

1,1,1,2-Tetrachloroethane

1,1,1-Trichloroethane

1,1,2,2-Tetrachloroethane

1,1,2-Trichloroethane

1,1-Dichloroethane

1,1-Dichloroethylene

1,2,3-Trichloropropane

1,2-Dibromo-3-chloropropane

1,2-Dibromoethane

1,2-Dichlorobenzene

1,2-Dichloroethane

1,2-Dichloropropane

1,3-Dichlorobenzene

1,4-Dichlorobenzene

1,4-Dioxane

2-Chloroethyl vinyl ether

tS

2-Hexanone

Acetone

Acetonitrile (Methyl cyanide)

Acrolein

Acrylonitrile

Allyl chloride

Aluminum, total recoverable

Antimony, total recoverable

Arsenic, total recoverable

Barium, total recoverable

Compliance

$101998 \quad 201998 \quad 301298 \quad \underline{401998}$ DFMod Unit Lab Filter

\begin{tabular}{|c|c|c|}
\hline$<5$ & $<5$ & $<5$ \\
\hline$<5$ & $<5$ & $<5$ \\
\hline$<5$ & $<5$ & $<5$ \\
\hline$<5$ & $<5$ & $<5$ \\
\hline$<5$ & $<5$ & $<5$ \\
\hline$<5$ & $<5$ & $<5$ \\
\hline$<5$ & $<5$ & $<5$ \\
\hline$<5$ & $<5$ & $<5$ \\
\hline \multirow[t]{2}{*}{$<5$} & $<5$ & $<5$ \\
\hline & $<5$ & $<5$ \\
\hline$<5$ & $<5$ & $<5$ \\
\hline \multirow[t]{2}{*}{$<5$} & $<5$ & $<5$ \\
\hline & & $<5$ \\
\hline \multirow[t]{3}{*}{$<5$} & $<5$ & $<5$ \\
\hline & & $<1000$ \\
\hline & $<5$ & $<5$ \\
\hline$<10$ & $<10$ & $<10$ \\
\hline$<10$ & $<10$ & $<10$ \\
\hline$<20$ & $<200$ & $<20$ \\
\hline$<20$ & $<10$ & $<10$ \\
\hline$<5$ & $<20$ & $<5$ \\
\hline \multirow[t]{3}{*}{$<10$} & $<10$ & $<10$ \\
\hline & & $<7.3$ \\
\hline & $<5$ & \\
\hline$<40$ & $<8$ & $<8$ \\
\hline 3.4 & 6.2 & $<6.8$ \\
\hline
\end{tabular}

Note: Flagging, dilution factors, modifiers, and laboratories are for fourth quartet998 data only. See Appendix B for flagging criteria. * $=$ exceeded holding time for fourth quarter 1998.

$+=$ exceded screening level or final primary drinking water standard for fourth quarter 998 .

Sanitary Landfill

D-60

Fourth Quarter 1998 and Annual Summary 
WSRC-TR-99-00011

Unclassified

WELL: LFW 43C

\section{ANALYTICAL DATA}

\section{H ST Analyte}

Benzene

Beryllium, total recoverable

Bromochloromethane

Bromodichloromethane

Bromoform

Bromomethane (Methyl bromide)

Cadmium, total recoverable

Carbon disulfide

Carbon tetrachloride

Chlorobenzene

Chloroethane

Chloroethene (Vinyl chloride)

Chloroform

Chloromethane (Methyl chloride)

Chloroprene

Chromium, total recoverable

Cobalt, total recoverable

Copper, total recoverable

Di-n-butyl phthalate

Dibromochloromethane

Dibromomethane (Methylene bromide)

Dichlorodifluoromethane

Dichloromethane (Methylene chloride)

Ethyl methacrylate

Ethylbenzene

Gross alpha

Iodomethane (Methyl iodide)

Iron, total recoverable

Isobutyl alcohol

Lead, total recoverable

Mercury, total recoverable

Methacrylonitrile

Methyl ethyl ketone

Methyl isobutyl ketone

Methyl methacrylate

Nickel, total recoverable

Pentachloroethane

Propionitrile

Selenium, total recoverable

Silver, total recoverable

Specific conductance

Styrene

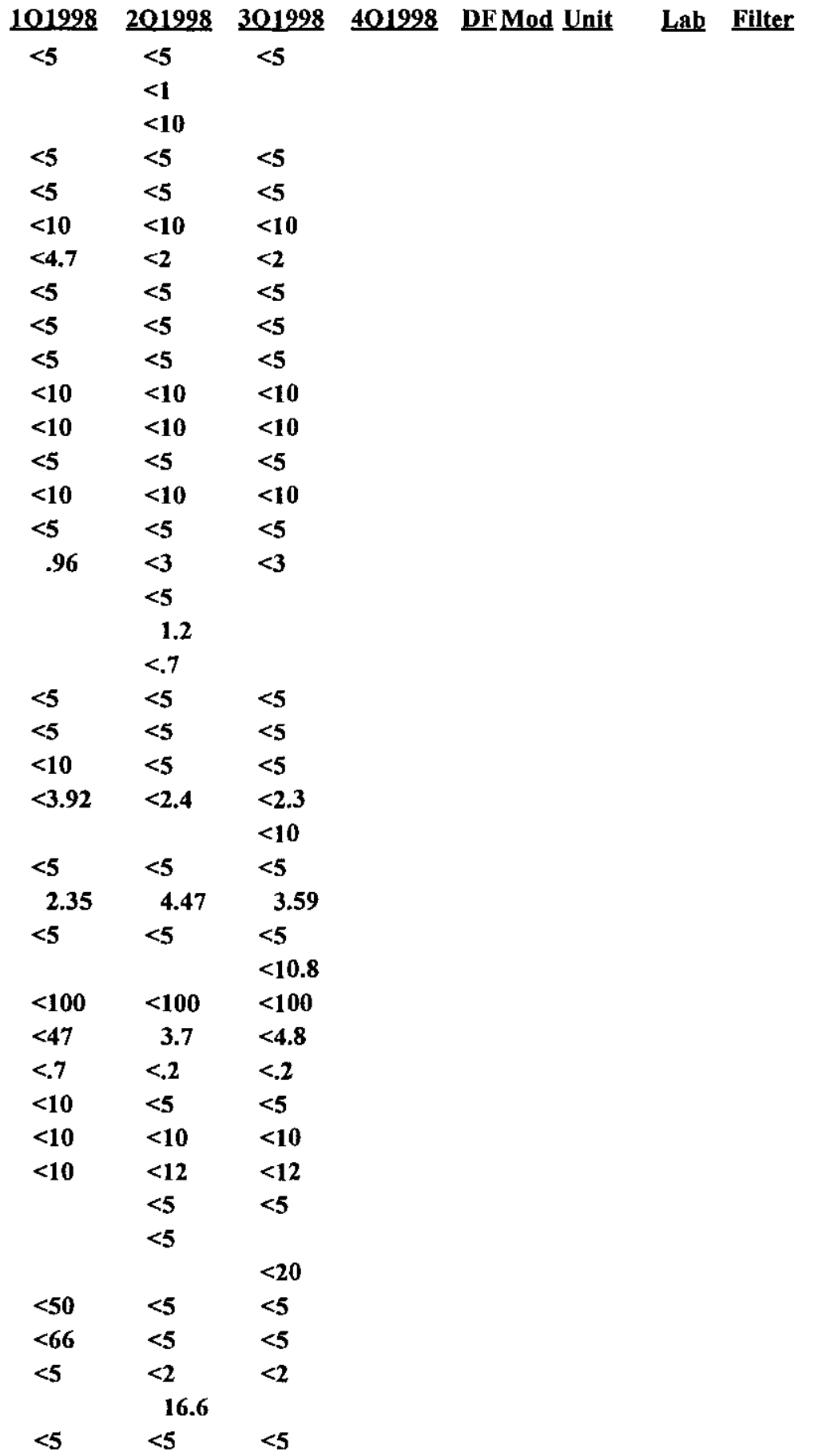

Note: Flagging, dilution factors, modifiers, and laboratories are for fourth quartet 998 data only. See Appendix B for flagging criteria. * = exceeded holding time for fourth quarter 1998.

$+=$ exceeded screening level or final primary drinking water standard for fourth quarter 998 . 
WSRC-TR-99-00011

Unclassified

WELL: LFW 43C

\section{ANALYTICAL DATA}

\section{H ST Analyte}

Tetrachloroethylene

Thallium, total recoverable

Toluene

Trichloroethylene

Trichlorofluoromethane

Tritium

Unknown

Vanadium, total recoverable

Vinyl acetate

Xylenes

Zinc, total recoverable

beta-Benzene hexachloride

cis-1,2-Dichloroethylene

cis-1,3-Dichloropropene

pH

trans-1,2-Dichloroethylene

trans-1,3-Dichloropropene

trans-1,4-Dichloro-2-butene

$\begin{array}{cccccc}101998 & 201998 & 301998 & 401998 & \text { Compliance } \\ <5 & <5 & <5 & & \text { DFod Unit } & \text { Lab Eilter } \\ & <5 & & \\ <5 & <5 & <5 \\ <5 & <5 & <5 \\ <5 & <5 & <5 \\ 1.3 & 1590 & <0 \\ & <6.05 & \\ & <2 & \\ <10 & <10 & <5 \\ <5 & <10 & <5 \\ & 10.8 & \\ & <.015 & \\ <5 & <5 & <5 \\ <5 & <5 & <5 \\ <5 & <5 & <5 \\ <20 & <5 & <5\end{array}$

\section{Compliance}
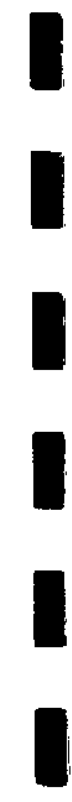

Note: Flagging, dilution factors, modifiers, and laboratories are for fourth quartet998 data only. See Appendix B for flagging criteria.

* = exceeded holding time for fourth quarter 1998.

+ exceeded screening level or final primary drinking water standard for fourth quarteri 998 . 
WELL: LFW 43D

\begin{tabular}{|c|c|c|c|c|c|c|}
\hline SRS Coord. & Lat/Longitude & Screen Zone Elevation & Top of Casing & Casing & Pump & Lation \\
\hline $\begin{array}{l}\text { N } 86443.2 \\
\text { E } 45244.5\end{array}$ & $\begin{array}{l}\text { 33.291 Deg N } \\
\text { 81.716 Deg W }\end{array}$ & 150.9 & sI & & $\mathbf{S}$ & U Steed Pond \\
\hline
\end{tabular}

SAMPLE DATE

\section{FIELD DATA}

Analyte
Water Elevation
Depth to water
pH
Sp. Conductance
Water temperature
Alkalinity as CaCO3
Turbidity
Volumes purged
Sampling code

ANALYTICAL DATA

\section{H ST Analyte}

1,1,1,2-Tetrachloroethane

1,1,1-Trichloroethane

1,1,2,2-Tetrachloroethane

1,1,2-Trichloroethane

1,1-Dichloroethane

1,1-Dichloroethylene

1,2,3-Trichloropropane

1,2-Dibromo-3-chloropropane

1,2-Dibromoethane

1,2-Dichlorobenzene

1,2-Dichloroethane

1,2-Dichloropropane

1,4-Dichlorobenzene

2-Hexanone

Acetone

Acetonitrile (Methyl cyanide)

Acrolein

Acrylonitrile

Allyl chloride

Aluminum, total recoverable

Antimony, total recoverable

Arsenic, total recoverable

Barium, total recoverable

Benzene

Beryllium, total recoverable

Bromochloromethane $\begin{array}{lll}12 / 05 / 97 & 03 / 16 / 98 & 06 / 09 / 98\end{array}$

$\begin{array}{lllll}101998 & \frac{201998}{162.1} & \underline{301998} & \underline{401998} & \text { Unit } \\ 162.8 & 167.98 & 164.6 & & \text { ft msl } \\ 38.8 & 32.92 & 36.3 & & \text { ft } \\ 5 & 5.2 & 4.8 & & \text { pH } \\ 12 & 16 & 18 & & \text { uS/cm } \\ 20 & 16 & 19.1 & & \text { deg. C } \\ 2 & 1 & 0 & & \text { mg/L } \\ .8 & .6 & .6 & & \text { NTU } \\ 8.89228 & 11.1054 & 8.54435 & & \text { well volumes }\end{array}$

$\begin{array}{cccccc}101998 & 201998 & 301998 & 401998 & \text { DFMod Unit } & \begin{array}{c}\text { Compliance } \\ \text { Lab Filter }\end{array} \\ <5 & <5 & <5 & & \\ <5 & <5 & <5 \\ <5 & <5 & <5 \\ <5 & <5 & <5 \\ <5 & <5 & <5 \\ <5 & <5 & <5 \\ <5 & <5 & <5 \\ <5 & <5 & <5 \\ <5 & <5 & <5 \\ & <5 & \\ <5 & <5 & <5 \\ <5 & <5 & <5 \\ <5 & <5 & <5 \\ <10 & <10 & <10 \\ <9.01 & <10 & <10 \\ <20 & <20 & <20 \\ <20 & <10 & <10 \\ <5 & <20 & <5 \\ <10 & <10 & <10 \\ & & <20.9 \\ <40 & <5 & <8 \\ 6.9 & 6.6 & <7.4 \\ <5 & <5 & <5 \\ & <1 & \\ & <10 & \\ & & \end{array}$

Note: Flagging, dilution factors, modifiers, and laboratories are for fourth quarte1998 data only. See Appendix B for flagging criteria.

* = exceeded holding time for fourth quarter 1998 .

$+=$ exceeded screening level or final primary drinking water standard for fourth quarten 1998 .

Sanitary Landfill

D-63

Fourth Quarter 1998 and Annual Summary 


\section{H ST Analyte}

Bromodichloromethane

Bromoform

Bromomethane (Methyl bromide)

Cadmium, total recoverable

Carbon disulfide

Carbon tetrachloride

Chlorobenzene

Chloroethane

Chloroethene (Vinyl chloride)

Chloroform

Chloromethane (Methyl chloride)

Chloroprene

Chromium, total recoverable

Cobalt, total recoverable

Copper, total recoverable

Di-n-butyl phthalate

Dibromochloromethane

Dibromomethane (Methylene bromide)

Dichlorodifluoromethane

Dichloromethane (Methylene chloride)

Ethylbenzene

Gross alpha

Iodomethane (Methyl iodide)

Iron, total recoverable

Isobutyl alcohol

Lead, total recoverable

Mercury, total recoverable

Methacrylonitrile

Methyl ethyl ketone

Methyl isobutyl ketone

Methyl methacrylate

Nickel, total recoverable

Propionitrile

Selenium, total recoverable

Silver, total recoverable

Specific conductance

Styrene

Tetrachloroethylene

Thallium, total recoverable

Toluene

Trichloroethylene

Trichlorofluoromethane

\section{8}

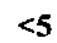

$<5$

$<10$

$<4.7$

$<5$

$<5$

$<5$

$<10$

$<10$

$<5$

$<10$

$<5$

$<5$

.88

201998

$<5$

301998

$<5$

$<5$

$<10$

$<10$

$<.3$

$<5$

$<5$

$<5$

$<10$

$<10$

$<5$

$<10$

$<5$

$<3$

$<3$

$<5$

2.4

$<.67$

$<5<5 \quad<5$

$<5<5<5$

$<10<5 \quad<5$

$<14.8<3.2<1.8$

$<5<5<5$

$1.06<.51 \quad 2.05$

$<5<5<5$

$<17$

$<100<100<100$

$<47<6.7<5$

$<.7<.2<.2$

$<10<5<5$

$<10 \quad<10 \quad<10$

$<10<12 \quad<12$

$<5<5$

$<5$

$\leq \mathbf{5 0} \ldots$

$\leq \mathbf{5}={ }_{-=} \leq \mathbf{5}$

$<5 \quad<5$

$<5<2 \quad<2$

11.6

$<5<5<5$

$<5<5<5$

$\begin{array}{ll}< & <5 \\ <5 & <5\end{array}$

$<5<5 \quad<5$

$<5<5 \quad<5$

Note: Flagging, dilution factors, modifiers, and laboratories are for fourth quartel 998 data only. See Appendix B for flagging criteria. $\star$ = excecded holding time for fourth quarter 1998 .

$+=$ exceeded screening level or final primary drinking water standard for fourth quarten 998 . 
WSRC-TR-99-00011

Unclassified

WELL: LFW 43D

ANALYTICAL DATA

H ST Analyte

Tritium

Vanadium, total recoverable

Vinyl acetate

Xylenes

Zinc, total recoverable

beta-Benzene hexachloride

cis-1,2-Dichloroethylene

cis-1,3-Dichloropropene

pH

trans-1,2-Dichloroethylene

trans-1,3-Dichloropropene

trans-1,4-Dichloro-2-butene

\begin{tabular}{|c|c|c|c|c|c|}
\hline \multirow[b]{2}{*}{101998} & \multirow[b]{2}{*}{201998} & \multirow[b]{2}{*}{301928} & \multirow[b]{2}{*}{401998} & \multirow[b]{2}{*}{ DEMod Unit } & Compliance \\
\hline & & & & & Lab Filter \\
\hline .97 & $<853$ & $<1.26$ & & & \\
\hline & .4 & & & & \\
\hline$<10$ & $<5$ & $<5$ & & & \\
\hline$<5$ & $<5$ & $<5$ & & & \\
\hline & 9.3 & & & & \\
\hline & $<.015$ & & & & \\
\hline & $<5$ & & & & \\
\hline$<5$ & $<5$ & $<5$ & & & \\
\hline & 5.42 & & & & \\
\hline$<5$ & & & & & \\
\hline$<5$ & $<5$ & $<5$ & & & \\
\hline$<\mathbf{2 0}$ & $<5$ & $<5$ & & & \\
\hline
\end{tabular}

Note: Flagging, dilution factors, modifiers, and laboratories are for fourth quarte1998 data only. See Appendix B for flagging criteria. * = exceeded holding time for fourth quarter 1998.

+ = exceeded screening level or final primary drinking water standard for fourth quarten 998 . 
WELL: LFW 45D

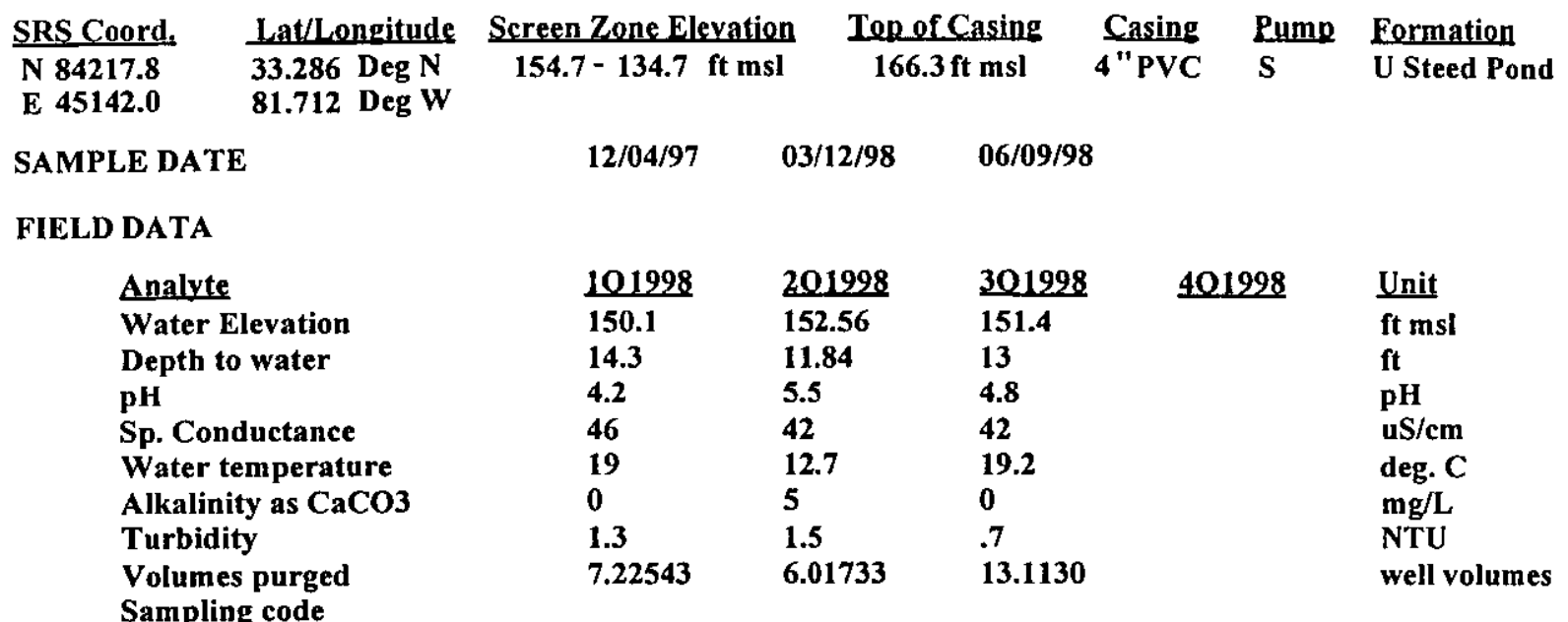

ANALYTICAL DATA
H ST Analyte

1,1,1,2-Tetrachloroethane

1,1,1-Trichloroethane

1,1,2,2-Tetrachloroethane

1,1,2-Trichloroethane

1,1-Dichloroethane

1,1-Dichloroethylene

1,2,3-Trichloropropane

1,2-Dibromo-3-chloropropane

1,2-Dibromoethane

1,2-Dichloroethane

1,2-Dichloropropane

1,4-Dichlorobenzene

2-Hexanone

Acetone

Acetonitrile (Methyl cyanide)

Acrolein

Acrylonitrile

Ally $\overline{\text { chiloride }}$

Aluminum, total recoverable

Arsenic, total recoverable

Barium, total recoverable

Benzene

Bromodichloromethane

Bromoform

Bromomethane (Methyl bromide)

Cadmium, total recoverable
Compliance

\section{DFMod Unit Lab Filter}

$<5<5<5$

$<5<5 \quad<5$

$<5<5 \quad<5$

$<5<5 \quad<5$

$<5 \quad<5 \quad<5$

$<5 \quad<5 \quad<5$

$<5<5 \quad<5$

$<5 \quad<5 \quad<5$

$<5 \quad<5 \quad<5$

$<5 \quad<5 \quad<5$

$<5<5 \quad<5$

$<5<5 \quad<5$

$<10<10<10$

$<10<10<10$

$<20<20<20$

$<20 \quad<10 \quad<10$

$<5<20<5$

$<10<<10=<10^{-}$

$<40 \quad<8 \quad<8$

$\begin{array}{lll}12.6 & 11 & 12.8\end{array}$

$<5<5<5$

$<5<5<5$

$<5<5<5$

$<10<10<10$

$<4.7<2<2$

Note: Flagging, dilution factors, modifiers, and laboratories are for fourth quartel998 data only. See Appendix $\mathbf{B}$ for flagging criteria.

* = exceeded holding time for fourth quarter 1998 .

$+=$ exceded screening level or final primary drinking water standard for fourth quarten 998 .

Sanitary Landfill

D-66

Fourth Quarter 1998 and Annual Summary 
WSRC-TR-99-00011

Unclassified

WELL: LFW 45D

ANALYTICAL DATA

\section{H SI Analyte}

Carbon disulfide

Carbon tetrachloride

Chlorobenzene

Chloroethane

Chloroethene (Vinyl chloride)

Chloroform

Chloromethane (Methyl chloride)

Chloroprene

Chromium, total recoverable

Dibromochloromethane

Dibromomethane (Methylene bromide)

Dichlorodifluoromethane

Dichloromethane (Methylene chloride)

Ethylbenzene

Gross alpha

Iodomethane (Methyl iodide)

Iron, total recoverable

Isobutyl alcohol

Lead, total recoverable

Mercury, total recoverable

Methacrylonitrile

Methyl ethyl ketone

Methyl isobutyl ketone

Methyl methacrylate

Propionitrile

Selenium, total recoverable

Silver, total recoverable

Styrene

Tetrachloroethylene

Toluene

Trichloroethylene

Trichlorofluoromethane

Tritium

Vinyl acetate

Xylenes

cis-1,3-Dichloropropene

trans-1,2-Dichloroethylene

trans-1,3-Dichloropropene

trans-1,4-Dichloro-2-butene

1

$101998 \quad \underline{201998}$

$<5$

$<5<5<5$

$<10<10 \quad<10$

$<10<10<10$

$<5<5<5$

$<10<10<10$

$<5<5<5$

$3.5<3<3$

$<5<5<5$

$<5<5<5$

$<10<5<5$

$<6.61<2.3<3$

$<5<5<5$

$6.56<1.59 \quad 8.58$

$<5<5<5$

33

$<100<100<100$

$<47<5 \quad 9.3$

$<.7 \quad .13 \quad .13$

$<10<5<5$

$<10 \quad<10 \quad<10$

$<10<12<12$

$<5<5$

$<50<5<5$

$<66<5<5$

$<5<2<2$

$<5<5<5$

$<5<5<5$

$<5<5<5$

$<5<5<5$

$\begin{array}{llll}2.09 & 2 & .89\end{array}$

$<1.55 \quad 1410<1.12$

$<10<5<5$

$<5<5<5$

$<5<5 \quad<5$

$<5$

$<5<5 \quad<5$

$<20<5 \quad<5$

Note: Flagging, dilution factors, modifiers, and laboratories are for fourth quartei 998 data only. See Appendix B for flagging criteria. * = exceeded holding time for fourth quarter 1998.

+ * exceded screening level or final primary drinking water standard for fourth quarteri 998 . 
WSRC-TR-99-00011

Unclassified

WELL: LFW 47D

\begin{tabular}{|c|c|c|c|c|c|c|}
\hline SRS Coord, & Lat/longitude & Screen Zone Elevation & Top of Casing & Casing & $\underline{\text { Pump }}$ & Formation \\
\hline $\begin{array}{l}\text { N } 83838.6 \\
\text { E } 45150.8\end{array}$ & $\begin{array}{l}\text { 33.285 Deg N } \\
\text { 81.711 } \text { Deg W }\end{array}$ & $154.7-134.9 \mathrm{ft} \mathrm{msl}$ & $161.7 \mathrm{ft} \mathrm{msl}$ & 4 "PVC & $\mathbf{S}$ & $\overline{\text { U Steed Pond }}$ \\
\hline
\end{tabular}

\section{FIELD DATA}

Analyte

Water Elevation

Depth to water

pH

Sp. Conductance

Water temperature

Alkalinity as $\mathrm{CaCO}$

Turbidity

Volumes purged

Sampling code

$\begin{array}{lllll}101998 & 201998 & 301998 & 401998 & \text { Unit } \\ 147 & 148.85 & 147.6 & 147.58 & \mathrm{ft} \mathrm{msl} \\ 12.7 & 10.85 & 12.1 & 12.12 & \mathrm{ft} \\ 4.4 & 5.8 & 5 & 4.8 & \mathrm{pH} \\ 40 & 21 & 38 & 53 & \text { uS/cm } \\ 18 & 13.1 & 19.1 & 22.9 & \text { deg. C } \\ 0 & 1 & 2 & 1 & \mathrm{mg} / \mathrm{L} \\ .7 & .6 & .8 & .6 & \text { NTU } \\ 5.83809 & 2.00703 & 7.25900 & 2.90756 & \text { well volumes }\end{array}$

\section{ANALYTICAL DATA}

H ST Analyte

1,1,1,2-Tetrachloroethane

1,1,1-Trichloroethane

1,1,2,2-Tetrachloroethane

1,1,2-Trichloroethane

1,1-Dichloroethane

1,1-Dichloroethylene

1,2,3-Trichloropropane

1,2-Dibromo-3-chloropropane

1,2-Dibromoethane

1,2-Dichlorobenzene

1,2-Dichloroethane

1,2-Dichloropropane

1,3-Dichlorobenzene

1,4-Dichlorobenzene

1,4-Dioxane

2-Hexanone

Acetone

Acetonitrile (Methyl cyanide)

Acrolein

Acrylonitrile

Allyl chloride

Aluminum, total recoverable

Arsenic, total recoverable

Barium, total recoverable

Benzene

Bromodichloromethane $\begin{array}{llll}12 / 04 / 97 & 03 / 12 / 98 & 06 / 09 / 98 & 09 / 30 / 98\end{array}$
Compliance

101998201998 301998 401998 DFMod Unit Lab Filter

$\begin{array}{lllllllll}< & <5 & <5 & <5 & 1 & \mathrm{U} & \mathrm{ug} / \mathrm{L} & \mathrm{EX} & <\mathrm{EQL}\end{array}$

$\begin{array}{lllllllll}< & <5 & <5 & <5 & 1 & \mathrm{U} & \mathrm{ug} / \mathrm{L} & \mathrm{EX} & <\mathrm{EQL}\end{array}$

$\begin{array}{lllllllll}< & <5 & <5 & <5 & 1 & \mathrm{U} & \mathrm{ug} / \mathrm{L} & \mathrm{EX} & <\mathrm{EQL}\end{array}$

$<5 \quad<5 \quad<5 \quad<5 \quad 1 \quad \mathrm{U} \quad \mathrm{ug} / \mathrm{L} \quad \mathrm{EX} \quad<\mathrm{EQL}$

$\begin{array}{lllllllll}< & <5 & <5 & <5 & 1 & \mathrm{U} & \mathrm{ug} / \mathrm{L} & \mathrm{EX} & <\mathrm{EQL}\end{array}$

$\begin{array}{lllllllll}< & <5 & <5 & <5 & 1 & \mathrm{U} & \mathrm{ug} / \mathrm{L} & \mathrm{EX} & <\mathrm{EQL}\end{array}$

$\begin{array}{lllllllll}< & <5 & <5 & <5 & 1 & \mathrm{U} & \mathrm{ug} / \mathrm{L} & \mathrm{EX} & <\mathrm{EQL}\end{array}$

$\begin{array}{lllllllll}<5 & <5 & <5 & <5 & \text { I } & \mathrm{U} & \mathrm{ug} / \mathrm{L} & \mathrm{EX} & <\mathrm{EQL}\end{array}$

$\begin{array}{lllllllll}< & <5 & <5 & <5 & \text { I } & \mathrm{U} & \mathrm{ug} / \mathrm{L} & \mathrm{EX} & <\mathrm{EQL}\end{array}$



$\begin{array}{lllllllll}<5 & <5 & <5 & <5 & 1 & \mathrm{U} & \mathrm{ug} / \mathrm{L} & \mathrm{EX} & <\mathrm{EQL}\end{array}$

$<5 \quad<5 \quad<5 \quad<5 \quad 1 \quad$ U $\quad$ ug/L $\quad \mathrm{EX} \quad<\mathrm{EQL}$

$\begin{array}{llllll}< & 1 & \mathrm{U} & \mathrm{ug} / \mathrm{L} & \mathrm{EX} & <\mathrm{EQL}\end{array}$

$<5 \quad<5 \quad<5 \quad<5 \quad 1 \quad$ U $\quad$ ug/L $\quad$ EX $<$ EQL

$<100011$ U ug/L EX $<$ EQL

$<10 \quad<10 \quad<10 \quad<5 \quad 1 \quad \mathrm{U} \quad$ ug/L $\quad \mathrm{EX} \quad<\mathrm{EQL}$



$<20<10 \quad<10 \quad<50 \quad 1 \quad \mathrm{U} \quad \mathrm{ug} / \mathrm{L} \quad \mathrm{EX} \quad<$ EQL

$<5 \quad<20 \quad<5 \quad<50 \quad 1 \quad$ U $\quad$ ug/L $\quad$ EX $\quad<$ EQL

$<10 \quad<10 \quad<10 \quad<10 \quad 1 \quad \mathrm{U} \quad \mathrm{ug} / \mathrm{L} \quad \mathrm{EX} \quad<\mathrm{EQL}$

$24.2<200 \quad 1 \quad \mathrm{U}$ ug/L $\mathrm{EX}<\mathrm{EQL}$

$<40 \quad<8 \quad<8 \quad<100 \quad 1 \quad \mathrm{U} \quad \mathrm{ug} / \mathrm{L} \quad \mathrm{EX} \quad<\mathrm{EQL}$

$\begin{array}{llllllllll}19.8 & 11.5 & 15.3 & 23 & \mathrm{ug} / \mathrm{L} & \mathrm{EX} & 23\end{array}$

$<5<<\quad<5 \quad<5 \quad 1 \quad \mathrm{U} \quad \mathrm{ug} / \mathrm{L} \quad \mathrm{EX}<\mathrm{EQL}$

$<5 \quad<5 \quad<5 \quad<5 \quad 1 \quad$ U $\quad$ ug/L $\quad \mathrm{EX}<\mathrm{EQL}$

Note: Flagging, dilution factors, modifiers, and laboratories are for fourth quartet 998 data only. See Appendix B for flagging criteria.

* $=$ exceeded holding time for fourth quarter 1998.

$+=$ exceded screening level or fingl primary drinking water standard for fourth quarten 998 .

Sanitary Landfill

D-68

Fourth Quarter 1998 and Annual Summary 
WSRC-TR-99-00011

Unclassified

\section{WELL: LFW 47D}

\section{ANALYTICAL DATA}

H ST Analyte

Bromoform

Bromomethane (Methyl bromide)

Cadmium, total recoverable

Carbon disulfide

Carbon tetrachloride

Chlorobenzene

Chloroethane

Chloroethene (Vinyl chloride)

Chloroform

Chloromethane (Methyl chloride)

Chloroprene

Chromium, total recoverable

Dibromochloromethane

Dibromomethane (Methylene bromide)

Dichlorodifluoromethane

Dichloromethane (Methylene chloride)

Ethyl methacrylate

Ethylbenzene

Gross alpha

Iodomethane (Methyl iodide)

Iron, total recoverable

Isobutyl alcohol

Lead, total recoverable

Mercury, total recoverable

Methacrylonitrile

Methyl ethyl ketone

Methyl isobutyl ketone

Methyl methacrylate

Pentachloroethane

Propionitrile

Selenium, total recoverable

Silver, total recoverable

Styrene

Tetrachloroethylene

Toluene

Trichloroethylene

Trichlorofluoromethane

Tritium

Vinyl acetate

Xylenes

cis-1,2-Dichloroethylene

cis-1,3-Dichloropropene

\section{8}

$<5$

$<10$

$<4.7$

$<5$

$<5$

$<5$

$<10$

$<10$

$<5$

$<10$

$<5$

$<7$

$<5$

$<5$

$<1$

$<6.5$$$
<5
$$

$$
<5
$$

$\begin{array}{rr}<100 \quad<100 \quad & 18.8 \\ <100\end{array}$

$<47 \quad<5 \quad 4.4$

$<.7 \quad<.2 \quad<.2$

$<10<5<5$

$<10 \quad<10 \quad<10$

$<10<12<12$

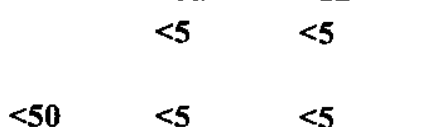

$\begin{array}{lll}<50 & <5 & <5 \\ <66 & <5 & <5 \\ <5 & <2 & <2\end{array}$

$<5<5<5<5$

$<5 \quad<5 \quad<5$

$<5 \quad<5 \quad<5$

$<5 \quad<5 \quad<5$

$<5<5 \quad<5$

$<.87 \quad 938$

$<10<5$

$<5<5$

2.12

$<5$

$<5$

$<5<5<5$
Compliance

Lab Filter

401998 DFMod Unit

$\mathbf{u g} / \mathbf{L}$

EX $<$ EQL

$\begin{array}{llllll}<5 & \mathbf{1} & \mathrm{U} & \mathrm{ug} / \mathrm{L} & \mathrm{EX} & <\mathrm{EQL} \\ <\mathbf{1 0} & \mathbf{1} & \mathrm{U} & \mathrm{ug} / \mathrm{L} & \mathrm{EX} & <\mathrm{EQL}\end{array}$

$\begin{array}{llllll}<5 & 1 & \mathbf{U} & \text { ug/L } & \text { EX } & <\mathrm{EQL}\end{array}$

$<5 \quad 1 \quad$ U $\quad$ ug/L $\quad$ EX $<$ EQL

$<5 \quad 1 \quad$ U $\quad$ ug/L $\quad$ EX $<$ EQL

$<10 \quad 1 \quad \mathrm{U} \quad \mathrm{ug} / \mathrm{L} \quad \mathrm{EX} \quad<$ EQL

$<5 \quad 1 \quad$ U $\quad$ ug/L $\quad$ EX $<$ EQL

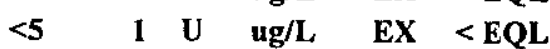

$<5 \quad 1 \quad$ U $\quad$ ug/L $\quad$ EX $<$ EQL

$<50 \quad 1 \quad$ U $\quad$ ug/L $\quad$ EX $<$ EQL

$\begin{array}{lllll}24 & 1 & \mathrm{ug} / \mathrm{L} & \mathrm{EX} & 24\end{array}$

$\begin{array}{lllllll}<5 & 1 & \text { U } & \text { ug/L } & \text { EX } & <\text { EQL }\end{array}$

$\begin{array}{lllllll}<5 & 1 & \text { U } & \text { ug/L } & \text { EX } & <\text { EQL }\end{array}$

$<5 \quad 1 \quad$ U $\quad$ ug/L $\quad$ EX $<$ EQL

$<\mathbf{1 0}$ 1 $U$ U

$<5 \quad 1 \quad$ U $\quad$ ug/L $\quad$ EX $<$ EQL

$\begin{array}{llllll}<5 & 1 & \mathbf{U} & \mathrm{ug} / \mathrm{L} & \mathrm{EX} & <\mathrm{EQL}\end{array}$

$\begin{array}{lllll}1.02 & 1 & \mathrm{pCi} / \mathrm{L} & \mathrm{TM} & 1.02\end{array}$

$\begin{array}{lllllll}< & 1 & \text { U } & \text { ug/L } & \text { EX } & <\text { EQL }\end{array}$

$\begin{array}{llllll}151 & 1 & \mathrm{~J} & \mathrm{ug} / \mathrm{L} & \mathrm{EX} & \text { NDD }\end{array}$

$<1500 \quad 1 \quad \mathrm{U} \quad$ ug/L $\quad$ EX $<$ EQL

$<100 \quad 1 \quad$ U $\quad$ ug/L $\quad$ EX $<$ EQL

$<.5 \quad 1 \quad \mathrm{U} \quad$ ug/L $\quad$ EX $<$ EQL

$<500 \quad 1 \quad$ U $\quad$ ug/L $\quad$ EX $<$ EQL

$\begin{array}{llllll}<10 & 1 & \text { U } & \text { ug/L } & \text { EX } & <\text { EQL }\end{array}$

$<5 \quad 1 \quad$ U $\quad$ ug/L $\quad$ EX $<$ EQL

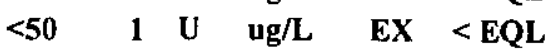

$<200 \quad 1 \quad$ U $\quad$ ug/L $\quad$ EX $<$ EQL

$<500 \quad 1$ U ug/L EX $<$ EQL

$<2001$ U ug/L EX $<$ EQL

$\begin{array}{llllll}<20 & 1 & \mathrm{U} & \mathrm{ug} / \mathrm{L} & \mathrm{EX} & <\mathrm{EQL}\end{array}$

$\begin{array}{llllll}<5 & 1 & \mathrm{U} & \mathbf{u g} / \mathrm{L} & \mathrm{EX} & <\mathrm{EQL}\end{array}$

$<5 \quad 1 \quad$ U $\quad$ ug/L $\quad$ EX $<$ EQL

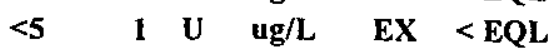

$<5 \quad 1 \quad$ U $\quad$ ug/L $/$ EX $\quad<$ EQL

$<5 \quad 1 \quad$ U $\quad$ ug/L $\quad$ EX $<$ EQL

$<1.18$ I UI $\mathrm{pCi} / \mathrm{ml}$ TM $<\mathrm{EQL}$

$<201$ U $\quad$ ug/L $/$ EX $<$ EQL

$<\mathbf{1 0} \quad 1 \quad \mathrm{U} \quad$ ug/L $\quad$ EX $<$ EQL

$\begin{array}{lllllll}< & 1 & \text { U } & \text { ug/L } & \text { EX } & <\text { EQL }\end{array}$

$<5 \quad 1 \quad \mathrm{U} \quad \mathrm{ug} / \mathrm{L} \quad$ EX $<$ EQL

Note: Flagging, dilution factors, modifiers, and laboratories are for fourth quartet998 data only. See Appendix B for fagging criteria.

* = exceeded holding time for fourth quarter 1998.

$+=$ exceeded screening level or final primary drinking water standard for fourth quarter 998 . 
WSRC-TR-99-00011

Unclassified

WELL: LFW 47D

\section{ANALYTICAL DATA}

\section{H ST Analyte}

trans-1,2-Dichloroethylene

trans-1,3-Dichloropropene

trans-1,4-Dichloro-2-butene

\begin{tabular}{|c|c|c|c|c|c|c|c|c|}
\hline & & & & & & & \multicolumn{2}{|c|}{ Complianc } \\
\hline 101998 & 201998 & 301998 & $\underline{401998}$ & DF & Mod & Unit & $\underline{\mathbf{L a b}}$ & Filter \\
\hline$<5$ & & & $<5$ & 1 & $\mathbf{U}$ & ug/L & $\mathbf{E X}$ & $<$ EQL \\
\hline$<5$ & $<5$ & $<5$ & $<5$ & 1 & $\mathbf{U}$ & ug/L & EX & $<\mathrm{EQL}$ \\
\hline$<\mathbf{2 0}$ & $<5$ & $<5$ & $<\mathbf{2 0}$ & 1 & $\mathbf{U}$ & ug/L & EX & $<$ EQL \\
\hline
\end{tabular}

\section{Compliance}

Note: Flagging, dilution factors, modifiers, and laboratories are for fourth quartei 998 data only. See Appendix B for flagging criteria.

* = exceeded holding time for fourth quarter 1998.

$+=$ exceeded screening level or final primary drinking water standard for fourth quarten 998 .

Sanitary Landfill

D-70

Fourth Quarter 1998 and Annual Summary 
WELL: LFW 48C

\begin{tabular}{|c|c|c|c|c|c|c|}
\hline $\begin{array}{l}\text { SRS Coord. } \\
\text { N } 83856.4 \\
\text { E } 45413.3\end{array}$ & $\begin{array}{l}\text { Lat/Longitude } \\
33.285 \text { Deg N } \\
81.710 \text { Deg W }\end{array}$ & $\frac{\text { Screen Zone Elevation }}{118.2-108.2 \mathrm{ft} \mathrm{msl}}$ & $\frac{\text { Top of Casing }}{169.3 \mathrm{ft} \mathrm{msl}}$ & $\frac{\text { Casing }}{4 \text { "PVC }}$ & $\frac{\text { Pump }}{S}$ & $\begin{array}{l}\text { Formation } \\
\text { M Steed Pond }\end{array}$ \\
\hline
\end{tabular}

SAMPLE DATE

\section{FIELD DATA}

Analyte

Water Elevation

Depth to water

pH

Sp. Conductance

Water temperature

Alkalinity as $\mathrm{CaCO}$

Turbidity

Volumes purged

Sampling code

\section{ANALYTICAL DATA}

\section{H ST Analyte}

1,1,1,2-Tetrachloroethane

1,1,1-Trichloroethane

1,1,2,2-Tetrachloroethane

1,1,2-Trichloroethane

1,1-Dichloroethane

1,1-Dichloroethylene

1,2,3-Trichloropropane

1,2-Dibromo-3-chloropropane

1,2-Dibromoethane

1,2-Dichloroethane

1,2-Dichloropropane

1,4-Dichlorobenzene

2-Hexanone

Acetone

Acetonitrile (Methyl cyanide)

Acrolein

Acrylonitrile

Allyl chloride

Arsenic, total recoverable

Barium, total recoverable

Benzene

Bromodichloromethane

Bromoform

Bromomethane (Methyl bromide)

Cadmium, total recoverable

Carbon disulfide
$12 / 04 / 97$

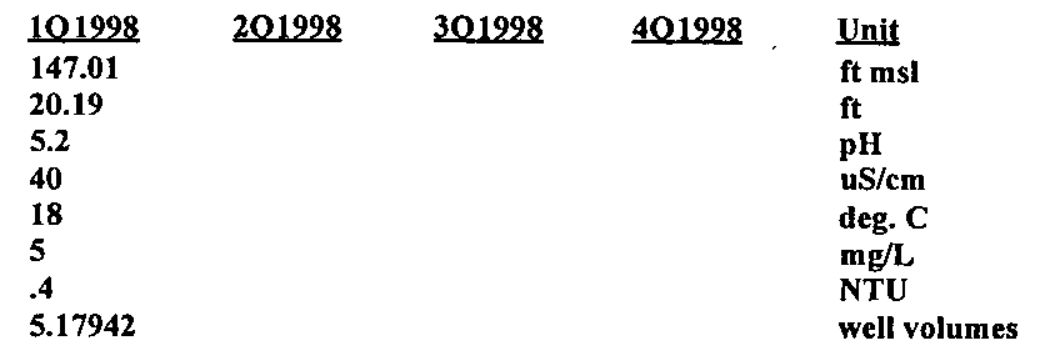

Compliance

101998201998 301998 401998 DEMad Unit Lab Filter

$<5$

$<5$

$<5$

$<5$

4.22

$<5$

$<5$

$<5$

$<5$

$<5$

$<5$

5.94

$<10$

$<7.41$

$<20$

$<20$

$<5$

$<10$

$<40$

29.5

$<5$

$<5$

$<5$

$<10$

$<4.7$

$<5$

Note: Flagging, dilution factors, modifiers, and laboratories are for fourth quartei 998 data only. See Appendix B for fagging criteria. * = exceeded holding time for fourth quarter 1998 .

$+=$ exceeded screening level or final primary drinking water standard for fourth quarten 998 .

Sanitary Landfill 
WELL: LFW 48C

\section{ANALYTICAL DATA}

\section{H ST Analyte}

Carbon tetrachloride

Chlorobenzene

Chloroethane

Chloroethene (Vinyl chloride)

Chloroform

Chioromethane (Methyl chloride)

Chloroprene

Chromium, total recoverable

Dibromochloromethane

Dibromomethane (Methylene bromide)

Dichlorodifluoromethane

Dichloromethane (Methylene chloride)

Ethylbenzene

Gross alpha

Iodomethane (Methyl iodide)

Isobutyl alcohol

Lead, total recoverable

Mercury, total recoverable

Methacrylonitrile

Methyl ethyl ketone

Methyl isobutyl ketone

Propionitrile

Selenium, total recoverable

Silver, total recoverable

Styrene

Tetrachloroethylene

Toluene

Trichloroethylene

Trichlorofluoromethane

Tritium

Vinyl acetate

Xylenes

cis-1,3-Dichloropropene:

trans-1,2-Dichloroethylene

trans-1,3-Dichloropropene

trans-1,4-Dichloro-2-butene
101998

Compliance

$<5$

$<5$

$<10$

1.61

$<5$

$<10$

$<5$

$<7$

$<5$

$<5$

$<10$

$<7.47$

$<5$

3.88

$<5$

$<100$

$<47$

1.59

$<10$

$<10$

$<10$

$<50$

$<66$

$<5$

$<5$

$<5$

$<5$

$<5$

$<5$

3.23

$<10$

$<5$

$\leq 5$

$<5$

$<5$

$<20$

Note: Flagging, dilution factors, modifiers, and taboratories are for fourth quartel998 data only. See Appendix B for flagging criteria. * = exceeded holding time for fourth quarter 1998.

$+=$ exceeded screening level or final primary drinking water standard for fourth quarter 998 . 
WSRC-TR-99-00011

Unclassified

WELL: LFW 56D

\begin{tabular}{|c|c|c|c|c|c|c|}
\hline SRS Coord. & Lat/Lengitude & Screen Zone Elevation & Top of Casing & Casing & Pump & Eormation \\
\hline $\begin{array}{l}\text { N } 83398.0 \\
\text { E } 45306.6\end{array}$ & $\begin{array}{l}\text { 33.284 Deg N } \\
\text { 81.709 Deg W }\end{array}$ & $151.4-131.3 \mathrm{ft} \mathrm{msl}$ & $158.1 \mathrm{ft} \mathrm{msl}$ & VC & $\mathbf{S}$ & Pond \\
\hline
\end{tabular}

$\begin{array}{llll}\text { SAMPLE DATE } & 12 / 03 / 97 & 03 / 12 / 98 & 06 / 09 / 98\end{array}$

FIELD DATA

Analyte

Water Elevation

Depth to water

pH

Sp. Conductance

Water temperature

Alkalinity as $\mathrm{CaCO}$

Turbidity

Volumes purged

$12 / 03 / 97$

$03 / 12 / 98$

$06 / 09 / 98$

Sampling code

$\begin{array}{lll}101998 & 201998 & 301998 \\ 142.84 & 143.88 & 143.3 \\ 13.06 & 12.02 & 12.6 \\ 4.2 & 4.6 & 4.9 \\ 20 & 22 & 22 \\ 17 & 15.2 & 18 \\ 0 & 1 & 0 \\ .7 & 6.2 & 1.4 \\ 13.6463 & 5.15694 & 6.97784\end{array}$

\begin{tabular}{ll}
401998 & Unit \\
\hline ft msl \\
ft \\
pH \\
uS/cm \\
deg. C \\
mg/L \\
NTU \\
well volumes
\end{tabular}

\section{ANALYTICAL DATA}

H ST Analyte

1,1,1,2-Tetrachloroethane

1,1,1-Trichloroethane

$1,1,2,2-$ Tetrachloroethane

1,1,2-Trichloroethane

1,1-Dichloroethane

1,1-Dichloroethylene

1,2,3-Trichloropropane

1,2-Dibromo-3-chloropropane

1,2-Dibromoethane

1,2-Dichloroethane

1,2-Dichloropropane

1,4-Dichlorobenzene

2-Hexanone

Acetone

Acetonitrile (Methyl cyanide)

Acrolein

Acrylonitrite

Allyl chloride

Aluminum, total recoverable

Arsenic, total recoverable

Barium, total recoverable

Benzene

Bromodichloromethane

Bromoform

Bromomethane (Methyl bromide)

Cadmium, total recoverable

\begin{tabular}{|c|c|c|}
\hline 101998 & 201998 & 301998 \\
\hline$<5$ & $<5$ & $<5$ \\
\hline$<5$ & $<5$ & $<5$ \\
\hline$<5$ & $<5$ & $<5$ \\
\hline$<5$ & $<5$ & $<5$ \\
\hline$<5$ & $<5$ & $<5$ \\
\hline$<5$ & $<5$ & $<5$ \\
\hline$<5$ & $<5$ & $<5$ \\
\hline$<5$ & $<5$ & $<5$ \\
\hline$<5$ & $<5$ & $<5$ \\
\hline$<5$ & $<5$ & $<5$ \\
\hline$<5$ & $<5$ & $<5$ \\
\hline$<5$ & $<5$ & $<5$ \\
\hline$<10$ & $<10$ & $<10$ \\
\hline$<10$ & $<3.2$ & $<10$ \\
\hline$<20$ & $<20$ & $<20$ \\
\hline$<20$ & $<10$ & $<10$ \\
\hline$<5$ & $<20$ & $<5$ \\
\hline$<10$ & $<10$ & $\begin{array}{c}<10 \\
83.1\end{array}$ \\
\hline$<40$ & $<8$ & $<8$ \\
\hline 7 & 5.1 & 6.4 \\
\hline$<5$ & $<5$ & $<5$ \\
\hline$<5$ & $<5$ & $<5$ \\
\hline$<5$ & $<5$ & $<5$ \\
\hline$<10$ & $<10$ & $<10$ \\
\hline$<4.7$ & $<2$ & $<2$ \\
\hline
\end{tabular}

Note: Flagging, dilution factors, modifiers, and laboratories are for fourth quartel998 data only. See Appendix B for flagging criteria.

* $=$ exceeded holding time for fourth quarter 1998 .

$+=$ exceeded screening level or final primary drinking water standard for fourth quarter1998 .

Compliance Lab Filter 
WELL: LFW 56D

\section{ANALYTICAL DATA}

\section{H ST Analyte}

Carbon disulfide

Carbon tetrachloride

Chlorobenzene

Chloroethane

Chloroethene (Vinyl chloride)

Chloroform

Chloromethane (Methyl chloride)

Chloroprene

Chromium, total recoverable

Dibromochloromethane

Dibromomethane (Methylene bromide)

Dichlorodifluoromethane

Dichloromethane (Methylene chloride)

Ethylbenzene

Gross alpha

Iodomethane (Methyl iodide)

Iron, total recoverable

Isobutyl alcohol

Lead, total recoverable

Mercury, total recoverable

Methacrylonitrile

Methyl ethyl ketone

Methyl isobutyl ketone

Methyl methacrylate

Propionitrile

Selenium, total recoverable

Silver, total recoverable

Styrene

Tetrachloroethylene

Toluene

Trichloroethylene

Trichlorofluoromethane

Tritium

Vinyl acetate

Xylenes

cis-1,3-Dichloropropene

trans-1,2-Dichloroethylene

trans-1,3-Dichloropropene

trans-1,4-Dichloro-2-butene
101998

$<5$

$<5$

$<5$

$<10$

$<10$

$<5$

$<10$

$<5$

.8

$<5$

$<5$

$<10$

$<7.62$

$<5$

1.49

$<5$

$<100$

$<47$

$<.7$

$<10$

$<10$

$<10$

$<50$

$<66$

$<5$

$<5$

$<5$

$<5$

$<5$

$<5$

1.3

$<10$

$<5$

$<5$

$<5$

$<5$

$<20$
201998

$<5$

$<5$

$$
<5
$$

$<10<10$

$<10<10$

$<5 \quad<5$

$<10<10$

$<5<5$

$.7<3$

$<5<5$

$<5<5$

$<5 \quad<5$

$<2.2<1.8$

$<5<5$

$<-.33 \quad 2.15$

$<5<5$

330

$<100$

$<5$

$<.2$

$<5$

$<10$

$<12$

$<5$

$<5$

$<5$

$<2$

$<5$

$<5$

$<5$

$<5$

$<5$

1080

$<5$

1.58

$<5$

$<5$

$<5$

$<5$

$<5$

$<5$
Compliance

Lab Eilter 
WELL: LFW 58D

\begin{tabular}{|c|c|c|c|c|c|c|}
\hline $\begin{array}{l}\text { SRS Coord, } \\
\text { N } 82940.6 \\
\text { E } 45700.2\end{array}$ & $\begin{array}{l}\text { Lat/Longitude } \\
\text { 33.284 Deg N } \\
\text { 81.708 Deg W }\end{array}$ & $\frac{\text { Screen Zone Elevation }}{147.6-127.5 \mathrm{ft} \mathrm{msl}}$ & $\frac{\text { Top of Casing }}{167.6 \mathrm{ft} \mathrm{msl}}$ & $\frac{\text { Casing }}{4 " P V C}$ & $\frac{\text { Pump }}{\mathrm{S}}$ & $\begin{array}{l}\text { Formation } \\
\text { U Steed Pond }\end{array}$ \\
\hline SAMPLE I & & $12 / 02 / 97$ & $06 / 0$ & & $4 / 98$ & \\
\hline
\end{tabular}

\section{FIELD DATA}

Analyte
Water Elevation
Depth to water
pH
Sp. Conductance
Water temperature
Alkalinity as CaCO3
Turbidity
Volumes purged
Sampling code

ANALYTICAL DATA

\section{H ST Analyte}

1,1,1,2-Tetrachloroethane

1,1,1-Trichloroethane

1,1,2,2-Tetrachloroethane

1,1,2-Trichloroethane

+ 1,1-Dichloroethane

1,1-Dichloroethylene

1,2,3-Trichloropropane

1,2-Dibromo-3-chloropropane

1,2-Dibromoethane

1,2-Dichlorobenzene

1,2-Dichloroethane

1,2-Dichloropropane

1,3-Dichlorobenzene

+ 1,4-Dichlorobenzene

1,4-Dioxane

2-Hexanone

Acetone

Acetonitrile (Methyl cyanide)

Acrolein

Acrylonitrile

Allyl chloride

Aluminum, total recoverable

Arsenic, total recoverable

Barium, total recoverable

+ Benzene

Bromodichloromethane

101998
139.7
25.9
5.2
140
14
24
8.8
.107351
$\mathrm{NX}$

201998
141.15
24.45

\begin{tabular}{|c|c|}
\hline 301998 & 401998 \\
\hline 140.08 & 140.09 \\
\hline 25.52 & 25.51 \\
\hline 5.4 & 5.6 \\
\hline 250 & 280 \\
\hline 21.5 & 23.8 \\
\hline 40 & 47 \\
\hline 4.1 & 2.7 \\
\hline 3.65937 & 13.1647 \\
\hline
\end{tabular}

Unit

ft msi

ft

pH

uS/cm

deg. C

$\mathrm{mg} / \mathrm{L}$

NTU

well volumes

Compliance

101998201998 301998 401998 DFMod Unit Lab Filter

\begin{tabular}{|c|c|c|c|c|c|c|c|}
\hline$<5$ & $<5$ & & & & & & \\
\hline 4.28 & 2.8 & $<5$ & 1 & $\mathbf{U}$ & ug/L & EX & $<$ EQL \\
\hline$<5$ & $<5$ & $<5$ & 1 & $\mathbf{U}$ & ug $/ \mathrm{L}$ & EX & $<\mathbf{E Q L}$ \\
\hline$<5$ & $<5$ & $<5$ & 1 & $\mathbf{U}$ & ug $/ \mathbf{L}$ & EX & $<\mathbf{E Q L}$ \\
\hline 67.7 & 130 & 92.3 & 1 & & $\mathbf{u g} / \mathbf{L}$ & EX & 92.3 \\
\hline 1.96 & 3.7 & $<5$ & 1 & $\mathbf{U}$ & $\mathbf{u g} / \mathbf{L}$ & EX & $<$ EQL \\
\hline$<5$ & $<5$ & & & & & & \\
\hline$<5$ & $<5$ & $<5$ & 1 & $\mathbf{U}$ & $\mathbf{u g} / \mathbf{L}$ & EX & $<$ EQL \\
\hline \multirow[t]{2}{*}{$<5$} & $<5$ & $<5$ & 1 & $\mathbf{U}$ & ug $/ \mathrm{L}$ & EX & $<\mathbf{E Q L}$ \\
\hline & & 2.84 & 1 & $\mathbf{J}$ & ug/L & $\mathbf{E X}$ & NDD \\
\hline$<5$ & 4.7 & $<5$ & 1 & $\mathbf{U}$ & $\mathrm{ug} / \mathrm{L}$ & EX & $<$ EQL \\
\hline \multirow[t]{2}{*}{1.59} & $<5$ & $<5$ & 1 & $\mathbf{U}$ & $\mathrm{ug} / \mathrm{L}$ & EX & $<$ EQL \\
\hline & & $<5$ & 1 & $\mathbf{v}$ & ug/L & EX & $<$ EQL \\
\hline \multirow[t]{2}{*}{34.2} & 170 & 173 & 1 & & ug/L & EX & 173 \\
\hline & & $<1000$ & 1 & $\mathbf{U}$ & $\mathrm{ug} / \mathrm{L}$ & EX & $<$ EQL \\
\hline$<10$ & $<10$ & $<5$ & 1 & $\mathbf{U}$ & $\mathbf{u g} / \mathrm{L}$ & EX & $<$ EQL \\
\hline$<10$ & $<5.8$ & $<20$ & 1 & $\mathbf{U}$ & ug/L & EX & $<$ EQL \\
\hline$<\mathbf{2 0}$ & $<20$ & $<500$ & 1 & $\mathbf{U}$ & ug/L & EX & $<\mathrm{EQL}$ \\
\hline$<\mathbf{2 0}$ & $<10$ & $<50$ & 1 & $\mathbf{U}$ & ug/L & EX & $<$ EQL \\
\hline$<5$ & $<5$ & $<50$ & 1 & $\mathbf{U}$ & $u g / L$ & EX & $<\mathrm{EQL}$ \\
\hline \multirow[t]{2}{*}{$<10$} & $<10$ & $<10$ & 1 & $\mathbf{U}$ & $\mathrm{ug} / \mathrm{L}$ & EX & $<\mathbf{E Q L}$ \\
\hline & 630 & $<200$ & 1 & $\mathbf{U}$ & ug/L & EX & $<\mathbf{E Q L}$ \\
\hline 6.1 & $<8$ & 47.7 & 1 & $\mathbf{J}$ & ug/L & EX & NDD \\
\hline 5.9 & 5.3 & 6.67 & 1 & $\mathbf{J}$ & ug/L & EX & NDD \\
\hline 3.94 & 24 & 13.3 & 1 & & ug/L & EX & 13.3 \\
\hline$<5$ & $<5$ & $<5$ & 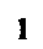 & U & $/ \mathrm{L}$ & $\mathrm{x}$ & $<$ EOL \\
\hline
\end{tabular}

Note: Flagging, dilution factors, modifiers, and laboratories are for fourth quarte1998 data only. See Appendix B for fagging criteria.

$*=$ exceeded holding time for fourth quarter 1998 .

$+=$ exceeded screening level or final primary drinking water standard for fourth quartert 998 .

Sanitary Landfill

D- 75

Fourth Quarter 1998 and Annual Summary 


\section{WELL: LFW 58D}

\section{ANALYTICAL DATA}

\section{H ST Analyte}

Bromoform

Bromomethane (Methyl bromide)

Cadmium, total recoverable

Carbon disulfide

Carbon tetrachloride

Chlorobenzene

Chloroethane

+ Chloroethene (Vinyl chloride)

Chloroform

Chloromethane (Methyl chloride)

Chloroprene

Chromium, total recoverable

Dibromochloromethane

Dibromomethane (Methylene bromide)

+ Dichlorodifluoromethane

+ Dichloromethane (Methylene chloride)

Ethyl methacrylate

Ethylbenzene

Gross alpha

Iodomethane (Methyl iodide)

+ Iron, total recoverable

Isobutyl alcohol

Lead, total recoverable

Mercury, total recoverable

Methacrylonitrile

Methyl ethyl ketone

Methyl isobutyl ketone

Methyl methacrylate

Pentachloroethane

Propionitrile

Selenium, total recoverable

Silver, total recoverable

Styrene
Tetrachloroethylene

Toluene

+ Trichloroethylene

+ Trichlorofluoromethane

Tritium

Vinyl acetate

Xylenes

cis-1,2-Dichloroethylene

cis-1,3-Dichloropropene
101298

$<5$

$<10$

$<4.7$

$<5$

$<5$

4.93

2.36

7.95

$<5$

$<10$

$<5$

$<2.8$

$<5$

$<5$

24.6

$<21.5$

$<5$

3.59

$<5$

$<100$

$<47$

$<.7$

$<10$

$<10$

$<10$

201998

301998

$<5$

3.6

$<2$

1.6

$<5$

22

6.7

40

$<5$

$<10$

$<5$

.9

$<5$

$<5$

15

$<16$

.61

3.33

11

7980

$<100$

11.9

.05
$<5$

$<5$

$<10$

$<12$

$<5$

5

$<5$

$<50$

$<66$

$<5$

$=<5$ -

5.25

$<5$

13.4

17.5

8.35

$<10$

11.4

$<5$

$<2$

$-<5$

2.6

$<5$

\section{8}

$<5$

$<5$

$<10$

$<5$

$<$

21.7

$<10$

15.1

$<5$

$<5$

$<50$

$<10$

$<5$

$<5$

$$
<5
$$$$
27.7
$$

$$
8.6
$$$$
<5
$$$$
<5
$$

8.36

$<5$

17900

$<1001 \mathrm{U}$ ug/L

$<.5 \quad 1 \quad \mathrm{U} \quad \mathrm{ug} / \mathrm{L}$

$<500 \quad 1 \quad \mathrm{U} \quad \mathrm{ug} / \mathrm{L}$

$\begin{array}{llll}<10 & 1 & \mathrm{U} & \mathrm{ug} / \mathrm{L}\end{array}$

$\begin{array}{llll}< & 1 & \mathrm{U} & \mathrm{ug} / \mathrm{L}\end{array}$

$<50 \quad 1 \quad \mathrm{U} \quad \mathrm{ug} / \mathrm{L}$

$<200 \quad 1 \quad \mathrm{U}$

ug/L

$<200 \quad 1 \quad \mathrm{U} \quad$ ug/L $\quad$ EX $\quad<$ EQL

$<20 \quad 1 \quad \mathrm{U} \quad \mathrm{ug} / \mathrm{L} \quad \mathrm{EX} \quad<\mathrm{EQL}$

$<5=-1_{==}$

$3.47 \quad 1$

$<5 \quad 1 \quad \mathrm{U}$

16.21

33.91

18.161

$<20$ I U

$11.8 \quad 1$

$21.9 \quad 1$

$<5$

Compliance

Lab Eilter

EX < EQL

EX $<$ EQL

EX < EQL

EX < EQL

EX < EQL

EX 21.7

EX < EQL

EX 15.1

EX $<$ EQL

EX $<$ EQL

EX < EQL

EX $<$ EQL

EX $<$ EQL

EX < EQL

EX 27.7

EX NDD

EX $<$ EQL

EX < EQL

TM NDD

EX < EQL

EX 17900

EX $<$ EQL

EX $<$ EQL

EX $<$ EQL

EX $<$ EQL

EX < EQL

EX $<$ EQL

EX $<$ EQL

$\mathbf{u g} / \mathbf{L}_{-} \quad \mathbf{E X}=\mathbf{E Q L}$

ug/L EX NDD

ug/L EX < EQL

$\mathrm{ug} / \mathrm{L} \quad \mathrm{EX} \quad 16.2$

ug/L EX 33.9

pCi/m! TM 18.16

ug/L EX < EQL

ug/L $\quad$ EX $\quad 11.8$

ug/L EX 21.9

ug/L EX < EQL

Note: Flagging, dilution factors, modifiers, and laboratories are for fourth quartet998 data only. See Appendix B for flagging criteria.

$*$ a exceeded holding time for fourth quarter 1998 .

+ = exceeded screening level or final primary drinking water standard for fourth quarten 998 . 
WSRC-TR-99-00011

Unclassified

WELL: LFW 58D

\section{ANALYTICAL DATA}

\section{H ST Analyte}

trans-1,2-Dichloroethylene trans-1,3-Dichloropropene trans-1,4-Dichloro-2-butene

10199

Comoliance
201998

301998

401998

$<5$

$<5$

$<5$

$<5$

$<20$
DEMod Unit

$1 \mathrm{U} u g / \mathrm{L}$

$1 \mathrm{U} u g / \mathrm{L}$

$1 \mathrm{U} u \mathrm{~g} / \mathrm{L}$
Lab Eilter

EX $<$ EQL

EX $<$ EQL

EX $<$ EQL

Note: Flagging, dilution factors, modifiers, and laboratories are for fourth quarte1998 data only. See Appendix B for nagging criteria. * = exceeded holding time for fourth quarter 1998.

$+=$ exceeded screening level or final primary drinking water standard for fourth quarten 998 . 
WELL: LFW 59D

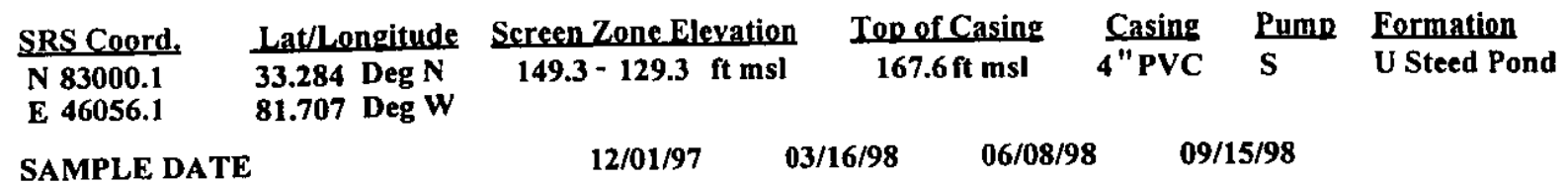

\section{FIELD DATA}

Analyte
Water Elevation
Depth to water
pH
Sp. Conductance
Water temperature
Alkalinity as CaCO3
Turbidity
Volumes purged
Sampling code

ANALYTICAL DATA

\section{H ST Analyte}

1,1,1,2-Tetrachloroethane

1,1,1-Trichloroethane

1,1,2,2-Tetrachloroethane

1,1,2-Trichloroethane

1,1-Dichloroethane

1,1-Dichloroethylene

1,2,3-Trichloropropane

1,2-Dibromo-3-chloropropane

1,2-Dibromoethane

1,2-Dichlorobenzene

1,2-Dichloroethane

1,2-Dichloropropane

1,3-Dichlorobenzene

1,4-Dichlorobenzene

1,4-Dioxane

2-Chloroethyl vinyl ether

2-Hexanone

Acetone

Acetonitrile (Methyl cyanide)

Acrolein

Acrylonitrile

Allyl chloride

+ Aluminum, total recoverable

Arsenic, total recoverable

Barium, total recoverable

Benzene

\begin{tabular}{|c|c|c|c|c|}
\hline 101998 & 201998 & 301998 & 401998 & Unit \\
\hline $141.2 \ldots$ & 129.4 & 141.61 & 142.33 & ft msl \\
\hline 24.1 & 35.9 & 23.69 & 22.97 & ft \\
\hline 4.2 & 4.8 & 4.2 & 4.8 & pH \\
\hline 26 & 20 & 23 & 22 & $\mathrm{uS} / \mathrm{cm}$ \\
\hline 20 & 21.7 & 21 & 20.2 & deg. C \\
\hline 0 & $\mathbf{0}$ & $\mathbf{0}$ & & $\mathbf{m g} / \mathbf{L}$ \\
\hline 3.1 & 5.2 & 1.6 & 5.6 & NTU \\
\hline 8.26606 & 29.2175 & 10.7469 & 5.46911 & well volumes \\
\hline
\end{tabular}

\begin{tabular}{|c|c|c|c|c|c|c|c|c|}
\hline \multirow{2}{*}{$\frac{101998}{<5}$} & \multirow{2}{*}{$\frac{201998}{<5}$} & \multirow{2}{*}{$\frac{301998}{<5}$} & \multirow{2}{*}{401998} & \multicolumn{3}{|c|}{ DEMod Unit } & \multicolumn{2}{|c|}{ Complianc } \\
\hline & & & & & & & & \\
\hline & $<5$ & $<5$ & $<5$ & 1 & $\mathbf{U}$ & ug/L & $\mathbf{E X}$ & $<\mathrm{EQL}$ \\
\hline$<5$ & $<5$ & $<5$ & $<5$ & 1 & $\mathbf{U}$ & ug $/ \mathrm{L}$ & $\mathbf{E X}$ & $<\mathbf{E Q L}$ \\
\hline$<5$ & $<5$ & $<5$ & $<5$ & 1 & $\mathbf{U}$ & $\mathbf{u g} / \mathbf{L}$ & EX & $<\mathrm{EQL}$ \\
\hline 1.34 & 1 & $<5$ & $<5$ & 1 & $\mathbf{U}$ & $u g / L$ & $\mathbf{E X}$ & $<$ EQL \\
\hline$<5$ & $<5$ & $<5$ & $<5$ & 1 & $\mathbf{U}$ & $\mathbf{u g} / \mathrm{L}$ & EX & $<$ EQL \\
\hline$<5$ & $<5$ & $<5$ & & & & & & \\
\hline$<5$ & $<5$ & $<5$ & $<5$ & 1 & $\mathbf{U}$ & $\mathbf{u g} / \mathbf{L}$ & EX & $<\mathrm{EQL}$ \\
\hline$<5$ & $<5$ & $<5$ & $<5$ & 1 & $\mathbf{U}$ & $\mathbf{u g} / \mathrm{L}$ & $\mathbf{E X}$ & $<\mathbf{E Q L}$ \\
\hline & & & $<5$ & 1 & $\mathbf{U}$ & ug/L & $\mathbf{E X}$ & $<\mathrm{EQL}$ \\
\hline$<5$ & $<5$ & $<5$ & $<5$ & 1 & $\mathbf{U}$ & ug/L & $\mathbf{E X}$ & $<$ EQL \\
\hline$<5$ & $<5$ & $<5$ & $<5$ & 1 & $\mathbf{U}$ & $\mathbf{u g} / \mathbf{L}$ & $\mathbf{E X}$ & $<\mathrm{EQL}$ \\
\hline & & & $<5$ & 1 & $\mathbf{U}$ & $\mathrm{ug} / \mathrm{L}$ & $\mathbf{E X}$ & $<$ EQL \\
\hline$<5$ & $<5$ & $<5$ & $<5$ & 1 & $\mathbf{U}$ & $\mathbf{u g} / \mathbf{L}$ & EX & $<$ EQL \\
\hline & & & $<1000$ & 1 & $\mathbf{U}$ & $\mathrm{ug} / \mathrm{L}$ & EX & $<$ EQL \\
\hline & $<5$ & & & & & & & \\
\hline$<10$ & $\leq 10$. & $<10$ & $<5$ & $=1$ & $\underline{\underline{U}}$ & ugg/L & EX & $<$ EQL \\
\hline$<3.92$ & $<10$ & $<10$ & $<20$ & 1 & $\mathbf{U}$ & $\mathrm{ug} / \mathrm{L}$ & $\mathbf{E X}$ & $<$ EQL \\
\hline$<20$ & $<200$ & $<20$ & $<500$ & 1 & $\mathbf{U}$ & ug/L & $\mathbf{E X}$ & $<$ EQL \\
\hline$<20$ & $<10$ & $<10$ & $<50$ & 1 & $\mathbf{U}$ & $\mathrm{ug} / \mathrm{L}$ & $\mathbf{E X}$ & $<\mathrm{EQL}$ \\
\hline$<5$ & $<10$ & $<5$ & $<50$ & 1 & $\mathbf{U}$ & $\mathbf{u g} / \mathbf{L}$ & EX & $<$ EQL \\
\hline$<10$ & $<10$ & $<10$ & $<10$ & 1 & $\mathbf{U}$ & ug/L & EX & $<$ EQL \\
\hline & & 145 & 119 & 1 & $\mathbf{J}$ & ug/L & EX & NDD \\
\hline 4.5 & $<8$ & $<8$ & $<100$ & 1 & $\mathbf{U}$ & $\mathrm{ug} / \mathrm{L}$ & $\mathbf{E X}$ & $<$ EQL \\
\hline 5.6 & 3.9 & 5.2 & 5.22 & 1 & $\mathbf{J}$ & $u g / L$ & EX & NDD \\
\hline$<5$ & $<5$ & $<5$ & $<5$ & 1 & $\mathbf{U}$ & $\mathrm{ug} / \mathrm{L}$ & EX & $<\mathrm{EQL}$ \\
\hline
\end{tabular}

Note: Flagging, dilution factors, modifiers, and laboratories are for fourth quartet 998 data only. See Appendir B for flagging criteria.

* = exceeded holding time for fourth quarter 1998 .

$+=$ exceeded screening level or final primary drinking water standard for fourth quarteri 998 . 
WSRC-TR-99-0001 1

Unclassified

WELL: LFW 59D

\section{ANALYTICAL DATA}

\section{H ST Analyte}

Bromodichloromethane

Bromoform

Bromomethane (Methyl bromide)

Cadmium, total recoverable

Carbon disulfide

Carbon tetrachloride

Chlorobenzene

Chloroethane

Chloroethene (Vinyl chloride)

Chloroform

Chloromethane (Methyl chloride)

Chloroprene

Chromium, total recoverable

Dibromochloromethane

Dibromomethane (Methylene bromide)

Dichlorodifluoromethane

Dichloromethane (Methylene chloride)

Ethyl methacrylate

Ethylbenzene

Gross alpha

Iodomethane (Methyl iodide)

+ Iron, total recoverable

Isobutyl alcohol

Lead, total recoverable

Mercury, total recoverable

Methacrylonitrile

Methyl ethyl ketone

Methyl isobutyl ketone

Methyl methacrylate

Pentachloroethane

Propionitrile

Selenium, total recoverable

Silver, total recoverable

Styrene

Tetrachloroethylene

Toluene

Trichloroethylene

Trichlorofluoromethane

Tritium

Vinyl acetate

Xylenes

cis-1,2-Dichloroethylene
$101998 \quad 201998 \quad 301998 \quad 401998$ DFMod $\mathrm{Vmit}$

$\begin{array}{lllllllll}< & <5 & <5 & <5 & 1 & \mathrm{U} & \mathrm{ug} / \mathrm{L} & \mathrm{EX} & <\mathrm{EQL}\end{array}$

$<5 \quad<5 \quad<5 \quad<5 \quad 1 \quad$ U $\quad$ ug/L $\quad$ EX $<$ EQL

$<10 \quad<10 \quad<10 \quad<5 \quad 1 \quad \mathrm{U}$ ug/L $\quad \mathrm{EX}<\mathrm{EQL}$

$<4.7 \quad<2 \quad<2 \quad<10 \quad 1 \quad \mathrm{U}$ ug/L $\quad \mathrm{EX}<\mathrm{EQL}$

$\begin{array}{lllllllll}55 & <5 & <5 & <5 & 1 & \mathrm{U} & \mathrm{ug} / \mathrm{L} & \mathrm{EX} & <\mathrm{EQL}\end{array}$

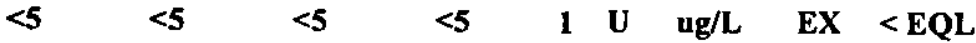

$<5 \quad<5 \quad<5 \quad<5 \quad 1 \quad$ U $\quad$ ug/L $\quad$ EX $<$ EQL

$<10 \quad<10 \quad<10 \quad<10 \quad$ I $\mathrm{U} \quad \mathrm{ug} / \mathrm{L} \quad \mathrm{EX}<\mathrm{EQL}$

$<10 \quad<10 \quad<10 \quad<5 \quad 1 \quad U \quad$ ug/L $\quad \mathrm{EX}<\mathrm{EQL}$

$<5 \quad<5 \quad<5 \quad<5 \quad 1 \quad \mathrm{U} \quad \mathrm{ug} / \mathrm{L} \quad \mathrm{EX}<\mathrm{EQL}$

$<10 \quad<10 \quad<10 \quad<5 \quad 1 \quad \mathrm{U} \quad \mathrm{ug} / \mathrm{L} \quad \mathrm{EX}<\mathrm{EQL}$

$<5 \quad<5 \quad<5 \quad<50 \quad 1 \quad$ U $\quad$ ug/h $\quad \mathrm{EX}<\mathrm{EQL}$

$<1.1 \quad 1.6 \quad<3 \quad r \quad<7.37 \quad 1 \quad \mathrm{U} \quad \mathrm{ug} / \mathrm{L} \quad \mathrm{EX} \quad<\mathrm{EQL}$

$<5 \quad<5 \quad<5 \quad<5 \quad 1 \quad \mathrm{U} \quad \mathrm{ug} / \mathrm{L} \quad \mathrm{EX} \quad<\mathrm{EQL}$

$<5 \quad<5 \quad<5 \quad<5 \quad 1 \quad \mathrm{U} \quad \mathrm{ug} / \mathrm{L} \quad \mathrm{EX}<\mathrm{EQL}$

$\begin{array}{lllllllll}1.72 & <5 & <5 & 3.17 & 1 & \mathrm{~J} & \mathrm{ug} / \mathrm{L} & \mathrm{EX} & \text { NDD }\end{array}$

$<3.14<2.5 \quad<3 \quad<10 \quad 1 \quad \mathrm{U} \quad \mathrm{ug} / \mathrm{L} \quad \mathrm{EX}<\mathrm{EQL}$

$<5 \quad 1 \quad$ U $\quad$ ug/L $\quad$ EX $<$ EQL

$<5 \quad 1 \quad$ U $\quad$ ug/L $/ \mathrm{EX}<$ EQL

$\begin{array}{lllll}2.86 & 1 & \mathrm{pCi} / \mathrm{L} & \mathrm{TM} & 2.86\end{array}$

$<5 \quad 1 \quad \mathrm{U} \quad \mathrm{ug} / \mathrm{L} \quad \mathrm{EX}<$ EQL

$\begin{array}{lllll}358 & 1 & \text { ug/L } & \text { EX } & 358\end{array}$

$<100 \quad 1 \quad U$ ug/L EX $<$ EQL

$\begin{array}{lllllll}<.5 & 1 & \mathrm{U} & \mathrm{ug} / \mathrm{L} & \mathrm{EX} & <\mathrm{EQL}\end{array}$

$<500 \quad 1 \quad \mathrm{U}$ ug/L $\mathrm{EX}<$ EQL

$<10 \quad 1 \quad U \quad$ ug/L EX $\quad<$ EQL

$<5 \quad 1 \quad$ U $\quad$ ug/L $\quad$ EX $<$ EQL

$<50 \quad 1 \quad \mathrm{U} \quad \mathrm{ug} / \mathrm{L} \quad \mathrm{EX} \quad<$ EQL

$<200 \quad 1 \quad$ U $\quad$ ug/L EX $<$ EQL

\begin{tabular}{|c|c|c|c|c|c|c|c|c|}
\hline$<50$ & $<5$ & $<5$ & & & & & 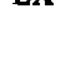 & 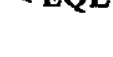 \\
\hline$<66$ & $<5$ & $<5$ & $<200$ & $\mathbf{1}$ & $\mathbf{U}$ & $\mathbf{u g} / \mathbf{L}$ & $\mathbf{E X}$ & $<$ EQL \\
\hline$<5$ & $<2$ & $<2$ & 12.4 & 1 & $\mathbf{J}$ & ug/L & $\mathbf{E X}$ & NDD \\
\hline$<5$ & $<5$ & $<5$ & $<5$ & 1 & $\mathbf{U}$ & ug $/ \mathrm{L}$ & EX & $<\mathbf{E Q}$ \\
\hline$<5$ & .81 & $<5$ & $<5$ & 1 & $\mathbf{U}$ & $\mathrm{ug} / \mathrm{L}$ & EX & $<\mathbf{E Q}$ \\
\hline$<5$ & $<5$ & $<5$ & $<5$ & 1 & $\mathbf{U}$ & $\mathrm{ug} / \mathrm{L}$ & $\mathbf{E X}$ & $<\mathbf{E C}$ \\
\hline$<5$ & .62 & $<5$ & $<5$ & 1 & $\mathbf{U}$ & ug/L & $\mathbf{E X}$ & $<\mathbf{E Q}$ \\
\hline 1.94 & 3 & $<5$ & $<5$ & 1 & $\mathbf{U}$ & ug/L & $\mathbf{E X}$ & $<\mathbf{E Q}$ \\
\hline$<20.54$ & .94 & $<.18$ & $<1.44$ & 1 & UI & $\mathrm{pCi} / \mathrm{ml}$ & TM & $<\mathbf{E Q}$ \\
\hline$<10$ & $<10$ & $<5$ & $<20$ & 1 & $\mathbf{U}$ & ug/L & $\mathbf{E X}$ & $<\mathbf{E Q}$ \\
\hline$<5$ & $<10$ & $<5$ & $<10$ & 1 & $\mathbf{U}$ & $\mathbf{u g} / \mathrm{L}$ & $\mathbf{E X}$ & $<\mathbf{E Q}$ \\
\hline & & & $<5$ & 1 & $\mathbf{U}$ & $\mathrm{ug} / \mathrm{L}$ & $\mathbf{E X}$ & \\
\hline
\end{tabular}

Note: Flagging, dilution factors, modifiers, and laboratories are for fourth quartei 998 data only. See Appendix B for flagging eriteria. * a exceeded holding time for fourth quarter 1998 .

+ a exceeded screening level or final primary drinking water standard for fourth quarten 998. 
WSRC-TR-99-00011

Unclassified

WELL: LFW 59D

ANALYTICAL DATA

H ST Analyte

cis-1,3-Dichloropropene trans-1,2-Dichloroethylene trans-1,3-Dichloropropene trans-1,4-Dichloro-2-butene

$\begin{array}{cc}101998 & 201998 \\ <5 & <5 \\ <5 & <5 \\ <5 & <5 \\ <20 & <5\end{array}$

$\begin{array}{cc}301998 & 401998 \\ <5 & <5 \\ & <5 \\ <5 & <5 \\ <5 & <20\end{array}$

$\begin{array}{llll} & & \\ 1 & U & u g / L \\ 1 & U & u g / L \\ 1 & U & u g / L \\ 1 & U & u g / L\end{array}$

Compliance

Lab Filter

EX $<$ EQL

EX $<$ EQL

EX $<$ EQL

....

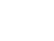


WSRC-TR-99-00011

Unclassified

WELL: LFW 60C

\begin{tabular}{|c|c|c|c|c|c|}
\hline d. & Lat/Longitude & $\frac{\text { Screen } Z \text { one Elevation }}{108.3-98.3 \mathrm{ft} \mathrm{msl}}$ & $\frac{\text { Top of Casing }}{157.2 \mathrm{ft} \text { msl }}$ & $\frac{\text { Casing }}{2 \text { "PVC }}$ & $\frac{P u}{y}$ \\
\hline
\end{tabular}
E 45711.9 81.707 Deg W

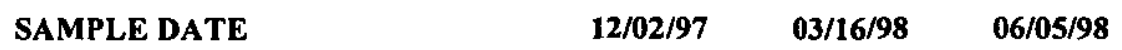

FIELD DATA

Analyte

Water Elevation

Depth to water

pH

Sp. Conductance

Water temperature

Alkalinity as $\mathrm{CaCO} 3$

Turbidity

Volumes purged

Sampling code

\begin{tabular}{lll}
101998 & 201998 & $\frac{301998}{135.6}$ \\
19.5 & -136.53 & 135.8 \\
5.2 & 18.57 & 19.3 \\
89 & 6.2 & 5.6 \\
17 & 74 & 84 \\
24 & 19 & 20.8 \\
4 & 20 & 20 \\
2.78569 & 1.5 & 2.7 \\
\hline
\end{tabular}

401998 Unit

ft msl

ft

pH

uS/cm

deg. $C$

$\mathbf{m g} / \mathbf{L}$

NTU

well volumes

\section{ANALYTICAL DATA}

\section{H ST Analyte}

1,1,1,2-Tetrachloroethane

1,1,1-Trichloroethane

1,1,2,2-Tetrachloroethane

1,1,2-Trichloroethane

1,1-Dichloroethane

1,1-Dichloroethylene

1,2,3-Trichloropropane

1,2-Dibromo-3-chloropropane

1,2-Dibromoethane

1,2-Dichloroethane

1,2-Dichloropropane

1,4-Dichlorobenzene

2-Hexanone

Acetone

Acetonitrile (Methyl cyanide)

Acrolein

Acrylonitrile

Allyl chloride

Aluminum, total recoverable

Arsenic, total recoverable

Barium, total recoverable

Benzene

Bromodichloromethane

Bromoform

Bromomethane (Methyl bromide)

Cadmium, total recoverable

\begin{tabular}{ccccc}
101998 & 201998 & 301998 & 401998 & \multicolumn{2}{c}{ Compliance } \\
$<5$ & $<5$ & $<5$ & \\
$<5$ & $<5$ & $<5$ \\
$<5$ & $<5$ & $<5$ \\
$<5$ & $<5$ & $<5$ & Lab Eilter \\
12.7 & 9.3 & 13 & \\
$<5$ & $<5$ & $<5$ \\
$<5$ & $<5$ & $<5$ \\
$<5$ & $<5$ & $<5$ \\
$<5$ & $<5$ & $<5$ \\
$<5$ & $<5$ & $<5$ \\
$<5$ & $<5$ & $<5$ \\
8.01 & 7.3 & 11 \\
$<10$ & $<10$ & $<10$ \\
$<5.3$ & $<10$ & $<3.5$ \\
$<20$ & $<20$ & $<20$ \\
$<20$ & $<10$ & $<10$ \\
$<5$ & $<20$ & $<5$ \\
$<10$ & $<10$ & $<10$ \\
$<40$ & $<8$ & $<8$ \\
7.1 & 5.2 & 5.2 \\
$<5$ & .55 & .79 \\
$<5$ & $\mathbf{2 . 9}$ & $<5$ \\
$<5$ & $<5$ & $<5$ \\
$<10$ & $<10$ & $<10$ \\
$<4.7$ & $<2$ & $<2$ \\
& & 6 \\
$<5$
\end{tabular}

Note: Flagging, dilution factors, modifiers, and laboratories are for fourth quartet 998 data only. See Appendix B for fagging criteria.

* = exceeded holding time for fourth quarter 1998.

t a exceeded screening level or final primary drinking water standard for fourth quarter 998 . 
WELL: LFW 60C

\section{ANALYTICAL DATA}

\section{H ST Analvte}

Carbon disulfide

Carbon tetrachloride

Chlorobenzene

Chloroethane

Chloroethene (Vinyl chloride)

Chloroform

Chloromethane (Methyl chloride)

Chloroprene

Chromium, total recoverable

Dibromochloromethane

Dibromomethane (Methylene bromide)

Dichlorodiffuoromethane

Dichloromethane (Methylene chloride)

Ethylbenzene

Gross alpha

lodomethane (Methyl iodide)

Iron, total recoverable

Isobutyl alcohol

Lead, total recoverable

Mercury, total recoverable

Methacrylonitrile

Methyl ethyl ketone

Methyl isobutyl ketone

Methyl methacrylate

Propionitrile

Selenium, total recoverable

Silver, total recoverable

Styrene

Tetrachloroethylene

Toluene

Trichloroethylene

Trichlorofluoromethane

Tritium

Vinyl acetate

Xylenes

cis-1,3-Dichloropropene

trans-1,2-Dichloroethylene

trans-1,3-Dichloropropene

trans-1,4-Dichloro-2-butene
101998

$<5$

$<5$

1.4

$<10$

2.87

$<5$

$<10$

$<5$

$<1.3$

$<5$

$<5$

1.6

$<8.42$

$<5$

1.34

$<5$

$<100$

$<47$

$<.7$

$<10$

$<10$

$<10$

$<5$

$<50<5$

$<66<5$

$<5<2$

$<5<5$

$<5$

$<5$

2

3.8

$-2$

$<10$

1.3

$<5$

$<5$

$<5$

$<20$

$<5$

\section{$201998 \quad 301998$}

$<5$

1.5

$<10$

4.1

2.6

$<10$

$<5$

.6

1.7

$<5$

$<5$

$<2.6$

$<5$

$<-.81$

$<5$

$<100$

7.9

$<.2$

$<5$

$<10$

$<12<12$

$<5<5$

$<5<5$

$<5<5$

$<2<2$

$<5<5$

$<5 \quad<5$

$<5 \quad .71$

$\begin{array}{ll}1.2 & 1.7\end{array}$

$\begin{array}{ll}1 & 2.9\end{array}$

$1870 \ldots 1.64$

$<5<5$

1.24

$<5<5$

$\begin{array}{ll}<5 & <5 \\ <5 & <5\end{array}$

$$
<5
$$

Compliance 401998 DEMod Unit Lab Filter

Nofe: Flagging, dilution factors, modifiers, and laboratories are for fourth quarte1998 date only. See Appendix B for fogging criteris. *- exceeded holding time for fourth quarter 1998.

$+=$ exceeded screening level or final primary drinking water standard for fourth quarten 998 . 
WELL: LFW 60D

\begin{tabular}{|c|c|c|c|c|c|c|}
\hline SRS Coord. & Lat/Lengitude & Screen Zone Elevation & Ton of Casing & Casing & Pump & Eormation \\
\hline $\begin{array}{l}\text { N } 82531.5 \\
\text { E } 45722.3\end{array}$ & $\begin{array}{l}\text { 33.283 Deg N } \\
\text { 81.707 } \text { Deg W }\end{array}$ & $143.8-123.8$ ft msl & $157.1 \mathrm{ft}$ msl & $4 " \mathrm{PVC}$ & $\mathbf{S}$ & U Steed Pond \\
\hline
\end{tabular}

SAMPLE DATE

FIELD DATA $\begin{array}{llll}12 / 04 / 97 & 03 / 16 / 98 & 06 / 09 / 98 & 09 / 16 / 98\end{array}$

$\begin{array}{lllll}101998 & 201998 & \frac{301998}{401998} & \text { Unit } \\ 136.2 & 137.5 & 136.32 & 136.72 & \text { ft msl } \\ 19 & 17.7 & 18.88 & 18.48 & \text { ft } \\ 4.6 & 5.2 & 4.4 & 4.8 & \text { pH } \\ 50 & 20 & 20 & 28 & \text { uS/cm } \\ 17 & 19 & 18.5 & 19.7 & \text { deg. C } \\ 0 & 4 & 1 & 1 & \text { mg/L } \\ 8.5 & .8 & 12.8 & 2 & \text { NTU } \\ .106601 & 5.66760 & .105714 & .205721 & \text { well volumes } \\ \text { NX } & & \text { NVX } & \text { NX } & \end{array}$

\section{ANALYTICAL DATA}

H ST Analyte

1,1,1,2-Tetrachloroethane

1,1,1-Trichloroethane

1,1,2,2-Tetrachloroethane

1,1,2-Trichloroethane

1,1-Dichloroethane

1,1-Dichloroethylene

1,2,3-Trichloropropane

1,2-Dibromo-3-chloropropane

1,2-Dibromoethane

1,2-Dichlorobenzene

1,2-Dichloroethane

1,2-Dichloropropane

1,3-Dichlorobenzene

1,4-Dichlorobenzene

1,4-Dioxane

2-Hexanone

Acetone

Acetonitrile (Methyl cyanide)

Acrolein

Acrylonitrile

Allyl chloride

Aluminum, total recoverable

Arsenic, total recoverable

Barium, total recoverable

Benzene

Bromodichloromethane

\begin{tabular}{|c|c|c|c|c|c|c|c|c|}
\hline & & & & & & & & omplia \\
\hline 101998 & 201998 & 301998 & 401998 & & Mod & 1 Unit & $\mathbf{L a b}$ & Filter \\
\hline$<5$ & & $<5$ & & & & & & \\
\hline$<5$ & & $<5$ & $<5$ & 1 & $\mathbf{U}$ & $\mathbf{u g} / \mathbf{L}$ & $\mathbf{E X}$ & < EQL \\
\hline$<5$ & & $<5$ & $<5$ & 1 & $\mathbf{U}$ & ug/L & $\mathbf{E X}$ & $<\mathbf{E Q L}$ \\
\hline$<5$ & & $<5$ & $<5$ & 1 & $\mathbf{U}$ & ug/L & EX & $<$ EQL \\
\hline 18.1 & & 3.8 & $<5$ & 1 & $\mathbf{U}$ & ug/L & $\mathbf{E X}$ & $<\mathbf{E Q L}$ \\
\hline$<5$ & & $<5$ & $<5$ & 1 & $\mathbf{U}$ & ug/L & $\mathbf{E X}$ & $<\mathbf{E Q L}$ \\
\hline$<5$ & & $<5$ & & & & & & \\
\hline$<5$ & & $<5$ & $<5$ & 1 & $\mathbf{U}$ & $\mathbf{u g} / \mathbf{L}$ & EX & $<$ EQL \\
\hline$<5$ & & $<5$ & $<5$ & 1 & $\mathbf{U}$ & ug/L & $\mathbf{E X}$ & $<$ EQL \\
\hline & & & $<5$ & 1 & $\mathbf{U}$ & ug/L & EX & $<$ EQL \\
\hline$<5$ & & $<5$ & $<5$ & 1 & $\mathbf{U}$ & $\mathbf{u g} / \mathbf{L}$ & $\mathbf{E X}$ & $<$ EQL \\
\hline$<5$ & & $<5$ & $<5$ & 1 & $\mathbf{U}$ & $\mathrm{ug} / \mathrm{L}$ & $\mathbf{E X}$ & $<$ EQL \\
\hline & & & $<5$ & 1 & $\mathbf{U}$ & ug/L & $\mathbf{E X}$ & $<$ EQL \\
\hline$<5$ & & $<5$ & $<5$ & 1 & $\mathbf{U}$ & ug/L & $\mathbf{E X}$ & $<$ EQL \\
\hline & & & $<1000$ & 1 & $\mathbf{U}$ & ug/L & $\mathbf{E X}$ & $<$ EQL \\
\hline$<10$ & & $<10$ & $<5$ & 1 & $\mathbf{U}$ & ug/L & $\mathbf{E X}$ & $<$ EQL \\
\hline$<10$ & & $<10$. & $<20$ & 1 & $\mathbf{U}$ & ug/L & $\mathbf{E X}$ & $<\mathrm{EQL}$ \\
\hline$<20$ & & $<20$ & $<500$ & 1 & $\mathbf{U}$ & ug/L & EX & $<$ EQL \\
\hline$<20$ & & $<10$ & $<50$ & 1 & $\mathbf{U}$ & ug/L & EX & $<$ EQL \\
\hline$<5$ & & $<5$ & $<50$ & 1 & $\mathbf{U}$ & ug/L & $\mathbf{E X}$ & $<\mathrm{EQL}$ \\
\hline$<10$ & & $<10$ & $<10$ & 1 & $\mathbf{U}$ & ug/L & $\mathbf{E X}$ & $<$ EQL \\
\hline & & 649 & $<200$ & 1 & $\mathbf{U}$ & ug/L & $\mathbf{E X}$ & $<$ EQL \\
\hline$<40$ & $<8$ & $<8$ & $<100$ & 1 & $\mathbf{U}$ & ug/L & EX & $<$ EQL \\
\hline 10.1 & 4.7 & 5.8 & $<10$ & 1 & $\mathbf{u}$ & ug/L & $\mathbf{E X}$ & $<$ EQL \\
\hline$<5$ & & $<5$ & $<5$ & 1 & $\mathbf{U}$ & ug/L & $\mathbf{E X}$ & $<$ EQL \\
\hline$<5$ & & $<5$ & $<5$ & 1 & $\mathbf{U}$ & ug/L & EX & $<$ EQL \\
\hline
\end{tabular}

Note: Flagging, dilution factors, modifiers, and laboratories are for fourth quarte1998 data only. See Appendix B for fagging criteria. - = exceeded holding time for fourth quarter 1998.

$+=$ exceeded sereening level or final primary drinking water standard for fourth quarten 998 .

Sanitary Landfill

D-83

Fourth Quarter 1998 and Annual Summary 
WSRC-TR-99-00011

Unclassified

WELL: LFW 60D

\section{ANALYTICAL DATA}

H ST Analyte

Bromoform

Bromomethane (Methyl bromide)

Cadmium, total recoverable

Carbon disulfide

Carbon tetrachloride

Chlorobenzene

Chloroethane

Chloroethene (Vinyl chloride)

Chloroform

Chloromethane (Methyl chloride)

Chloroprene

Chromium, total recoverable

Dibromochloromethane

Dibromomethane (Methylene bromide)

Dichlorodifluoromethane

Dichloromethane (Methylene chloride)

Ethyl methacrylate

Ethylbenzene

Gross alpha

Iodomethane (Methyl iodide)

Iron, total recoverable

Isobutyl alcohol

Lead, total recoverable

Mercury, total recoverable

Methacrylonitrile

Methyl ethyl ketone

Methyl isobutyl ketone

Methyl methacrylate

Pentachloroethane

Propionitrile

Selenium, total recoverable

Silver, total recoverable

Styrene

Tetrachloroethylene

Toluene

Trichloroethylene

Trichlorofluoromethane

Tritium

Vinyl acetate

Xylenes

cis-1,2-Dichloroethylene

cis-1,3-Dichloropropene
101998

$<5$

$<10$

$<4.7$

1.89

$<5$.

$<5$

$<10$

$<10$

$<5$

$<10$

$<5$

2.6

$<5$

$<5$

5.63

$<7.77$

$<5$

1.32

$<5$

$<100$

15.9

2.48

$<10$

$<10$

$<10$

$<12$

50

$<2$

$<50$
$201998 \quad 301998$

$<5$

$<10$

$<2$

$<5$

$<5$

$<5$

$<10$

$<10$

$<5$

$<10$

$<5$

$<3$

$<5$

$<5$

$<5$

$<3.1$

$<5$

$<16$

$<5$

531

$<100$

17.9

.17

$<5$

$<10$

$<12$

$<5$

$<66<$

$<5$

$-\leq 5$

1

$<5$

4.45

15.2

4.6

$<10$

$<5$

$<5$

$<5<$

$<2$

$<5$

$<5$
$<2$

$<5$
$<5$

$<5$

$<5$

$<5$

$<5$

1350

2.08

$<5$

$<5$

$<5$
401998 DEMod Unit

$<5 \quad 1 \mathrm{U}$ ug/L

$<5 \quad 1 \quad \mathrm{U} \quad \mathrm{ug} / \mathrm{L}$

$<10 \quad 1 \quad \mathrm{U} \quad \mathrm{ug} / \mathrm{L}$

$<5 \quad 1 \quad \mathrm{U} \quad \mathrm{ug} / \mathrm{L}$

$<5 \quad 1 \quad$ U $\quad$ ug/L

$<5 \quad 1 \quad U \quad u g / L$

$<10 \quad 1$ U ug/L

$<5 \quad 1 \quad \mathrm{U} \quad \mathrm{ug} / \mathrm{L}$

$\begin{array}{llll}<5 & 1 & \mathbf{U} & \mathrm{ug} / \mathrm{L}\end{array}$

$<5 \quad 1 \quad \mathrm{U} \quad \mathrm{ug} / \mathrm{L}$

$<50 \quad 1 \quad U \quad u g / L$

$<10 \quad 1 \quad \mathrm{U} \quad \mathrm{ug} / \mathrm{L}$

$<5 \quad 1 \quad \mathrm{U} \quad \mathrm{ug} / \mathrm{L}$

$<5 \quad 1 \quad \mathrm{U} \quad \mathrm{ug} / \mathrm{L}$

$<5 \quad 1 \quad \mathrm{U} \quad \mathrm{ug} / \mathrm{L}$

$<10 \quad 1 \quad \mathrm{U} \quad \mathrm{ug} / \mathrm{L}$

$<5$

$<5$

.95

$<5$

$<200$

$1 \mathrm{U}$

ug/L

ug/L

pCi/L

ug/L

ug/L

$<100$

.221

$<500$

$<10$

$<5$

$<5$

$<50$

Compliance

Lab Filter

EX < EQL

EX < EQL

EX < EQL

EX < EQL

EX $<$ EQL

EX $<$ EQL

EX < EQL

EX < EQL

EX $<$ EQL

EX < EQL

EX < EQL

EX < EQL

EX < EQL

EX $<$ EQL

EX < EQL

EX < EQL

EX $<$ EQL

EX < EQL

TM .95

EX < EQL

EX $<$ EQL

ug/L EX $<$ EQL

ug/L EX NDD

ug/L $\quad$ EX $<$ EQL

ug/L EX $<$ EQL

ug/L EX $<$ EQL

ug/L EX $<$ EQL

ug/L EX $<$ EQL

$<200 \quad 1 \quad \mathrm{U} \quad \mathrm{ug} / \mathrm{L} \quad \mathrm{EX}<\mathrm{EOL}$

$<201$ U $\quad$ ug/L EX $\quad$ EQL

$\leq 5 \ldots$. 1 U . ug/L EX $\leq \mathrm{EQL}$

$<5 \quad 1$ U ug/L EX $<$ EQL

$<5 \quad 1$ U ug/L EX $<$ EQL

$<5 \quad 1 \quad$ U ug/L EX $\quad$ EQL

$\begin{array}{llllll}2.93 & 1 & \mathrm{~J} & \mathrm{ug} / \mathrm{L} & \mathrm{EX} & \text { NDD }\end{array}$

$\begin{array}{lllll}1.7 & 1 & \mathrm{pCi} / \mathrm{ml} & \mathrm{TM} & 1.7\end{array}$

$<20 \quad 1 \quad \mathrm{U}$ ug/L EX $<$ EQL

$<10 \quad 1 \quad \mathrm{U} \quad$ ug/L $\quad$ EX $<$ EQL

$1.7 \quad$ J ug/L EX NDD

$<5 \quad 1 \quad$ U $\quad$ ug/L $\quad$ EX $<$ EQL

Note: Flagging, dilution factors, modifiers, and laboratories are for fourth quartet 998 data only. See Appendix B for fagging criteria.

" = exceeded holding time for fourth quarter 1998.

+- exceeded screening level or final primary drinking water standard for fourth quarter1998 . 
WELL: LFW 60D

ANALYTICAL DATA

H ST Analvte

trans-1,2-Dichloroethylene trans-1,3-Dichloropropene trans-1,4-Dichloro-2-butene
101998

$<5$

$<5$

$<20$

\section{8}

301998

\section{8}

$<5$

$<5$

$<5$
Compliance

$\begin{array}{llll}\text { DFMod Unit } & \text { Lab } & \text { Filter } \\ 1 & \mathrm{U} & \text { ug/L } & \text { EX }\end{array}$

$1 \mathrm{U}$ ug/L EX $<$ EQL

$1 \mathrm{U}$ ug/L EX $<$ EQL

Note: Flagging, dilution factors, modifiers, and laboratories are for fourth quarte1998 data only. See Appendix B for fagging criteria. * = exceeded holding time for fourth quarter 1998.

+- exceeded screening level or final primary drinking water standard for fourth quarteri 998 . 
WSRC-TR-99-00011

Unclassified

WELL: LFW 61D

\begin{tabular}{|c|c|c|c|c|c|c|}
\hline$\frac{\text { SRS Coord }}{\text { N 83089.1 }}$ & $\frac{\text { Lat/Longitude }}{33.285 \text { Deg N }}$ & $\frac{\text { Screen Zone Elevation }}{150.4-130.3 \mathrm{ft} \mathrm{msl}}$ & $\frac{\text { Top of Casing }}{168.3 \mathrm{ft} \mathrm{msl}}$ & $\frac{\text { Casing }}{4 \text { "PVC }}$ & $\begin{array}{l}\text { Pump } \\
\text { S }\end{array}$ & $\begin{array}{l}\text { Eormation } \\
\text { U Steed Pond }\end{array}$ \\
\hline
\end{tabular}

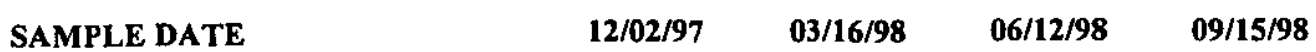

FIELD DATA

\begin{tabular}{|c|c|c|c|c|c|}
\hline Anaiyte & 101998 & 201998 & 301998 & 401998 & Unit \\
\hline Water Elevation & ․ 142.64 & 145.51 & 143.16 & 143.4 & ft msl \\
\hline Depth to water & 23.76 & 20.89 & 23.24 & 23 & \\
\hline pH & 6.2 & 5.5 & 5.3 & 5.7 & pH \\
\hline Sp. Conductance & 260 & 160 & 130 & 120 & $\mathrm{uS} / \mathrm{cm}$ \\
\hline Water temperature & 19 & 20.4 & 20.9 & 21.9 & deg. $C$ \\
\hline Alkalinity as $\mathrm{CaCO} 3$ & 121 & 43 & 32 & 44 & $\mathrm{mg} / \mathbf{L}$ \\
\hline Turbidity & 3.5 & 2.1 & 2.7 & 1.8 & NTU \\
\hline Volumes purged & 19.2690 & 7.12748 & 7.22949 & 4.67480 & well volumes \\
\hline
\end{tabular}

\section{ANALYTICAL DATA}

\section{H ST Analyte}

1,1,1,2-Tetrachloroethane 1,1,1-Trichloroethane 1,1,2,2-Tetrachloroethane 1,1,2-Trichloroethane

+ 1,1-Dichloroethane 1,1-Dichloroethylene 1,2,3-Trichloropropane 1,2-Dibromo-3-chloropropane 1,2-Dibromoethane

1,2-Dichlorobenzene

1,2-Dichloroethane

1,2-Dichloropropane

1,3-Dichjorobenzene

1,4-Dichlorobenzene

1,4-Dioxane

2-Chloroethyl vinyl ether 2-Hexanone

\section{Acetone}

Acetonitrile (Methyl cyanide)

Acrolein

Acrylonitrile

Allyl chloride

Aluminum, total recoverable

Arsenic, total recoverable

Barium, total recoverable

Benzene 
WSRC-TR-99-00011

Unclassified

WELL: LFW 61D

\section{ANALYTICAL DATA}

H ST Analyte

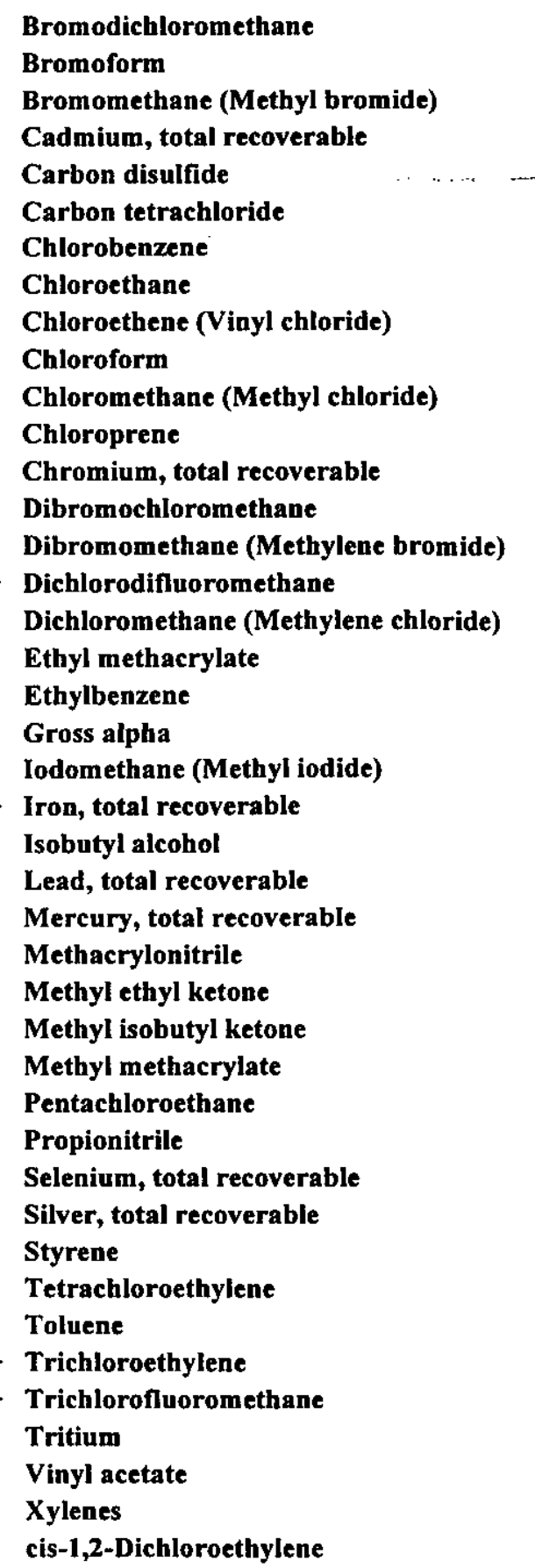

\section{8}



$$
<5
$$$$
<10
$$

$<4.7$

$<5$ -

$<5$

$<5$

$<10$

$<10$

$<5$

1.63

$<5$

$<7$

$<5$

$<5$

38.5

$<9.31$

$$
19.9
$$$$
2.2
$$$$
<5
$$

$<47$

$<.7$

$<10$

$<80.1$

$<17.2$

$<25$

$<50 \quad<25$

$<66<5$

$<5<2$

$<5 \quad<25$

8.312 .2

$6.12<25$

$26.2 \quad 10$

$14.8<25$

3.64

$<10$

\section{8}

$<25$
$<25$

$<50$

$<25$

$<25$

$<25$

$<50$

$<50$

$<25$

$<50$
$<25$

1.2

$<25$

$<25$

17

$<14$

$<25$

2.1

$<25$

$<5$

$<.2$

$<25$

$<50$

$<60$

5

25
2.2
25
10
25
302

3020
$<25$

$<25$

\section{8}

$<5<5$

$<5<5$

$<10<5$

$<2<10$

$<5 \quad<5$

$<5<5$

$<5<5$

$<10<10$

$<10<5$

$<5<5$

$<10$

$<5$

$<3$

$<5$

$<5$

17

$<4.6$

$<10$

2.1

4.32

$<5$

23000

$<100$

6.2

.06

$<5$

$<10$

$<1.8$

$<5$

$<20$

$<5$

$<5$

$<2$

$<5$

3.1

.68

12

7.1

2.39

$<5$

15
$<5$
Compliance

Lab Filter

EX < EQL

EX $<$ EQL

EX $<$ EQL

EX $<$ EQL

EX < EQL

EX $<$ EQL

EX < EQL

EX < EQL

EX < EQL

EX < EQL

EX < EQL

EX < EQL

EX < EQL

EX $<$ EQL

EX < EQL

EX 18.7

EX $<$ EQL

EX < EQL

EX NDD

TM $\quad 5.78$

EX < EQL

EX 19200

192001

$<100 \quad 1 \quad$ U $\quad$ ug/L $\quad$ EX $\quad<$ EQL

$<.5 \quad 1 \quad \mathrm{U} \quad \mathrm{ug} / \mathrm{L} \quad \mathrm{EX} \quad<$ EQL

$<500 \quad 1 \quad$ U $\quad$ ug/L $\quad$ EX $<$ EQL

$<10 \quad 1 \quad$ U $\quad$ ug/L $\quad$ EX $<$ EQL

$<5 \quad 1 \quad \mathrm{U} \quad \mathrm{ug} / \mathrm{L} \quad$ EX $<$ EQL

$<50 \quad 1 \quad$ U $\quad$ ug/L $\quad$ EX $<$ EQL

$<2001 \mathrm{I}$ U ug/L EX $<$ EQL

$<200 \quad 1 \quad$ U $\quad$ ug/L $\quad$ EX $\quad<$ EQL

$<20 \quad 1 \quad$ U $\quad \mathrm{ug} / \mathrm{L} \quad \mathrm{EX} \quad \mathrm{EQL}$

$<5 \quad 1 \quad$ U $\quad$ ug/L $\quad$ EX $<$ EQL

$\begin{array}{llllll}3.94 & 1 & J & u g / L & \text { EX } & \text { NDD }\end{array}$

$<5 \quad 1 \quad$ U $\quad$ ug/L $\quad$ EX $\quad<$ EQL

$\begin{array}{lllll}14.4 & 1 & \mathrm{ug} / \mathrm{L} & \mathrm{EX} & 14.4\end{array}$

$\begin{array}{lllll}16 & 1 & \mathrm{ug} / \mathrm{L} & \mathrm{EX} & 16\end{array}$

$\begin{array}{lllll}2.56 & 1 & \mathrm{pCi} / \mathrm{ml} & \mathrm{TM} & \mathbf{2 . 5 6}\end{array}$

$<20 \quad 1 \quad$ U $\quad$ ug/L $\quad$ EX $<$ EQL

$\begin{array}{lllll}17.3 & 1 & \text { ug/L } & \text { EX } & 17.3\end{array}$

$<5 \quad 1 \quad \mathrm{U} \quad \mathrm{ug} / \mathrm{L} \quad \mathrm{EX} \quad<\mathrm{EQL}$

Note: Flagging, dilution factors, modifiers, and taboratories are for fourth quarte1998 data only. See Appendix B for fagging criteria. * = exceeded holding time for fourth quarter 1998 .

+ + exceeded screening level or final primary drinking water standard for fourth quarten 998 .

Sanitary Landfill

D-87

Fourth Quarter 1998 and Annual Summary 
WELL: LFW 610

\section{ANALYTICAL DATA}

\section{H ST Analvte}

cis-1,3-Dichloropropene trans-1,2-Dichloroethylene trans-1,3-Dichloropropene trans-1,4-Dichloro-2-butene
101998

$<5$

$<5$

$<5$

$<20$

$\begin{array}{ccc}201998 & 301998 & 401998 \\ <25 & <5 & <5 \\ & <5 & <5 \\ <25 & <5 & <5 \\ <25 & <5 & <20\end{array}$

Compliance

Lab Filter

EX $<$ EQL

EX $<$ EQL

EX $<$ EQL

EX $<$ EQL

Note: Flagging, dilution factors, modifiers, and laboratories are for fourth quartet 998 data only. See Appendix B for fagging criteria. * = exceeded holding time for fourth quarter 1998.

$+=$ exceeded screening level or final primary drinking water standard for fourth quartent 998 . 
WELL: LFW 62C

\begin{tabular}{|c|c|c|c|c|c|c|}
\hline SRS Coord. & Lat/Longitude & Screen Zone Elevation & Top of Casing & Casing & Pump & Formation \\
\hline $\begin{array}{l}\text { N } 83012.7 \\
\text { E } 45906.7\end{array}$ & $\begin{array}{ll}\text { 33.284 } & \text { Deg N } \\
\text { 81.707 } & \text { Deg W }\end{array}$ & $118.4-108.4 \mathrm{ft} \mathrm{msl}$ & $165.5 \mathrm{ft} \mathrm{msl}$ & 4"PVC & $\mathbf{S}$ & M Steed Pond \\
\hline
\end{tabular}

SAMPLE DATE

FIELD DATA

Analyte

Water Elevation

Depth to water

pH

Sp. Conductance

Water temperature

Alkalinity as $\mathrm{CaCO} 3$

Turbidity

Volumes purged

Sampling code

\section{ANALYTICAL DATA}

\section{H $\underline{\text { ST Analyte }}$}

1,1,1,2-Tetrachloroethane

1,1,1-Trichloroethane

1,1,2,2-Tetrachloroethane

1,1,2-Trichloroethane

1,1-Dichloroethane

1,1-Dichloroethylene

1,2,3-Trichloropropane

1,2-Dibromo-3-chloropropane

1,2-Dibromoethane

1,2-Dichloroethane

1,2-Dichloropropane

1,4-Dichlorobenzene

2-Hexanone

Acetone

Acetonitrile (Methyl cyanide)

Acrolein

10.1998

23.2

4.4

80

19

0

2.6

$\mathbf{5 . 2 6 1 1 7}$

10

10 $\begin{array}{lll}201998 & 301998 & 401998\end{array}$

Unit

ft msl

fit

pH

uS/cm

deg. C

mg/L

NTU

well volumes

\section{Acrylonitrile}

Allyl chloride

Arsenic, total recoverable

Barium, total recoverable

Benzene

Bromodichloromethane

Bromoform

Bromomethane (Methyl bromide)

$$
\begin{aligned}
& <5 \\
& <5 \\
& <5 \\
& <5 \\
& 45.6 \\
& 1.62 \\
& <5 \\
& <5 \\
& <5 \\
& 5.22 \\
& <5 \\
& 35.9 \\
& <10 \\
& <10 \\
& <20 \\
& <20 \\
& <5 \\
& <10 \\
& 4.1 \\
& 10.4 \\
& 3.14 \\
& <5 \\
& <5 \\
& <10 \\
& <4.7 \\
& <5
\end{aligned}
$$

Carbon disulfide

Note: Flagging, ditution factors, modifiers, and laboratories are for fourth quarte1998 data only. See Appendix B for flagging criteria. * = exceeded holding time for fourth quarter 1998 .

$+=$ exceeded screening level or final primary drinking water standard for fourth quarten 998 . 
WELL: LFW 62C

\section{ANALYTICAL DATA}

\section{H ST Analyte}

Carbon tetrachloride

Chlorobenzene

Chloroethane

Chloroethene (Vinyl chloride)

Chloroform

Chloromethane (Methyl chloride)

Chloroprene

Chromium, total recoverable

Dibromochloromethane

Dibromomethane (Methylene bromide)

Dichlorodifluoromethane

Dichloromethane (Methylene chloride)

Ethylbenzene

Gross alpha

Iodomethane (Methyl iodide)

Isobutyl alcohol

Lead, total recoverable

Mercury, total recoverable

Methacrylonitrile

Methyl ethyl ketone

Methyl isobutyl ketone

Propionitrile

Selenium, total recoverable

Silver, total recoverable

Styrene

Tetrachloroethylene

Toluene

Trichloroethylene

Trichlorofluoromethane

Tritium

Vinyl acetate

Xylenes

cis-1,3-Dichloropropene

trans-1,2-Dichloroethylene

trans-1,3-Dichloropropene

trans-1,4-Dichloro-2-butene
101998

201998

301998

$<5$

1.29

$<10$

$<10$

$<5$

$<10$

$<5$

$<1.7$

$<5$

$<5$

11.9

$<22.3$

$<5$

6.13

$<5$

$<100$

$<47$

3.21

$<10$

$<10$

$<10$

$<50$

$<66$

$<5$

$<5$

2

$<5$

13.2

2.9

17.2

$<10$

4.04

$\leq 5$

$<5$

$<5$

$<20$

\section{Compliance}

Lab Eilter 
WSRC-TR-99-00011

Unciassified

WELL: LFW 62D

\begin{tabular}{|c|c|c|c|c|c|c|}
\hline SRS Coord. & Lat/Longitude & Screen Zone Elevation & Top of Casing & Casing & Pump & Eormation \\
\hline $\begin{array}{l}\text { N } 82991.6 \\
\text { E } 45922.9\end{array}$ & $\begin{array}{l}\text { 33.284 Deg N } \\
\text { 81.707 Deg W }\end{array}$ & 127.6 & 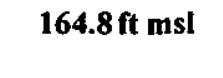 & C & & $\mathbf{U}$ \\
\hline
\end{tabular}

SAMPLE DATE

$\begin{array}{llll}12 / 03 / 97 & 03 / 16 / 98 & 06 / 12 / 98 & 09 / 15 / 98\end{array}$

FIELD DATA

Analyte

Water Elevation

Depth to water

pH

Sp. Conductance

Water temperature

Alkalinity as $\mathrm{CaCO3}$

Turbidity

Volumes purged

Sampling code

$\begin{array}{lllll}101998 & 201998 & 301998 & 401998 & \text { Unit } \\ 141.05 & 143.4 & 141.5 & 141.79 & \text { ft msl } \\ 21.55 & 19.2 & 21.1 & 20.81 & \text { ft } \\ 4.4 & 5.3 & 4.3 & 5.2 & \text { pH } \\ 420 & 110 & 130 & 88 & \text { uS/cm } \\ 15 & 19.3 & 26.5 & 24 & \text { deg. C } \\ 0 & 0 & 1 & & \text { mg/L } \\ 18.9 & 2 & 13.2 & 14.5 & \text { NTU } \\ .097405 & 2.54065 & 0 & .186015 & \text { well volumes } \\ \text { NX } & & \text { NSX } & \text { NX } & \end{array}$

ANALYTICAL DATA

H ST Analyte

1,1,1,2-Tetrachloroethane

1,1,1-Trichloroethane

1,1,2,2-Tetrachloroethane

1,1,2-Trichloroethane

+ 1,1-Dichloroethane

1,1-Dichloroethylene

1,2,3-Trichloropropane

1,2-Dibromo-3-chloropropane

1,2-Dibromoethane

1,2-Dichlorobenzene

1,2-Dichloroethane

1,2-Dichloropropane

1,3-Dichlorobenzene

1,4-Dichlorobenzene

1,4-Dioxane

2-Hexanone

Acetone

Acetonitrile (Methyl cyanide)

Acrolein

Acrylonitrile

Allyl chloride

+ Aluminum, total recoverable

Arsenic, total recoverable

Barium, total recoverable

+ Benzene

Bromodichloromethane

\begin{tabular}{|c|c|c|c|c|c|c|c|c|}
\hline & & & & & & & & apli \\
\hline 101998 & 201998 & 301998 & 401998 & DE & Me & Unit & Lab & Filter \\
\hline$<5$ & & $<5$ & & & & & & \\
\hline 2.99 & & 2.1 & 2.13 & 1 & $\mathbf{J}$ & ug $/ \mathbf{L}$ & EX & NDD \\
\hline$<5$ & & $<5$ & $<5$ & 1 & $\mathbf{U}$ & ug/L & EX & $<$ EQL \\
\hline$<5$ & & $<5$ & $<5$ & 1 & $\mathbf{U}$ & ug/L & EX & $<$ EQL \\
\hline 119 & & 82 & 79.1 & 1 & & $\mathbf{u g} / \mathbf{L}$ & EX & 79.1 \\
\hline 3.15 & & 2.3 & 2.86 & 1 & $\mathbf{J}$ & $\mathrm{ug} / \mathrm{L}$ & EX & NDD \\
\hline$<5$ & & $<5$ & & & & & & \\
\hline$<5$ & & $<5$ & $<5$ & 1 & $\mathbf{U}$ & $\mathrm{ug} / \mathrm{L}$ & EX & $<$ EQL \\
\hline$<5$ & & $<5$ & $<5$ & 1 & $\mathbf{U}$ & ug/L & EX & $<\mathbf{E Q L}$ \\
\hline & & & 1.74 & 1 & $\mathbf{J}$ & ug/L & $\mathbf{E X}$ & NDD \\
\hline 5.38 & & 3.8 & 3.74 & 1 & $\mathbf{J}$ & $\mathbf{u g} / \mathbf{L}$ & EX & NDD \\
\hline 2.1 & & $<5$ & $<5$ & 1 & $\mathbf{U}$ & $\mathrm{ug} / \mathrm{L}$ & EX & $<$ EQL \\
\hline & & & $<5$ & 1 & $\mathbf{U}$ & ug/L & EX & $<\mathbf{E Q L}$ \\
\hline 86 & & 66 & 59 & 1 & & $u g / L$ & EX & 59 \\
\hline & & & $<1000$ & 1 & $\mathbf{U}$ & ug/L & EX & $<$ EQL \\
\hline$<10$ & & $<10$ & $<5$ & 1 & $\mathbf{U}$ & $\mathrm{ug} / \mathrm{L}$ & $\mathbf{E X}$ & $<$ EQL \\
\hline$<10$ & & $<6$ & $<20$ & 1 & $\mathbf{U}$ & ug/L & $\mathbf{E X}$ & $<\mathbf{E Q L}$ \\
\hline$<\mathbf{2 0}$ & & $<20$ & $<500$ & 1 & $\mathbf{U}$ & ug/L & EX & $<\mathbf{E Q L}$ \\
\hline$<20$ & & $<10$ & $<50$ & 1 & $\mathbf{U}$ & ug/L & EX & $<$ EQL \\
\hline$<5$ & & $<5$ & $<50$ & 1 & $\mathbf{U}$ & $\mathrm{ug} / \mathrm{L}$ & $\mathbf{E X}$ & $<$ EQL \\
\hline$<10$ & & $<10$ & $<10$ & 1 & $\mathbf{u}$ & ug/L & EX & $<$ EQL \\
\hline & & 369 & 861 & 1 & & $u g / L$ & EX & 861 \\
\hline$<40$ & $<8$ & $<8$ & $<100$ & 1 & $\mathbf{U}$ & $u g / L$ & EX & $<\mathbf{E Q L}$ \\
\hline 7.8 & 4.3 & 6.5 & 7.22 & 1 & $\mathbf{J}$ & $u g / L$ & EX & NDD \\
\hline 25.6 & & 12 & 11 & 1 & & ug $/ \mathrm{L}$ & EX & 11 \\
\hline$<5$ & & $<5$ & $<5$ & 1 & $\mathbf{U}$ & ug/L & EX & $<\mathrm{EQL}$ \\
\hline
\end{tabular}

Note: Flagging, dilution factors, modifiers, and laboratories are for fourth quartet 998 data only. See Appendix B for flagging eriteria.

* - exceeded holding time for fourth quarter 1998.

$+=$ exceeded screening level or fnal primary drinking water standard tor fourth quarten 998 .

Sanitary Landfill

D-91

Fourth Quarter 1998 and Annual Summary 
WELL: LFW 62D

\section{ANALYTICAL DATA}

H ST Analyte

Bromoform
Bromomethane (Methyl bromide)
Cadmium, total recoverable
Carbon disulfide
Carbon tetrachloride
Chlorobenzene
Chloroethane
Chloroethene (Vinyl chloride)
Chloroform
Chloromethane (Methyl chloride)
Chloroprene
Chromium, total recoverable
Dibromochloromethane
Dibromomethane (Methylene bromide)
Dichlorodifluoromethane
Dichloromethane (Methylene chloride)
Ethyl methacrylate
Ethylbenzene
Gross alpha
Iodomethane (Methyl iodide)
Iron, total recoverable
Isobutyl alcohol
Lead, total recoverable
Mercury, total recoverable
Methacrylonitrile
Methyl ethyl ketone
Methyl isobutyl ketone
Methyl methacrylate
Pentachloroethane
Propionitrile
(a)

Selenium, total recoverable

Sijver, total recoverable

+- Styrene $=$
+ Tetrachloroethylene

Toluene

+ Trichloroethylene

+ Trichlorofluoromethane

Tritium

Vinyl acetate

Xylenes

+ cis-1,2-Dichloroethylene

cis-1,3-Dichioropropene

\section{8}

$<5$

$<10$

$<4.7$

5.43

$<5$

23.9

3.79

15.6

$<5$

$<10$

$<5$

5.2

$<5$

$<5$

19.9

$<26.2$

$<5$

6.5

$<5$

$<100$

8.6

.526

$<10$

$<10$

$<10$

$<50$

$<66<5<5$

$<5<2<2$

$=<5$

$$
3.43
$$

$<5$

18

34.5

17.25

$<10$

28.4

$<5$
201998

301998

$<5$

$<10$

$<2$

4.6

$<5$

21

$<10$

7.8

$<5$

$<10$

$<5$

2.1

$<5$

$<5$

12

$<13$

$<5$

1.72

3.91

$<5$

660

$<100$

4.1

.16

$<5$

$<10$

$<12$

$<5$

$<5$

$<5$

$<2$

$<5=-$

3.5

$<5$

12

26

8.37

$<5$

8.1

$<5$

401998

$$
<5
$$

$<5$

$$
<5
$$$$
<10
$$

8.92

$<5$

21.8

$<10$

$<5$

$<5$

$<5$

1

$<50 \quad 1 \mathrm{U}$

$<6.96$

$<5 \quad 1 \quad U$

$<5$

9.27

10.31

$$
<5 \quad 1 \quad \text { U }
$$$$
<5 \quad 1 \quad
$$$$
7.671
$$$$
<5
$$$$
<5
$$

$\begin{array}{lll}<100 & 1 & U \\ .352 & 1 & J \\ <500 & 1 & U \\ <10 & 1 & U \\ <5 & 1 & U \\ <50 & 1 & U \\ <200 & 1 & U\end{array}$

$$
\begin{array}{ll}
\text { ug/ } \\
\text { ug/L } \\
\text { ug/L } \\
\text { ug/L } \\
\text { ug/L } \\
\text { ug/L }
\end{array}
$$

$<200 \quad 1 \quad \mathrm{U} \quad \mathrm{ug} / \mathrm{L} \quad \mathrm{EX} \quad<$ EQL

$<20 \quad 1 \quad$ U $\quad$ ug/L EX $<$ EQL

$<5-\ldots 1-\mathbf{U}_{-} \mathbf{u g} / \mathbf{L}=-\mathbf{E X}=<\mathbf{E Q L}$

$\begin{array}{lllll}7.05 & 1 & \mathrm{ug} / \mathrm{L} & \mathrm{EX} & \mathbf{7 . 0 5}\end{array}$

$\begin{array}{llllll}<5 & 1 & \mathrm{U} & \mathrm{ug} / \mathrm{L} & \mathrm{EX} & <\mathrm{EQL}\end{array}$

$\begin{array}{llllll}19.3 & 1 & & \text { ug/L } & \text { EX } & 19.3\end{array}$

$\begin{array}{lllll}27.9 & 1 & \mathrm{ug} / \mathrm{L} & \mathrm{EX} & 27.9\end{array}$

$\begin{array}{lllll}10.69 & 1 & \mathrm{pCi} / \mathrm{ml} & \mathrm{TM} & 10.69\end{array}$

$<20 \quad 1 \quad$ U $\quad$ ug/L $\quad$ EX $<$ EQL

$\begin{array}{llllll}9.31 & 1 & \mathrm{~J} & \mathrm{ug} / \mathrm{L} & \mathrm{EX} & \mathrm{NDD}\end{array}$

$\begin{array}{lllll}75.2 & 1 & \text { ug/L } & \text { EX } & \mathbf{7 5 . 2}\end{array}$

$\begin{array}{llllll}<5 & 1 & \text { U } & \text { ug/L } & \text { EX } & <\text { EQL }\end{array}$

Note: Flagging, dilution factors, modifiers, and laboratories are for fourth quarte1998 data only. See Appendix B for fagging criteria.

* = exceeded holding time for fourth quarter 1998.

$+=$ exceeded screening level or nnal primary drinking water standard for fourth quartert 998 . 
WELL: LFW 62D

\section{ANALYTICAL DATA}

H ST Analyte

$$
\begin{aligned}
& \text { trans-1,2-Dichloroethylene } \\
& \text { trans-1,3-Dichloropropene } \\
& \text { trans-1,4-Dichloro-2-butene }
\end{aligned}
$$

\section{8}

$<5$

$<5$

$<20$
Compliance

Lab Filter

EX $<$ EQL

EX $<$ EQL

EX $<$ EQL

Note: Flagging, dilution factors, modifiers, and laboratories are for fourth quartet 998 data only. See Appendix B for fagging criteria.

* = exceded holding time for fourth quarter 1998.

$+=$ exceeded screening level or final primary drtnking water standard for fourth quarteri998 . 
WELL: LFW 63B

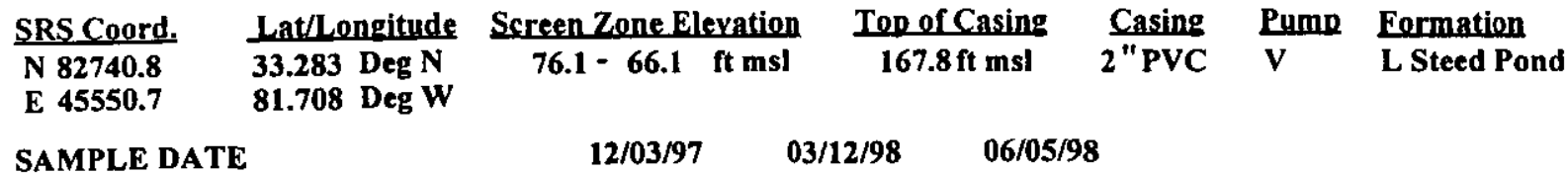

\section{FIELD DATA}

Analyte
Water Elevation
Depth to water
pH
Sp. Conductance
Water temperature
Alkalinity as CaCO3
Turbidity
Volumes purged
Sampling code

ANALYTICAL DATA

$\begin{array}{lll}101998 & 201998 & \frac{301998}{138.15} \\ 137.8 & -138.97 & 27.75 \\ 28.1 & 26.93 & 4 \\ 3.4 & 2.8 & 58 \\ 66 & 54 & 20 \\ 17 & 17 & 0 \\ 0 & 0 & .5 \\ 6 & .4 & 4.61749\end{array}$

$\begin{array}{ll}401998 & \text { Unit } \\ \text { ft ms! } \\ \text { ft } \\ \text { pH } \\ \text { uS/cm } \\ \text { deg. C } \\ \text { mg/L } \\ \text { NTU } \\ \text { well volumes }\end{array}$

Compliance

H ST Analyte

1,1,1,2-Tetrachloroethane

1,1,1-Trichloroethane

1,1,2,2-Tetrachloroethane

1,1,2-Trichloroethane

1,1-Dichloroethane

1,1-Dichloroethylene

1,2,3-Trichloropropane

1,2-Dibromo-3-chloropropane

1,2-Dibromoethane

1,2-Dichloroethane

1,2-Dichloropropane

1,4-Díchlorobenzene

2-Hexanone

Acetone

Acetonitrile (Methyl cyanide)

Acrolein

Acrylonitrile

Allyl chloride

Aluminum, total recoverable

Arsenic, total recoverable

Barium, total recoverable

Benzene

Bromodichloromethane

Bromoform

Bromomethane (Methyl bromide)

Cadmium, total recoverable

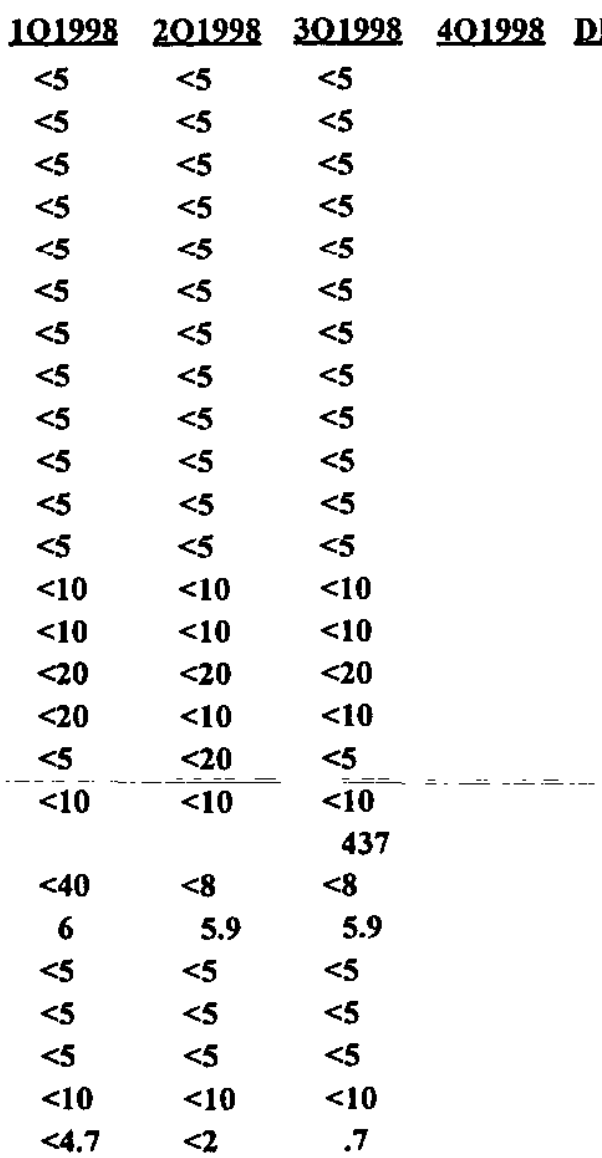

\section{FMod Unit Lab Eilter}

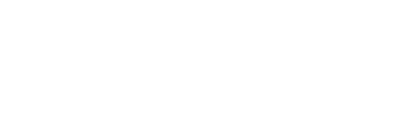

Note: Flagging, dilution factors, modifiers, and laboratories are for fourth quartet 998 data only. See Appendix B for fagging criteria.

* = exceeded holding time for fourth quarter 1998.

$+=$ exceed ed screening level or final primary drinking water standard for fourth quarteri 998 .

Sanitary Landfill 
WSRC-TR-99-00011

Unclassified

WELL: LFW 63B

\section{ANALYTICAL DATA}

H ST Analyte

Carbon disulfide

Carbon tetrachloride

Chlorobenzene

Chloroethane

Chloroethene (Vinyl chloride)

Chloroform

Chloromethane (Methyl chloride)

Chloroprene

Chromium, total recoverable

Dibromochloromethane .

Dibromomethane (Methylene bromide)

Dichlorodifluoromethane

Dichloromethane (Methylene chloride)

Ethylbenzene

Gross alpha

Iodomethane (Methyl iodide)

Iron, total recoverable

Isobutyl alcohol

Lead, total recoverable

Mercury, total recoverable

Methacrylonitrile

Methyl ethyl ketone

Methyl isobutyl ketone

Methyl methacrylate

Propionitrile

Selenium, total recoverable

Silver, total recoverable

Styrene

Tetrachloroethylene

Toluene

Trichloroethylene

Trichlorofluoromethane

Tritium

Vinyl acetate

Xylenes

cis-1,3-Dichloropropene

trans-1,2-Dichloroethylene

trans-1,3-Dichloropropene

trans-1,4-Dichloro-2-butene

\begin{tabular}{|c|c|c|}
\hline 101998 & 201998 & 301998 \\
\hline$<5$ & $<5$ & $<5$ \\
\hline$<5$ & $<5$ & $<5$ \\
\hline$<5$ & $<5$ & $<5$ \\
\hline$<10$ & $<10$ & $<10$ \\
\hline$<10$ & $<10$ & $<10$ \\
\hline$<5$ & $<5$ & $<5$ \\
\hline$<10$ & $<10$ & $<10$ \\
\hline$<5$ & $<5$ & $<5$ \\
\hline .87 & $<3$ & .6 \\
\hline$<5$ & $<5$ & $<5$ \\
\hline$<5$ & $<5$ & $<5$ \\
\hline$<10$ & $<5$ & $<5$ \\
\hline$<24.8$ & $<2.3$ & $<2.3$ \\
\hline$<5$ & $<5$ & $<5$ \\
\hline 9.51 & 3.02 & 5.89 \\
\hline$<5$ & $<5$ & $\begin{array}{l}<5 \\
10.3\end{array}$ \\
\hline$<100$ & $<100$ & $<100$ \\
\hline$<47$ & $<5$ & 7.7 \\
\hline$<.7$ & $<.2$ & $<.2$ \\
\hline$<10$ & $<5$ & $<5$ \\
\hline$<10$ & $<10$ & $<10$ \\
\hline \multirow[t]{2}{*}{$<10$} & $<12$ & $<12$ \\
\hline & $<5$ & $<5$ \\
\hline$<50$ & $<5$ & $<5$ \\
\hline$<66$ & $<5$ & $<5$ \\
\hline$<5$ & $<2$ & $<2$ \\
\hline$<5$ & $<5$ & $<5$ \\
\hline$<5$ & 1.2 & $<5$ \\
\hline$<5$ & $<5$ & $<5$ \\
\hline$<5$ & $<5$ & $<5$ \\
\hline$<5$ & $<5$ & $<5$ \\
\hline$<.31$ & $<249$ & $<-.22$ \\
\hline$<10$ & $<5$ & $<5$ \\
\hline$<5$ & $<5$ & $<5$ \\
\hline$<5$ & $<5$ & $<5$ \\
\hline \multicolumn{3}{|l|}{$<5$} \\
\hline$<5$ & $<5$ & $<5$ \\
\hline$<20$ & $<5$ & $<5$ \\
\hline
\end{tabular}

Note: Flagging, dilution factors, modifiers, and laboratories are for fourth quartei998 data only. See Appendix B for nagging criteria. * e exceeded holding time for fourth quarter 1998 .

$+=$ exceeded screening level or final primary drinking water standard for fourth quarter1998 .

Sanitary Landfill 
WELL: LFW 63C

\begin{tabular}{|c|c|c|c|c|c|c|}
\hline SRS Coord. & Lat/Longitude & Screen Zone Elevation & Top of Casing & Casing & Pump & Eormation \\
\hline $\begin{array}{l}\mathbf{N} \\
\mathbf{E}\end{array}$ & $\begin{array}{ll}33.283 & \text { Deg } N \\
\text { 81.708 } & \text { Deg } W\end{array}$ & $2-96.2$ ft msl & 1 & C & $\mathbf{V}$ & nd \\
\hline
\end{tabular}

$\begin{array}{llll}\text { SAMPLE DATE } & 12 / 03 / 97 & 03 / 11 / 98 & 06 / 05 / 98\end{array}$

\section{FIELD DATA}

\section{Analyte \\ Water Elevation \\ Depth to water \\ pH \\ Sp. Conductance \\ Water temperature \\ Alkalinity as $\mathrm{CaCO} 3$ \\ Turbidity \\ Volumes purged \\ Sampling code}

$\begin{array}{lllll}101998 & 201998 & 301998 & \text { 401998 } & \text { Unit } \\ 137.64-\ldots- & 138.9 & 138.05 & & \text { ft msl } \\ 28.36 & 27.1 & 27.95 & & \mathrm{ft} \\ 4.2 & 3.6 & 4.4 & \mathrm{pH} \\ 32 & 32 & 34 & \text { uS/cm } \\ 17 & 19 & 19.9 & \text { deg. C } \\ 0 & 0 & 0 & \mathrm{mg} / \mathrm{L} \\ .2 & .3 & .5 & \text { NTU } \\ 3.08099 & 6.53310 & 10.6829 & \text { well volumes }\end{array}$

\section{ANALYTICAL DATA}

\section{H ST Analyte}

1,1,1,2-Tetrachloroethane

1,1,1-Trichloroethane

1,1,2,2-Tetrachloroethane

1,1,2-Trichloroethane

1,1-Dichloroethane

1,1-Dichloroethylene

1,2,3-Trichloropropane

1,2-Dibromo-3-chloropropane

1,2-Dibromoethane

1,2-Dichloroethane

1,2-Dichloropropane

1,4-Dichlorobenzene

2-Hexanone

Acetone

Acetonitrile (Methyl cyanide)

Acrolein

Acrylonitrile

Allyl chloride

Aluminum, total recoverable

Arsenic, total recoverable

Barium, total recoverable

Benzene

Bromodichloromethane

Bromoform

Bromomethane (Methyl bromide)

Cadmium, total recoverable

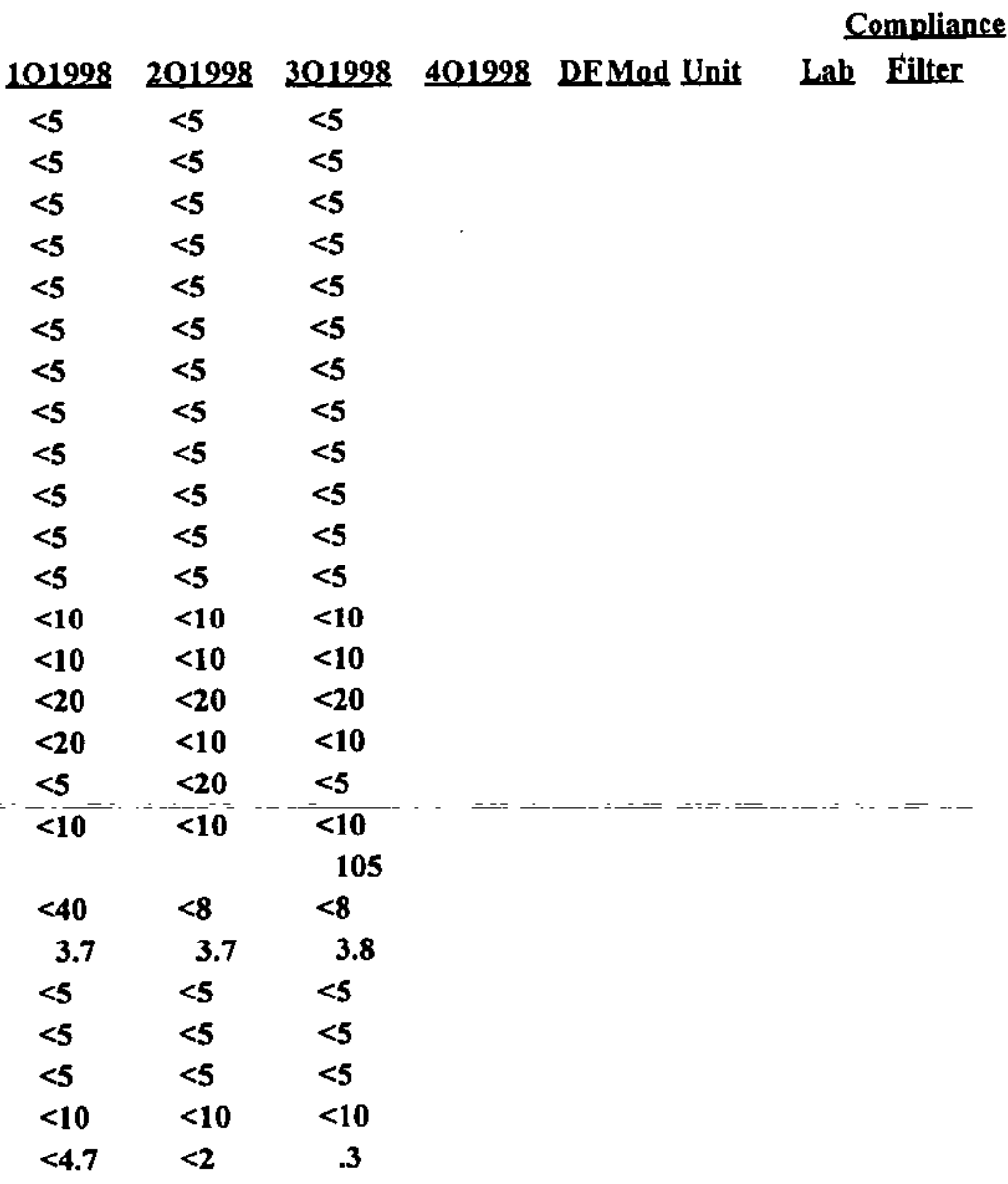

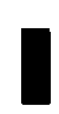

I
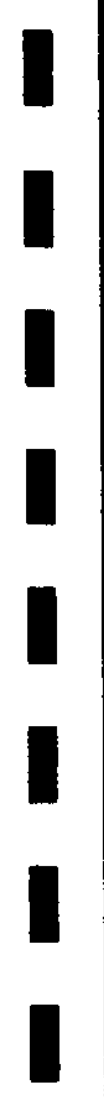



Note: Flagging, dilution factors, modifiers, and laboratories are for fourth quartet998 data only. See Appendix B for flagging criteria.

* exceeded holding time for fourth quarter 1998.

$+=$ exceeded screening level or final primary drinking water standard for fourth quarten 998.

Sanitary Landfill

D-96

Fourth Quarter 1998 and Annual Summary 
WELL: LFW 63C

\section{ANALYTICAL DATA}

H ST Analyte

Carbon disulfide

Carbon tetrachloride

Chlorobenzene

Chloroethane

Chloroethene (Vinyl chloride)

Chloroform

Chloromethane (Methyl chloride)

Chloroprene

Chromium, total recoverable

Dibromochloromethane

Dibromomethane (Methylene bromide)

Dichlorodifluoromethane

Dichloromethane (Methylene chloride)

Ethylbenzene

Gross alpha

Iodomethane (Methyl iodide)

Iron, total recoverable

Isobutyl alcohol

Lead, total recoverable

Mercury, total recoverable

Methacrylonitrile

Methyl ethyl ketone

Methyl isobutyl ketone

Methyl methacrylate

Propionitrile

Selenium, total recoverable

Silver, total recoverable

Styrene

Tetrachloroethylene

Toluene

Trichloroethylene

Trichlorofluoromethane

Tritium

Vinyl acetate

Xylenes

cis-1,3-Dichloropropene

trans-1,2-Dichloroethylene

trans-1,3-Dichloropropene

trans-1,4-Dichloro-2-butene
101998

$<5$

$<5$

$<5$

$<10$

$\leq 10$

$<5$

$<10$

$<5$

$<7$

$<5$

$<5$

$<10$

$<6.82$

$<5$

6.38

$<5$

$<100$

$<47$

$<.7$

$<10$

$<10$

$<10$

$<50$

$<66$

$<5$

$<5$

$<5$

$<5$

$<5$

$<5$

1.19

$<10$

$<5$

$<5$

$<5$

$<5$

$<20$
201998

$<5$

$<5$

$<5$

$<10$

$<1$

$<5$

$<10$

$<5$

$<3$

$<5$

$<5$

$<5$

$<2$

$<5$

1.12

$<5$

301998

401998

DEMod Unit

Compliance

$<5$

$<5$

$<5$

$<10$

$<10$

$<5$

$<10$

$<5$

$<3$

$<5$

$<5$

$<5$

$<2.4$

$<5$

4.27

$<5$

5.7

$<100<100$

$<5<5$

$<.2<.2$

$<5<5$

$<10<10$

$<12<12$

$<5<5$

$<5 \quad<5$

$<5 \quad<5$

$<2<2$

$<5 \quad<5$

$<5<5$

$<5 \quad<5$

$<5<5$

$<5<5$

$907<.04$

$<5<5$

$<5<5$

$<5<5$

$<5<5$

$<5<5$

Note: Flagging, dilution factors, modifiers, and laboratories are for tourth quartei 998 data only. See Appendix B tor fagging criteria. * $=$ exceeded holding time for fourth quarter 1998.

$+=$ exceded screening level or final primary drinking water standard for fourth quarten 998 . 
WELL: LFW 63D

\begin{tabular}{|c|c|c|c|c|c|c|}
\hline$\frac{\text { SRS Coord, }}{\text { N } 82751.8}$ & $\frac{\text { Lat/Longitude }}{33.283 \text { Deg } N}$ & $\frac{\text { Screen Zone Elevation }}{146.4-126.4 \mathrm{ft} \mathrm{msl}}$ & $\frac{\text { Top of Casing }}{168.3 \mathrm{ft} \mathrm{msl}}$ & $\frac{\text { Casing }}{2 " \text { PVC }}$ & Pump & $\begin{array}{l}\text { Eormation } \\
\text { U Steed Pond }\end{array}$ \\
\hline $\begin{array}{l}\text { N } 82751.8 \\
\text { E } 45569.1\end{array}$ & 81.708 Deg W & & & & & \\
\hline
\end{tabular}

\begin{tabular}{|c|c|c|}
\hline MPLE & $12 / 02 / 97$ & $03 / 16 / 98$ \\
\hline
\end{tabular}

\section{FIELD DATA}

Analyte
Water Elevation
Depth to water
pH
Sp. Conductance
Water temperature
Alkalinity as CaCO3
Turbidity
Volumes purged
Sampling code

ANALYTICAL DATA

H ST Analyte

1,1,1,2-Tetrachloroethane

1,1,1-Trichloroethane

1,1,2,2-Tetrachloroethane

1,1,2-Trichloroethane

1,1-Dichloroethane

1,1-Dichloroethylene

1,2,3-Trichloropropane

1,2-Dibromo-3-chloropropane

1,2-Dibromoethane

1,2-Dichlorobenzene

1,2-Dichloroethane

1,2-Dichloropropane

1,3-Dichlorobenzene

1,4-Dichlorobenzene

1,4-Dioxane

2-Hexanone

Acetone

Acetonitrile (Methyl cyanide)

Acrolein

Acrylonitrile

Allyl chloride

Aluminum, total recoverable

Arsenic, total recoverable

Barium, total recoverable

Benzene

Bromodichloromethane

\begin{tabular}{|c|c|c|c|c|}
\hline 101998 & 201998 & 301998 & 401998 & Unit \\
\hline $138 \ldots$ & 139.3 & 138.35 & 138.5 & ft msl \\
\hline 28.2 & 26.9 & 27.85 & 27.7 & fit \\
\hline 5.4 & 5.2 & 4.6 & 5 & pH \\
\hline 44 & 46 & 32 & 19 & $\mathbf{u S} / \mathbf{c m}$ \\
\hline 20 & 19 & 19.5 & 19.7 & deg. $C$ \\
\hline 9 & 10 & 0 & & $\mathrm{mg} / \mathrm{L}$ \\
\hline 1 & .7 & .3 & .5 & NTU \\
\hline $\begin{array}{l}21.3637 \\
\text { tV }\end{array}$ & 30.4878 & 15.1897 & 6.01168 & well volumes \\
\hline
\end{tabular}

Comoliance 101998201998301998 401998 DFMod Unit Lab Filter

\begin{tabular}{|c|c|c|c|c|c|c|c|c|}
\hline$<5$ & $<5$ & $<5$ & & & & & & \\
\hline$<5$ & 4.9 & 3.2 & $<5$ & 1 & $\mathbf{U}$ & ug/L & EX & $<\mathrm{EQL}$ \\
\hline$<5$ & $<5$ & $<5$ & $<5$ & 1 & $\mathbf{U}$ & $\mathrm{ug} / \mathrm{L}$ & $\mathbf{E X}$ & $<$ EQL \\
\hline$<5$ & $<5$ & $<5$ & $<5$ & 1 & $\mathbf{U}$ & ug/L & EX & $<$ EQL \\
\hline 4.34 & 10 & 6.1 & 1.78 & 1 & $\mathbf{J}$ & ug/L & EX & NDD \\
\hline$<5$ & $<5$ & $<5$ & $<5$ & 1 & $\mathbf{U}$ & ug/L & EX & $<$ EQL \\
\hline$<5$ & $<5$ & $<5$ & & & & & & \\
\hline$<5$ & $<5$ & $<5$ & $<5$ & 1 & $\mathbf{U}$ & ug/L & EX & $<\mathbf{E Q L}$ \\
\hline \multirow[t]{2}{*}{$<5$} & $<5$ & $<5$ & $<5$ & 1 & $\mathbf{U}$ & $\mathrm{ug} / \mathrm{L}$ & EX & $<\mathrm{EQL}$ \\
\hline & & & $<5$ & 1 & $\mathbf{U}$ & $\mathrm{ug} / \mathrm{L}$ & EX & $<$ EQL \\
\hline$<5$ & $<5$ & $<5$ & $<5$ & 1 & $\mathbf{U}$ & ug/L & EX & < EQL \\
\hline \multirow[t]{2}{*}{$<5$} & $<5$ & $<5$ & $<5$ & 1 & $\mathbf{U}$ & ug/L & EX & $<$ EQL \\
\hline & & & $<5$ & 1 & $\mathbf{U}$ & $\mathbf{u g} / \mathrm{L}$ & $\mathbf{E X}$ & $<\mathrm{EQL}$ \\
\hline \multirow[t]{2}{*}{10.1} & 12 & 7.5 & $<5$ & 1 & $\mathbf{U}$ & $\mathbf{u g} / \mathbf{L}$ & EX & $<$ EQL \\
\hline & & & $<1000$ & 1 & $\mathbf{U}$ & ug/L & EX & $<\mathbf{E Q L}$ \\
\hline$<10$ & $<10$ & $<10$ & $<5$ & 1 & $\mathbf{U}$ & $\mathbf{u g} / \mathbf{L}$ & EX & $<\mathrm{EQL}$ \\
\hline$\leq 10$ & $\leq 10$ & $<10$ & $\leq 20$ & 1 & $\underline{\mathbf{U}}$ & $\underline{\mathbf{u g}} / \underline{\mathbf{L}}$ & $\underline{\underline{E X}}$ & $<$ EQL \\
\hline$<20$ & $<20$ & $<20$ & $<500$ & 1 & $\bar{U}$ & ug/L & $\overline{\mathbf{E X}}$ & $<\mathbf{E Q L}$ \\
\hline$<20$ & $<10$ & $<10$ & $<50$ & 1 & $\mathbf{U}$ & ug/L & EX & $<\mathrm{EQL}$ \\
\hline$<5$ & $<20$ & $<5$ & $<50$ & 1 & $\mathbf{U}$ & ug/L & EX & $<\mathrm{EQL}$ \\
\hline \multirow[t]{2}{*}{$<10$} & $<10$ & $<10$ & $<10$ & 1 & $\mathbf{U}$ & $\mathbf{u g} / \mathbf{L}$ & EX & $<$ EQL \\
\hline & & 23.1 & $<200$ & 1 & $\mathbf{U}$ & $\mathbf{u g} / \mathbf{L}$ & EX & $<$ EQI \\
\hline$<40$ & $<8$ & $<8$ & $<100$ & 1 & $\mathbf{U}$ & ug/L & EX & $<$ EQI \\
\hline 3.3 & 3.4 & 3.2 & 3.66 & 1 & $\mathbf{J}$ & $\mathbf{u g} / \mathbf{L}$ & $\mathbf{E X}$ & NDD \\
\hline$<5$ & .76 & .56 & $<5$ & 1 & $\mathbf{U}$ & $u g / L$ & EX & $<\mathbf{E Q}$ \\
\hline$<5$ & $<5$ & $<5$ & $<5$ & 1 & $\mathbf{U}$ & ug/L & EX & $<\mathbf{E Q}$ \\
\hline
\end{tabular}

Note: Flagging, dilution factors, modifiers, and laboratories are for fourth quarte1998 data only. See Appendix B for fagging criteria.

* = exceeded holding time for fourth quarter 1998.

$+=$ exceeded screening level or final primary drinking water standard for fourth quarter1998 . 
WSRC-TR-99-00011

Unclassified

WELL: LFW 63D

\section{ANALYTICAL DATA}

\section{H ST Analyte}

Bromoform

Bromomethane (Methyl bromide)

Cadmium, total recoverable

Carbon disulfide

Carbon tetrachloride

Chlorobenzene

Chlorocthane

Chloroethene (Vinyl chloride)

Chloroform

Chloromethane (Methyl chloride)

Chloroprene

Chromium, total recoverable

Dibromochloromethane

Dibromomethane (Methylene bromide)

+ Dichlorodifluoromethane

Dichloromethane (Methylene chloride)

Ethyl methacrylate

Ethylbenzene

Gross alpha

Iodomethane (Methyl iodide)

Iron, total recoverable

Isobutyl alcohol

Lead, total recoverable

Mercury, total recoverable

Methacrylonitrile

Methyl ethyl ketone

Methyl isobutyl ketone

Methyl methacrylate

Pentachloroethane

Propionitrile

Selenium, total recoverable

Silver, total recoverable

Styrene

Tetrachloroethylene

Toluene

Trichloroethylene

+ Trichlorofluoromethane

Tritium

Vinyl acetate

Xylenes

cis-1,2-Dichloroethylene

cis-1,3-Dichloropropene
101998

$<4.7$

$<5$

$<5$

4.22

$<10<10$

9.89

$<5$

1.48

$<5<5$

$<7 \quad<$

$<5<5$

$<5<5$

$21.4 \quad 28$

$<7.74$

$<10$

$<5$

1.83

$<5$

$<10$

$<47$

$<.7$

$<10$

$<10$

$<10$

\section{$<5$}

$<-.31$

$<5$

1.04

$<5$

12.1

$<100$

$<100$

3.9

.05

$<5$

$<10$

$<12$

$<5$

8.4

.05

$<5$

$<10$

$<12$

$<5$

$<50$

$<6$

$<5$

$<5$

$<5$

$<5$

$<5$

24.9

1.99

$<10$

$<5$

$$
<5
$$

$<5$
$<5$

$<5$$$
<2
$$

$<5$

1

1
$<5$

2.3

27

1710

$<5$

$<5$

$<5$
Compliance

$\begin{array}{llllll}01998 & \text { DEMod Unit } & \text { Lab } & \text { Eilter } \\ <5 & 1 & \text { U } & \text { ug/L } & \text { EX } & <\text { EQL }\end{array}$

$<5 \quad 1 \quad$ U $\quad$ ug/L $\quad$ EX $<$ EQL

$<10 \quad 1 \quad$ U $\quad$ ug/L $\quad$ EX $\quad$ EQL

$\begin{array}{lllllll}< & 1 & \text { U } & \text { ug/L } & \text { EX } & <\text { EQL }\end{array}$

$<5 \quad 1 \quad$ U $\quad$ ug/L $\quad$ EX $<$ EQL

$\begin{array}{lllllll}< & 1 & \mathrm{U} & \mathrm{ug} / \mathrm{L} & \mathrm{EX} & <\mathrm{EQL}\end{array}$

$<10 \quad 1 \quad \mathrm{U} \quad \mathrm{ug} / \mathrm{L} \quad \mathrm{EX} \quad<$ EQL

$<5 \quad 1 \quad$ U $\quad$ ug/L $\quad$ EX $<$ EQL

$<5 \quad 1 \quad \mathrm{U} \quad \mathrm{ug} / \mathrm{L} \quad \mathrm{EX} \quad<$ EQL

$\begin{array}{llllll}3.75 & 1 & \mathrm{~J} & \mathrm{ug} / \mathrm{L} & \mathrm{EX} & \mathrm{NDD}\end{array}$

$<50 \quad 1 \quad$ U $\quad$ ug/L $\quad$ EX $<$ EQL

$<10$ I U ug/L EX $<$ EQL

$\begin{array}{lllllll}< & 1 & \mathrm{U} & \mathrm{ug} / \mathrm{L} & \mathrm{EX} & <\mathrm{EQL}\end{array}$

$\begin{array}{lllllll}< & 1 & \text { U } & \text { ug/L } & \text { EX } & <\text { EQL }\end{array}$

$\begin{array}{lllll}18.1 & 1 & \mathrm{ug} / \mathrm{L} & \mathrm{EX} & 18.1\end{array}$

$<10 \quad 1 \quad$ U $\quad$ ug/L $/$ EX $<$ EQL

$<5 \quad$ I $\quad$ U $\quad \mathrm{ug} / \mathrm{L} \quad$ EX $<$ EOL

$<5 \quad 1 \quad$ U $\quad$ ug/L $\quad$ EX $<$ EQL

$\begin{array}{lllll}.99 & 1 & \mathrm{pCi} / \mathrm{L} & \mathrm{TM} & .99\end{array}$

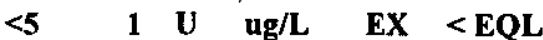

$<21.8 \quad 1 \quad$ U $\quad$ ug/L $\quad$ EX $\quad<$ EQL

$<100 \quad 1 \quad$ U $\quad$ ug/L $\quad$ EX $\quad<$ EQL

$<.5 \quad 1 \quad$ U $\quad$ ug/L $\quad$ EX $<$ EQL

$<500 \quad 1 \quad$ U $\quad$ ug/L $\quad$ EX $\quad<$ EQL

$<10 \quad 1 \quad$ U $\quad$ ug/L $\quad$ EX $<$ EQL

$\begin{array}{llllll}<5 & 1 & \text { U } & \mathrm{ug} / \mathrm{L} & \mathrm{EX} & <\mathrm{EQL}\end{array}$

$<50 \quad 1 \quad$ U $\quad$ ug/L $\quad$ EX $<$ EQL

$<200 \quad 1 \quad$ U $\quad$ ug/L $\quad$ EX $<$ EQL

$<200 \quad 1 \quad$ U $\quad$ ug/L $\quad$ EX $\quad<$ EQL

$<2011 \quad$ U $\quad$ ug/L $\quad$ EX $<$ EQL

$<5 \quad 1 \quad$ U $\quad$ ug/L $\quad$ EX $<$ EQL

$<5 \quad 1 \quad$ U $\quad$ ug/L $\quad$ EX $<$ EQL

$<5 \quad 1 \quad$ U $\quad$ ug/L $\quad$ EX $<$ EQL

$\begin{array}{llllll}<5 & 1 & \text { U } & \text { ug/L } & \text { EX } & <\text { EQL }\end{array}$

$\begin{array}{lllll}11.6 & 1 & \mathrm{ug} / \mathrm{L} & \mathrm{EX} & 9.84\end{array}$

$<.78 \quad 1$ UI $\mathrm{pCi} / \mathrm{ml}$ TM $<\mathrm{EQL}$

$<20 \quad 1 \quad$ U $\quad$ ug/L $\quad$ EX $<$ EQL

$<10 \quad 1 \quad$ U ug/L EX $<$ EQL

$\begin{array}{llllllllll}<5 & & 1 & \text { U } & \text { ug/L } & \text { EX } & <\text { EQL }\end{array}$

$<5 \quad 1 \quad$ U $\quad$ ug/L $\quad$ EX $<$ EQL

Note: Flagging, dilution factors, modifiers, and laboratories are for fourth quartei998 data only. See Appendix B for flagging criteria.

* = exceeded holding time for fourth quarter 1998.

$+=$ exceeded screening level or final primary drinking water standard for fourth quarten 998 . 
WSRC-TR-99-00011

Unclassified

WELL: LFW 63D

ANALYTICAL DATA

\section{H ST Analyte}

trans-1,2-Dichloroethylene trans-1,3-Dichloropropene trans-1,4-Dichloro-2-butene

\section{8}

$<5$

$<5$

$<20$
201998

301998

$<5$

$<5$
$<5$

$<5$
Compliance

401998

$<5$

$<5$

$<20$
DEMod Unit

$1 \mathrm{U}$

$1 \mathrm{U}$

$1 \mathrm{U}$
ug/L

ug/L

ug/L
Lab Filter

EX $<$ EQL

EX $<$ EQL

EX $<$ EQL

Note: Flagging, dilution factors, modifiers, and laboratories are for fourth quartel 998 data only. See Appendix B for flagging criteria. - se exceeded holding time for fourth quarter 1998.

+ = exceeded screening level or fnal prtmary drinking water standard for fourth quarten 998 .

Sanitary Landfill

D-100

Fourth Quarter 1998 and Annual Summary 
WELL: LFW 64C

\begin{tabular}{|c|c|c|c|c|c|c|}
\hline SRS Coord. & Lat/Longitude & Screen Zone Eleyation & Top of Casing & Casing & Pume & Eormation \\
\hline $\begin{array}{l}\text { N 82744.8 } \\
\text { E 45271.3 }\end{array}$ & $\begin{array}{l}\text { 33.283 Deg N } \\
\text { 81.708 Deg W }\end{array}$ & $93.0-83.0 \mathrm{ft} \mathrm{msl}$ & $152.2 \mathrm{ft} \mathrm{msl}$ & $\overline{2 " P V C}$ & $\mathrm{v}$ & eed Pond \\
\hline
\end{tabular}

SAMPLE DATE

$\begin{array}{lll}12 / 03 / 97 & 03 / 12 / 98 & 06 / 08 / 98\end{array}$

\section{FIELD DATA}

\section{Analyte}

Water Elevation

Depth to water

pH

Sp. Conductance

Water temperature

Alkalinity as $\mathrm{CaCO} 3$

Turbidity

Volumes purged

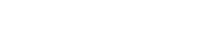

Sampling code

$\begin{array}{lllll}\frac{201998}{137.65} & \frac{201998}{138.41} & \frac{301998}{137.75} & 401998 & \text { Unit } \\ 12.35 & 11.59 & 12.25 & & \text { ft msl } \\ 3.4 & 3.2 & 4.1 & & \text { ft } \\ 82 & 64 & 77 & & \text { uS } / \mathrm{cm} \\ 19 & 16.3 & 18.2 & & \text { deg. C } \\ 0 & 0 & 0 & & \mathrm{mg} / \mathrm{L} \\ .2 & .4 & .2 & & \text { NTU } \\ 2.35965 & 3.81031 & 2.35551 & & \text { well volumes }\end{array}$

\section{ANALYTICAL DATA}

\section{H ST Analyte}

1,1,1,2-Tetrachloroethane

1,1,1-Trichloroethane

1,1,2-Trichloroethane

1,1-Dichloroethane

1,1-Dichloroethylene

1,2,3-Trichloropropane

1,2-Dibromo-3-chloropropane

1,2-Dibromoethane

1,2-Dichloroethane

1,2-Dichloropropane

1,4-Dichlorobenzene

2-Hexanone

Acetone

Acetonitrile (Methyl cyanide)

Acrolein

Acrylonitrile

Allyl chloride

Aluminum, total recoverable

Arsenic, total recoverable

Barium, total recoverable

Benzene

Bromodichloromethane

Bromoform

Bromomethane (Methyl bromide)

Cadmium, total recoverable
1,1,2,2-Tetrachloroethane

$\begin{array}{ccccc}101998 & 201998 & 301998 & \text { 401998 DFMod Unit Lab Filter } \\ <5 & <5 & <5 & & \\ <5 & <5 & <5 \\ <5 & <5 & <5 \\ <5 & <5 & <5 \\ <5 & <5 & <5 \\ <5 & <5 & <5 \\ <5 & <5 & <5 \\ <5 & <5 & <5 \\ <5 & <5 & <5 \\ <5 & <5 & <5 \\ <5 & <5 & <5 \\ <5 & <5 & <5 \\ <10 & <10 & <10 \\ <10 & <10 & <10 \\ <20 & <20 & <20 \\ <20 & <10 & <10 \\ <5 & <20 & <5 \\ <10 & <10 & <10 \\ <40 & <8 & <8 \\ 8.1 & 6.5 & 6.9 \\ <5 & <5 & <5 \\ <5 & <5 & <5 \\ <5 & <5 & <5 \\ <10 & <10 & <10 \\ .75 & .5 & .6 \\ & & 731 \\ <5\end{array}$

Note: Flagging, dilution factors, modifiers, and laboratories are for fourth quarte1 998 data only. See Appendix B for Magging criteria. * = exceeded holding time for fourth quarter 1998.

$+=$ exceeded screening level or final primary drinking water standard for fourth quarten 998 .

Sanitary Landfill

D-101

Fourth Quarter 1998 and Annual Summary 
WELL: LFW 64C

\section{ANALYTICAL DATA}

H ST Analyte

Carbon disulfide

Carbon tetrachloride

Chlorobenzene

Chloroethane

Chloroethene (Vinyl chloride)

Chloroform

Chloromethane (Methyl chloride)

Chloroprene

Chromium, total recoverable

Dibromochloromethane

Dibromomethane (Methyiene bromide)

Dichlorodifluoromethane

Dichloromethane (Methylene chloride)

Ethylbenzene

Gross alpha

Iodomethane (Methyl iodide)

Iron, total recoverable

Isobutyl alcohol

Lead, total recoverable

Mercury, total recoverable

Methacrylonitrile

Methyl ethyl ketone

Methyl isobutyl ketone

Methyl methacrylate

Propionitrile

Selenium, total recoverable

Silver, total recoverable

Styrene

Tetrachloroethylene

Toluene

Trichloroethylene

Trichlorofluoromethane

Tritium

Vinyl acetate

Xylenes

cis-1,3-Dichloropropene

trans-1,2-Dichloroethylene

trans-1,3-Dichloropropene

trans-1,4-Dichloro-2-butene
101998

$<5$

$<5$

$<10 \quad<10<10$

$-510<10<10$

$<5<5<5$

$<10<10 \quad<10$

$<5<5<5$

$<7 \quad .7<3$

$<5<5<5$

$<5<5<5$

$<10<5<5$

$<6.5<2.1<2.4$

$<5<5<5$

$\begin{array}{lll}13.51 & 5.74 \quad 10.43\end{array}$

$<5<5<5$

12

$<100<100<100$

$<47<5<5$

$<.7 \quad<.2 \quad<.2$

$<10<5<5$

$<10 \quad<10 \quad<10$

$<10<12<12$

$<5<5$

$<50<5<5$

$<66<5<5$

$<5<2 \quad<2$

$<5<5 \quad<5$

$<5<5 \quad<5$

$<5<5 \quad<5$

$<5<5<5$

$<5<5<5$

$<524 \quad<.02$.

$<5<5$

$<5 \quad<5$

$<5<5$

$<5<5$

$<5$

$<5<5<5$

$<20<5 \quad<5$
Compliance

Lab Eilter 
WELL: LFW 64D

\begin{tabular}{|c|c|c|c|c|c|c|}
\hline$\frac{\text { SRS Coord. }}{\text { N } 82737.8}$ & $\frac{\text { Lat/Longitude }}{33.283 \text { Deg N }}$ & $\frac{\text { Screen Z One Elevation }}{135.2-115.2 \mathrm{ft} \mathrm{msl}}$ & $\frac{\text { Top of Casing }}{152.2 \mathrm{ft} \mathrm{msl}}$ & $\frac{\text { Casing }}{2 \text { "PVC }}$ & $\frac{\text { Pump }}{V}$ & $\begin{array}{l}\text { Eormation } \\
\text { U Steed Pond }\end{array}$ \\
\hline
\end{tabular}

\section{FIELD DATA}

Analyte

Water Elevation

Depth to water

pH

Sp. Conductance

Water temperature

Alkalinity as $\mathrm{CaCO} 3$

Turbidity

Volumes purged

Sampling code

$\begin{array}{lllll}101998 & 201998 & 301998 & \frac{401998}{\text { Unit }} & \text { for } \\ 137.8 & -138.75 & 138.05 & 138.07 & \text { ft msl } \\ 12.4 & 11.45 & 12.15 & 12.13 & \text { ft } \\ 5.4 & 5.2 & 4.9 & 5.2 & \text { pH } \\ 62 & 42 & 48 & 55 & \text { uS/cm } \\ 20 & 17 & 19 & 19.2 & \text { deg. C } \\ 6 & 6 & 5 & 6 & \text { mg/L } \\ .7 & .3 & .3 & .7 & \text { NTU } \\ 11.8977 & 9.54608 & 17.6670 & 2.94213 & \text { well volumes } \\ \text { tV } & & & & \end{array}$

\section{ANALYTICAL DATA}

H ST Analvte

1,1,1,2-Tetrachloroethane

1,1,1-Trichloroethane

1,1,2,2-Tetrachloroethane

1,1,2-Trichloroethane

1,1-Dichloroethane

1,1-Dichloroethylene

1,2,3-Trichloropropane

1,2-Dibromo-3-chloropropane

1,2-Dibromoethane

1,2-Dichlorobenzene

1,2-Dichloroethane

1,2-Dichloropropane

1,3-Dichlorobenzene

1,4-Dichlorobenzene

1,4-Dioxane

2-Hexanone

Acetone

Acetonitrile (Methyl cyanide)

Acrolein

Acrylonitrile

Allyl chloride

Aluminum, total recoverable

Arsenic, total recoverable

Barium, total recoverable

Benzene

Bromodichloromethane

\begin{tabular}{|c|c|c|c|c|c|c|c|c|}
\hline \multirow{2}{*}{$\frac{101998}{<5}$} & \multirow{2}{*}{$\frac{201998}{<5}$} & \multirow{2}{*}{$\frac{301998}{<5}$} & \multirow[t]{2}{*}{401998} & \multicolumn{3}{|c|}{ DFMod Unit } & Lab & Filter \\
\hline & & & & & & & & \\
\hline$<5$ & $<5$ & $<5$ & $<5$ & 1 & $\mathbf{U}$ & ug/L & $\mathbf{E X}$ & $<$ EQL \\
\hline$<5$ & $<5$ & $<5$ & $<5$ & 1 & $\mathbf{U}$ & $\mathbf{u g} / \mathrm{L}$ & $\mathbf{E X}$ & $<$ EQL \\
\hline$<5$ & $<5$ & $<5$ & $<5$ & 1 & $\mathbf{U}$ & ug/L & EX & $<\mathbf{E Q L}$ \\
\hline 5.49 & 2.2 & 3.8 & 3.33 & 1 & $\mathbf{J}$ & $\mathbf{u g} / \mathbf{L}$ & $\mathbf{E X}$ & NDD \\
\hline$<5$ & $<5$ & $<5$ & $<5$ & 1 & $\mathbf{U}$ & $\mathbf{u g} / \mathbf{L}$ & EX & $<$ EQL \\
\hline$<5$ & $<5$ & $<5$ & & & & & & \\
\hline$<5$ & $<5$ & $<5$ & $<5$ & 1 & $\mathbf{U}$ & $\mathbf{u g} / \mathbf{L}$ & $\mathbf{E X}$ & $<$ EQL \\
\hline$<5$ & $<5$ & $<5$ & $<5$ & 1 & $\mathbf{U}$ & ug/L & EX & $<$ EQL \\
\hline & & & $<5$ & 1 & $\mathbf{U}$ & ug/L & EX & < EQL \\
\hline$<5$ & $<5$ & $<5$ & $<5$ & 1 & $\mathbf{U}$ & $\mathbf{u g} / \mathrm{L}$ & EX & < EQL \\
\hline$<5$ & $<5$ & $<5$ & $<5$ & 1 & $\mathbf{U}$ & ug/L & EX & $<$ EQL \\
\hline & & & $<5$ & 1 & $\mathbf{U}$ & $\mathbf{u g} / \mathrm{L}$ & EX & $<$ EQL \\
\hline 17.5 & 5.1 & 7.5 & 8.49 & 1 & & ug/L & $\mathbf{E X}$ & 8.49 \\
\hline & & & $<1000$ & 1 & $\mathbf{U}$ & ug/L & EX & $<$ EQL \\
\hline$<10$ & $<10$ & $<10$ & $<5$ & 1 & $\mathbf{U}$ & ug/L & $\mathbf{E X}$ & $<$ EQL \\
\hline$<10$ & $<10$ & $<4.5$ & $<20$ & 1 & $\mathbf{U}$ & ug/L & EX & $<$ EQL \\
\hline$<20$ & $<20$ & $<20$ & $<500$ & 1 & $\mathbf{U}$ & $\mathbf{u g} / \mathrm{L}$ & $\mathbf{E X}$ & $<$ EQL \\
\hline$<20$ & $<10$ & $<10$ & $<50$ & 1 & $\mathbf{U}$ & $\mathbf{u g} / \mathbf{L}$ & EX & $<\mathbf{E Q L}$ \\
\hline$<5$ & $<\mathbf{2 0}$ & $<5$ & $<50$ & 1 & $\mathbf{U}$ & $\mathbf{u g} / \mathrm{L}$ & $\mathbf{E X}$ & $<$ EQL \\
\hline$<10$ & $<10$ & $<10$ & $<10$ & 1 & $\mathbf{U}$ & $\mathbf{u g} / \mathbf{L}$ & $\mathbf{E X}$ & $<$ EQL \\
\hline & & 24.4 & $<200$ & 1 & $\mathbf{U}$ & ug/L & $\mathbf{E X}$ & $<$ EQL \\
\hline$<40$ & $<8$ & $<8$ & $<100$ & 1 & $\mathbf{U}$ & ug/L & EX & $<\mathrm{EQL}$ \\
\hline 8.8 & 6.1 & 6.9 & 5.89 & 1 & $\mathbf{J}$ & $\mathrm{ug} / \mathrm{L}$ & EX & NDD \\
\hline$<5$ & $<5$ & $<5$ & $<5$ & 1 & $\mathbf{U}$ & ug/L & EX & $<$ EQL \\
\hline$<5$ & $<5$ & $<5$ & $<5$ & 1 & $\mathbf{U}$ & ug/L & $\mathbf{E X}$ & $<\mathbf{E Q L}$ \\
\hline
\end{tabular}

Note: Flagging, dilution factors, modifiers, and laboratories are for fourth quartet 998 data only. See Appendix B tor Ragging criteria.

* = exceeded holding time for fourth quarter 1998 .

$+=$ exceeded screening level or final primary drinking water standard for fourth quarten 998 .

Sanitary Landfill

D- 103

Fourth Quarter 1998 and Annual Summary 
WSRC-TR-99-00011

Unclassified

WELL: LFW 64D

\section{ANALYTICAL DATA}

H ST Analyte

Bromoform

Bromomethane (Methyl bromide)

Cadmium, total recoverable

Carbon disulfide

Carbon tetrachloride

Chlorobenzene

Chloroethane

+ Chloroethene (Vinyl chloride)

Chloroform

Chloromethane (Methyl chloride)

Chloroprene

Chromium, total recoverable

Dibromochloromethane

Dibromomethane (Methylene bromide)

Dichlorodifluoromethane

Dichloromethane (Methylene chloride)

Ethyl methacrylate

Ethylbenzene

Gross alpha

Iodomethane (Methyl iodide)

Iron, total recoverable

Isobutyl alcohol

Lead, total recoverable

Mercury, total recoverable

Methacrylonitrile

Methyl ethyl ketone

Methyl isobutyl ketone

Methyl methacrylate

Pentachloroethane

Propionitrile

Selenium, total recoverable

Silver, total recoverable

Styrene

Tetrachloroethylene

Toluene

Trichloroethylene

Trichlorofluoromethane

Tritium

Vinyl acetate

Xylenes

cis-1,2-Dichloroethylene

cis-1,3-Dichloropropene

\section{$101998 \quad 201998$}

$<$

$<10$

$<4.7$

$<5$

$-<5$

8.5

$<10$

20.2

$<5$

1.3

$<$

$<1.1$

$<5$

$<5$

1.69

$<7.57$

$<5$

$<5<5 \quad<5$

$1.29<-.45$

$<5$

$<100$

$<47$

$<.7$

$<10$

$<10$

$<10$

$$
<5
$$

$<5$

$<12$

$<5$

$<50$

$<66$

$<5$

-55 . .

$<5$

$<5$

$<5$

$<5$

17.29

$<10$

$<5$

$<5$
$<100$

$<5$

.05

$<10$

301998

$<5$

$<10$

.4

$<5$

$<5$

2.6

$<10$

$<10$

$<5$

$<10$

$<5$

$<3$

$<5$

$<5$

$<5$

$<3$

$<5$

1.26

$<5$

12

$<100$

4.3

$<.2$

$<5$

$<10$

$<12$

$<5$

$<5 \quad<5$

$<5<5$

$<2<2$

$<5=-<5$

$<5<5$

$<5<5$

$<5<5$

$<5$

6930

$<5$

$<5$

$<5$
401998

$<5$

$<5$

$<10$

$<5$

$<5$

3.25

$<10$

9.14

$<5 \quad 1 \quad$ U $\quad u g / L$

$<50 \quad 1 \quad$ U $u g / L$

$\begin{array}{lllll}<10 & 1 & \mathrm{U} & \mathrm{ug} / \mathrm{L}\end{array}$

$\begin{array}{lllll}<5 & 1 & \mathrm{U} & \mathrm{ug} / \mathrm{L}\end{array}$

$<5 \quad 1 \quad$ U $\quad$ ug/L

$<5 \quad 1 \quad$ U

$<10 \quad 1 \quad U$

$<5 \quad 1 \quad$ U

$<5 \quad 1 \quad$ U

1.431

$<5$

$<16$

$$
\text { (1) }
$$

$<100 \quad 1 \quad$ U $\quad$ ug/L $\quad$ EX $\quad<$ EQL

$<.5 \quad 1 \quad \mathrm{U} \quad \mathrm{ug} / \mathrm{L} \quad \mathrm{EX} \quad<\mathrm{EQL}$

$<500 \quad 1 \quad$ U $\quad$ ug/L $\quad$ EX $<$ EQL

$<10 \quad 1 \quad \mathrm{U} \quad \mathrm{ug} / \mathrm{L} \quad \mathrm{EX}<\mathrm{EQL}$

$<5 \quad 1 \quad$ U $\quad$ ug/L $\quad$ EX $<$ EQL

$<50$ I U Ug/L EX $<$ EOL

$<200 \quad 1 \quad \mathrm{U} \quad \mathrm{ug} / \mathrm{L} \quad \mathrm{EX}<\mathrm{EQL}$

$\begin{array}{lllllll}< & 200 & 1 & \mathrm{U} & \mathrm{ug} / \mathrm{L} & \mathrm{EX} & <\mathrm{EQL}\end{array}$

$2<20 \quad 1 \quad$ U $\quad$ ug/L $\quad$ EX $<$ EQL

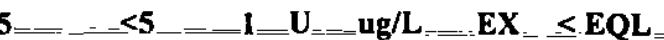

$<5 \quad 1 \quad$ U $\quad$ ug/L $\quad$ EX $<$ EQL

$<5 \quad 1 \quad$ U $\quad u g / L \quad$ EX $<$ EQL

$<5 \quad 1 \quad$ U $\quad$ ug/L $\quad$ EX $<$ EQL

$<5 \quad 1 \quad$ U $\quad$ ug/L $\quad$ EX $<$ EQL

$\begin{array}{lllll}8.19 & 1 & \mathrm{pCi} / \mathrm{ml} & \mathrm{TM} & 8.19\end{array}$

$<20 \quad 1 \quad$ U $\quad$ ug/h $\quad$ EX $<$ EQL

$<10 \quad 1 \quad \mathrm{U}$ ug/L EX $<$ EQL

$\begin{array}{llllll}4.96 & 1 & \mathrm{~J} & \mathrm{ug} / \mathrm{L} & \mathrm{EX} & \mathrm{NDD}\end{array}$

$<5 \quad 1 \quad$ U $\quad$ ug/L $\quad$ EX $<$ EQL

Note: Flagging, dilution factors, modifiers, and laboratories are for fourth quarted 998 data only. See Appendix B for fagging criteria.

* = exceeded hoiding time for fourth quarter 1998.

$+=$ exceeded screening level or final primary drinking water standard for fourth quarten 998 .

Sanitary Landfill

D-104

Fourth Quarter 1998 and Annual Summary 
WSRC-TR-99-00011

Unclassified

WELL: LFW 64D

ANALYTICAL DATA

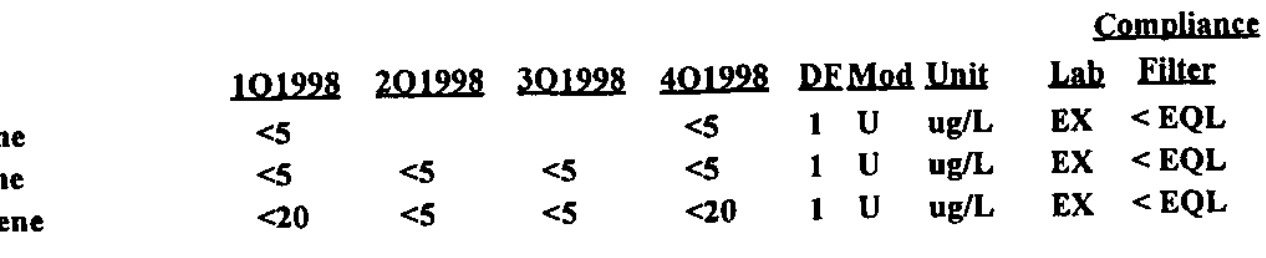

\section{H ST Analyte}

trans-1,2-Dichloroethylene trans-1,3-Dichloropropene trans-1,4-Dichloro-2-butene

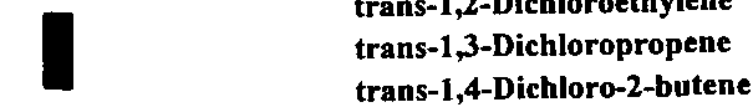

Note: Flagging, dilution tactors, modifiers, and laboratories are for fourth quarte1998 date only. See Appendix B for fiagging criteria. * = exceeded holding time for fourth quarter 1998.

+ - exceeded screening level or final primary drinking water standard for fourth quarter 998.

Sanitary Landfill

D- 105

Fourth Quarter 1998 and Annual Summary 
WELL: LFW 65B



FIELD DATA

Analyte

Water Elevation

Depth to water

pH

Sp. Conductance

Water temperature

Alkalinity as $\mathrm{CaCO}$

Turbidity

Volumes purged

Sampling code

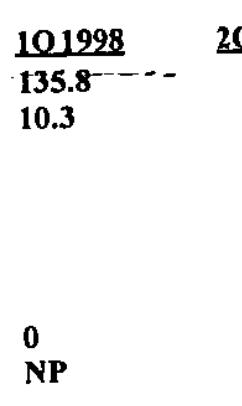

\section{ANALYTICAL DATA}

\section{H ST Analyte}

1,1,1,2-Tetrachloroethane

1,1,1-Trichloroethane

1,1,2,2-Tetrachloroethane

1,1,2-Trichloroethane

1,1-Dichloroethane

1,1-Dichloroethylene

1,2,3-Trichloropropane

1,2-Dibromo-3-chloropropane

1,2-Dibromoethane

1,2-Dichloroethane

1,2-Dichloropropane

1,4-Dichlorobenzene

2-Hexanone

Acetone

Acetonitrile (Methyl cyanide)

Acrolein

Acrylonitrile - = =

Allyl chloride

Aluminum, total recoverable

Arsenic, total recoverable

Barium, total recoverable

Benzene

Bromodichloromethane

Bromoform

Bromomethane (Methyl bromide)

Cadmium, total recoverable

\begin{tabular}{|c|c|c|}
\hline \multirow[t]{2}{*}{101998} & 201998 & 301998 \\
\hline & & $<5$ \\
\hline & & $<5$ \\
\hline & & $<5$ \\
\hline & & $<5$ \\
\hline & & $<5$ \\
\hline · & & $<5$ \\
\hline & & $<5$ \\
\hline & & $<5$ \\
\hline & & $<5$ \\
\hline & & $<5$ \\
\hline & & $<5$ \\
\hline & & $<5$ \\
\hline & & $<10$ \\
\hline & & $<10$ \\
\hline & & $<20$ \\
\hline & & $<10$ \\
\hline$-\ldots$ & . - - & $\leq 5$ \\
\hline & & $<10$ \\
\hline & & 281 \\
\hline & & $<8$ \\
\hline & & 7.1 \\
\hline & & $<5$ \\
\hline & & $<5$ \\
\hline & & $<5$ \\
\hline & & $<10$ \\
\hline & & .5 \\
\hline
\end{tabular}

\section{Compliance}

ft msl

ft

pH

uS/cm

deg. C

$\mathbf{m g} / \mathbf{L}$

NTU

well volumes

Note: Flagging, dilution factors, modifiers, and laboratories are for fourth quartel 998 data only. See Appendix B for fiagging criteria. - = exceeded holding time for fourth quarter 1998.

+- exceeded screening level or final primary drinking water standard for fourth quarten 998 . 
WELL: LFW 65B

ANALYTICAL DATA

H ST Analyte

101998201998

Carbon disulfide

Carbon tetrachtoride

$<5$

$<2<5$

Chlorobenzene $\quad<5$

Chloroethane $\quad<10$

Chloroethene (Vinyl chloride) . ........... $<10$

Chloroform $\quad<5$

Chloromethane (Methyl chloride) $\quad<10$

Chloroprene

Chromium, total recoverable

Dibromochloromethane

Dibromomethane (Methylene bromide)

Dichlorodifluoromethane

Dichloromethane (Methylene chloride)

$<5$

1.6

$<5$

$<5$

$<5$

$<2.1$

Ethylbenzene

$<5$

Gross alpha

Iodomethane (Methyl iodide)

$<5$

Iron, total recoverable

Isobutyl alcohol

47.4

Lead, total recoverable

Mercury, total recoverable

Methacrylonitrile

$<.2$

Methyl ethyl ketone

$<5$

Methyl isobutyl ketone

$<10$

Methyl methacrylate

$<12$

Propionitrile

$<5$

Selenium, total recoverable

$<5$

Silver, total recoverable

$<5$

Styrene

$<2$

Tetrachloroethylene

$<5$

$<5$

Toluene

$<5$

Trichloroethylene

$<5$

Trichlorofluoromethane

Tritium

$<-.72$

Vinyl acetate

$<5$

Xylenes

$<5$

cis-1,3-Dichloropropene

$<5$

trans-1,3-Dichloropropene

$<5$

trans-1,4-Dichloro-2-butene

$<5$

Note: Flagging, dilution factors, modifiers, and laboratories are for fourth quartei 998 data only. See Appendix B for flagging criteria. * a exceeded holding time for fourth quarter 1998.

+ = exceeded sereening level or final primary drinking water standard for fourth quarter1998 .

Sanitary Landfill

D- 107

Fourth Quarter 1998 and Annual Summary 
WELL: LFW 65C

\begin{tabular}{|c|c|c|c|c|c|c|}
\hline SRS Coord. & Lat/Longitude & Screen Zone Elevation & Top of Casing & Casing & Pump & Eormation \\
\hline $\begin{array}{l}\text { N } 82592.9 \\
\text { E } 46064.4\end{array}$ & $\begin{array}{l}\text { 33.284 Deg N } \\
\text { 81.706 Deg W }\end{array}$ & $96.1-86.1$ ft msl & 148.2 ft msl & $\checkmark \mathrm{C}$ & $\mathbf{V}$ & ond \\
\hline
\end{tabular}

SAMPLE DATE

FIELD DATA

Analyte

Water Elevation

Depth to water

pH

Sp. Conductance

Water temperature

Alkalinity as $\mathrm{CaCO} 3$

Turbidity

Volumes purged

Sampling code

\section{ANALYTICAL DATA}

H ST Analvte

1,1,1,2-Tetrachloroethane

1,1,1-Trichloroethane

1,1,2,2-Tetrachloroethane

1,1,2-Trichloroethane

1,1-Dichloroethane

1,1-Dichloroethylene

1,2,3-Trichloropropane

1,2-Dibromo-3-chloropropane

1,2-Dibromoethane

1,2-Dichloroethane

1,2-Dichloropropane

1,4-Dichlorobenzene

2-Hexanone

Acetone

Acetonitrile (Methyl cyanide)

Acrolein

Acrylonitrile

Allyl chloride

Aluminum, total recoverable

Arsenic, total recoverable

Barium, total recoverable

Benzene

Bromodichloromethane

Bromoform

Bromomethane (Methyl bromide)

Cadmium, total recoverable $\begin{array}{lll}12 / 03 / 97 & 03 / 11 / 98 & 06 / 04 / 98\end{array}$

\begin{tabular}{|c|c|c|c|}
\hline 101998 & 201998 & $301998 \quad 401998$ & Unit \\
\hline 135.6 & 136.85 & 135.75 & $\overline{\text { ft msl }}$ \\
\hline 10.5 & 9.25 & 10.35 & ft \\
\hline 3.8 & 4.4 & 4.6 & pH \\
\hline 32 & 32 & 33 & $\mathrm{uS} / \mathrm{cm}$ \\
\hline 17 & 17 & 20.2 & $\operatorname{deg} . C$ \\
\hline $\mathbf{0}$ & $\mathbf{0}$ & $\mathbf{0}$ & $\mathrm{mg} / \mathrm{L}$ \\
\hline .3 & $\mathbf{3}$ & .3 & NTU \\
\hline 2.48157 & 8.76849 & 5.42005 & well volumes \\
\hline
\end{tabular}

Comoliance

\section{DFMod Unit Lab Filter}

$<5<5<5$

$<5<5<5$

$<5<5<5$

$<5<5 \quad<5$

$<5<5 \quad<5$

$<5<5<5$

$<5<5<5$

$<5<5 \quad<5$

$<5<5<5$

$<5<5<5$

$<5<5 \quad<5$

$<5<5<5$

$<10$ <10 $<10$

$<10<10<10$

$<20<20 \quad<20$

$<20<10 \quad<10$

$<5<20<5$

$<10<<10<10$

$<40<8<8$

$\begin{array}{lll}8.6 & 7.8 & 8.1\end{array}$

$<5<5<5$

$<5<5<5$

$<5<5<5$

$<10<10<10$

$<4.7 \quad .6 \quad .4$

Note: Flagging, dilution factors, modifiers, and laboratories are for fourth quarte1998 data only. See Appendix B for Aagging criteria.

- = exceeded holding time for fourth quarter1998.

+ = exceeded screening level or final primary drinking water standard for fourth quartern 998 .

Fourth Quarter 1998 and Annual Summary 
WSRC-TR-99-00011

Unclassified

WELL: LFW 65C

\section{ANALYTICAL DATA}

\section{H ST Analyte}

Carbon disulfide

Carbon tetrachloride

Chlorobenzene

Chloroethane

Chloroethene (Vinyl chloride)

Chloroform

Chloromethane (Methyl chloride)

Chloroprene

Chromium, total recoverable

Dibromochloromethane

Dibromomethane (Methylene bromide)

Dichlorodinuoromethane

Dichloromethane (Methylene chloride)

Ethylbenzene

Gross alpha

Iodomethane (Methyl iodide)

Iron, total recoverable

Isobutyl alcohol

Lead, total recoverable

Mercury, total recoverable

Methacrylonitrile

Methyl ethyl ketone

Methyl isobutyl ketone

Methyl methacrylate

Propionitrile

Selenium, total recoverable

Silver, total recoverable

Styrene

Tetrachloroethylene

Toluene

Trichloroethylene

Trichlorofluoromethane

Tritium

Vinyl acetate

Xylenes

cis-1,3-Dichloropropene

trans-1,2-Dichloroethylene

trans-1,3-Dichloropropene

trans-1,4-Dichloro-2-butene
101998

$<5$

$<5<5<5$

$<5<5<5$

$<10<10<10$

$\leq 10<10<10$

$<5<5<5$

$<10<10<10$

$<5<5 \quad<5$

$\begin{array}{lll}.9 & 1.4 & .8\end{array}$

$<5<5<5$

$<5<5<5$

$<10<5<5$

$<6.42<2.1<2.3$

$<5<5 \quad<5$

$6.32<.39 \quad 5.19$

$<5<5<5$

9.7

$<100<100<100$

$<47 \quad 15.6<5$

$<.7<.2<.2$

$<10<5<5$

$<10<10<10$

$<10<12<12$

$<5<5$

$<50<5<5$

$<66<5<5$

$<5<2<2$

$<5<5<5$

$<5<5<5$

$<5<5<5$

$<5<5<5$

$<5<5<5$

$.72<410<.22$

$<10<5<5$

$<5<5 \quad<5$

$<5<5<5$

$\begin{array}{lll}<5 & <5 & <5\end{array}$

$<20<5 \quad<5$
Compliance Lab Eilter 
WELL: LFW 65D

\begin{tabular}{|c|c|c|c|c|c|c|}
\hline SRS Coord, & Lat/Longitude & Screen Zone Elevation & Ton of Casing & Casing & Pump & Eormation \\
\hline $\begin{array}{l}\text { N } 82598.4 \\
\text { E } 46071.8\end{array}$ & $\begin{array}{l}\text { 33.284 Deg N } \\
\text { 81.706 Deg W }\end{array}$ & $131.5-111.5 \mathrm{ft} \mathrm{msl}$ & $148.4 \mathrm{ft} \mathrm{msl}$ & 2 "PVC & $\mathrm{V}$ & Pond \\
\hline
\end{tabular}
E 46071.8 $81.706 \operatorname{Deg} W$

SAMPLE DATE

FIELD DATA

Analyte

Water Elevation

Depth to water

pH

Sp. Conductance

Water temperature

Alkalinity as $\mathrm{CaCO}$

Turbidity

Volumes purged

Sampling code

\section{ANALYTICAL DATA}

\section{H ST Analyte}

1,1,1,2-Tetrachloroethane

1,1,1-Trichloroethane

1,1,2,2-Tetrachlorocthane

1,1,2-Trichloroethane

+ 1,1-Dichloroethane

1,1-Dichloroethylene

1,2,3-Trichloropropane

1,2-Dibromo-3-chloropropane

1,2-Dibromoethane

1,2-Dichlorobenzene

1,2-Dichloroethane

1,2-Dichloropropane

1,3-Dichlorobenzene

1,4-Dichlorobenzene

1,4-Dioxane

2-Hexanone

Acetonitrile (Methyl cyanide)

Acrolein

Acrylonitrile

Allyl chloride

Aluminum, total recoverable

Arsenic, total recoverable

Barium, total recoverable

Benzene

Bromodichloromethane
Acetone

$\begin{array}{llll}12 / 03 / 97 & 03 / 12 / 98 & 06 / 09 / 98 & 09 / 14 / 98\end{array}$

\begin{tabular}{|c|c|c|c|c|}
\hline 101998 & 201998 & 301998 & 401998 & Unit \\
\hline 136.35 & 138.35 & 136.35 & 136.4 & ft msl \\
\hline 9.95 & 7.95 & 9.95 & 9.9 & ft \\
\hline 4 & 4.6 & 4.6 & 4.7 & pH \\
\hline 22 & 20 & 24 & 23 & $\mathbf{u S} / \mathbf{c m}$ \\
\hline 17 & 15 & 18.3 & 20.8 & deg. $C$ \\
\hline $\mathbf{0}$ & 0 & 0 & & $\mathrm{mg} / \mathrm{L}$ \\
\hline $\begin{array}{l}.3 \\
2.48880\end{array}$ & $\begin{array}{l}.5 \\
673996\end{array}$ & $\begin{array}{l}.7 \\
10.1815\end{array}$ & $\begin{array}{l}.5 \\
11.9693\end{array}$ & NTU \\
\hline
\end{tabular}

Compliance

\section{$101998201998 \quad 301998$ 401998 DFMod Unit Lab Filter}

\begin{tabular}{|c|c|c|c|c|c|c|c|c|}
\hline$<5$ & $<5$ & $<5$ & & & & & & \\
\hline 3.24 & $<5$ & 1.6 & $<5$ & 1 & $\mathbf{U}$ & $\mathbf{u g} / \mathbf{L}$ & $\mathbf{E X}$ & $<$ EQL \\
\hline$<5$ & $<5$ & $<5$ & $<5$ & 1 & $\mathbf{U}$ & $\mathrm{ug} / \mathrm{L}$ & EX & $<$ EQL \\
\hline$<5$ & $<5$ & $<5$ & $<5$ & 1 & $\mathbf{U}$ & ug/L & $\mathbf{E X}$ & $<$ EQL \\
\hline 22.2 & 14 & 14 & 10.8 & 1 & & $\mathbf{u g} / \mathrm{L}$ & $\mathbf{E X}$ & 10.8 \\
\hline 1.03 & $<5$ & .74 & $<5$ & 1 & $\mathbf{U}$ & ug/L & $\mathbf{E X}$ & $<\mathrm{EQL}$ \\
\hline$<5$ & $<5$ & $<5$ & & & & & & \\
\hline$<5$ & $<5$ & $<5$ & $<5$ & 1 & $\mathbf{U}$ & ug/L & $\mathbf{E X}$ & $<$ EQL \\
\hline \multirow[t]{2}{*}{$<5$} & $<5$ & $<5$ & $<5$ & 1 & $\mathbf{U}$ & ug/L & $\mathbf{E X}$ & $<\mathrm{EQL}$ \\
\hline & & & $<5$ & 1 & $\mathbf{U}$ & $\mathbf{u g} / \mathbf{L}$ & EX & $<$ EQL \\
\hline$<5$ & $<5$ & $<5$ & $<5$ & 1 & $\mathbf{U}$ & ug/L & $\mathbf{E X}$ & $<$ EQL \\
\hline \multirow[t]{2}{*}{.983} & $<5$ & $<5$ & $<5$ & 1 & $\mathbf{U}$ & $\mathbf{u g} / \mathbf{L}$ & $\mathbf{E X}$ & $<$ EQL \\
\hline & & & $<5$ & 1 & $\mathbf{U}$ & $\mathbf{u g} / \mathbf{L}$ & $\mathbf{E X}$ & $<\mathrm{EQL}$ \\
\hline \multirow[t]{2}{*}{$<5$} & $<5$ & $<5$ & 1.71 & 1 & $\mathbf{J}$ & ug/L & $\mathbf{E X}$ & NDD \\
\hline & & & $<1000$ & 1 & $\mathbf{U}$ & ug/L & EX & $<$ EQL \\
\hline$<10$ & $<10$ & $<10$ & $<5$ & 1 & $\mathbf{U}$ & ug/L & $\mathbf{E X}$ & $<\mathrm{EQL}$ \\
\hline$<10$ & $<10$ & $<10$ & $<20$ & 1 & $\mathbf{U}$ & ug/L & $\mathbf{E X}$ & $<$ EQL \\
\hline$<20$ & $<\overline{\overline{20}}$ & $<\mathbf{2 0}$ & $<500$ & $\mathbf{1}^{-}$ & $\mathbf{U}^{-}$ & $\mathbf{u g} / \mathrm{L}$ & $\mathbf{E} \mathbf{X}$ & $<$ EQL \\
\hline$<20$ & $<10$ & $<10$ & $<50$ & 1 & $\mathbf{U}$ & ug/L & $\mathbf{E X}$ & $<\mathrm{EQL}$ \\
\hline$<5$ & $<\mathbf{2 0}$ & $<5$ & $<50$ & 1 & $U$ & ug/L & EX & $<\mathrm{EQL}$ \\
\hline \multirow[t]{2}{*}{$<10$} & $<10$ & $<10$ & $<10$ & 1 & $\mathbf{U}$ & ug/L & $\mathbf{E X}$ & $<\mathrm{EQL}$ \\
\hline & & 36.7 & $<200$ & 1 & $\mathbf{U}$ & $\mathbf{u g} / \mathbf{L}$ & EX & $<\mathbf{E Q L}$ \\
\hline$<40$ & $<8$ & $<8$ & $<100$ & 1 & $\mathbf{U}$ & $\mathbf{u g} / \mathbf{L}$ & EX & $<\mathrm{EQL}$ \\
\hline 3.2 & 3.1 & 3.5 & 4.33 & 1 & $\mathbf{J}$ & ug $/ \mathrm{L}$ & EX & NDD \\
\hline$<5$ & $<5$ & $<5$ & $<5$ & 1 & $\mathbf{U}$ & $\mathbf{u g} / \mathbf{L}$ & $\mathbf{E X}$ & $<\mathrm{EQL}$ \\
\hline$<5$ & $<5$ & $<5$ & $<5$ & 1 & $\mathbf{U}$ & ug/L & $\mathbf{E X}$ & $<$ EQL \\
\hline
\end{tabular}

Note: Flagging, dilution factors, modifiers, and laboratories are for fourth quartet 998 data only. See Appendix B tor hagging criteria.

* = exceeded holding time for fourth quarter 1998.

+ - exceeded screening level or final primary drinking water standard for fourth quarten 998 .

Sanitary Landfill

D-110

Fourth Quarter 1998 and Annual Summary 
WSRC-TR-99-00011

Unclassified

WELL: LFW 65D

\section{ANALYTICAL DATA}

\section{H ST Analyte}

Bromoform

Bromomethane (Methyl bromide)

Cadmium, total recoverable

Carbon disulfide

Carbon tetrachloride

Chlorobenzene

Chloroethane

Chloroethene (Vinyl chloride)

Chloroform

Chloromethane (Methyl chloride)

Chloroprene

Chromium, total recoverable

Dibromochloromethane

Dibromomethane (Methylene bromide)

Dichlorodifluoromethane

Dichloromethane (Methylene chloride)

Ethyl methacrylate

Ethylbenzene

Gross alpha

lodomethane (Methyl iodide)

Iron, total recoverable

Isobutyl alcohol

Lead, total recoverable

Mercury, total recoverable

Methacrylonitrile

Methyl ethyl ketone

Methyl isobutyl ketone

Methyl methacrylate

Pentachloroethane

Propionitrile

Selenium, total recoverable

Silver, total recoverable

Styrene

Tetrachloroethylene

Toluene

+ Trichloroethylene

+ Trichlorofluoromethane

Tritium

Unknown

Vinyl acetate

Xylenes

cis-1,2-Dichloroethylene

\section{401998 DEMod Unit}

$<5$

$<10$

$<5$

$<5$

$<10$

$<4.7$

$<10$

.6

$<5$

$<\frac{5}{5}$

$<5$

$<10$

$<5$

$<10$

$<5$

$<7$

$<5$

$<5$

9.9

$<7.72$

$<2$
$<5$

$<5$
$<5$

$<5$
$<5$

$<5$
$<10$

$<10$
$<10$

$<5$

$<10$

$<5$

.7

$<5$

$<5$

7.1

$<3.5$

$<5$

1.37

$<5$

$<5$$$
<-.97
$$

$<5$

$<100$

100

$<47$

$<5$

$<.7$

$<5$

$<10$

.07

$<5$

$<10$

$<10$

$<10$

$<12$

$<5$

$<50$

$<66$

$<5$

$<5$

$<5$

3.97

$<5$

9.77

25.7

3.29

$<10$

$<5$

$<5 \quad 1 \quad \mathrm{U} \quad \mathrm{ug} / \mathrm{L}$

$<5 \quad 1 \quad \mathrm{U} \quad \mathrm{ug} / \mathrm{L}$

$<10 \quad 1 \quad U \quad$ ug/L

$<5 \quad 1$ U ug/L

$<5 \quad 1 \quad U$

$<10 \quad 1 \mathrm{U}$

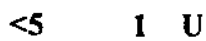

$<5 \quad 1 \quad \mathrm{U}$

$<5 \quad 1 \quad$

$<50$

4.29

$<5$

$<5$

4.5

$<3.2$

9.02

$<10$

$<5 \quad 1$

$<5 \quad 1 \quad$ U

1.47

$<5$

16.8

$<100$

7.8

.09

$<5$

$<10$

$<12$

$<5$

121

ng/L

ug/L

ug/L

ug/L

ug/L

ug/L

ug/L

ug/L

ug/L

$\mathbf{u g} / \mathbf{L}$

ug/L

ug/L

ug/L

pCi/L

ug/L

ug/L

$<45.22$

$<5<5$

$<5$
$<5$

$<5 \quad 1 \quad \mathrm{U} \quad \mathrm{ug} / \mathrm{L}$

Compliance

Lab Filter

EX $<$ EQL

EX $<$ EQL

EX $<$ EQL

EX $<$ EQL

EX $<$ EQL

EX $<$ EQL

EX $<$ EQL

EX $<$ EQL

EX $<$ EQL

EX $<$ EQL

EX $<$ EQL

EX NDD

EX $<$ EQL

EX $<$ EQL

EX 9.02

EX $<$ EQL

EX $<$ EQL

EX $<$ EQL

TM NDD

EX $<$ EQL

EX NDD

$\begin{array}{llllll}<100 & 1 & \mathbf{U} & \text { ug/L } & \text { EX } & <\text { EQL }\end{array}$

$<.5 \quad 1 \quad U \quad$ ug/L $\quad$ EX $<$ EQL

$<500 \quad 1 \quad U$ ug/L EX $<$ EQL

$<10$ I U ug/L EX $<$ EQL

$\begin{array}{llllll}<5 & 1 & \mathrm{U} & \mathrm{ug} / \mathrm{L} & \mathrm{EX} & <\text { EQL }\end{array}$

$<50 \quad 1 \quad$ U $\quad$ ug/L $\quad$ EX $<$ EQL

$<200 \quad 1 \quad \mathrm{U}$ ug/L EX $<$ EQL

$<200 \quad 1 \quad U \quad$ ug/L $\quad$ EX $<$ EQL

$<20 \quad 1 \quad \mathrm{U}$ ug/L $\mathrm{EX}<$ EQL

$<5 \quad 1 \quad \mathrm{U} \quad$ ug/L $\quad$ EX $<$ EQL

$<5 \quad 1 \quad$ U $\quad$ ug/L $\quad$ EX $<$ EQL

$<5 \quad 1 \quad U \quad$ ug/L EX $<$ EQL

$\begin{array}{lllll}5.09 & 1 & \mathrm{ug} / \mathrm{L} & \mathrm{EX} & 5.09\end{array}$

$24.3 \quad 1 \quad$ ug/L EX 24.3

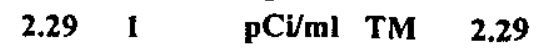

$\begin{array}{cccccc}<20 & 1 & \text { U } & \text { ug/L } & \text { EX } & <\text { EQL } \\ <10 & 1 & \text { U } & \text { ug/L } & \text { EX } & <\text { EQL } \\ 2.6 & 1 & \text { J } & \text { ug/L } & \text { EX } & \text { NDD }\end{array}$

Note: Flagging, dilution factors, modifiers, and laboratories are for fourth quartel998 data only. See Appendix B for flagging criteria. * = exceeded holding time for fourth quarter 1998.

$+=$ exceeded screening level or final primary drinking water standard for fourth quarten 998 . 
WSRC-TR-99-00011

Unclassified

WELL: LFW 65D

ANALYTICAL DATA

H ST Analvte

cis-1,3-Dichloropropene

trans-1,2-Dichloroethylene

trans-1,3-Dichloropropene

trans-1,4-Dichloro-2-butene

\begin{tabular}{|c|c|c|c|c|c|c|c|c|}
\hline \multirow{2}{*}{101998} & \multirow[b]{2}{*}{201998} & \multirow[b]{2}{*}{301998} & \multirow[b]{2}{*}{401998} & \multirow{2}{*}{\multicolumn{2}{|c|}{ DEMod }} & \multicolumn{3}{|c|}{ Complianc } \\
\hline & & & & & & Unit & Lab & Filter \\
\hline$<5$ & $<5$ & $<5$ & $<5$ & 1 & $\mathbf{U}$ & ug/L & EX & $<$ EQL \\
\hline$<5$ & & & $<5$ & I & $\mathbf{U}$ & ug/L & $\mathbf{E X}$ & $<$ EQL \\
\hline$<5$ & $<5$ & $<5$ & $<5$ & 1 & $\mathbf{U}$ & ug/L & $\mathbf{E X}$ & $<\mathbf{E Q L}$ \\
\hline$<20$ & $<5$ & $<5$ & $<20$ & 1 & $\mathbf{U}$ & ug/L & EX & $<$ EQL \\
\hline
\end{tabular}

Note: Flagging, dilution factors, modifiers, and laboratories are for fourth quartel 998 data only. See Appendix B for fagging eriteria.

* exceeded holding time for fourth quarter 1998.

+ = exceeded screening level or final primary drinking water standard for fourth quarteri 998 . 
WSRC-TR-99-00011

Unclassified

WELL: LFW 66D

\begin{tabular}{|c|c|c|c|c|c|c|}
\hline SRS Coord. & Lat/Longitude & Screen Zone Elevation & Top of Casing & Casing & Pump & Eormation \\
\hline N 828 & 33.284 Deg N & $21.8 \mathrm{ft}$ & $1617 \mathrm{ft} \mathrm{msl}$ & $2 " \mathrm{PVC}$ & $\mathbf{V}$ & d Pond \\
\hline
\end{tabular}
E 46173.7 81.706 Deg W

\section{SAMPLE DATE}

FIELD DATA

Analyte

Water Elevation

Depth to water

pH

Sp. Conductance

Water temperature

Alkalinity as $\mathrm{CaCO}$

Turbidity

Volumes purged

Sampling code

\section{ANALYTICAL DATA}

\section{H ST Analyte}

1,1,1,2-Tetrachloroethane

1,1,1-Trichloroethane

1,1,2,2-Tetrachloroethane

1,1,2-Trichloroethane

1,1-Dichloroethane

1,1-Dichloroethylene

1,2,3-Trichloropropane

1,2-Dibromo-3-chloropropane

1,2-Dibromoethane

1,2-Dichloroethane

1,2-Dichloropropane

1,4-Dichlorobenzene

2-Hexanone

Acetone

Acetonitrile (Methyl cyanide)

Acrolein

Acrylonitrile

Allyl chloride

Arsenic, total recoverable

Barium, total recoverable

Benzene

Bromodichloromethane

Bromoform

Bromomethane (Methyl bromide)

Cadmium, total recoverable

Carbon disulfide
$12 / 04 / 97$

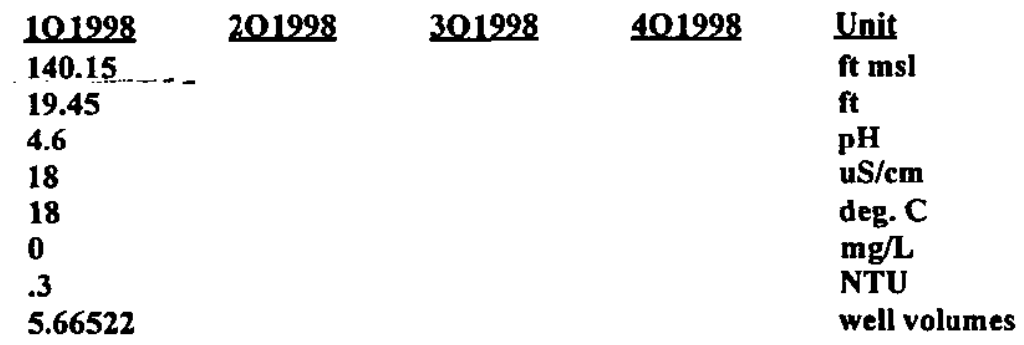

Compliance

101998201998301998 401998 DFMod Unit Lab Eilter

$<5$

$<5$

$<5$

$<5$

$<5$

$<5$

$<5$

$<5$

$<5$

$<5$

$<5$

$<10$

$<7.3$

$<20$

$<20$

$<5$

$<10$

$<40$

2

$<5$

$<5$

$<5$

$<10$

$<4.7$

$<5$

Note: Flagging, dijution factors, modifiers, and laboratories are for fourth quartei998 data only. See Appendix B for flagging criteria. * = exceeded holding time for fourth quarter 1998.

$+=$ exceeded screening level or final primary drinking water standard for fourth quarten 998 . 
WELL: LFW 66D

\section{ANALYTICAL DATA}

\section{H ST Analyte}

Carbon tetrachloride

Chlorobenzene

Chloroethane

Chloroethene (Vinyl chloride)

Chloroform

Chloromethane (Methyl chloride)

Chloroprene

Chromium, total recoverable

Dibromochloromethane

Dibromomethane (Methylene bromide)

Dichlorodifluoromethane

Dichloromethane (Methylene chloride)

Ethylbenzene

Gross alpha

Iodomethane (Methyl iodide)

Isobutyl alcohol

Lead, total recoverable

Mercury, total recoverable

Methacrylonitrile

Methyl ethyl ketone

Methyl isobutyl ketone

Propionitrile

Selenium, total recoverable

Silver, total recoverable

Styrene

Tetrachloroethylene

Toluene

Trichloroethylene

Trichlorofluoromethane

Tritium

Vinyl acetate

Xylenes

_cis=1 3-Dichloropropene

trans-1,2-Dichloroethylene

trans-1,3-Dichloropropene

trans-1,4-Dichloro-2-butene
101998

$<5$

$<5$

$<10$

$<10$

$<5$

$<10$

$<5$

1.1

$<5$

$<5$

$<10$

$<7.03$

$<5$

2.34

$<5$

$<100$

$<47$

$<.7$

$<10$

$<10$

$<10$

$<50$

$<66$

$<5$

$<5$

$<5$

$<5$

$<5$

$<5$

$<1.17$

$<10$

$<5$

$\leq 5$

$<5$

$<5$

$<20$
Compliance

Lab Filter 
WELL: LFW 67B

\begin{tabular}{|c|c|c|c|c|c|c|}
\hline SRS Coord. & Lat/Longitude & Screen Zone Elevation & Top of Casing & Casing & Pump & Formation \\
\hline $\begin{array}{l}\text { N } 82847.1 \\
\text { E } 46517.1\end{array}$ & $\begin{array}{l}\text { 33.285 Deg } N \\
\text { 81.705 Deg } W\end{array}$ & $65.6-55.6 \mathrm{ft} \mathrm{msl}$ & & 2 & $\mathbf{V}$ & Pond \\
\hline
\end{tabular}

SAMPLE DATE

$12 / 04 / 97 \quad 03 / 12 / 98 \quad 06 / 05 / 98$

\section{FIELD DATA}

Analyte

Water Elevation

Depth to water

pH

Sp. Conductance

Water temperature

Alkalinity as $\mathrm{CaCO} 3$

Turbidity

Volumes purged

$12 / 04 / 97 \quad 03 / 12 / 98$

$06 / 05 / 98$

Sampling code

\section{ANALYTICAL DATA}

\section{H ST Analyte}

1,1,1,2-Tetrachloroethane

1,1,1-Trichloroethane

1,1,2,2-Tetrachloroethane

1,1,2-Trichloroethane

1,1-Dichloroethane

1,1-Dichloroethylene

1,2,3-Trichloropropane

1,2-Dibromo-3-chloropropane

1,2-Dibromoethane

1,2-Dichloroethane

1,2-Dichloropropane

1,4-Dichlorobenzene

2-Hexanone

Acetone

Acetonitrile (Methyl cyanide)

Acrolein

Acrylonitrile

Allyl chloride

Aluminum, total recoverable Arsenic, total recoverable Barium, total recoverable

Benzene

Bromodichloromethane

Bromoform

Bromomethane (Methyl bromide)

Cadmium, total recoverable

\begin{tabular}{lll}
101998 & 201998 & $\frac{301998}{136.7}$ \\
\hline 136.7 & -137.73 & 137 \\
3.6 & 17.67 & 18.4 \\
60 & 4.1 & 4 \\
19 & 48 & 59 \\
0 & 17.5 & 20 \\
.3 & 0 & 0 \\
2.63204 & .4 & .2 \\
\hline
\end{tabular}

401998

Unit

ft msi

ft

pH

$\mathbf{u S} / \mathbf{c m}$

deg. C

mg/L

NTU

well volumes

\section{Compliance}

$\begin{array}{ccccc}101998 & 201998 & 301998 & 401998 & \text { DEMod Unit Lab Filter } \\ <5 & <5 & <5 & \\ <5 & <5 & <5 \\ <5 & <5 & <5 \\ <5 & <5 & <5 \\ <5 & <5 & <5 \\ <5 & <5 & <5 \\ <5 & <5 & <5 \\ <5 & <5 & <5 \\ <5 & <5 & <5 \\ <5 & <5 & <5 \\ <5 & <5 & <5 \\ <5 & <5 & <5 \\ <10 & <10 & <10 \\ <10 & <10 & <3.9 \\ <20 & <20 & <20 \\ <20 & <10 & <10 \\ <5 & <20 & <5 \\ <10 & <10 & <10 \\ & & 315 \\ <40 & <8 & <8 \\ 6 & 5.5 & 5.5 \\ <5 & <5 & <5 \\ <5 & <5 & <5 \\ <5 & <5 & <5 \\ <10 & <10 & <10 \\ <4.7 & <2 & .4\end{array}$

Note: Flagging, dilution factors, modifiers, and laboratories are for fourth quartel998 data only. See Appendix B for flagging criteria.

* $=$ exceeded holding time for fourth quarter 1998.

$+=$ exceeded screening level or final primary drinking water standard for fourth quartern 998 .

Sanitary Landfill

D-115

Fourth Quarter 1998 and Annual Summary 
WELL: LFW 67B

\section{ANALYTICAL DATA}

\section{H ST Analyte}

Carbon disulfide

Carbon tetrachloride

Chlorobenzene

Chloroethane

Chloroethene (Vinyl chloride)

Chloroform

Chloromethane (Methyl chloride)

Chloroprene

Chromium, total recoverable

Dibromochloromethane

Dibromomethane (Methylene bromide)

Dichlorodifluoromethane

Dichloromethane (Methylene chloride)

Ethylbenzene

Gross alpha

Iodomethane (Methyl iodide)

Iron, total recoverable

Isobutyl alcohol

Lead, total recoverable

Mercury, total recoverable

Methacrylonitrile

Methyl ethyl ketone

Methyl isobutyl ketone

Methyl methacrylate

Propionitrile

Selenium, total recoverable

Silver, total recoverable

Styrene

Tetrachloroethylene

Toluene

Trichloroethylene

Trichlorofluoromethane

Tritium

Vinyl acetate

Xylenes

cis-1,3-Dichloropropene

trans-1,2-Dichloroethylene

trans-1,3-Dichloropropene

trans-1,4-Dichloro-2-butene
101998

201998

$<5$

$<5$

$<5<5$

$<5<5$

$<10<10$

$-<10<10$

$<5<5$

$<10<10$

$<5<5$

$.86<3$

$<5<5$

$<5<5$

$<10<5$

$<7.01$

$<5$

8.49

$<5$

$<1.8$

$<5$

2.93

$<5$

301998

$<5$

$<5$

$<5$

$<10$

$<10$

$<5$

$<10$

$<5$

$<3$

$<5$

$<5$

$<5$

$<2$

$<5$

7.54

$<5$

8.9

$<100<100<100$

$<47 \quad<5 \quad<5$

$<.7<.2<.2$

$<10<5<5$

$<10<10 \quad<10$

$<10<12<12$

$<5<5$

$<50<5<5$

$<66<5<5$

$<5<2 \quad<2$

$<5 \quad<5 \quad<5$

$<5<5<5$

$<5 \quad<5 \quad<5$

$<5<5<5$

$<5 \quad<5 \quad<5$

$<.56 \ldots<351$ _ $<\leq-.34$

$<10<5 \quad<5$

$<5<5 \quad<5$

$<5<5 \quad<5$

$<5$

$<5 \quad<5 \quad<5$

$<20<5 \quad<5$
Compliance Lab Filter 
WSRC-TR-99-00011

Unclassified

WELL: LFW 67C

\begin{tabular}{|c|c|c|c|c|c|c|}
\hline SRS Coord, & Lat/Longitude & Screen Zone Elevation & Top of Casing & Casing & Pump & Eormation \\
\hline $\begin{array}{l}\text { N 82844.2 } \\
\text { E } 46527.5\end{array}$ & $\begin{array}{l}\text { 33.285 Deg } N \\
\text { 81.705 } \text { Deg W }\end{array}$ & $96.1-86.1 \mathrm{ft} \mathrm{msl}$ & $157.1 \mathrm{ft} \mathrm{msl}$ & VC & & $\mathbf{M}$ \\
\hline
\end{tabular}

SAMPLE DATE

$\begin{array}{llll}12 / 02 / 97 & 03 / 12 / 98 & 06 / 09 / 98 & 09 / 14 / 98\end{array}$

\section{FIELD DATA}

Analvte

Water Elevation

Depth to water

pH

Sp. Conductance

Water temperature

Alkalinity as $\mathrm{CaCO} 3$

Turbidity

Volumes purged

Sampling code

\begin{tabular}{lllll}
101998 & $\frac{201998}{30}$ & $\frac{301998}{136.53}$ & $\underline{401998}$ & Unit \\
\hline 136.6 & 136.6 & 18.6 & ft msl \\
18.3 & 17 & 18.37 & 18.3 & ft \\
6.4 & 6.2 & 6 & 6.2 & pH \\
660 & 600 & 520 & 490 & uS/cm \\
20 & 18 & 20.4 & 21 & deg. C \\
193 & 232 & 217 & 172 & mg/L \\
1.3 & .7 & 1 & .5 & NTU \\
6.01657 & 5.30714 & 4.63429 & 10.6447 & well volumes \\
$t V$ & & & &
\end{tabular}

\section{ANALYTICAL DATA}

\section{H ST Analyte}

1,1,1,2-Tetrachloroethane

1,1,1-Trichloroethane

1,1,2,2-Tetrachloroethane

1,1,2-Trichloroethane

+ 1,1-Dichloroethane

1,1-Dichloroethylene

1,2,3-Trichloropropane

1,2-Dibromo-3-chloropropane

1,2-Dibromoethane

1,2-Dichlorobenzene

1,2-Dichloroethane

1,2-Dichloropropane

1,3-Dichlorobenzene

1,4-Dichlorobenzene

1,4-Dioxane

2-Hexanone

Acetone

Acetonitrile (Methyl cyanide)

Acrolein

Acrylonitrile

Allyl chloride

Aluminum, total recoverable

+ Arsenic, total recoverable

Barium, total recoverable

+ Benzene

Bromodichloromethane

\begin{tabular}{|c|c|c|c|c|c|c|c|c|}
\hline & & & & & & & & mpliar \\
\hline 01998 & $\underline{201998}$ & 301998 & $\underline{401998}$ & & Mod & Unit & Lab & Eilter \\
\hline$<25$ & $<25$ & $<5$ & & & & & & \\
\hline$<25$ & $<25$ & $<5$ & $<5$ & 1 & $\mathbf{U}$ & $\mathbf{u g} / \mathbf{L}$ & EX & $<\mathrm{EQL}$ \\
\hline$<25$ & $<25$ & $<5$ & $<5$ & 1 & $\mathbf{u}$ & ug/L & $\mathbf{E X}$ & $<$ EQL \\
\hline$<25$ & $<25$ & $<5$ & $<5$ & 1 & $\mathbf{U}$ & ug/L & $\mathbf{E X}$ & < EQL \\
\hline 147 & 120 & 140 & 114 & 1 & & $\mathbf{u g} / \mathbf{L}$ & EX & 114 \\
\hline$<25$ & $<25$ & 2.2 & $<5$ & 1 & $\mathbf{U}$ & $\mathrm{ug} / \mathrm{L}$ & EX & < EQL \\
\hline$<25$ & $<25$ & $<5$ & & & & & & \\
\hline$<25$ & $<25$ & $<5$ & $<5$ & 1 & $\mathbf{U}$ & ug/L & EX & $<$ EQL \\
\hline$<25$ & $<25$ & $<5$ & $<5$ & 1 & $\mathbf{U}$ & ug/L & $\mathbf{E X}$ & $<$ EQL \\
\hline & & & $<5$ & 1 & $\mathbf{U}$ & ug/L & $\mathbf{E X}$ & $<$ EQL \\
\hline$<25$ & $<25$ & 1.8 & $<5$ & 1 & $\mathbf{U}$ & ug/L & $\mathbf{E X}$ & $<\mathbf{E Q L}$ \\
\hline$<25$ & $<25$ & 3.3 & $<5$ & 1 & $\mathbf{U}$ & ug/L & $\mathbf{E X}$ & $<$ EQL \\
\hline & & & $<5$ & 1 & $\mathbf{U}$ & ug/L & $\mathbf{E X}$ & $<$ EQL \\
\hline 44.5 & 43 & 40 & 44.9 & 1 & & $\mathbf{u g} / \mathbf{L}$ & $\mathbf{E X}$ & 44.9 \\
\hline & & & $<1000$ & 1 & $\mathbf{U}$ & $\mathbf{u g} / \mathbf{L}$ & $\mathbf{E X}$ & $<$ EQL \\
\hline$<50$ & $<50$ & $<10$ & $<5$ & 1 & $\mathbf{U}$ & ug/L & $\mathbf{E X}$ & $<$ EQL \\
\hline$<43.6$ & 130 & $<15$ & 12.5 & 1 & $\mathbf{J}$ & ug/L & EX & NDD \\
\hline$<100$ & $<100$ & $<20$ & $<500$ & 1 & $\mathbf{U}$ & ug/L & EX & < EQL \\
\hline$<100$ & $<50$ & $<10$ & $<50$ & 1 & $\mathbf{U}$ & ug/L & $\mathbf{E X}$ & < EQL \\
\hline$<25$ & $<25$ & $<5$ & $<50$ & 1 & $\mathbf{U}$ & $\mathbf{u g} / \mathbf{L}$ & EX & $<$ EQL \\
\hline$<50$ & $<50$ & $<10$ & $<10$ & 1 & $\mathbf{U}$ & ug/L & EX & $<\mathbf{E Q L}$ \\
\hline & & 12 & $<200$ & 1 & $\mathbf{U}$ & ug/L & EX & $<$ EQL \\
\hline 39.2 & 32.4 & 37.1 & 68.8 & 1 & $\mathbf{J}$ & ug/L & EX & NDD \\
\hline 12 & 11.7 & 14.1 & 15.8 & 1 & & ug/L & $\mathbf{E X}$ & 15.8 \\
\hline 10.2 & 8.9 & 8.4 & 7.29 & 1 & & ug/L & EX & 7.29 \\
\hline$<25$ & $<25$ & $<5$ & $<5$ & 1 & $\mathbf{U}$ & ug/L & EX & $<$ EQL \\
\hline
\end{tabular}

Note: Flagging, dilution factors, modifiers, and laboratories are for fourth quartei 998 data only. See Appendix B for flagging criterla.

* exceeded holding time for fourth quarter 1998.

+ - exceeded screening level or final primary drinking water standard for fourth quarter 998 .

Sanitary Landfill

D-117

Fourth Quarter 1998 and Annual Summary 
WSRC-TR-99-00011

Unclassified

WELL: LFW 67C

\section{ANALYTICAL DATA}

\begin{tabular}{|c|c|c|c|c|c|c|c|c|c|c|}
\hline \multicolumn{2}{|c|}{ H SI Analyte } & 101998 & 201998 & 301998 & 401998 & \multicolumn{2}{|c|}{ DEMod } & \multirow{2}{*}{$\frac{\text { Unit }}{\text { ug/L }}$} & \multirow{2}{*}{$\frac{\mathbf{L a b}}{\mathbf{E X}}$} & Filter \\
\hline & Bromoform & $<25$ & $<25$ & $<5$ & $<5$ & 1 & $\mathbf{U}$ & & & $<\mathbf{E Q L}$ \\
\hline & Bromomethane (Methyl bromide) & $<50$ & $<\mathbf{5 0}$ & 1.9 & $<5$ & 1 & $\mathbf{U}$ & $\mathbf{u g} / \mathbf{L}$ & $\mathbf{E X}$ & $<$ EQL \\
\hline & Cadmium, total recoverable & $<4.7$ & $<2$ & $<.3$ & $<10$ & 1 & $\mathbf{U}$ & ug/L & $\mathbf{E X}$ & $<$ EQL \\
\hline & Carbon disulfide & $<25$ & $<\mathbf{2 5}$ & $<5$ & $<5$ & 1 & $\mathbf{U}$ & ug/L & $\mathbf{E X}$ & $<\mathbf{E Q L}$ \\
\hline & Carbon tetrachloride & $\ldots \leq 25$ & $<25$ & $<5$ & $<5$ & 1 & $\mathbf{U}$ & $\mathbf{u g} / \mathrm{L}$ & $\mathbf{E X}$ & $<$ EQL \\
\hline & Chlorobenzene & $<25$ & 2.9 & 1.7 & 1.25 & 1 & $\mathbf{J}$ & $\mathbf{u g} / \mathbf{L}$ & $\mathbf{E X}$ & NDD \\
\hline & Chloroethane & $<\mathbf{5 0}$ & $<\mathbf{5 0}$ & $<10$ & $<10$ & 1 & $\mathbf{U}$ & ug/L & EX & $<$ EQL \\
\hline+ & Chloroethene (Vinyl chloride) & $<50$ & 3.9 & 3.3 & 41.3 & 1 & & ug/L & $\mathbf{E X}$ & 41.3 \\
\hline & Chloroform & $<25$ & $<25$ & $<5$ & $<5$ & 1 & $\mathbf{U}$ & $\mathbf{u g} / \mathbf{L}$ & $\mathbf{E X}$ & $<$ EQL \\
\hline & Chloromethane (Methyl chloride) & $<50$ & $<\mathbf{5 0}$ & $<10$ & $<5$ & 1 & $\mathbf{U}$ & ug/L & $\mathbf{E X}$ & $<\mathrm{EQL}$ \\
\hline & Chloroprene & $<25$ & $<25$ & $<5$ & $<50$ & 1 & $\mathbf{U}$ & $\mathbf{u g} / \mathbf{L}$ & $\mathbf{E X}$ & $<$ EQL \\
\hline & Chromium, total recoverable & $<7$ & $<3$ & $<3$ & $<10$ & 1 & $\mathbf{U}$ & ug/L & EX & $<$ EQL \\
\hline & Dibromochloromethane & $<25$ & $<\mathbf{2 5}$ & $<5$ & $<5$ & 1 & $\mathbf{U}$ & Ig/L & $\mathbf{E X}$ & $<$ EQL \\
\hline & Dibromomethane (Methylene bromide) & $<25$ & $<25$ & $<5$ & $<5$ & 1 & $\mathbf{U}$ & ug/L & $\mathbf{E X}$ & $<$ EQL \\
\hline+ & Dichlorodifluoromethane & 14.2 & 19 & 11 & 14.2 & 1 & & $\mathbf{u g} / \mathrm{L}$ & $\mathbf{E X}$ & 14.2 \\
\hline & Dichloromethane (Methylene chloride) & $<50.4$ & $<14$ & $<2.7$ & $<10$ & 1 & $\mathbf{U}$ & $\mathbf{u g} / \mathbf{L}$ & EX & $<$ EQL \\
\hline & Ethyl methacrylate & & & & $<5$ & 1 & $\mathbf{U}$ & $\mathbf{u g} / \mathbf{L}$ & EX & $<\mathbf{E Q L}$ \\
\hline & Ethylbenzene & 54.8 & 50 & 48 & 53.6 & $\mathbf{1}$ & & ug/L & $\mathbf{E X}$ & 53.6 \\
\hline & Gross alpha & 7.46 & $<-4.94$ & 7.67 & 5.73 & 1 & $\mathbf{J}$ & $\mathrm{pCi} / \mathrm{L}$ & TM & NDD \\
\hline & Iodomethane (Methyl iodide) & $<25$ & 23 & $<5$ & $<5$ & 1 & $\mathbf{U}$ & $\mathbf{u g} / \mathrm{L}$ & $\mathbf{E X}$ & $<$ EQL \\
\hline+ & Iron, total recoverable & & & 65300 & 60200 & 1 & & $\mathbf{u g} / \mathrm{L}$ & $\mathbf{E X}$ & 60200 \\
\hline & Isobutyl alcohol & $<500$ & $<500$ & $<100$ & & & & & & \\
\hline & Lead, total recoverable & $<47$ & $<5$ & $<5$ & $<100$ & 1 & $\mathbf{U}$ & ug/L & $\mathbf{E X}$ & $<$ EQL \\
\hline & Mercury, total recoverable & $<.7$ & $<.2$ & $<.2$ & $<.5$ & 1 & $\mathbf{U}$ & ug/L & $\mathbf{E X}$ & $<$ EQL \\
\hline & Methacrylonitrile & $<50$ & $<25$ & $<5$ & $<500$ & 1 & $\mathbf{U}$ & ug/L & $\mathbf{E X}$ & $<$ EQL \\
\hline & Methyl ethyl ketone & $<50$ & $<50$ & $<10$ & $<10$ & 1 & $\mathbf{U}$ & $\mathbf{u g} / \mathbf{L}$ & $\mathbf{E X}$ & $<$ EQL \\
\hline & Methyl isobutyl ketone & $<50$ & $<11$ & $<6.8$ & $<5$ & 1 & $\mathbf{U}$ & ug/L & $\mathbf{E X}$ & $<$ EQL \\
\hline & Methyl methacrylate & & $<25$ & $<5$ & $<50$ & 1 & $\mathbf{U}$ & $\mathbf{u g} / \mathrm{L}$ & $\mathbf{E X}$ & $<$ EQL \\
\hline & Pentachloroethane & & & & $<\mathbf{2 0 0}$ & 1 & $\mathbf{U}$ & $\mathrm{ug} / \mathrm{L}$ & $\mathbf{E X}$ & $<\mathbf{E Q L}$ \\
\hline & Propionitrile & $<250$ & $<25$ & $<5$ & & & & & & \\
\hline & Selenium, total recoverable & $<66$ & $<5$ & $<5$ & $<200$ & 1 & $\mathbf{U}$ & $\mathrm{Ig} / \mathrm{L}$ & $\mathbf{E X}$ & $<$ EQL \\
\hline & Silver, total recoverable & $<5$ & $<2$ & $<2$ & $<\mathbf{2 0}$ & 1 & $\mathbf{U}$ & $\mathbf{u g} / \mathbf{L}$ & $\mathbf{E X}$ & $<$ EQL \\
\hline & $=$ Styrene ${ }_{-}=-\ldots$ & $\leq \mathbf{2 5}$ & $\leq \mathbf{2 5}$ & $=.86$ & $\leq 5$ & 1 & $\underline{\mathbf{U}}$ & _ug/L & EXX & $\leq \mathbf{E Q L}$ \\
\hline & Tetrachloroethylene & $<25$ & $<25$ & .84 & $<5$ & 1 & $\mathbf{U}$ & $\mathbf{u g} / \mathrm{L}$ & $\mathbf{E X}$ & $<$ EQL \\
\hline & Toluene & 114 & 98 & 99 & 92.7 & 1 & & ug/L & $\mathbf{E X}$ & 92.7 \\
\hline+ & Trichloroethylene & 14.4 & 16 & 14 & 9.52 & 1 & & ug/L & EX & 9.52 \\
\hline & Trichlorofluoromethane & $<25$ & 21 & 15 & $<5$ & 1 & $\mathbf{U}$ & $\mathbf{u g} / \mathrm{L}$ & $\mathbf{E X}$ & $<$ EQL \\
\hline+ & Tritium & 26.91 & 25400 & 33.83 & 34.53 & 1 & & pCi/ml & TM & $>\mathbf{2 0}$ \\
\hline & Vinyl acetate & $<50$ & $<25$ & $<5$ & $<20$ & 1 & $\mathbf{U}$ & ug/L & $\mathbf{E X}$ & $<$ EQL \\
\hline & Xylenes & 185 & 120 & 110 & 116 & 1 & & Ig/L & $\mathbf{E X}$ & 116 \\
\hline+ & cis-1,2-Dichloroethylene & & & & 201 & $\mathbf{1}$ & & $g / L$ & $\mathbf{E X}$ & 201 \\
\hline & cis-1,3-Dichloropropene & $<25$ & $<25$ & $<5$ & $<5$ & 1 & $\mathbf{U}$ & ug/L & EX & $<\mathbf{E Q L}$ \\
\hline
\end{tabular}

Note: Flagging, dilution factors, modifiers, and laboratories are for fourth quartet 998 data only. See Appendix B for ilagging criteria.

* - exceeded holding time for fourth quarter 1998 .

+ - exceeded screening level or final primary drinking water standard for fourth quartert 998 .

Sanitary Landfitl

D-118

Fourth Quarter 1998 and Annual Summary 
WSRC-TR-99-00011

Unclassified

WELL: LFW 67C

\section{ANALYTICAL DATA}

\section{H ST Analyte}

trans-1,2-Dichloroethylene

trans-1,3-Dichloropropene

trans-1,4-Dichloro-2-butene

\section{$101998 \quad 201998$}

$<25$

$<25$

$<100$
$201998 \quad \underline{301998}$

$<25$

$<25$

$<5$

$<5$
401998

$<5$
Compliance

Lab Eilter

EX $<$ EQL

EX $<$ EQL

EX $<$ EQL

Note: Flagging, dilution factors, modifiers, and laboratories are for fourth quartet 998 data only. See Appendix B for fagging criteria. * = exceeded holding time for fourth quarter 1998.

$+=$ exceeded screening level or final primary drinking water standard for fourth quarten998 .

Sanitary Landfill

D-119

Fourth Quarter 1998 and Annual Summary 
WELL: LFW 67D



\section{ANALYTICAL DATA}

\section{H ST Analyte}

1,1,1,2-Tetrachloroethane

1,1,1-Trichloroethane

1,1,2,2-Tetrachloroethane

1,1,2-Trichloroethane

+ 1,1-Dichloroethane

+ 1,1-Dichloroethylene

1,2,3-Trichloropropane

1,2-Dibromo-3-chloropropane

1,2-Dibromoethane

1,2-Dichlorobenzene

1,2-Dichloroethane

1,2-Dichloropropane

1,3-Dichlorobenzene

1,4-Dichlorobenzene

1,4-Dioxane

2-Hexanone

Acetone

Acetonitrile (Methyl cyanide)

Acrolein

Acrylonitrile

Allyl chloride

Aluminum, total recoverable

Arsenic, total recoverable

Barium, total recoverable

Benzene

Bromodichloromethane
101998

$<5$

$4.77 \quad 3.2 \quad 5.1$

$<5<5 \quad<25$

$<5 \quad<5 \quad<25$

$31.8 \quad 43 \quad 26$

$1.69<25$

$<5<5<25$

$<5<5 \quad<25$

$<5<5<25$

$<5 \quad<5 \quad<25$

$1.12 \quad 1.7<25$

$1.35 \quad 2.1<25$

$<10<10 \quad<50 \quad<5 \quad 1 \quad$ UJ ug/L EX $<$ EQL $<5.97 \quad<10 \quad<36 \quad<20 \quad 1 \quad$ UJ ug/L EX $\quad<$ EQL

$<20<20<100$

$<20<10<50$

$<5 \quad<20 \quad<25$

$<10<10$

$<50$

54.2

$<40 \quad<8$

$\begin{array}{lll}6 & 7.7\end{array}$

$<8$

7.7

$\begin{array}{lll}<5 & <5 & <25 \\ <5 & <5 & <25\end{array}$
Compliance

Lab Eilter

$\begin{array}{lllllr}18.8 & 1 & \mathrm{~J} & \mathrm{ug} / \mathrm{L} & \text { EX } & \text { NDD } \\ <5 & 1 & \mathrm{UJ} & \mathrm{ug} / \mathrm{L} & \mathrm{EX} & <\text { EQL } \\ <5 & 1 & \mathrm{UJ} & \mathrm{ug} / \mathrm{L} & \mathrm{EX} & <\text { EQL } \\ 36.3 & 1 & \mathrm{~J} & \mathrm{ug} / \mathrm{L} & \text { EX } & \text { NDD } \\ 8.71 & 1 & \mathrm{~J} & \mathrm{ug} / \mathrm{L} & \mathrm{EX} & \text { NDD }\end{array}$

$<5 \quad 1 \quad$ UJ $\quad$ ug/L $\quad$ EX $<$ EQL

$<5 \quad 1 \quad$ UJ ug/L EX $<$ EQL

$<5 \quad 1$ UJ ug/L EX $\quad$ EQL

$<5 \quad 1$ UJ ug/L EX $<$ EQL

$<5 \quad 1$ UJ ug/L EX $<$ EQL

$<5 \quad 1$ UJ ug/L EX $\quad$ EQL

$\begin{array}{llllll}1.15 & 1 & \mathrm{~J} & \mathrm{ug} / \mathrm{L} & \mathrm{EX} & \text { NDD }\end{array}$

$<10001$ UJ ug/L EX < EQL

$<500 \quad 1 \quad \mathrm{UJ} \quad \mathrm{ug} / \mathrm{L} \quad \mathrm{EX}<\mathrm{EQL}$

$<501$ UJ ug/L EX $<$ EQL

$<501$ UJ ug/L EX $<$ EQL

$<101$ UJ ug/L EX $<$ EQL

$<2001 \mathrm{U} \quad \mathrm{ug} / \mathrm{L} \quad$ EX $<$ EQL

$<100 \quad 1 \mathrm{U}$ ug/L EX $<$ EQL

$\begin{array}{llllll}9.78 & 1 & \mathrm{~J} & \mathrm{ug} / \mathrm{L} & \mathrm{EX} & \mathrm{NDD}\end{array}$

$<5 \quad 1 \quad$ UJ $\mathrm{ug} / \mathrm{L} \quad$ EX $<$ EQL

$<5 \quad 1$ UJ $\mathrm{ug} / \mathrm{L} \quad \mathrm{EX}<\mathrm{EQL}$

Note: Flagging, dilution factors, modifiers, and laboratories are for fourth quartet 998 data only. See Appendix B for flagging criteria.

$\star=$ exceeded holding time for fourth quarter1998.

+ = exceeded screening level or final primary drinking water standard for fourth quarten 998.

Sanitary Landfill 
WSRC-TR-99-00011

Unclassified

WELL: LFW 67D

\section{ANALYTICAL DATA}

\begin{tabular}{|c|c|c|c|c|c|c|c|c|c|c|}
\hline \multicolumn{2}{|c|}{ H ST Analyte } & 101998 & 201998 & 301998 & 401998 & \multicolumn{2}{|c|}{ DEMod } & Unit & Lab & Eilter \\
\hline & Bromoform & $<5$ & $<5$ & $<25$ & $<5$ & 1 & UJ & $\mathbf{u g} / \mathrm{L}$ & EX & $<$ EQL \\
\hline & Bromomethane (Methyl bromide) & $<10$ & $<10$ & $<\mathbf{5 0}$ & $<5$ & 1 & $\mathbf{U} \mathbf{J}$ & ug/L & EX & $<$ EQL \\
\hline & Cadmium, total recoverable & $<4.7$ & $<2$ & $<2$ & $<10$ & 1 & $\mathbf{U}$ & $\mathrm{ug} / \mathrm{L}$ & $\mathbf{E X}$ & $<$ EQL \\
\hline & Carbon disulfide & $<\mathbf{5}$ & $<\mathbf{5}$ & $<25$ & $<\mathbf{5}$ & $\mathbf{1}$ & UJ & $\mathrm{ug} / \mathrm{L}$ & EX & $<\mathbf{E Q L}$ \\
\hline & Carbon tetrachloride & $\leq \mathbf{5}$ & $<5$ & $<\mathbf{2 5}$ & $<5$ & 1 & UJ & ug/L & EX & $<$ EQL \\
\hline & Chlorobenzene & $<5$ & $<5$ & $<25$ & $<\mathbf{5}$ & 1 & $\mathbf{U J}$ & ug/L & $\mathbf{E X}$ & $<\mathbf{E Q L}$ \\
\hline & Chloroethane & $<10$ & $<10$ & $<50$ & $<10$ & 1 & UJ & ug/L & $\mathbf{E X}$ & $<\mathbf{E Q L}$ \\
\hline & Chloroethene (Vinyl chloride) & $<10$ & $<10$ & $<\mathbf{5 0}$ & $<5$ & 1 & UJ & $\mathrm{ug} / \mathrm{L}$ & EX & $<$ EQL \\
\hline & Chloroform & $<5$ & $<5$ & $<25$ & $<5$ & 1 & $\mathbf{U J}$ & $\mathrm{ug} / \mathrm{L}$ & EX & $<$ EQL \\
\hline & Chloromethane (Methyl chloride) & $<10$ & $<10$ & 5.8 & $<5$ & 1 & $\mathbf{U} \mathbf{J}$ & $\mathrm{ug} / \mathrm{L}$ & EX & $<$ EQL \\
\hline & Chloroprene & $<5$ & $<5$ & $<25$ & $<\mathbf{5 0}$ & 1 & $\mathbf{U J}$ & $\mathrm{ug} / \mathrm{L}$ & EX & $<$ EQL \\
\hline & Chromium, total recoverable & $<7$ & $<3$ & 1.4 & 3.68 & 1 & $\mathbf{J}$ & ug/L & $\mathbf{E X}$ & NDD \\
\hline & Dibromochloromethane & $<5$ & $<5$ & $<25$ & $<5$ & 1 & $\mathbf{U} \mathbf{J}$ & $\mathrm{ug} / \mathrm{L}$ & $\mathbf{E X}$ & $<$ EQL \\
\hline & Dibromomethane (Methylene bromide) & $<5$ & $<\mathbf{5}$ & $<25$ & $<5$ & 1 & $\mathbf{U} \mathbf{J}$ & ug/L & EX & $<\mathbf{E Q L}$ \\
\hline+ & Dichlorodifluoromethane & 18.2 & 25 & 12 & 43.9 & 1 & $\mathbf{J}$ & ug/L & EX & NDD \\
\hline & Dichloromethane (Methylene chloride) & $<8.02$ & $<3.7$ & $<49$ & $<10$ & 1 & UJ & $\mathrm{ug} / \mathrm{L}$ & EX & $<$ EQL \\
\hline & Ethyl methacrylate & & & & $<5$ & 1 & UJ & ug/L & EX & $<$ EQL \\
\hline & Ethylbenzene & 1.98 & 3.7 & $<25$ & $<5$ & $\mathbf{1}$ & UJ & ug/L & EX & $<$ EQL \\
\hline & Gross alpha & 3.75 & $<1.53$ & 2.89 & 7.64 & 1 & $\mathbf{J}$ & $\mathrm{pCi} / \mathrm{L}$ & TM & NDD \\
\hline & lodomethane (Methyl iodide) & $<\mathbf{5}$ & $<5$ & $<25$ & $<5$ & 1 & UJ & ug/L & $\mathbf{E X}$ & $<$ EQL \\
\hline+ & Iron, total recoverable & & & 71.6 & 447 & 1 & & ug/h & EX & 447 \\
\hline & Isobutyl alcohol & $<100$ & $<100$ & $<500$ & & & & & & \\
\hline & Lead, total recoverable & $<47$ & $<5$ & $<5$ & $<100$ & 1 & $\mathbf{U}$ & $\mathbf{u g} / \mathbf{L}$ & EX & $<$ EQL \\
\hline & Mercury, total recoverable & $<.7$ & .06 & .05 & $<.5$ & 1 & $\mathbf{u}$ & $\mathbf{u g} / \mathrm{L}$ & $\mathbf{E X}$ & $<$ EQL \\
\hline & Methacrylonitrile & $<10$ & $<5$ & $<25$ & $<500$ & 1 & UJ & ug/L & $\mathbf{E X}$ & $<$ EQL \\
\hline & Methyl ethyl ketone & $<10$ & $<10$ & $<\mathbf{5 0}$ & $<10$ & 1 & UJ & ug/L & EX & $<$ EQL \\
\hline & Methyl isobutyl ketone & $<10$ & $<12$ & $<60$ & $<5$ & 1 & UJ & ug/L & $\mathbf{E X}$ & $<$ EQL \\
\hline & Methyl methacrylate & & $<5$ & $<25$ & $<50$ & 1 & UJ & ug/L & $\mathbf{E X}$ & $<\mathbf{E Q L}$ \\
\hline & Pentachloroethane & & & & $<\mathbf{2 0 0}$ & 1 & $\mathbf{U J}$ & ug/L & $\mathbf{E X}$ & $<\mathbf{E Q L}$ \\
\hline & Propionitrile & $<\mathbf{5 0}$ & $<5$ & $<25$ & & & & & & \\
\hline & Selenium, total recoverable & $<66$ & $<5$ & $<5$ & $<\mathbf{2 0 0}$ & 1 & $\mathbf{U}$ & $\mathbf{u g} / \mathrm{L}$ & $\mathbf{E X}$ & $<$ EQL \\
\hline & Silver, total recoverable & $<5$ & $<2$ & $<2$ & $<20$ & 1 & $\mathbf{U}$ & $\mathrm{ug} / \mathrm{L}$ & $\mathbf{E X}$ & $<$ EQL \\
\hline & Styrene & $<5$ & $<5$ & $<25$ & $<5$ & 1 & $\mathbf{U} \mathbf{J}$ & $\mathrm{ug} / \mathrm{L}$ & $\mathbf{E X}$ & $<\mathbf{E Q L}$ \\
\hline & Tetrachloroethylene & 3.17 & 3.6 & $<25$ & 4.07 & 1 & $\mathbf{J}$ & ug/L & $\mathbf{E X}$ & NDD \\
\hline & Toluene & $<5$ & $<5$ & $<25$ & $<5$ & 1 & UJ & $u g / L$ & EX & $<$ EQL \\
\hline+ & Trichloroethylene & 12.2 & 17 & 7.4 & 11.6 & 1 & $\mathbf{J}$ & ug/L & $\mathbf{E X}$ & NDD \\
\hline+ & Trichlorofluoromethane & 40.1 & 11 & 11 & 21.9 & 1 & $\mathbf{J}$ & ug/L & $\mathbf{E X}$ & NDD \\
\hline & Tritium & 1.91 & 2060 & 6.14 & 3.66 & 1 & & $\mathrm{pCi} / \mathrm{ml}$ & $\mathrm{TM}$ & 3.66 \\
\hline & Vinyl acetate & $<10$ & $<5$ & $<25$ & $<20$ & 1 & UJ & $g / L$ & $\mathbf{E X}$ & $<$ EQL \\
\hline & Xylenes & 16.9 & 34 & 7.1 & 5.06 & 1 & $\mathbf{J}$ & ug/L & $\mathbf{E X}$ & NDD \\
\hline & cis-1,2-Dichloroethylene & & & & $<5$ & 1 & UJ & $\mathrm{ug} / \mathrm{L}$ & EX & $<\mathbf{E Q L}$ \\
\hline & cis-1,3-Dichloropropene & $<5$ & $<5$ & $<25$ & $<5$ & 1 & UJ & $g / L$ & EX & $<\mathbf{E Q L}$ \\
\hline
\end{tabular}

Note: Flagging, dilution factors, modifiers, and labontories are for fourth quartei 998 data only. See Appendix B for Angging criteria. * = exceeded holding time for fourth quarter 1998.

+ exceeded screening level or final primary drinking water standard for fourth quarten 998 . 
WELL: LFW 67D

\section{ANALYTICAL DATA}

\section{H ST Analyte}

trans-1,2-Dichloroethylene trans-1,3-Dichloropropene trans-1,4-Dichloro-2-butene

$\begin{array}{ccc}101998 & 201998 & 301998 \\ <5 & & \\ <5 & <5 & <25 \\ <20 & <5 & <25\end{array}$

\begin{tabular}{|c|c|c|c|}
\hline 401998 & & Mo & Uni \\
\hline$<5$ & 1 & UJ & $g / L$ \\
\hline$<5$ & 1 & UJ & $g / 1$ \\
\hline$<20$ & 1 & $\mathbf{U} \mathbf{J}$ & $\mathrm{ug} / \mathrm{L}$ \\
\hline
\end{tabular}

\section{Compliance}

Lab Eilter

EX $<$ EQL

EX $<$ EQL

EX $<$ EQL

Note: Flagging, dilution factors, modifiers, and laboratories are for fourth quartei998 data only. See Appendix B for nagging eriteria. * = exceeded holding time for fourth quarter 1998 .

+ - exceeded sereening level or final primary drinking water standard for fourth quarten 998. 
WSRC-TR-99-00011

Unclassified

WELL: LFW 68D

\begin{tabular}{|c|c|c|c|c|c|c|}
\hline SRS Coord, & Lat/Longitude & Screen Zone Elevation & Top of Casing & Casing & Pump & Eormation \\
\hline $\begin{array}{l}\text { N } 83031.6 \\
\text { E } 46868.0\end{array}$ & $\begin{array}{l}\text { 33.286 Deg } N \\
\text { 81.705 Deg } W\end{array}$ & $144.6-124.6 \mathrm{ft} \mathrm{msl}$ & 161.4 ft ms! & VC & $\mathbf{V}$ & U Steed Pond \\
\hline
\end{tabular}

$\begin{array}{llll}\text { SAMPLE DATE } & 12 / 08 / 97 & 03 / 12 / 98 & 06 / 05 / 98\end{array}$

\section{FIELD DATA}

\section{Analyte \\ Water Elevation \\ Depth to water \\ pH \\ Sp. Conductance \\ Water temperature \\ Alkalinity as $\mathrm{CaCO3}$ \\ Turbidity \\ Volumes purged \\ Sampling code}

\begin{tabular}{lllll}
101998 & 201998 & $\frac{301998}{401998}$ & Unit \\
\hline 141.06 & -145.59 & 141.62 & & ft msl \\
18.34 & 13.81 & 17.78 & & ft \\
4.6 & 4.3 & 5 & pH \\
38 & 38 & 52 & US/cm \\
19 & 16.6 & 19.9 & deg. C \\
0 & 0 & 0 & mg/L \\
.8 & .8 & .8 & NTU \\
14.5337 & 8.48725 & 6.09115 & well volumes
\end{tabular}

\section{ANALYTICAL DATA}

\section{H ST Analyte}

1,1,1,2-Tetrachloroethane

1,1,1-Trichloroethane

1,1,2,2-Tetrachloroethane

1,1,2-Trichloroethane

1,1-Dichloroethane

1,1-Dichloroethylene

1,2,3-Trichloropropane

1,2-Dibromo-3-chloropropane

1,2-Dibromoethane

1,2-Dichloroethane

1,2-Dichloropropane

1,4-Dichlorobenzene

2-Hexanone

Acetone

Acetonitrile (Methyl cyanide)

Acrolein

Acrylonitrile

Allyl chloride

Aluminum, total recoverable

Arsenic, total recoverable

Barium, total recoverable

Benzene

Bromodichloromethane

Bromoform

Bromomethane (Methyl bromide)

Cadmium, total recoverable

$\begin{array}{ccc}101998 & 201998 & 301998 \\ <5 & <5 & <5 \\ <5 & <5 & <5 \\ <5 & <5 & <5 \\ <5 & <5 & <5 \\ <5 & <5 & <5 \\ <5 & <5 & <5 \\ <5 & <5 & <5 \\ <5 & <5 & <5 \\ <5 & <5 & <5 \\ <5 & <5 & <5 \\ <5 & <5 & <5 \\ <5 & <5 & <5 \\ <10 & <10 & <10 \\ <10 & 18 & <10 \\ <20 & <20 & <20 \\ <20 & <10 & <10 \\ <5 & <20 & <5 \\ <10 & <10 & <10 \\ <40 & <8 & <8 \\ 6.6 & 5.3 & 5 \\ <5 & <5 & <5 \\ <5 & <5 & <5 \\ <5 & <5 & <5 \\ <10 & <10 & <10 \\ <4.7 & <2 & 1 \\ & <5 \\ <5 & & 45 \\ <5 & 55\end{array}$

Note: Flagging, dilution factors, modifiers, and laboratories are for fourth quartet 998 data only. See Appendix B for fagging criteria. * = exceeded holding time for fourth quarter 1998 .

$+=$ exceeded screening level or final primary drinking water standard for fourth quarter1998 .

Sanitary Landfill

D-123

Fourth Quarter 1998 and Annual Summary 


\section{ANALYTICAL DATA}

H ST Analvte

Carbon disulfide

Carbon tetrachloride

Chlorobenzene

Chloroethane

Chloroethene (Vinyl chloride)

Chloroform

Chloromethane (Methyl chloride)

Chloroprene

Chromium, total recoverable

Dibromochloromethane

Dibromomethane (Methylene bromide)

Dichlorodifluoromethane

Dichloromethane (Methylene chloride)

Ethylbenzene

Gross alpha

Iodomethane (Methyl iodide)

Iron, total recoverable

Isobutyl alcohol

Lead, total recoverable

Mercury, total recoverable

Methacrylonitrile

Methyl ethyl ketone

Methyl isobutyl ketone

Methyl methacrylate

Propionitrile

Selenium, total recoverable

Silver, total recoverable

Styrene

Tetrachloroethylene

Toluene

Trichloroethylene

Trichlorofluoromethane

Tritium

Vinyl acetate

Xylenes

cis-1,3-Dichloropropene

trans-1,2-Dichloroethylene

trans-1,3-Dichloropropene

trans-1,4-Dichloro-2-butene
101998

$<5$

$<5<5<5$

$<5<5<5$

$<10<10<10$

$\leq 10<10<10$

$<5<5<5$

$<10<10<10$

$<5<5 \quad<5$

$1.7<3 \quad 1.2$

$<5<5<5$

$<5<5<5$

$<10<5<5$

$<18.2<2<3$

$<5<5<5$

$\begin{array}{lll}1.37 & 3.66 \quad 4.63\end{array}$

$<5<5 \quad<5$

1790

$<100<100<100$

$<47<5 \quad 15$

$.263 \quad .07<.2$

$<10<5<5$

$<10<10<10$

$<10<12<12$

$<5<5$

$<50<5<5$

$<66<5<5$

$<5<2<2$

$<5<5 \quad<5$

$<5 \quad<5 \quad<5$

$<5<5 \quad<5$

$<5<5 \quad<5$

$<5<5 \quad<5$

$.98 \ldots$

$<10<5<5$

$<5<5 \quad<5$

$<5 \quad<5 \quad<5$

$<5$

$<5<5 \quad<5$

$<20<5<5$
Compliance

Lab Eilter 
WELL: LFW 69C

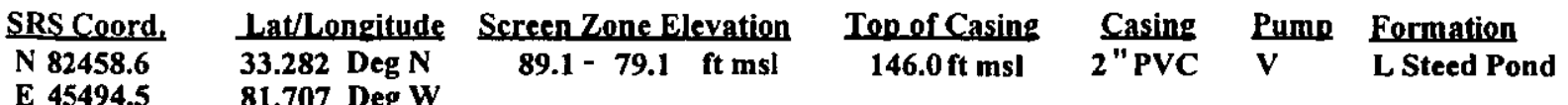

SAMPLE DATE

FIELD DATA

Analyte

Water Elevation

Depth to water

pH

Sp. Conductance

Water temperature

Alkalinity as $\mathrm{CaCO} 3$

Turbidity

Volumes purged

Sampling code $\begin{array}{lll}12 / 04 / 97 & 03 / 12 / 98 & 06 / 05 / 98\end{array}$

\begin{tabular}{|c|c|c|c|c|}
\hline 101998 & 201998 & 301998 & 401998 & Unit \\
\hline 135.49 & 136.26 & 135.73 & & ft msl \\
\hline 8.61 & 7.84 & 8.37 & & ft \\
\hline 3.8 & 3.6 & 3.9 & & pH \\
\hline 54 & 46 & 50 & & $\mathrm{uS} / \mathrm{cm}$ \\
\hline 17 & 17 & 19.7 & & deg. $C$ \\
\hline 0 & 0 & 0 & & $\mathrm{mg} / \mathbf{L}$ \\
\hline .2 & .4 & .2 & & NTU \\
\hline 2.51058 & 4.64596 & 3.54207 & & well volumes \\
\hline
\end{tabular}

ANALYTICAL DATA

H ST Analyte

1,1,1,2-Tetrachloroethane

1,1,1-Trichloroethane

1,1,2,2-Tetrachloroethane

1,1,2-Trichloroethane

1,1-Dichloroethane

1,1-Dichloroethylene

1,2,3-Trichloropropane

1,2-Dibromo-3-chloropropane

1,2-Dibromoethane

1,2-Dichloroethane

1,2-Dichloropropane

1,4-Dichlorobenzene

2-Hexanone

Acetone

Acetonitrile (Methyl cyanide)

Acrolein

Acrylonitrile

Allyl chloride

Aluminum, total recoverable

Arsenic, total recoverable

Barium, total recoverable

Benzene

Bromodichloromethane

Bromoform

Bromomethane (Methyl bromide)

Cadmium, total recoverable

$\begin{array}{ccc}101998 & 201998 & 301998 \\ <5 & <5 & <5 \\ <5 & <5 & <5 \\ <5 & <5 & <5 \\ <5 & <5 & <5 \\ <5 & <5 & <5 \\ <5 & <5 & <5 \\ <5 & <5 & <5 \\ <5 & <5 & <5 \\ <5 & <5 & <5 \\ <5 & <5 & <5 \\ <5 & <5 & <5 \\ <5 & <5 & <5 \\ <10 & <10 & <10 \\ <8.67 & <10 & <4.4 \\ <20 & <20 & <20 \\ <20 & <10 & <10 \\ <5 & <20 & <5 \\ <10 & <10 & <10 \\ & & 321 \\ <40 & <8 & <8 \\ 6 & 6.1 & 6 \\ <5 & <5 & <5 \\ <5 & <5 & <5 \\ <5 & <5 & <5 \\ <10 & <10 & <10 \\ .49 & <2 & .5\end{array}$

Compliance Lab Eilter

Note: Flagging, dilution factors, modifiers, and laboratories are for fourth quartel998 data only. See Appendix B for fagging criteria.

* = exceeded holding time for fourth quarter 1998 .

+ = exceeded screening level or final primary drinking water standard for fourth quarten 998 . 
WEIL: LFW 69C

ANALYTICAL DATA

H ST Analyte

Carbon disulfide

Carbon tetrachloride

Chlorobenzene

Chloroethane

Chloroethene (Vinyl chloride)

Chloroform

Chloromethane (Methyl chloride)

Chloroprene

Chromium, total recoverable

Dibromochloromethane

Dibromomethane (Methylene bromide)

Dichlorodifluoromethane

Dichloromethane (Methylene chloride)

Ethylbenzene

Gross alpha

lodomethane (Methyl iodide)

Iron, total recoverable

Isobutyl alcohol

Lead, total recoverable

Mercury, total recoverable

Methacrylonitrile

Methyl ethyl ketone

Methyl isobutyl ketone

Methyl methacrylate

Propionitrile

Selenium, total recoverable

Silver, total recoverable

Styrene

Tetrachloroethylene

Toluene

Trichloroethylene

Trichlorofluoromethane

Tritium

Vinyl acetate

Xylenes

cis-1,3-Dichloropropene

trans-1,2-Dichloroethylene

trans-1,3-Dichloropropene

trans-1,4-Dichloro-2-butene
101998

$<5$

$<5$

$<5$

$<10$

$<10$

$<5$

$<10$

$<5$

$<7$

$<5$

$<5$

$<10$

$<5.54$

$<5$

13.73

$<5$

$<100$

$<47$

$<7$

$<.7$

$<10$

$<10$

$<50$

$<66$

$<5$

$<5$

$<5$

$<5$

$<5$

$<5$

$<.77$

$<10$

$<5$

$<5$

$<5$

$<5$
$<5$

$<20$
201998

$<5$

$<5$

$<5$

$<10$

$<10$

$<5$

$<10$

$<5$

$<3$

$<5$

$<5$

$<5$

$<2$

$<5$

5.14

$<5$

301998

401998

Compliance

$<5$

$<5$

$<5$

$<10$

$<10$

$<5$

$<10$

$<5$

1.2

$<5$

$<5$

$<5$

$<3$

$<5$

12.25

$<5$

15.3

$<100<100$

$<5<5$

$<.2 \quad<.2$

$<5 \quad<5$

$<10<10$

$<12<12$

$<5<5$

$<5<5$

$<5<5$

$<2 \quad<2$

$<5<5$

$<5<5$

$<5<5$

$<5 \quad<5$

$<5<5$

$813 \ldots<.21$

$<5<5$

$<5<5$

$<5<5$

$<5<5$

$<5<5$

Note: Flagging, dilution factors, modifiers, and laboratories are for fourth quartel998 data only. See Appendix B for flagging criteria. * = exceeded holding time for fourth quarter 1998 .

+ - exceeded screening level or final primary drinking water standard for fourth quarten 998 .

Sanitary Landfill

D-126

Fourth Quarter 1998 and Annual Summary 
WELL: LFW 69D

\begin{tabular}{|c|c|c|c|c|c|c|}
\hline SRS Coord. & Lat/Longitude & Screen Zone Elevation & Top of Casing & Casing & Pump & Eormation \\
\hline $\begin{array}{l}\text { N } 824 \\
\text { E } 455\end{array}$ & $\begin{array}{ll}\text { 33.282 } & \text { Deg } N \\
\text { 81.707 } & \text { Deg } W\end{array}$ & $139.0-119.0$ ft msl & & $2 " \mathrm{PVC}$ & $\mathbf{V}$ & \\
\hline
\end{tabular}

\section{FIELD DATA}

Analyte
Water Elevation
Depth to water
pH
Sp. Conductance
Water temperature
Alkalinity as CaCO3
Turbidity
Volumes purged
Sampling code

\section{ANALYTICAL DATA}

\section{H ST Analvte}

1,1,1,2-Tetrachloroethane 1,1,1-Trichloroethane 1,1,2,2-Tetrachloroethane 1,1,2-Trichloroethane 1,1-Dichloroethane 1,1-Dichloroethylene 1,2,3-Trichloropropane 1,2-Dibromo-3-chloropropane 1,2-Dibromoethane

1,2-Dichlorobenzene 1,2-Dichloroethane 1,2-Dichloropropane 1,3-Dichlorobenzene

1,4-Dichlorobenzene

1,4-Dioxane

2-Hexanone

Acetone

Acetonitrile (Methyl cyanide)

Acrolein

Acrylonitrile

Allyl chloride

Aluminum, total recoverable Arsenic, total recoverable Barium, total recoverable Benzene Bromodichloromethane

$\begin{array}{lllll}101998 & 201998 & \frac{301998}{401998} & \text { Unit } \\ .135 .5 & -136.5 & 135.64 & 135.65 & \text { ft msl } \\ 8.5 & 7.5 & 8.36 & 8.35 & \text { ft } \\ 4.4 & 5 & 5 & 5.1 & \text { pH } \\ 58 & 48 & 53 & 52 & \text { uS/cm } \\ 17 & 16 & 23.4 & 27.3 & \text { deg. C } \\ 0 & 10 & 3 & 8 & \text { mg/L } \\ 3.9 & 2 & 7.4 & .8 & \text { NTU } \\ 11.1461 & 11.5107 & 8.78517 & 12.6829 & \text { well volumes }\end{array}$

Compliance

\begin{tabular}{|c|c|c|c|c|c|c|c|c|}
\hline 101998 & 201998 & 301998 & 401998 & \multicolumn{3}{|c|}{ DFMod Unit } & Lab & Filter \\
\hline$<5$ & $<5$ & $<5$ & & & & & & \\
\hline$<5$ & $<5$ & $<5$ & $<5$ & 1 & $\mathbf{U}$ & ug/L & $\mathbf{E X}$ & $<$ EQL \\
\hline$<5$ & $<5$ & $<5$ & $<5$ & 1 & $\mathbf{U}$ & $u g / L$ & EX & $<\mathrm{EQL}$ \\
\hline$<5$ & $<5$ & $<5$ & $<5$ & $\mathbf{1}$ & $\mathbf{U}$ & ug/L & EX & $<\mathbf{E Q L}$ \\
\hline 10.2 & 3.3 & 9 & 5.56 & 1 & & ug/L & EX & 5.56 \\
\hline$<5$ & $<5$ & $<5$ & $<5$ & 1 & $\mathbf{U}$ & $\mathbf{u g} / \mathbf{L}$ & EX & $<$ EQL \\
\hline$<5$ & $<5$ & $<5$ & & & & & & \\
\hline$<5$ & $<5$ & $<5$ & $<5$ & 1 & $\mathbf{U}$ & $\mathbf{u g} / \mathrm{L}$ & $\mathbf{E X}$ & $<\mathrm{EQL}$ \\
\hline$<5$ & $<5$ & $<5$ & $<5$ & 1 & $\mathbf{U}$ & $\mathbf{u g} / \mathbf{L}$ & $\mathbf{E X}$ & $<$ EQL \\
\hline & & & $<5$ & 1 & $\mathbf{U}$ & $\mathrm{ug} / \mathrm{L}$ & EX & $<\mathbf{E Q L}$ \\
\hline$<5$ & $<5$ & $<5$ & $<5$ & 1 & $\mathbf{U}$ & $\mathbf{u g} / \mathbf{L}$ & EX & $<$ EQL \\
\hline$<5$ & $<5$ & $<5$ & $<5$ & 1 & $\mathbf{U}$ & $\mathbf{u g} / \mathbf{L}$ & EX & < EQL \\
\hline & & & $<5$ & 1 & $\mathbf{U}$ & ug/L & EX & $<$ EQL \\
\hline 30.1 & 9.9 & 21 & 19 & 1 & & ug $/ \mathrm{L}$ & EX & 19 \\
\hline & & & $<1000$ & 1 & $\mathbf{U}$ & $\mathrm{ug} / \mathrm{L}$ & EX & $<$ EQL \\
\hline$<10$ & $<10$ & $<10$ & $<5$ & 1 & $\mathbf{U}$ & ug/L & EX & $<$ EQL \\
\hline$<10$ & $<10$ & $<10$ & $<20$ & 1 & $\mathbf{U}$ & ug/L & EX & $<$ EQL \\
\hline$<20$ & $<20$ & $<20$ & $<500$ & 1 & $\mathbf{U}$ & $\mathrm{ug} / \mathrm{L}$ & EX & $<\mathbf{E Q L}$ \\
\hline$<20$ & $<10$ & $<10$ & $<50$ & 1 & $\mathbf{U}$ & $\mathbf{u g} / \mathbf{L}$ & EX & < EQL \\
\hline$<5$ & $<20$ & $<5$ & $<50$ & 1 & $\mathbf{U}$ & $\mathbf{u g} / \mathbf{L}$ & EX & $<$ EQL \\
\hline$<10$ & $<10$ & $<10$ & $<10$ & 1 & $\mathbf{U}$ & ug/L & EX & < EQL \\
\hline & & 110 & $<200$ & 1 & $\mathbf{U}$ & ug/L & EX & $<\mathrm{EQL}$ \\
\hline 8.4 & $<8$ & $<8$ & $<100$ & 1 & $\mathbf{U}$ & ug/L & EX & $<$ EQL \\
\hline 10.1 & 23.2 & 8 & 9.78 & 1 & $\mathbf{J}$ & ug/L & EX & NDD \\
\hline 1.05 & $<5$ & .64 & $<5$ & 1 & $\mathbf{U}$ & ug/L & EX & $<$ EQL \\
\hline$<5$ & $<5$ & $<5$ & $<5$ & 1 & $\mathbf{U}$ & ug/L & EX & $<$ EQL \\
\hline
\end{tabular}

Note: Flagging, dilution factors, modifiers, and laboratories are for fourth quartet 998 data only. See Appendix B for flagging criteria. * s exceeded holding time for fourth quarter 1998.

$+=$ exceeded screening level or final primary drinking water standard for fourth quarten998 . 
WELL: LFW 69D

\section{ANALYTICAL DATA}

H ST Analyte

Bromoform

Bromomethane (Methyl bromide)

Cadmium, total recoverable

Carbon disulfide

Carbon tetrachloride

Chlorobenzene

Chloroethane

+ Chloroethene (Vinyl chloride)

Chloroform

Chloromethane (Methyl chloride)

Chloroprene

Chromium, total recoverable

Dibromochloromethane

Dibromomethane (Methylene bromide)

Dichlorodifluoromethane

Dichloromethane (Methylene chloride)

Ethyl methacrylate

Ethylbenzene

Gross alpha

lodomethane (Methyl iodide)

+ Iron, total recoverable

Isobutyl alcohol

Lead, total recoverable

Mercury, total recoverable

Methacrylonitrile

Methyl ethyl ketone

Methyl isobutyl ketone

Methyl methacrylate

Pentachloroethane

Propionitrile

Selenium, total recoverable

Silver, total recoverable

Styrene

Tetrachloroethylene

Toluene

Trichloroethylene

Trichlorofluoromethane

Tritium

Vinyl acetate

Xylenes

cis-1,2-Dichloroethylene

cis-1,3-Dichloropropene

\section{$101998 \quad 201998 \quad 301998$}

$<5$

$<10$

$<4.7$

$<5$

$<5$.

9.25

$<10$

18.5

$<5$

$<10$

$<5$

$<2.4$

$<5$

$<5$

1.32

$<6.95$

$$
<5
$$

$<10$

$<5$

$<10$

$<2$

$<5$

$<5$

3.8

$<10$

7.8

$<5$

$<10$

$<5$

$<3$

$<3$

$<5$

$<5$

6.95

$<5$

1.21

$<5$

$<100<100$

$<47$

$<.7$

$<10$

$<10$

$<10$

$<5$

$<5$

3140

$<100$

$<5$

13.1

$<.2$

$<5$

$<10$

$<5$

$<10$

$<12$

$<5$

$<12$

$<5$
401998

$<5$

$<5 \quad 1 \quad \mathrm{U} \quad \mathrm{ug} / \mathrm{L}$

$<10 \quad 1 \quad \mathrm{U} \quad \mathrm{ug} / \mathrm{L}$

$<5 \quad 1 \quad$ U ug/L

$<5 \quad 1 \quad U \quad u g / L$

5.921 ug/L

$<10 \quad 1 \mathrm{U} \quad \mathrm{ug} / \mathrm{L}$

7.991

$<5$

$<5$

$<5$
$<50$

$<50$

$<10$

$<5$

$<5$

$$
<5
$$$$
<10
$$

$$
<5
$$

$$
<5
$$

$<50<5<5$

$<66<5<5$

$<5<2<2$

$<5$

$<5<5$

$<5<5$

$<5 \quad<5$

$<5 \quad<5$

8.72

5120

$<1$

$<5$

$$
<5
$$

$<5$

$<5$

$<5$

$\leq 5$

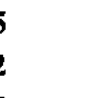$$
\begin{aligned}
& <200 \\
& <20 \\
& <5 \\
& <5 \\
& <5 \\
& <5 \\
& <5 \\
& 8.07 \\
& <20 \\
& <10 \\
& 2.71 \\
& <5
\end{aligned}
$$

Compliance

Lab Filter

EX $<$ EQL

EX < EQL

EX $<$ EQL

EX $<$ EQL

EX $<$ EQL

EX 5.92

EX < EQL

EX 7.99

EX < EQL

EX < EQL

EX < EQL

EX $<$ EQL

EX $<$ EQL

EX < EQL

EX $<$ EQL

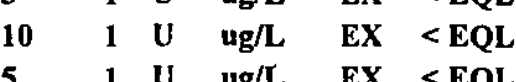

$<5$ I U $\quad$ ug/L $\quad$ EX $<$ EQL

$<.791$ UIJ $\mathrm{pCi} / \mathrm{L} \quad \mathrm{TM}<\mathrm{EQL}$

$<5 \quad 1 \quad \mathrm{U} \quad \mathrm{ug} / \mathrm{L} \quad \mathrm{EX}<\mathrm{EQL}$

$\begin{array}{lllll}629 & 1 & \mathrm{ug} / \mathrm{L} & \mathrm{EX} & 629\end{array}$

$<100 \quad 1 \quad U$ ug/L EX $<$ EQL

$<.5 \quad 1 \quad \mathrm{U} \quad \mathrm{ug} / \mathrm{L} \quad \mathrm{EX}<\mathrm{EQL}$

$<5001 \mathrm{U} \quad \mathrm{ug} / \mathrm{L} \quad \mathrm{EX}<\mathrm{EQL}$

$<10 \quad 1 \quad \mathrm{U} \quad \mathrm{ug} / \mathrm{L} \quad \mathrm{EX}<\mathrm{EQL}$

$<5 \quad 1 \quad$ U $\quad$ ug/L $\quad$ EX $<$ EQL

$<50 \quad 1 \quad$ U $\quad$ ug/L $\quad$ EX $<$ EQL

$<2001 \mathrm{U}$ ug/L EX $<$ EQL

Note: Flagging, dilution factors, modifiers, and laboratories are for fourth quartet 998 data only. See Appendix B for flagging criteria.

* - exceeded holding time for fourth quarter 1998.

+- exceeded screening level or final primary drinking water standard for fourth quarten 998 .

Sanitary Landfill 
WSRC-TR-99-00011

Unclassified

WELL: LFW 69D

\section{ANALYTICAL DATA}

\section{H ST Analyte}

trans-1,2-Dichloroethylene trans-1,3-Dichloropropene trans-1,4-Dichloro-2-butene

\begin{tabular}{lccccccccc}
101998 & 201998 & 301998 & 401998 & \multicolumn{1}{c}{ DEMod Unit } & Lab & Eilter \\
$<5$ & & & $<5$ & 1 & U & ug/L & EX & $<$ EQL \\
$<5$ & $<5$ & $<5$ & $<5$ & 1 & U & ug/L & EX & $<$ EQL \\
$<20$ & $<5$ & $<5$ & $<20$ & 1 & U & ug/L & EX & $<$ EQL
\end{tabular}

Note: Flagging, dilution factors, modifiers, and laboratories are for fourth quartei998 data only. See Appendix B for Aagging criteria. * = exceeded holding time for fourth quarter 1998.

+ = exceeded screening level or final primary drinking water standard for fourth quarter1998 . 
WELL: LFW 71B

\begin{tabular}{|c|c|c|c|c|c|c|}
\hline \multirow{2}{*}{$\begin{array}{l}\text { SRS Coord, } \\
\text { N } 82616.7 \\
\text { E } 46340.4\end{array}$} & Lat/Longitude & Screen Zone Elevation & $\frac{\text { Ton of Casing }}{147.0 \mathrm{ft} \mathrm{msl}}$ & \multirow{2}{*}{$\frac{\text { Casing }}{2 " \text { PVC }}$} & \multirow{2}{*}{$\frac{\text { Pump }}{v}$} & \multirow{2}{*}{$\begin{array}{l}\text { Formation } \\
\text { L Steed Pond }\end{array}$} \\
\hline & $\begin{array}{l}\text { 33.284 Deg N } \\
\text { 81.705 Deg W }\end{array}$ & $67.0-57.0$ ft msl & $147.0 \mathrm{ft}$ msl & & & \\
\hline SAMPI & & 3/97 & $1 / 98$ & & & \\
\hline
\end{tabular}

\section{FIELD DATA}

Analyte
Water Elevation
Depth to water
pH
Sp. Conductance
Water temperature
Alkalinity as CaCO3
Turbidity
Volumes purged
Sampling code

ANALYTICAL DATA

H ST Analyte

1,1,1,2-Tetrachloroethane

1,1,1-Trichloroethane

1,1,2,2-Tetrachloroethane

1,1,2-Trichloroethane

1,1-Dichloroethane

1,1-Dichloroethylene

1,2,3-Trichloropropane

1,2-Dibromo-3-chloropropane

1,2-Dibromoethane

1,2-Dichlorobenzene

1,2-Dichloroethane

1,2-Dichloropropane

1,3-Dichlorobenzene

1,4-Dichlorobenzene

1,4-Dioxane

2-Hexanone

2-Picoline

Acetone

Acetonitrile (Methyl cyanide)

Acrolein

Acrylonitrile

Allyl chloride

Aluminum, total recoverable

Arsenic, total recoverable

Barium, total recoverable

Benzene

$\begin{array}{lll}101998 & \frac{201998}{136.4} & \frac{301998}{135.57} \\ 135.4 & -13.4 & 9.33 \\ 9.5 & 8.5 & 4.2 \\ 4.2 & 3.8 & 50 \\ 48 & 50 & 20.6 \\ 19 & 17 & 0 \\ 0 & 0 & .3 \\ 1 & .3 & 4.15726\end{array}$

Unit
ft msl
ft
pH
uS/cm
deg. C
mg/L
NTU
well volumes

$\begin{array}{ll}401998 & \text { Unit } \\ & \text { ft msl } \\ \text { ft } \\ \text { pH } \\ \text { uS/cm } \\ \text { deg. C } \\ \text { mg/L } \\ \text { NTU } \\ \text { well volumes }\end{array}$

Compliance

101998201998301998 401998 DEMod Unit Lab Filter

\begin{tabular}{|c|c|c|}
\hline$<5$ & $<5$ & $<5$ \\
\hline$<5$ & $<5$ & $<5$ \\
\hline$<5$ & $<5$ & $<5$ \\
\hline$<5$ & $<5$ & $<5$ \\
\hline$<5$ & $<5$ & $<5$ \\
\hline$<5$ & $<5$ & $<5$ \\
\hline$<5$ & $<5$ & $<5$ \\
\hline$<5$ & $<5$ & $<5$ \\
\hline$<5$ & $<5$ & $<5$ \\
\hline \multicolumn{3}{|l|}{$<1$} \\
\hline$<5$ & $<5$ & $<5$ \\
\hline$<5$ & $<5$ & $<5$ \\
\hline \multicolumn{3}{|l|}{$<1$} \\
\hline$<5$ & $<5$ & $<5$ \\
\hline \multicolumn{3}{|l|}{$<1$} \\
\hline$<10$ & $<10$ & $<10$ \\
\hline \multicolumn{3}{|l|}{$<1$} \\
\hline$<1 \overline{0}$ & $<10$ & $<10$ \\
\hline$<20$ & $<20$ & $<20$ \\
\hline$<20$ & $<10$ & $<10$ \\
\hline$<5$ & $<20$ & $<5$ \\
\hline$<10$ & $<10$ & $\begin{array}{r}<10 \\
309\end{array}$ \\
\hline$<40$ & $<8$ & $<8$ \\
\hline 5 & 4.2 & 4.5 \\
\hline$<5$ & $<5$ & $<5$ \\
\hline
\end{tabular}

Note: Flagging, dilution factors, modifiers, and laboratories are for fourth quartet998 data only. See Appendix B for nagging criteria. * = exceeded holding time for fourth quarter 1998 .

$+=$ exceeded screening level or final primary drinking water standard for fourth quarten 998 .

Sanitary Landfill

D- 130

Fourth Quarter 1998 and Annual Summary 
WELL: LFW 71B

\section{ANALYTICAL DATA}

H ST Analvte

Bromodichloromethane

Bromoform

Bromomethane (Methyl bromide)

Cadmium, total recoverable

Carbon disulfide

Carbon tetrachloride

Chlorobenzene

Chloroethane

Chloroethene (Vinyl chloride)

Chloroform

Chloromethane (Methyl chloride)

Chloroprene

Chromium, total recoverable

Dibromochloromethane

Dibromomethane (Methylene bromide)

Dichlorodifluoromethane

Dichloromethane (Methylene chloride)

Ethyl methacrylate

Ethylbenzene

Gross alpha

Iodomethane (Methyl iodide)

Iron, total recoverable

Isobutyl alcohol

Lead, total recoverable

Mercury, total recoverable

Methacrylonitrile

Methyl ethyl ketone

Methyl isobutyl ketone

Methyl methacrylate

Pentachloroethane

Propionitrile

Selenium, total recoverable

Silver, total recoverable

Styrene

Tetrachloroethylene

Toluene

Trichloroethylene

Trichlorofluoromethane

Tritium

Vinyl acetate

Xylenes

cis-1,3-Dichloropropene
101998

$<5$

$<5$

$<10$

$<4.7$

55 .

$<5$

$<5$

$<10$

$<10$

$<5$

$<10$

$<5$

3.6

$<5$

$<5$

$<10$

$<5.58$

$<1$

$<5$

$<.15$

$<5$

$<100$

$<47$

$<.7$

$<10$

$<10$

$<10$

$<1$

$<1$

$<50$

$<66$

$<5$

$<5$

$<5$

$<5$

$<5$

$<5$

$<.34$

$<10$

$<5$

$<5$

\section{8}

$<5$

$<5$

$<10$

$<2$

$<5$

$<5$

$<5$

$<10$

$<10$

$$
<5
$$

$<10$

$<5$

.7

$<5$

$<5$

$<5$

$<2.2$

$<5$

4.19

$<5$

10.09

$<5$

18.9

$<100<100$

$<5 \quad 3.6$

$<.2 \quad<.2$

$<5<5$

$<10 \quad<10$

$<12<12$

$<5 \quad<5$

$<5<5$

$<5<5$

$<2<2$

$<5 \quad<5$

$<5<5$

$<5<5$

$<5<5$

$<5<5$

$<307<-1.14$

$<5<5$

$<5 \quad<5$

$<5 \quad<5$

Note: Flagging, dilution factors, modifiers, and laboratories are for fourth quartel998 data only. See Appendix B for flagging criteria.

* = exceeded holding time for fourth quarter 1998 .

+ wexceded screening level or final primary drinking water standard for fourth quarter 998 .

Sanitary Landfill

Fourth Quarter 1998 and Annual Summary 
WELL: LFW 71B

\section{ANALYTICAL DATA}

\section{H ST Analyte}

trans-1,2-Dichloroethylene trans-1,3-Dichloropropene

trans-1,4-Dichloro-2-butene

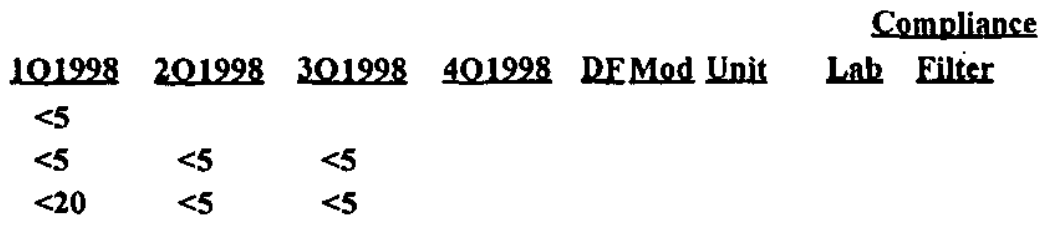

\section{Compliance}

Note: Flagging, dilution factors, modifiers, and laboratories are for fourth quartel998 data only. See Appendix B for fiagging criteria. - exceeded holding time for fourth quarter 1998 .

+ - exceeded screening level or final primary drinking water standard for fourth quarten 998 . 
WSRC-TR-99-00011

Unclassified

WELL: LFW 71C

\begin{tabular}{|c|c|c|c|c|c|c|}
\hline SRS Coord. & Lat/Longitude & Screen Zone Elevation & Ton of Casing & Casing & Pump & Eormation \\
\hline $\begin{array}{l}\text { N } 82615.8 \\
\text { E } 46329.8\end{array}$ & $\begin{array}{l}\text { 33.284 Deg } N \\
\text { 81.705 Deg } W\end{array}$ & $90.4-80.4$ ft msl & $147.2 \mathrm{ft}$ msl & $2 " \mathrm{PVC}$ & V & M Steed Pond \\
\hline
\end{tabular}

SAMPLE DATE

$\begin{array}{lll}12 / 03 / 97 & 03 / 11 / 98 & 06 / 04 / 98\end{array}$

FIELD DATA

Analyte

Water Elevation

Depth to water

pH

Sp. Conductance

Water temperature

Alkalinity as $\mathrm{CaCO} 3$

Turbidity

Volumes purged

Sampling code

$\begin{array}{lll}\frac{101998}{135.55} & \frac{201998}{136.4} & \frac{301998}{135.7} \\ 9.65 & -8.8 & 9.5 \\ & 4 & 4.5 \\ & 40 & 38 \\ & 17 & 20.2 \\ & 0 & 0 \\ 0 & .3 & .2 \\ \text { NP } & 6.72834 & 4.15017\end{array}$

401998

Unit

ft msl

ft

pH

uS/cm

deg. C

mg/L

NTU

well volumes

ANALYTICAL DATA

H ST Analyte

1,1,1,2-Tetrachloroethane

1,1,1-Trichloroethane

1,1,2,2-Tetrachloroethane

1,1,2-Trichloroethane

1,1-Dichloroethane

1,1-Dichloroethylene

1,2,3-Trichloropropane

1,2-Dibromo-3-chloropropane

1,2-Dibromoethane

1,2-Dichloroethane

1,2-Dichloropropane

1,4-Dichlorobenzene

2-Hexanone

Acetone

Acetonitrile (Methyl cyanide)

Acrolein

Acrylonitrile

Allyl chloride

Aluminum, total recoverable

Arsenic, total recoverable

Barium, total recoverable

Benzene

Bromodichloromethane

Bromoform

101998

\section{8}

301998

401998 DEMod Unit

Compliance

$<5<5$

$<5<5$

$<5 \quad<5$

$<5<5$

$<5<5$

$<5<5$

$<5<5$

$<5<5$

$<5<5$

$<5<5$

$<5<5$

$<5 \quad<5$

$<10<10$

$<10<10$

$<20<20$

$<10<10$

$<20<5$

$<10<10$

$<8<8$

$11.3 \quad 7.4$

$<5<5$

$<5<5$

$<5 \quad<5$

$<10<10$

Bromomethane (Methyl bromide)

$<2 \quad .3$

Note: Flagging, dilution factors, modifiers, and laboratories are for fourth quartel998 data only. See Appendix B for fagging eriteria. - exceeded holding time for fourth quarter 1998 .

$+=$ exceeded screening level or final primary drinking water standard for fourth quarten 998 . 
WELL: LFW 71C

ANALYTICAL DATA

H ST Analyte

Carbon disulfide

Carbon tetrachloride

Chlorobenzene

Chloroethane

Chloroethene (Vinyl chloride)

Chloroform

Chloromethane (Methyl chloride)

Chloroprene

Chromium, total recoverable

Dibromochloromethane

Dibromomethane (Methylene bromide)

Dichlorodifluoromethane

Dichloromethane (Methylene chloride)

Ethylbenzene

Gross alpha

Iodomethane (Methyl iodide)

Iron, total recoverable

Isobutyl alcohol

Lead, total recoverable

Mercury, total recoverable

Methacrylonitrile

Methyl ethyl ketone

Methyl isobutyl ketone

Methyl methacrylate

Propionitrile

Selenium, total recoverable

Silver, total recoverable

Styrene

Tetrachloroethylene

Toluene

Trichloroethylene

Trichlorofiuoromethane

-Tritium-

Vinyl acetate

Xylenes

cis-1,3-Dichloropropene

trans-1,3-Dichloropropene

trans-1,4-Dichloro-2-butene
101998

\section{8}

$<5$

$<5$

$<5$

$<10$

$<10$

$<5$

$<10$

$<5$

$1.1 \quad 1.1$

$<5<5$

$<5 \quad<5$

$<5<5$

$<2.2<3.2$

$<5<5$

3.619 .44

$<5<5$

13.8

$<100<100$

$<5<5$

$<.2<.2$

$<5<5$

$<10<10$

$<12<12$

$<5<5$

$<5<5$

$<5<5$

$<2<2$

$<5 \quad<5$

$<5 \quad<5$

$<5<5$

$<5<5$

$<5<5$

$<810_{-}=<.11$

$<5<5$

$<5 \quad<5$

$<5<5$

$<5<5$

$<5 \quad<5$

Note: Flagging, dilution factors, modifiers, and laboratories are for fourth quartel998 data only. See Appendix B for flagging crtteria. * = exceeded holding time for fourth quarter 1998.

+ - exceeded screening level or final primary drinking water standard for fourth quarten 998 .

Sanitary Landfill

D-134

Fourth Quarter 1998 and Annual Summary 
WSRC-TR-99-00011

Unclassified

WELL: LFW 71D

\begin{tabular}{|c|c|c|c|c|c|c|}
\hline SRS Coord. & Lat/Longitude & Screen Zone Elevation & Ton of Casing & Casing & Pump & Eormation \\
\hline $\begin{array}{l}\text { N } 82615.1 \\
\text { E } 46319.8\end{array}$ & $\begin{array}{l}\text { 33.284 Deg N } \\
\text { 81.705 Deg W }\end{array}$ & $135.5-115.5 \mathrm{ft} \mathrm{msl}$ & $147.4 \mathrm{ft} \mathrm{msl}$ & $2 "$ PVC & $\mathrm{V}$ & U Steed Pond \\
\hline SAMPLE D & & $12 / 03 / 97$ & $06 /$ & & & \\
\hline
\end{tabular}

\section{FIELD DATA}

Analyte
Water Elevation
Depth to water
pH
Sp. Conductance
Water temperature
Alkalinity as CaCO3
Turbidity
Volumes purged
Sampling code

\section{ANALYTICAL DATA}

H ST Analyte

1,1,1,2-Tetrachloroethane

1,1,1-Trichloroethane

1,1,2,2-Tetrachloroethane

1,1,2-Trichloroethane

1,1-Dichloroethane

1,1-Dichloroethylene

1,2,3-Trichloropropane

1,2-Dibromo-3-chloropropane

1,2-Dibromoethane

1,2-Dichloroethane

1,2-Dichloropropane

1,4-Dichlorobenzene

2-Hexanone

Acetone

Acetonitrile (Methyl cyanide)

Acrolein

Acrylonitrile

Allyl chloride

Aluminum, total recoverable

Arsenic, total recoverable

Barium, total recoverable

Benzene

Bromodichloromethane

Bromoform

Bromomethane (Methyl bromide)

Cadmium, total recoverable

$\begin{array}{lllll}101998 & 201998 & 301998 & \text { 401998 } & \text { Unit } \\ 135.4 & 137 & 135.2 & & \text { ft msl } \\ 10.1 & 8.5 & 10.3 & & \text { ft } \\ 4.6 & 5 & 4.5 & \text { pH } \\ 22 & 24 & 21 & \text { uS/cm } \\ 17 & 14 & 18.3 & \text { deg. C } \\ 0 & 4 & 0 & \text { mg/L } \\ .2 & .9 & .2 & \text { NTU } \\ 3.63616 & 25.2762 & 16.9377 & \text { well volumes }\end{array}$

Compliance

$\begin{array}{ccccc}101998 & 201998 & 301998 & \text { 401998 DEMod Unit Lab Filter } \\ <5 & <5 & <5 & \\ <5 & <5 & <5 \\ <5 & <5 & <5 \\ <5 & <5 & <5 \\ <5 & <5 & <5 \\ <5 & <5 & <5 \\ <5 & <5 & <5 \\ <5 & <5 & <5 \\ <5 & <5 & <5 \\ <5 & <5 & <5 \\ <5 & <5 & <5 \\ <5 & <5 & <5 \\ <10 & <10 & <10 \\ <10 & <10 & <6.6 \\ <20 & <20 & <20 \\ <20 & <10 & <10 \\ <5 & <20 & <5 \\ <10 & <10 & <10 \\ <40 & <8 & <8 \\ 7.5 & 7.6 & 5.1 \\ <5 & <5 & <5 \\ <5 & <5 & <5 \\ <5 & <5 & <5 \\ <10 & <10 & <10 \\ <4.7 & <2 & .4 \\ & & 30.7 \\ <5\end{array}$

Note: Flagging, dilution factors, modifiers, and laboratories are for fourth quartel 998 dat only. See Appendix B for nagging criteria.

* axceeded holding time for fourth quarter 1998.

$+=$ exceeded screening level or final primary drinking water standard for fourth quarteri 998 .

Sanitary Landfill

D- 135

Fourth Quarter 1998 and Annual Summary 
WELL: LFW 71D

\section{ANALYTICAL DATA}

\section{H ST Analyte}

Carbon disulfide

Carbon tetrachloride

Chlorobenzene

Chloroethane

Chloroethene (Vinyl chloride)

Chloroform

Chloromethane (Methyl chloride)

Chloroprene

Chromium, total recoverable

Dibromochloromethane

Dibromomethane (Methylene bromide)

Dichlorodifluoromethane

Dichloromethane (Methylene chloride)

Ethylbenzene

Gross alpha

Iodomethane (Methyl iodide)

Iron, total recoverable

Isobutyl alcohol

Lead, total recoverable

Mercury, total recoverable

Methacrylonitrile

Methyl ethyl ketone

Methyl isobutyl ketone

Methyl methacrylate

Propionitrile

Selenium, total recoverable

Silver, total recoverable

Styrene

Tetrachloroethylene

Toluene

Trichloroethylene

Trichlorofluoromethane

Tritium

Vinyl acetate

Xylenes

cis-1,3-Dichloropropene

trans-1,2-Dichloroethylene

trans-1,3-Dichloropropene

trans-1,4-Dichloro-2-butene
101998

201998

$<5$

$<5$

$<5$

$<10$

$\ldots+10$

$<5$

$<10$

$<5$

1.2

$<5$

$<5$

$<10$

$<6.5$

$<5$

$<.19$

$<5$

\section{$<100$}

$<100$
$<47$

$<47$
$<7$

$<.7$

$<10$

$<10$

$<10$

$<50$

$<66$

$<5$

$<5$

$<5$

$<5$

$<5$

$<5$

1.15

$<10$

$<5$

$<5$

$<5$

$<5$

$<20$

\section{$<5$}

$<5$

$<5$

$<10$

$<10$

$<5$

$<10$

$<5$

$<3$

$<5$

$<5$

$<5$

$<2.1$

$<5$

$<-.66$

$<5$

301998

$<5$

$<5$

$<5$

$<10$

$<10$

$<5$

$<10$

$<5$

1.4

$<5$

$<5$

$<5$

$<3$

$<5$

$<-.01$

$<5$

9.2

$<100$

$<100$

$<5$

$<.2$

$<5$

$<10$

$<12$

$<5$

$<5$

$<5$

$<2$

$<5$

$<5$

$<5$

$<5$

$<5$

1260

$<5$

$<5$

$<5$

$<5$

$<5$

$<5$

$<.2$

$<5$

$<10$

$<12$

$<5$

$<5$

$<5$

$<5$

$<5$

$<5$

$<5$

$<5$

$\leq 1.22$

$<5$

$<5$

$<5$

$<5$

$<5$
Compliance

401998 DEMod Unit Lab Filter

Note: Flagging, dilution factors, modifiers, and laboratories are for fourth quartei998 data only. See Appendix B for fagging criteria. * = exceeded holding time for fourth quarter 1998.

+ - exceeded screening level or final primary drinking water standard for fourth quarten 998 . 
WSRC-TR-99-00011

Unclassified

WELL: LFW 74C

\section{SRS Coord. Lat/Longitude \\ N 85813.8 \\ E 45097.8

33.289 Deg N
81.715 Deg W

SAMPLE DATE

FIELD DATA

Analvte

Water Elevation

Depth to water

pH

Sp. Conductance

Water temperature

Alkalinity as $\mathrm{CaCO3}$

Turbidity

Volumes purged

Sampling code

\section{ANALYTICAL DATA}

\section{H ST Analyte}

1,1,1,2-Tetrachloroethane

1,1,1-Trichloroethane

1,1,2,2-Tetrachloroethane

1,1,2-Trichloroethane

1,1-Dichloroethane

1,1-Dichloroethylene

1,2,3-Trichloropropane

1,2-Dibromo-3-chloropropane

1,2-Dibromoethane

1,2-Dichlorobenzene

1,2-Dichloroethane

1,2-Dichloropropane

1,3-Dichlorobenzene

1,4-Dichlorobenzene

1,4-Dioxane

2-Chloroethyl vinyl ether

2-Hexanone

Acetone

Acetonitrile (Methyl cyanide)

Acrolein

Acrylonitrile

Allyl chloride

Aluminum, total recoverable

Antimony, total recoverable

Arsenic, total recoverable

Barium, total recoverable

\section{Screen Zone Elevation Top of Casing Casing Pump Eormation 116.0 - $101.0 \mathrm{ft} \mathrm{msl}$ \\ 213.6 ft msl \\ 4"PVC \\ S \\ M Steed Pond}

$02 / 02 / 98$



Comoliance

$101998201998 \quad 301998 \quad \underline{401998}$ DFMod Unit Lab Filter

$<5$

$<5$

$<5$

$<5$

$<5$

$<5$

$<5$

$<5$

$<5$

$<5$

$<5$

$<5$

$<5$

$<5$

$<1000$

$<5$

$<10$

$<10$

$<20$

$<10$

$<20$

$<10$

$<100$

$<5$

$<8$

5.2

Note: Flagging, dilution factors, modifiers, and laboratories are for fourth quartei998 data only. See Appendix B for flagging criteria. * - exceeded holding time for fourth quarter 1998.

$+\infty$ exceded screening level or final primany drinking water standard for fourth quarten 998 . 
WELL: LFW 74C

\section{ANALYTICAL DATA}

\section{H ST Analyte}

Benzene

Beryllium, total recoverable

Bromochloromethane

Bromodichloromethane

Bromoform

Bromomethane (Methyl bromide)

Cadmium, total recoverable

Calcium, total recoverable

Carbon disulfide

Carbon tetrachloride

Chlorobenzene

Chloroethane

Chloroethene (Vinyl chloride)

Chloroform

Chloromethane (Methyl chloride)

Chloroprene

Chromium, total recoverable

Cobalt, total recoverabie

Copper, total recoverable

Di-n-butyl phthalate

Dibromochloromethane

Dibromomethane (Methylene bromide)

Dichlorodifluoromethane

Dichloromethane (Methylene chloride)

Ethyl methacrylate

Ethylbenzene

Iodomethane (Methyl iodide)

Iron, total recoverable

Isobutyl alcohol

Lead, total recoverable

Magnesium, total recoverable

Manganese, total recoverable

Methacrylonitrile

Methyl ethyl ketone

Methyl isobutyl ketone

Methyl methacrylate

Nickel, total recoverable

Pentachloroethane

Potassium, total recoverable

Propionitrile

Selenium, total recoverable

Silver, total recoverable
101998

20

$<5$

$<1$

$<10$

$<5$

$<5$

$<10$

$<2$

$<1410$

$<5$

$<5$

$<5$

$<10$

$<10$

$<5$

$<10$

$<5$

$<3$

$<5$

2.3

$<10$

$<5$

$<5$

$<5$

$<2.3$

$<10$

$<5$

$<5$

$<25.6$

$<100$

$<5$

$<656$

2.81

$\leq 5$

$<10$

$<12$

$<5$

$<5$

$<20$

$<1000$

$<5$

$<5$

$<2$
Compliance

\section{401998 DFMod Unit Lab Filter}

Note: Flagging, dilution factors, modifiers, and laboratories are for fourth quartel 998 data only. See Appendix B for flagging crtteria. * - exceeded holding time for fourth quarter 1998.

+ - exceeded screening level or final primary drinking water standard for fourth quarter1998 . 
WELL: LFW 74C

\section{ANALYTICAL DATA}

H ST Analvte

Sodium, total recoverable

Specific conductance

Styrene

Tetrachloroethylene

Thallium, total recoverable

Toluene

Trichloroethylene

Trichlorofluoromethane

Tritium

Vanadium, total recoverable

Vinyl acetate

Xylenes

Zinc, total recoverable beta-Benzene hexachloride cis-1,2-Dichloroethylene cis-1,3-Dichloropropene pH

trans-1,2-Dichloroethylene trans-1,3-Dichloropropene trans-1,4-Dichloro-2-butene

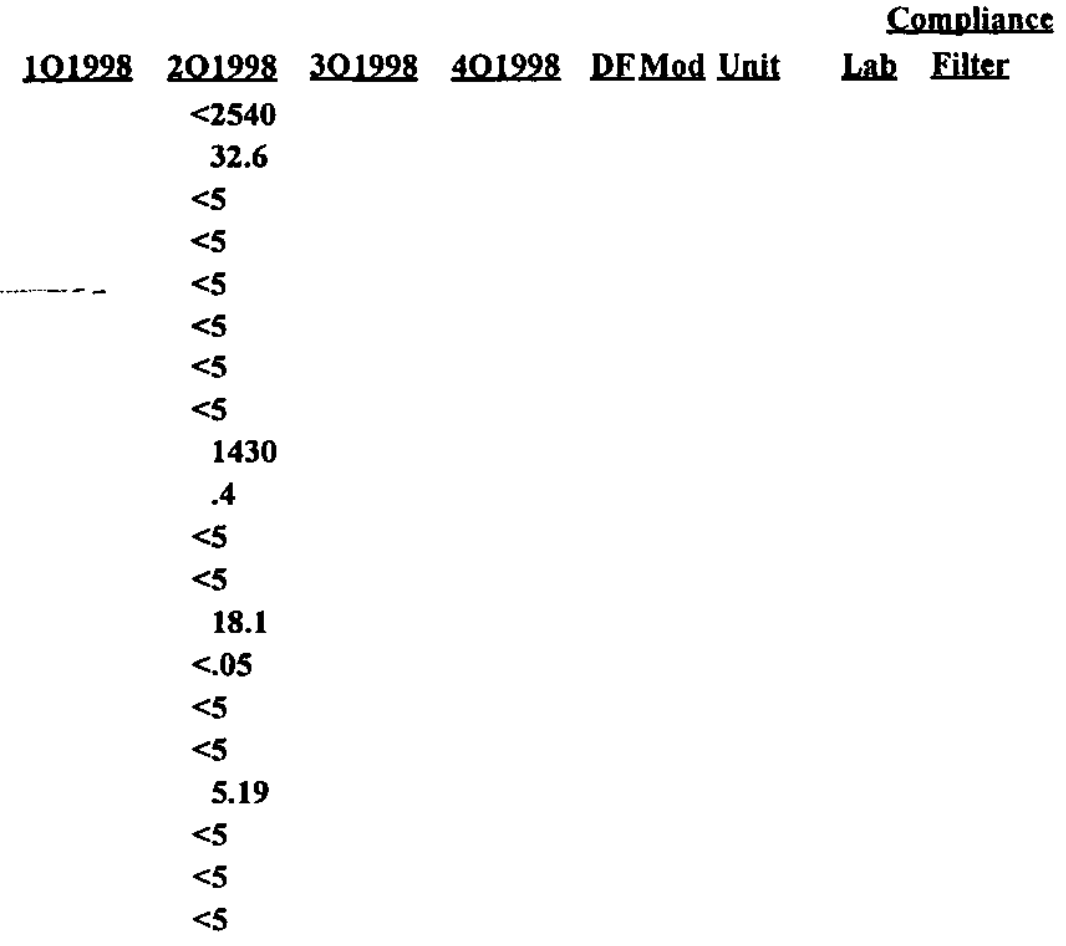

Note: Flagging, dilution factors, modifiers, and laboratories are for fourth quarte1998 data only. See Appendix B for fagging criteria. * = exceeded holding time for fourth quarter 1998.

$+=$ exceeded screening level or final primary drinking water standard for fourth quarter 998 .

Sanitary Landfill

D-139

Fourth Quarter 1998 and Annual Summary 
WELL: LFW 74D

\begin{tabular}{|c|c|c|c|c|c|c|}
\hline SRS & Lat/Longitude & Screen Zone Elevation & Top of Casing & Casing & Pump & Formation \\
\hline $\begin{array}{l}\text { N } 8 \\
\text { E }\end{array}$ & $\begin{array}{ll}33.289 & \text { Deg N } \\
81.715 & \text { Deg } W\end{array}$ & $.7-152.7$ ft msl & 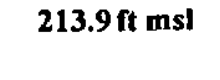 & 4"PVC & $\mathbf{S}$ & ond \\
\hline
\end{tabular}

\section{SAMPLE DATE}

\section{FIELD DATA}

Analyte
Water Elevation
Depth to water
pH
Sp. Conductance
Water temperature
Alkalinity as CaCO3
Turbidity
Volumes purged
Sampling code

\section{ANALYTICAL DATA}

\section{H ST Analyte}

1,1,1,2-Tetrachloroethane 1,1,1-Trichloroethane 1,1,2,2-Tetrachloroethane 1,1,2-Trichloroethane 1,1-Dichloroethane 1,1-Dichloroethylene 1,2,3-Trichloropropane 1,2-Dibromo-3-chloropropane 1,2-Dibromoethane 1,2-Dichlorobenzene 1,2-Dichloroethane 1,2-Dichloropropane 1,4-Dichlorobenzene 2-Hexanone $\begin{array}{ll}101998 & 201998 \\ \ldots \quad \ldots & 160.85 \\ & 50.85 \\ & 4.5 \\ & 23 \\ & 18.2 \\ & 0 \\ & 1.9 \\ & 8.68976\end{array}$ 301998 401998

Unit ft msl ft pH uS/cm deg. C $\mathrm{mg} / \mathrm{L}$ NTU well volumes

101998

201998 $<5$

1.1

$<5$

$<5$

$<5$

$<5$

$<5$

$<5$

$<5$

$<5$

$<5$

$<5$

$<5$

$<10$

50

Acetonitrile (Methyl cyanide) $\quad<20$

Acrolein

Acrylonitrile

Compliance

Allyl chloride

Antimony, total recoverable

$<10$

Arsenic, total recoverable

Barium, total recoverable

Benzene

$<20$

$<10$

$<5$

$<8$

4

$<5$

Beryllium, total recoverable

$<1$

Bromochloromethane

$<10$

Bromodichloromethane

$<5$

Note: Flagging, dilution factors, modifiers, and laboratories are for fourth quartel 998 data only. See Appendix B for fagging criteria.

- a exceeded holding time for fourth quarter 1998 .

+ = exceded screening level or final primary drinking water standard for fourth quarterf 998 . 
WSRC-TR-99-00011

Unclassified

WELL: LFW 74D

ANALYTICAL DATA

H ST Analyte

Bromoform

Bromomethane (Methyl bromide)

Cadmium, total recoverable

Carbon disulfide

Carbon tetrachloride

Chlorobenzene

Chloroethane

Chloroethene (Vinyl chloride)

Chloroform

Chloromethane (Methyl chloride)

Chloroprene

Chromium, total recoverable

Cobalt, total recoverable

Copper, total recoverable

Di-n-butyl phthalate

Dibromochloromethane

Dibromomethane (Methylene bromide)

Dichlorodifluoromethane

Dichloromethane (Methylene chloride)

Ethylbenzene

Iodomethane (Methyl iodide)

Isobutyl alcohol

Lead, total recoverable

Methacrylonitrile

Methyl ethyl ketone

Methyl isobutyl ketone

Methyl methacrylate

Nickel, total recoverable

Propionitrile

Selenium, total recoverable

Silver, total recoverable

Specific conductance

Styrene

Tetrachloroethylene

Thallium, total recoverable

Toluene

Trichloroethylene

Trichlorofluoromethane

Tritium

Unknown

Vanadium, total recoverable

Vinyl acetate
$101998 \quad 201$

$$
<10
$$

$<2$

$<5$

$<5$

$<5$

$<10$

$<10$

$<5$

$<10$

$<5$

$<3$

$<5$

$<1.5$

$<5$

$<5$

$<5$

$<2.1$

$<5$

$<5$

$<100$

3.8

$<5$

$<10$

$<12$

$<5$

$<5$

$<5$

$<5$

$<2$

17.4

$<5$

$<5$

$<5$

$<5$

$<5$

11

1550

$<41.06$

.4

$<5$

Compliance

998301998401998 DFMod Unit Lab Eilter


WSRC-TR-99-00011

Unclassified

WELL: LFW 74D

\section{ANALYTICAL DATA}

H ST Analyte

Xylenes

Zinc, total recoverable beta-Benzene hexachloride cis-1,2-Dichloroethylene cis-1,3-Dichloropropene pH

trans-1,3-Dichloropropene trans-1,4-Dichloro-2-butene

101998
201998
$<5$
19.9
$<.015$
$<5$
$<5$
5.2
$<5$
$<5$

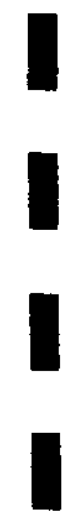

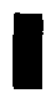

I

I

I

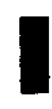

I

Note: Flagging, dilution factors, modifiers, and laboratories are for fourth quarteil 998 data only. See Appendix B for fingging criteria.

* = exceeded holding time for fourth quarter 1998 .

+- exceeded screening level or final primary drinking water standard for fourth quarten 1998 .

Sanitary Landfill

D-142

Fourth Quarter 1998 and Annual Summary 
WELL: LFW 75C

\begin{tabular}{|c|c|c|c|c|c|c|}
\hline SRS Coord. & Lat/longitude & Screen Zone Elevation & Top of Casing & Casing & Pump & Eormation \\
\hline $\begin{array}{l}\text { N } 85856.8 \\
\text { E } 45357.0\end{array}$ & $\begin{array}{l}33.290 \text { Deg } N \\
81.714 \text { Deg W }\end{array}$ & $115.6-100.6 \mathrm{ft}$ mst & $197.8 \mathrm{ft} \mathrm{msl}$ & 4"PVC & $\mathbf{S}$ & I Pond \\
\hline
\end{tabular}

SAMPLE DATE

FIELD DATA

Analyte

Water Elevation

Depth to water

pH

Sp. Conductance

Water temperature

Alkalinity as $\mathrm{CaCO} 3$

Turbidity

Volumes purged

Sampling code

\section{ANALYTICAL DATA}

\section{H ST Analvte}

1,1,1,2-Tetrachloroethane

1,1,1-Trichloroethane

1,1,2,2-Tetrachloroethane

1,1,2-Trichloroethane

1,1-Dichloroethane

1,1-Dichloroethylene

1,2,3-Trichloropropane

1,2-Dibromo-3-chloropropane

1,2-Dibromoethane

1,2-Dichlorobenzene

1,2-Dichloroethane

1,2-Dichloropropane

1,4-Dichlorobenzene

2-Hexanone

Acetone

Acetonitrile (Methyl cyanide)

Acrolein

Acrylonitrile

Allyl chloride

Antimony, total recoverable

Arsenic, total recoverable

Barium, total recoverable

Benzene

Beryllium, total recoverable

Bromochloromethane

Bromodichloromethane
$02 / 02 / 98$

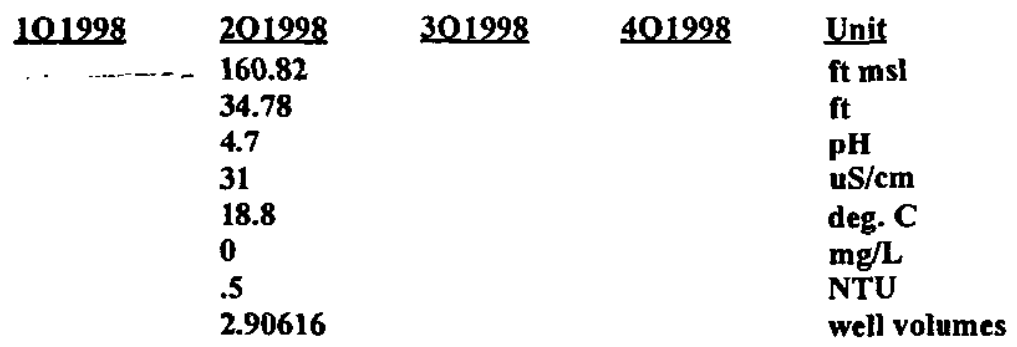

Compliance

$101998201998 \quad 301998 \quad 401998$ DEMod Unit Lab Filter

$<5$

$<5$

$<5$

$<5$

$<5$

$<5$

$<5$

$<5$

$<5$

$<5$

$<5$

$<5$

$<5$

$<10$

$<10$

$<20$

$<10$

$<20$

$<10$

$<5$

$<8$

6.2

$<5$

$<1$

$<10$

$<5$

Note: Flagging, dilution factors, modifiers, and laboratories are for fourth quarteil998 data only. See Appendix B for flagging criteria. * exceeded holding time for fourth quarter1998.

$+=$ exceeded screening level or final primary drinking water standard for fourth quartert 998 . 
WSRC-TR-99-00011

Unclassified

WELL: LFW 75C

ANALYTICAL DATA

H ST Analyte

Bromoform

Bromomethane (Methyl bromide)

Cadmium, total recoverable

Carbon disulfide

Carbon tetrachloride

Chlorobenzene

Chloroethane

Chloroethene (Vinyl chloride)

Chloroform

Chloromethane (Methyl chloride)

Chloroprene

Chromium, total recoverable

Cobalt, total recoverable

Copper, total recoverable

Di-n-butyl phthalate

Dibromochloromethane

Dibromomethane (Methylene bromide)

Dichlorodifluoromethane

Dichloromethane (Methylene chloride)

Ethylbenzene

Iodomethane (Methyl iodide)

Isobutyl alcohol

Lead, total recoverable

Methacrylonitrile

Methyl ethyl ketone

Methyl isobutyl ketone

Methyl methacrylate

Nickel, total recoverable

Propionitrile

Selenium, total recoverable

Silver, total recoverable

Specific conductance

Styrene

Tetrachloroethylene

Thallium, total recoverable

Toluene

Trichloroethylene

Trichlorofluoromethane

Tritium

Vanadium, total recoverable

Vinyl acetate

Xylenes

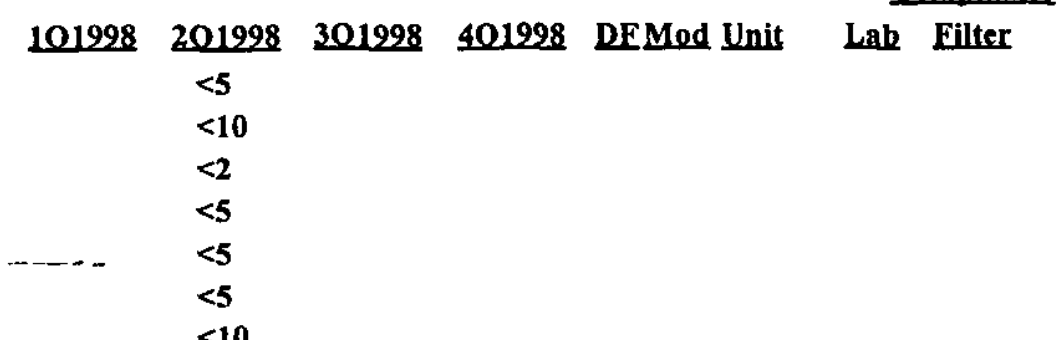

$<10$

$<10$

$<5$

$<10$

$<5$

$<3$

$<5$

$<3$

$<.93$

$<5$

$<5$

$<5$

$<2.3$

$<5$

$<5$

$<100$

$<5$

$<5$

$<10$

$<12$

$<5$

$<5$

$<5$

$<5$

$<2$

31

$\leq 5$.

$<5$

$<5$

$<5$

$<5$

$<5$

952

$<2$

$<5$

$<5$

Note: Flagging, dilution factors, modifiers, and laboratories are for fourth quartei 998 data only. See Appendix B for fagging criteria. - exceeded holding time for fourth quarter 1998 .

$+=$ exceeded screening level or final primary drinking water standard for fourth quarten 998 .

Sanitary Landfill 
WSRC-TR-99-00011

Unclassified

WELL: LFW 75C

\section{ANALYTICAL DATA}

H ST Analvte

Zinc, total recoverable. beta-Benzene hexachloride

cis-1,2-Dichloroethylene cis-1,3-Dichloropropene

pH

trans-1,3-Dichloropropene

trans-1,4-Dichloro-2-butene
Compliance

101998201998301998401998 DEMed Unit Lab Filter

38.4

$<.015$

$<5$

$<5$

5.05

$<5$

$<5$

Note: Flagging, dilution factorz, smodifiers, and laboratories are for fourth quartel998 data only. See Appendix B for flagging criterta.

* a exceeded holding time for fourth quarter 1998.

+ - exceeded screening level or final primary drinking water standard for fourth quarten 998 .

Sanitary Landfill

D-145

Fourth Quarter 1998 and Annual Summary 
WSRC-TR-99-00011

Unclassified

WELL: LFW $75 D$

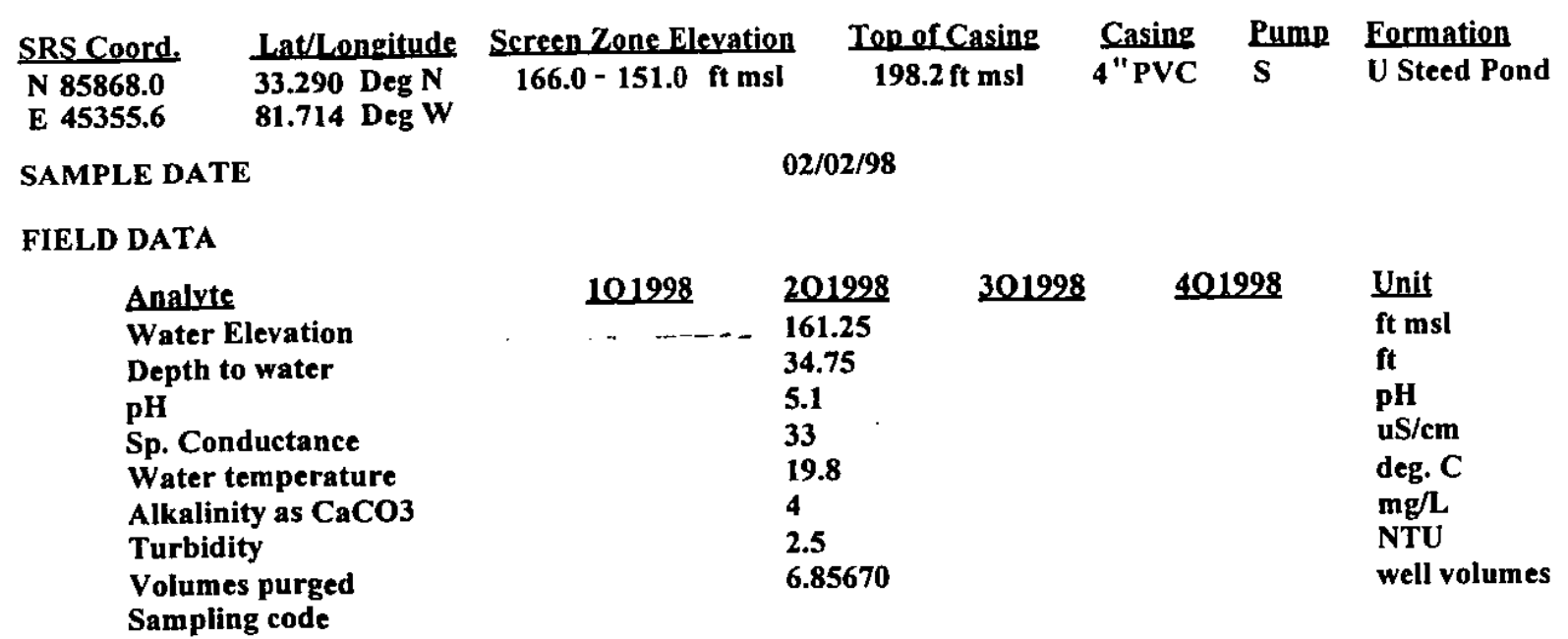

ANALYTICAL DATA

Compliance

H ST Analyte

1,1,1,2-Tetrachloroethane

1,1,1-Trichloroethane

1,1,2,2-Tetrachloroethane

1,1,2-Trichloroethane

1,1-Dichloroethane

1,1-Dichloroethylene

1,2,3-Trichloropropane

1,2-Dibromo-3-chloropropane

1,2-Dibromoethane

1,2-Dichlorobenzene

1,2-Dichloroethane

1,2-Dichloropropane

1,4-Dichlorobenzene

2-Hexanone

Acetone

Acetonitrile (Methyl cyanide)

Acrolein

Acrylonitrile

Allyl chloride

Antimony, total recoverable

Arsenic, total recoverable

Barium, total recoverable

Benzene

Beryllium, total recoverable

Bromochloromethane

Bromodichloromethane

\section{$101998201998301998 \quad \underline{401998}$ DEMOd Unit Lab Filter}

$<5$

$<5$

$<5$

$<5$

$<5$

$<5$

$<5$

$<5$

$<5$

$<5$

$<5$

$<5$

$<5$

$<10$

$<10$

$<20$

$=-\quad----=\frac{<10}{<20}$

$<10$

$<5$

$<8$

9.9

$<5$

$<1$

$<10$

$<5$

Note: Fiagging, dilution factors, modifiers, and laboratories are for fourth quartei998 data only. See Appendix B for flagging criteria. * exceeded holding time for fourth quarter 1998.

$+=$ exceeded screening level or final primary drinking water standard for fourth quarten 998 . 
WELL: LFW 75D

\section{ANALYTICAL DATA}

H ST Analyte

Bromoform

Bromomethane (Methyl bromide)

Cadmium, total recoverable

Carbon disulfide

Carbon tetrachloride

Chlorobenzene

Chloroethane

Chloroethene (Vinyl chloride)

Chloroform

Chloromethane (Methyl chloride)

Chloroprene

Chromium, total recoverable

Cobalt, total recoverable

Copper, total recoverable

Di-n-butyl phthalate

Dibromochloromethane

Dibromomethane (Methylene bromide)

Dichlorodifluoromethane

Dichloromethane (Methylene chloride)

Ethylbenzene

Iodomethane (Methyl iodide)

Isobutyl alcohol

Lead, total recoverable

Methacrylonitrile

Methyl ethyl ketone

Methyl isobutyl ketone

Methyl methacrylate

Nickel, total recoverable

Propionitrile

Selenium, total recoverable

Silver, total recoverable

Specific conductance

Styrene

Tetrachloroethylene

Thallium, total recoverabie

Toluene

Trichloroethylene

Trichlorofluoromethane

Tritium

Vanadium, total recoverable

Vinyl acetate

Xylenes
$101998201998301998 \quad 401998$ DEMod Unit Lab Filter

$<5$

$<10$

.3

$<5$

$<5$

$<5$

$<10$

$<10$

$<5$

$<10$

$<5$

$<3$

$<5$

$<3$

$<.98$

$<5$

$<5$

$<5$

$<2.1$

$<5$

$<5$

$<100$

6.9

$<5$

$<10$

$<12$

$<5$

$<5$

$<5$

$<5$

$<2$

31.6

$<5$

$<5$

$<5$

$<5$

$<5$

2.8

1270

$<2$

$<5$

$<5$

Note: Flagging, dilution factors, modifiers, and laboratories are for fourth quarte1998 data only. See Appendix B for fagging criteria. * = exceeded holding time for fourth quarter 1998.

+ = exceeded screening level or final primary drinking water standard for fourth quarter 998 . 
WSRC-TR-99-00011

Unclassified

WELL: LFW 75D

\section{ANALYTICAL DATA}

\section{H ST Analyte}

101998

Zinc, total recoverable beta-Benzene hexachloride cis-1,2-Dichloroethylene cis-1,3-Dichloropropene

$$
\text { pH }
$$

trans-1,3-Dichloropropene

trans-1,4-Dichloro-2-butene

$\begin{array}{rr}101998 & 20199 \\ & 33.5 \\ & <.015 \\ & <5 \\ & <5 \\ -- & 5.85 \\ & <5 \\ & <5\end{array}$

Compliance Lab Filter
33.5

$<.015$

$<5$

5.85

$<5$
$<5$

Note: Flagging, dilution factors, modifiers, and laboratories are for fourth quartet 998 data only. See Appendix B for fiagging criteria. - exceeded holding time for fourth quarter 1998 .

+ - exceeded screening level or final primary drinking water standard for fourth quarten 998 . 
WELL: LFW 76

\begin{tabular}{|c|c|c|c|c|c|c|}
\hline SRS Coord. & Lat/Longitude & Screen Zone Elevation & Top of Casing & Casing & $\mathbf{P u}$ & Form \\
\hline $\begin{array}{l}\text { N } 856 \\
\text { E } 447\end{array}$ & $\begin{array}{ll}\text { 33.288 } & \text { Deg } N \\
\text { 81.715 } & \text { Deg } W\end{array}$ & $157.9-142.9 \mathrm{ft} \mathrm{msl}$ & & $2 " \mathrm{PVC}$ & & \\
\hline
\end{tabular}

SAMPLE DATE

FIELD DATA

Analyte

Water Elevation

Depth to water

pH

Sp. Conductance

Water temperature

Alkalinity as $\mathrm{CaCO} 3$

Turbidity

Volumes purged

Sampling code
$02 / 17 / 98$

$\begin{array}{lll}101998 & 201998 & 301998 \\ & 157.85 & \\ & 61.55 \\ 5 & 89 \\ & 22.2 \\ 11 & 14.6 \\ & 5.24146 \\ & N X\end{array}$

$09 / 26 / 98$

\begin{tabular}{ll}
401998 & Unit \\
\hline 158.89 & ft msl \\
60.51 & ft \\
4.7 & pH \\
18 & uS/cm \\
21 & deg. C \\
2 & mg/L \\
10.4 & NTU \\
.329776 & well volumes \\
NX &
\end{tabular}

\section{ANALYTICAL DATA}

\section{H ST Analvte}

1,1,1,2-Tetrachloroethane

1,1,1-Trichloroethane

1,1,2,2-Tetrachloroethane

1,1,2-Trichloroethane

1,1-Dichloroethane

1,1-Dichloroethylene

1,2,3-Trichloropropane

1,2,4,5-Tetrachlorobenzene

1,2,4-Trichlorobenzene

1,2-Dibromo-3-chloropropane

1,2-Dibromoethane

1,2-Dichlorobenzene

1,2-Dichloroethane

1,2-Dichloropropane

1,3,5-Trinitrobenzene

1,3-Dichlorobenzene

1,3-Dinitrobenzene

1,4-Dichlorobenzene

1,4-Dioxane

1,4-Naphthoquinone

1-Naphthylamine

2,3,4,6-Tetrachlorophenol

2,4,5-Trichlorophenol

2,4,6-Trichlorophenol

2,4-Dichlorophenol

2,4-Dimethyl phenol
101998

\section{8}

301998

$<5$

$<5$

$<5$

$<5$

$<5$

$<5$

$<5$

$<5$

$<5$

$<5$

$<5$

$<5$

$<5$

Compliance

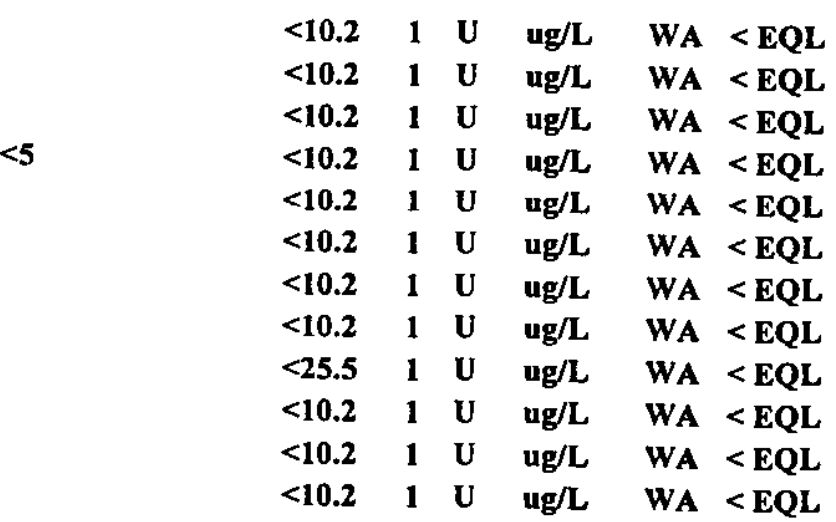

Note: Flagging, dilution factors, modifiers, and laboratories are for fourth quartei998 data only. See Appendix B for flagging criteria.

* exceeded holding time for fourth quarter 1998

+ - exceeded screening level or final primary drinking water standard for fourth quarten 998. 
WELL: LFW 76

\section{ANALYTICAL DATA}

H ST Analyte

2,4-Dinitrophenol

2,4-Dinitrotoluene

2,6-Dichlorophenol

2,6-Dinitrotoluene

2-Acetylaminofluorene

2-Chloronaphthalene

2-Chlorophenol

2-Hexanone

2-Methyl-4,6-dinitrophenol

2-Methylnaphthaiene

2-Naphthylamine

2-Nitrophenol

2-Picoline

2-sec-Butyl-4,6-dinitrophenol

3,3"-Dichlorobenzidine

3,3"-Dimethylbenzidine

3-Methylcholanthrene

4-Aminobiphenyl

4-Bromophenyl phenyl ether

4-Chloro-m-cresol

4-Chloroaniline

4-Chlorophenyl phenyl ether

4-Nitrophenol

4-Nitroquinoline-1-oxide

5-Nitro-0-toluidine

7,12-Dimethylbenz(a)anthracene

Acenaphthene

Acenaphthylene

Acetone

Acetonitrile (Methyl cyanide)

Acetophenone

Acrolein

Acrylonitrile

Allyl chloride

Aniline

Anthracene

Antimony, total recoverable

Aramite

Arsenic, total recoverable

Barium, total recoverable

Benzene

Benzo(a)anthracene $\begin{array}{lllll}101998 & 201998 \quad 301998 & 401998 & \text { DFMod Unit }\end{array}$

$<25.5 \quad 1 \quad$ U $\quad u g / L$

$<10.2 \quad 1 \quad \mathrm{U} \quad$ ug/L

$<10.2 \quad 1 \quad \mathrm{U} \quad \mathrm{ug} / \mathrm{L}$

$<10.2 \quad 1 \quad U \quad u g / L$

$<10.21 \mathrm{U} \quad \mathrm{ug} / \mathrm{L}$

$<10.2 \quad 1 \quad \mathrm{U} \quad \mathrm{ug} / \mathrm{L}$

$<10.2 \quad 1 \quad \mathrm{U} \quad \mathrm{ug} / \mathrm{L}$

$<10$

$<25.5 \quad 1 \quad$ U $\quad u g / L \quad$ WA $<$ EQL

$<10.2 \quad 1 \quad$ U $\quad$ ug/L $\quad$ WA $<$ EQL

$<10.2 \quad 1 \quad$ U $\quad$ ug/L $\quad$ WA $<$ EQL

$<10.2 \quad 1 \quad$ U $\quad$ ug/L WA $<$ EQL

$<10.2$ I $U$ U

$<51 \quad 1 \quad$ U $\quad$ ug/L $\quad$ WA $<$ EQL

$<10.2$ I $U$ U $\quad$ ug/L $\quad$ WA $<$ EQL

$<10.2$ I U $\quad$ ug/L $\quad$ WA $<$ EQL

$<10.211$ U ug/L WA $<$ EQL

$<10.2 \quad 1 \quad$ U $\quad$ ug/L $\quad$ WA $<$ EQL

$<10.21 \mathrm{U} \quad$ ug/L $\quad$ WA $<\mathrm{EQL}$

$<10.2 \quad 1 \quad$ U $\quad$ ug/L $\quad$ WA $<$ EQL

$<10.2 \quad 1 \quad \mathrm{U} \quad$ ug/L $\quad$ WA $<$ EQL

$<10.2 \quad 1 \quad \mathrm{U} \quad \mathrm{ug} / \mathrm{L} \quad$ WA $<$ EQL

$<25.51 \mathrm{U} \quad \mathrm{ug} / \mathrm{L} \quad$ WA $<$ EQL

$<20.4 \quad 1 \quad U \quad$ ug/L $\quad$ WA $<$ EQL

$<10.2 \quad 1 \quad \mathrm{U} \quad \mathrm{ug} / \mathrm{L} \quad$ WA $<$ EQL

$<10.2 \quad 1 \quad \mathrm{U} \quad \mathrm{ug} / \mathrm{L} \quad$ WA $<$ EQL

$<10.2 \quad 1 \quad \mathrm{U} \quad \mathrm{ug} / \mathrm{L} \quad$ WA $<$ EQL

$<10.2 \quad 1 \quad \mathrm{U} \quad \mathrm{ug} / \mathrm{L} \quad$ WA $<$ EQL

$<20$

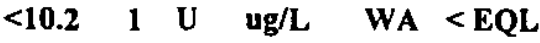

$<10$

$\leq 20$

$<10$

$<5$

$<8$

3.3

$<5$

Note: Flagging, dilution factors, modifiers, and laboratories are for fourth quarted 998 data only. See Appendix B for fiagging criteria.

= exceeded holding time for fourth quarter 1998 .

+ - exceeded screening level or final primary drinking water standard for fourth quartex 998 . 


\section{WELL: LFW 76}

\section{ANALYTICAL DATA}

\section{H ST Analyte}

Benzo(a)pyrene

Benzo(b)fluoranthene

Benzo(g,h,i)perylene

Benzo(k)fluoranthene

Benzoic acid

Benzyl alcohol

Beryllium, total recoverable

Bis(2-chloroethoxy) methane

Bis(2-chloroethyl) ether

Bis(2-chloroisopropyl) ether

Bis(2-ethylhexyl) phthalate

Bromochloromethane

Bromodichloromethane

Bromoform

Bromomethane (Methyl bromide)

Butylbenzyl phthalate

Cadmium, total recoverable

Carbon disulfide

Carbon tetrachloride

Chlorobenzene

Chlorobenzilate

Chloroethane

Chloroethene (Vinyl chloride)

Chloroform

Chloromethane (Methyl chloride)

Chloroprene

Chromium, total recoverable

Chrysene

Cobalt, total recoverable

Copper, total recoverable

Di-n-butyl phthalate

Di-n-octyl phthalate

Diallate

Dibenz(a,h)anthracene

Dibenzofuran

Dibromochloromethane

Dibromomethane (Methylene bromide)

Dichlorodifluoromethane

Dichloromethane (Methylene chloride)

Diethyl phthalate

Dimethoate

Dimethyl phthalate

\section{8 \\ 201998 \\ 301998}

40199

DEMod Unit

Compliance

$<10.2 \quad 1 \quad U \quad u g / L \quad$ WA $<$ EQL

$<10.2 \quad 1 \quad \mathrm{U} \quad \mathrm{ug} / \mathrm{L} \quad$ WA $<$ EQL

$<10.211$ U $\quad$ ug/L $\quad$ WA $<$ EQL

$<25.5 \quad 1 \quad \mathrm{U} \quad \mathrm{ug} / \mathrm{L} \quad$ WA $<$ EQL

$<10.2 \quad 1 \quad \mathrm{U} \quad \mathrm{ug} / \mathrm{L} \quad$ WA $<$ EQL

$<1$

$<10$

$<5$

$<5$

$<10$

.5

$<5$

$<5$

$<5$

$<10$

$<10$

$<5$

$<10$

$<5$

11.8

$<10.2 \quad 1 \quad$ U $\quad$ ug/L $\quad$ WA $<$ EQL

$<10.2 \quad 1 \quad U \quad$ ug/L $\quad$ WA $<$ EQL

$<10.2 \quad 1 \quad$ U ug/L WA $<$ EQL

$<.78 \quad 1 \quad \mathrm{U} \quad$ ug/L $\quad$ WA $<$ EQL

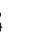

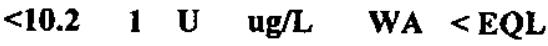

$<5$

2.8

$<10$

$<5$

$<5$

$<5$

$<2.1$

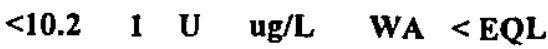

$\begin{array}{llllll}<10.2 & 1 & \mathrm{U} & \mathrm{ug} / \mathrm{L} & \text { WA } & <\mathrm{EQL}\end{array}$

$<.586 \quad 1 \quad \mathrm{~J}$ ug/L WA NDD

$<10.2 \quad 1 \quad$ U $\quad$ ug/L $\quad$ WA $<$ EQL

$<10.2 \quad 1 \quad$ U ug/L WA $<$ EQL

$<10.2 \quad 1 \quad \mathrm{U} \quad \mathrm{ug} / \mathrm{L} \quad$ WA $<$ EQL

$<10.21 \mathrm{U} \quad$ ug/L WA $<$ EQL

Note: Flagging, dilution factors, modifiers, and laboratories are for fourth quartet998 data only. See Appendix B for flagging criteria.

* = exceeded holding time for fourth quarter 1998.

+ a exceeded screening level or final primary drinking water standard for fourth quarten 998 . 
WSRC-TR-99-00011

Unclassified

WELL: LFW 76

\section{ANALYTICAL DATA}

H ST Analyte

Diphenylamine

Disulfoton

Ethyl methacrylate

Ethyl methanesulfonate

Ethylbenzene

Famphur

Fluoranthene

Fluorene

Hexachlorobenzene

Hexachlorobutadiene

Hexachlorocyclopentadiene

Hexachloroethane

Hexachlorophene

Hexachloropropene

Indeno(1,2,3-c,d)pyrene

Iodomethane (Methyl iodide)

Isobutyl alcohol

Isophorone

Isosafrole

Lead, total recoverable

Methacrylonitrile

Methapyrilene

Methyl ethyl ketone

Methyl isobutyl ketone

Methyl methacrylate

Methyl methanesulfonate

N-Nitrosodi-n-butylamine

$\mathrm{N}$-Nitrosodiethylamine

N-Nitrosodimethylamine

$\mathrm{N}$-Nitrosodiphenylamine

N-Nitrosodipropylamine

N-Nitrosomethylethylamine

$\mathrm{N}$-Nitrosomorpholine

N-Nitrosopiperidine

N-Nitrosopyrrolidine

Naphthalene

Nickel, total recoverable

Nitrobenzene

0,0,0-Triethyl phosphorothioate

Parathion ethyl

Parathion methyl

Pentachlorobenzene
101998

$201998 \quad 301998$

$<5$

$<5$

$<100$

$<10.2 \quad 1 \quad$ U $\quad$ ug/L $\quad$ WA $<$ EQL

$<10.2$ I U ug/L WA $<$ EQL

6.4
$<5$

$<10$

$<12$

$<5$

19.1
Compliance

401998 DEMod Unit

$<10.2 \quad 1 \quad \mathrm{U}$ ug/L

$<.51 \quad 1 \mathrm{U}$ ug/L

$<10.21 \mathrm{U} \quad \mathrm{ug} / \mathrm{L}$

$<10.2 \quad 1 \quad \mathrm{U} \quad \mathrm{ug} / \mathrm{L}$

$<1.33 \quad 1 \quad \mathrm{U} \quad \mathrm{ug} / \mathrm{L}$

$<10.2 \quad 1 \quad$ U $\quad$ ug/L

$<10.2 \quad 1 \quad \mathrm{U} \quad \mathrm{ug} / \mathrm{L}$

$<10.2 \quad 1 \quad \mathrm{U} \quad \mathrm{ug} / \mathrm{L}$

$<10.2 \quad 1 \quad$ U $\quad$ ug/L

$<10.2 \quad 1 \quad \mathrm{U} \quad \mathrm{ug} / \mathrm{L}$

$<10.2$ I U ug/L

$<102 \quad 1 \quad$ U $\quad$ ug/L

$<10.2 \quad 1 \quad$ U $\quad$ ug/L

$<10.21 \mathrm{U} \quad \mathrm{ug} / \mathrm{L}$

WA $<$ EQL

WA $<$ EQL

WA $<$ EQL

WA $<$ EQL

WA $<$ EQL

WA $<$ EQL

WA $<$ EQL

WA $<$ EQL

WA $<$ EQL

WA $<$ EQL
$<10.2 \quad 1 \quad \mathrm{U} \quad$ ug/L $\quad$ WA $<$ EQL

$<10.2 \quad 1 \quad$ U $\quad$ ug/L $\quad$ WA $<$ EQL

$<10.2 \quad 1 \quad \mathrm{U} \quad$ ug/L $\quad$ WA $<$ EQL

$<10.2 \quad 1 \quad$ U $\quad$ ug/L $\quad$ WA $<$ EQL

$<10.2 \quad 1 \quad \mathrm{U}$ ug/L $\quad$ WA $<$ EQL

$<10.2 \quad 1 \quad$ U $\quad u g / L \quad$ WA $<$ EQL

$<10.2 \quad 1 \quad \mathrm{U}$ ug/L $\quad$ WA $<$ EQL

$<10.2 \quad 1 \quad$ U $\quad$ ug/L $\quad$ WA $<$ EQL

$<10.2 \quad 1 \quad \mathrm{U} \quad$ ug/L $\quad$ WA $<$ EQL

$\leq 10.2 \quad 1 \quad \mathrm{U}_{\text {U }}$ ug/L_ WA $<$ EQL

$<51 \quad 1 \quad U \quad u g / L \quad W A<E Q L$

$<10.2 \quad 1 \quad \mathrm{U}$ ug/L $\quad$ WA $<$ EQL

$<10.2$ I U ug/L WA $<$ EQL

$\begin{array}{llllll}<10.2 & 1 & \mathrm{U} & \mathrm{ug} / \mathrm{L} & \text { WA } & <\mathrm{EQL}\end{array}$

$<.51 \quad 1$ U ug/L WA $<$ EQL

$<.51 \quad 1 \quad$ U ug/L WA $<$ EQL

$<.51 \quad 1 \quad \mathrm{U}$ ug/L WA $<$ EQL

$<10.21 \mathrm{U}$ ug/L WA $<$ EQL

Note: Flagging, dilution factors, modifiers, and laboratories are for fourth quartei 998 data only. See Appendix B for flagging criteria.

- =xceeded holding time for fourth quarter 1998.

+ = exceeded screening level or final primary drinking water standard for fourth quarter 998 .

Sanitary Landfil!

D- 152

Fourth Quarter 1998 and Annual Summary 
WSRC-TR-99-00011

Unclassified

WELL: LFW 76

ANALYTICAL DATA

\section{H ST Analyte}

Pentachloroethane

Pentachloronitrobenzene

Pentachlorophenol

Phenacetin

Phenanthrene

Phenol

Phorate

Pronamid

Propionitrile

Pyrene

Pyridine

Safrole

Selenium, total recoverable

Silver, total recoverable

Specific conductance

Styrene

Sulfotepp

Tetrachloroethylene

Thallium, total recoverable

Thionazin

Toluene

Trichloroethylene

Trichlorofluoromethane

Tritium

Unknown 13

Unknown 15

Unknown 16

Unknown 17

Vanadium, total recoverable

Vinyl acetate

Xylenes

Zinc, total recoverable

a,a-Dimethylphenethylamine beta-Benzene hexachloride cis-1,2-Dichloroethylene cis-1,3-Dichloropropene m-Cresol (3-Methylphenol) m-Nitroaniline o-Cresol (2-Methylphenol)

o-Nitroaniline

o-Toluidine

p-Cresol (4-Methylphenol)

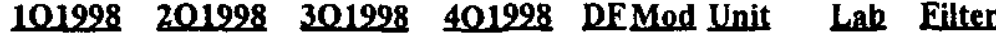

$<10.2 \quad 1 \quad \mathrm{U} \quad \mathrm{ug} / \mathrm{L} \quad$ WA $<$ EQL

$<51 \quad 1 \quad$ U $\quad$ ug/L $\quad$ WA $<$ EQL

$<25.5 \quad 1 \quad$ U $\quad$ ug/L WA $<$ EQL

$<10.2 \quad 1 \quad$ U $\quad$ ug/L $\quad$ WA $<$ EQL

$<10.2 \quad 1 \quad$ U ug/L WA $<$ EQL

$<10.2 \quad 1 \quad$ U $\quad$ ug/L $\quad$ WA $<$ EQL

$<1.02 \quad 1 \quad \mathrm{U} \quad \mathrm{ug} / \mathrm{L} \quad$ WA $<$ EQL

$<10.2$ I U ug/L WA $<$ EQL

$<5$

$<5$

$<2$

49.6

$<5$

$<5$

$<5$

$\begin{array}{llllll}<10.2 & 1 & \mathrm{U} & \mathrm{ug} / \mathrm{L} & \text { WA } & <\mathrm{EQL}\end{array}$

$<10.2 \quad 1 \quad$ U $\quad$ ug/L $\quad$ WA $<$ EQL

$<10.2 \quad 1 \quad U \quad u g / L \quad$ WA $<$ EQL

$<5$

$<5$

$<5$

829

$<4.27$

$<5.08$

$<4.67$

$<8.79$

2.9

$<5$

$<5$

18.8

$<.015$

$<5$

$<5$

Note: Flagging, dilution factors, modifiers, and laboratories are for fourth quartel998 data only. See Appendix B for fagging criteria.

* = exceeded holding time for fourth quarter 1998.

$+=$ exceeded screening level or final primary drinking water standard for fourth quarter1998 . 
WSRC-TR-99-00011

Unclassified

WELL: LFW 76

ANALYTICAL DATA

H ST Analvte

101998

$201998 \quad 301998$

401998 DEMod Unit

Compliance

p-Dimethylaminoazobenzene

p-Nitroaniline

p-Phenylenediamine

pH

6.84

trans-1,3-Dichloropropene

$<5$

trans-1,4-Dichloro-2-butene

$<5$

$\begin{array}{llllll}<10.2 & 1 & \mathrm{U} & \mathrm{ug} / \mathrm{L} & \text { WA } & <\mathrm{EQL} \\ <25.5 & \mathrm{I} & \mathrm{U} & \mathrm{ug} / \mathrm{L} & \text { WA } & <\mathrm{EQL} \\ <10.2 & 1 & \mathrm{U} & \mathrm{ug} / \mathrm{L} & \text { WA } & <\mathrm{EQL}\end{array}$

$<10.2 \quad 1 \quad \mathrm{U} \quad \mathrm{ug} / \mathrm{L} \quad \mathrm{WA}_{\mathrm{A}}<\mathrm{EQL}$

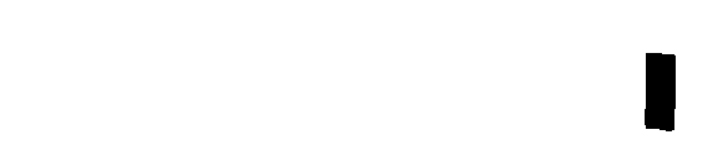

Note: Flagging, dilution factors, modifiers, and laboratories are for fourth quartel 998 data only. See Appendix B for fagging criteria.

$\star$ = exceeded holding time for fourth quarter 1998.

+ exceeded screening level or final primary drinking water standard for fourth quarter 998 .

Sanitary Landfill

D- 154

Fourth Quarter 1998 and Annual Summary 
WSRC-TR-99-00011

Unclassified

WELL: LFW 77

\begin{tabular}{|c|c|c|c|c|c|c|}
\hline SRS Coord. & Lat/Longitude & Screen Zone Elevation & Ton of Casing & Casing & Pump & Eormation \\
\hline N 86461.7 & & $159.2-144.2 \mathrm{ft} \mathrm{msl}$ & $222.7 \mathrm{ft} \mathrm{msl}$ & 2"PVC & $\mathbf{V}$ & d Pond \\
\hline
\end{tabular}

E 44866.5 81.717 Deg W

$02 / 06 / 98$

SAMPLE DATE

FIELD DATA

Analyte

Water Elevation

Depth to water

pH

Sp. Conductance

Water temperature

Alkalinity as $\mathrm{CaCO} 3$

Turbidity

Volumes purged

Sampling code

ANALYTICAL DATA

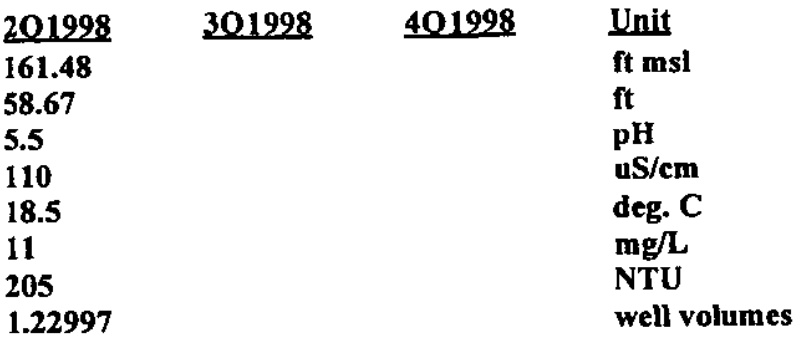

\section{H ST Analyte}

1,1,1,2-Tetrachloroethane

1,1,1-Trichloroethane

$\begin{array}{ll}101998 & 201998 \\ & 161.48 \\ & 58.67 \\ & 5.5 \\ & 110 \\ 18.5 \\ 11 \\ 205 \\ 1.22997 \\ \mathrm{NX}\end{array}$

$\frac{\text { Ton of Casing }}{222.7 \mathrm{ft} \text { msl }} \quad \frac{\text { Casing }}{\text { 2"PVC }} \quad \underset{V}{\text { Pump }} \frac{\text { Eormation }}{\text { U Steed Pond }}$

1,1,2,2-Tetrachloroethane

1,1,2-Trichloroethane

1,1-Dichloroethane

1,1-Dichloroethylene

1,2,3-Trichloropropane

1,2-Dibromo-3-chloropropane

1,2-Dibromoethane

1,2-Dichlorobenzene

1,2-Dichloroethane

1,2-Dichloropropane

1,4-Dichlorobenzene

2-Hexanone

Acetone

Acetonitrile (Methyl cyanide)

Acrolein

Acrylonitrile

Allyl chloride

Benzene

Bromochloromethane

Bromodichloromethane

Bromoform

Bromomethane (Methyl bromide)

Carbon disulfide

Carbon tetrachloride



Note: Flagging, dilution factors, modifiers, and laboratories are for fourth quarte1998 data onfy. See Appendix B for flagging criteria.

* =xceeded holding time for fourth quarter 1998 .

$+=$ exceeded screening level or final primary drinking water standard for fourth quarter1998.

Sanitary Landfill

D- 155

Fourth Quarter 1998 and Annual Summary 
WELL: LFW 77

\section{ANALYTICAL DATA}

H ST Analyte

Chlorobenzene

Chloroethane

Chloroethene (Vinyl chloride)

Chloroform

Chloromethane (Methyl chloride)

Chloroprene

Di-n-butyl phthalate

Dibromochloromethane

Dibromomethane (Methylene bromide)

Dichlorodifluoromethane

Dichloromethane (Methylene chloride)

Ethylbenzene

Iodomethane (Methyl iodide)

Isobutyl alcohol

Methacrylonitrile

Methyl ethyl ketone

Methyl isobutyl ketone

Methyl methacrylate

Propionitrile

Specific conductance

Styrene

Tetrachloroethylene

Toluene

Trichloroethylene

Trichlorofluoromethane

Tritium

Vinyl acetate

Xylenes

beta-Benzene hexachloride

cis-1,2-Dichloroethylene

cis-1,3-Dichloropropene

pH

trans-1,3-Dichloropropene
$101998 \quad 201998$

$<5$

$<10$

$<10$

$<5$

$<10$

$<5$

$<10$

$<5$

$<5$

$<5$

$<3.4$

$<5$

$<5$

$<100$

$<5$

$<10$

$<12$

$<5$

$<5$

60.6

$<5$

$<5$

$<5$

$<5$

$<5$

$<668$

$<5$

$<5$

$<.015$

$<5$

$<5$

6.02

trans-1,4-Dichloro-2-butene

\section{Compliance}

Lab Eilter 
WSRC-TR-99-00011

Unclassified

WELL: LFW 78

\begin{tabular}{|c|c|c|c|c|c|c|}
\hline SRS Coord. & Lat/Longitude & Screen Zone Elevation & Top of Casing & Casing & Pumo & Eormation \\
\hline $\begin{array}{l}\text { N } 86064.9 \\
\text { E } 44726.5\end{array}$ & $\begin{array}{l}\text { 33.289 Deg N } \\
\text { 81.716 Deg W }\end{array}$ & $164.9-149.9$ ft msl & $238.9 \mathrm{ft} \mathrm{msl}$ & 2"PVC & $\mathbf{v}$ & U Steed Pond \\
\hline
\end{tabular}

SAMPLE DATE

$02 / 17 / 98$

FIELD DATA

Analute

Water Elevation

Depth to water

pH

Sp. Conductance

Water temperature

Alkalinity as $\mathrm{CaCO}$

Turbidity

Volumes purged

Sampling code

$\begin{array}{lll}101998 & 201998 & 301998 \\ \cdots \quad & 159.6 \\ & 76.82 \\ & 5.5 \\ & 55 \\ & 27.8 \\ & 6 \\ & 14 \\ & 13.0161 \\ & \mathrm{NX}\end{array}$

$\underline{401998}$

Unit

ft msi

ft

pH

$\mathrm{uS} / \mathrm{cm}$

deg. $C$

mg/L

NTU

well volumes

ANALYTICAL DATA

H ST Analyte

1,1,1,2-Tetrachloroethane

101998

20199

301998

401998

Comoliance

1,1,1-Trichloroethane

$<5$

$<5$

$\begin{array}{ll}1,1,2,2-\text { Tetrachloroethane } & <5 \\ 1,1,2-\text { Trichloroethane } & <5\end{array}$

1,1-Dichloroethane $\quad<5$

1,1-Dichloroethylene $\quad<5$

1,2,3-Trichloropropane $<5$

1,2-Dibromo-3-chloropropane $\quad<5$

1,2-Dibromoethane $\quad<5$

1,2-Dichlorobenzene $<5$

1,2-Dichloroethane $\quad<5$

1,2-Dichloropropane $\quad<5$

1,4-Dichlorobenzene $\quad<5$

2-Hexanone $\quad<10$

Acetone $\quad<10$

Acetonitrile (Methyl cyanide) $\quad<20$

Acrolein $\quad<10$

Acrylonitrile $\quad<20$

Allyl chloride $\quad<10$

Antimony, total recoverable $\quad<5$

Arsenic, total recoverable $\quad<8$

Barium, total recoverable 5

Benzene $\quad<5$

Beryllium, total recoverable $\quad<1$

Bromochloromethane $\quad<10$

Bromodichloromethane $\quad<5$

Note: Flagging, dilution factors, modifiers, and laboratories are for fourth quartei 998 data only. See Appendix B for flagging criteria.

* = exceeded holding time for fourth quarter 1998 .

$+=$ exceeded screening level or final primary drinking water standard for fourth quarten 998 .

Sanitary Landfil

D- 157

Fourth Quarter 1998 and Annual Summary 
WSRC-TR-99-00011

Unclassified

WELL: LFW 78

\section{ANALYTICAL DATA}

H ST Analyte

101998

201998

301998401998 DEMod Unit

Compliance

Bromoform

$<5$

Bromomethane (Methyl bromide)

$<10$

Cadmium, total recoverable

$<2$

Carbon disulfide

$<5$

Carbon tetrachloride $\quad \ldots \ldots+\ldots$

Chlorobenzene

$<5$

Chloroethane

$<10$

Chloroethene (Vinyl chloride)

$<10$

Chloroform

$<5$

Chloromethane (Methyl chloride)

$<10$

Chloroprene

$<5$

Chromium, total recoverable

Cobalt, total recoverable

$<5$

Copper, total recoverable

Di-n-butyl phthalate

$<.55$

Dibromochloromethane

$<5$

Dibromomethane (Methylene bromide)

$<5$

Dichlorodifluoromethane

$<5$

Dichloromethane (Methylene chloride)

$<2.3$

Ethylbenzene

$<5$

Iodomethane (Methyl iodide)

$<5$

Isobutyl alcohol

$<100$

Lead, total recoverable

Methacrylonitrile

$<5$

Methyl ethyl ketone

$<10$

Methyl isobutyl ketone

$<12$

Methyl methacrylate

$<5$

Nickel, total recoverable

Propionitrile

$<5$

Selenium, total recoverable

$<5$

Silver, total recoverable

$<2$

Specific conductance

Styrene

$<5$

Tetrachloroethylene

$<5$

Thallium, total recoverable

$<5$

Toluene

$<5$

Trichloroethylene

$<5$

Trichlorofluoromethane 24

Tritium

Unknown 15

Unknown 17

$<5.68$

Vanadium, total recoverable

Note: Flagging, dilution factors, modifiers, and laboratories are for fourth quartei 998 data only. See Appendix B for nagging eriteria. * = exceeded holding time for fourth quarter 1998 .

$+=$ exceeded screening level or final primary drinking water standard for fourth quartert 998 .

Sanitary Landfil!

D- 158

Fourth Quarter 1998 and Annual Summary 
WSRC-TR-99-00011

Unclassified

WELL: LFW 78

ANALYTICAL DATA

\section{H ST Analyte}

Vinyl acetate

Xylenes

Zinc, total recoverable beta-Benzene hexachloride cis-1,2-Dichloroethylene cis-1,3-Dichloropropene pH

trans-1,3-Dichloropropene trans-1,4-Dichloro-2-butene
Compliance

101998201998301998 401998 DFMod Unit Lab Eilter

$<5$

25.8

$<.015$

$<5$

$<5$

6.48

$<5$

$<5$

Note: Flagging, dilution factors, modifiers, and laboratories are for fourth quartei 998 data only. See Appendix B for fagging criteria. * a exceeded holding time for fourth quarter 1998.

+ = exceeded screening level or final primary drinking water standard for fourth quarten 998 . 


\section{Appendix E}

\section{Data Quality/Usability Assessment}


WSRC-TR-99-00011

Unclassified

THIS PAGE LEFT BLANK INTENTIONALLY.

THS PAGELEFT BLANK INTENTIONALLY. 


\section{Data Quality/Usability Assessment}

Quality assurance/quality control (QA/QC) procedures relating to accuracy and precision of analyses performed on groundwater samples are followed in the field and laboratory and are reviewed prior to publication of results. The review by the Environmental Protection Department/Environmental Monitoring Section (EPD/EMS) of the volume of analytical data acquired each quarter and presented in various reports is an ongoing process; its review of the QA/QC data cannot be completed in time to meet the deadlines for the reports required by the Resource Conservation and Recovery Act and associated regulations. Other site and regulatory personnel can obtain further information on the data quality and usability in a variety of ways, including those described below.

\section{Data Qualification}

The contract laboratories continually assess their own accuracy and precision according to U.S. Environmental Protection Agency (EPA) guidelines. They submit sample- or batch-specific QAVC information either at the same time as analytical results or in quarterly summaries. Properly defined and used result modifiers (also referred to as qualifiers) can be a key component in assessing data usability. Result modifiers designed by EPD/EMS and used by the primary laboratories are presented in Appendix D.

\section{Assessment of Accuracy of the Data}

Accuracy, or the neamess of the reported result to the true concentration of a constituent in a sample, can be assessed in several ways.

A laboratory's general accuracy can be judged by analysis of results obtained from known samples. The non-radionuclide contract laboratories analyze commercial reference samples every quarter at EPD/EMS' request. The results of these analyses are presented in the EPD/EMS groundwater monitoring quarterly reports. The primary laboratories also seek or maintain state certification by participating periodically in performance studies; reference samples and analysis of results are provided by EPA. Results of these studies also are published in the EPD/EMS quarterly reports.

Analysis of blanks provides a tool for assessing the accuracy of both sampling and laboratory analysis. Results for all field blanks for the quarter can be found in the EPD/EMS quarterly reports. Any field or laboratory blanks that exceed established minimums are identified in the same reports, in tables associating them with groundwater samples analyzed in the same batches.

Surrogates, organic compounds similar in chemical behavior to the compounds of interest but not normally found in environmental samples, are used to monitor the effect of the matrix on the accuracy of analyses for organic parameters. For example, for analyses of volatile organics by EPA Method 8240, three surtogate compounds are added to all samples and blanks in each analytical batch. In analyses of semivolatile organics, three acid compounds and three base/neutral compounds are used. Two surrogates are used in organochlorine pesticides analyses. Percent recoveries for surrogate analyses are calculated by laboratory personnel, reported to EPD/EMS, reviewed, and entered into the database, but they are not published. If recoveries are not within specified limits, the laboratory is expected to reanalyze the samples or attach qualifiers to the data identifying the anomalous results.

Sample-specific accuracy for both organic and inorganic parameters can be assessed by examination of matrix spike/matrix spike duplicate results. A sample is analyzed unspiked to determine a baseline set of values. A second portion of the sample is spiked with known concentrations of compounds appropriate to the analyses being performed, typically five volatile organic compounds for volatile organics analyses, eleven semivolatile compounds for semivolatiles, six pesticide compounds for pesticides, all metals for metals analyses by SW-846 methods (EPA, 1986), and a known quantity of cyanide for cyanide analysis. The percentage of the spike compound that is recovered (i.e., measured in excess of the value obtained for the unspiked sample) is a direct measure of analytical accuracy. EPA requires matrix spike/matrix spike duplicates to be run at least once per 20 samples of similar matrix. 
Matrix spike/matrix spike duplicate results are reported to EPD/EMS but are not published. For organic compounds, according to EPA guidelines, no action is taken on the basis of matrix spike/matrix spike duplicate data alone (i.e., no result modifiers are assigned solely on the basis of matrix spike results); however, the results can indicate if a laboratory is having a systematic problem in the analysis of one or more analytes.

In the case of organic compounds, such as metals, the matrix spike sample analysis provides information about the effect of each sample matrix on the digestion and measurement methodology. Data qualifiers assigned by the laboratories on the basis of the percentage of spike recovery are reported in the published results tables.

\section{Assessment of Precision}

Precision of the analyses, or agreement of a set of replicate results among themselves, is assessed through the use of duplicates initiated by the laboratory and blind replicates provided by EPD/EMS. The results of duplicate and replicate analyses are presented in those results tables of the quarterly reports which report only one quarter of data, usually during first, second, and third quarters. Duplicate and replicate results are not presented in results tables that report more than one quarter of data, generally provided in the fourth quarter reports. In this case, the results' tables instead present only the highest result for each analyte for each quarter of the year.

The laboratories assess the precision by calculating the relative percent difference (RPD) for each pair of laboratory-initiated duplicate results. One of the contract laboratories uses a data qualifier (J3) to modify metals analyses when the RPD for laboratory duplicates is greater than 20 percent.

Additional statistical comparisons of laboratory duplicate and blind duplicate results, both intraand inter-laboratory, are presented in the EPD/EMS quarterly reports. The calculation used for these reports is the mean relative difference (MRD) which is similar to EPA's RPD except that the $M R D$ is the average of all the RPD values from one laboratory for each compound (intralaboratory MRD) or all the RPD values from all laboratories for each compound (inter-laboratory $M R D$ ), during one quarter. Because detection limits may vary among samples, the MRD requires calculation of a reference detection limit, which is the detection limit at the $90^{\text {th }}$ percentile of the array of limits in the population of all duplicate and replicate analyses for a given analyte during a particular quarter. The MRD is not method-specific.

\section{Method-Specific Accuracy and Precision}

The contract laboratories' EPA approved laboratory procedures include QAVC requirements as an integral part of the methods. Thus, knowledge of the method used in obtaining data is an important component of determining data usability. EPA has conducted extensive research and development on the methods approved for the analysis of water and wastewater; information on the accuracy and precision of a method is available from EPA publications, as is full information on required QA/QC procedures. A listing of the methods used by the primary laboratories during fourth quarter 1998 is given below along with the source for the method description. Many, if not all, of these sources include presentations of representative accuracy and precision results.

Methods Used by the Contract Laboratories

\begin{tabular}{lll} 
Method & Used to Analyze & Source \\
\hline EPA120.1 & Specific conductance & \\
EPA150.1 & pH & EPA EMSL $=1983$ \\
EPA160.1 & Total dissolved solids & EPA EMSL, 1983 \\
EPA160.2 & Total dissolved solids, total suspended solids & EPA EMSL, 1983 \\
EPA180.1 & Turbidity & EPA EMSL, 1983 \\
EPA200.7 & Metals & EPA EMSL, 1983 \\
EPA204.2 & Antimony & EPA EMSL, 1983 \\
EPA206.2 & Arsenic & EPA EMSL, 1983 \\
EPA239.2 & Lead & EPA EMSL, 1983 \\
EPA245.1 & Mercury & EPA EMSL, 1983 \\
EPA270.2 & Selenium & EPA EMSL, 1983 \\
EPA279.2 & Thallium & EPA EMSL, 1983 \\
EPA300.0 & Chloride, nitrite, nitrate & EPA EMSL, 1983 \\
& & EPA EMSL, 1983
\end{tabular}


WSRC-TR-98-00011

Unclassified

\begin{tabular}{|c|c|c|}
\hline Method & Used to Analyze & Source \\
\hline EPA310.1 & Alkalinity & EPA EMSL, 1983 \\
\hline EPA325.2 & Chloride & EPA EMSL, 1983 \\
\hline EPA335.3 & Cyanide & EPA EMSL, 1983 \\
\hline EPA340.2 & Fluoride & EPA EMSL, 1983 \\
\hline EPA353.1 & Nitrogen, nitrate-nitrite & EPA EMSL, 1983 \\
\hline EPA353.2 & Nitrogen, nitrate, nitrite, or combined & EPA EMSL, 1983 \\
\hline EPA365.1 & Phosphorus, all forms (reported as total phosphates) & EPA EMSL, 1983 \\
\hline EPA365.2 & Phosphorus, all forms (reported as total phosphates) & EPA EMSL, 1983 \\
\hline EPA376.2 & Sulfide & EPA EMSL, 1983 \\
\hline EPA413.1 & Oil \& grease & EPA EMSL, 1983 \\
\hline EPA415.1 & $\begin{array}{l}\text { Dissolved organic carbon, total inorganic carbon, } \\
\text { total organic carbon }\end{array}$ & EPA EMSL, 1983 \\
\hline EPA418.1 & Total petroleum hydrocarbons & EPA EMSL, 1983 \\
\hline EPA420.2 & Phenols & EPA EMSL, 1983 \\
\hline EPA900.0 & Gross alpha, nonvolatile beta & EPA EMSL, 1980 \\
\hline EPA900.1 & Total alpha-emitting radium & EPA EMSL, 1980 \\
\hline EPA906.0 & Tritium & EPA EMSL, 1980 \\
\hline EPA6010 & Metals & EPA, 1986 \\
\hline EPA7041 & Antimony & EPA, 1986 \\
\hline EPA7060 & Arsenic & EPA, 1986 \\
\hline EPA7421 & Lead & EPA, 1986 \\
\hline EPA7470 & Metals & EPA, 1986 \\
\hline EPA7740 & Selenium & EPA, 1986 \\
\hline EPA7841 & Thallium & EPA, 1986 \\
\hline EPA8010 & Chlorinated volatile organics & EPA, 1986 \\
\hline EPA8080 & Organochlorine pesticides and PCBs & EPA, 1986 \\
\hline EPA8150 & Chlorinated herbicides & EPA, 1986 \\
\hline EPA8240 & GCMS volatiles & EPA, 1986 \\
\hline EPA8270 & GCMS semivolatiles & EPA, 1986 \\
\hline EPA8280 & Dioxins and furans & EPA, 1986 \\
\hline EPA9012 & Cyanide & EPA, 1986 \\
\hline EPA9020 & Total organic halogens & EPA, 1986 \\
\hline EPA9020A & Total organic halogens & EPA, 1986 \\
\hline EPA9030 & Sulfide & EPA, 1986 \\
\hline EPA9060 & $\begin{array}{l}\text { Dissolved organic carbon, total inorganic carbon, } \\
\text { total organic carbon }\end{array}$ & EPA, 1986 \\
\hline
\end{tabular}

An example of available method-specific QA/QC information is that for the analysis of metals by EPA Method 6010/200.7 (EPA, 1986/EPA EMSL, 1983). The primary laboratories, General Engineering Laboratories (GE) and Roy F. Weston, Inc. (Weston), use this inductively coupled plasma (ICP) atomic emission spectrometric method.

The following precision and accuracy data are based on the experience of seven laboratories that applied the ICP technique to acid-distilled water matrices that had been spiked with various metal concentrates. (Note: Not all seven laboratories analyzed all 14 elements.) The references give results for samples having three concentration ranges; the results here are for samples having the lowest values, similar to actual groundwater results for SRS.

ICP Precision and Accuracy Data

\begin{tabular}{|c|c|c|c|}
\hline Element & True value $(\mu \mathrm{g} / \mathrm{L})$ & $\begin{array}{l}\text { Mean Reported } \\
\text { Value (쌔 })\end{array}$ & $\begin{array}{l}\text { Mean percent } \\
\underline{\mathrm{RSD}^{2}}\end{array}$ \\
\hline Aluminum & 60 & 62 & 33 \\
\hline Arsenic & 22 & 19 & 23 \\
\hline Beryllium & 20 & 20 & 9.8 \\
\hline Cadmium & 2.5 & 2.9 & 16 \\
\hline Chromium & 10 & 10 & 18 \\
\hline Cobalt & 20 & 20 & 4.1 \\
\hline
\end{tabular}


WSRC-TR-98-00011 Unclassified

\begin{tabular}{llll} 
Element & \multicolumn{1}{c}{} & Mean Reported & - Mean percent \\
Copper & 11 & Value $(\mu \mathrm{g} / \mathrm{L})$ & $\underline{\text { RSD }}$ \\
Iron & 20 & 11 & 40 \\
Lead & 24 & 19 & 15 \\
Manganese & 15 & 30 & 32 \\
Nickel & 30 & 15 & 6.7 \\
Selenium & 6 & 28 & 11 \\
Vanadium & 70 & 8.5 & 42 \\
Zinc & 16 & 69 & 2.9 \\
\end{tabular}

"Relative standard deviation. In EPA (1986), the column heading is Mean Standard Deviation (\%).

As another example, EPA Method 601/8010 (EPA, 1991/EPA, 1986) is used by both GE and Weston for analyses of halogenated volatile organics. In the presentation of the method in both references, the following table gives method-specific accuracy and precision as functions of concentration. Contract laboratories are expected to achieve or at least approach these limits.

Accuracy and Precision as Functions of Concentration for EPA Method 601/8010

\begin{tabular}{|c|c|c|c|}
\hline Parameter & $\begin{array}{l}\text { Accuracy as } \\
\text { recovery, } X^{n}(\mu \mathrm{g} / \mathrm{L})\end{array}$ & $\begin{array}{l}\text { Single analyst } \\
\text { precision }(\mu \mathrm{g} / \mathrm{L})^{b}\end{array}$ & $\begin{array}{l}\text { Overall } \\
\text { precision }(\mu \mathrm{g} / \mathrm{L})^{c}\end{array}$ \\
\hline Bromodichloromethane & $1.12 C-1.02^{d}$ & $0.11 X+0.04^{e}$ & $0.20 X+1.00$ \\
\hline Bromoform & $0.96 C-2.05$ & $0.12 X+0.58$ & $0.21 X+2.41$ \\
\hline Bromomethane & $0.76 C-1.27$ & $0.28 X+0.27$ & $0.36 X+0.94$ \\
\hline Carbon tetrachloride & $0.98 C-1.04$ & $0.15 X+0.38$ & $0.20 X+0.39$ \\
\hline Chlorobenzene & $1.00 C-1.23$ & $0.15 \times-0.02$ & $0.18 X+1.21$ \\
\hline Chloroethane & $0.99 C-1.53$ & $0.14 \times-0.13$ & $0.17 X+0.63$ \\
\hline 2-Chloroethyl vinyl ether & $1.00 \mathrm{C}$ & $0.20 \mathrm{X}$ & $0.35 \mathrm{X}$ \\
\hline Chloroform & $0.93 C-0.39$ & $0.13 X+0.15$ & $0.19 \times-0.02$ \\
\hline Chloromethane & $0.77 C+0.18$ & $0.28 \times-0.31$ & $0.52 X+1.31$ \\
\hline Dibromochloromethane & $0.94 C+2.72$ & $0.11 X+1.10$ & $0.24 X+1.68$ \\
\hline 1,2-Dichlorobenzene & $0.93 C+1.70$ & $0.20 X+0.97$ & $0.13 X+6.13$ \\
\hline 1,3-Dichlorobenzene & $0.95 C+0.43$ & $0.14 X+2.33$ & $0.26 X+2.34$ \\
\hline 1,4-Dichlorobenzene & $0.93 C-0.09$ & $0.15 X+0.29$ & $0.20 X+0.41$ \\
\hline 1,1-Dichloroethane & $0.95 C-1.08$ & $0.09 X+0.17$ & $0.14 X+0.94$ \\
\hline 1,2-Dichloroethane & $1.04 C-1.06$ & $0.11 X+0.70$ & $0.15 X+0.94$ \\
\hline 1,1-Dichloroethene & $0.98 C-0.87$ & $0.21 \times-0.23$ & $0.29 \times-0.40$ \\
\hline trans-1,2-Dichloroethene & $0.97 C-0.16$ & $0.11 X+1.46$ & $0.17 X+1.46$ \\
\hline $\begin{array}{l}\text { Dichloromethane } \\
\text { (Methylene chloride) }\end{array}$ & $0.91 C-0.93$ & $0.11 X+0.33$ & $0.21 X+1.43$ \\
\hline 1,2-Dichloropropane $\mathrm{e}^{\mathrm{f}}$ & $1.00 C$ & $0.13 X$ & $0.23 \mathrm{X}$ \\
\hline cis-1,3-Dichloropropene ${ }^{f}$ & $1.00 \mathrm{C}$ & $0.18 \mathrm{X}$ & $0.32 X$ \\
\hline trans-1,3-Dichloropropene ${ }^{f}$ & $1.00 \mathrm{C}$ & $0.18 X$ & $0.32 X$ \\
\hline $1,1,2,2-$ Tetrachloroethane & $0.95 C+0.19$ & $0.14 X+2.41$ & $0.23 X+2.79$ \\
\hline Tetrachloroethylene & $0.94 C+0.06$ & $0.14 X+0.38$ & $0.18 \mathrm{X}+2.21$ \\
\hline 1,1,1-Trichloroethane & $0.90 C-0.16$ & $0.15 X+0.04$ & $0.20 X+0.37$ \\
\hline $1,1,2$-Trichloroethane & $0.86 C+0.30$ & $0.13 X-0.14$ & $0.19 X+0.67$ \\
\hline Trichloroethylene & $0.87 C+0.48$ & $0.13 X-0.03$ & $0.23 X+0.30$ \\
\hline Trichlorofluoromethane & $0.89 C-0.07$ & $0.15 X+0.67$ & $0.26 X+0.91$ \\
\hline Vinyl chloride & $0.97 C-0.36$ & $0.13 X+0.65$ & $0.27 X+0.40$ \\
\hline
\end{tabular}


d $C=$ true value for the concentration, in $\mu \mathrm{g} / \mathrm{L}$.

- $\mathrm{x}=$ average recovery found for measurements of samples containing a concentration of $C$, in $\mu \mathrm{g} / \mathrm{L}$.

$f$ Estimates based on performance of a single laboratory.

\section{References Cited}

EPA (U.S. Environmental Protection Agency), 1986. Test Methods for Evaluating Solid Waste (SW-846), Volume IA-IC. Washington, DC.

EPA (U.S. Environmental Protection Agency), 1991. Guidelines Establishing Test Procedures for the Analysis of Pollutants, Code of Federal Regulations, Title 40, Part 136, Appendix A. Washington, DC.

EPA EMSL (U.S. Environmental Protection Agency, Environmental Monitoring and Systems Laboratory), 1980. Prescribed Procedures for Measurement of Radioactivity in Drinking Water, EPA-6000/4-80-032. Cincinnati, OH.

EPA EMSL (U.S. Environmental Protection Agency, Environmental Monitoring and Systems Laboratory), 1983. Methods for Chemical Analysis of Water and Wastes. Cincinnati, $\mathrm{OH}$.

EPA EMSL (U.S. Environmental Protection Agency, Environmental Monitoring and Systems Laboratory), 1991. Test Method, The Determination of Inorganic Anions in Water by Ion Chromatography-Method 300.0. Cincinnati, $\mathrm{OH}$. 
WSRC-TR-99-00011

Unclassified

\section{Appendix F}

\section{Time Series Plots}


1, 1, 1 - TRI CHLOROE T HA NE WE L L L F W 8

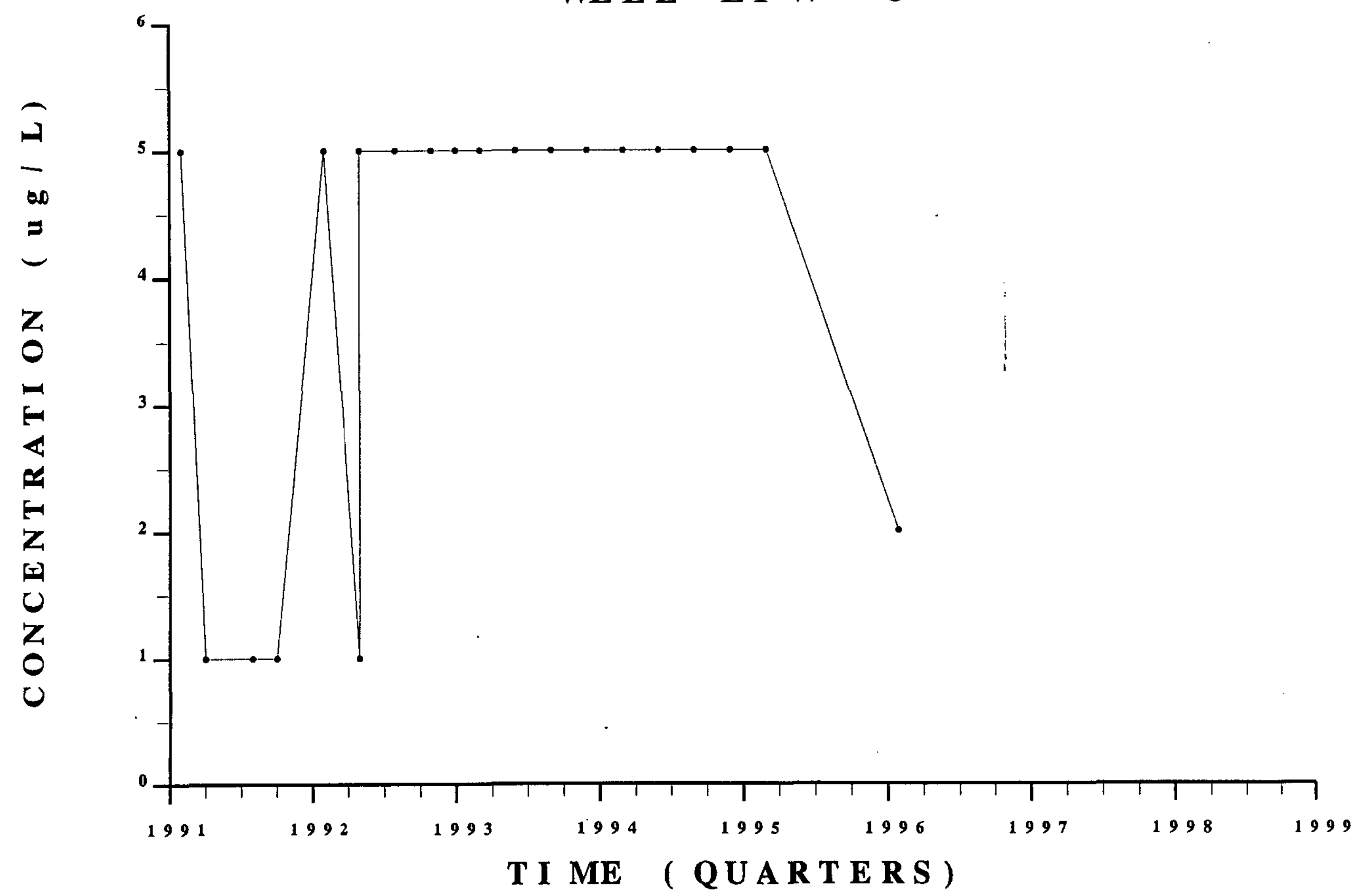

WS R C - T R - $99-000011$ Unc l a s s i f i e d 


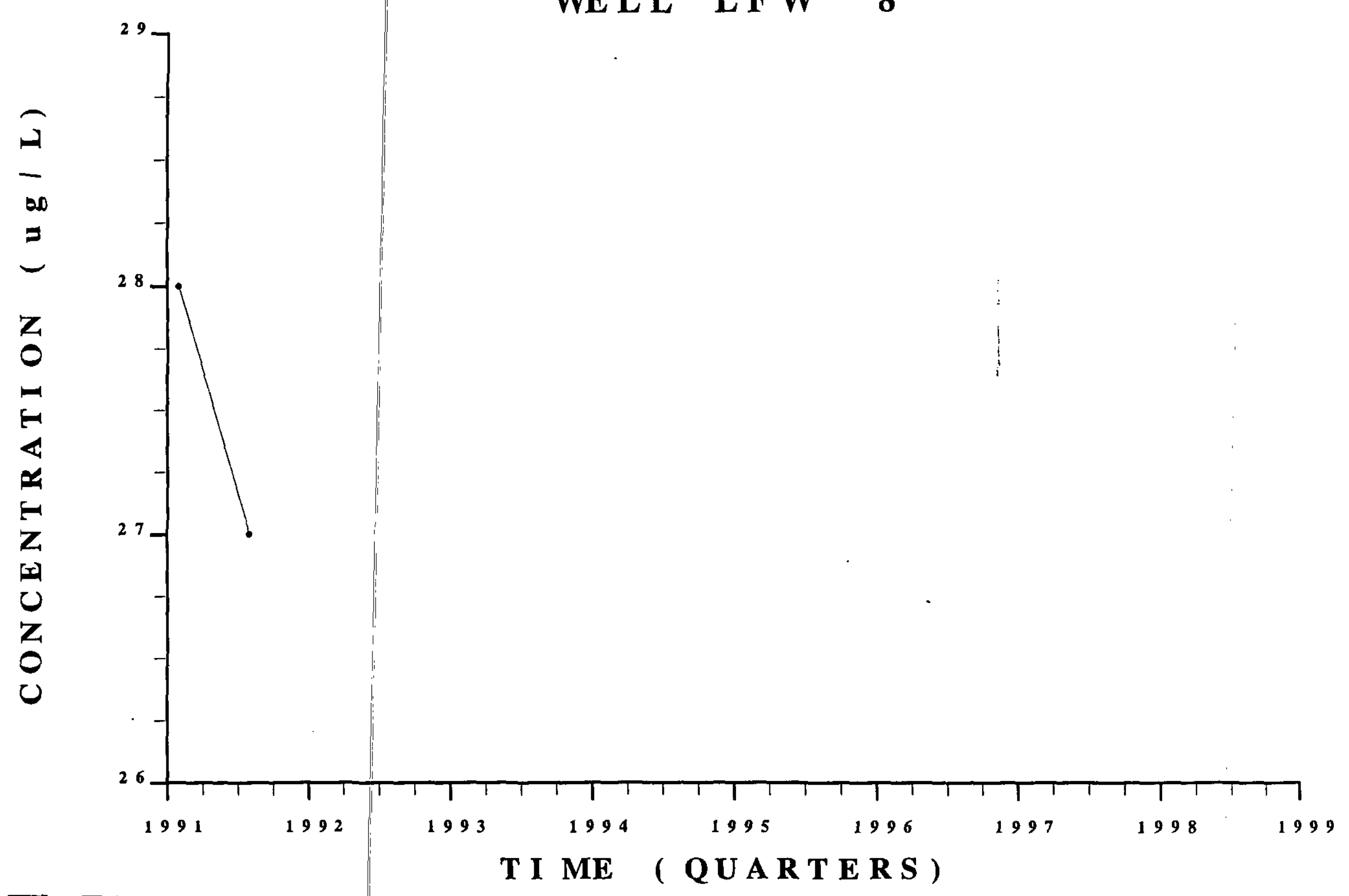

1,4 - D I C H L O R O B E NZ E N E WE L L L F W 8
WS R C - T R - $99-00011$ Unc l a s s i f i ed 

$\begin{array}{llllllllllll}\text { E } & \mathbf{T} & \mathbf{H} & \mathbf{Y} & \mathbf{L} & \mathbf{B} & \mathbf{E} & \mathbf{N} & \mathbf{Z} & \mathbf{E} & \mathbf{N} & \mathbf{E}\end{array}$ WE L L L F W 8
WS R C - T R - 99-0 00011 Unc 1 as s if i ed

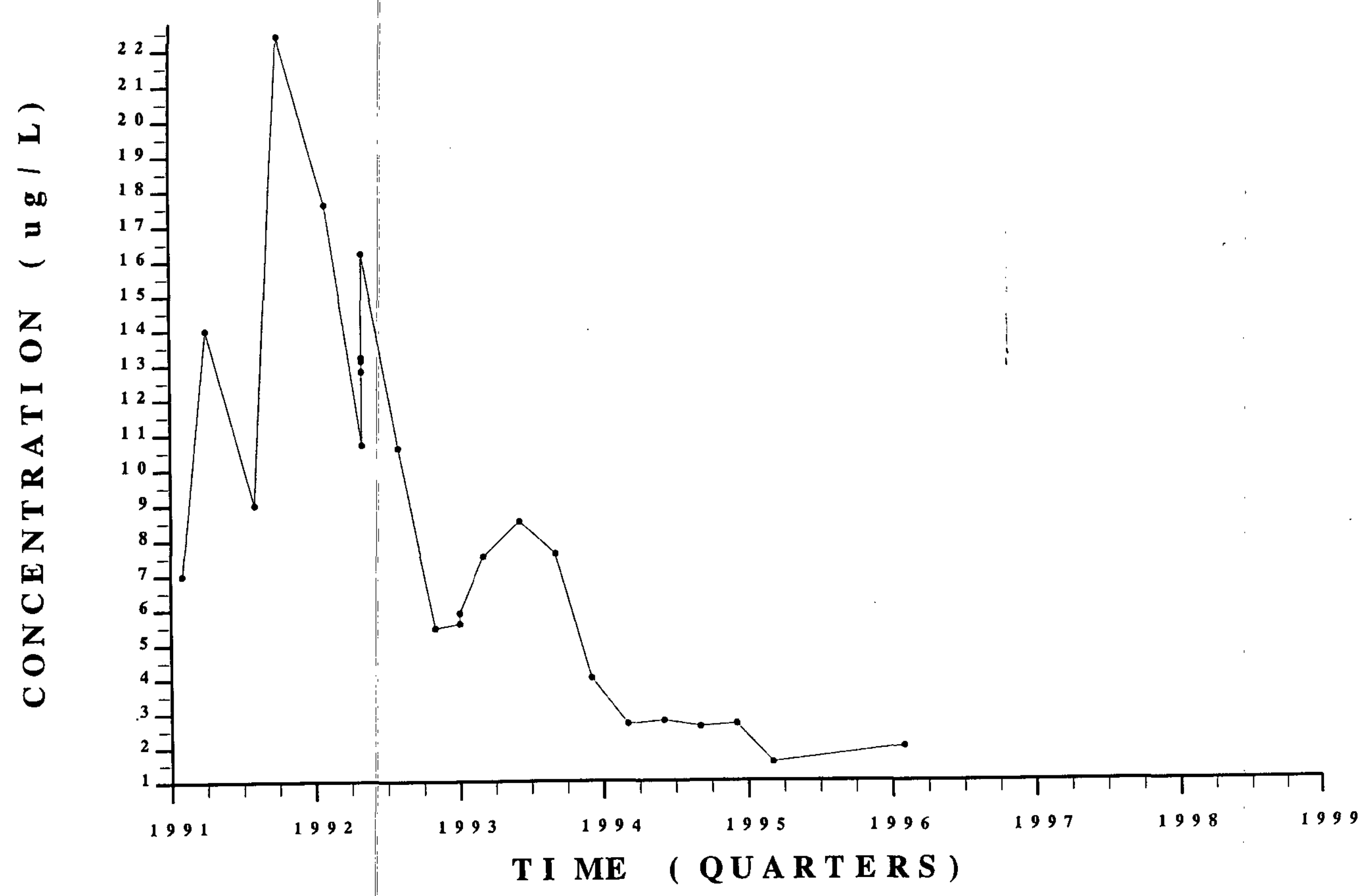




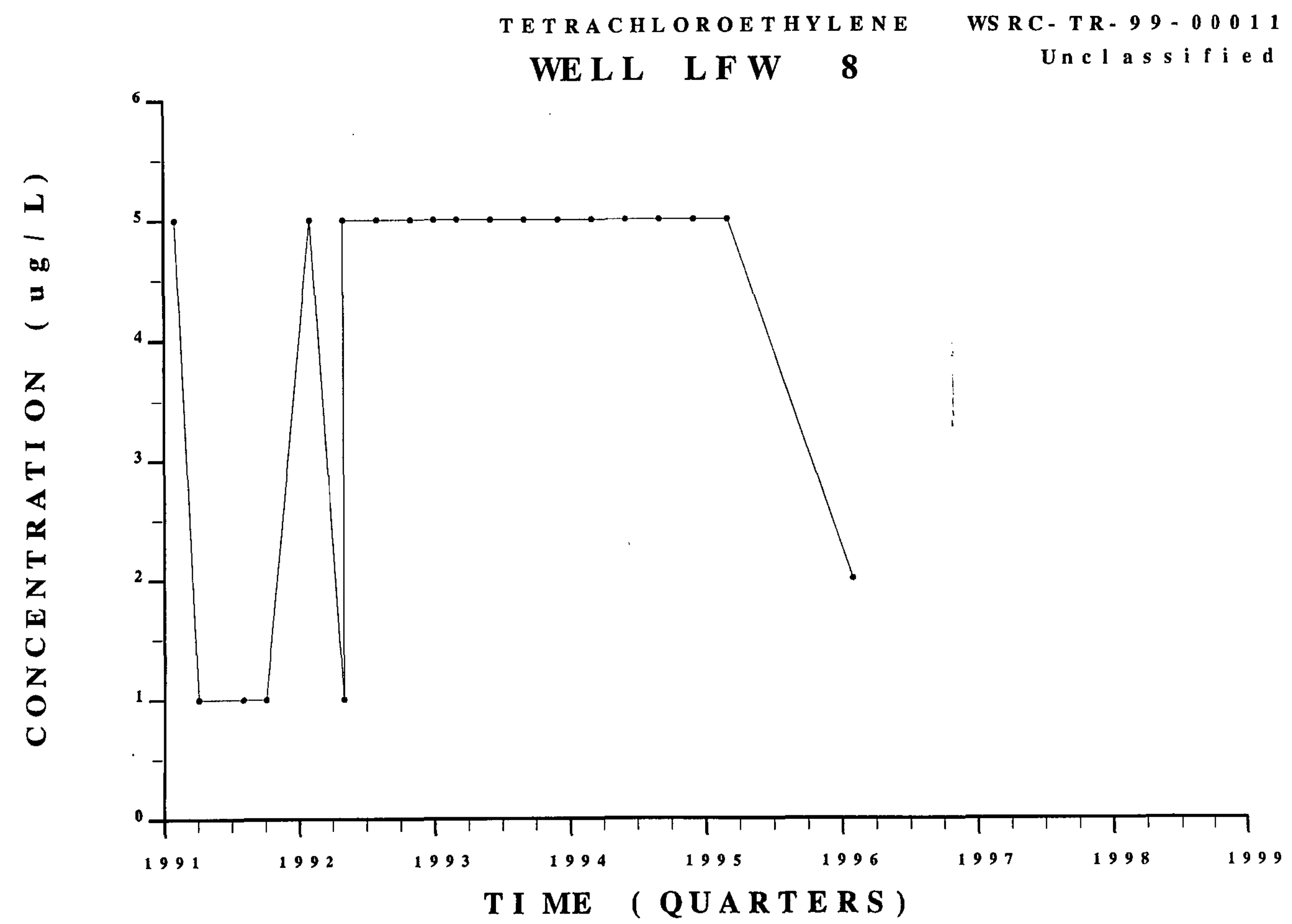

Sanitar v landfill

Fourth Ouarter.

1998

\& 1998

Su mma $r v$ 
T R I C H L O R O E T H Y L E N E WE L L L F W 8

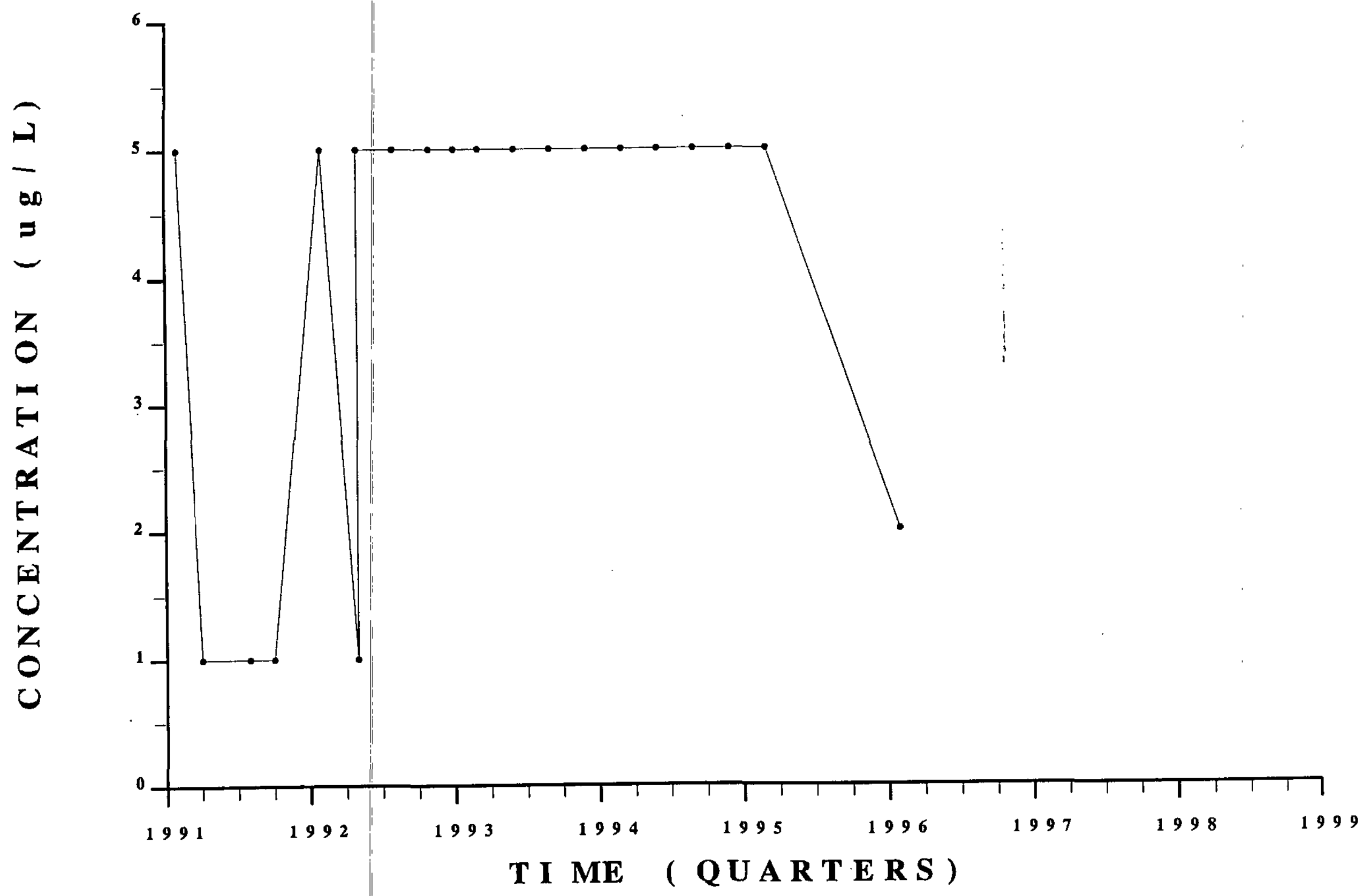

Sanitarv Landfil 


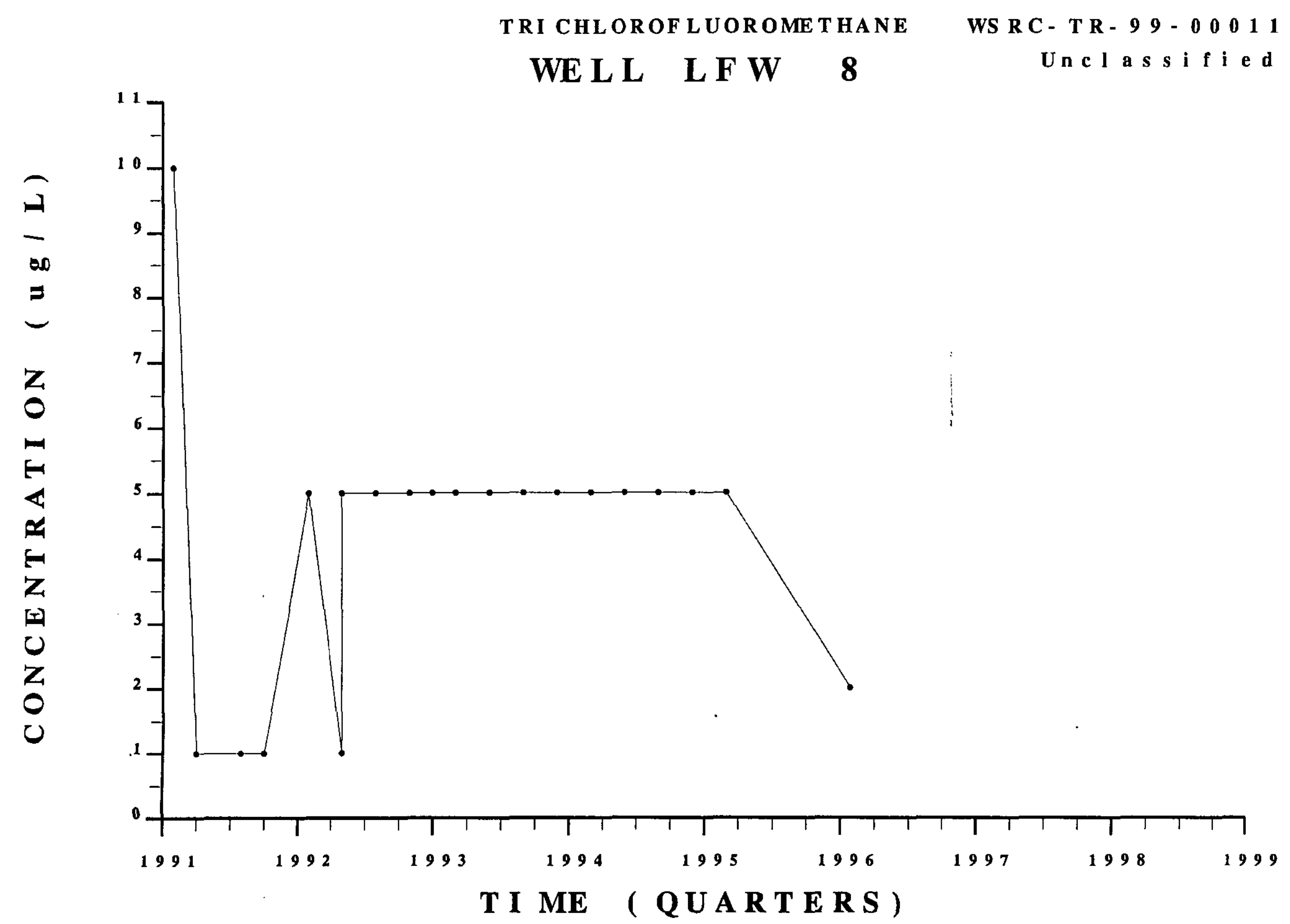

Sani tar v Landfi I I

Fourth ouarter.

$1998 \quad \& \quad 1998$

Summar 


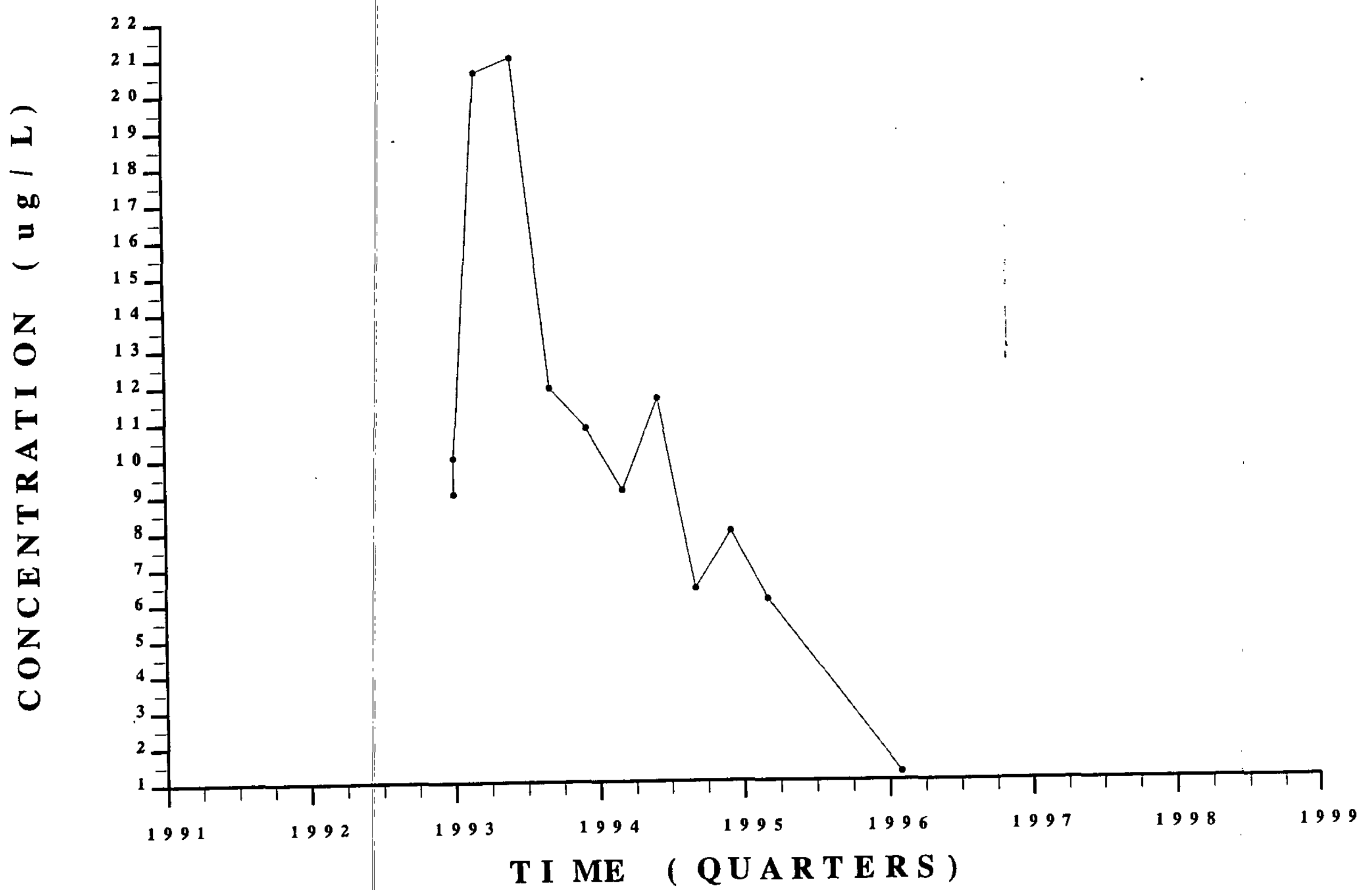

Snnitarv I,andfil 


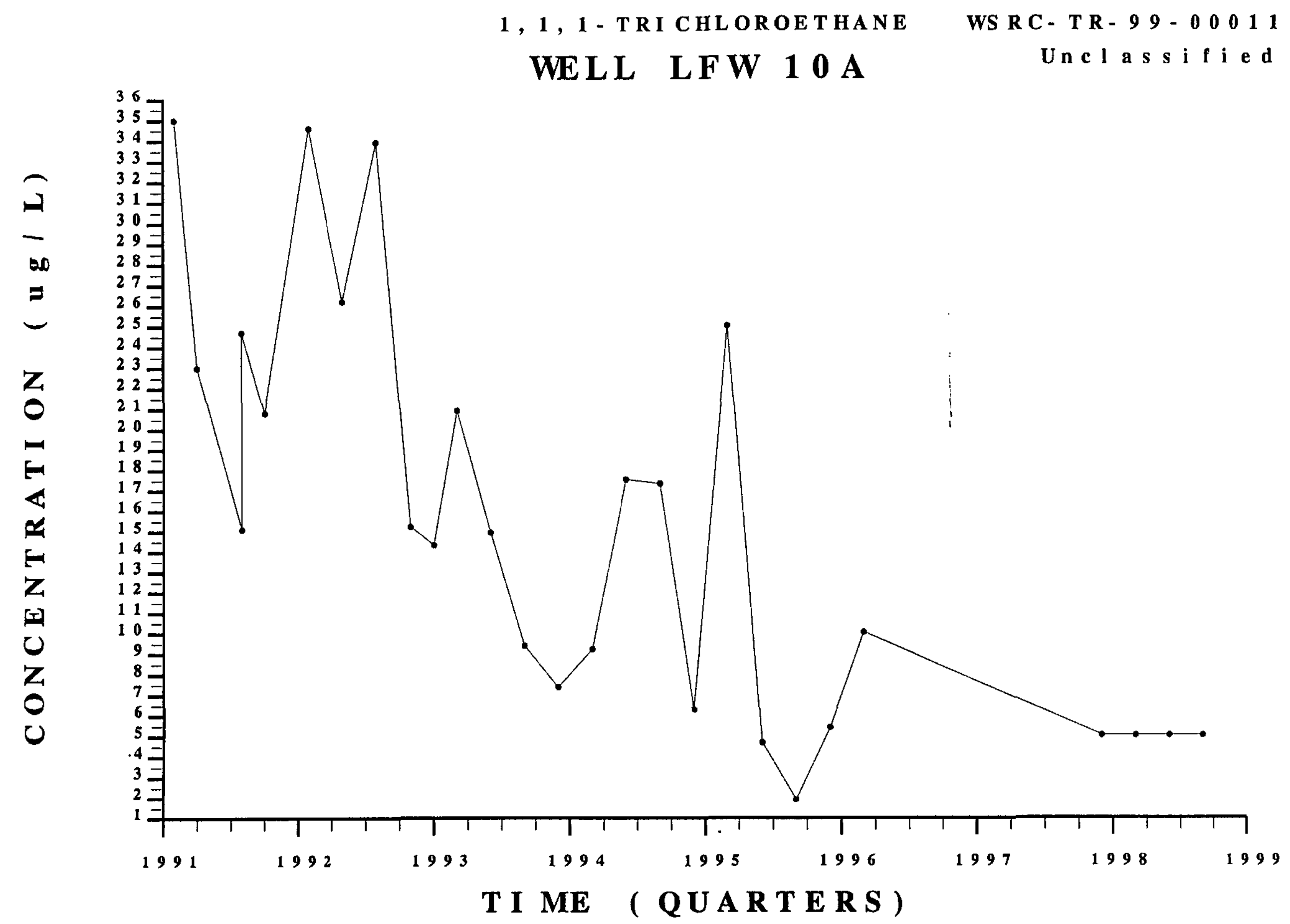

Sa n i a r v Land f i 1 l

Fourth Ouarter.

1998

\& 1998

Su mma r v 


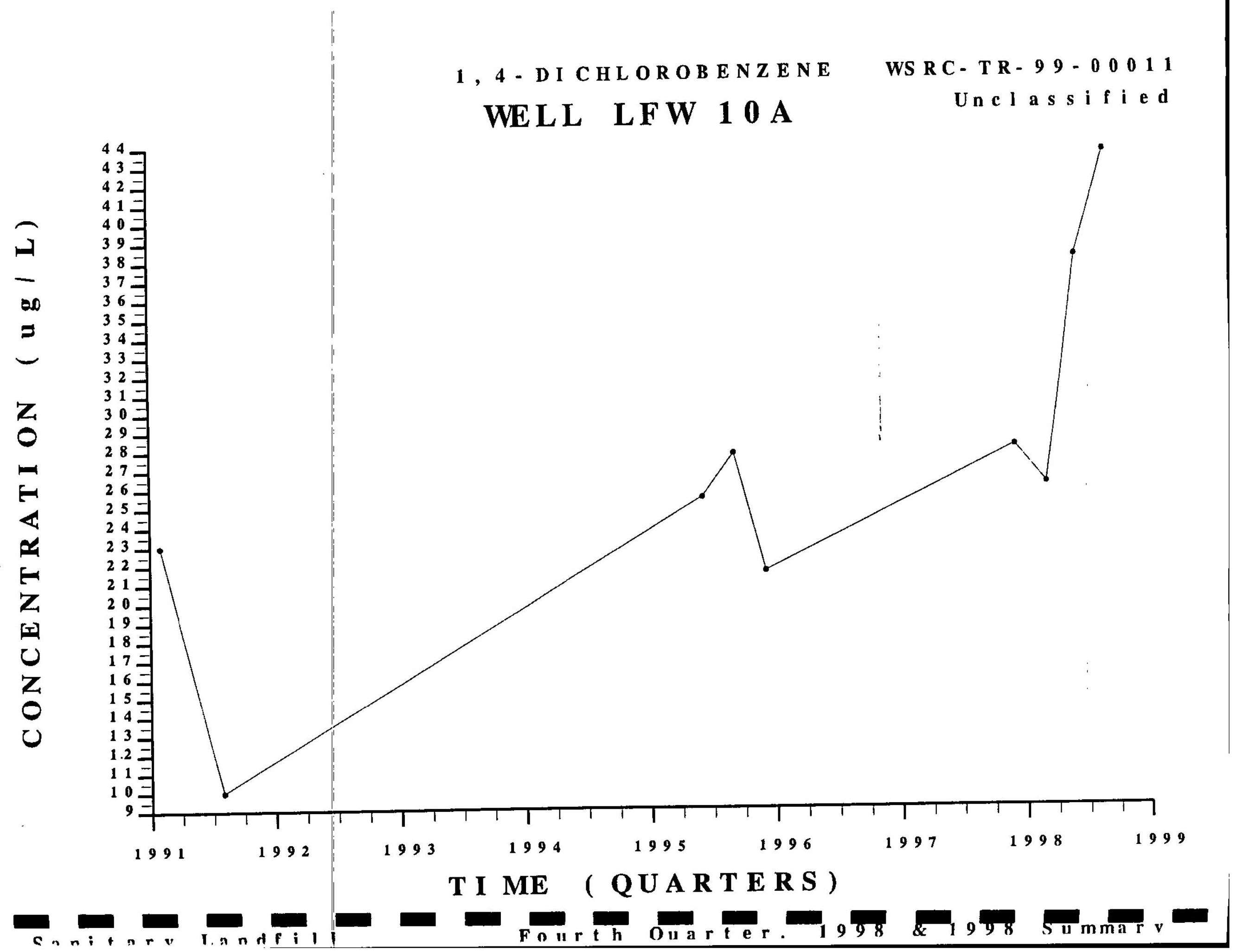




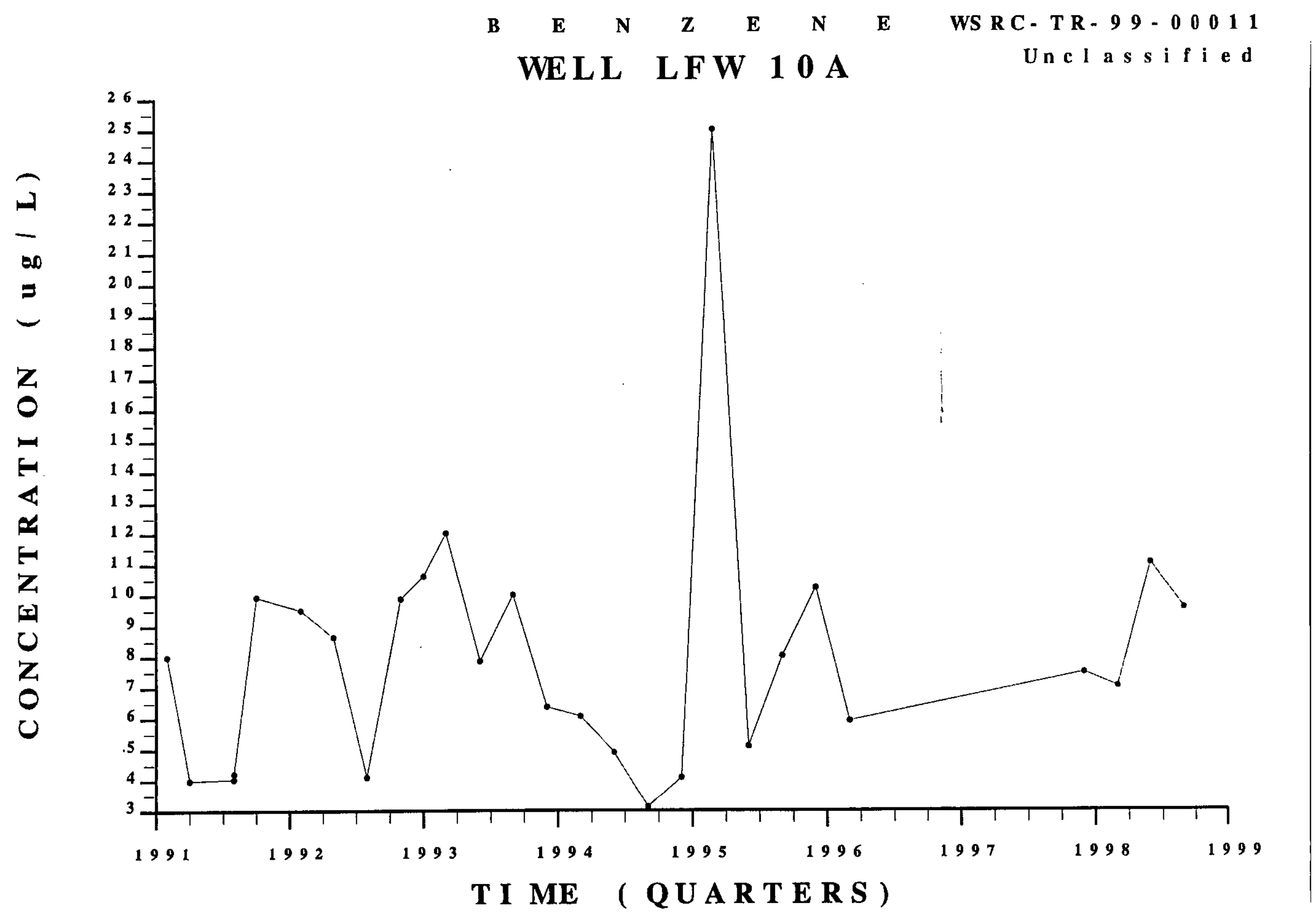

$S$ a $n$ i t a r v L a n d f i I I

Fourth Ouarter. 1998 \& 1998 Summarv 


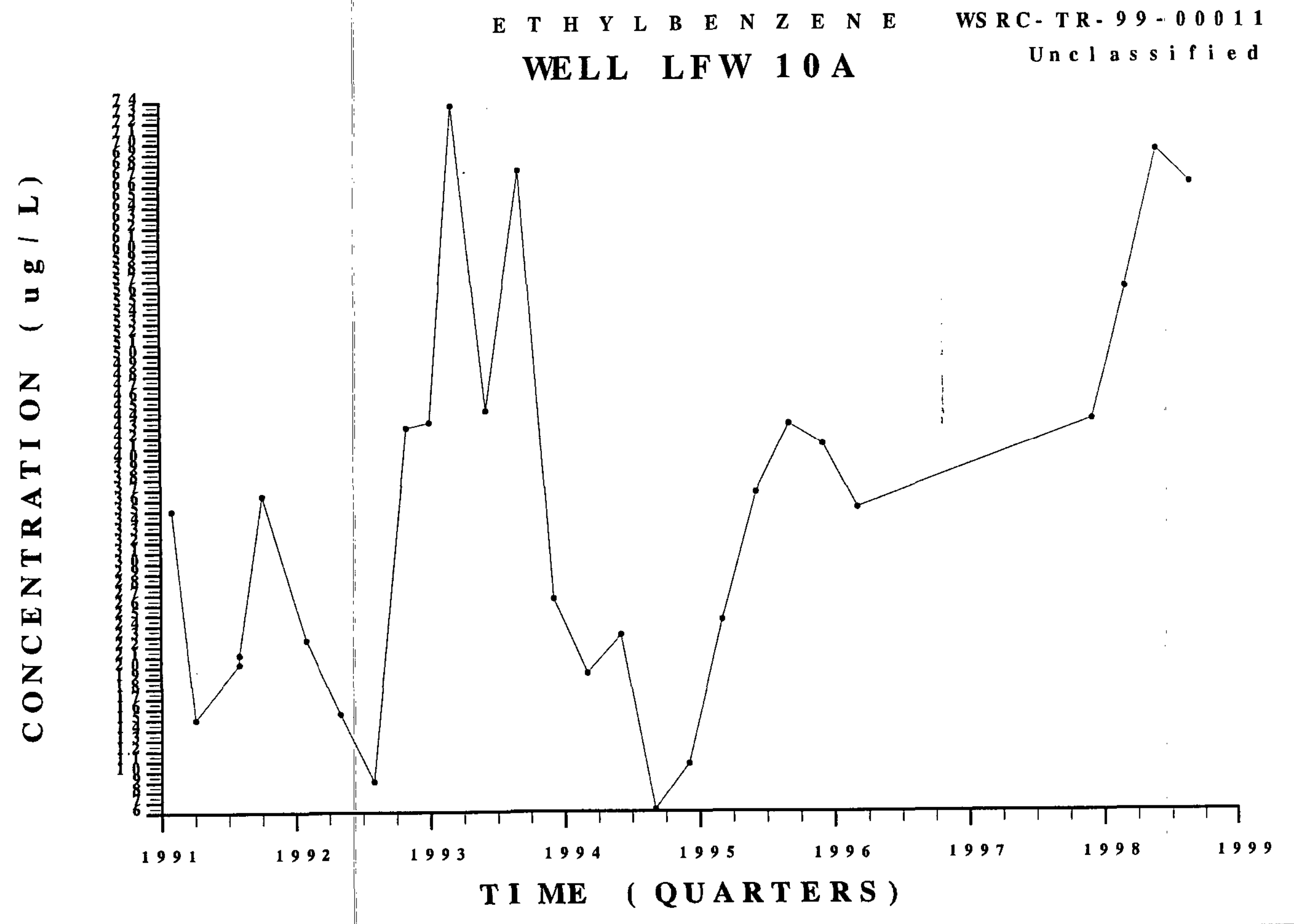




T E T RA C HLO R O E T H Y L N E WELL LFW 10 A
WS R C - T R - $99-00011$ Unc l a s s i f i e d

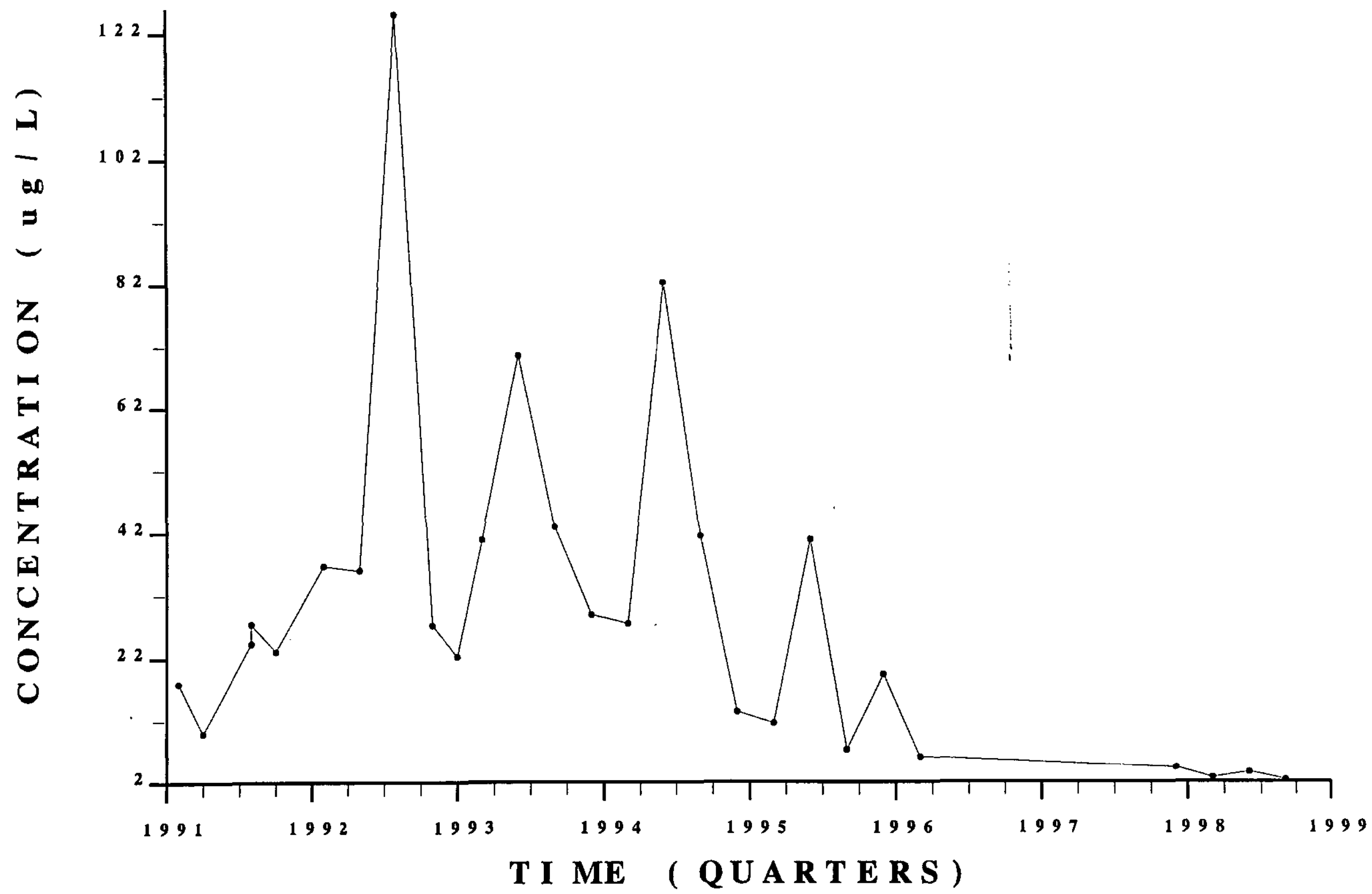

Sanitar v Landfill Fourth Ouater. 1998 \& 1998 S umma $r$ v 
T R I C H L O R O E T H Y L E N E WELL LFW $10 A$
WS R C - TR - $99-00011$

Unc l as s if i ed

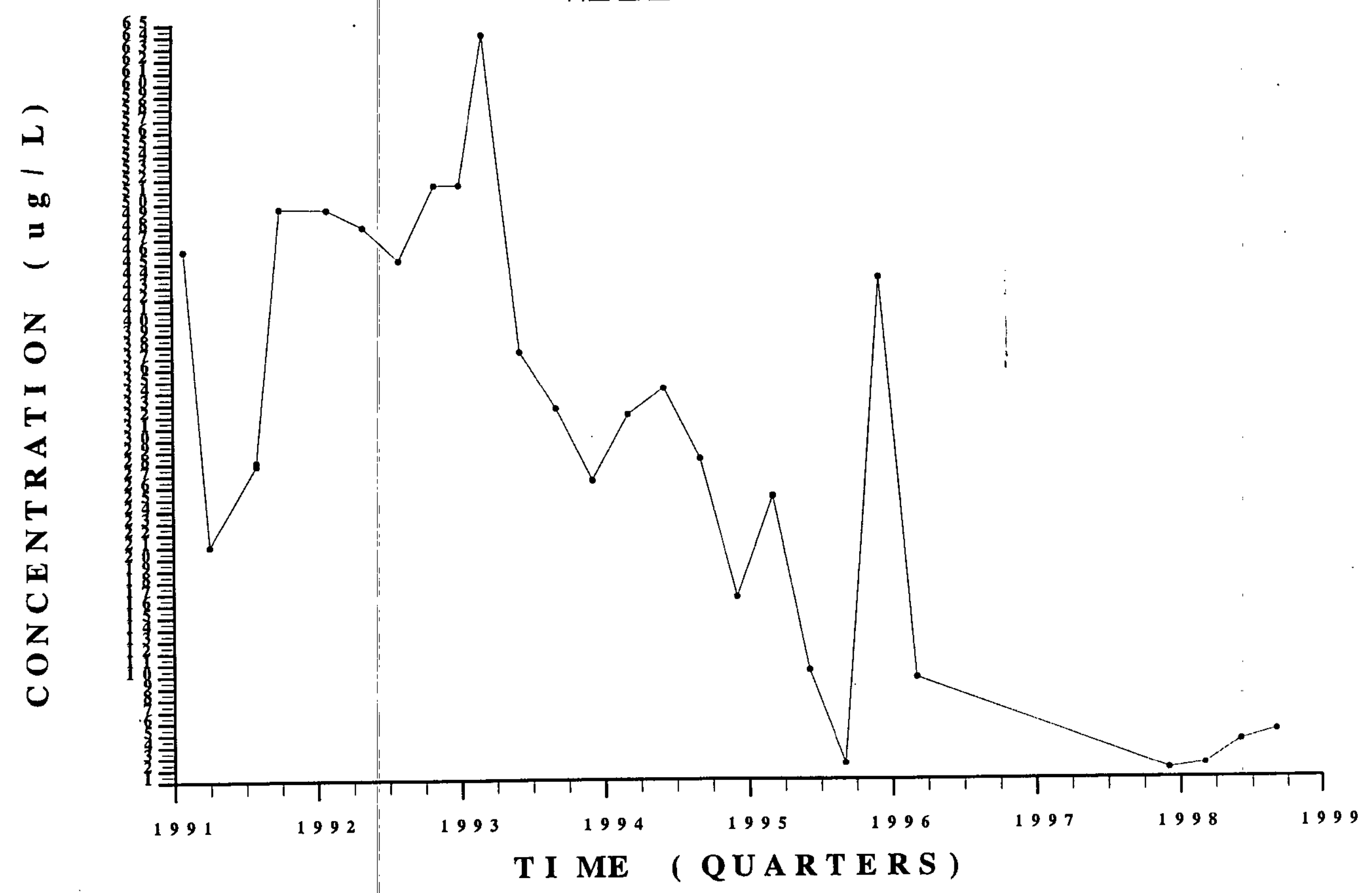

Santarv सandT1 
T RI CHLOROF L UOROME T HANE WELL LFW 10 A
WS R C - T R - 9 9-0 00011

Un c l a s s i f i e d

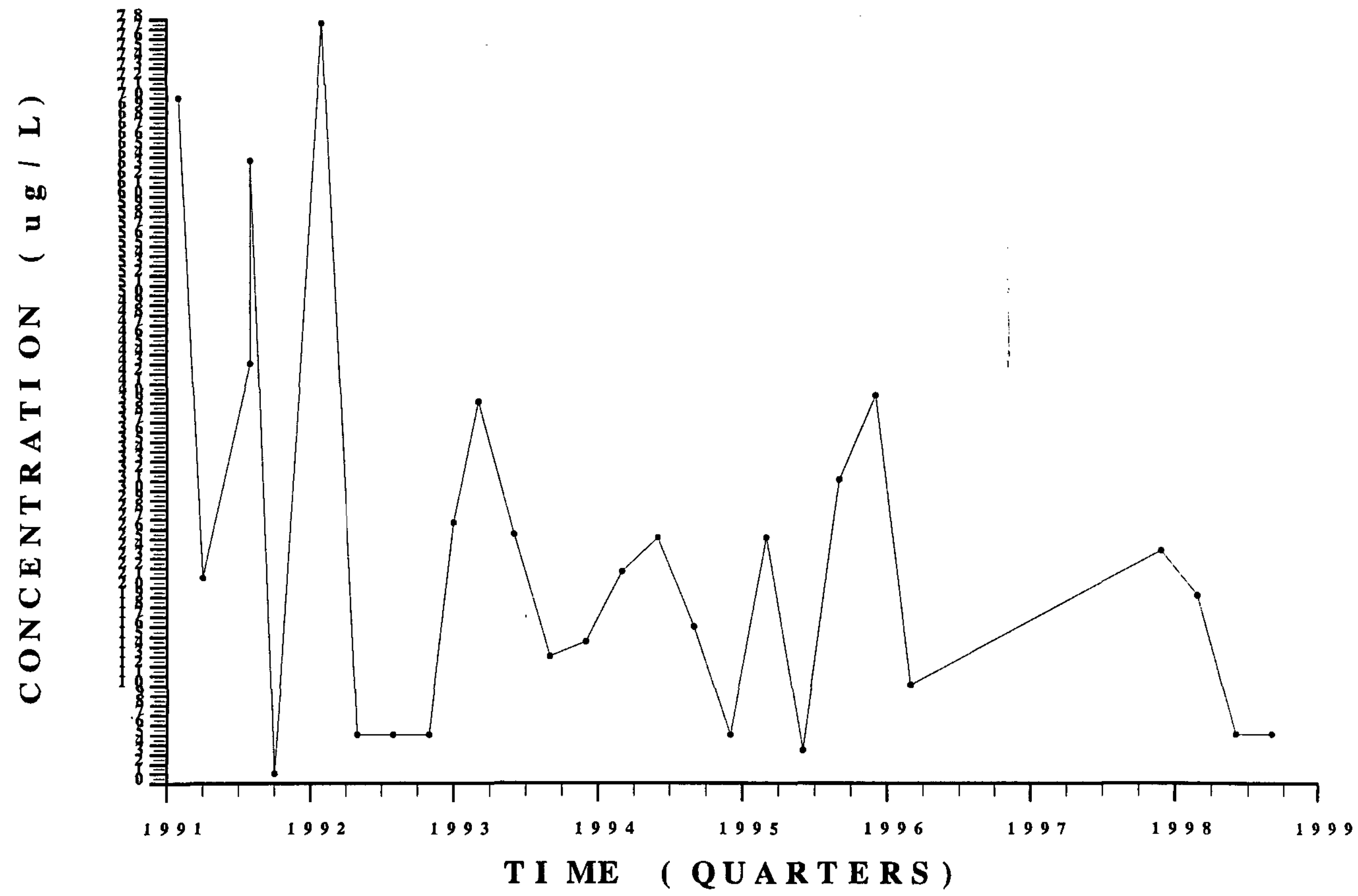

Sanitar v Landfil I

Four h

Ou a $r$ e $r$.

1998

\& 1998

S u ma r v 


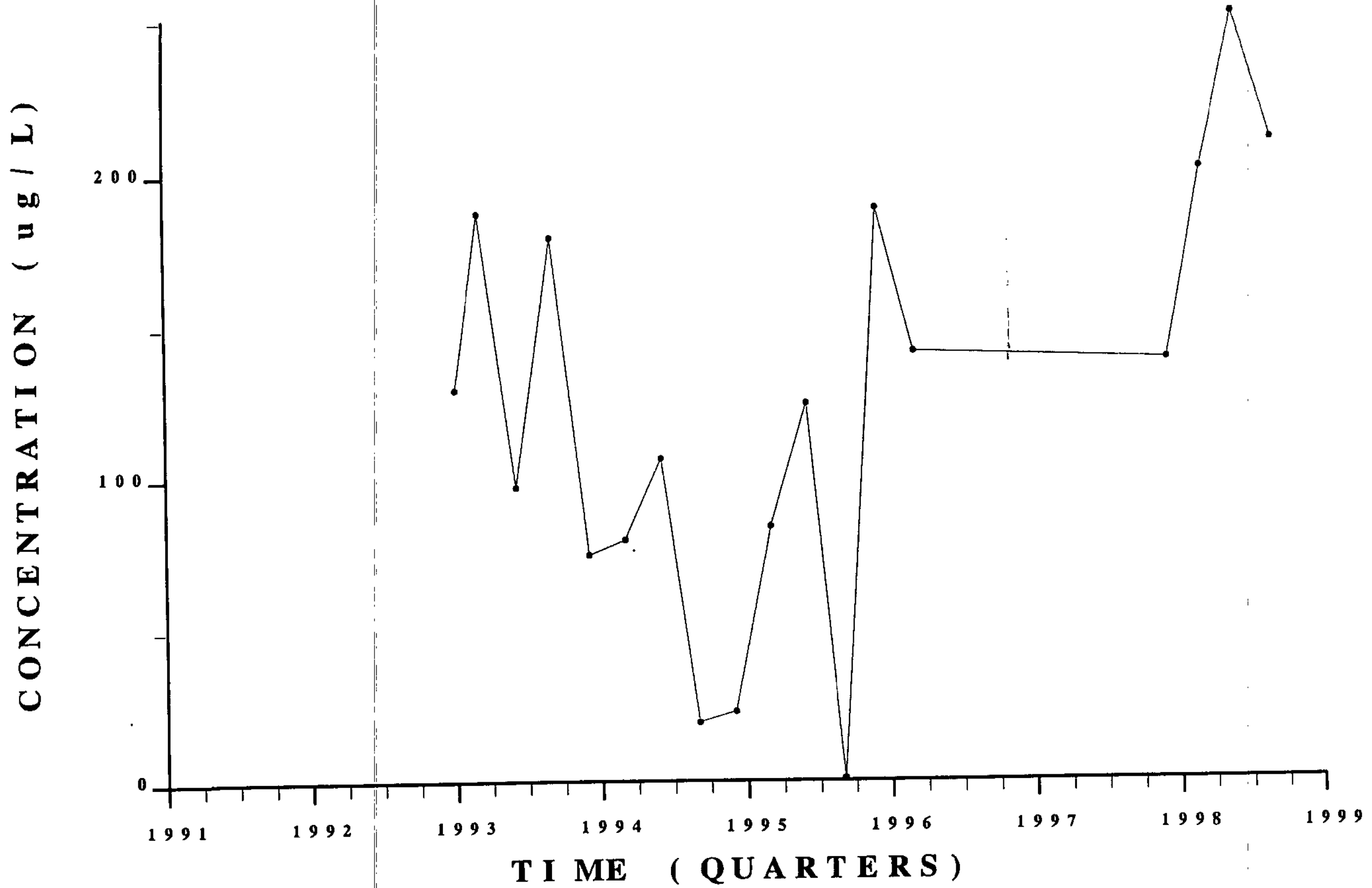

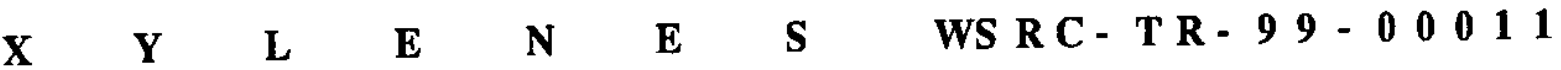
WELL LWW 10 Unclassifid 


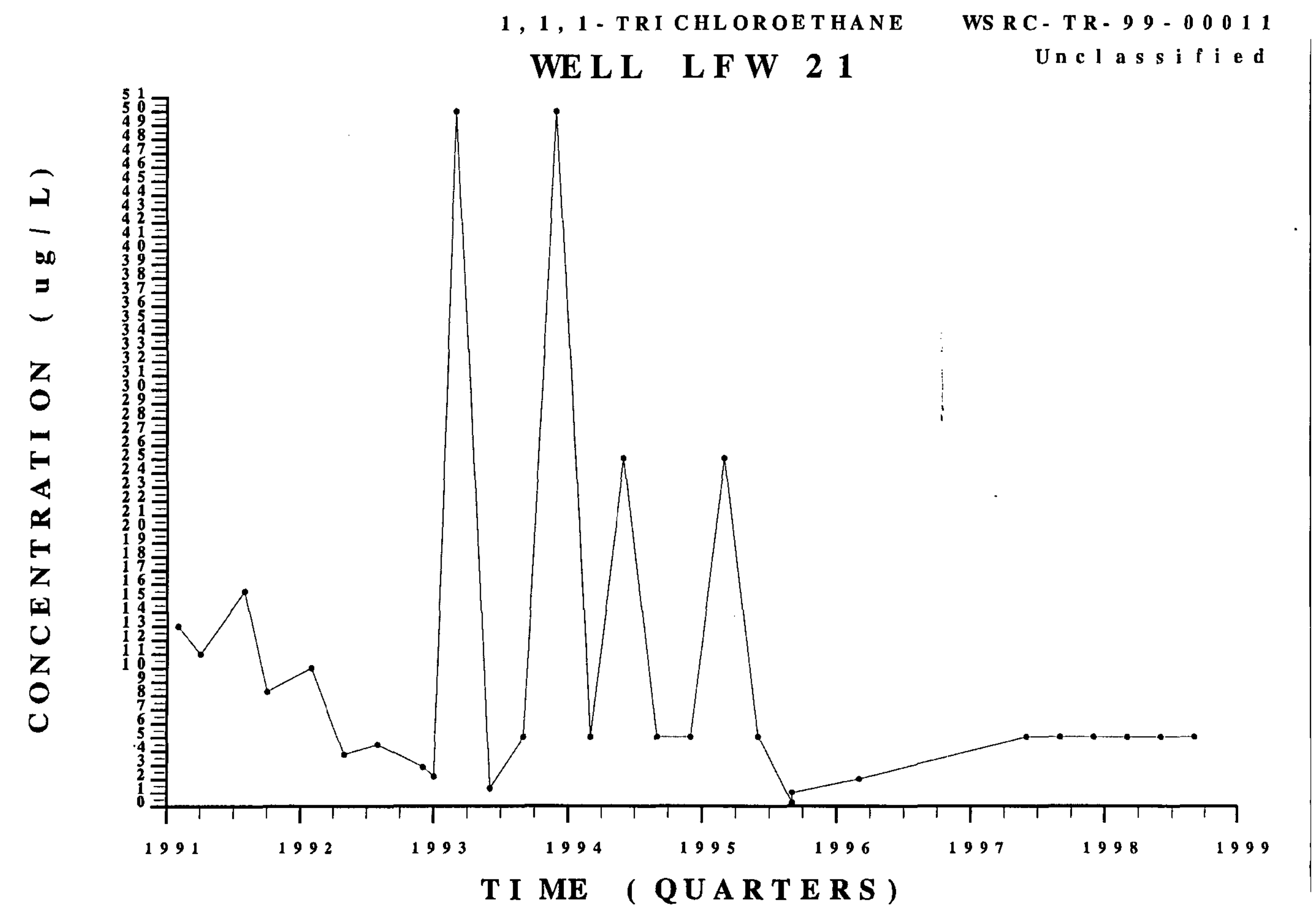

S a n i t a r v Landf i l l

Fourth ouarter.

1998

\& 1998

S u mma $r v$ 
1, 4- D I C HL OR O B E NZE NE WS RC-TR- $99-000011$ WELL LFW $21 \quad$ Unclas s if i ed

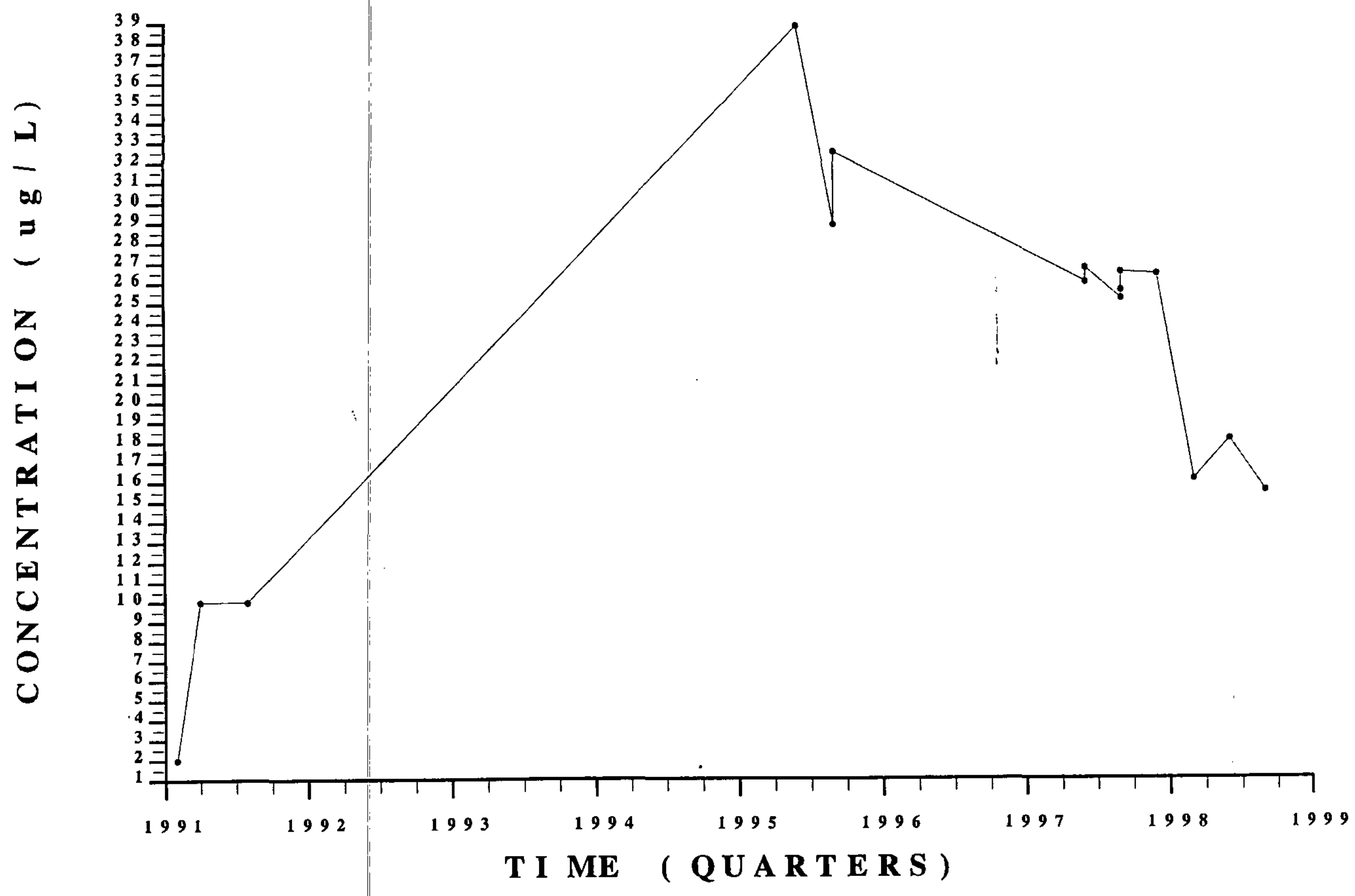

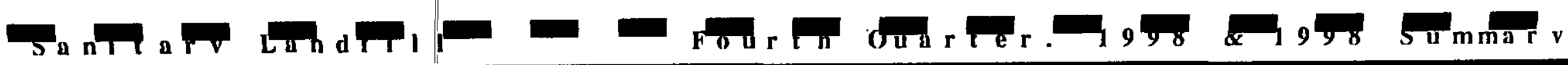




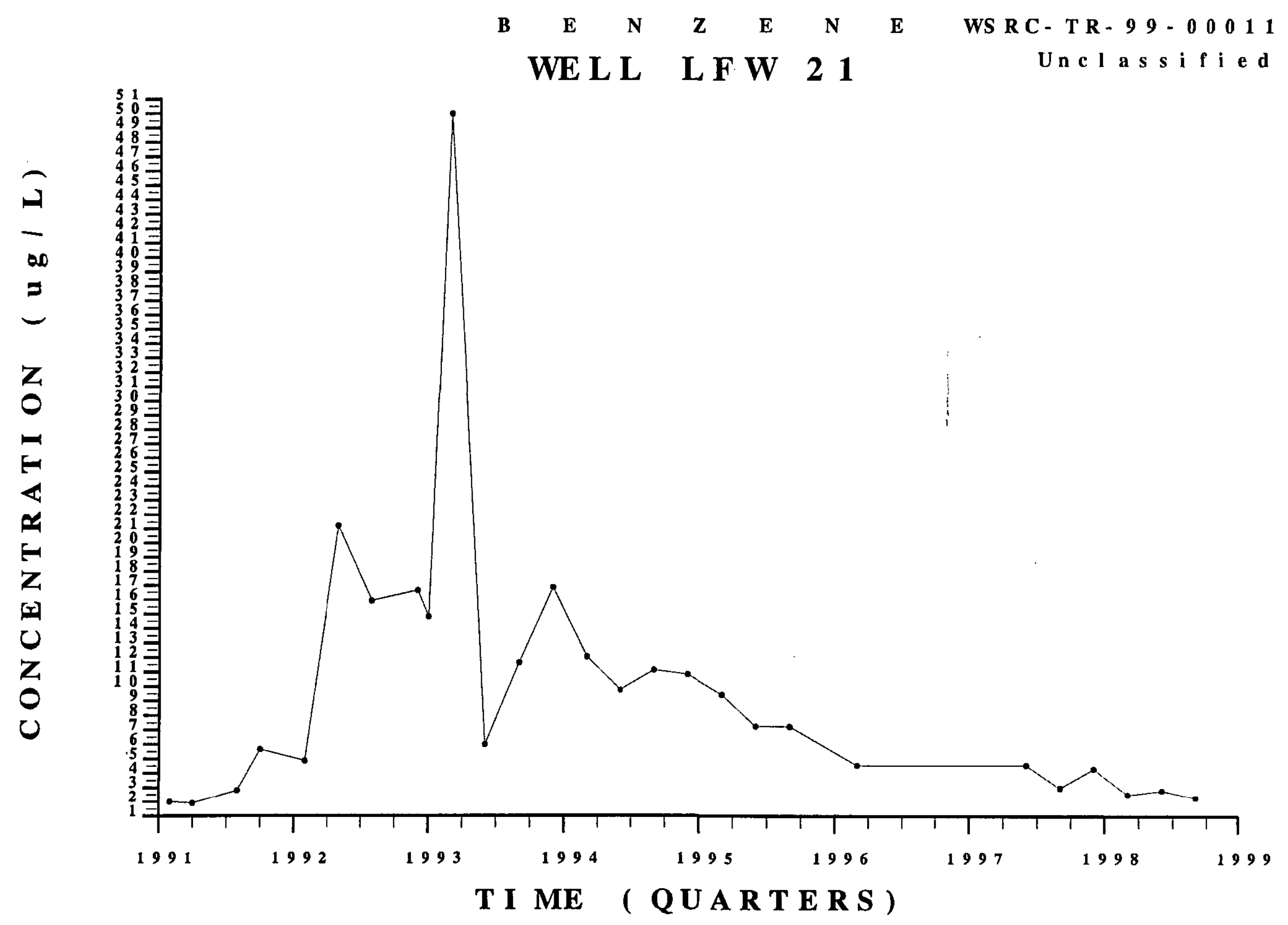

Sa ni t a r v La nd f i l I

Fourth Ouarter.

$1998 \quad \& \quad 1998 \quad S u m m a r v$ 


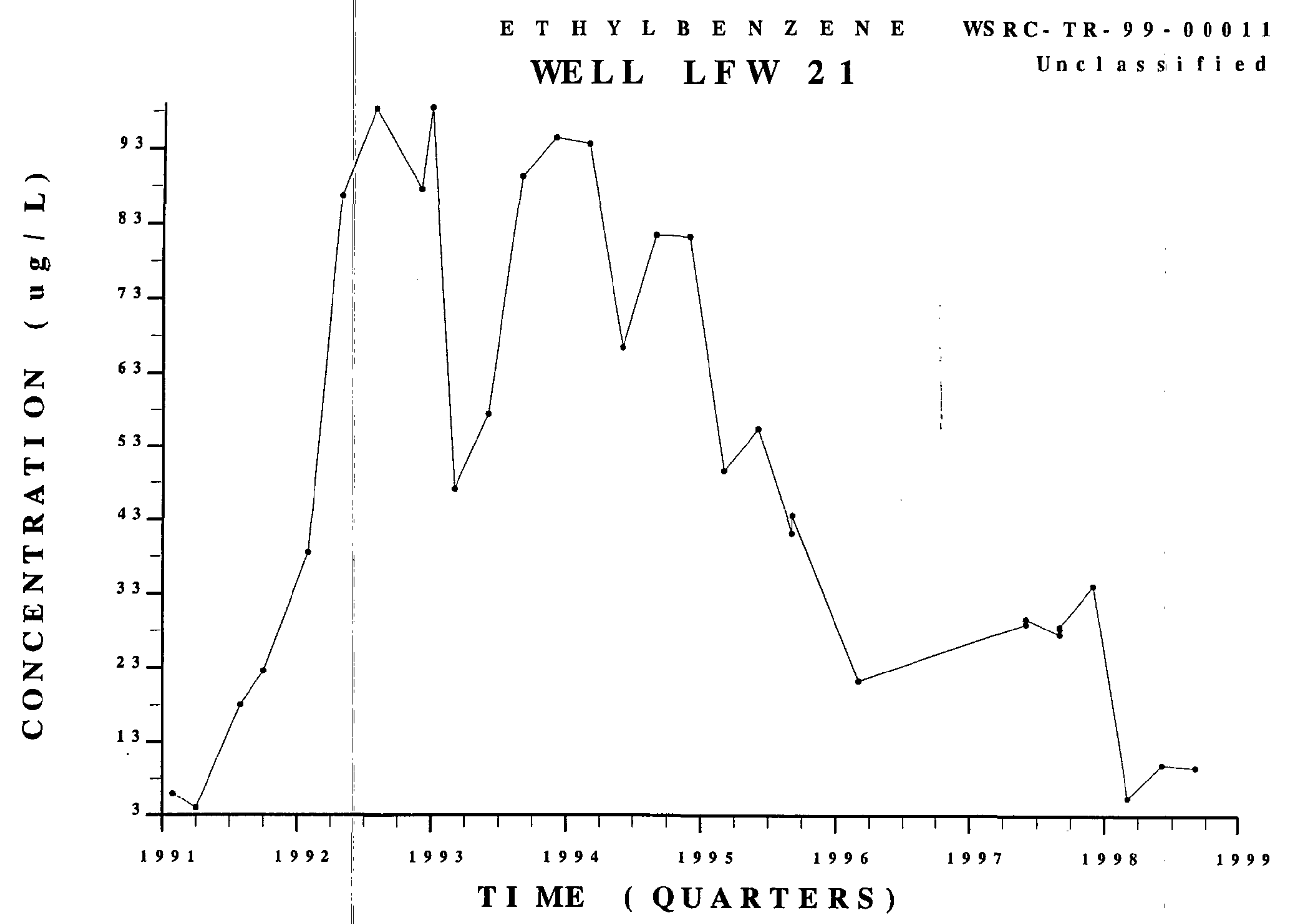




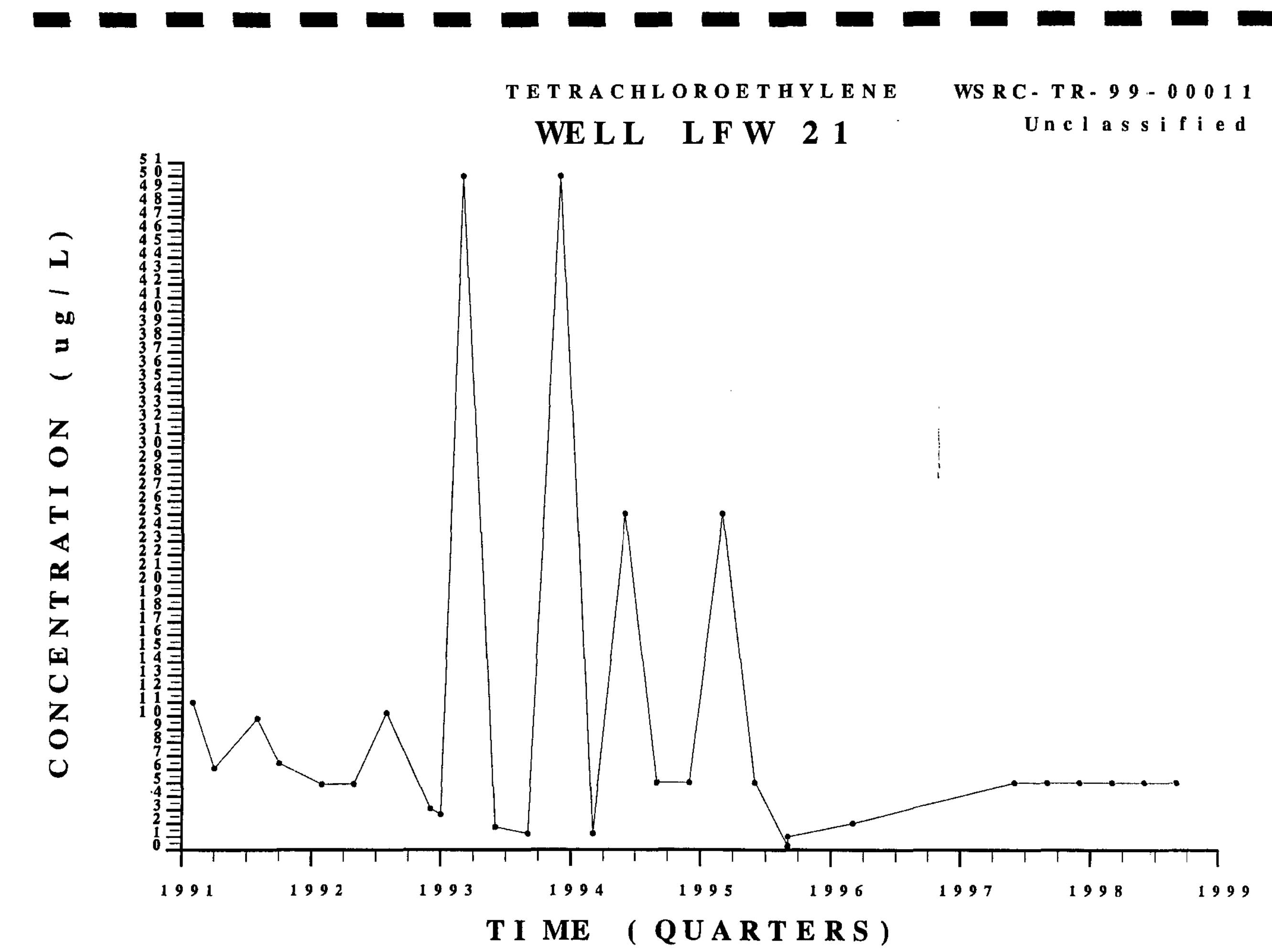

Sa ni t a r v La nd f i l l

Fourth Ouarter. 1998 \& 1998 Summar 


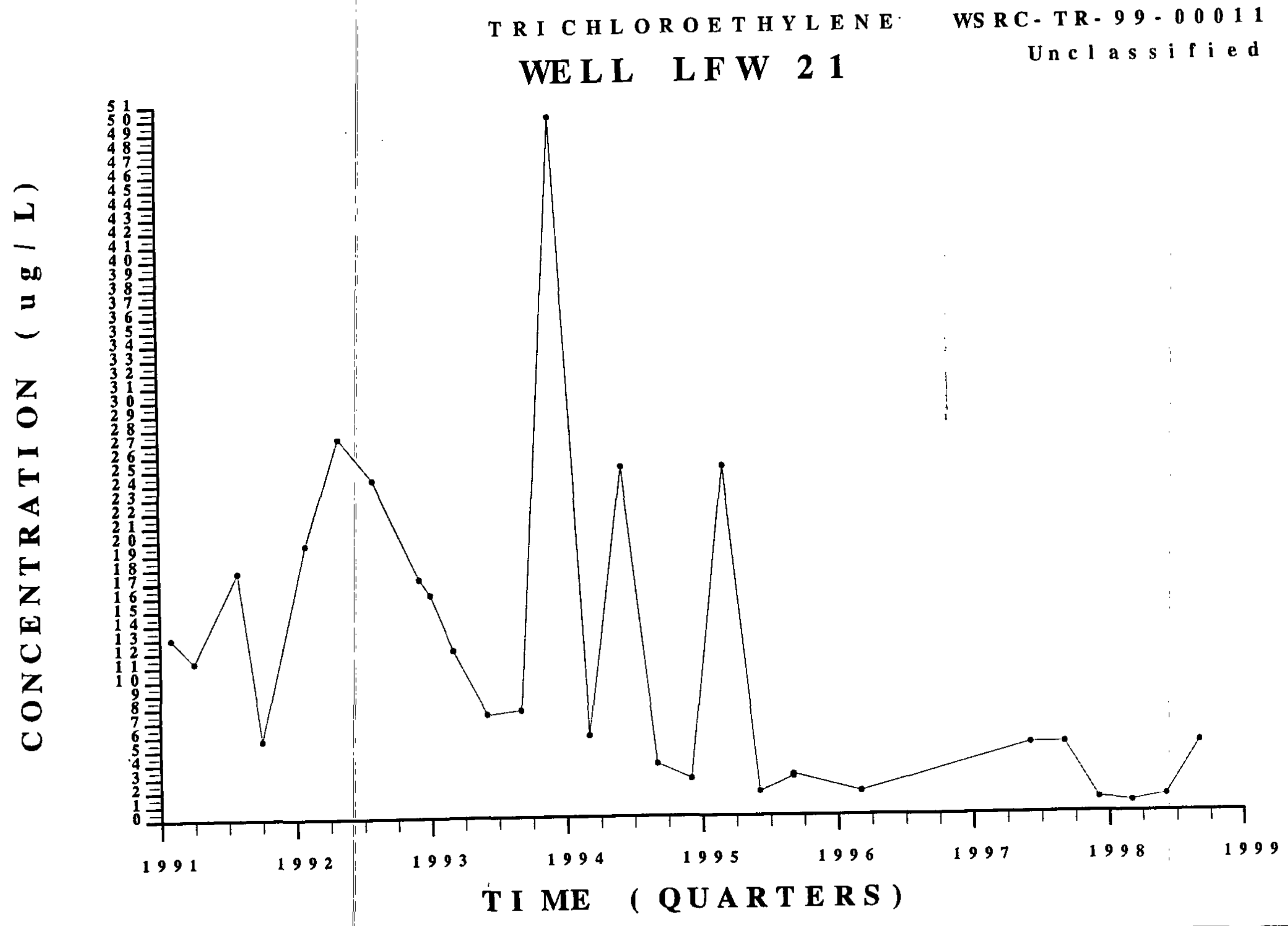

Cnnitnrv 


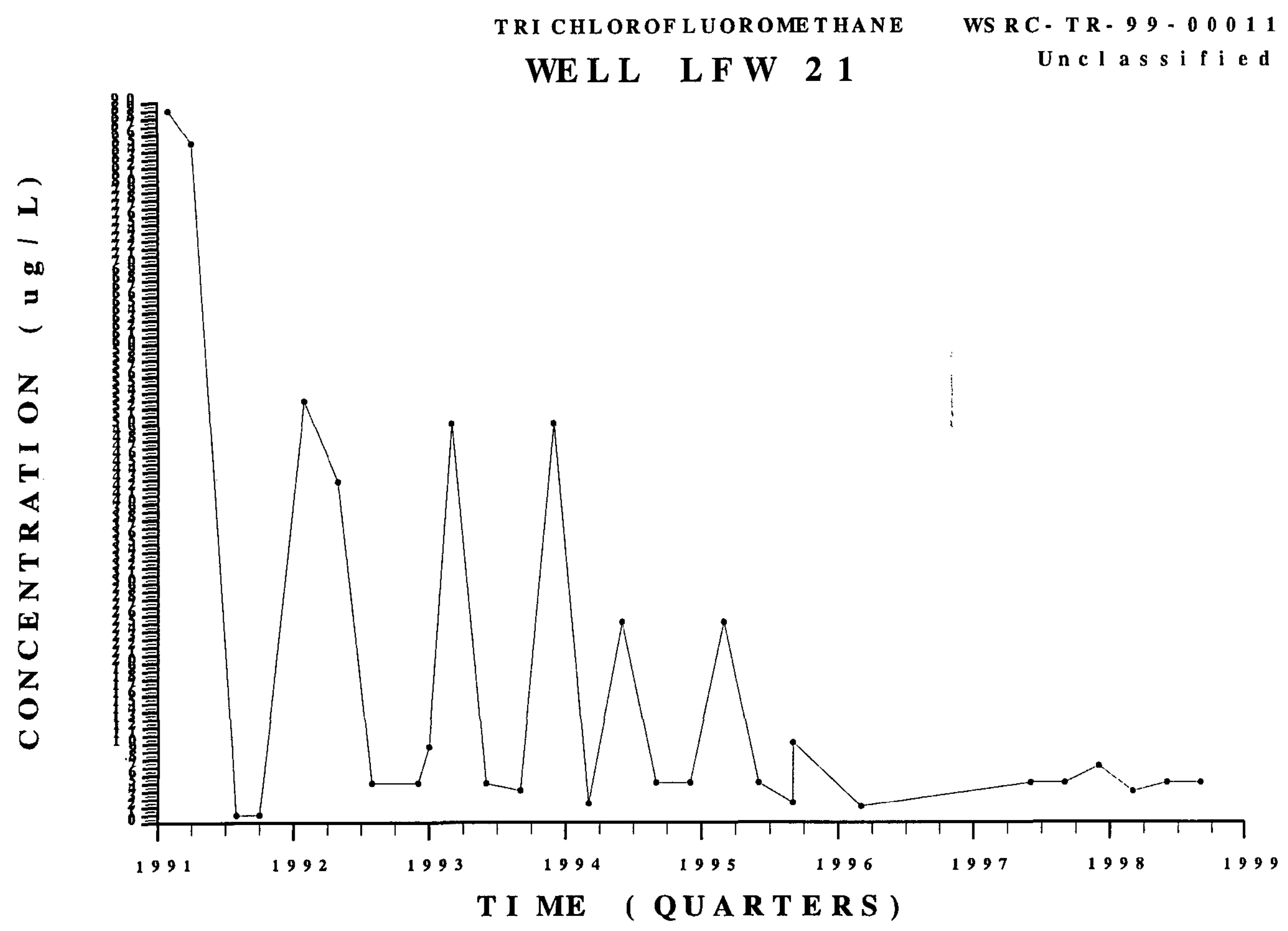

Sa n i t a r Land f i l l Fourth Ouarter.

1998

\& 1998

S u m ma r v 


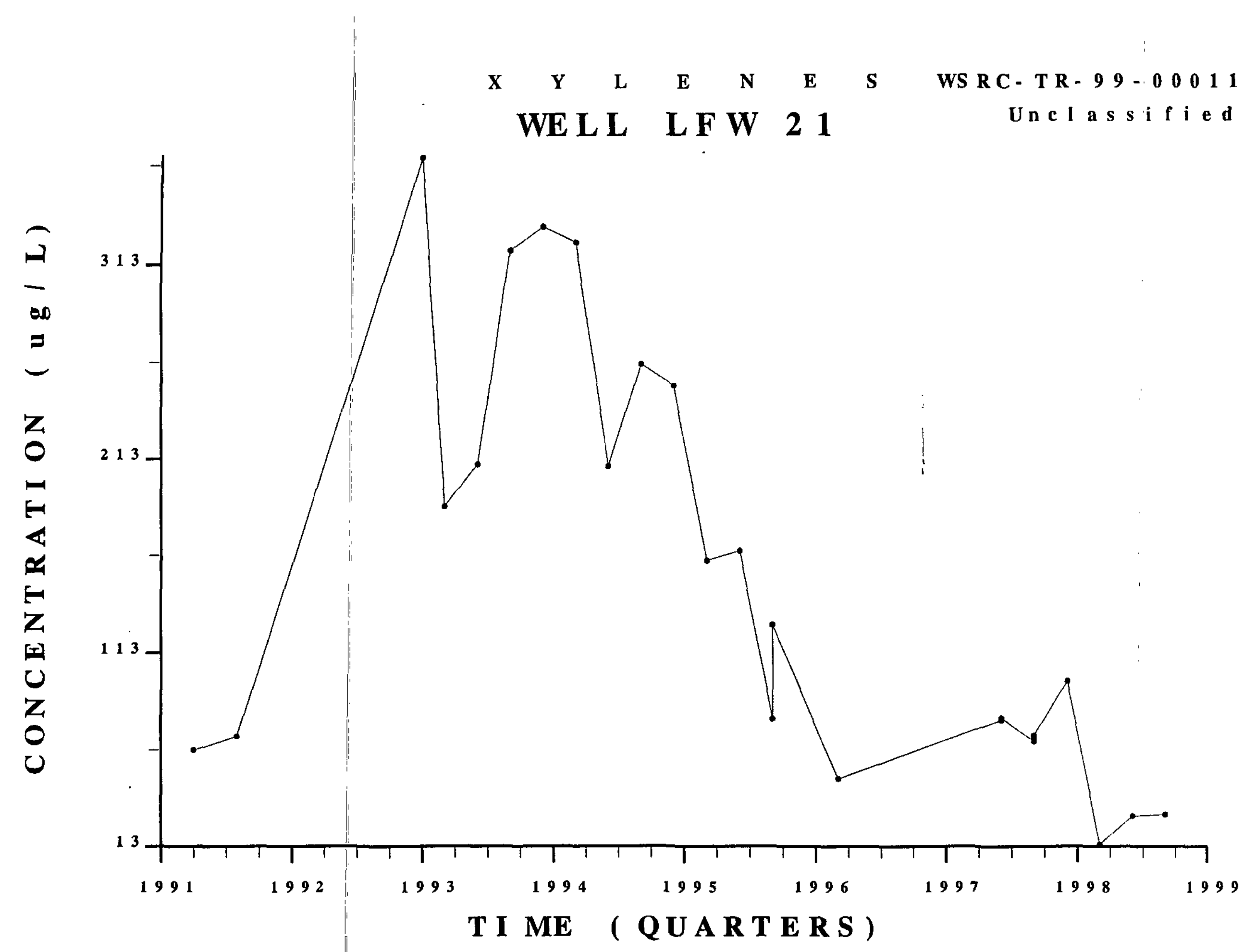

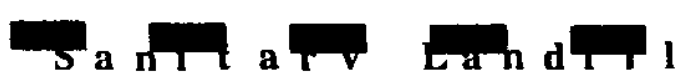




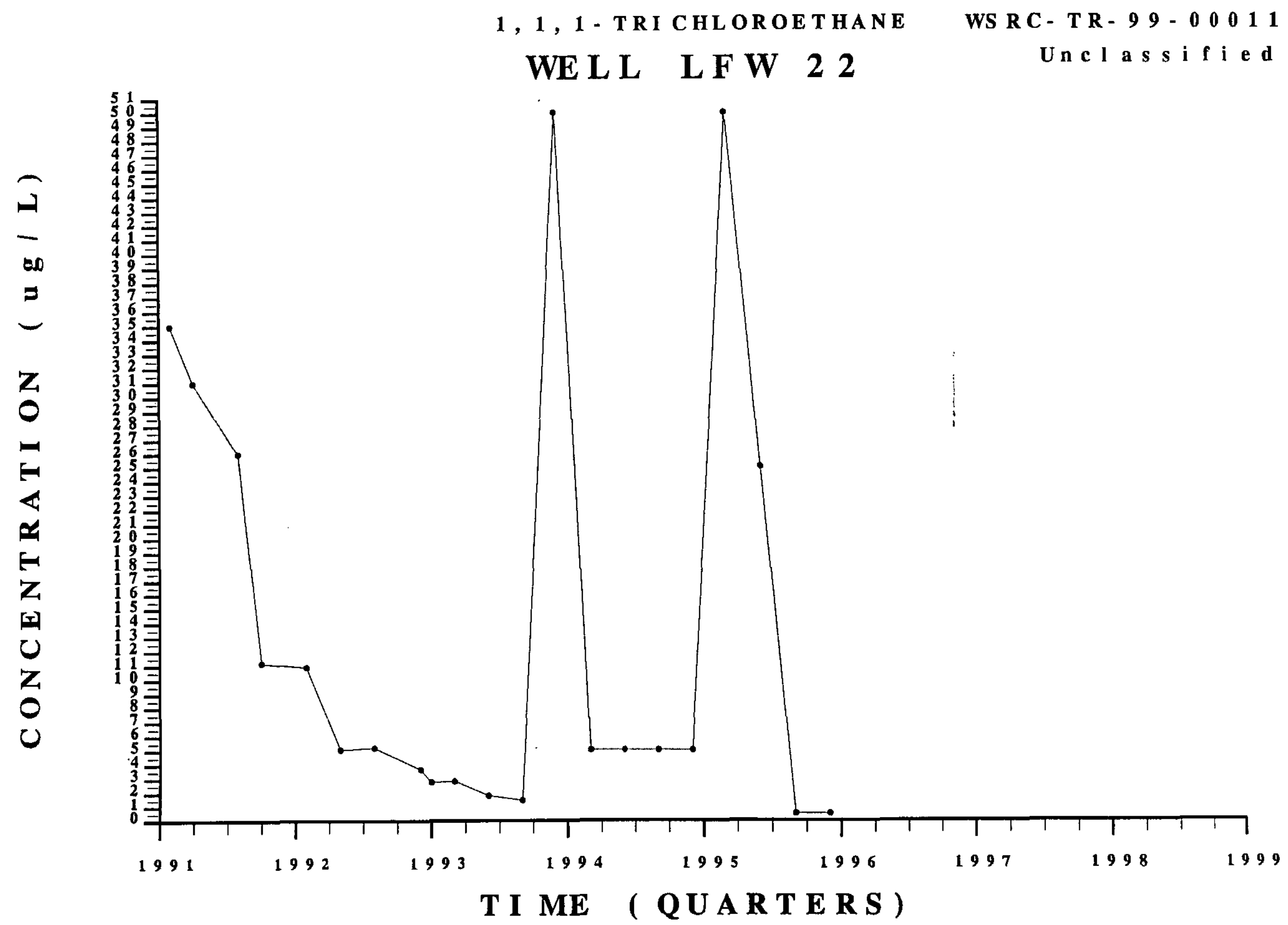

San i t a r v La ndf i l l

Fourth Ouarter.

\& 1998

Summa $\mathbf{r}$ 
1, 4 - D I C H L O R O B E NZ E N E WE L L L F W 22

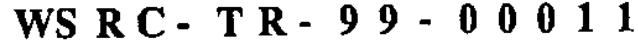

Unc l a s s i f i ed








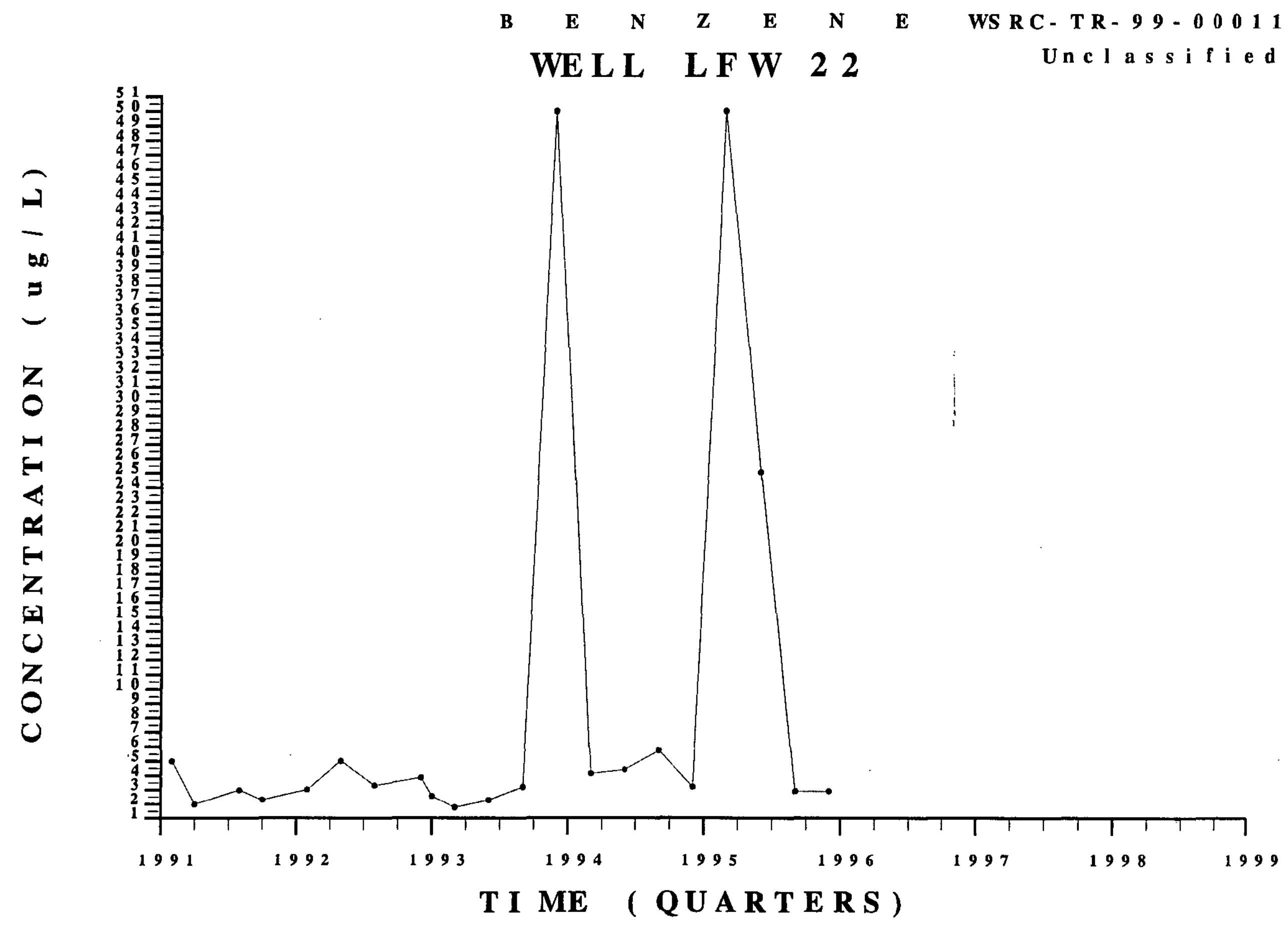

Sanitar $y$ Landf i l l

Fourth ouarter.

1998

\& 1998

Su mar v 


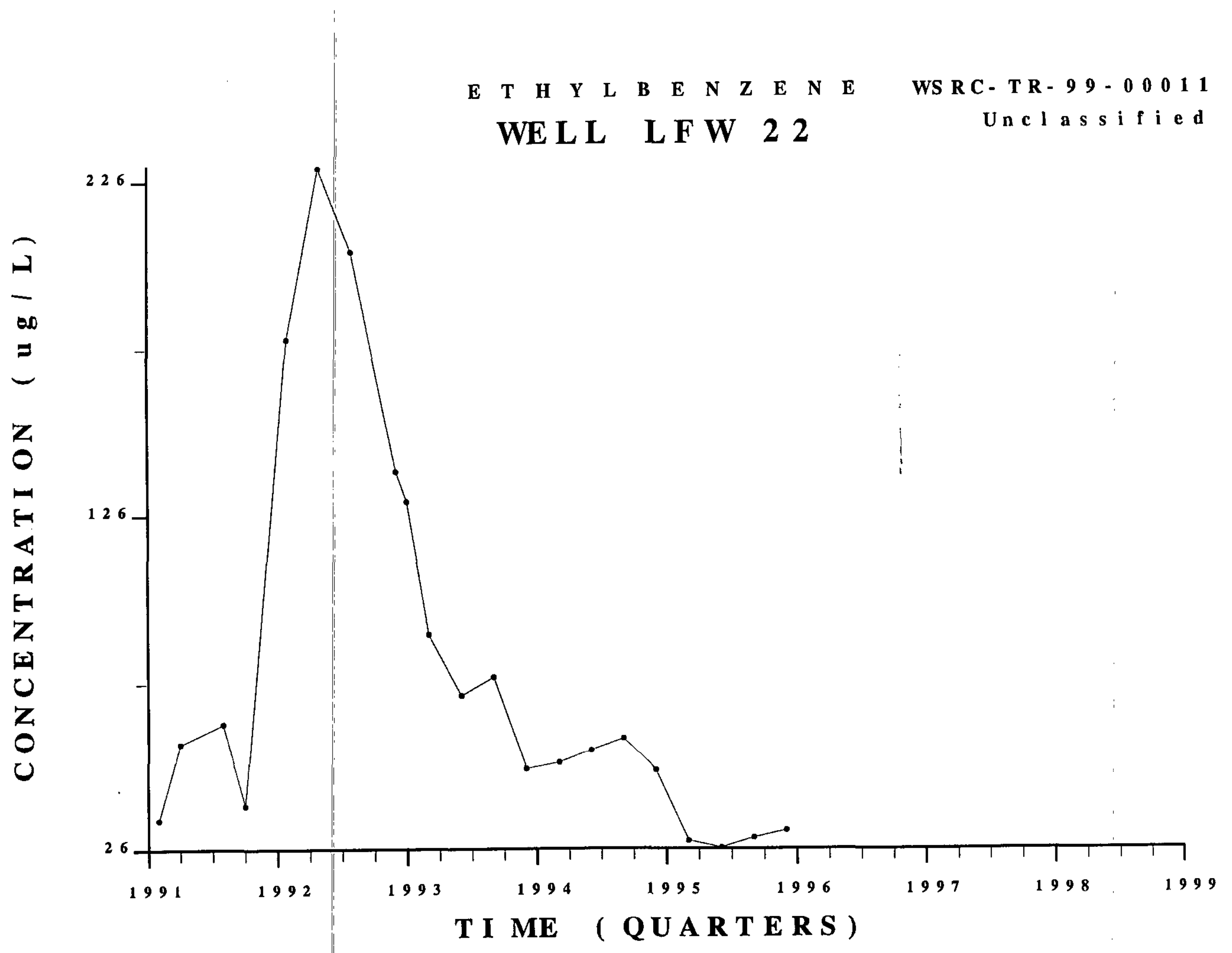

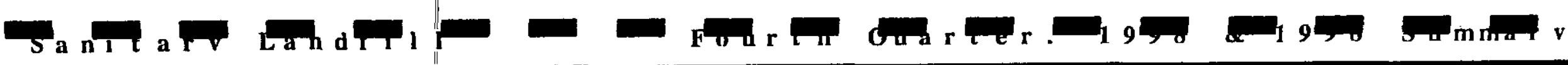


TETRA CHLOROETHYLEN E WE L L L F W 22

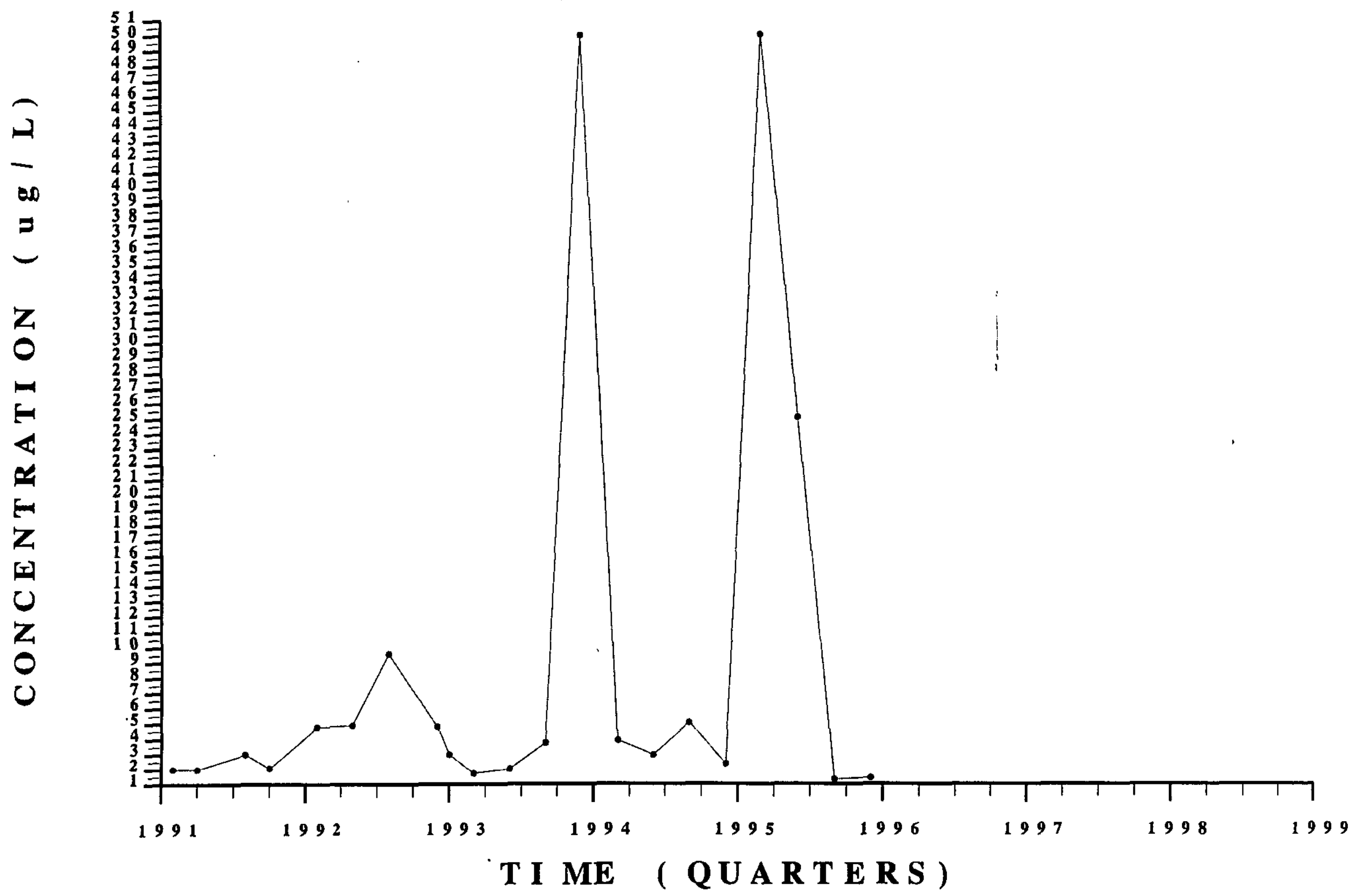

Fourth Ouarter.

1998

$\& \quad 1998$

S u m ma $\mathrm{r}$
WS R C - T R - $99-00011$ Unc l a s $s$ i f i e d Sanitar v Land fill l 


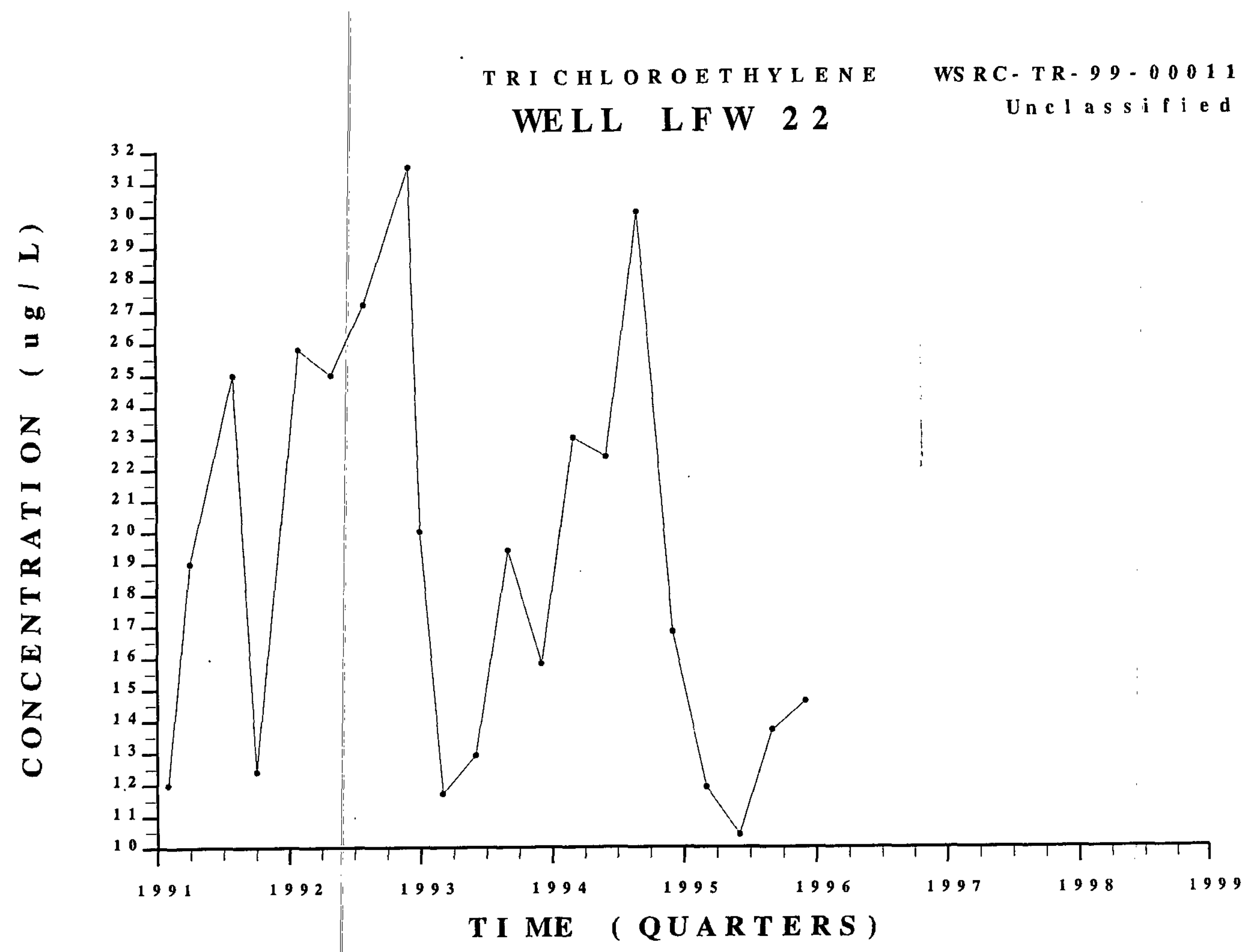




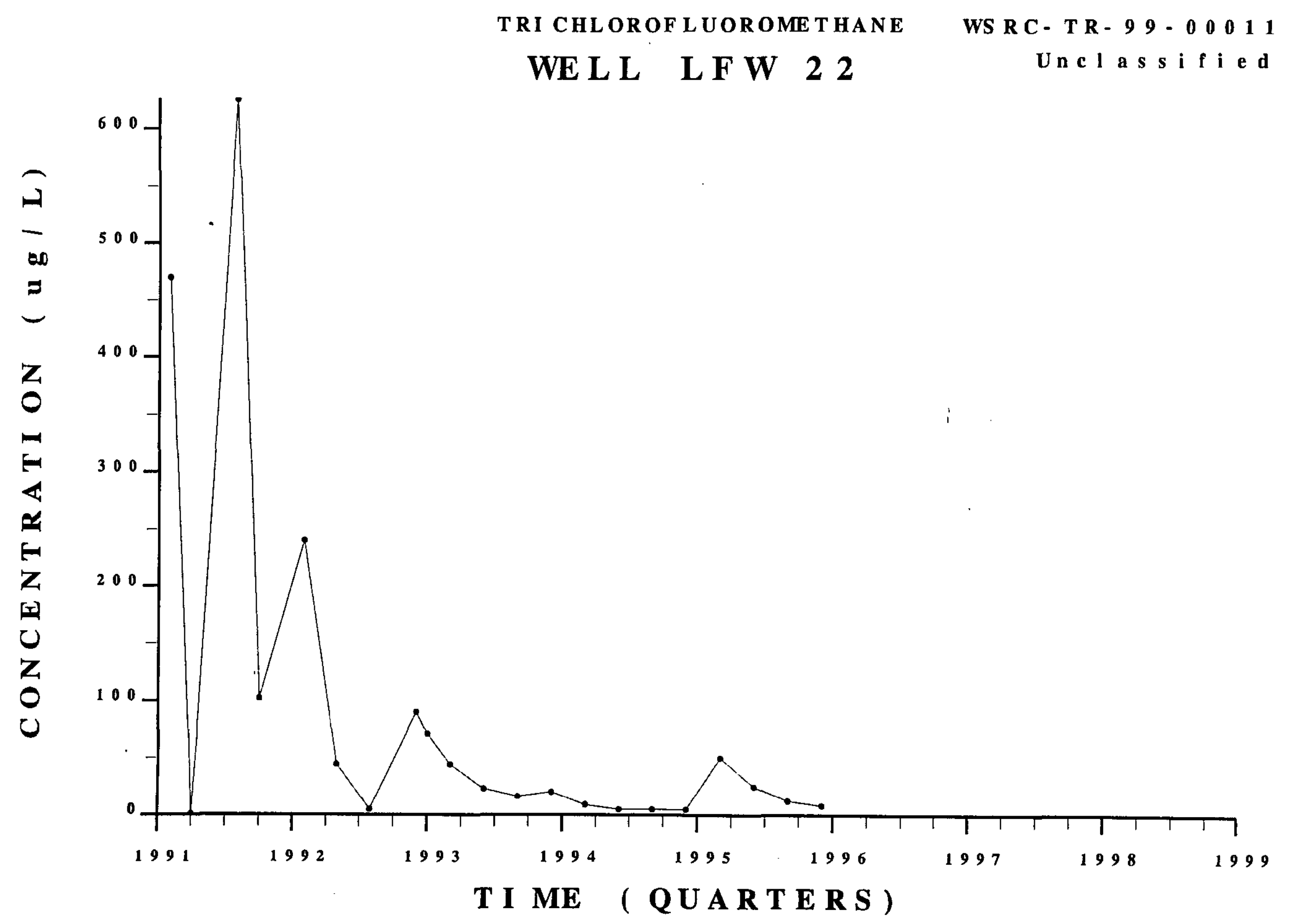

THANE

WE L L L F W 22

Sanit ar $r$ Landfi I l 


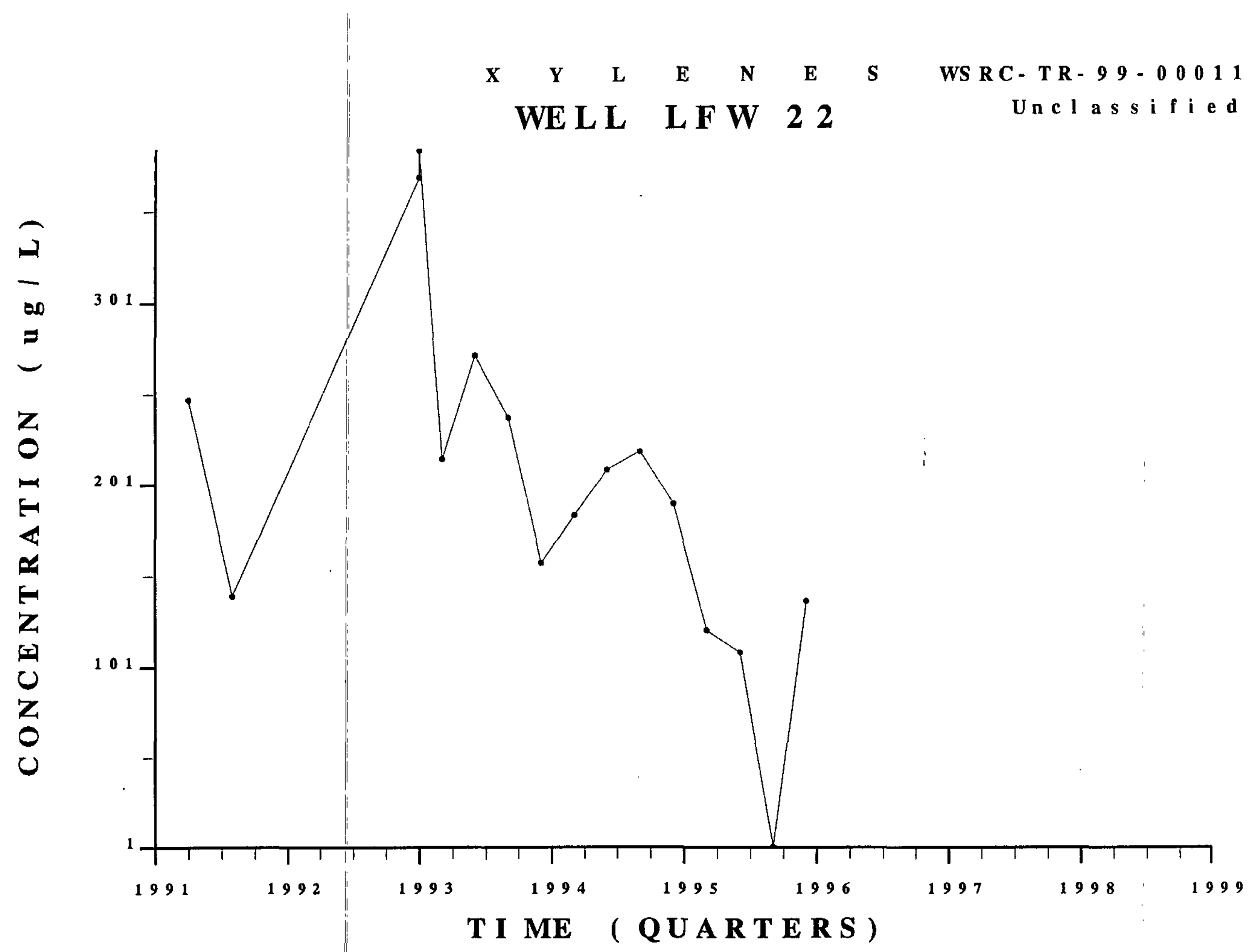

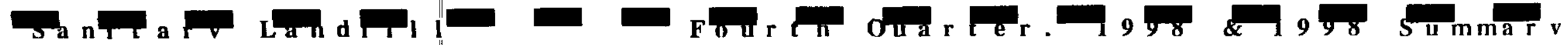


$1,1,1$ - TRI CHLOROETHANE WELL L F W 36
WS R C - TR- $99-00011$ Unc l a s s i f i ed

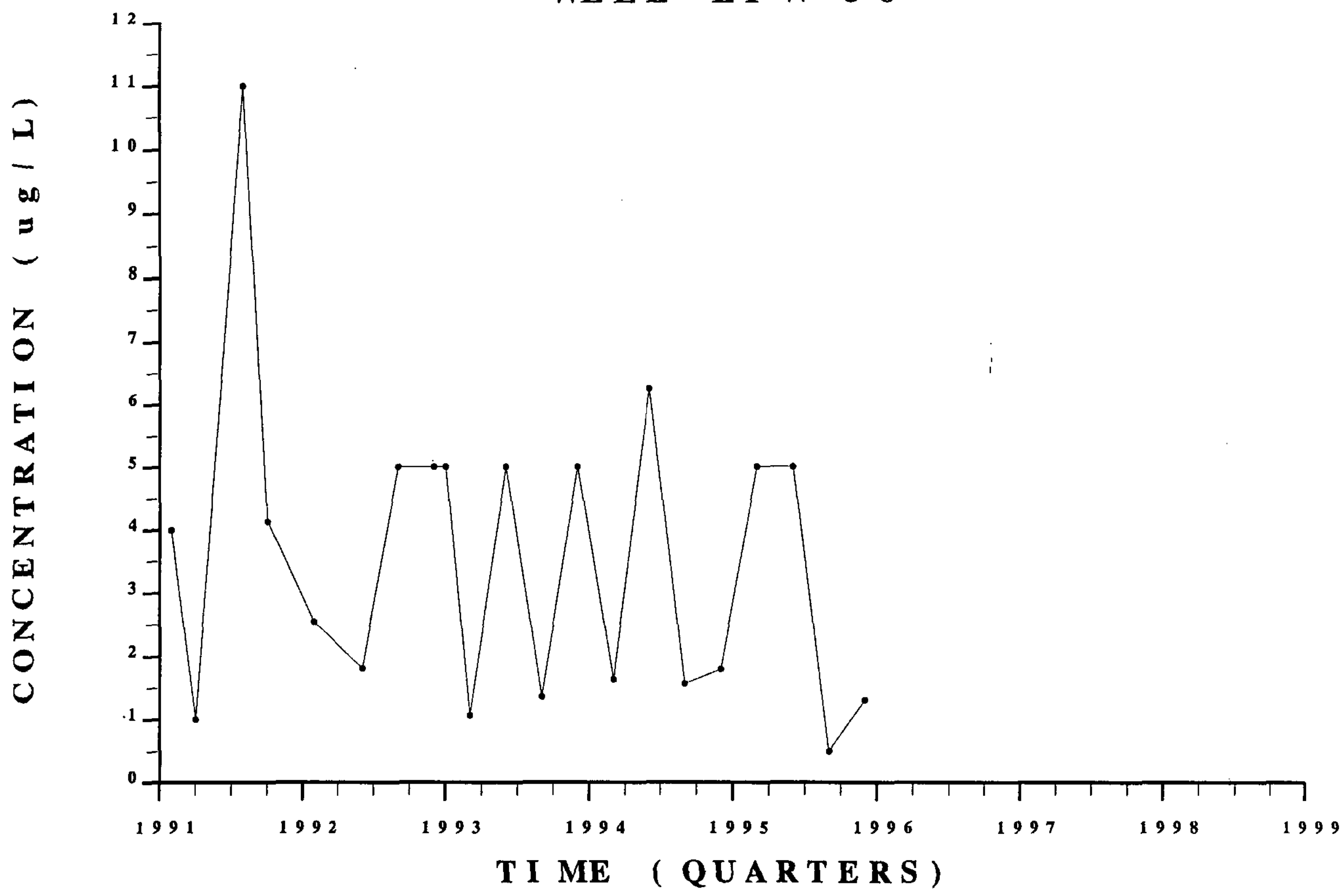

Sanitar $r$ Landf i l l Fourth Ouarter. 1998 \& 1998 S u mma $r$ v 
1, 4 - D I C H L O R O B E N Z E N E WE L L L F W 36

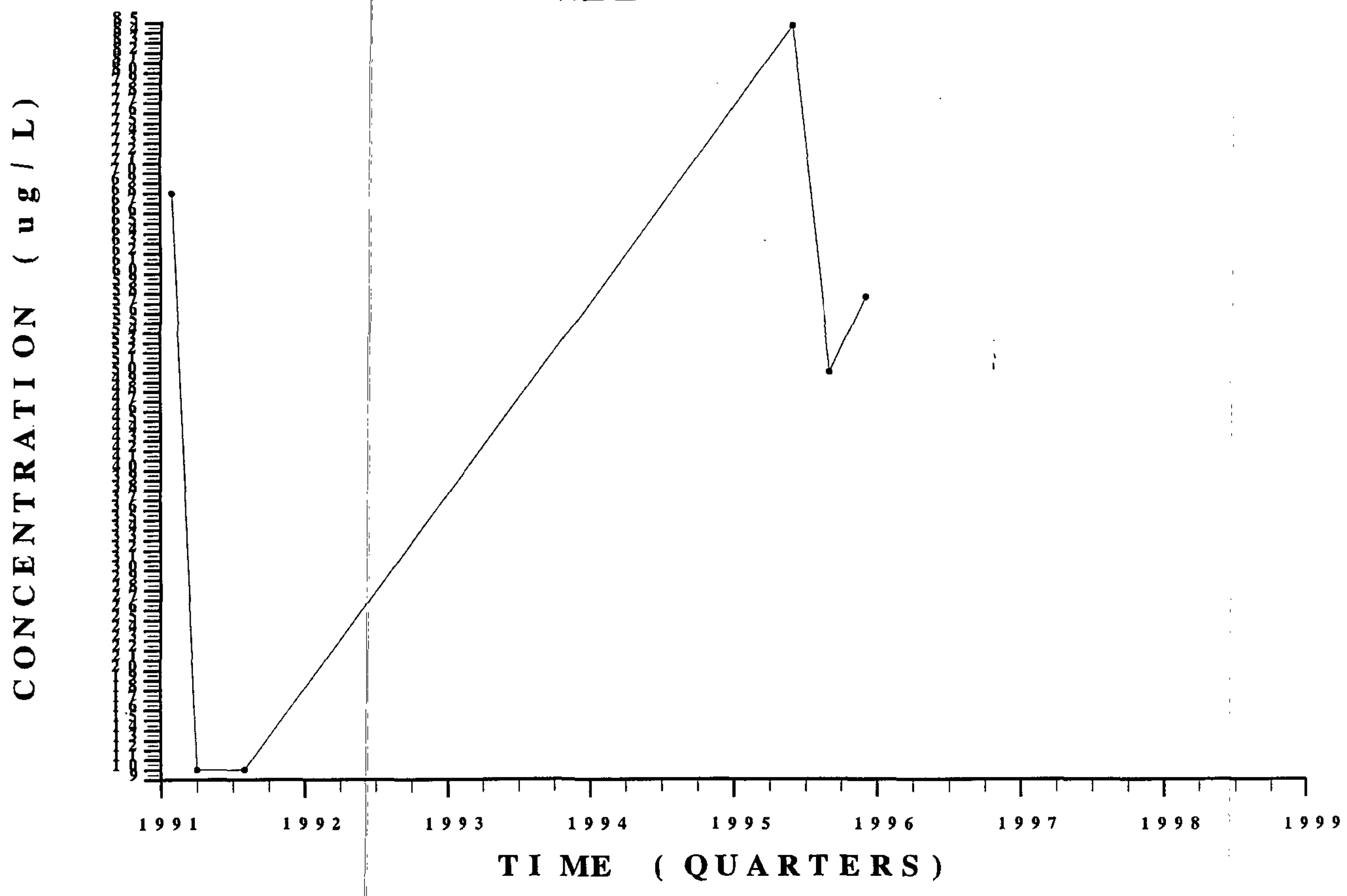




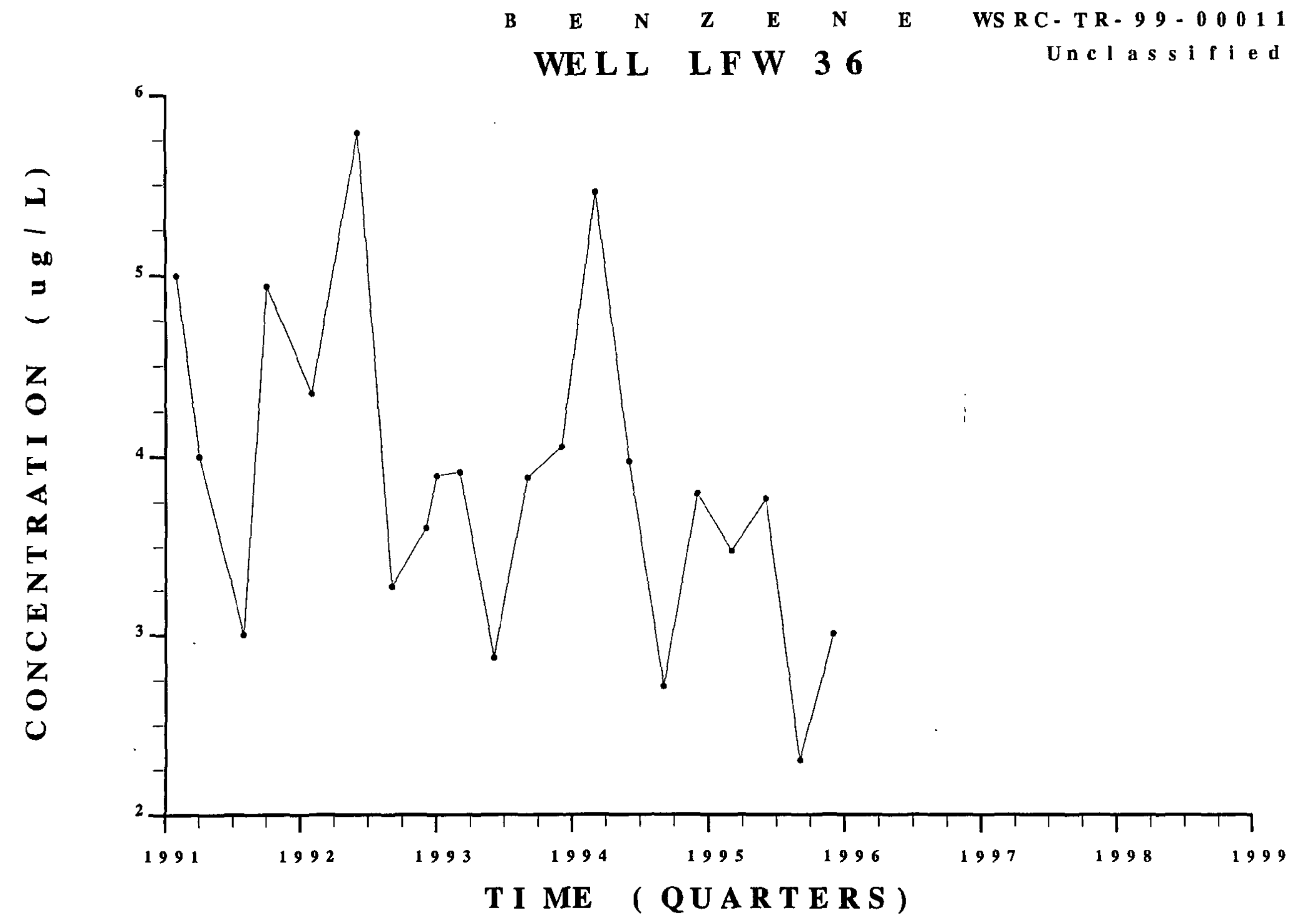

Sanitary Landfil 1 Fourth Ouarter. $1998 \& 1998$ S u mma r v 


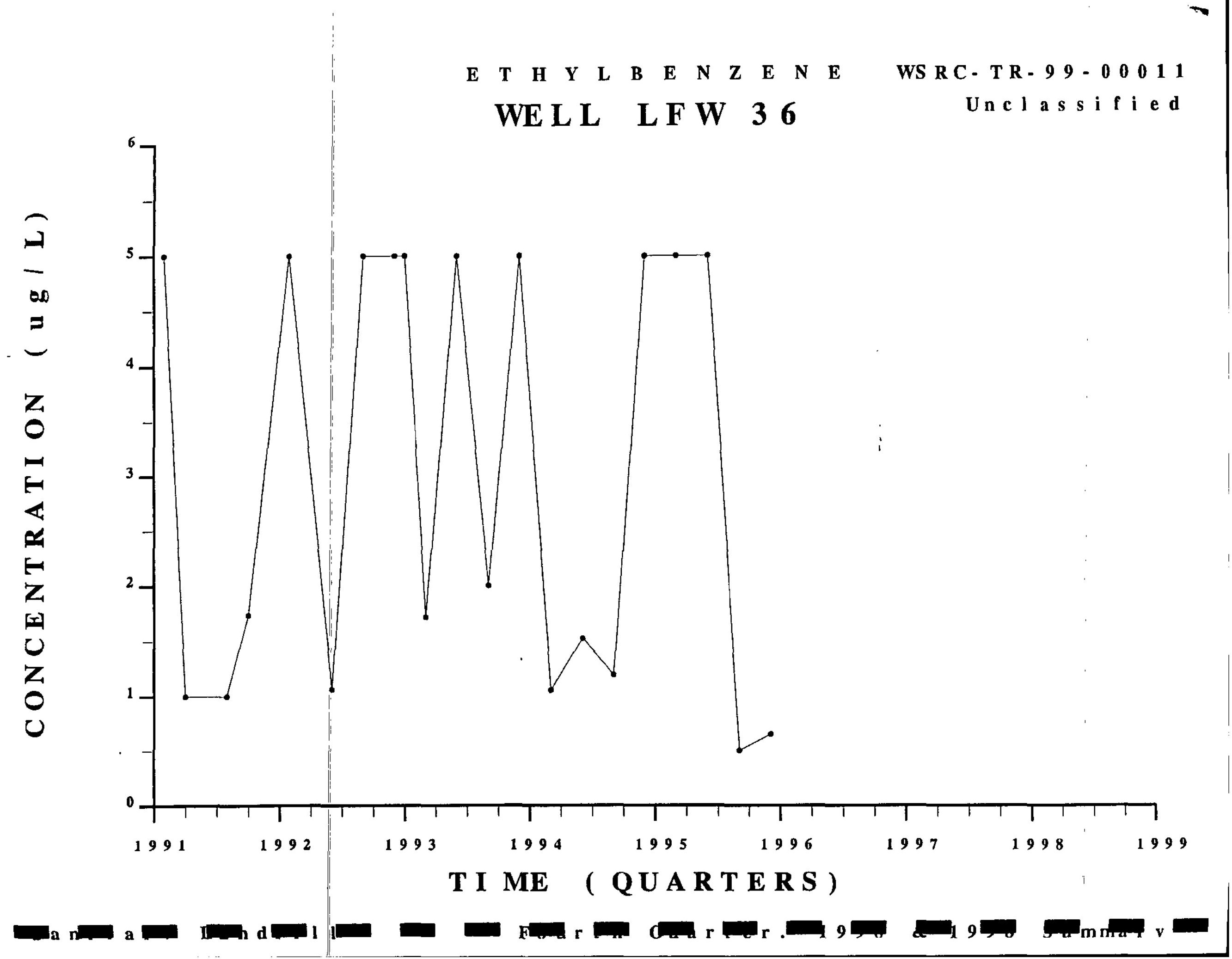


T ET RA CHLOR O E T H YL E N E WE L L L F W 36

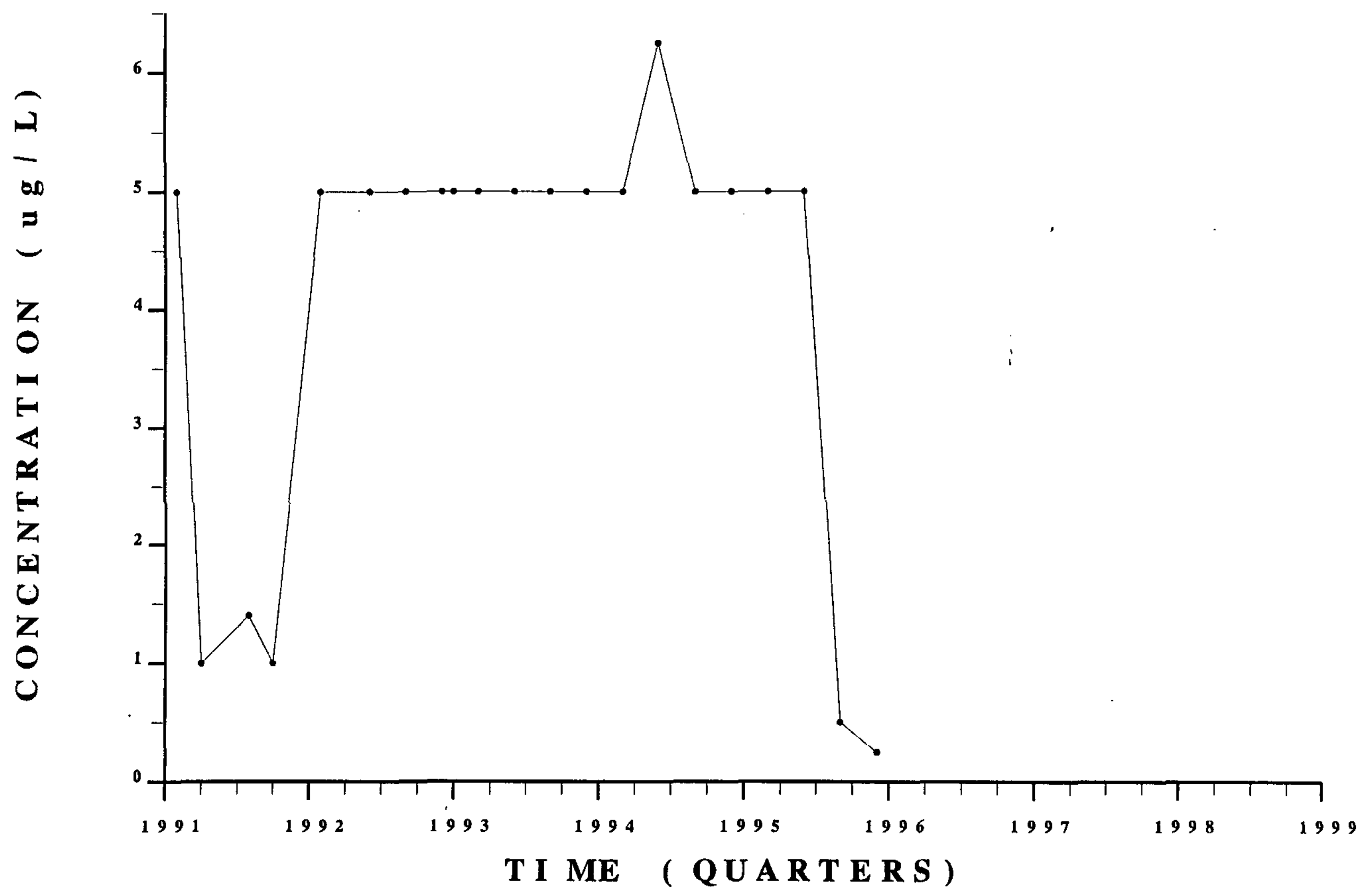




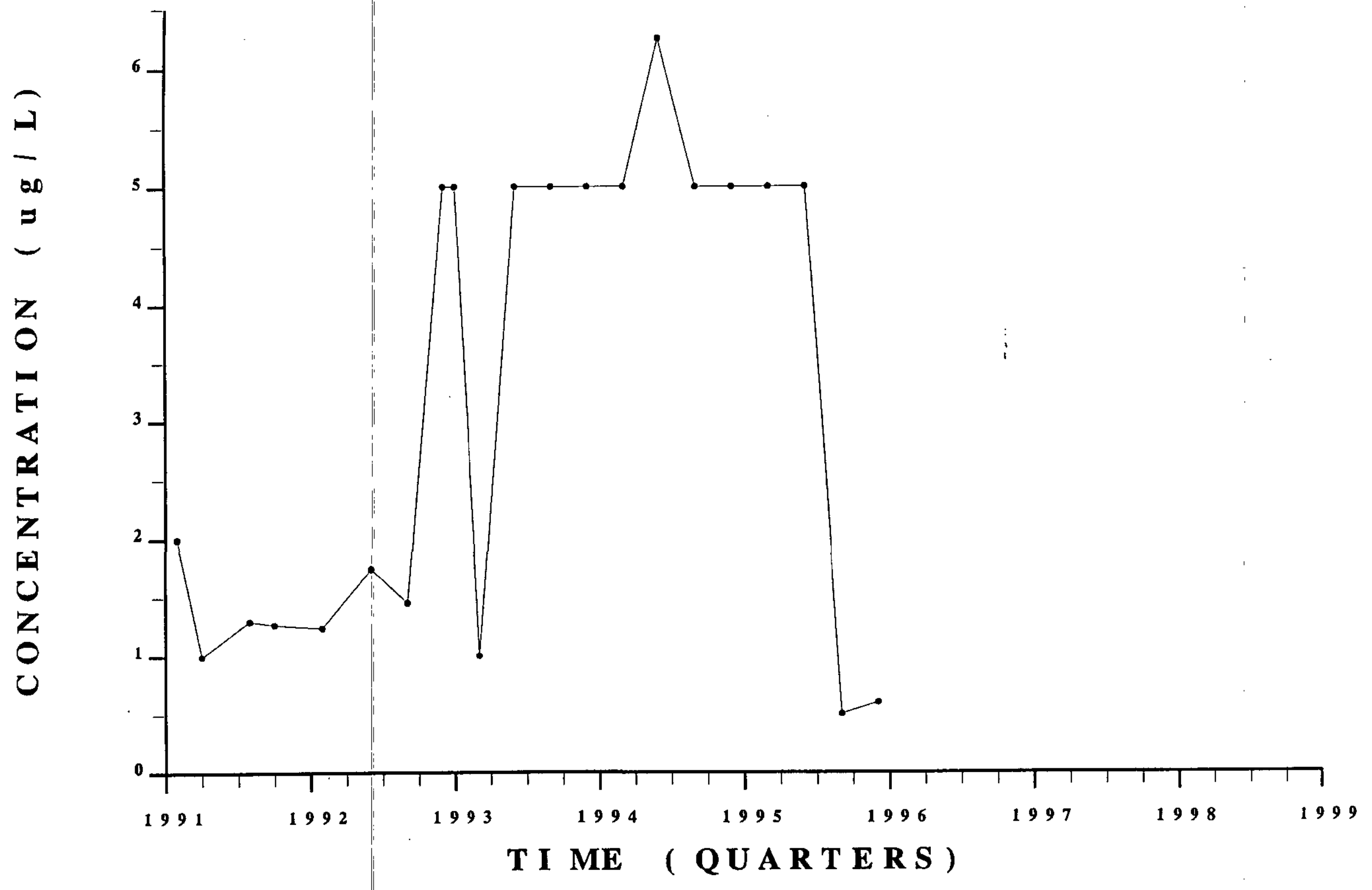

T R I C H L O R O E T H Y L E N E WE L L L F W 36 Unclass i f i ed 
TRI CHLOROF LUOROMET HANE

WE L L L F W 36
WS R C - T R - $99-\begin{array}{llllll}0 & 0 & 0 & 1 & 1\end{array}$ Unc l a s s i f i ed

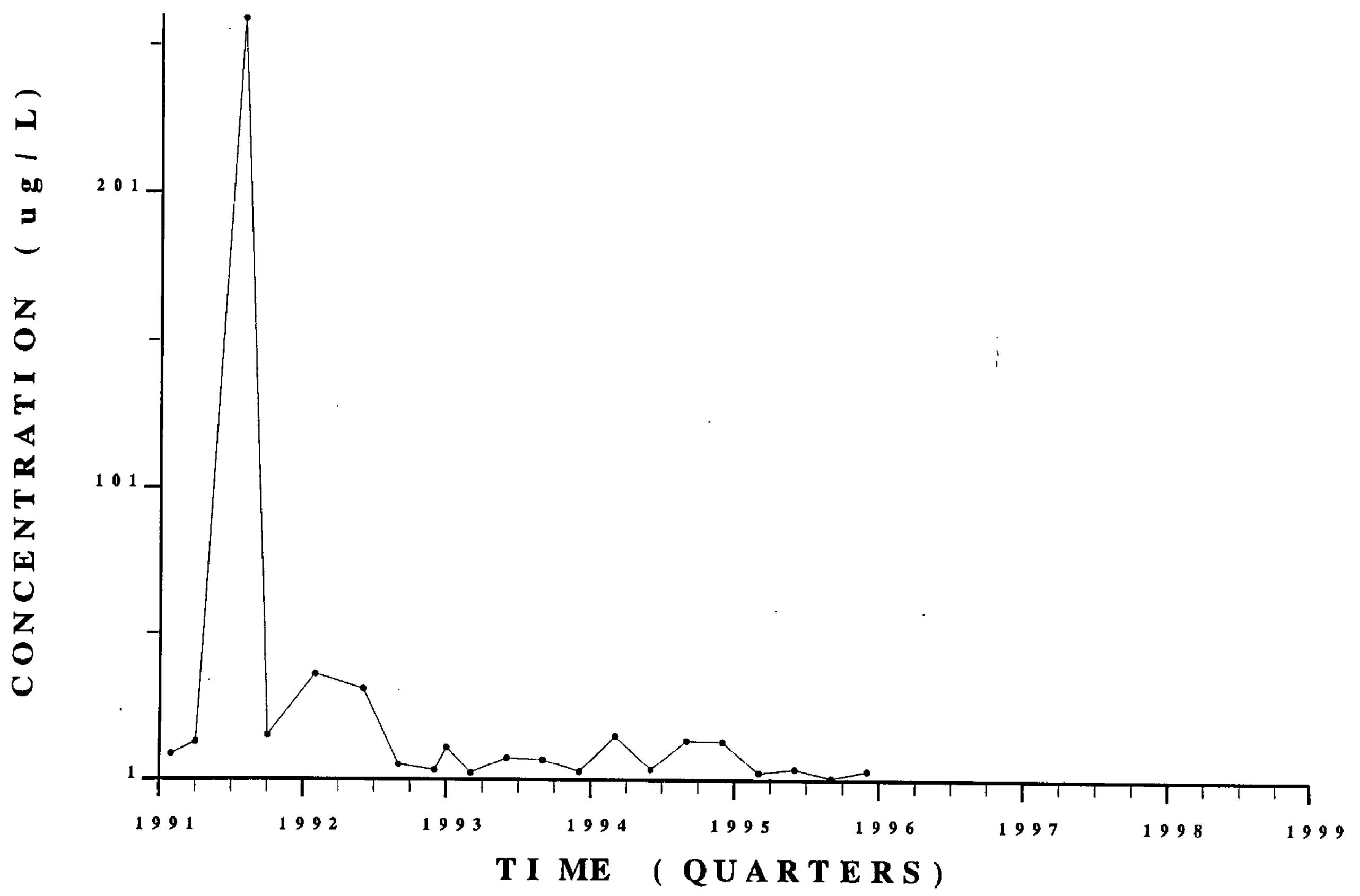

Sanitar $v$ Landfil I

Fourth ouarter.

$1998 \& \quad \& 998$

S I $\mathrm{mmar}$ 


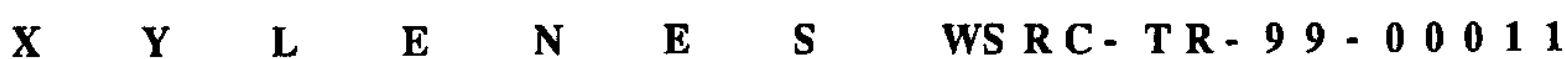
WE L L L F W 36

Unc l a s s i f i ed






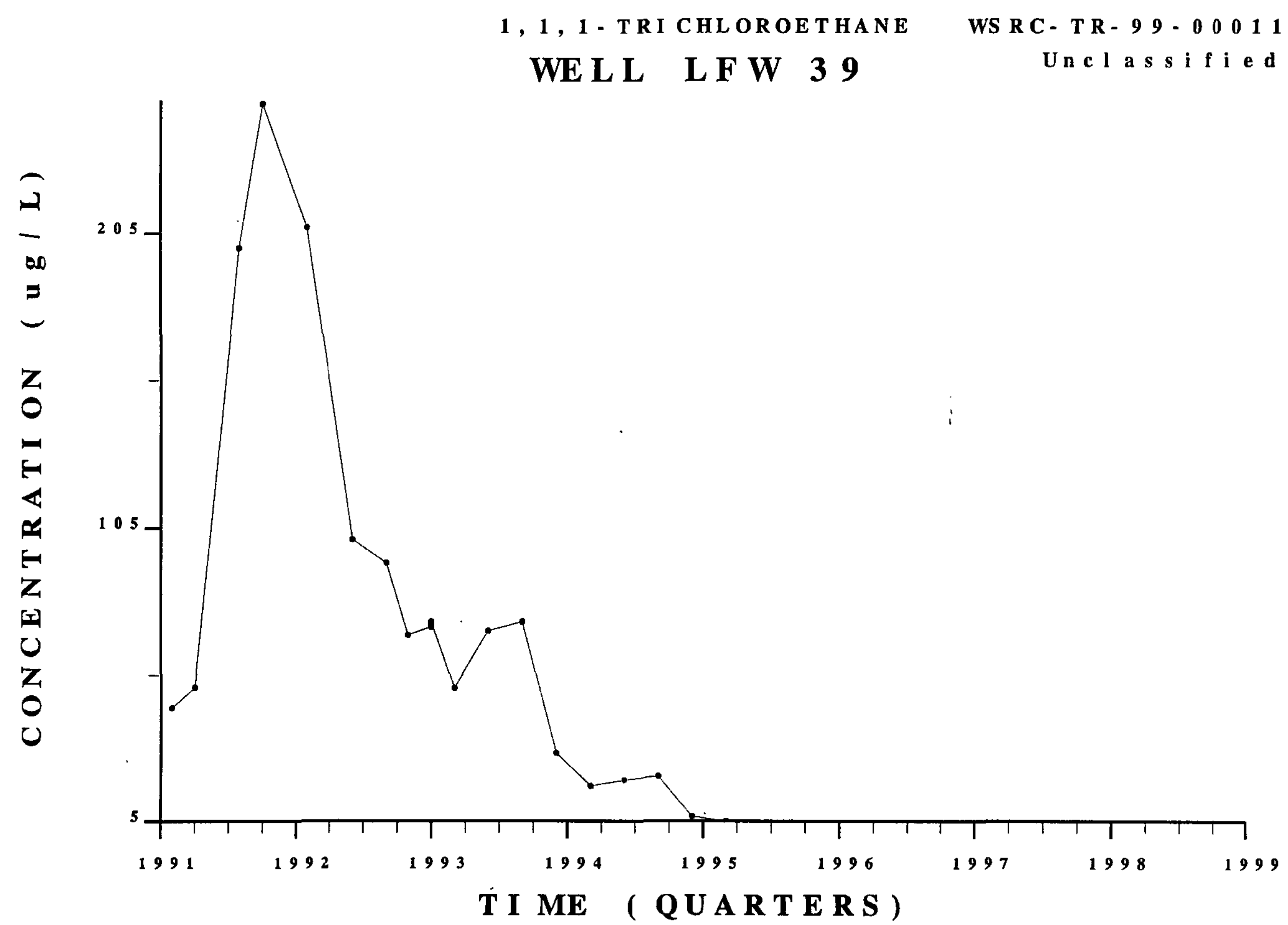

Sanitar v landfill

Fourth ouarter. 1998 \& 1998 Summarv 


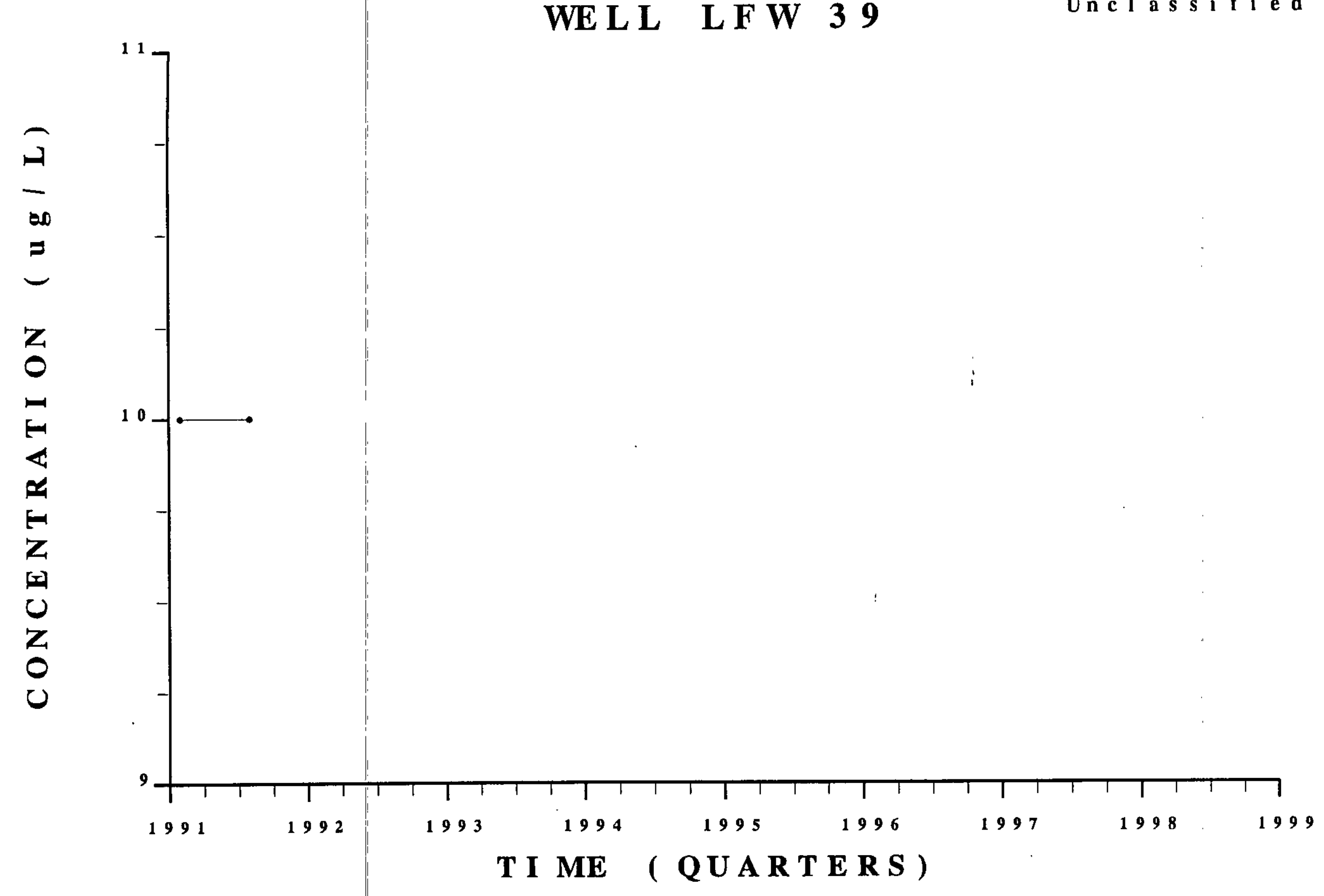

1, 4 - D I C H L O R O B E N Z E N E WE L L L F W 39
WS R C - TR- $99-00011$ Unclass if i ed 


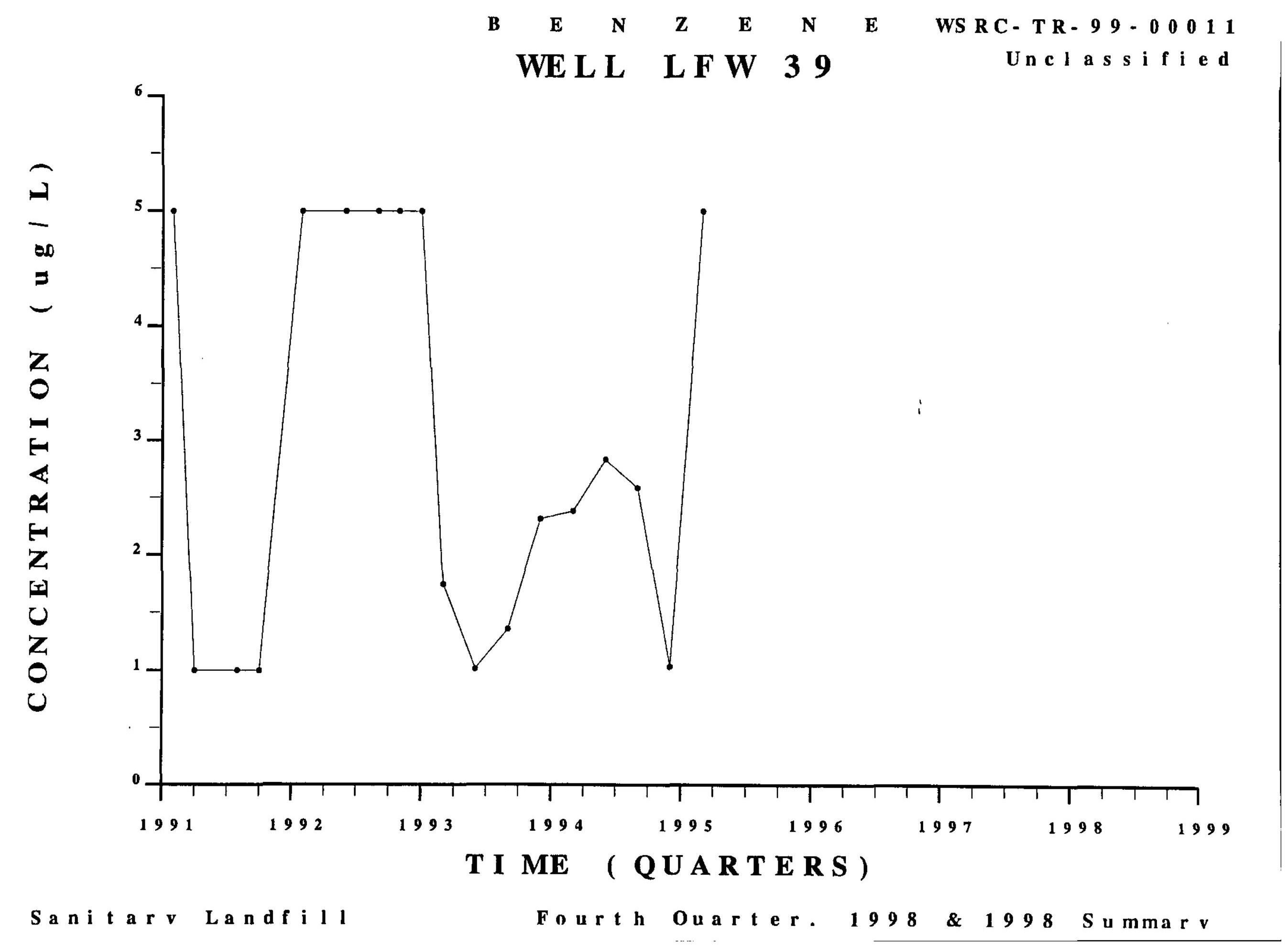




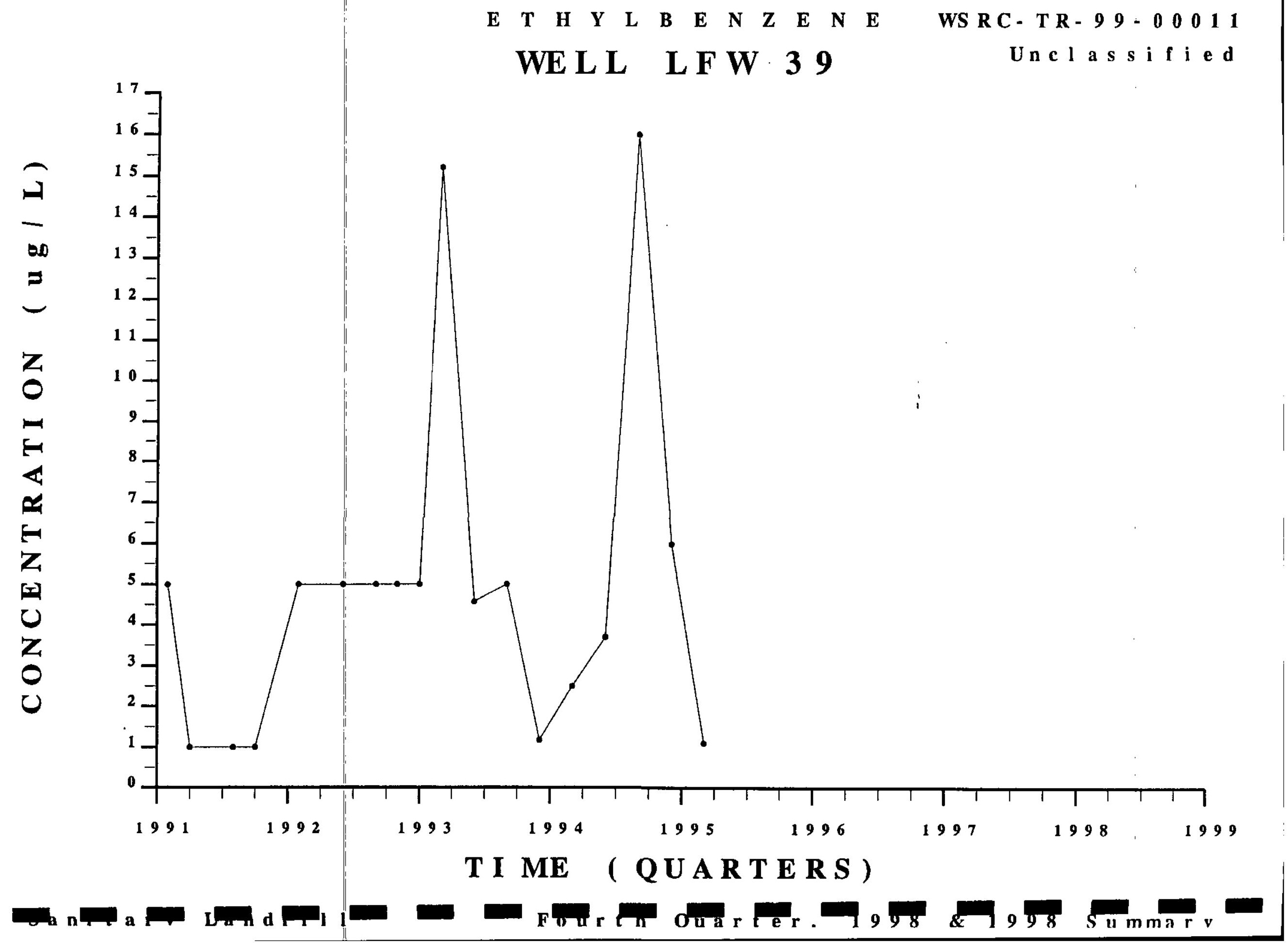


T E T RA CHLOROE THYL E NE WE L L L F W 39
WS R C - T R- $99-00011$

Unclas s i f i ed

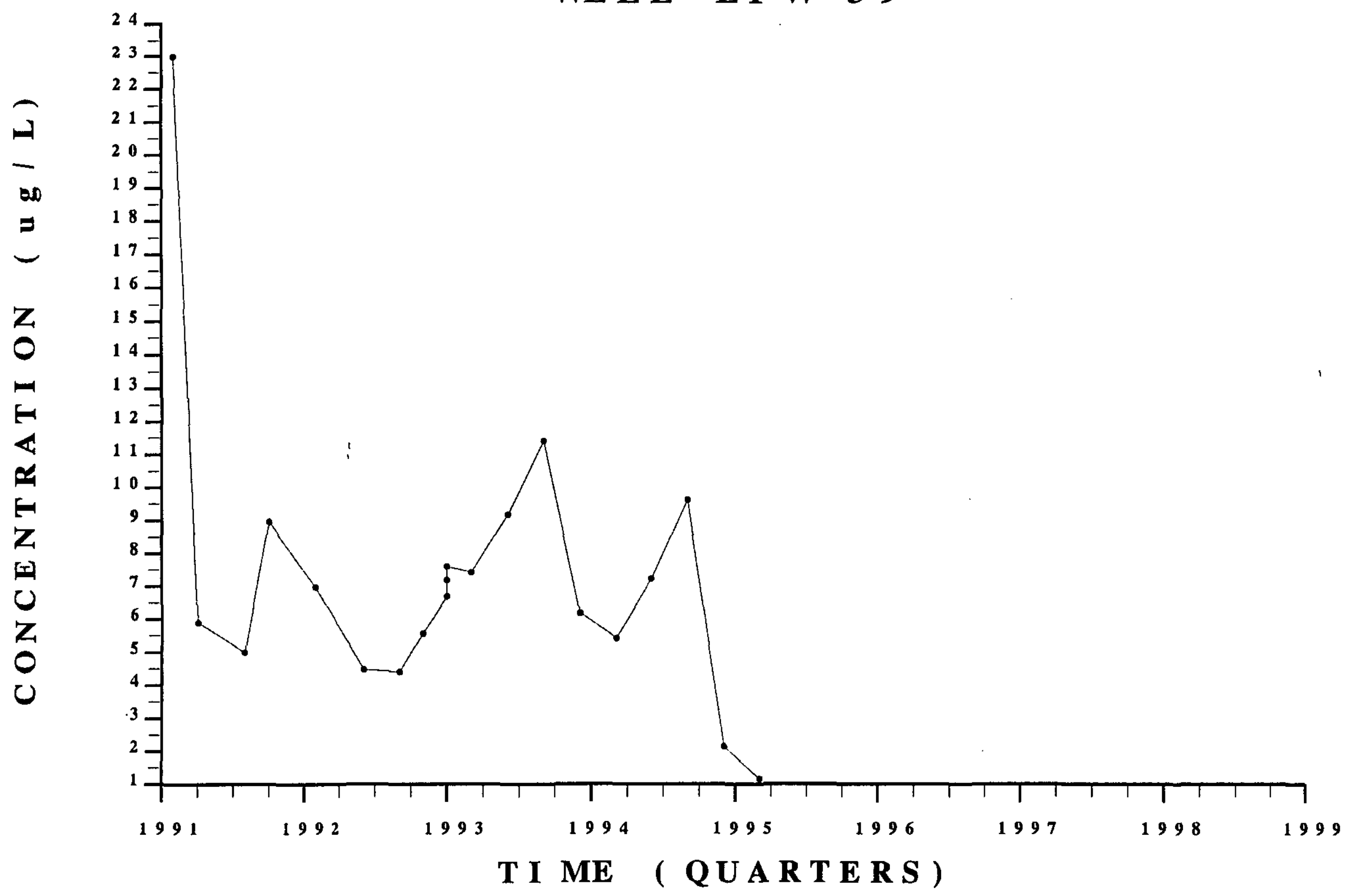

Sanit ar v Landfill Fourth Ouarter. 1998 \& 1998 Summar 


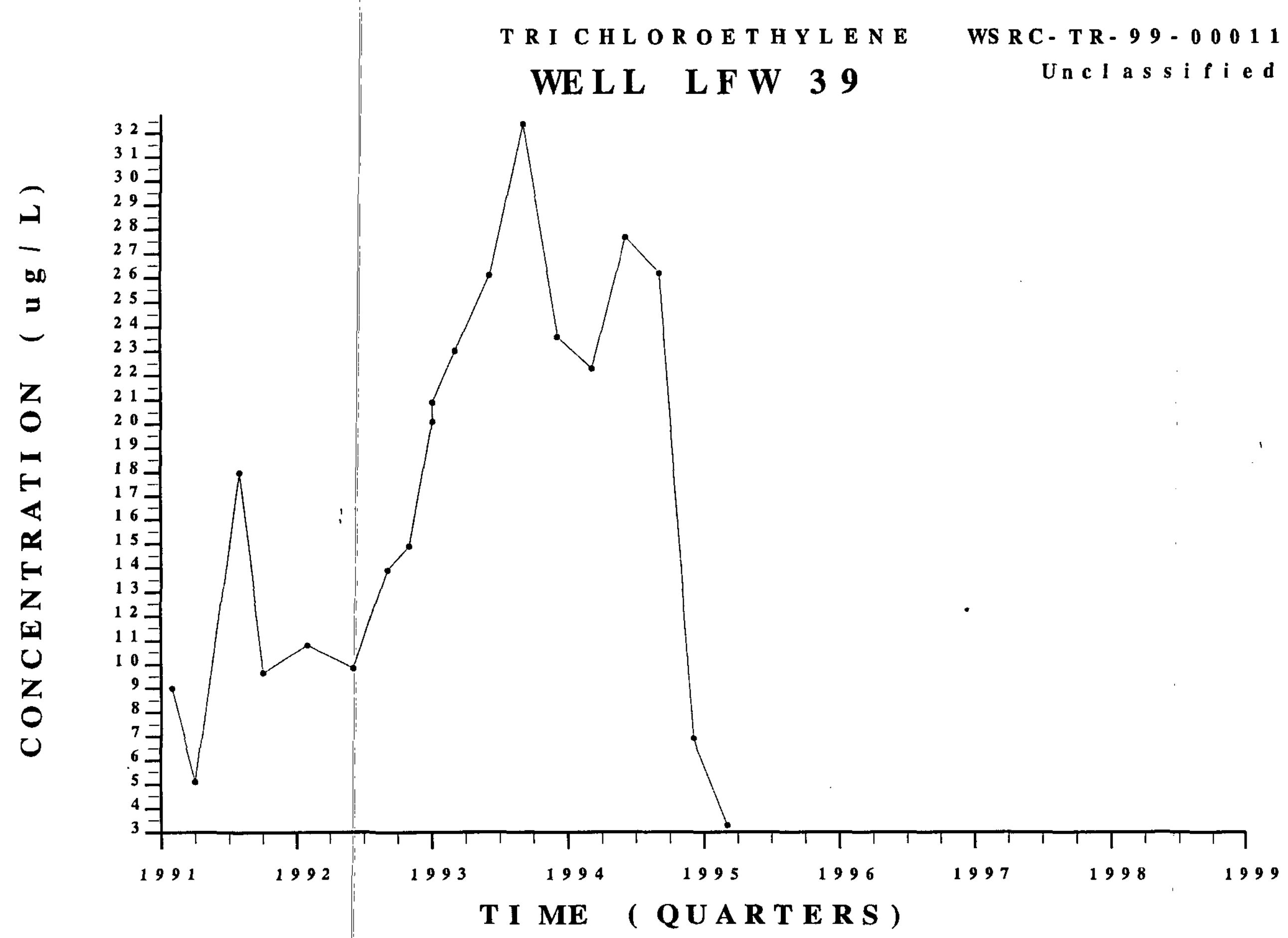

Sanitarv Landfil 
TRI CHL OROF L UOROME THANE

WE L L L F W 39
WS R C - T R - $99-000011$

Unc l a s s i fi ed

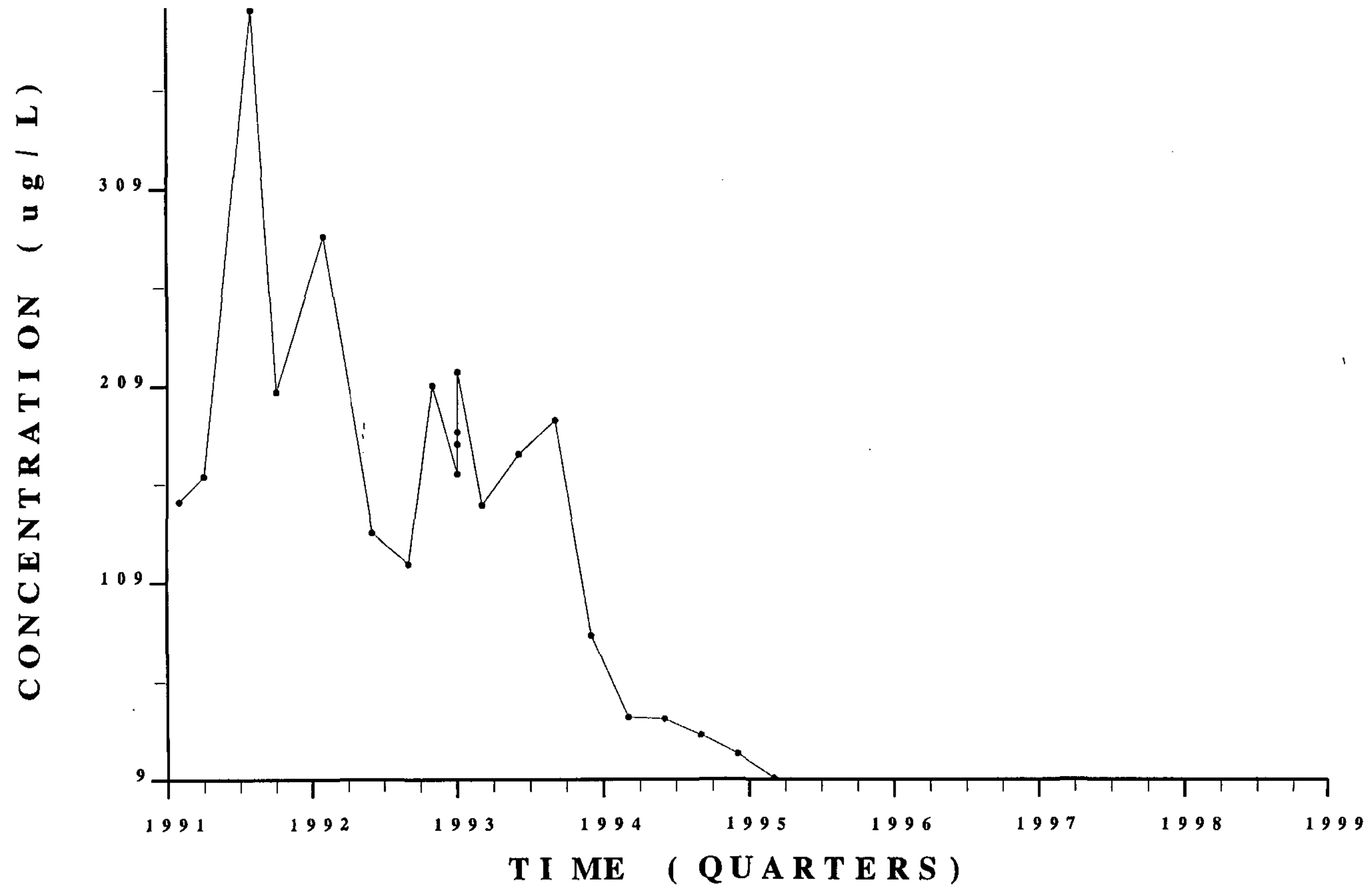

Sanitary Landfil I

Fourth ouarter.

$1998 \& \quad 1998$ S u mmar v 


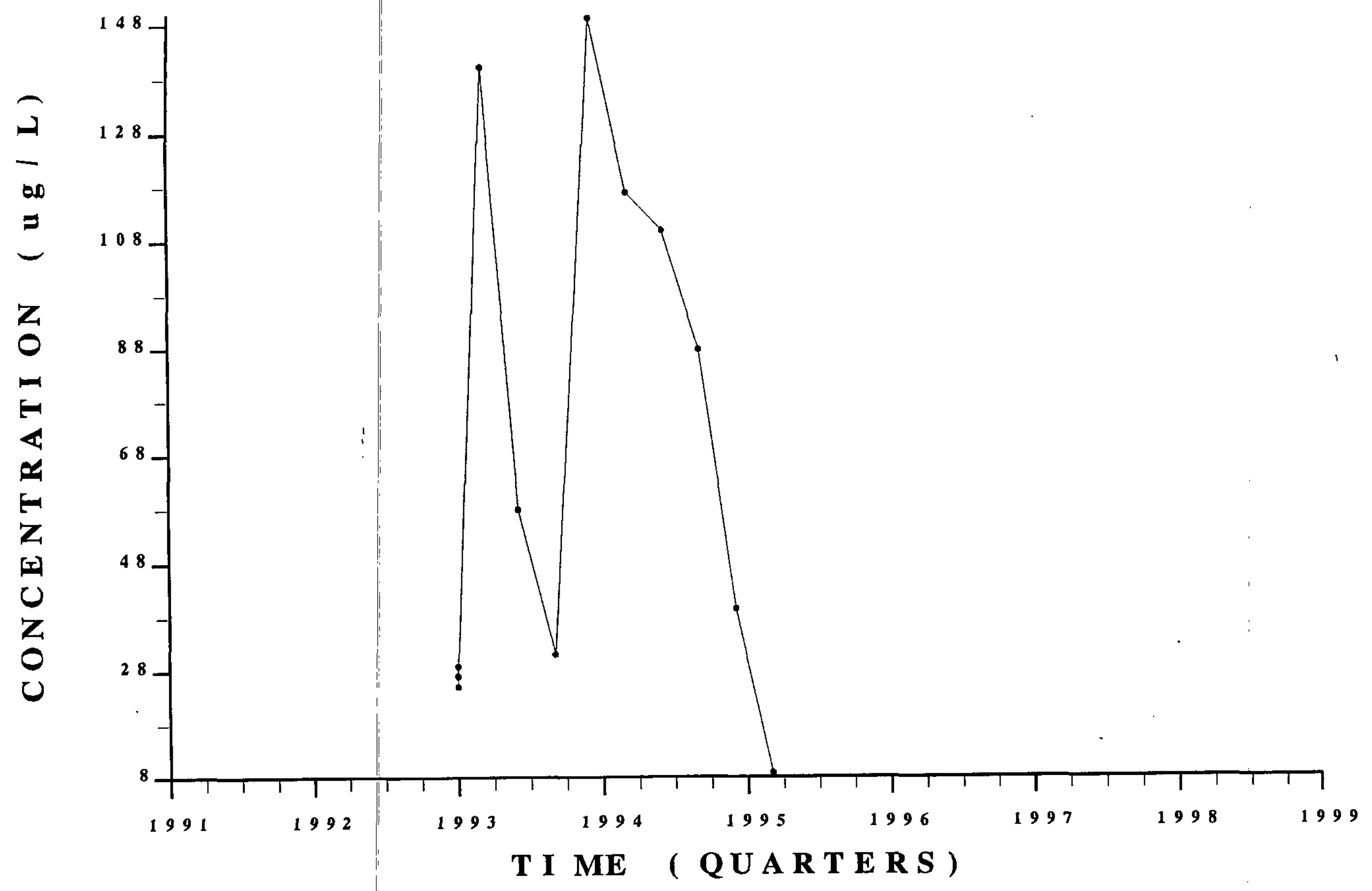

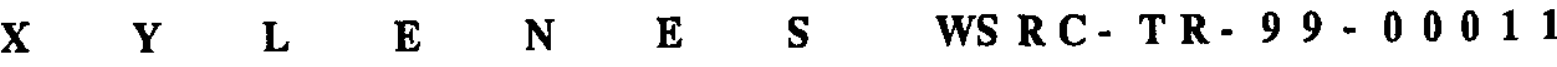
WELL LFW 39 Unclassified 
$1,1,1$ - T R I CHLOROE T HA N E WELL LFW 48 D

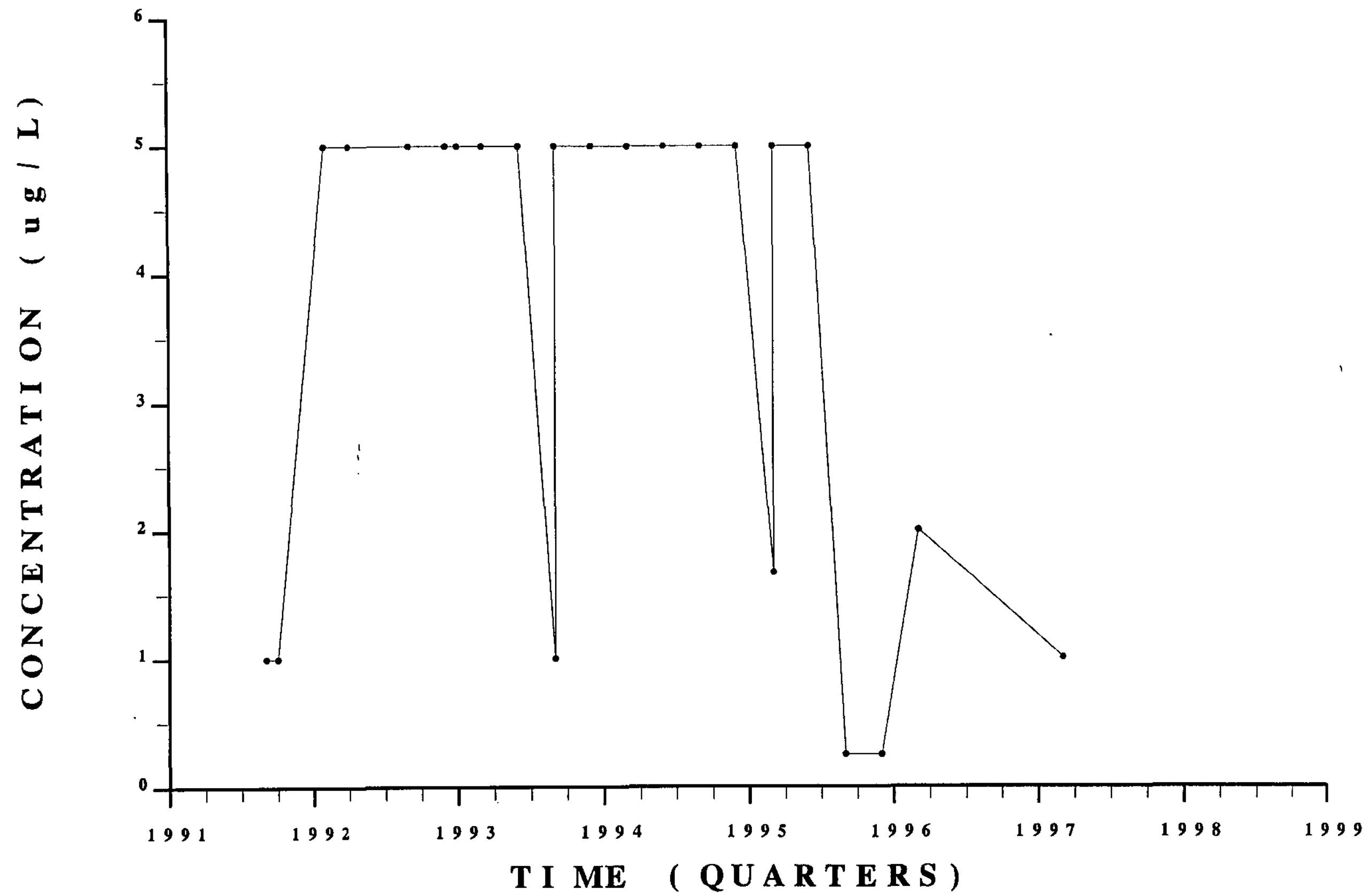

Fourthouarter.
\& 1998

S u mmar v 


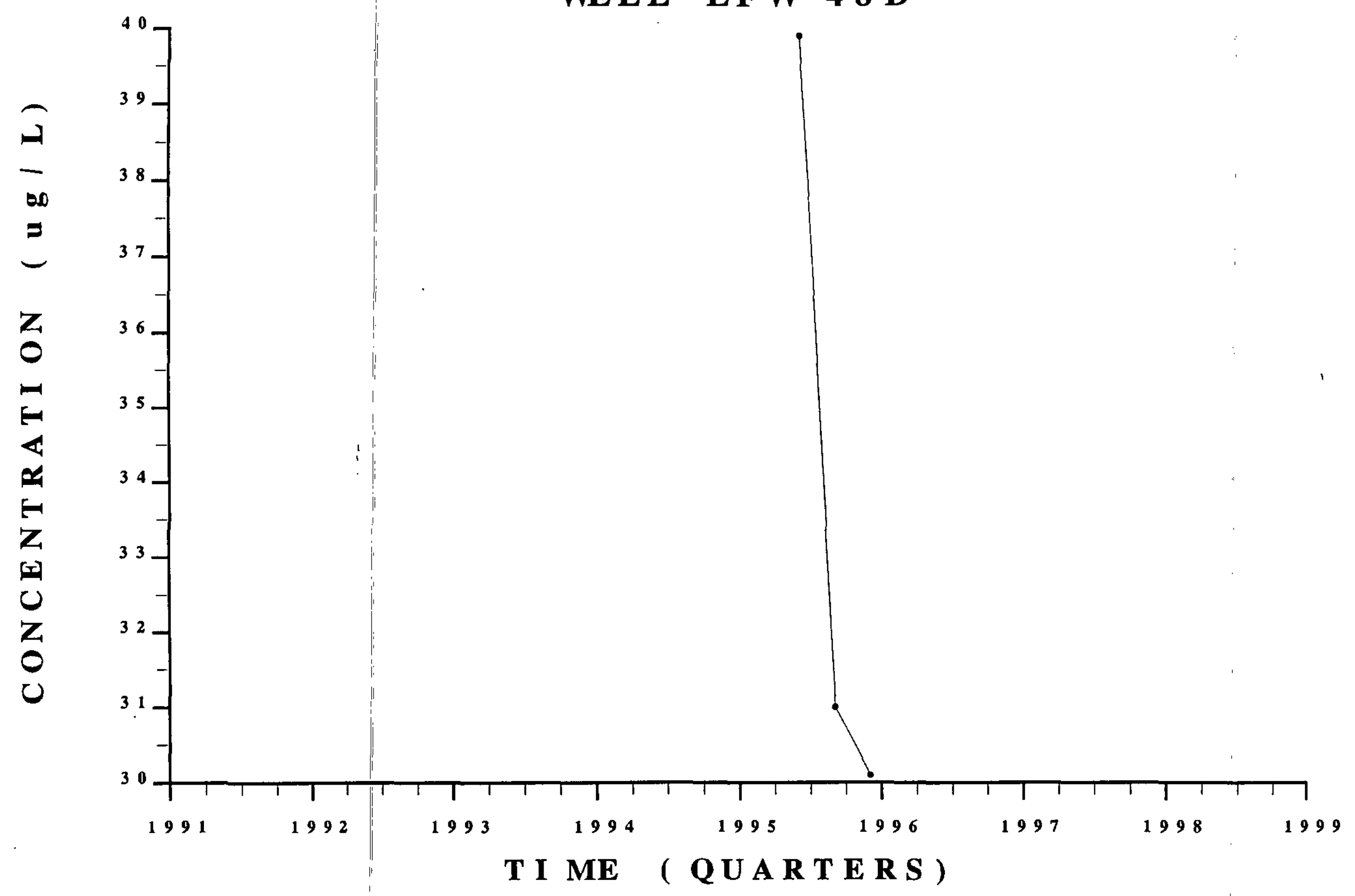

1,4 - D I C HL ORO B E N Z E N E WELL LFW 48 D
WS RC-TR- $99-000011$ Unclass if i ed 


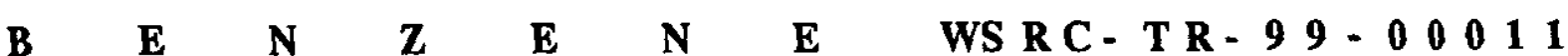
WELL LFW 48 D






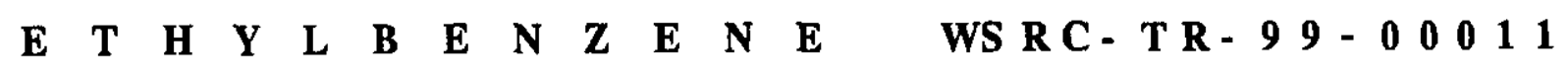
WELL LFW 48 D

Unclass if i ed

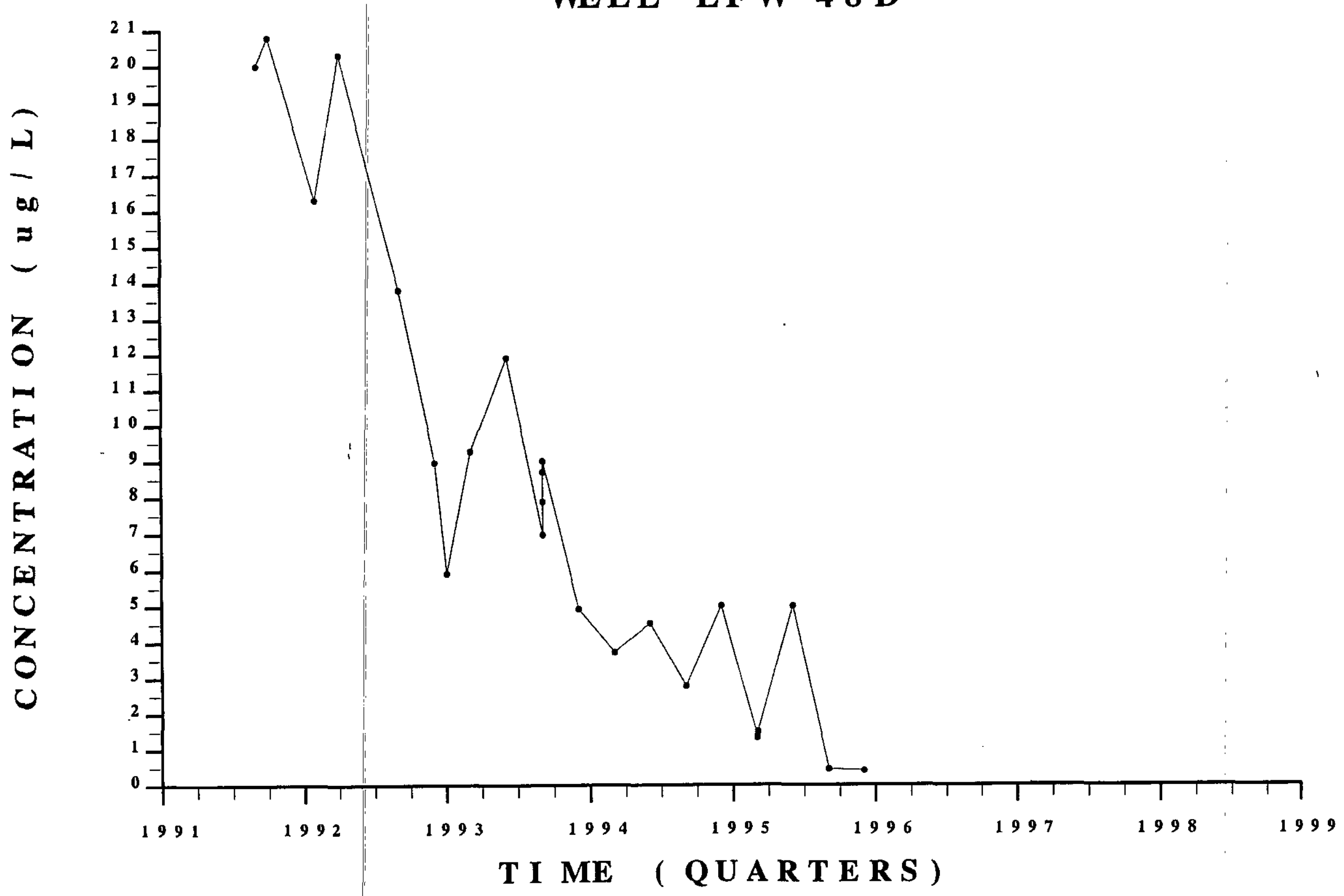

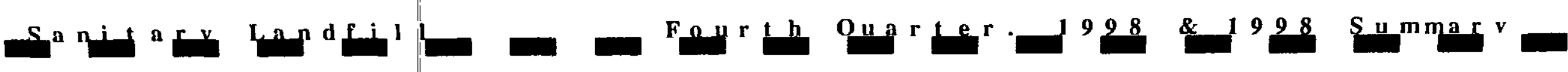


T E T R A C HLOROE T HYL E NE WELL LFW 48 D

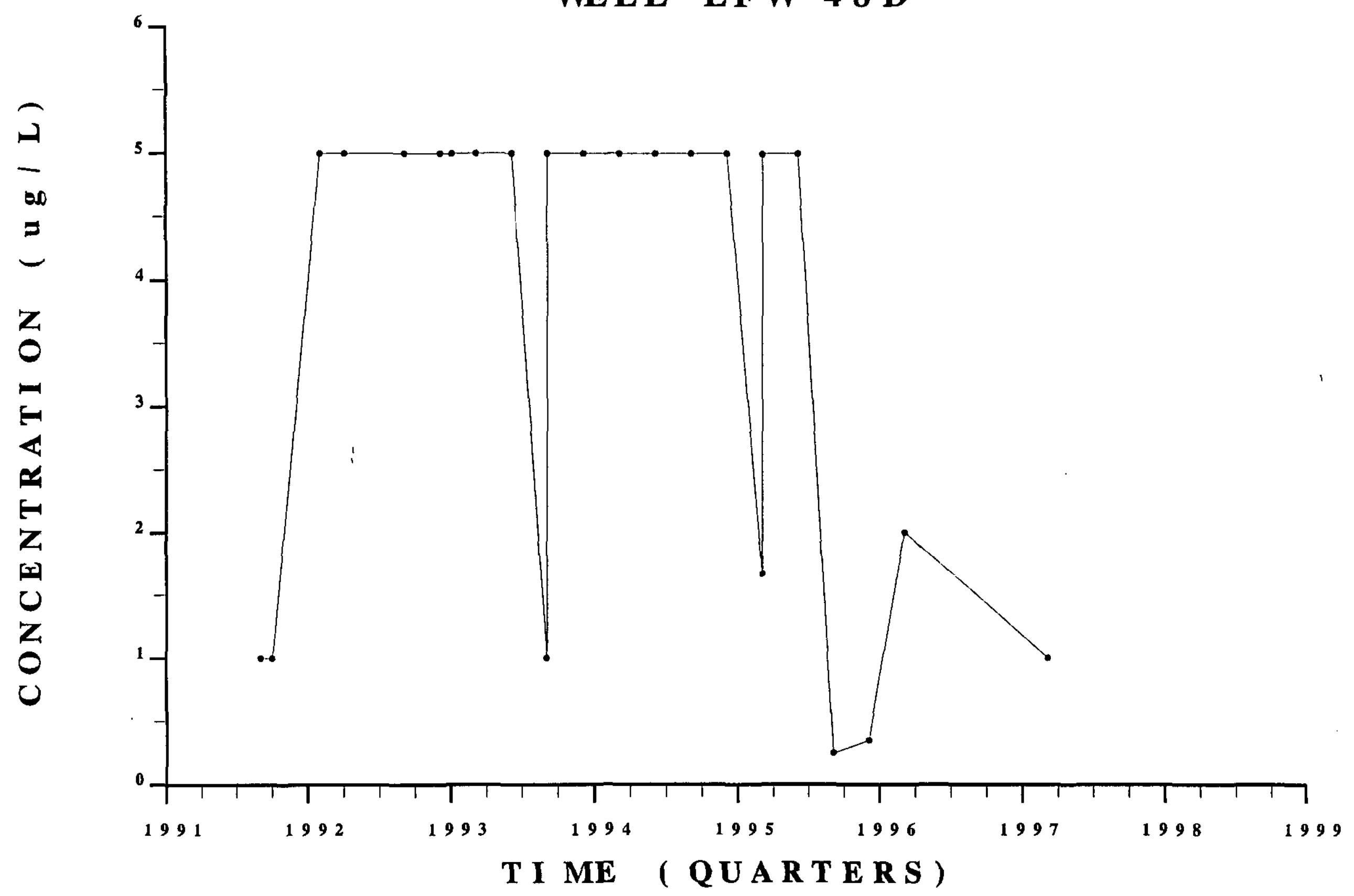

Sanitary Landfill
Fourth Ouarter. 1998 \& 1998 Summar 
TR I CHLOROE THYL N E WELL LFW 48 D
WS R C - T R - $99-000011$

Unclassified

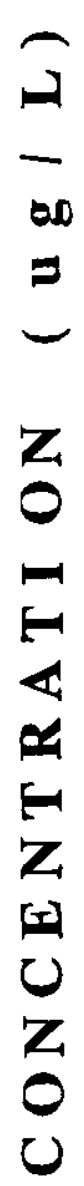

6
5

$-$

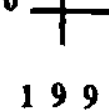

1991

1994

1995

1997

1998

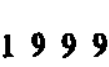

T I ME ( QU A R T E R S ) 
T R I CHLOROF LUOROME T HANE

WELL LFW 4 8 D

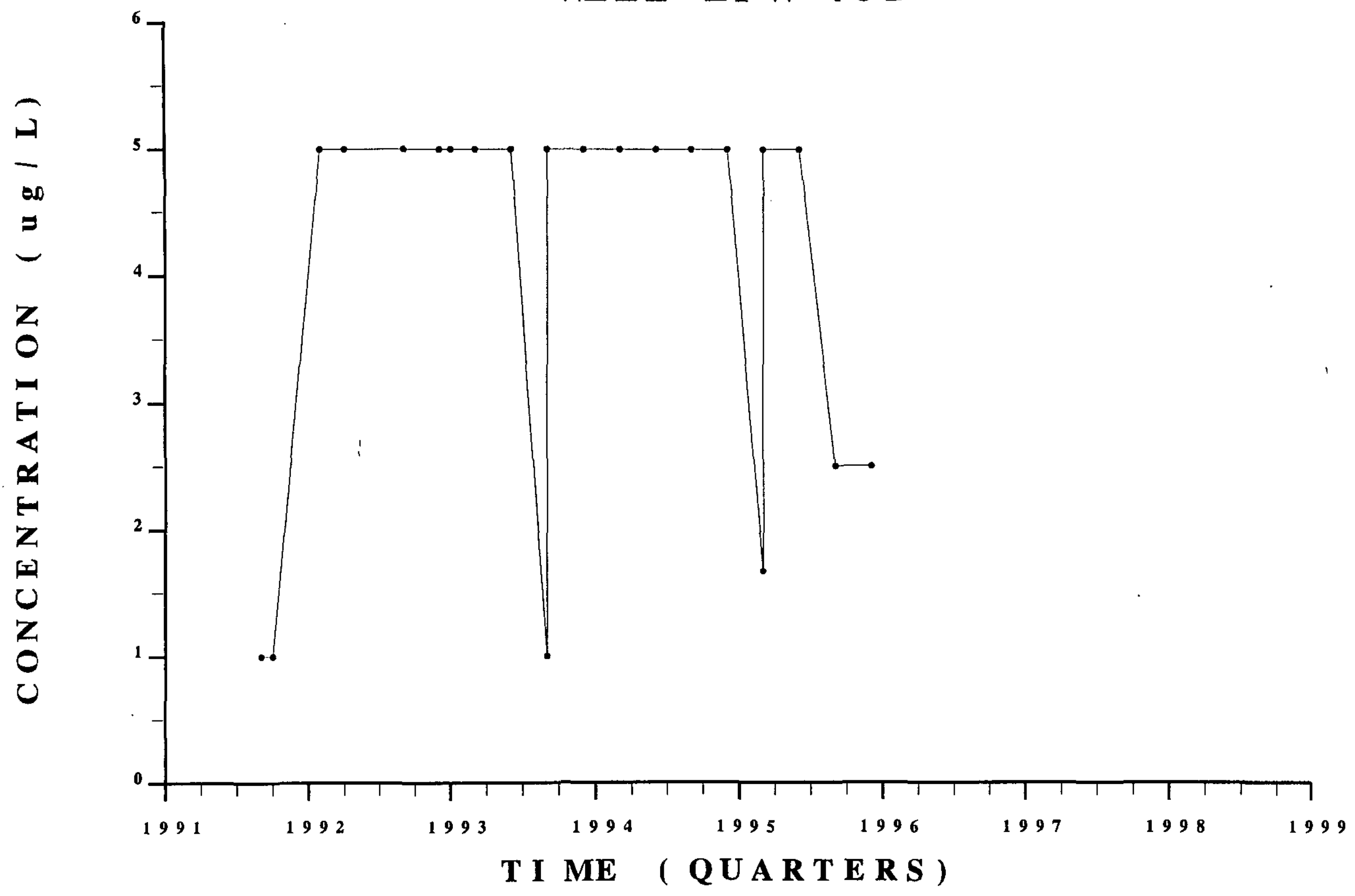

S a n i t a r v Landf i l i
Fourth Ouarter.

1998

\& 1998

S u mma r v 


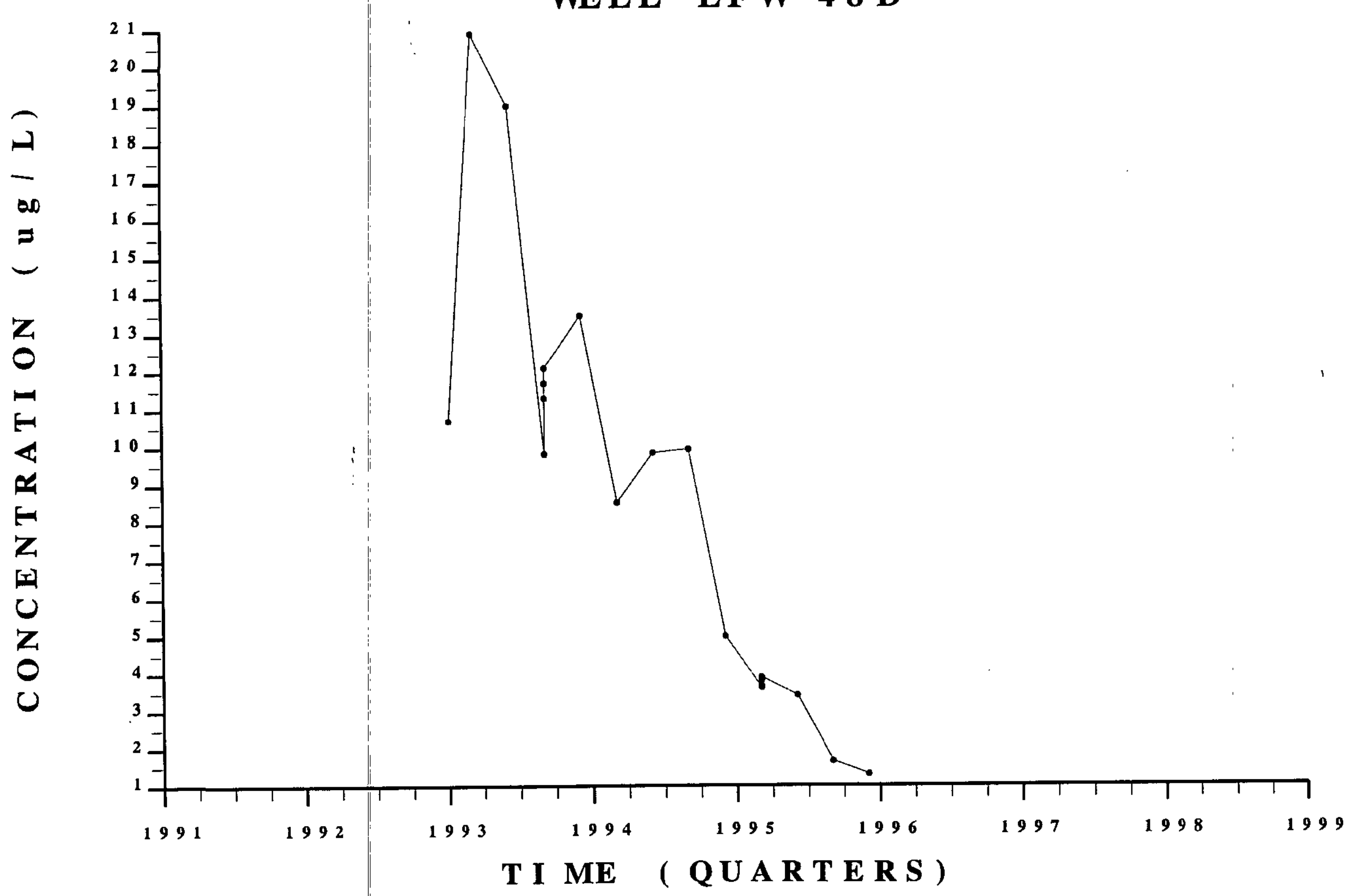

Sanitary Landfil 
$1,1,1$ - TRI CHLOROE THANE WELL LFW 58 D

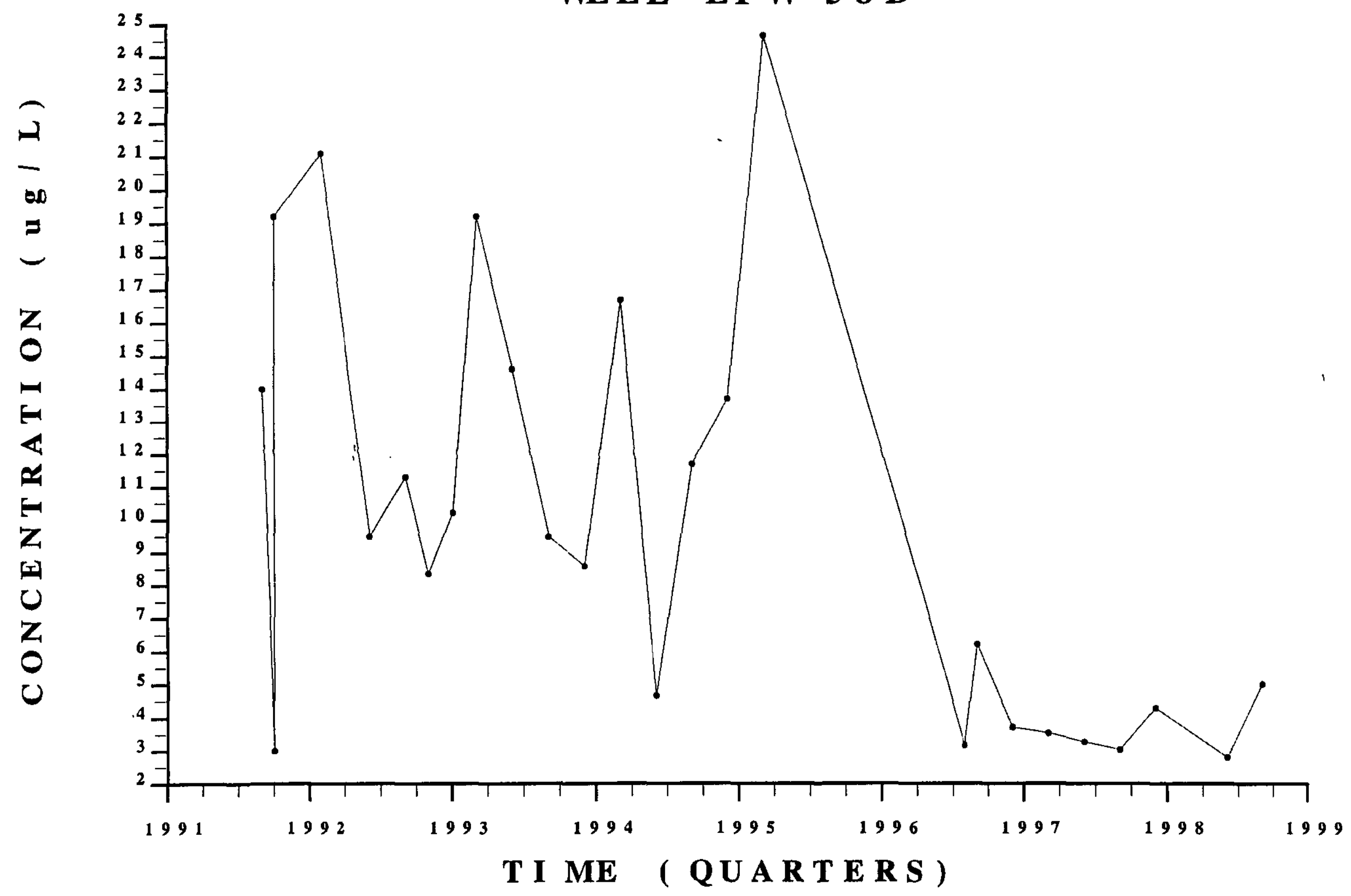

Sanitar $\mathbf{L}$ a n d f i I I
Fourth ouarter. 1998 \& 1998 Summar 


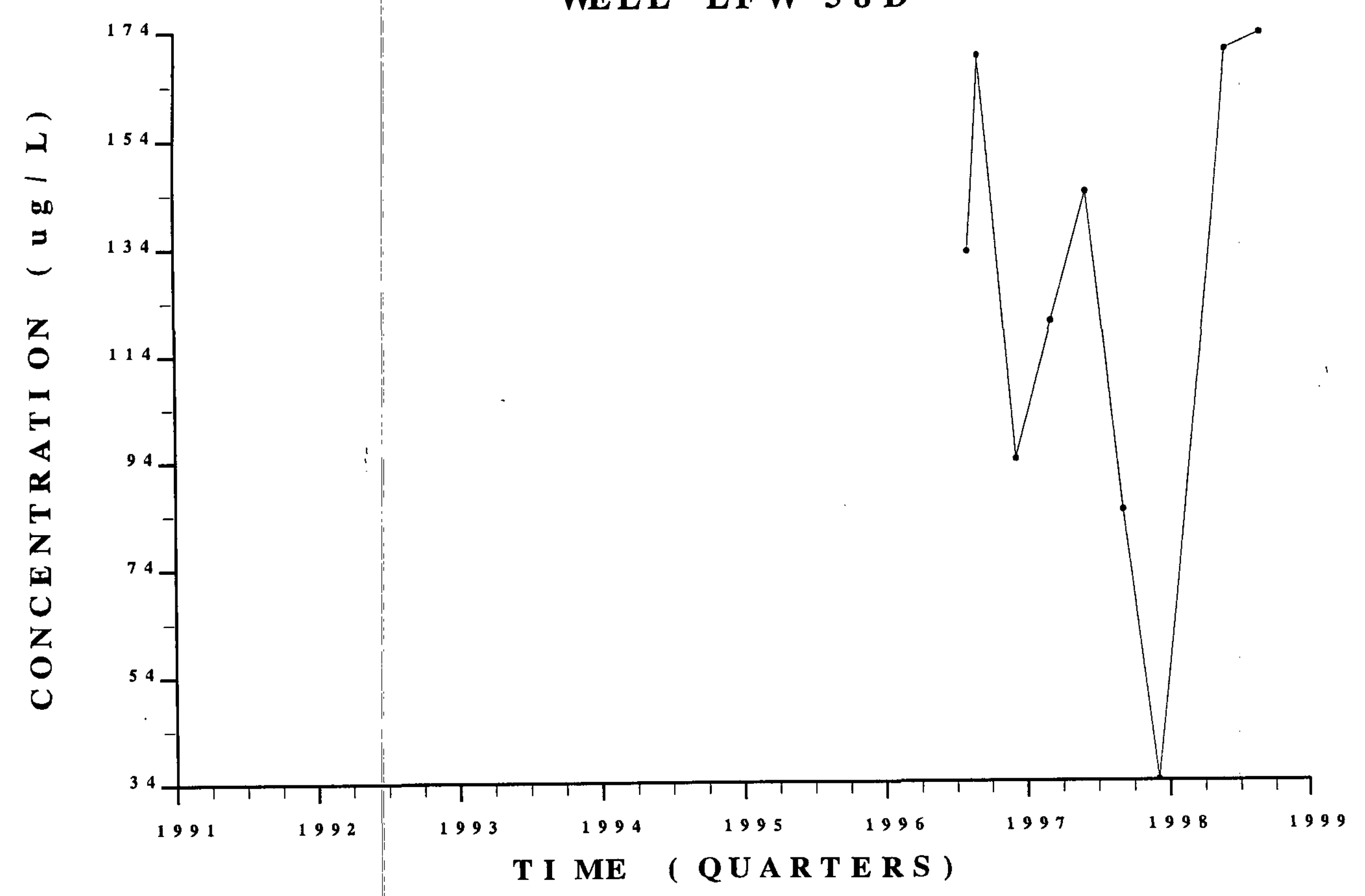

1,4-D I C HLOROB ENZENE WS R C-TR-9 9 - 000011 WELL LFW 58 D Unclass i f i ed

Sanitarv Landfil 


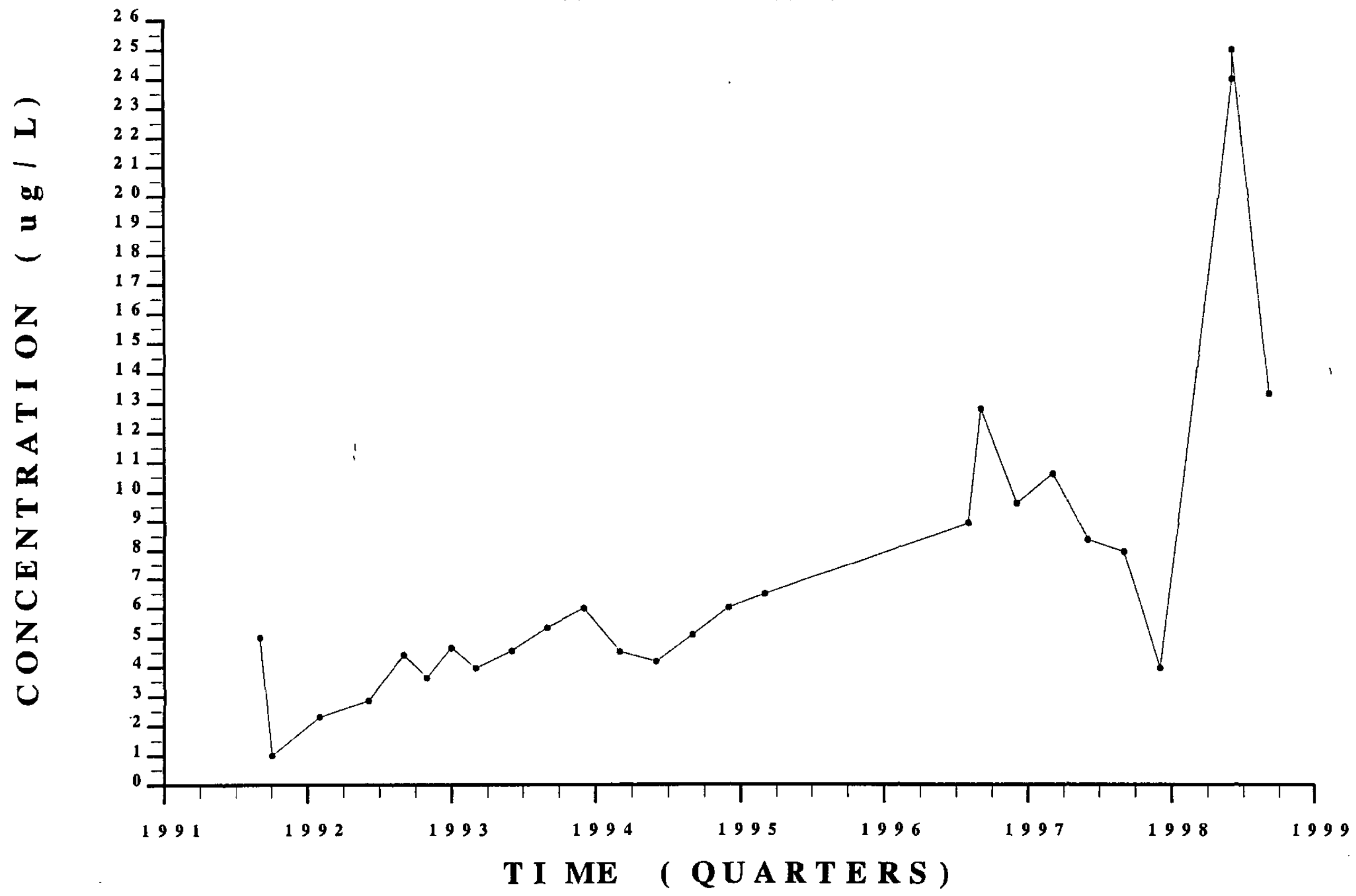

Sanitary Landfill

Fourth Ouarter.

199

\& 1998

S u mmar 


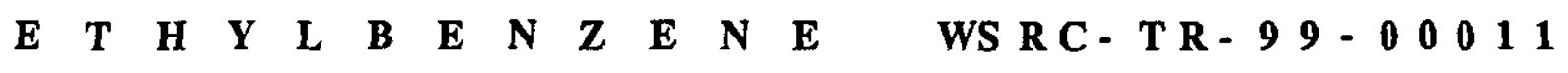
WELL LFW 58 D

Unclass i f i ed



Sa pif ary Landfil 
TE T RA C HLOROE THYLE N E WELL LFW 58 D

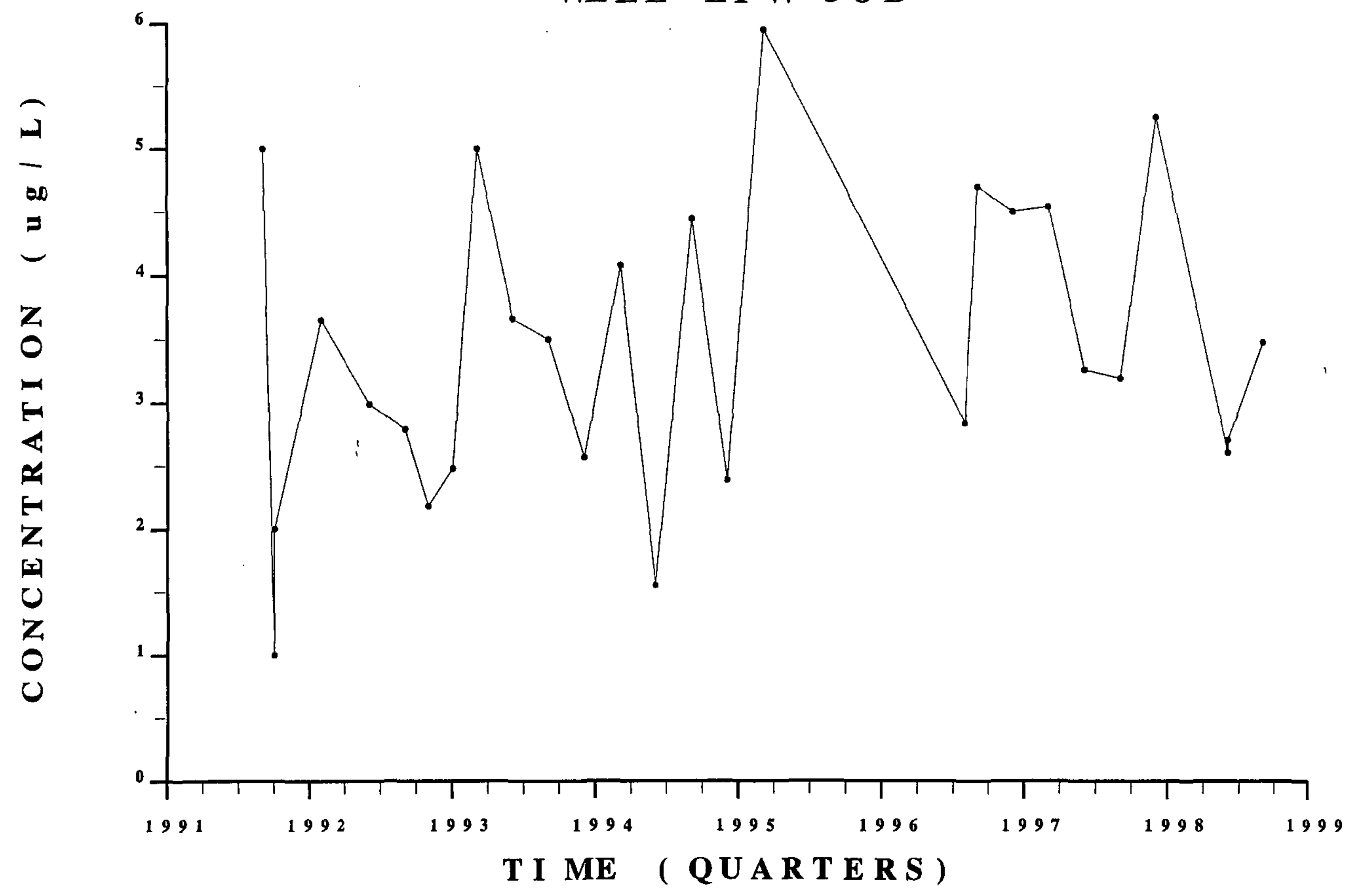

Sani t a r v Landfill
Fourth Ouarter. 1998 \& 1998 Summarv 
T R I C H L O R O E T H Y L E N E WELL LFW 58 D

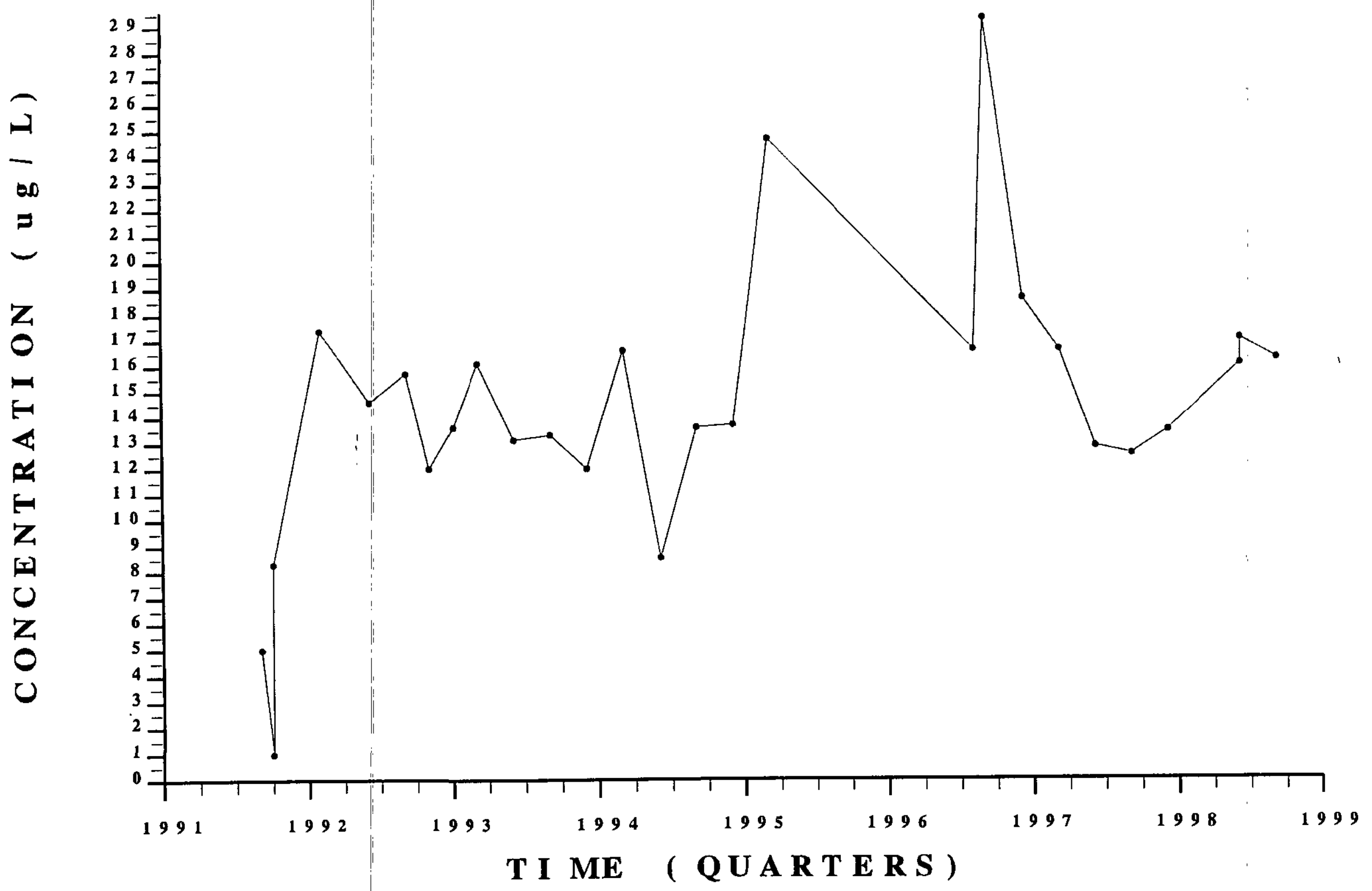

tand $i_{i}$
WS R C - T R - $999-000011$

Unc l a s s i f i ed 
TR I CHL OROF LUOROME T HANE

WE LL LF W 58 D

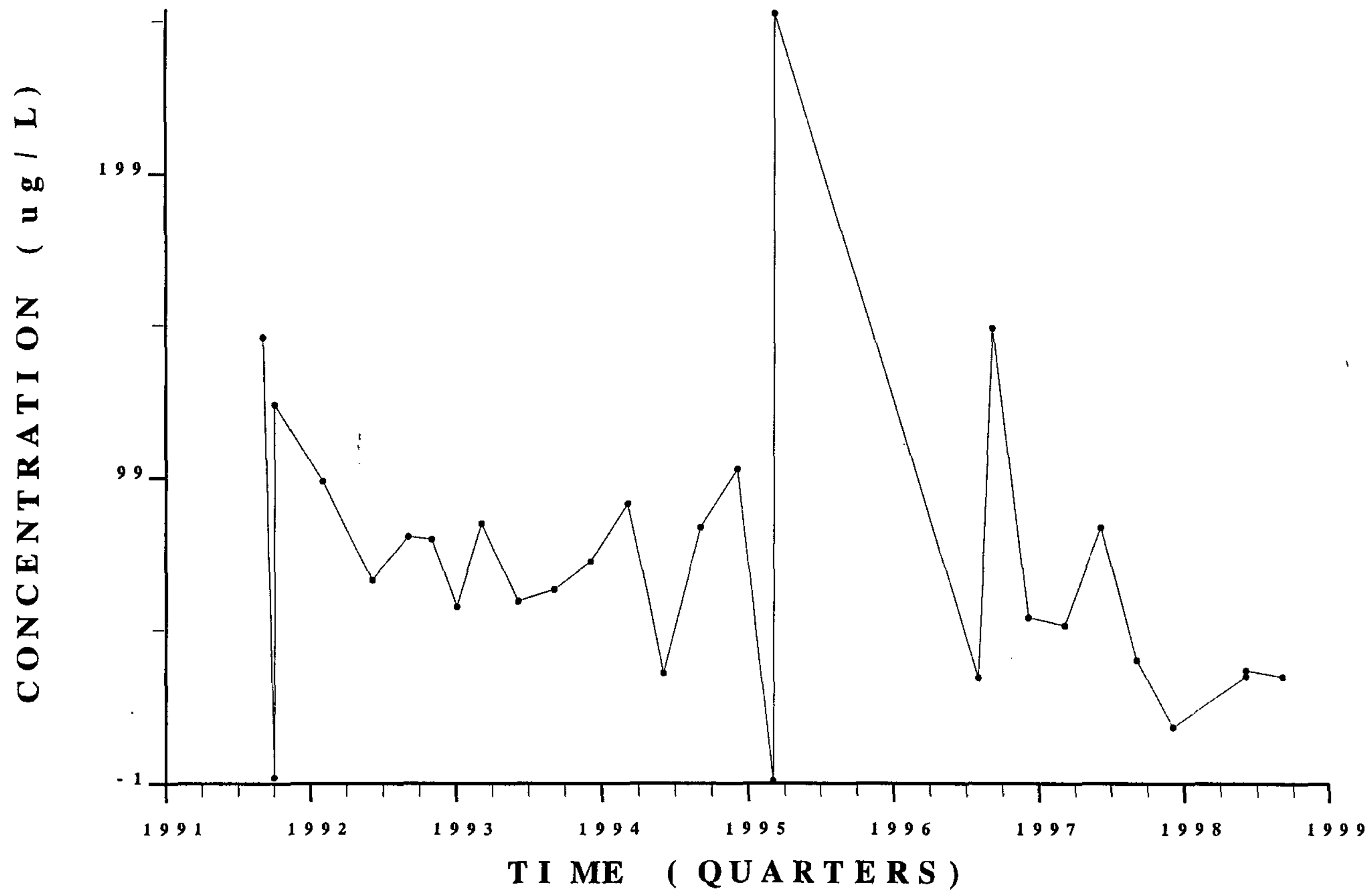

San i t a r v L n d f i l I
Fourth ouarter.

1998 \& 1998 S u mmar v 


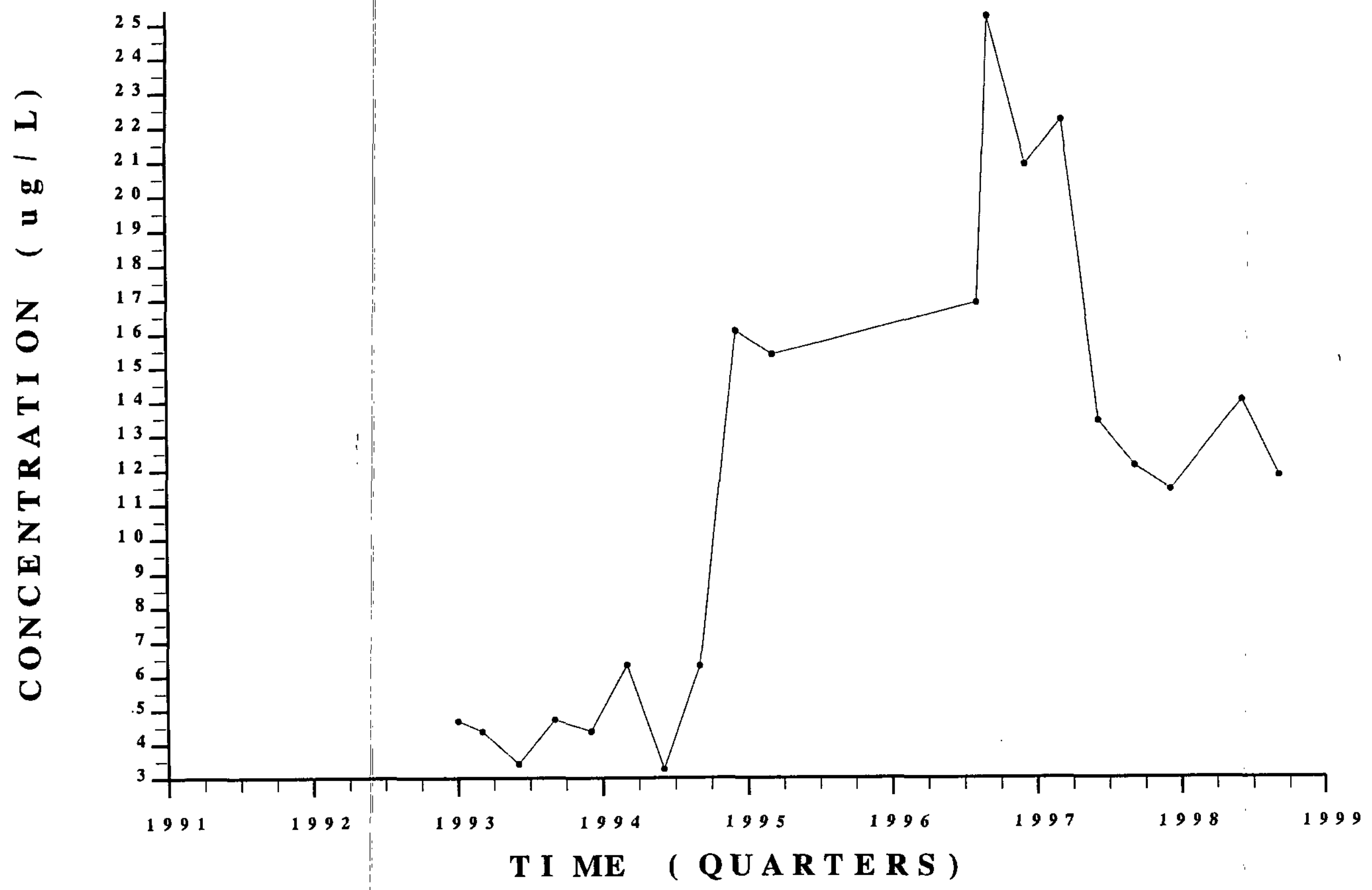

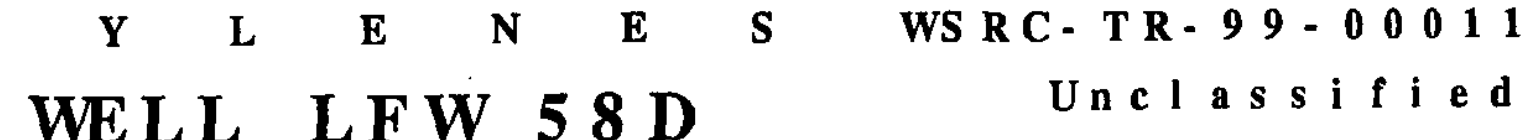


$1,1,1$ - TRI CHLOROE THANE WELL LFW 59 D
WS R C - T R - $99-00011$

Unc l a s s i f i ed

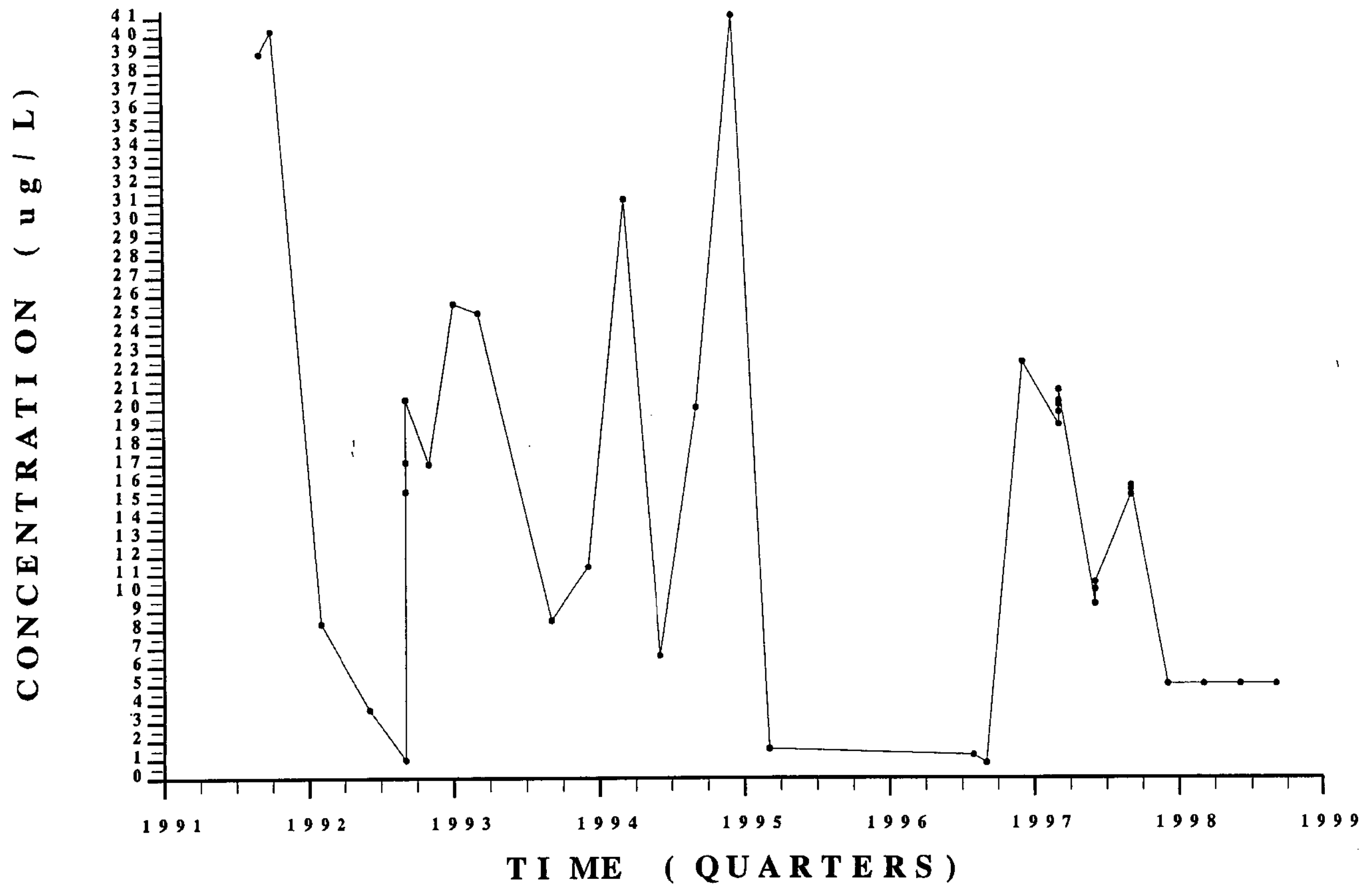

S a n i a r v land f I l

Fourth Ouarter.

\& 1998

Su mmar v 


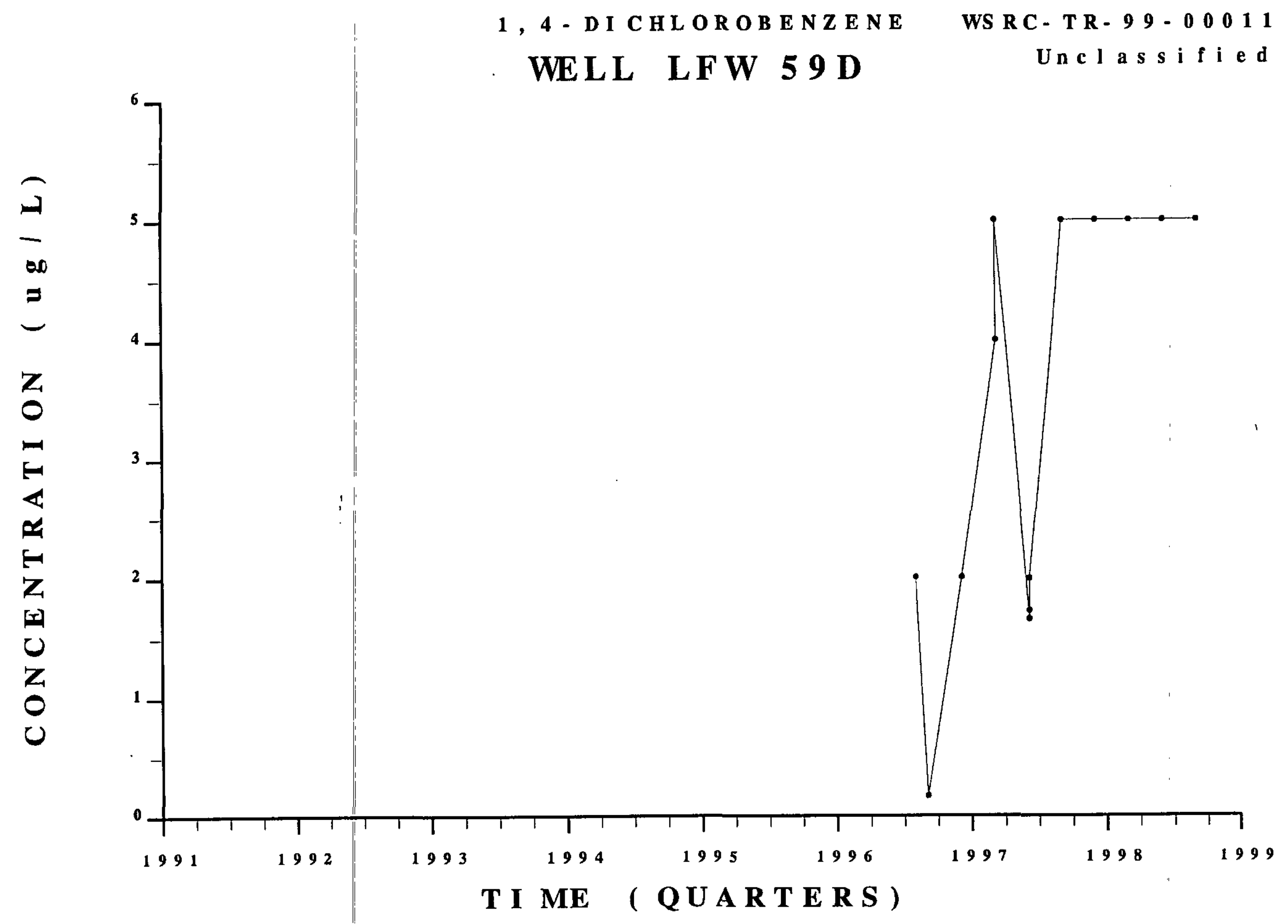

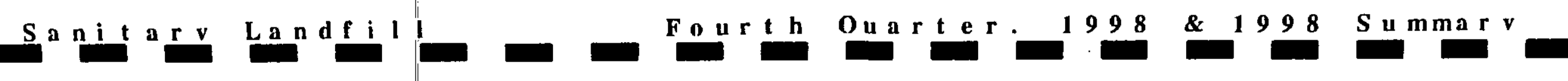




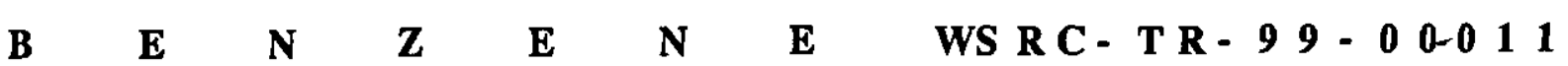
WELL LFW 59 D

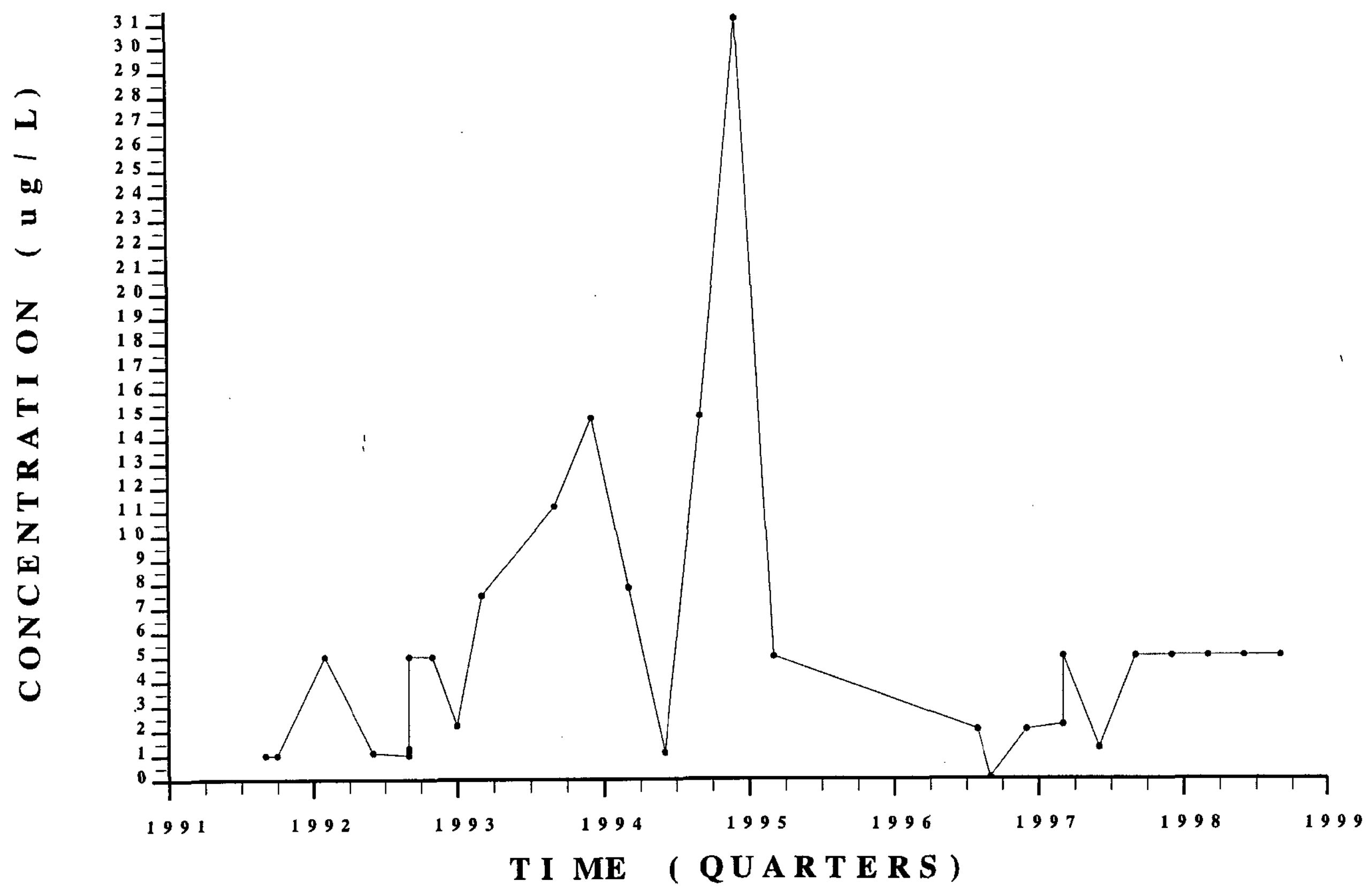

$S$ a n i t a r v l a n d f i l l

Fourth Ouart e r. $\begin{array}{llllll}1998 & \& & 1998 & \text { Summar }\end{array}$ 


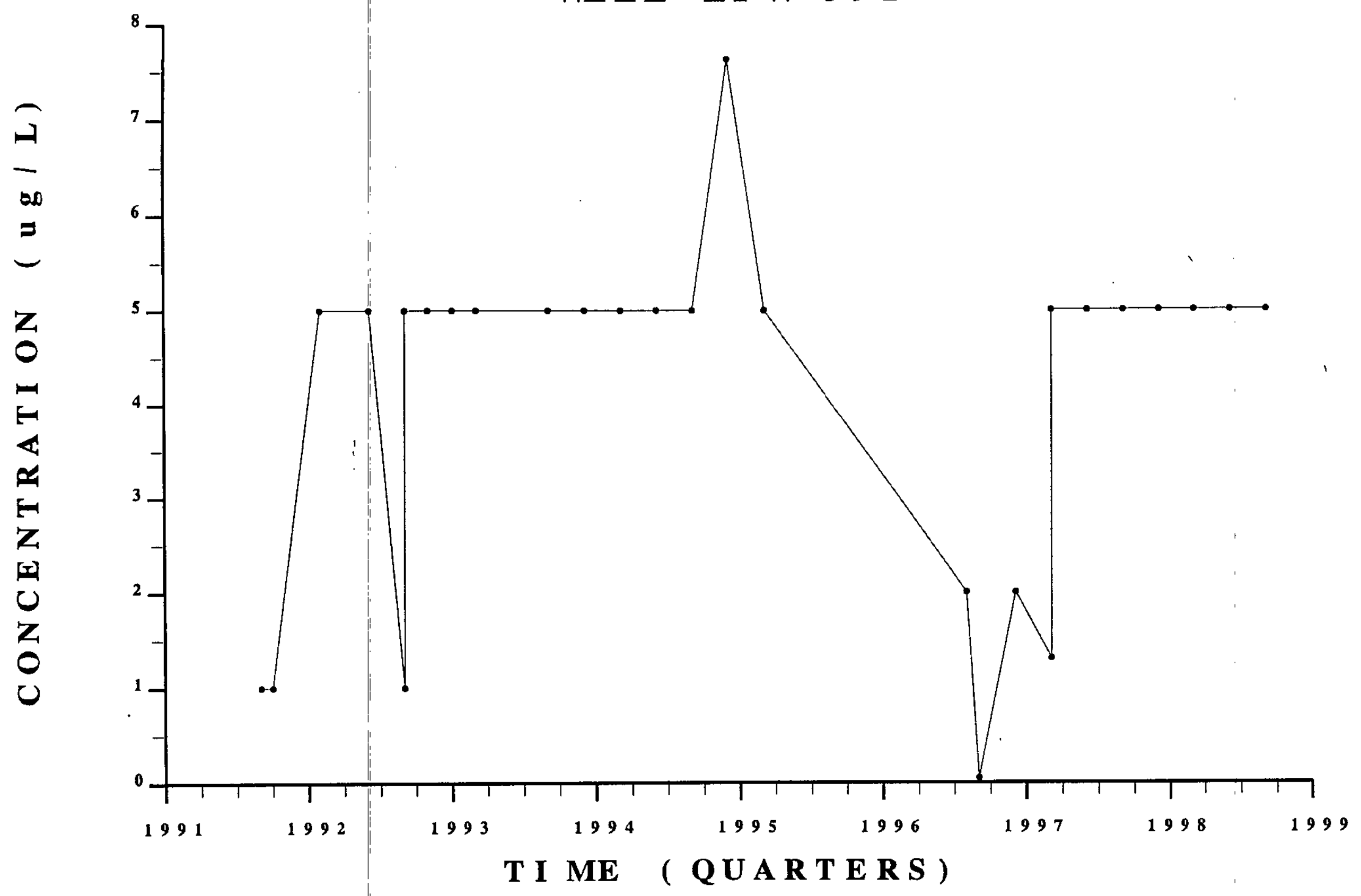

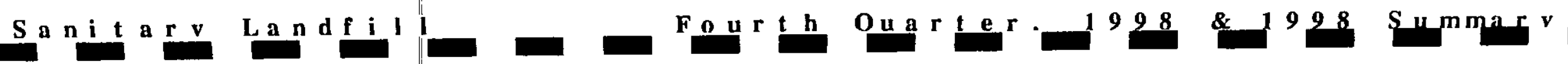


T E T RA CHLOROE THYL E N E WELL LFW 59 D
WS R C - T R - $99-0 \begin{array}{lllll}0 & 0 & 0 & 1\end{array}$ Un c l a s s i f i e d

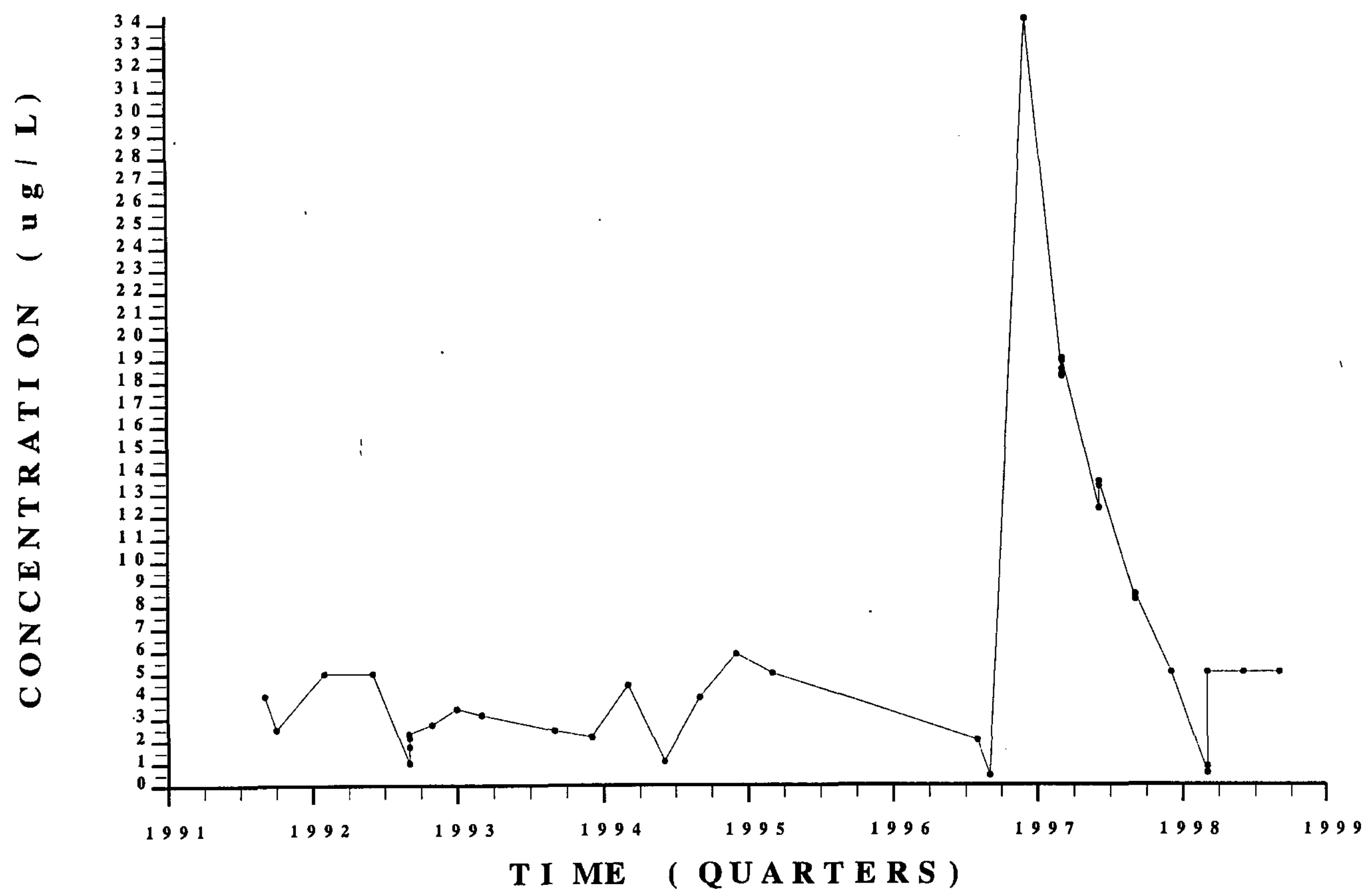

S a n i t a r v La ndf i l l
Fourth Ouarter. $1998 \quad \& \quad 1998$ Summar 


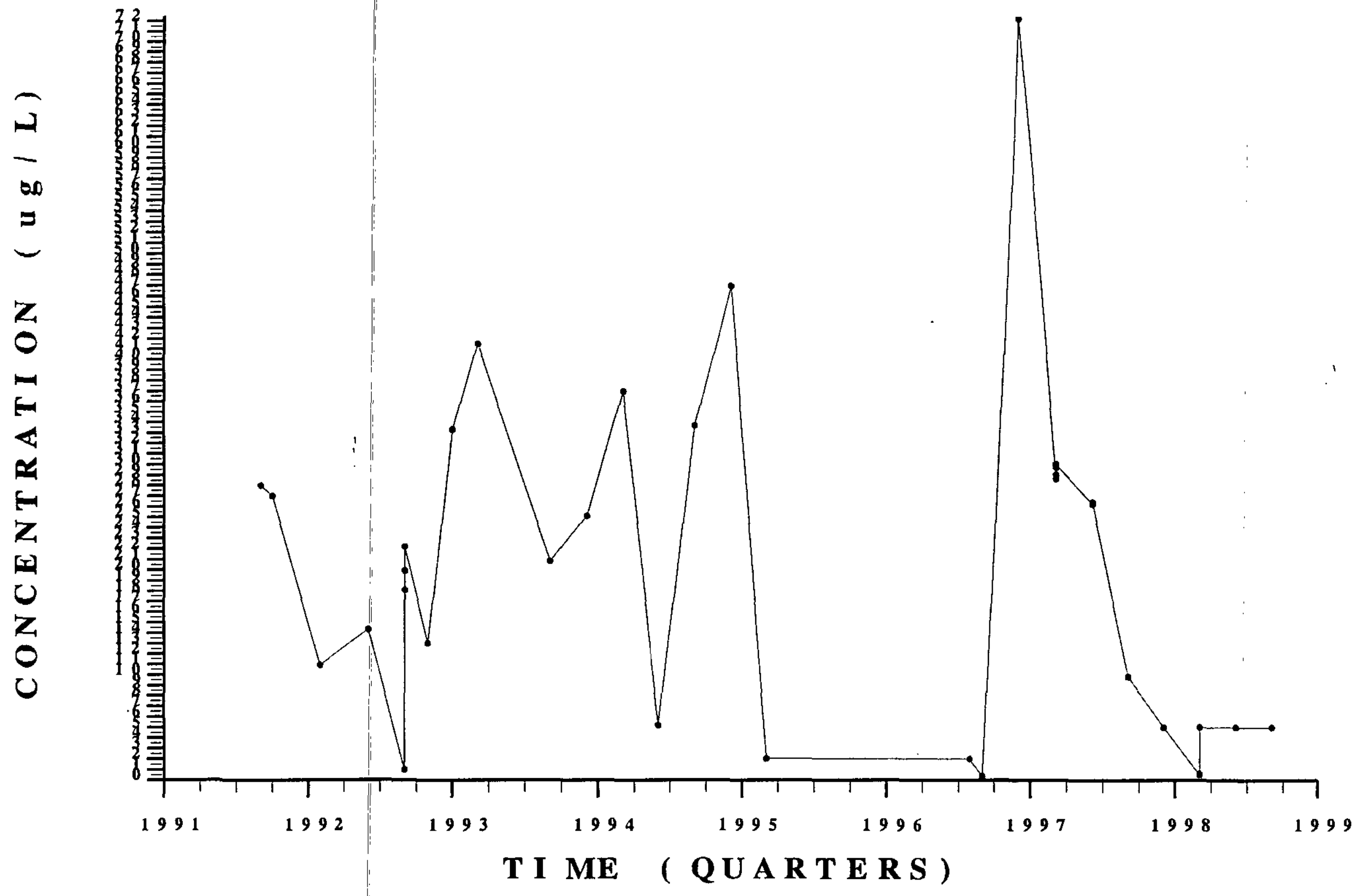


TRI CHL OROF L UOROME THANE WELL LFW 59 D
WS R C - T R - $99-000011$ Unclass i fi ed

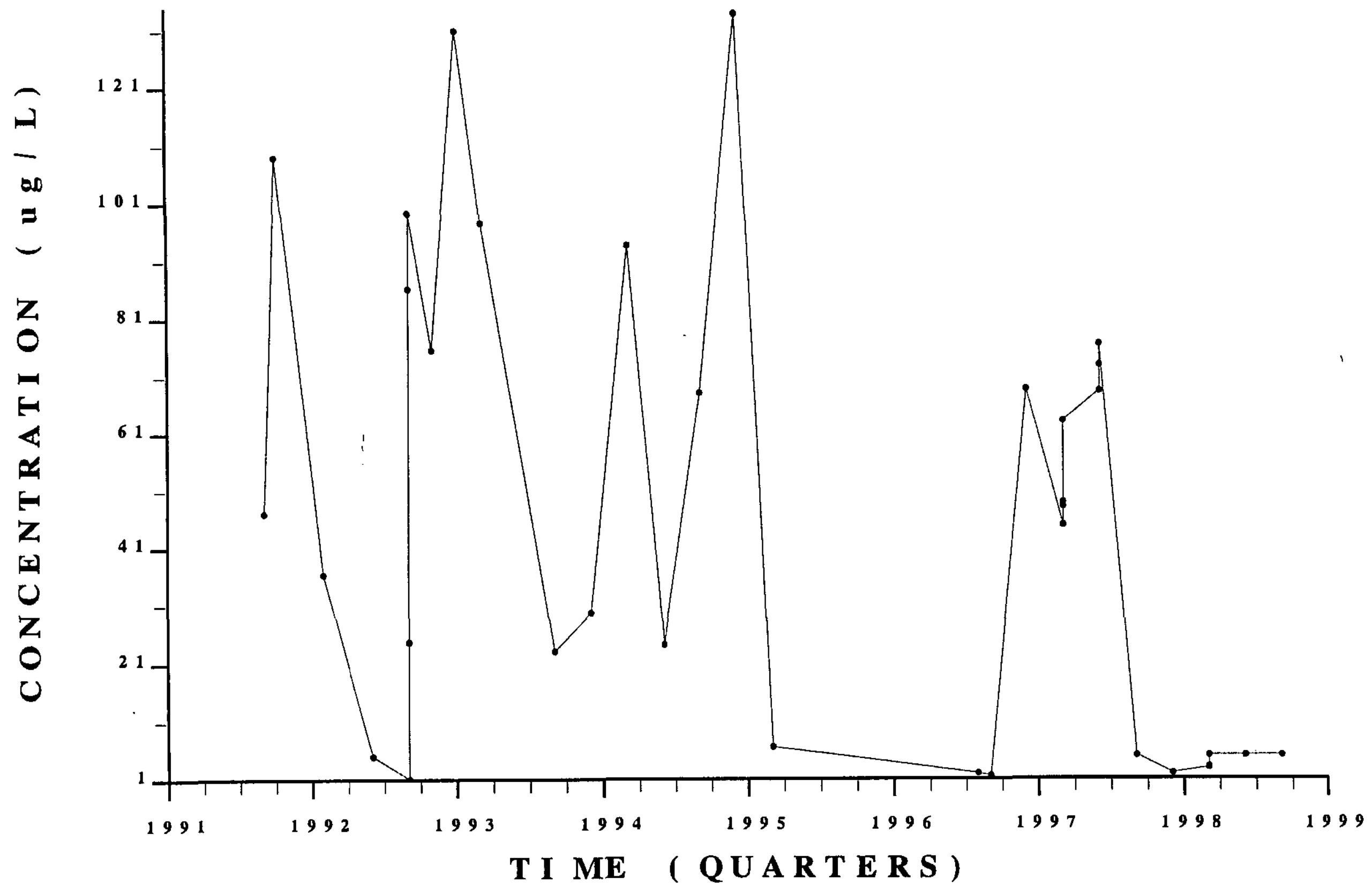

Sanitar Landfill

Fourth Ou a $r$ e $r$.

1998 \& 1998 S ummar 


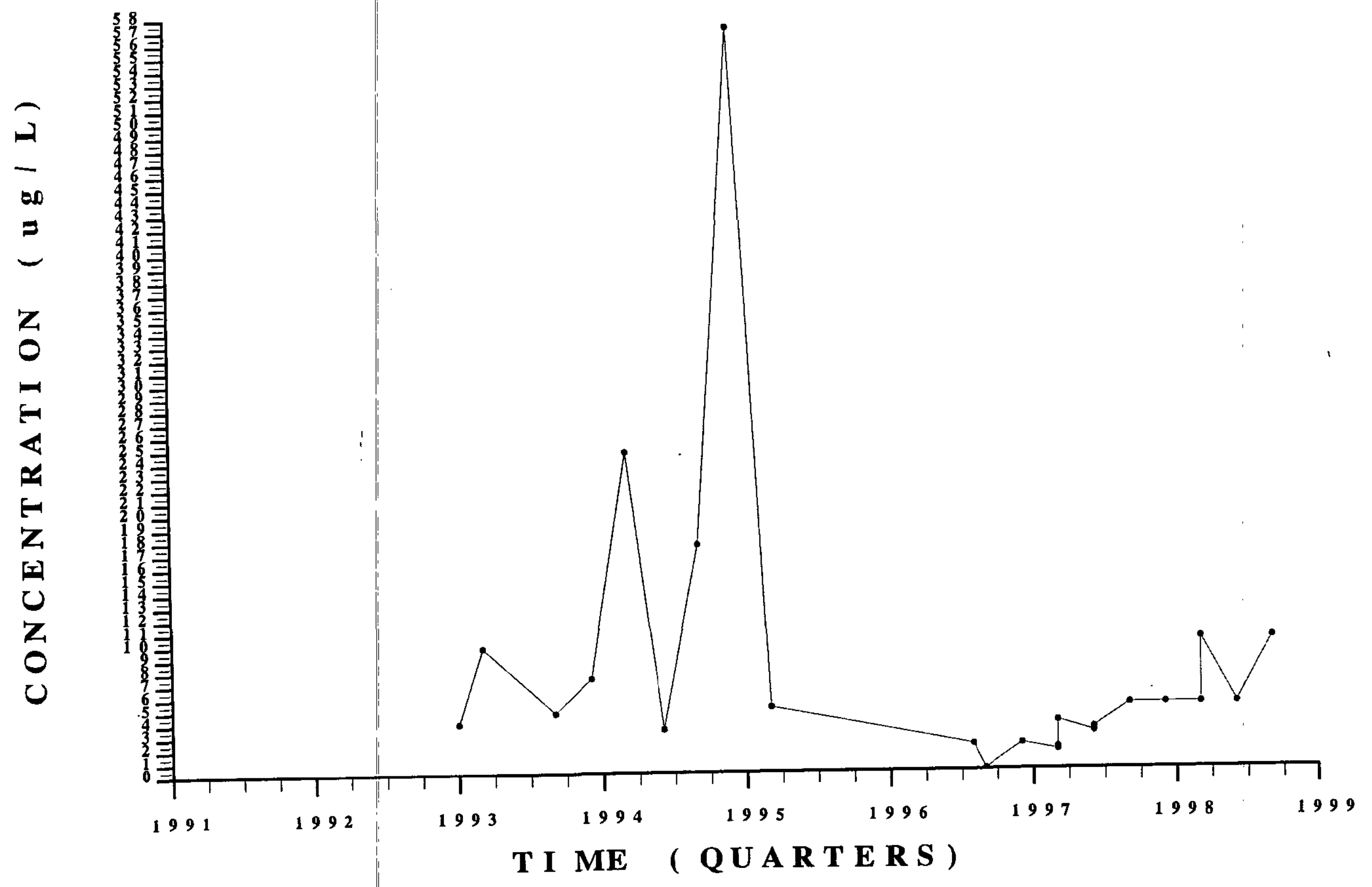

- 


\section{$1,1,1$ - T R I CHLOROE THANE} WELL LFW $61 \mathrm{C}$

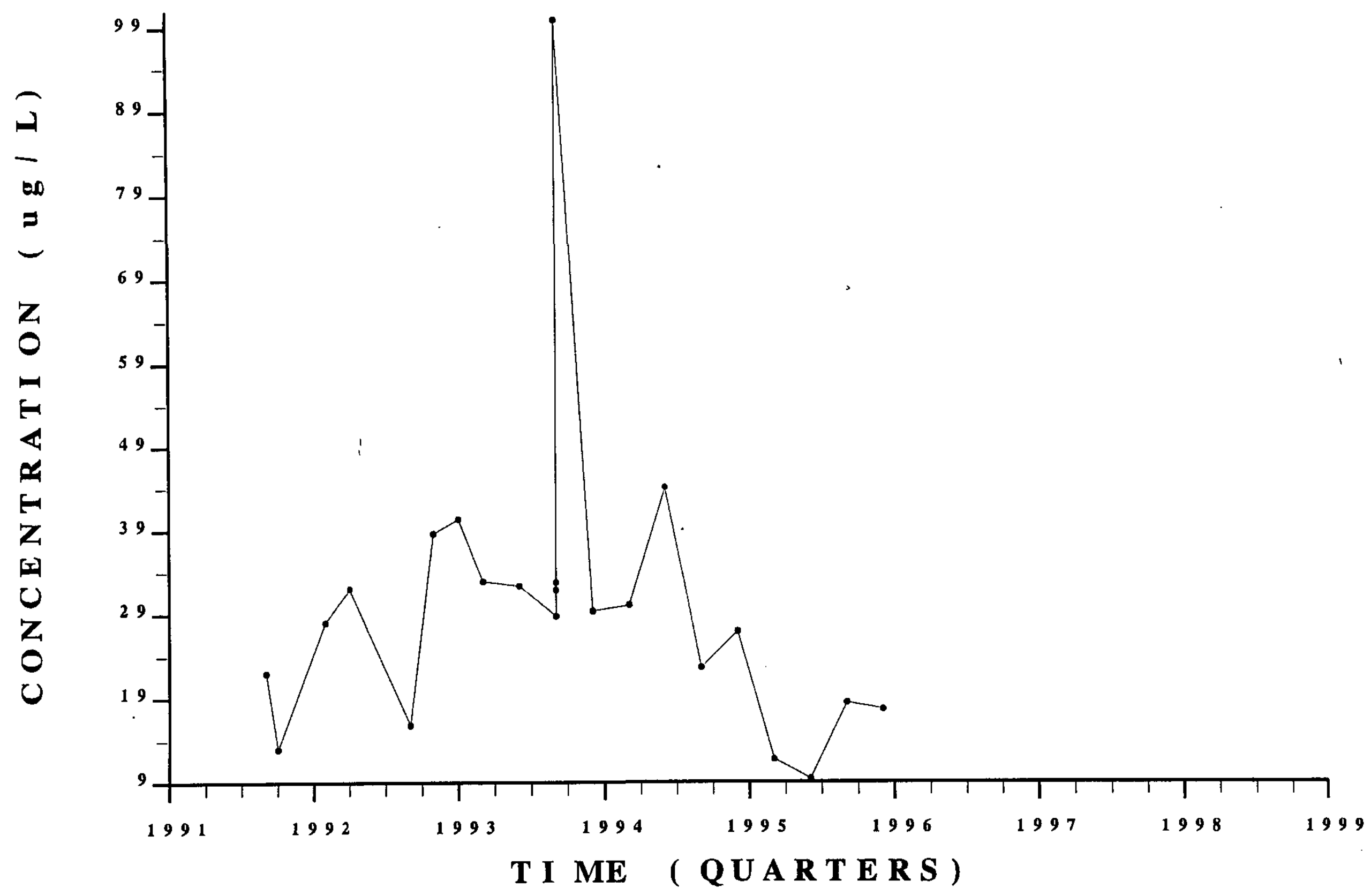

Fourth ouarter.

1998 


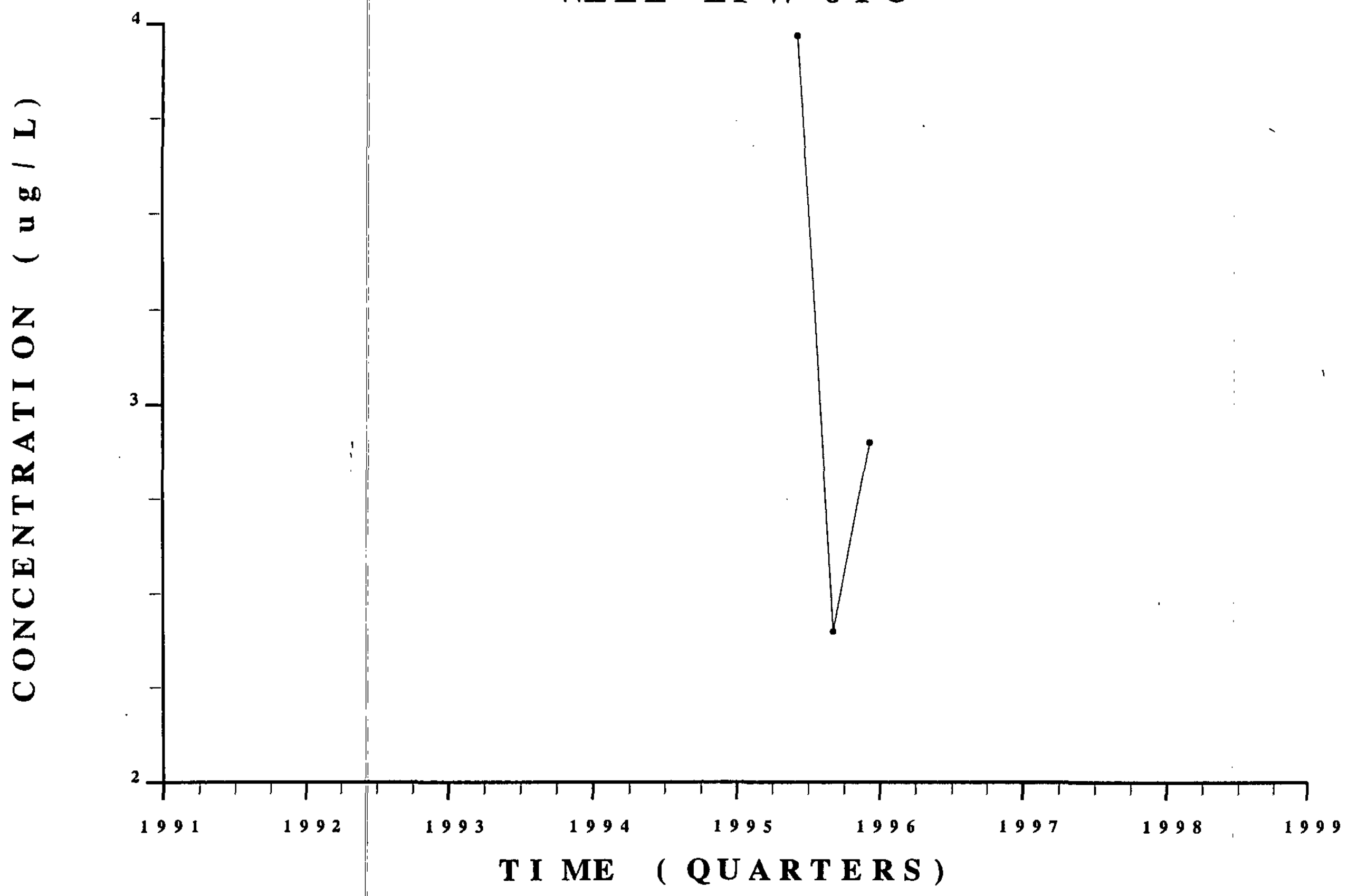

1, 4 - D I C HL ORO B E N Z E N E WELL LFW $61 \mathrm{C}$

WS R C - TR - 99-000011 Unc l a s s i f i ed

San i t a r v L a n d f i l Four $r$ Ou a r t e r. 


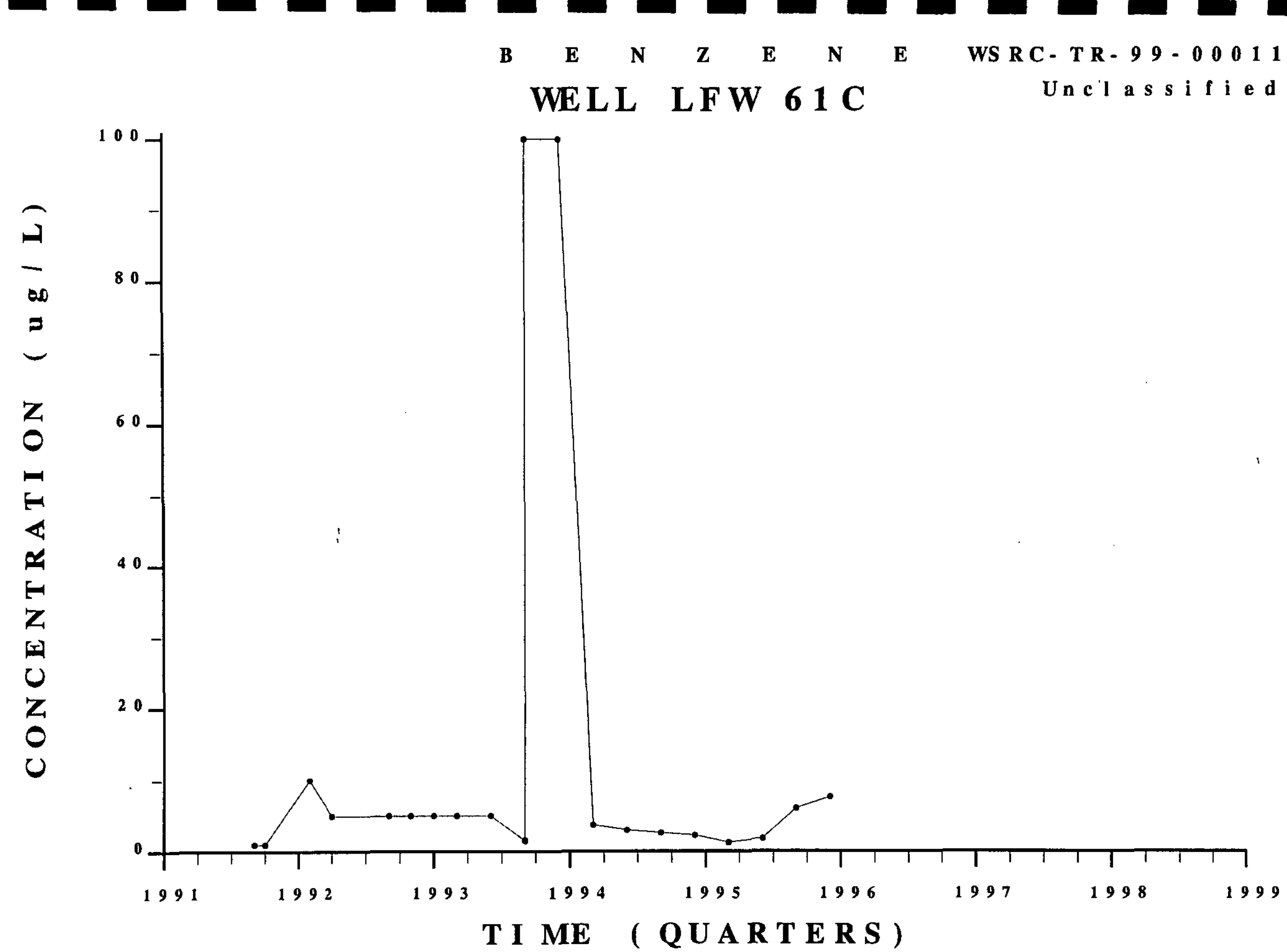




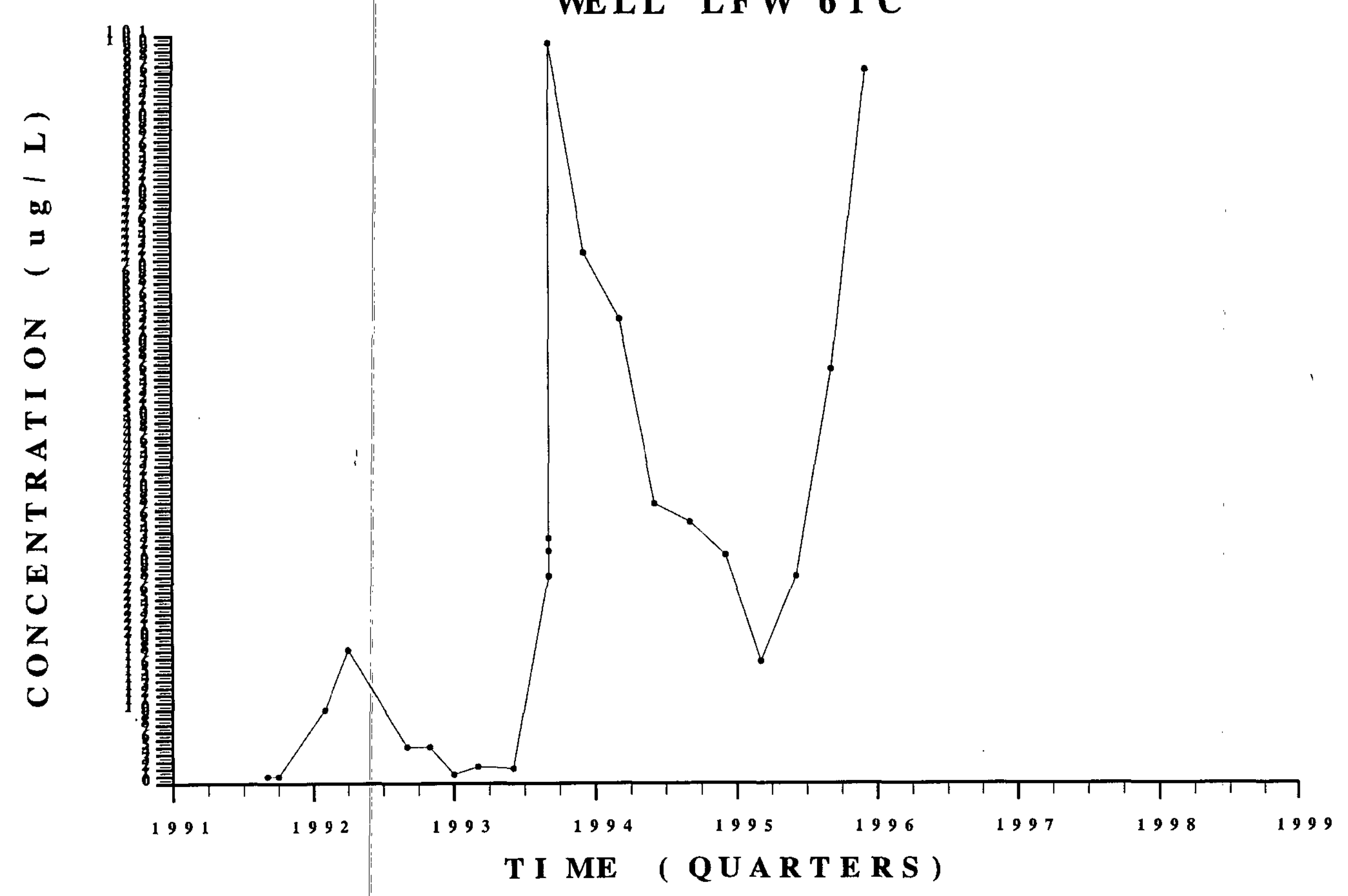

Sanitary Land f i l l

Fourth Ouarter.

1998

\& 1998

S u m ma $\mathbf{v}$ 


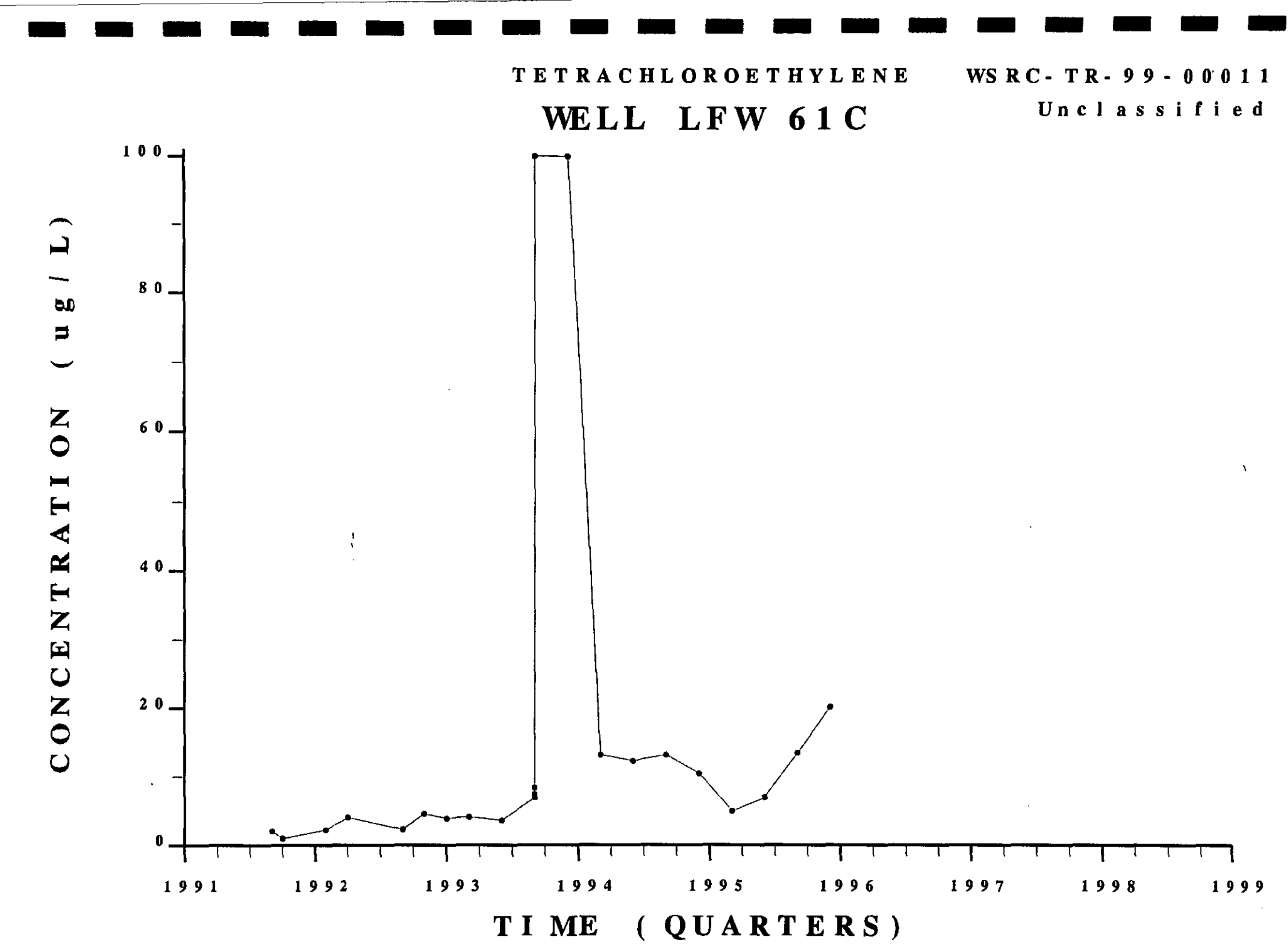

\section{WELL LFW 61 C}

Sanitar $r$ Landf i I l

Fourth Ouarter. 1998 \& 1998 Summar 


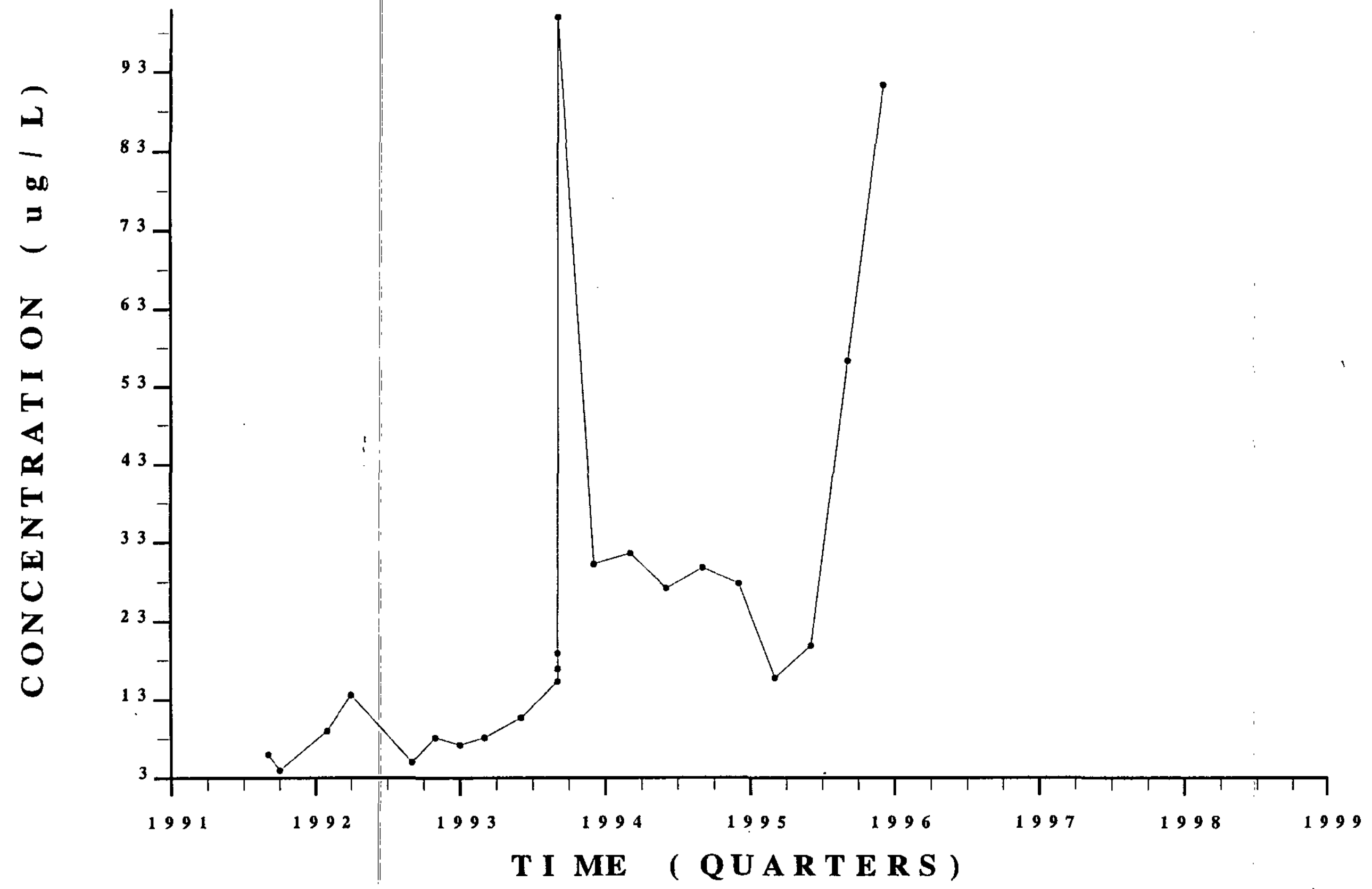

Sanitarv Landfili 
T RI CHL OROF LUOROME THANE WELL LFW $61 \mathrm{C}$
WS R C - T R - $99-0 \begin{array}{lllll}0 & 0 & 1 & 1\end{array}$ Unc l a s s i f i e d

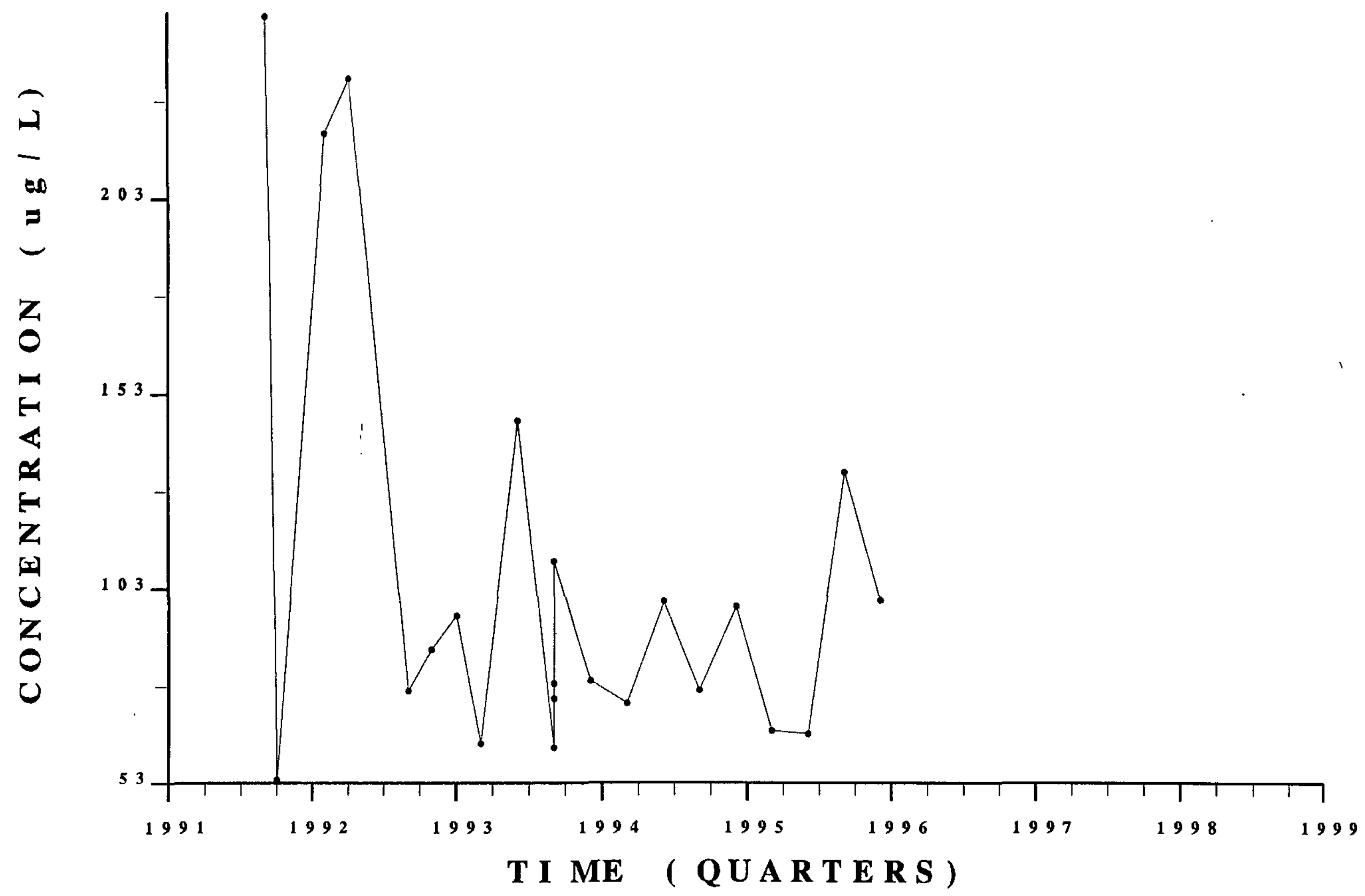

S a n i t a r v La n d f i l l Fourt h Ouarter. 1998 \& 1998 S и mmar 


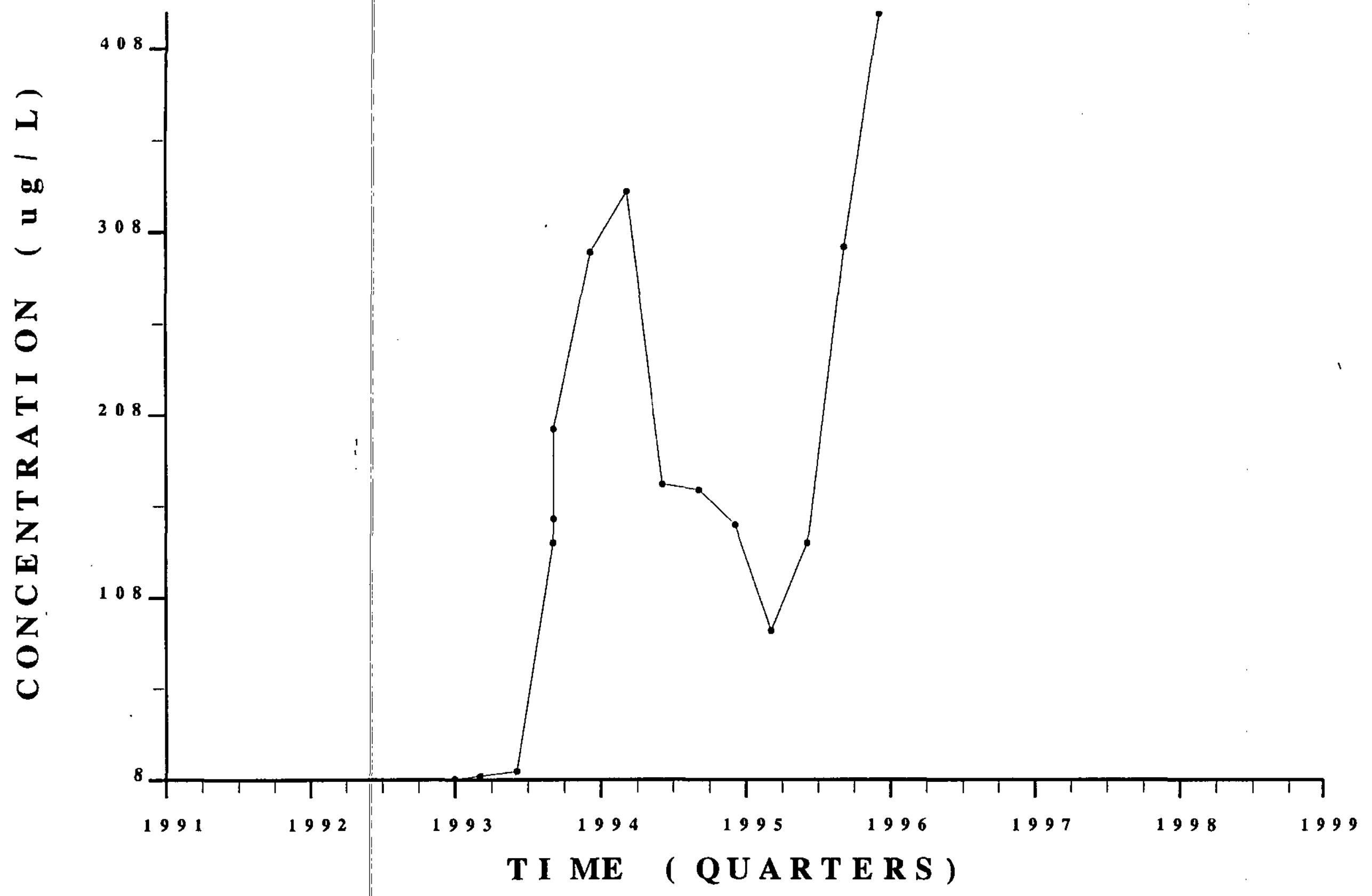

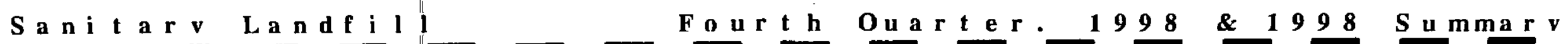




\section{$1,1,1-T R I$ C HL OROE THANE}

WELL LFW 61 D

WS R C - T R - $99-00011$

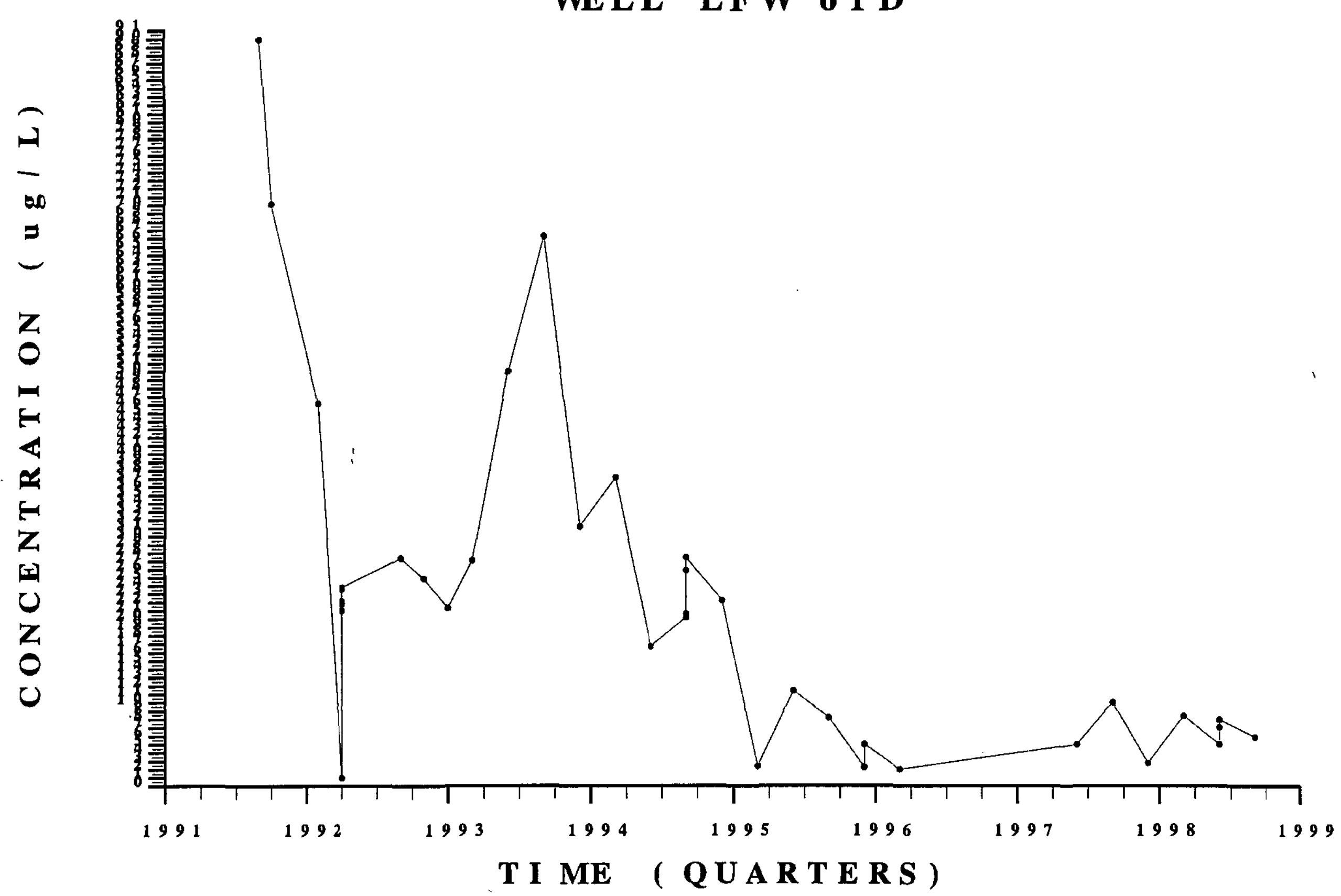

San i t a r v Landf i l I Fourth Ouarter. 1998 \& 1998 Summarv 


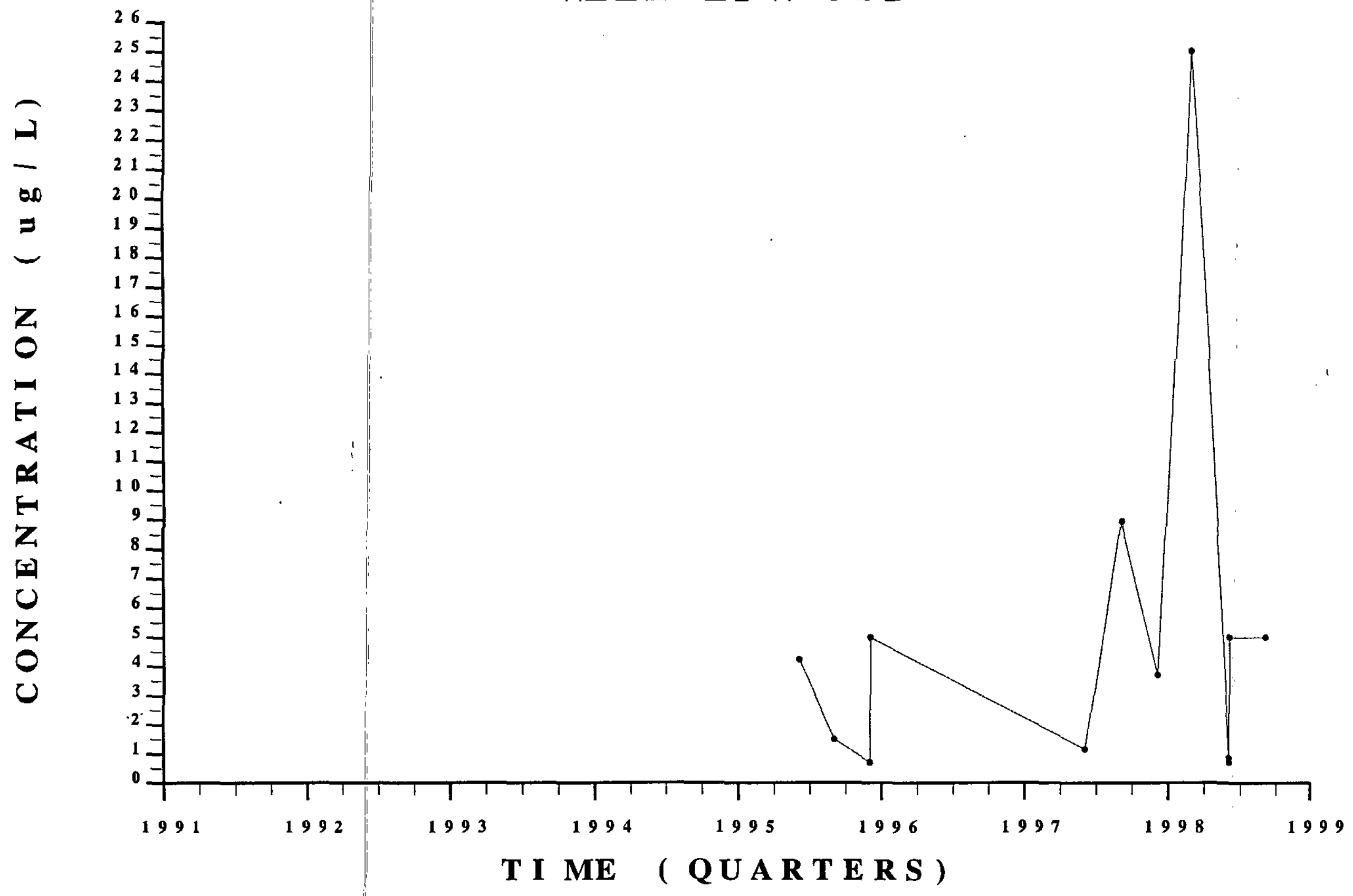

, 4 - D I C HLOROB E NZ E NE WS R C - T R- $99-0$ - 001011 WELL LFW 61 D Unc I a s s i f i e d

Sanit ary Landfi 


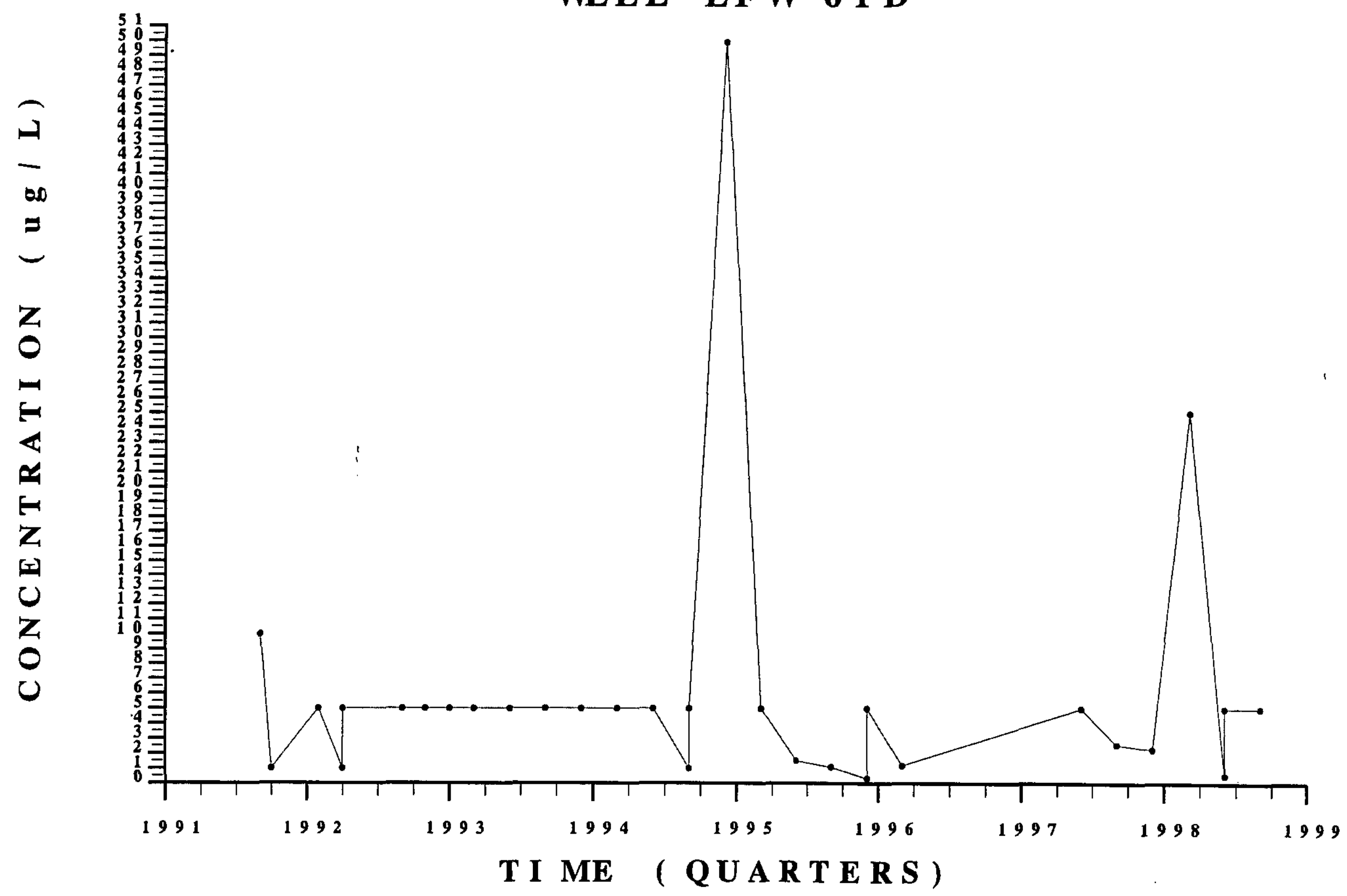

Sanitar $r$ Landfill

Fourth Ouarter. 1998 \& 1998 Summar 





WELL LFW 61 D

Unc l a s s i f i e d

T I ME ( Q U A R T R R S )

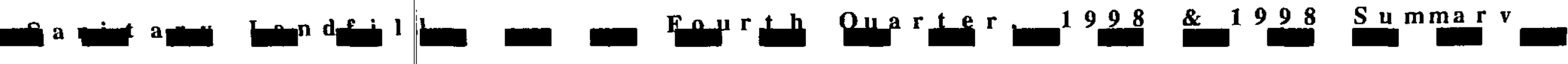




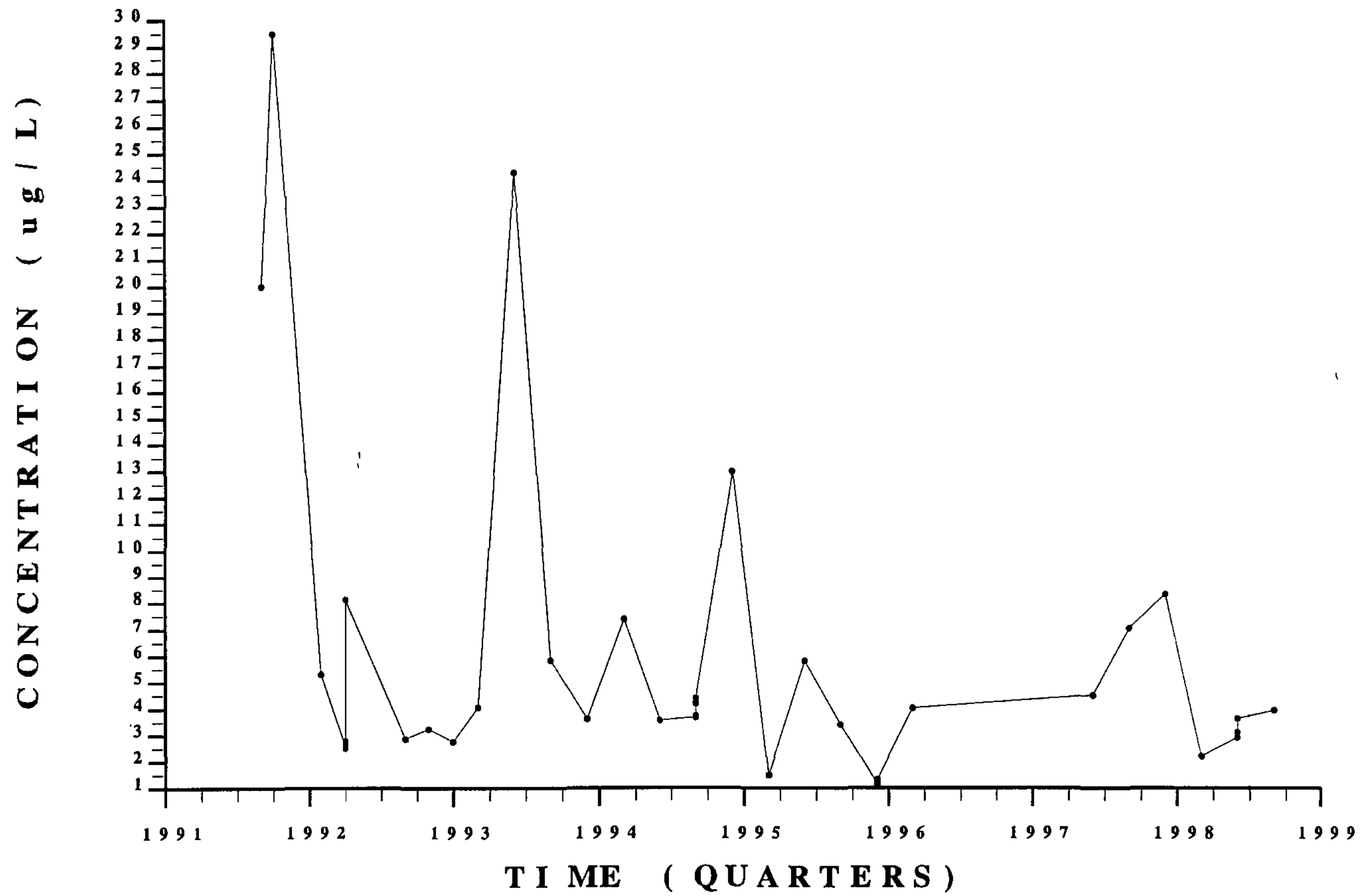

S a n i t a r v L a n d f i I l

Fourth Ouarter 


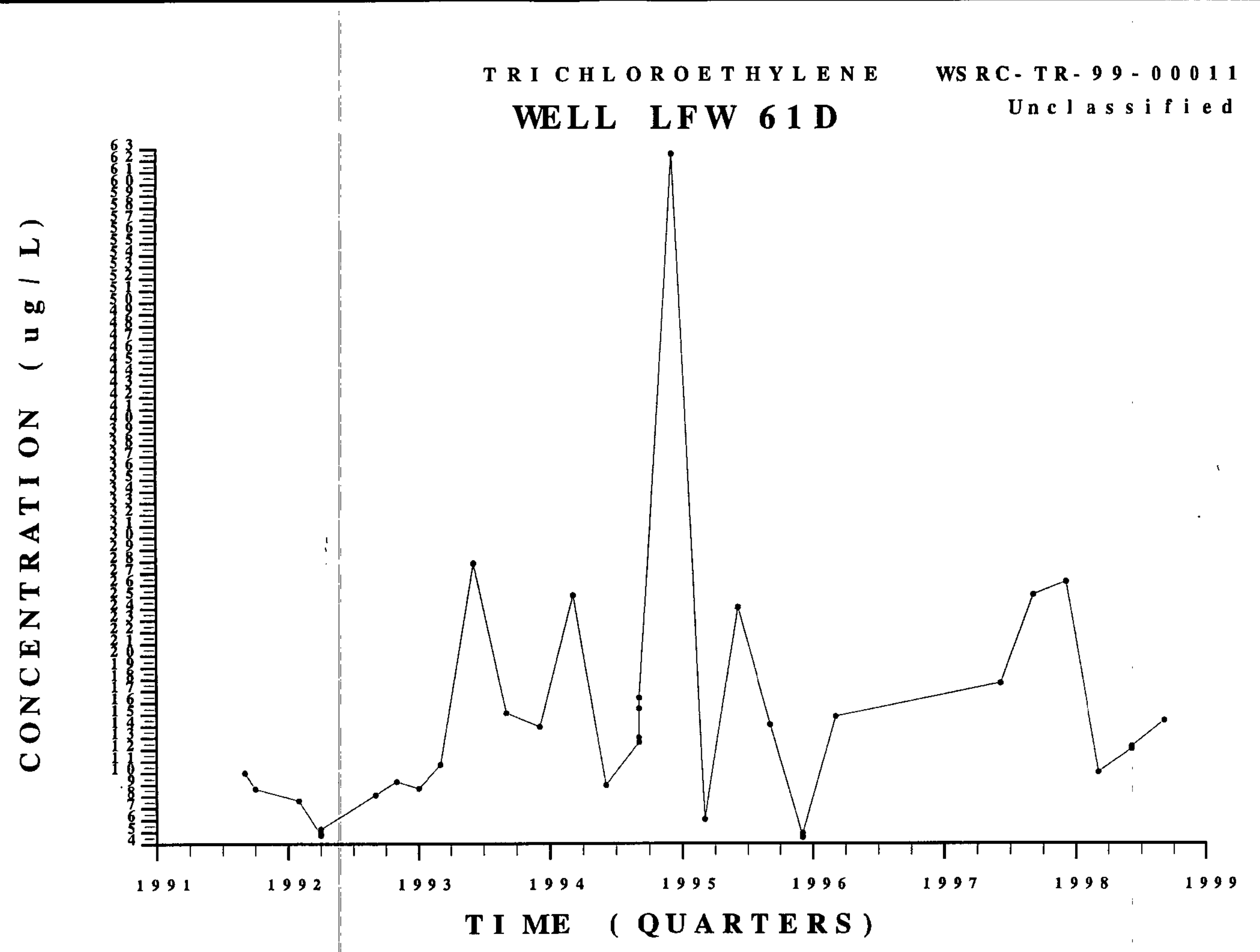


TRI CHLOROF LUOROME THANE WELL LFW 61 D
WS R C - T R - $99-00011$ Unc l a s s i f i ed

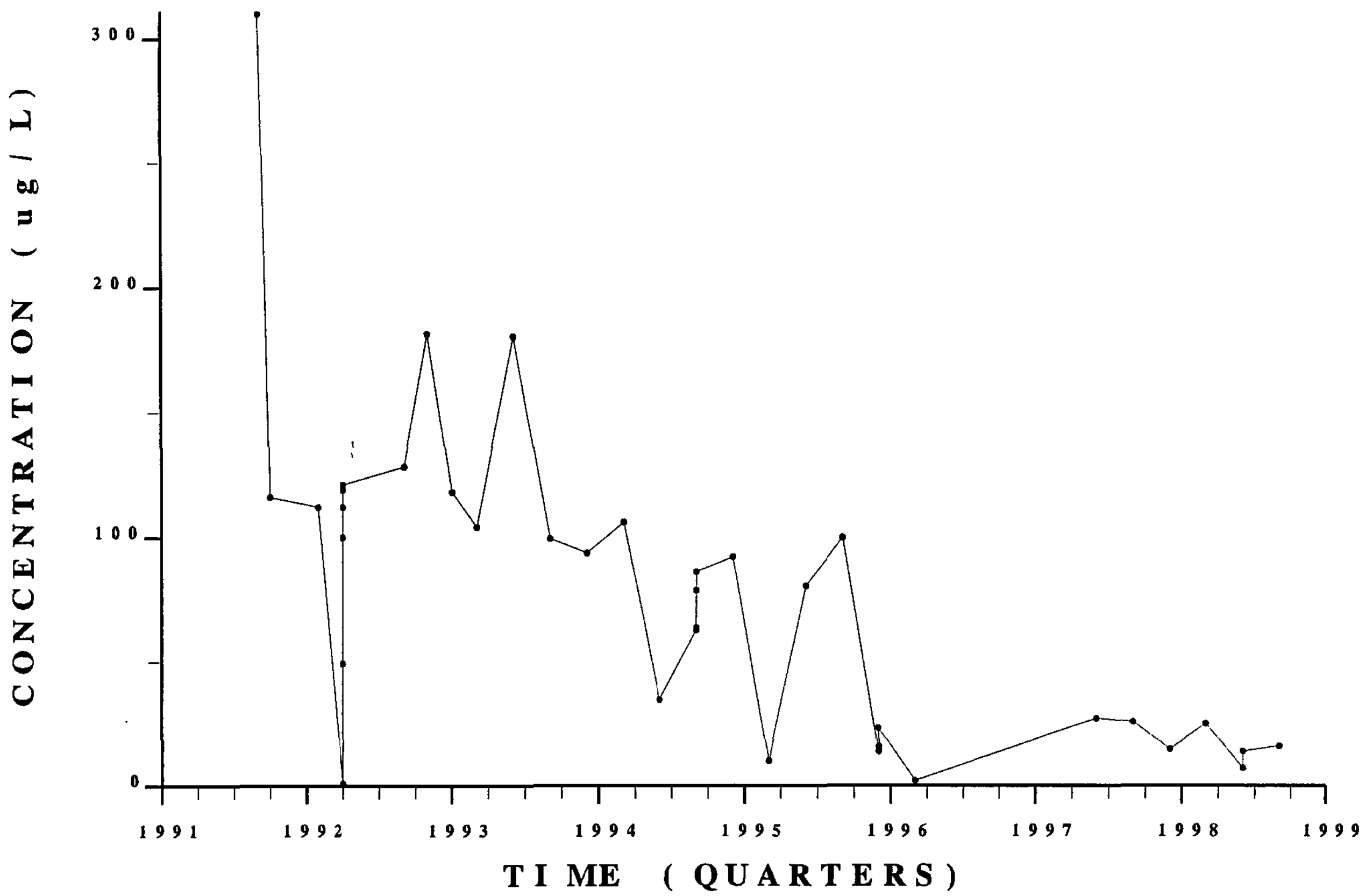




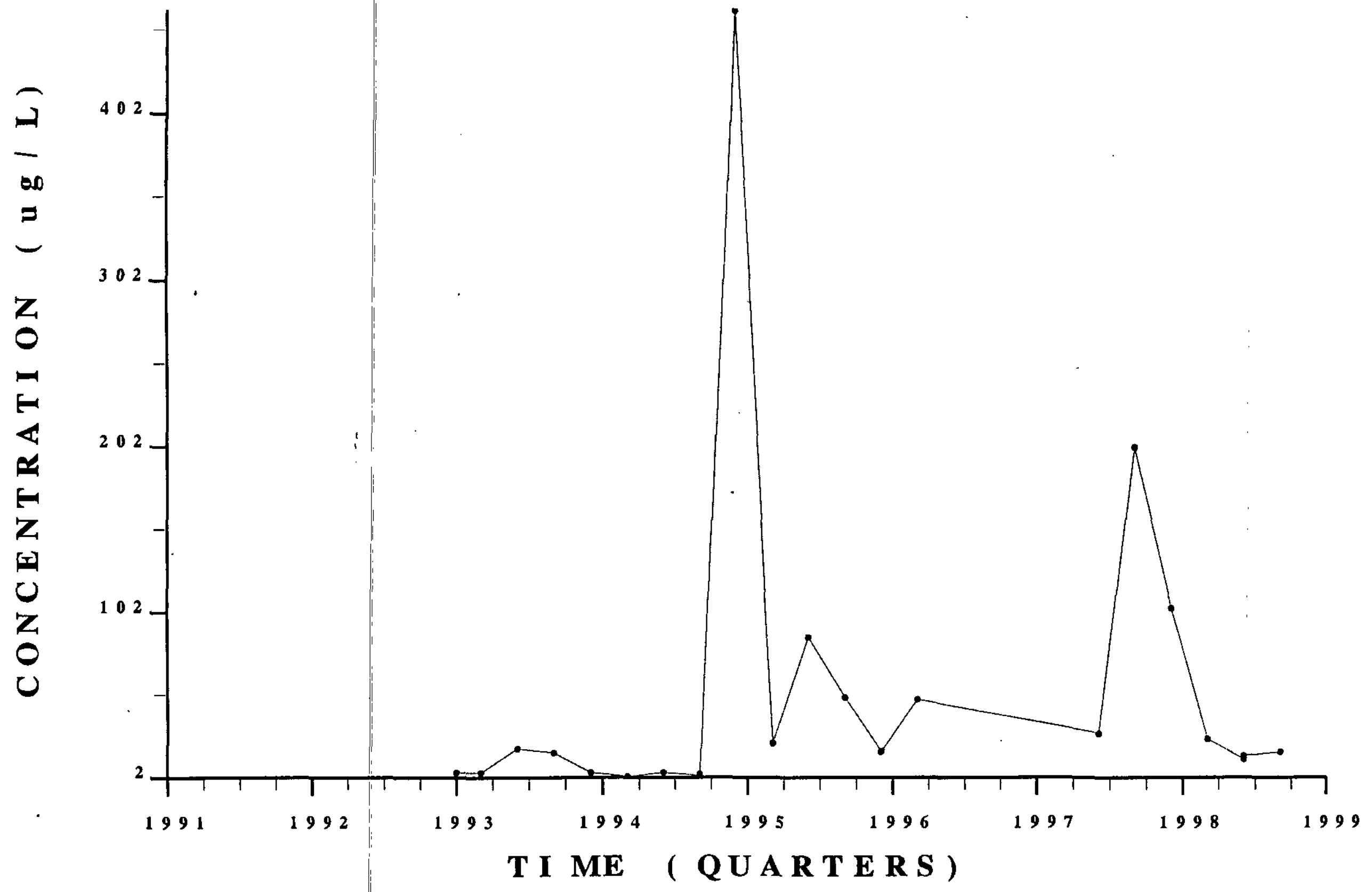

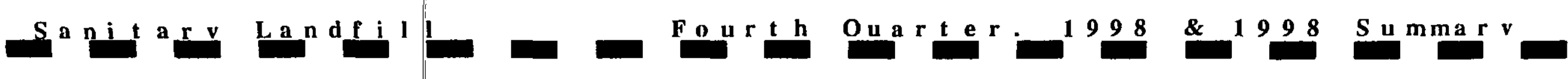


WSRC-TR-99-00011

Unclassified

\section{Appendix G}

\section{Hydrographs}


WSRC-TR-99-00011

Unclassified

THIS PAGE LEFT BLANK INTENTIONALLY. 
H Y D R O G R A P H WE L L L F W 6

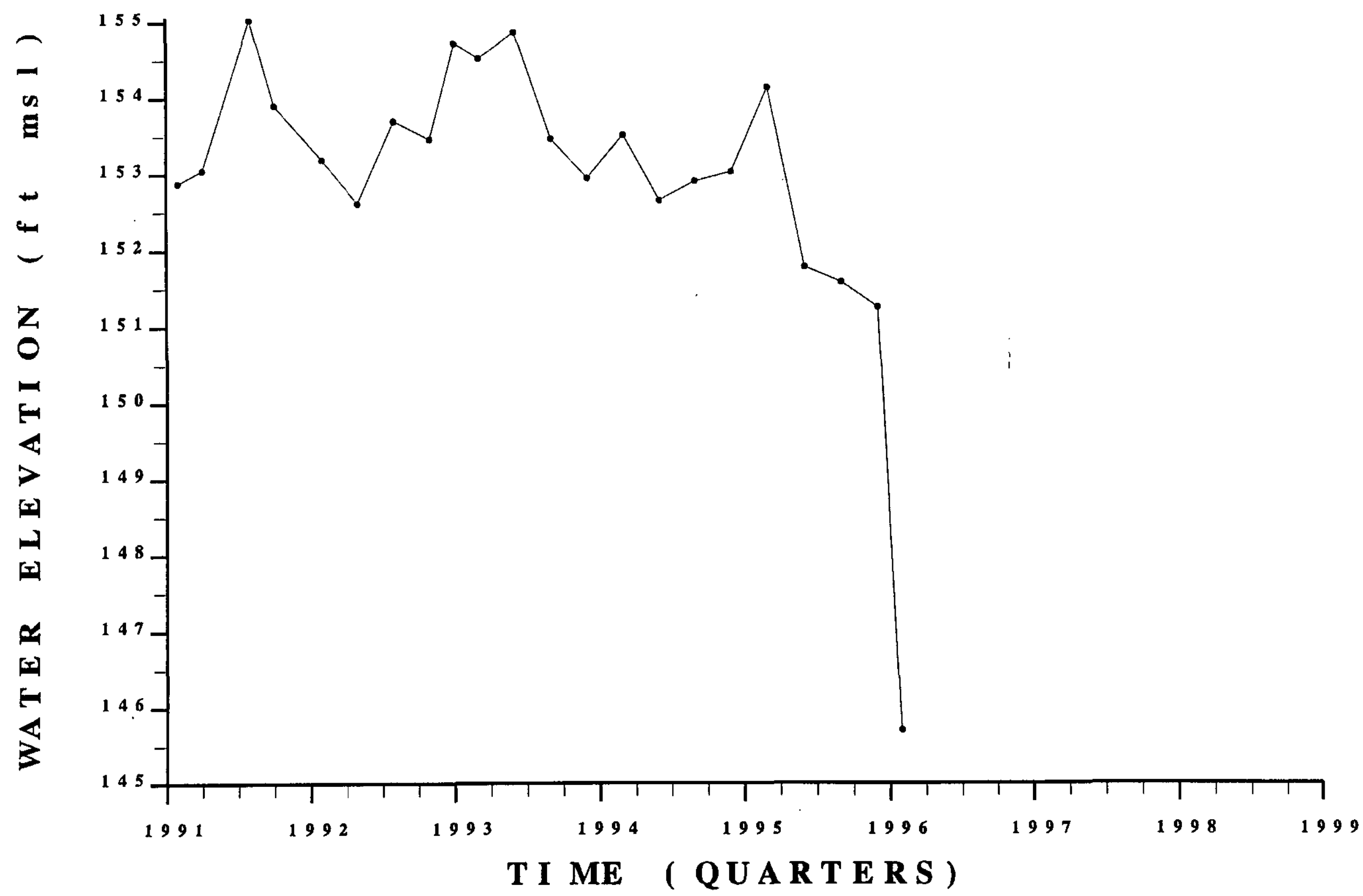

WS R C - T R - $99-\begin{array}{llllll}0 & 0 & 0 & 1 & 1\end{array}$ Unc l a s s i f i ed 


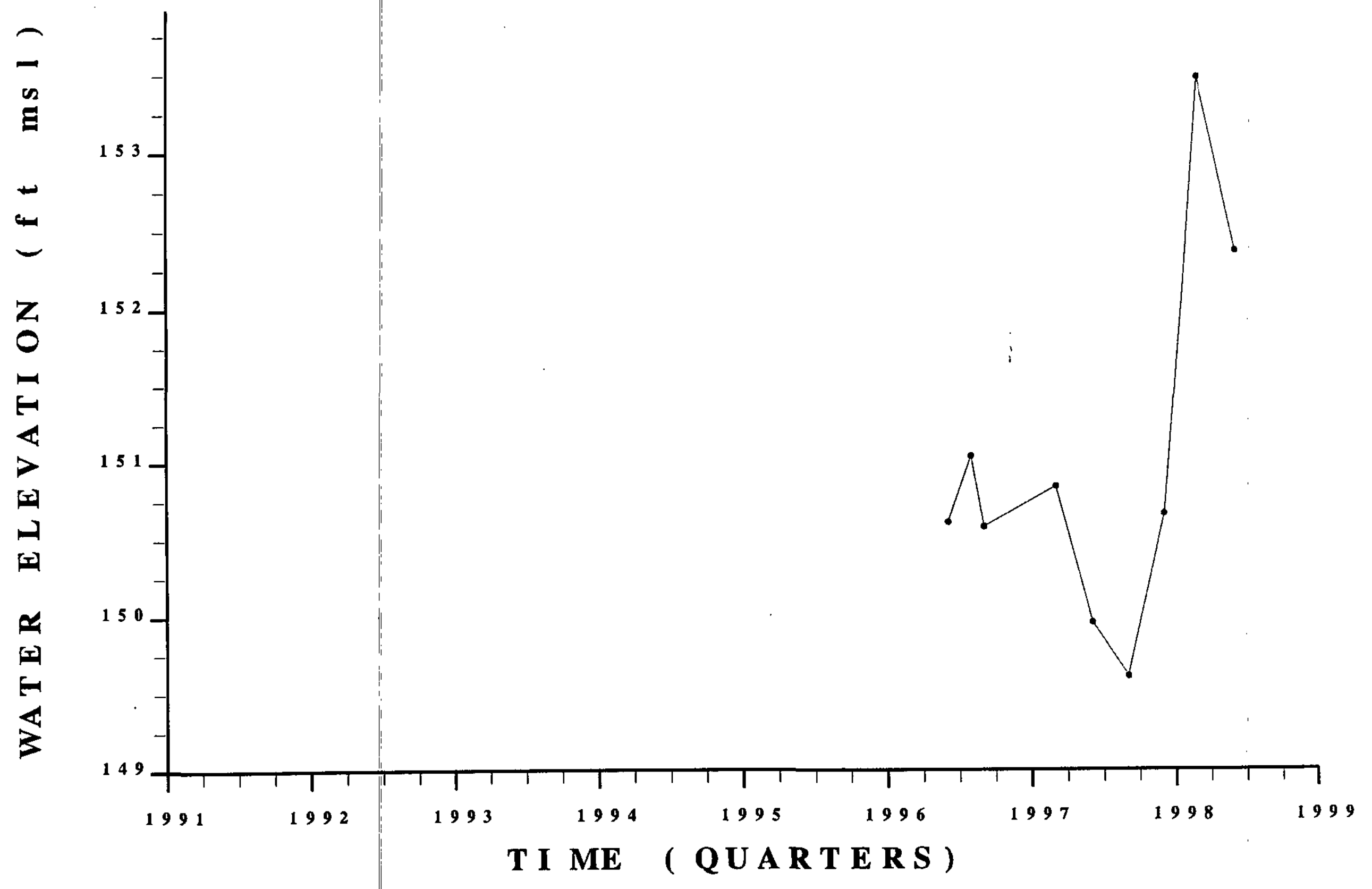

HY D R O G R A P H WELL LFW 6 R
WS R C - T R - 99-0 00011

Unclass if i ed 
H Y D R O G R A P H WE L L L F W 7
WS R C - T R - $99-00011$ Unc l a s s i f i ed

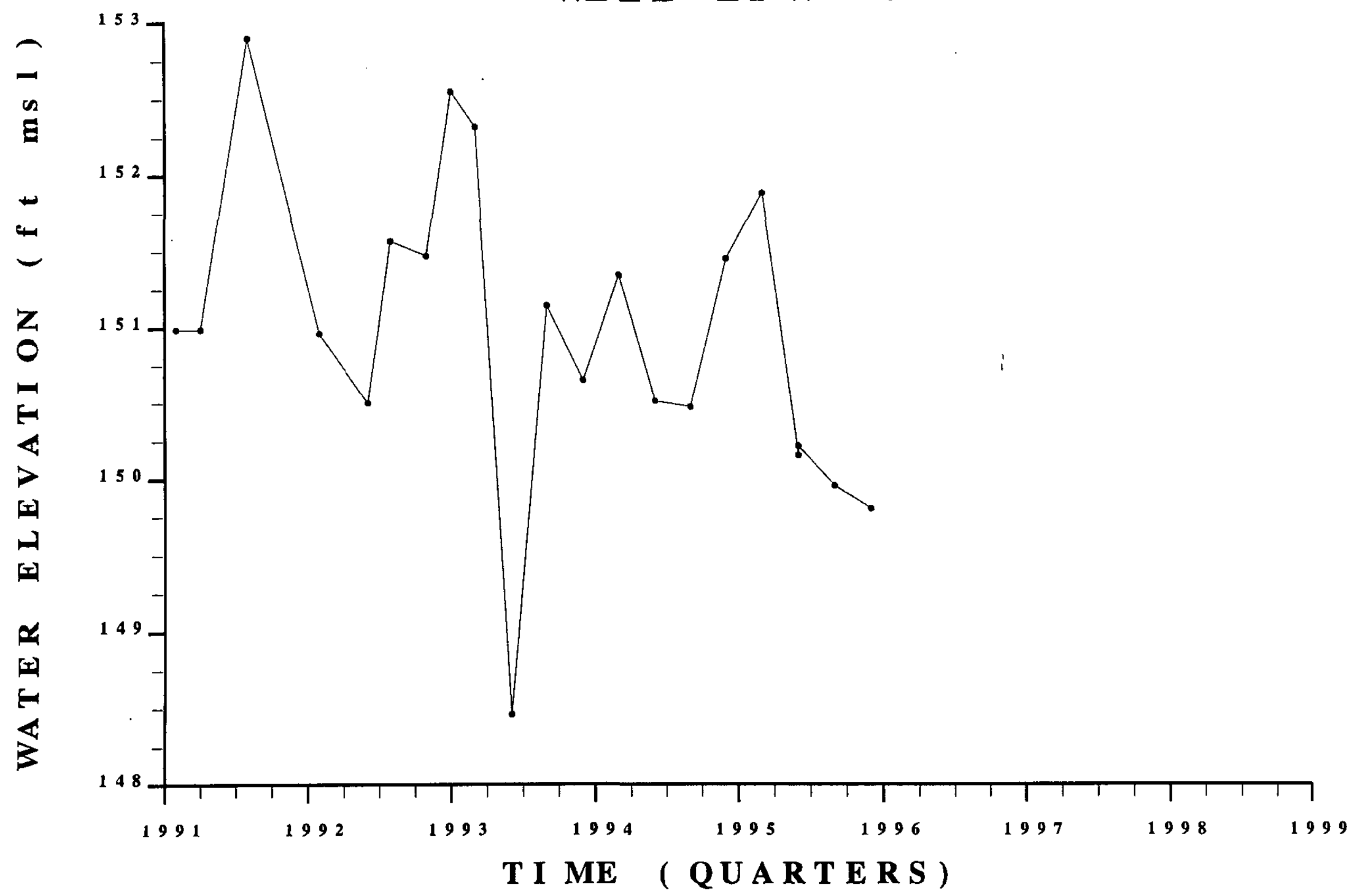

S a n i t a r v L a n d f i l l

Fourth Ouarter. 1998 \& 1998 Summarv 
HY D R O G R A P H WE L L L F W 8
WS R C - T R - $99-000011$ Unc l a s s i f i e d




H Y D R O G R A P H WELL LF W 8 R
WS R C - T R - $99-00011$

Un c l a s s i f i e d

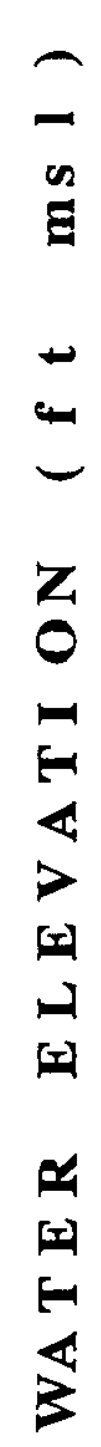$$
-
$$

14

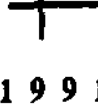
T I ME ( QUARTERS)

Sanitar a landfill

Fourth Ouarter. 1998 \& 1998 Summarv 
$\begin{array}{ll}-1 & 0 \\ 0 & 0 \\ 0 & - \\ 0 & - \\ 1 & 0 \\ 0 & 0 \\ 0 & = \\ 1 & 0 \\ 1 & 5 \\ 0 & 0 \\ 0 & 0 \\ 3 & \end{array}$




HY D R O G R A P H

WE L L L F W 16
WS R C - TR- $99-00011$ Unc l a s s i fi ed

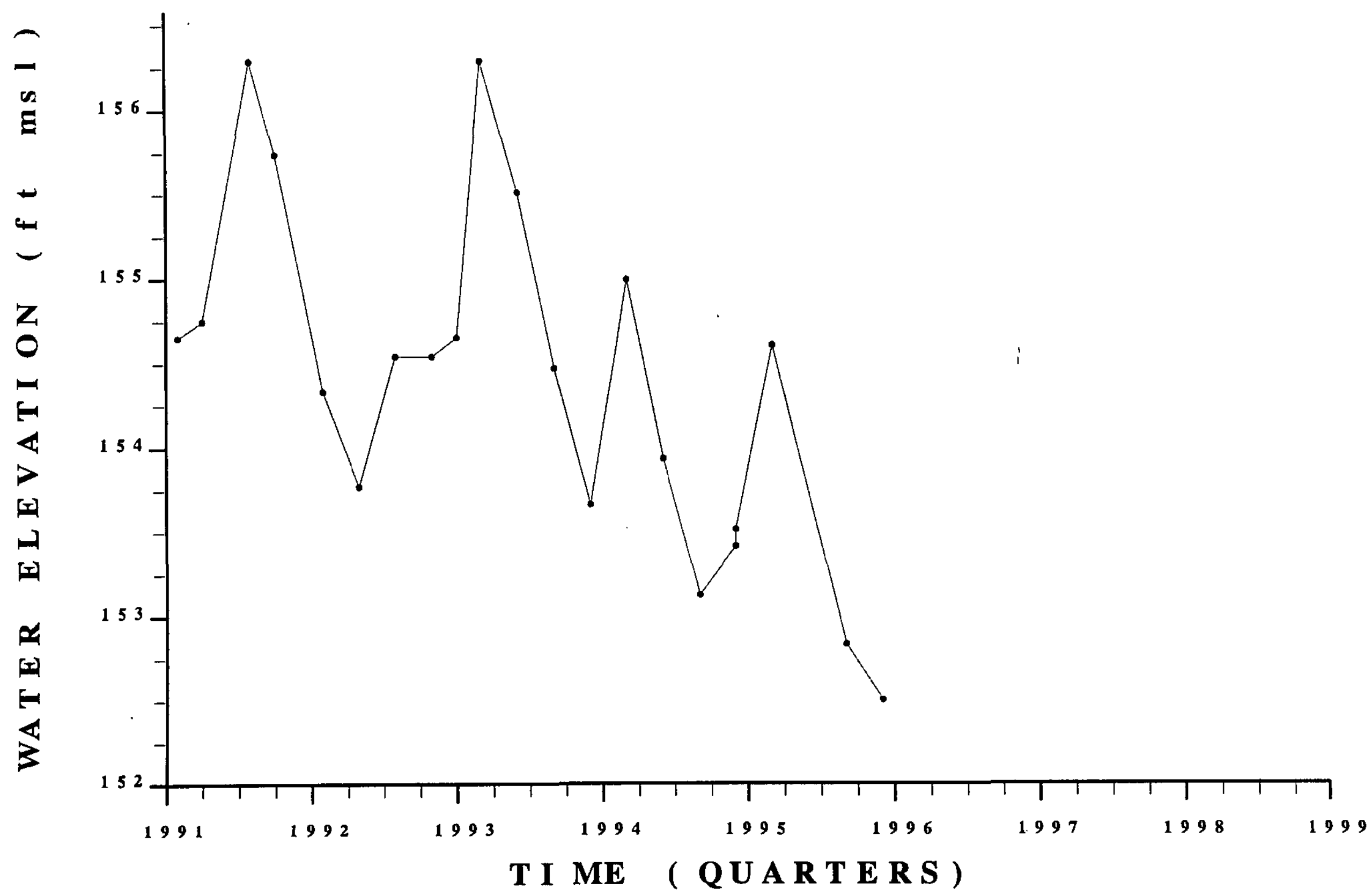

Sanitary Landfil I

Fourth Ouarter. 1998 \& 1998 Summarv 
HY D R O G R A P H

WE L L L F W 17
WS R C - T R - $99-000011$ Unc l a s s i f i e d

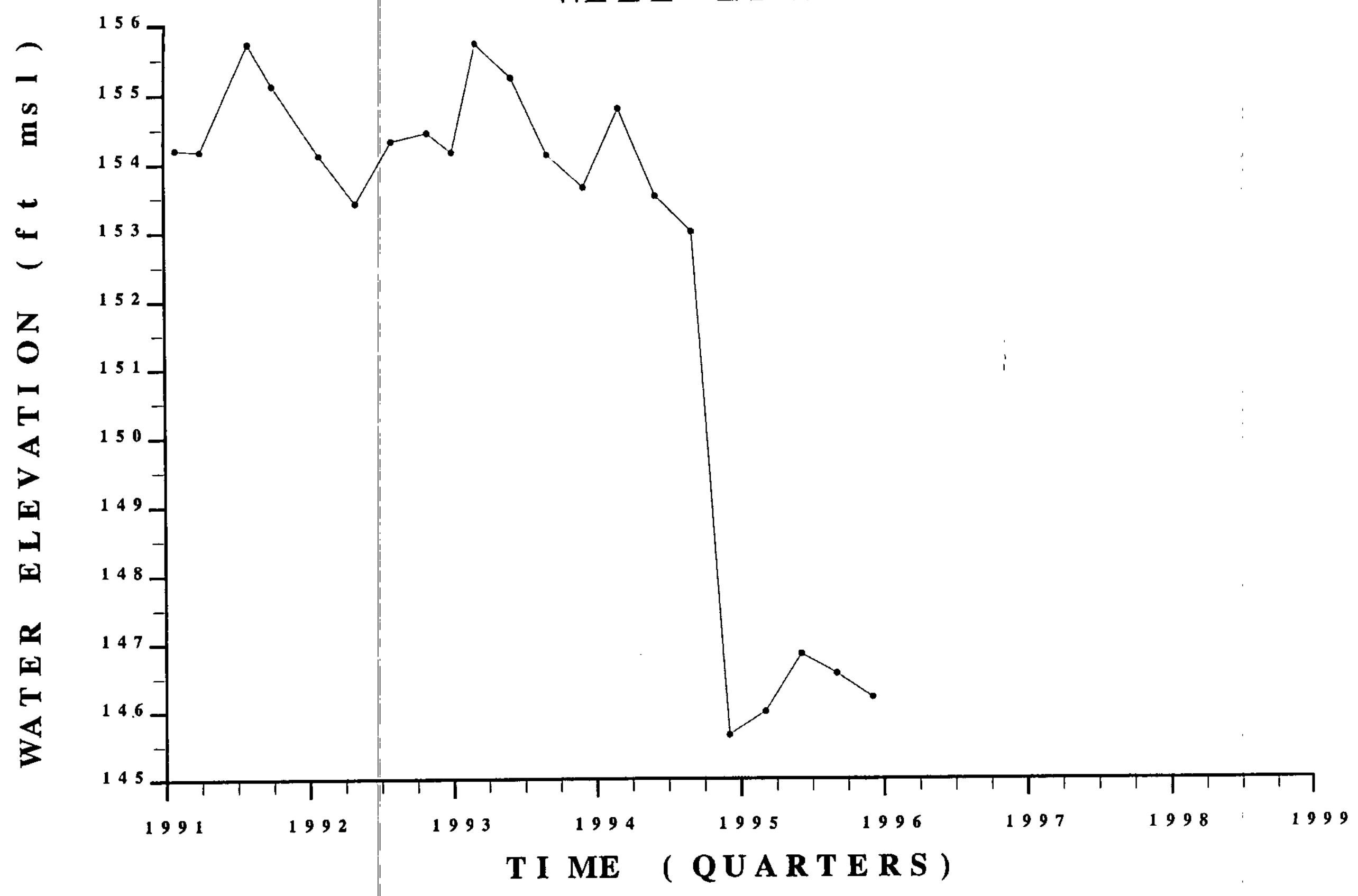


H Y D R O G R A P H WE L L L F W 18

WS R C - T R - $99-000011$ Un c l a s s i f i e d

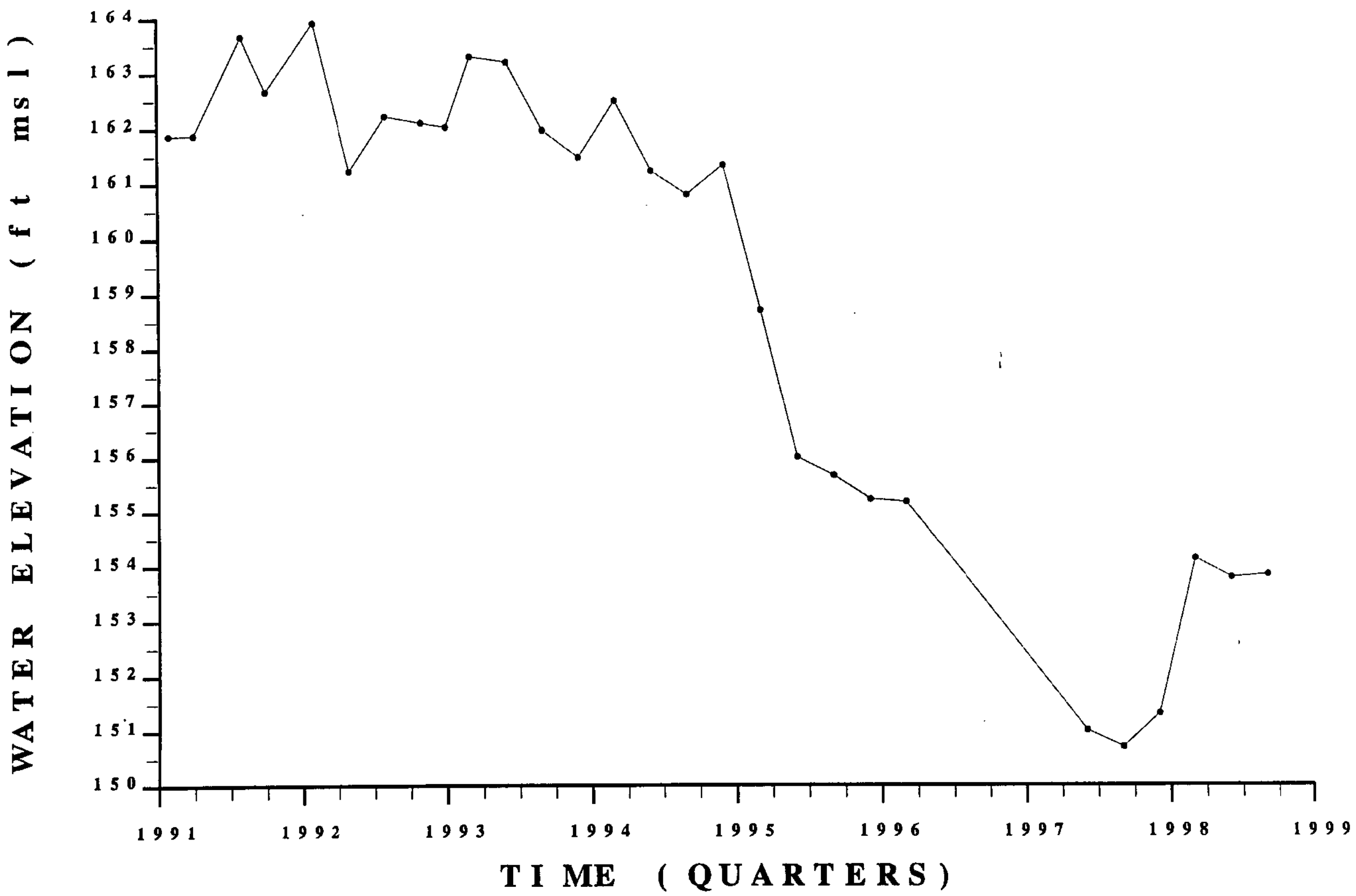

S a n i t a r v L a n d f i l l

Fo urth Ouarter. 199

\& 1998 S u mar v 
H Y D R O G R A P H WE L L L F W 19

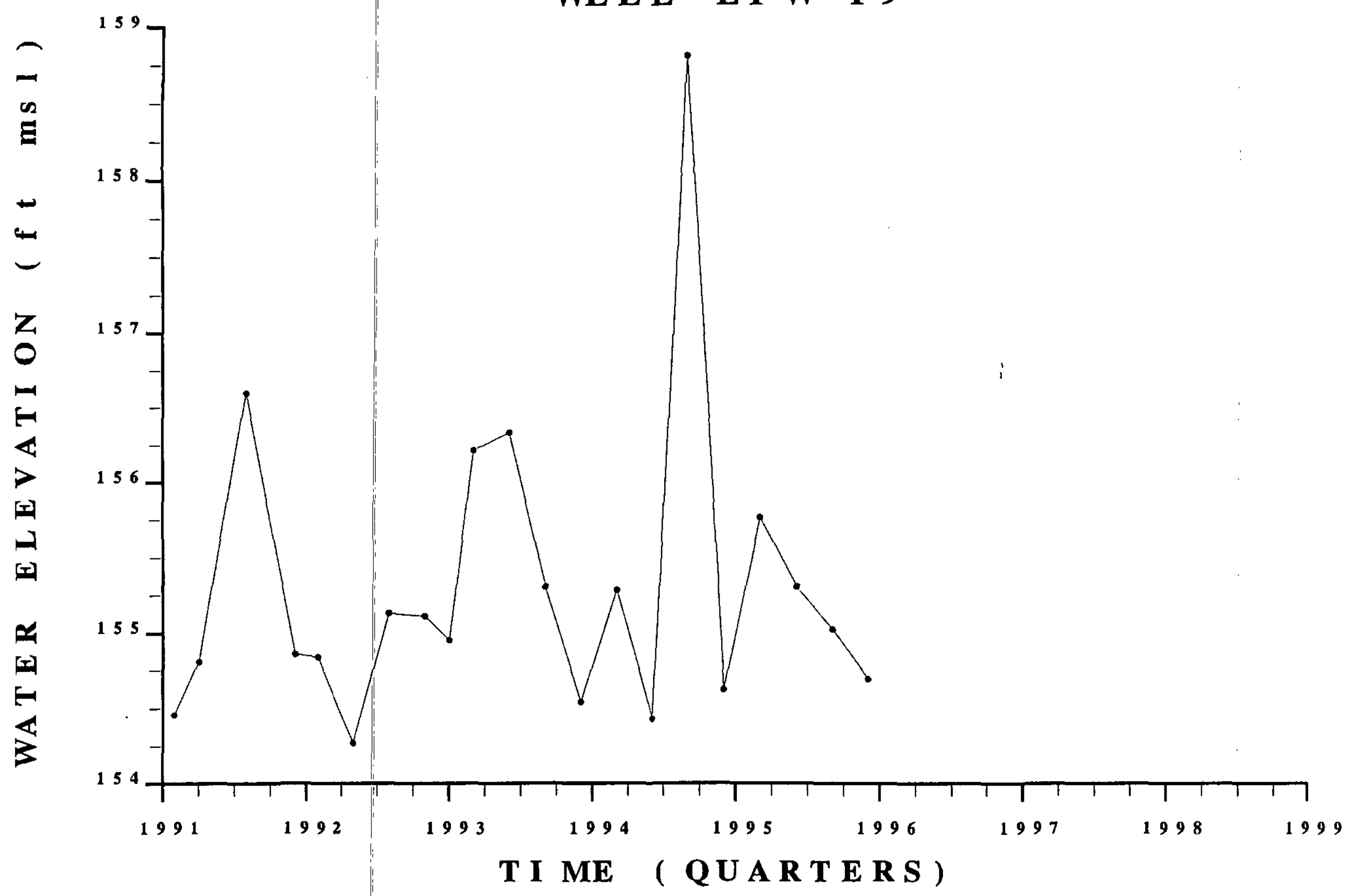

WS R C - T R - $99-000011$ Unc l a s s i f i ed 
H Y D R O G R A P H WE L L L F W 20
WS R C - T R-99-00011 Unc l a s s i f i ed

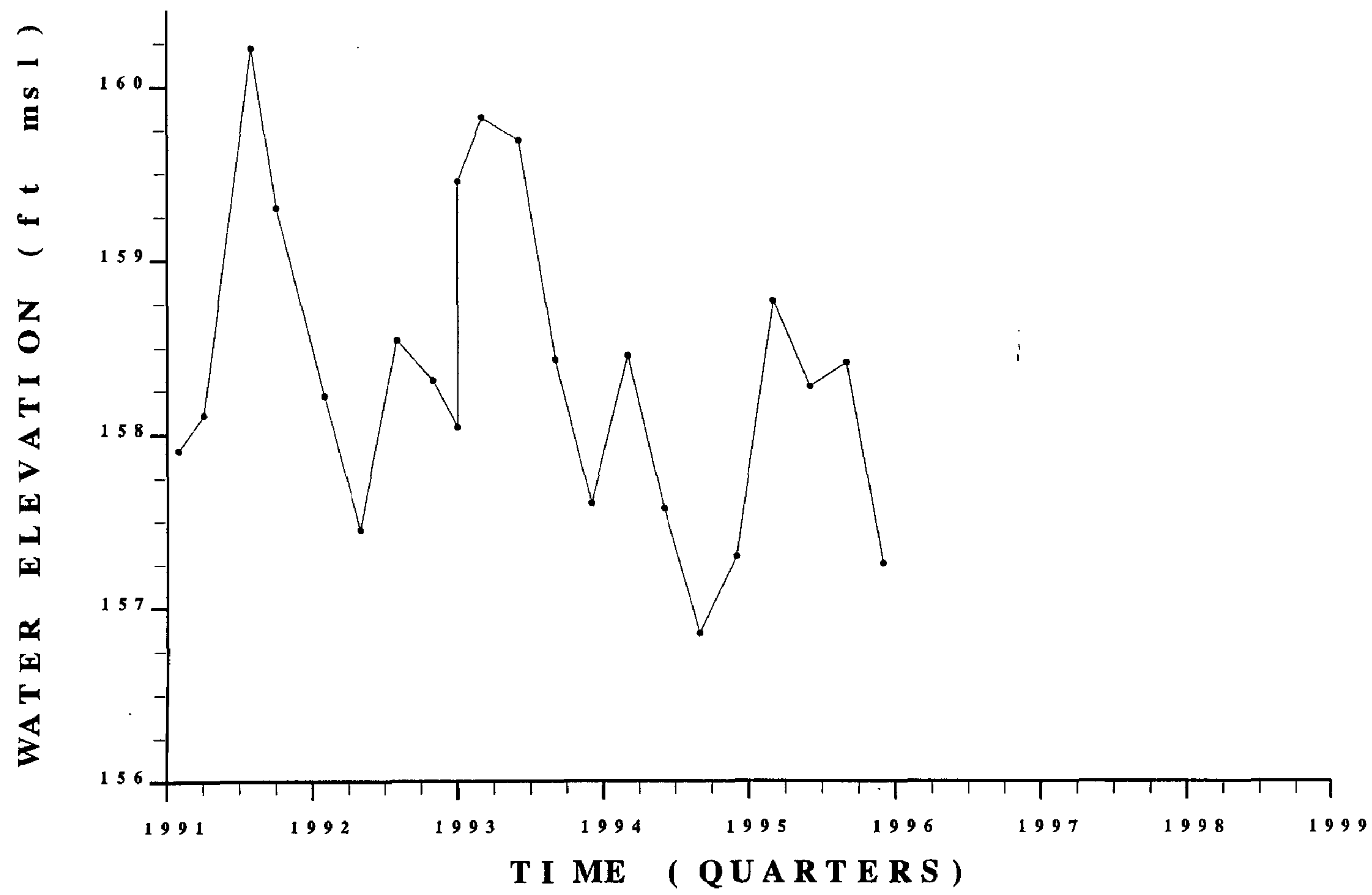

S a n i t a r v La n d f i l l

Fourt h Ou a $r$ e $r$.

1998

\& 1998

S u m ma r 


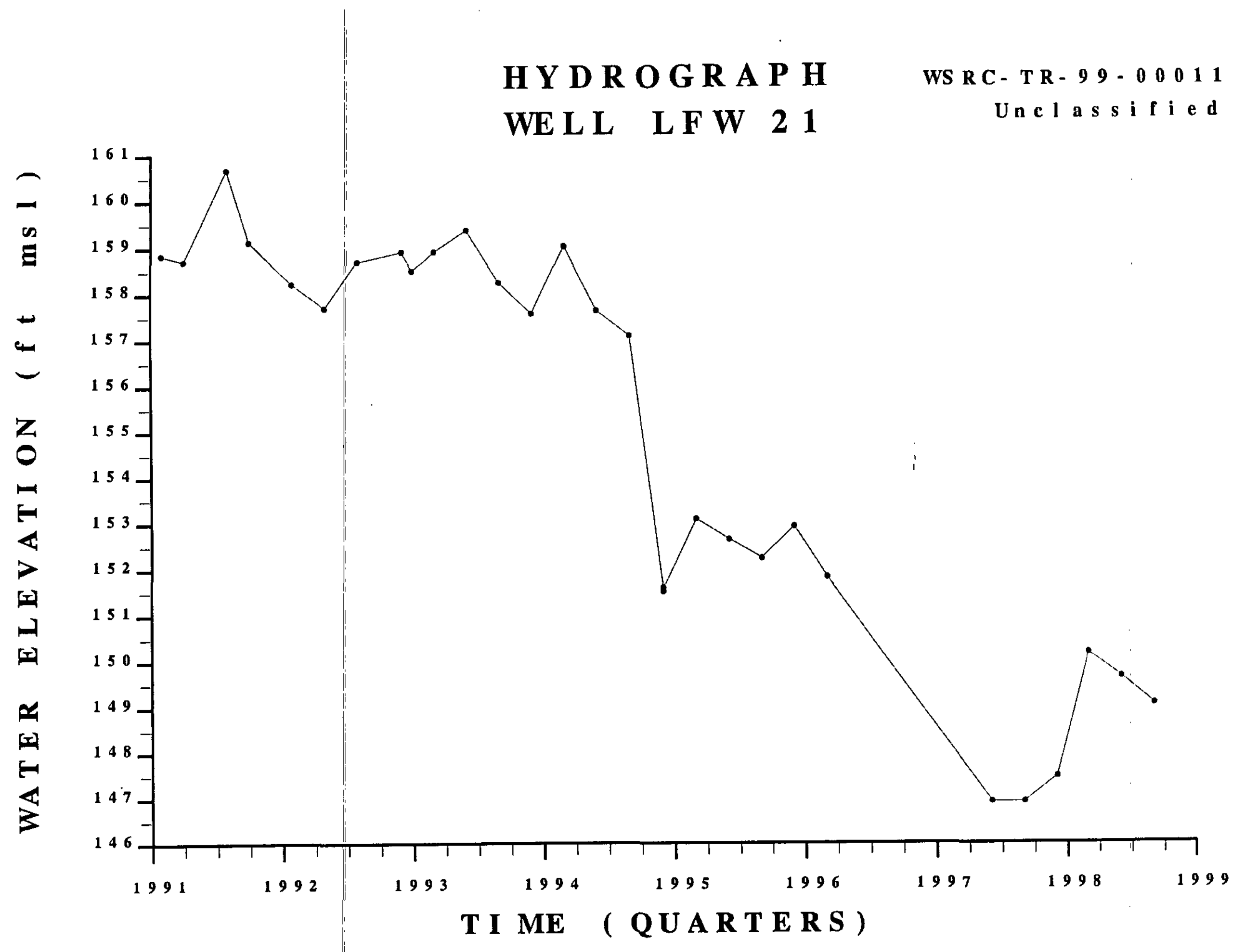


H Y D R O G R A P H WE L L L F W 22
WS R C - T R- 9 9:- 00011 Unclass i f i ed

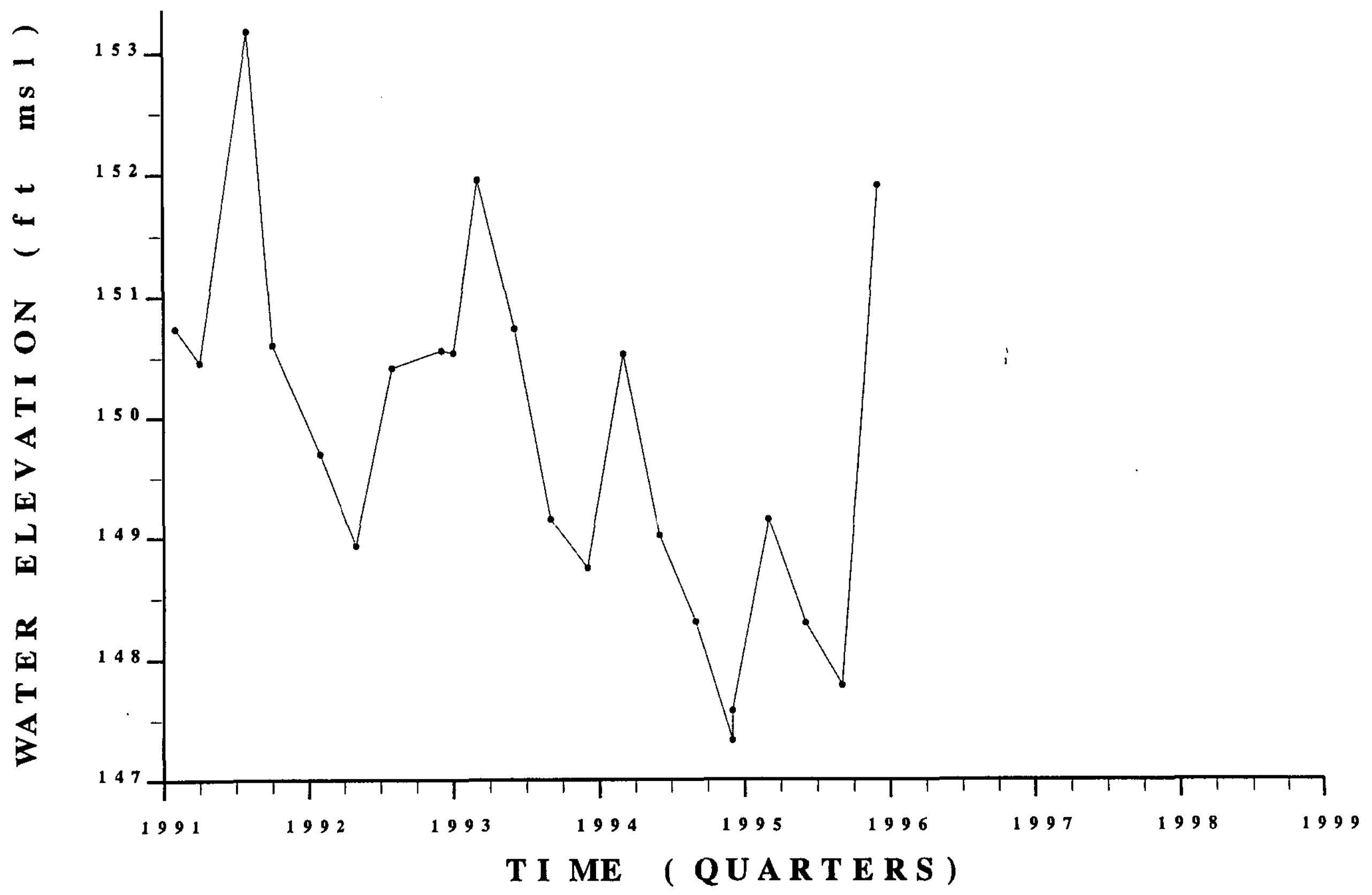

S a n i t a r v L a n d f i l l l

Fourth Ouarter. 1998 \& 1998 Summar 


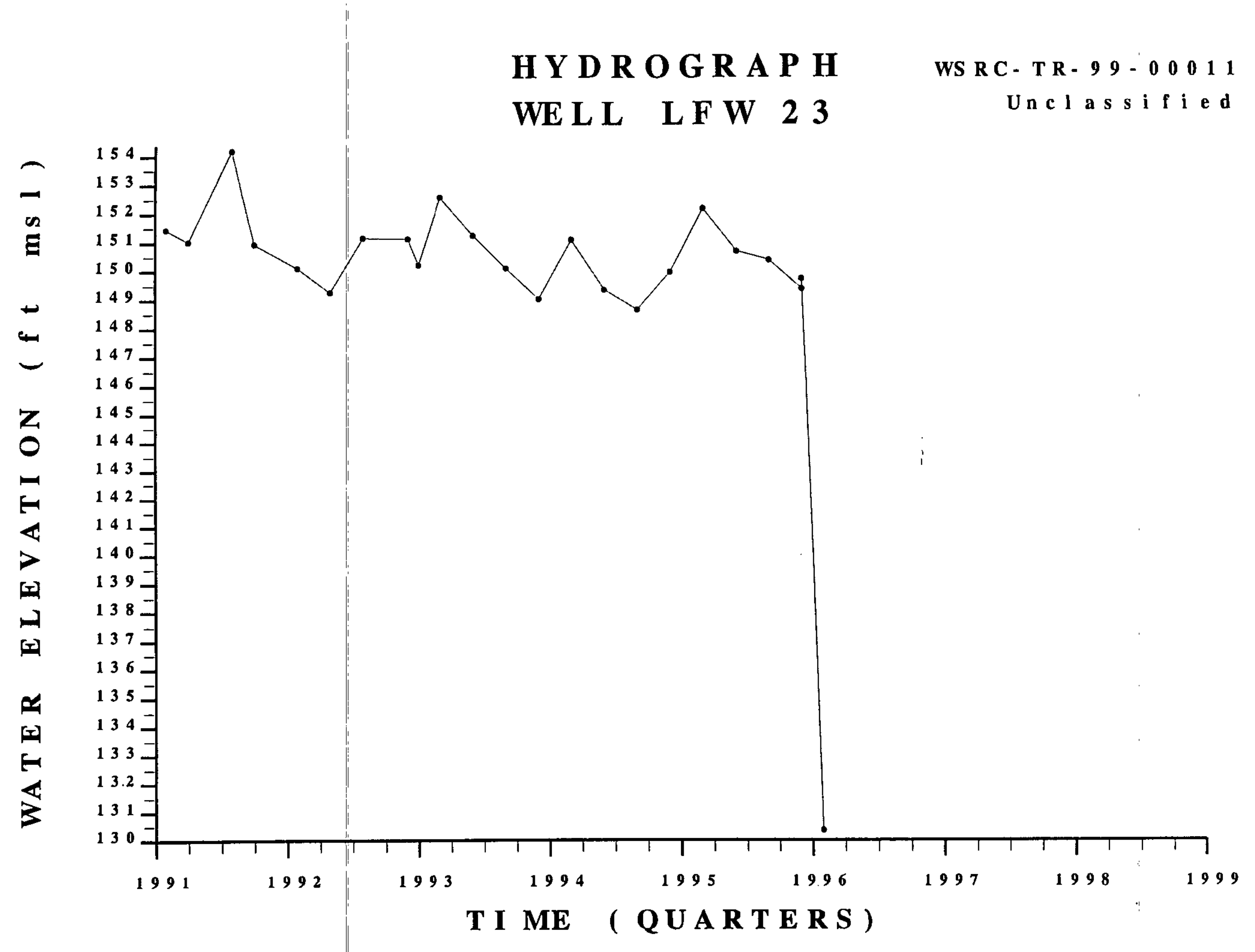


HY D R O G R A P H

\section{WELL LFW 23 R}

WS R C - T R - 99-0 00011

Un c 1 a s s i f i e d

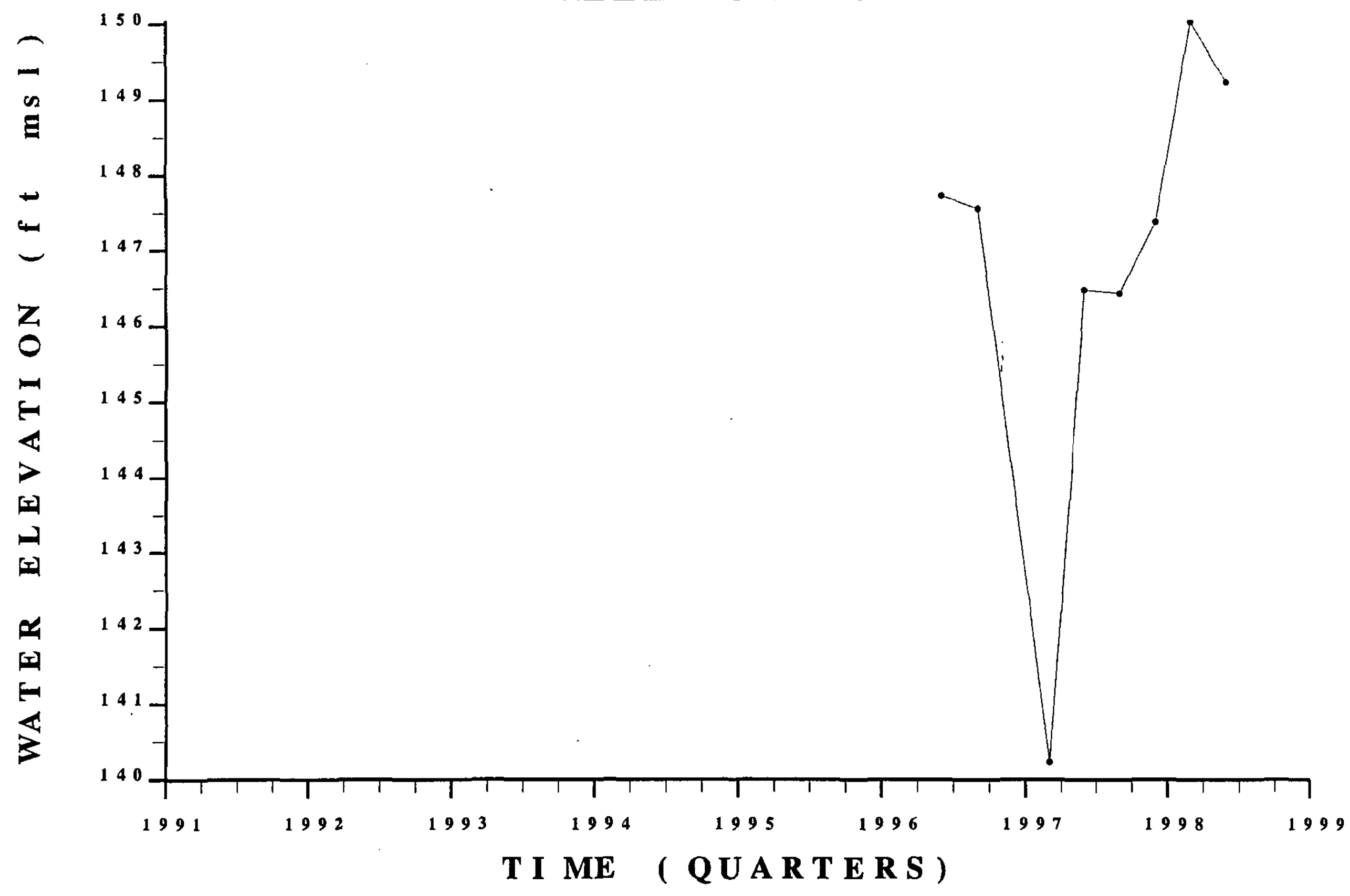

$S$ an i t a r v Landf i I l

Fourth Ouarter.

$1998 \quad \& \quad 1998$

S u m ma r 


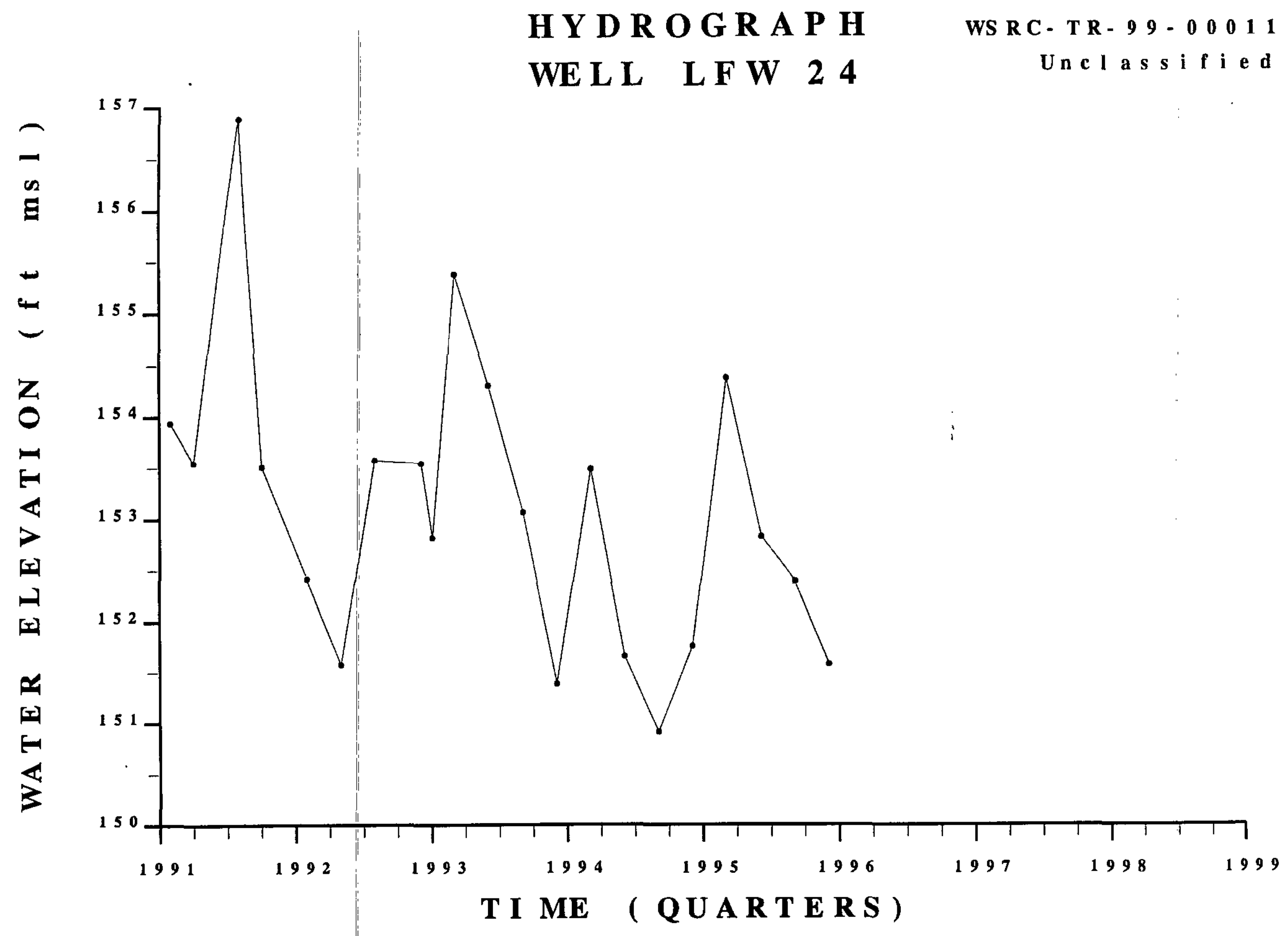




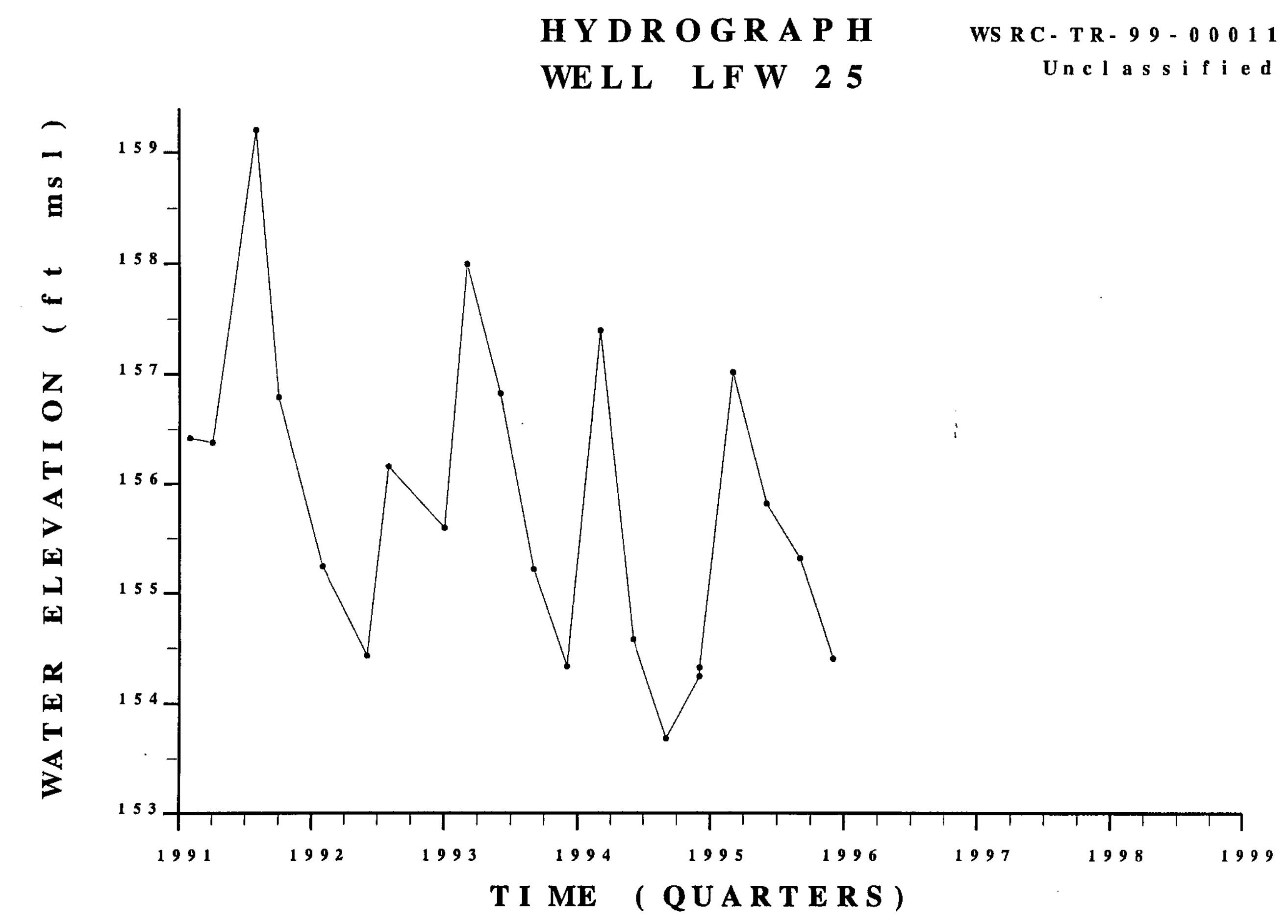


H Y D R O G R A P H WE L L L F W 26
WS R C - T R - $99-0$ - 00011 Unc l a s s i f i e d

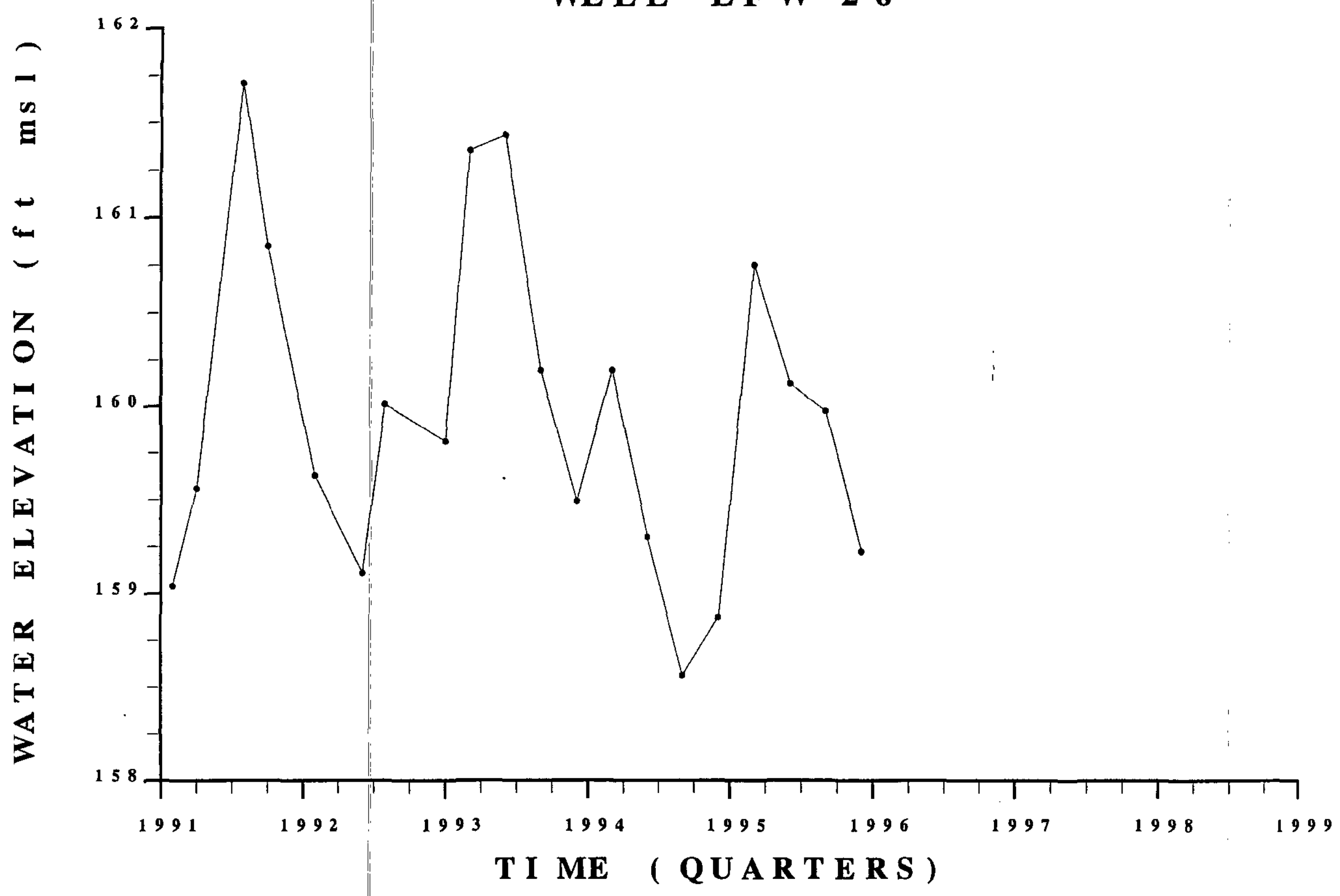




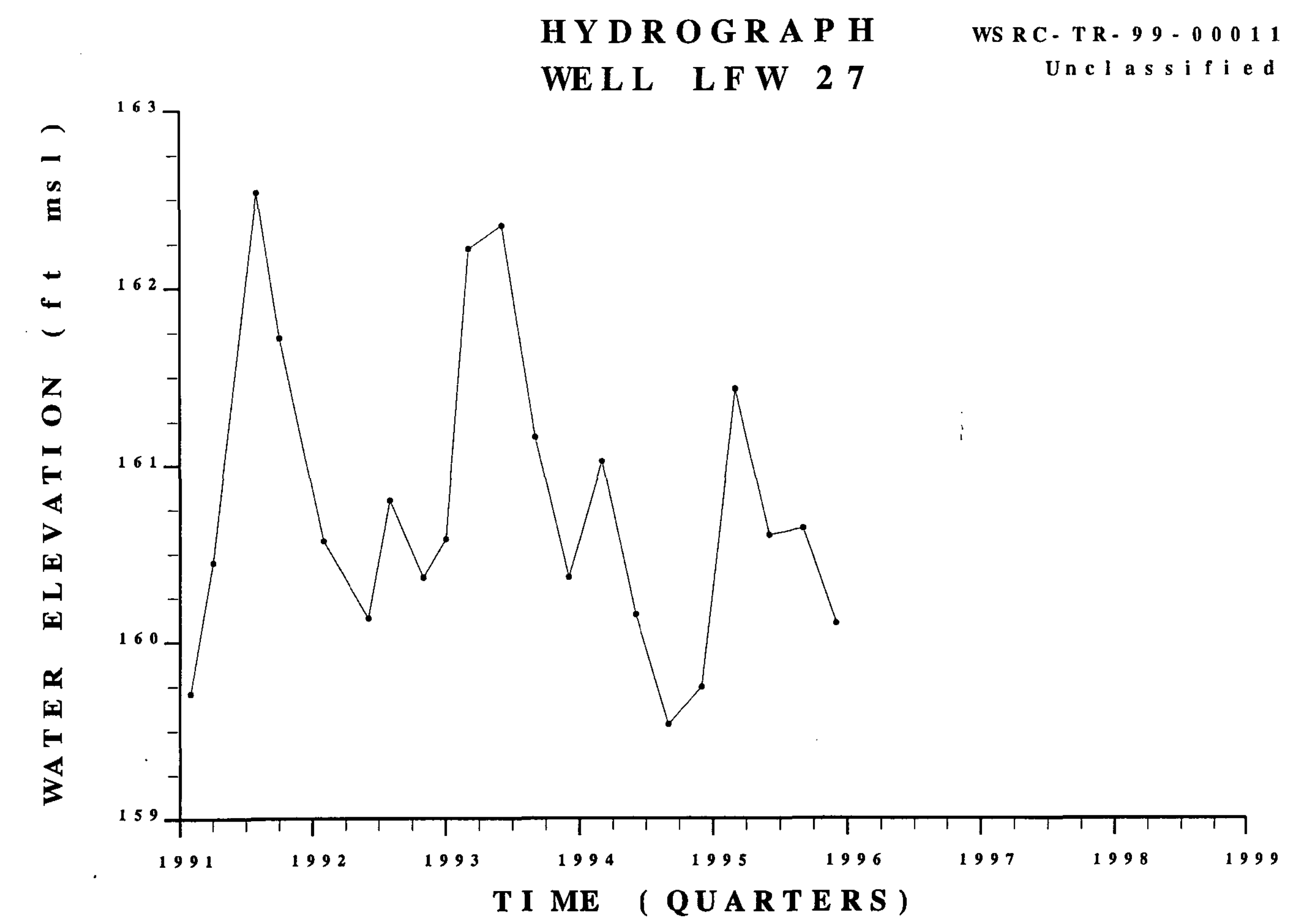




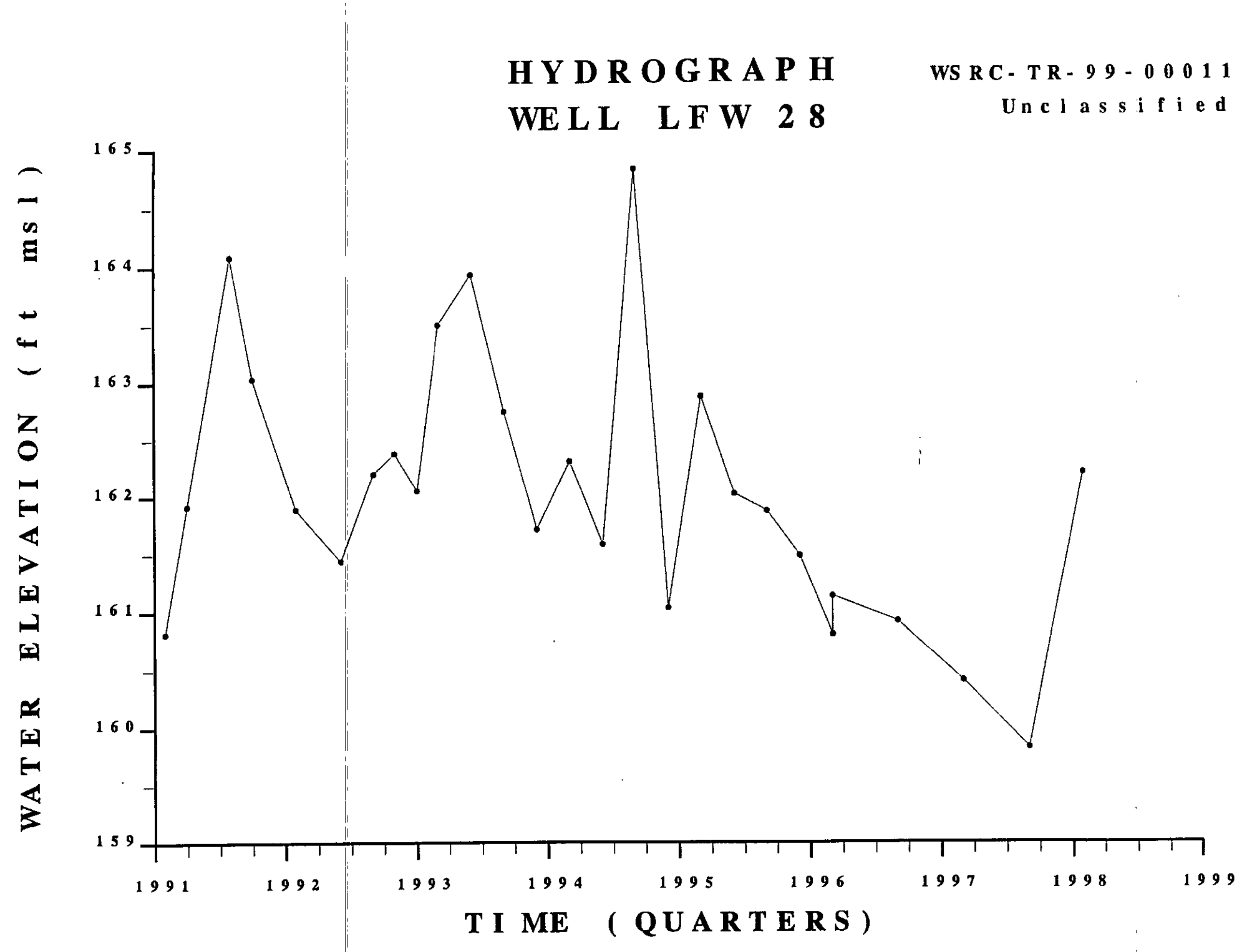


HY D R O G R A P H

WE L L L F W 29
WS R C - T R - $99-0 \begin{array}{lllll}0 & 0 & 1 & 1\end{array}$

Unclassified

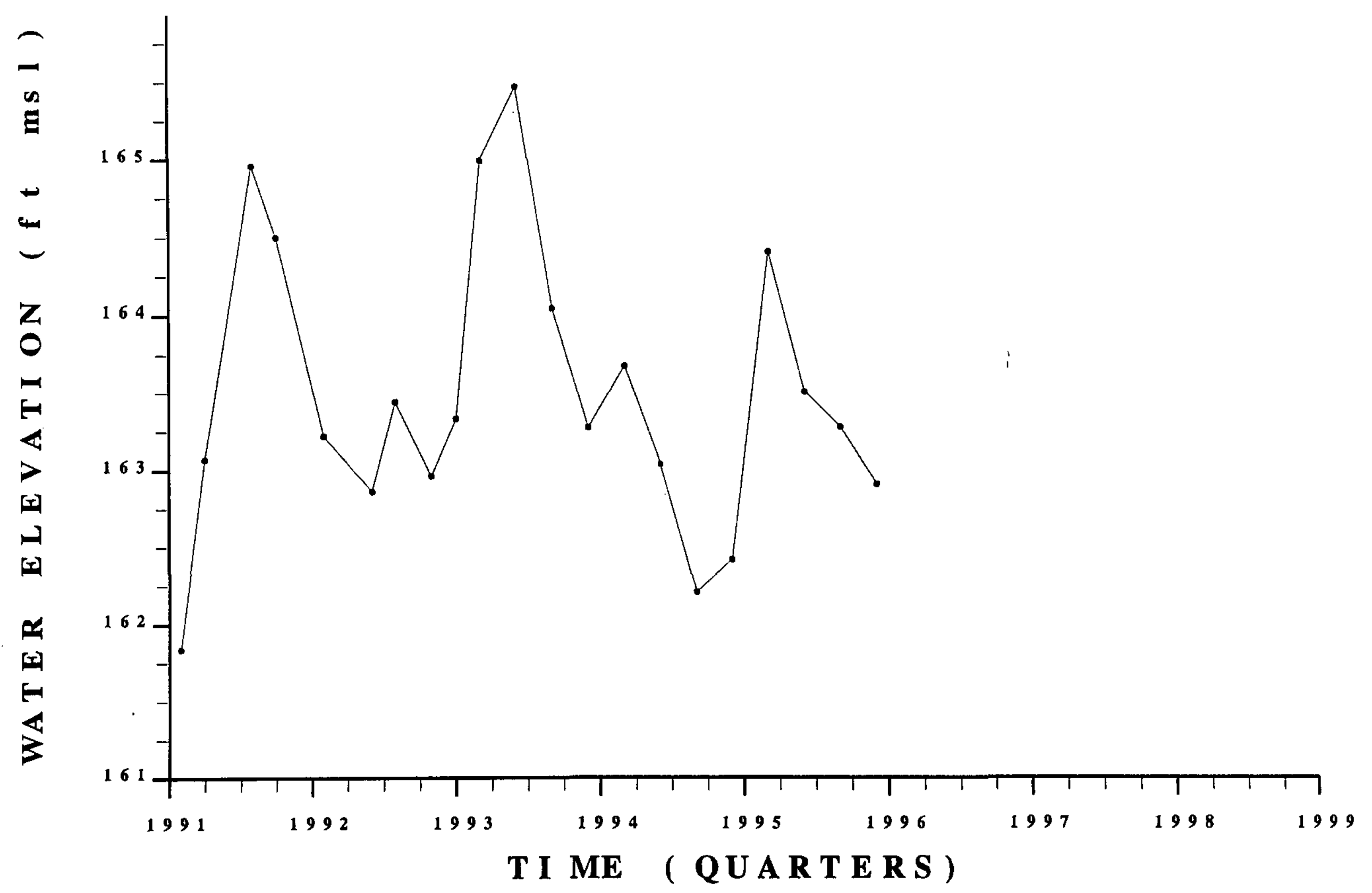

S a n i t a r v Landf i l l

Fourth Ouarter. 1998 \& 1998 Summar 


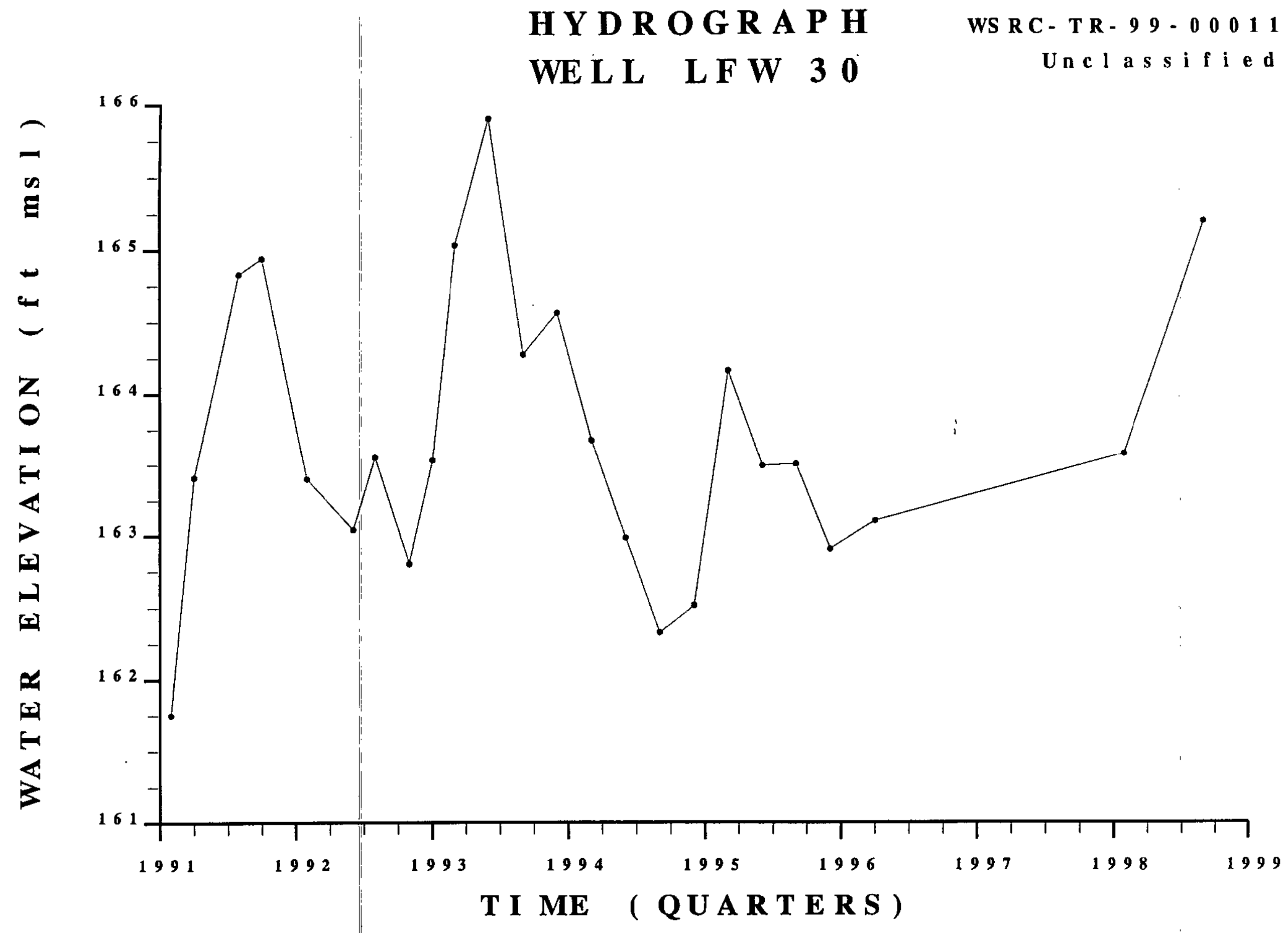




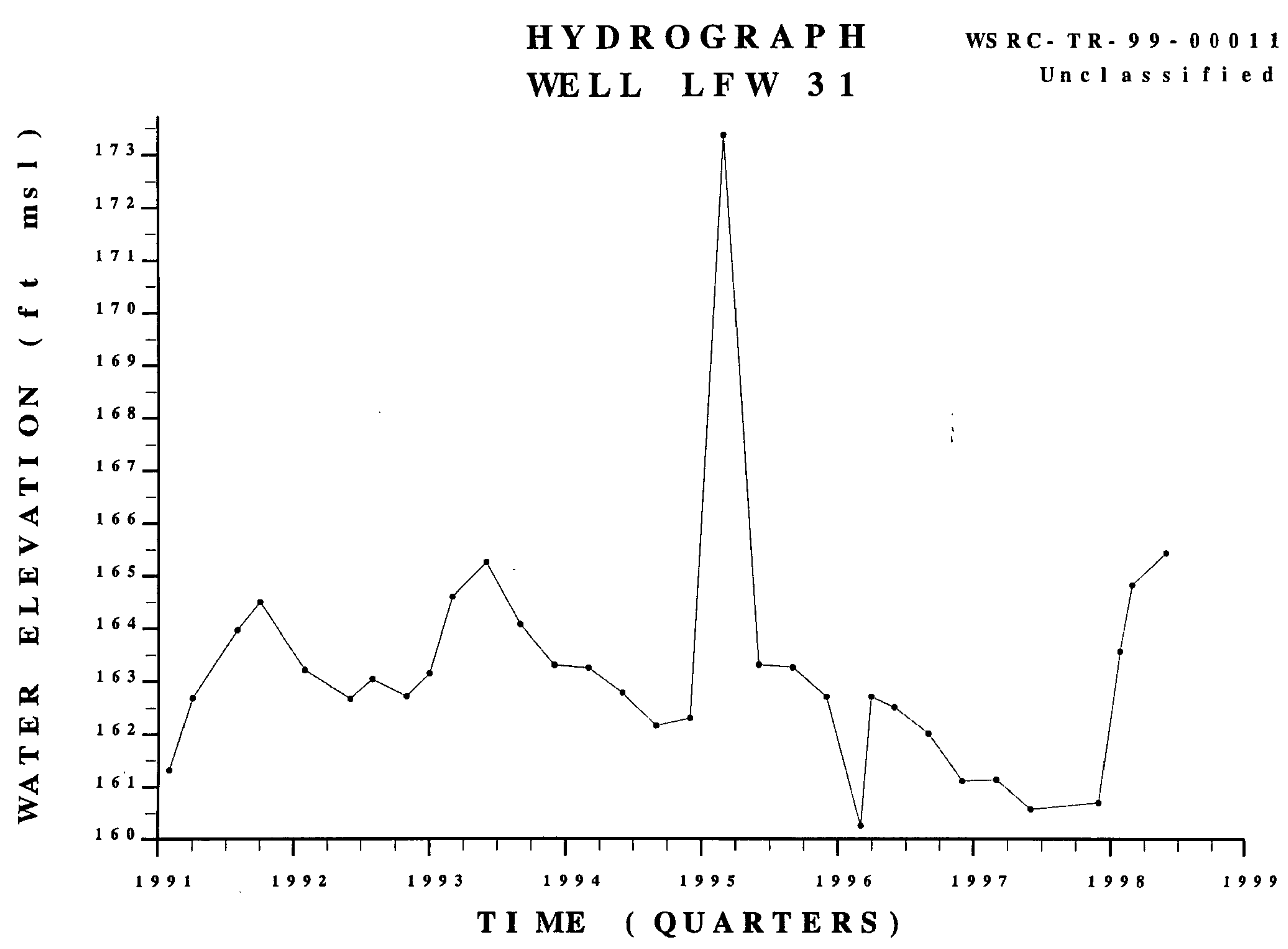

S a n i t a r v La n d f i l I

Fourth Ouarter.

$1998 \quad \& \quad 1998 \quad S$ ummar 
HY D R O G R A P H

WE L L L F W 32
WS R C - T R - $99-0 \begin{array}{lllll}0 & 0 & 1 & 1\end{array}$ Unc l a s s i f i ed

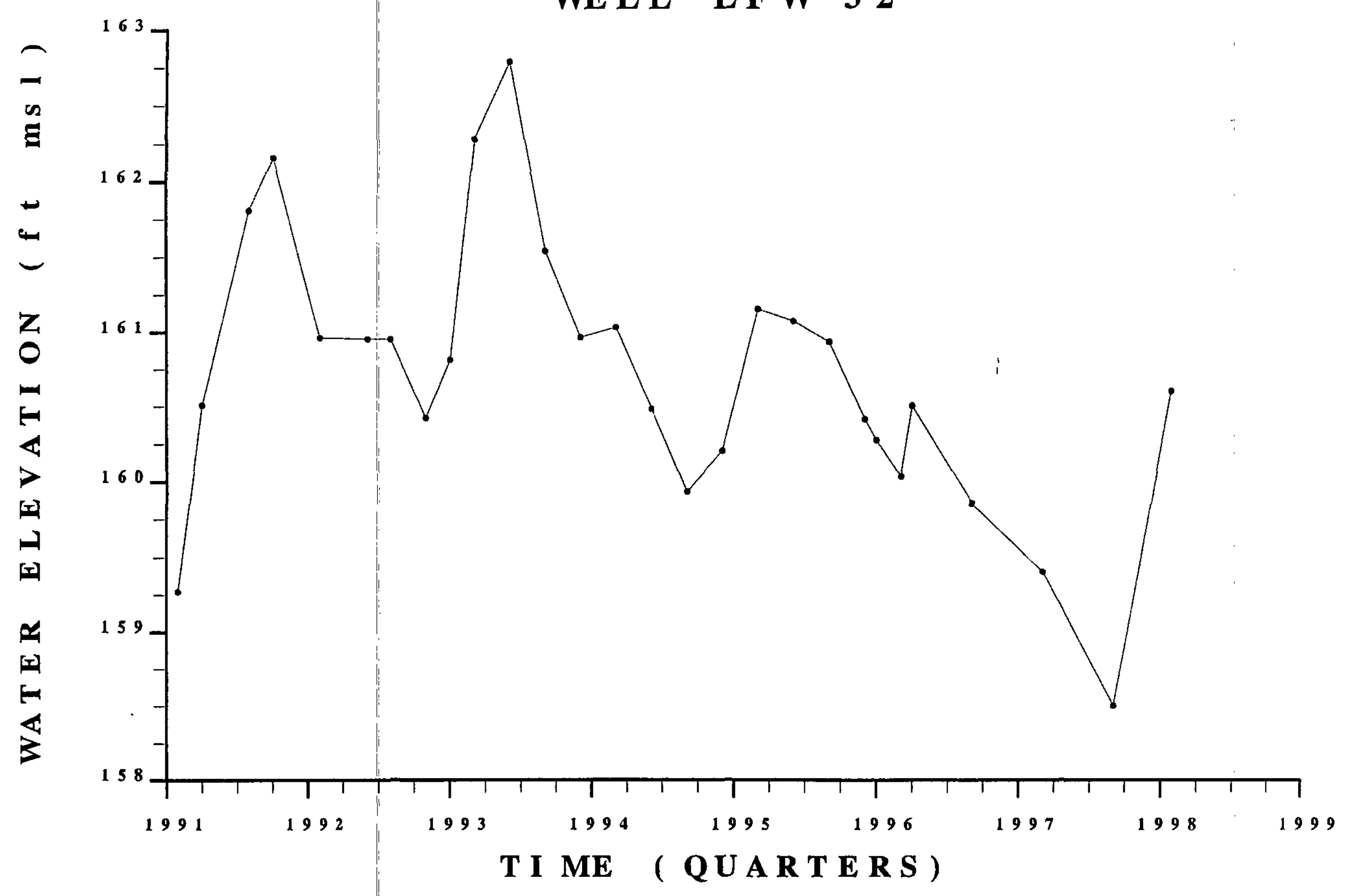


H Y D R O G R A P H WELL LFW 32 C
WS R C - T R - $99-000011$

Unc l a s s i f i e d

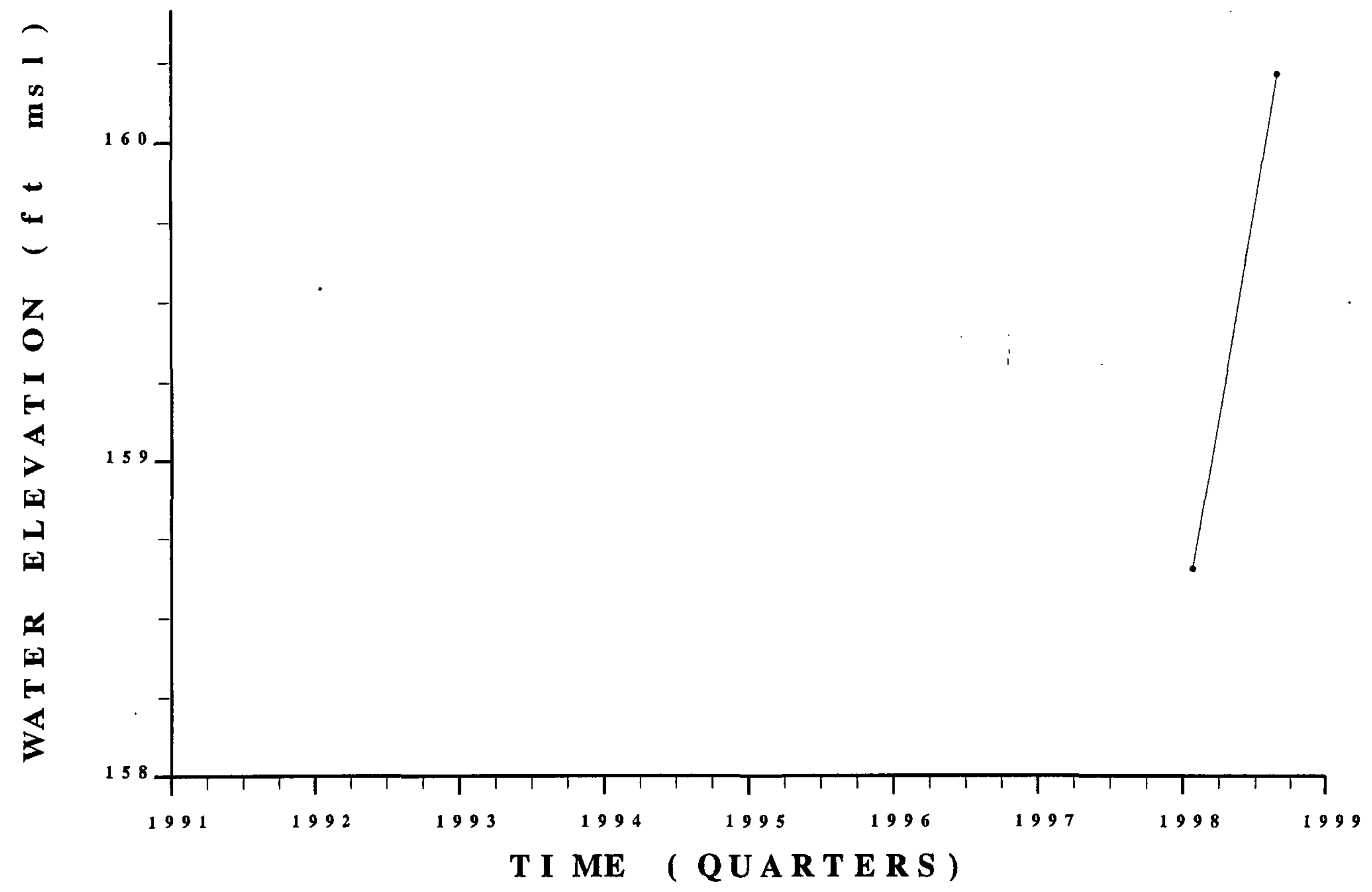

S a n i t a r v La nd f i l l

Fourth Ouarter. $1998 \quad \& \quad 1998$ Summarv 
HY D R O G R A P H WE L L L F W 33
WS R C - T R - $999-000011$ Unc l a s s i f i e d

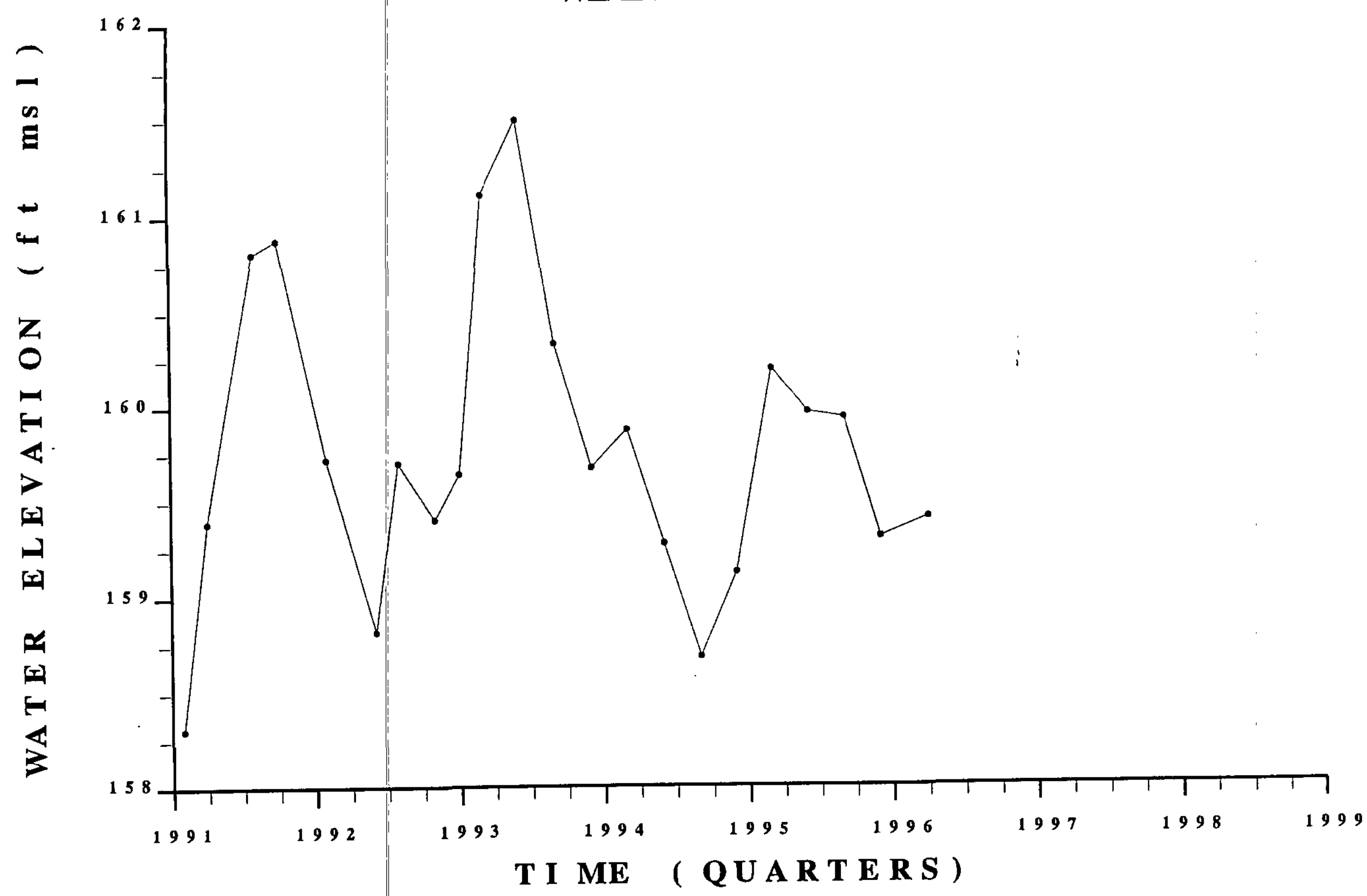


H Y D R O G R A P H WE L L L F W 34
WS R C - T R - $99-00011$ Unc l a s s i f i ed

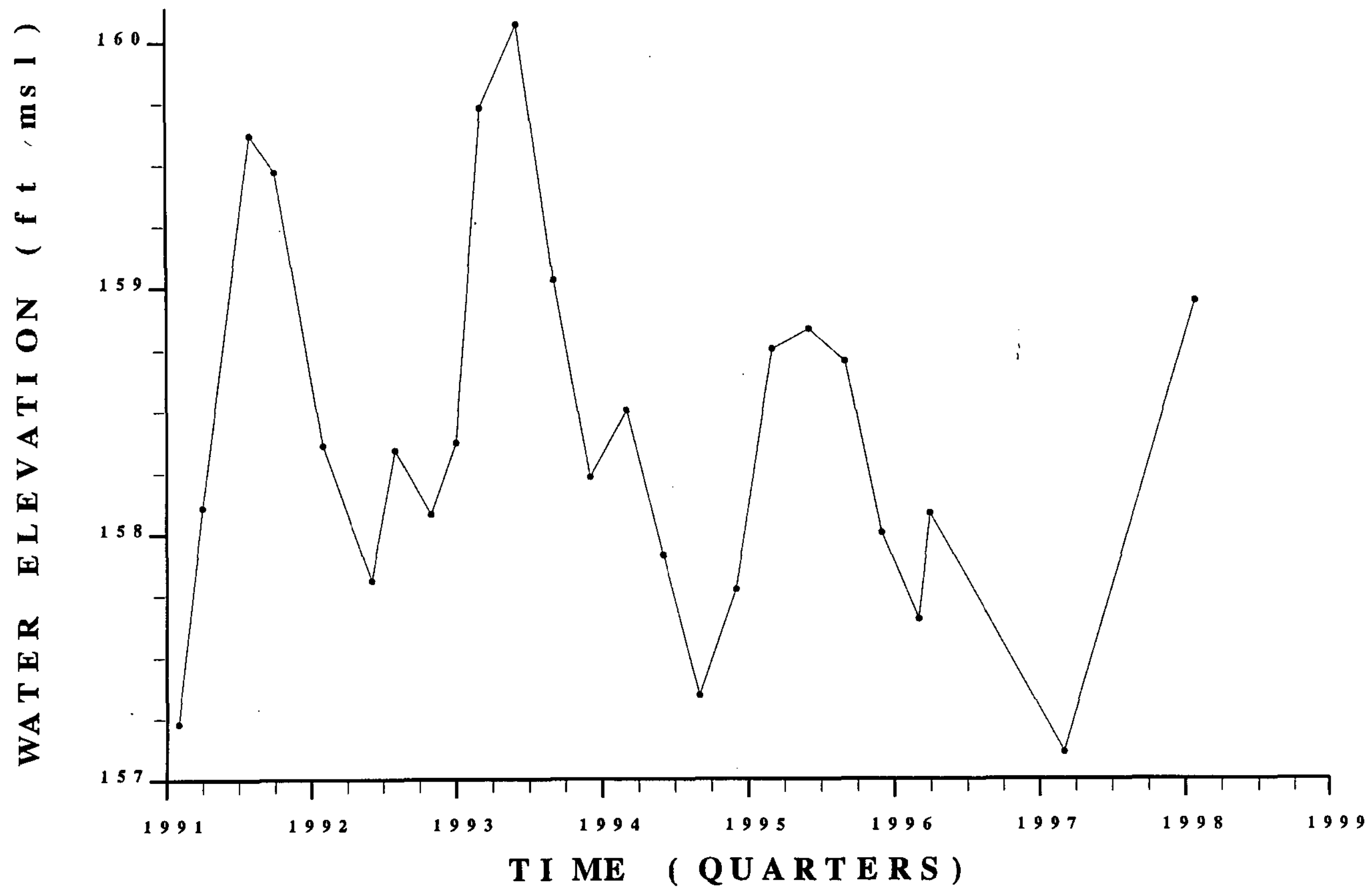

$S$ an i t a r v La nd f i l l

Fourt h Ou a r t e $r$.

1998

\& 1998

$S \mathbf{u m m a} \mathbf{r}$ 
H Y D R O G R A P H WE L L L F W 35

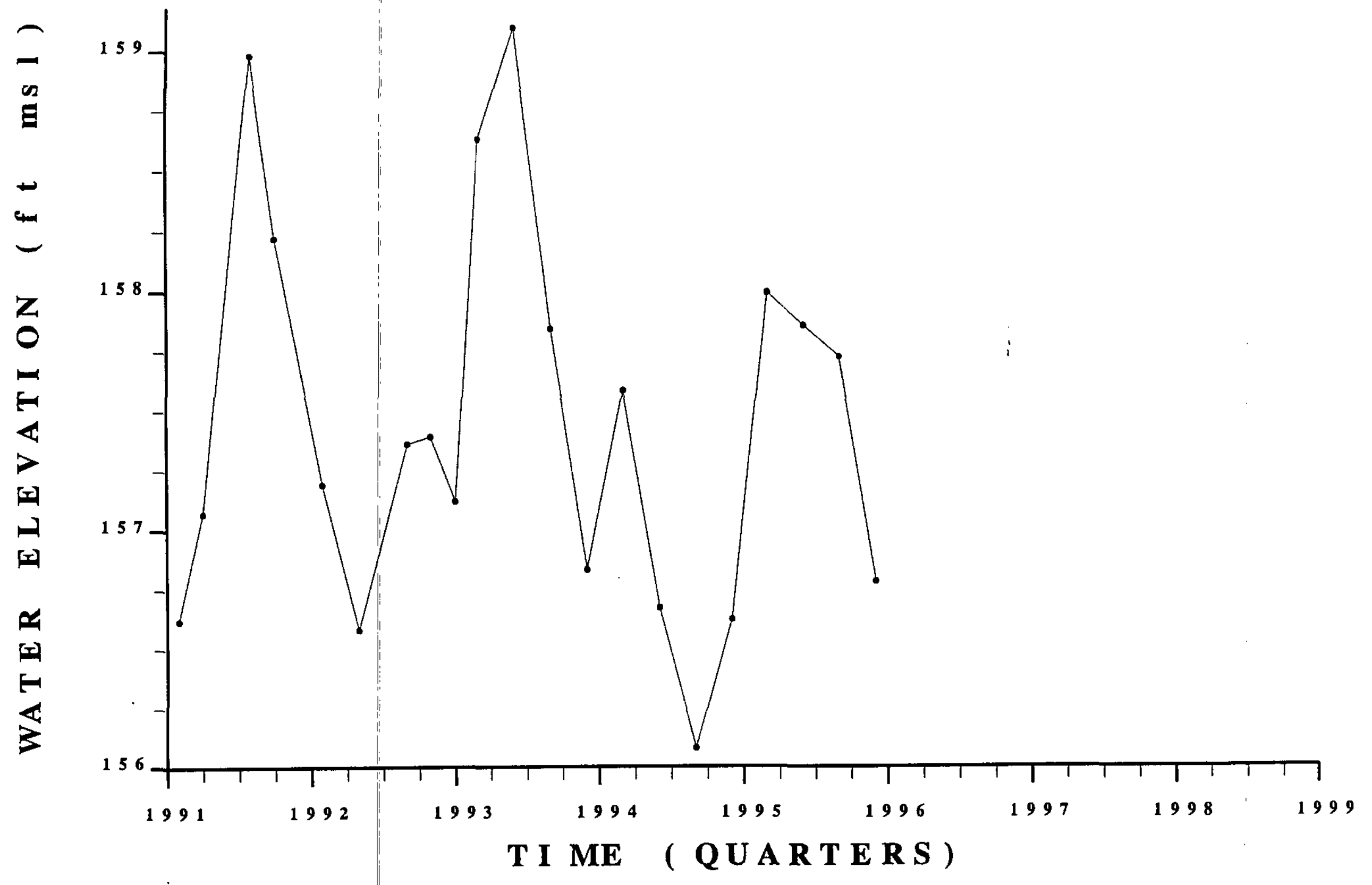


H Y D R O G R A P H WE L L L F W 36
WS R C - T R - $99-00011$ Unc I a s s i f i ed

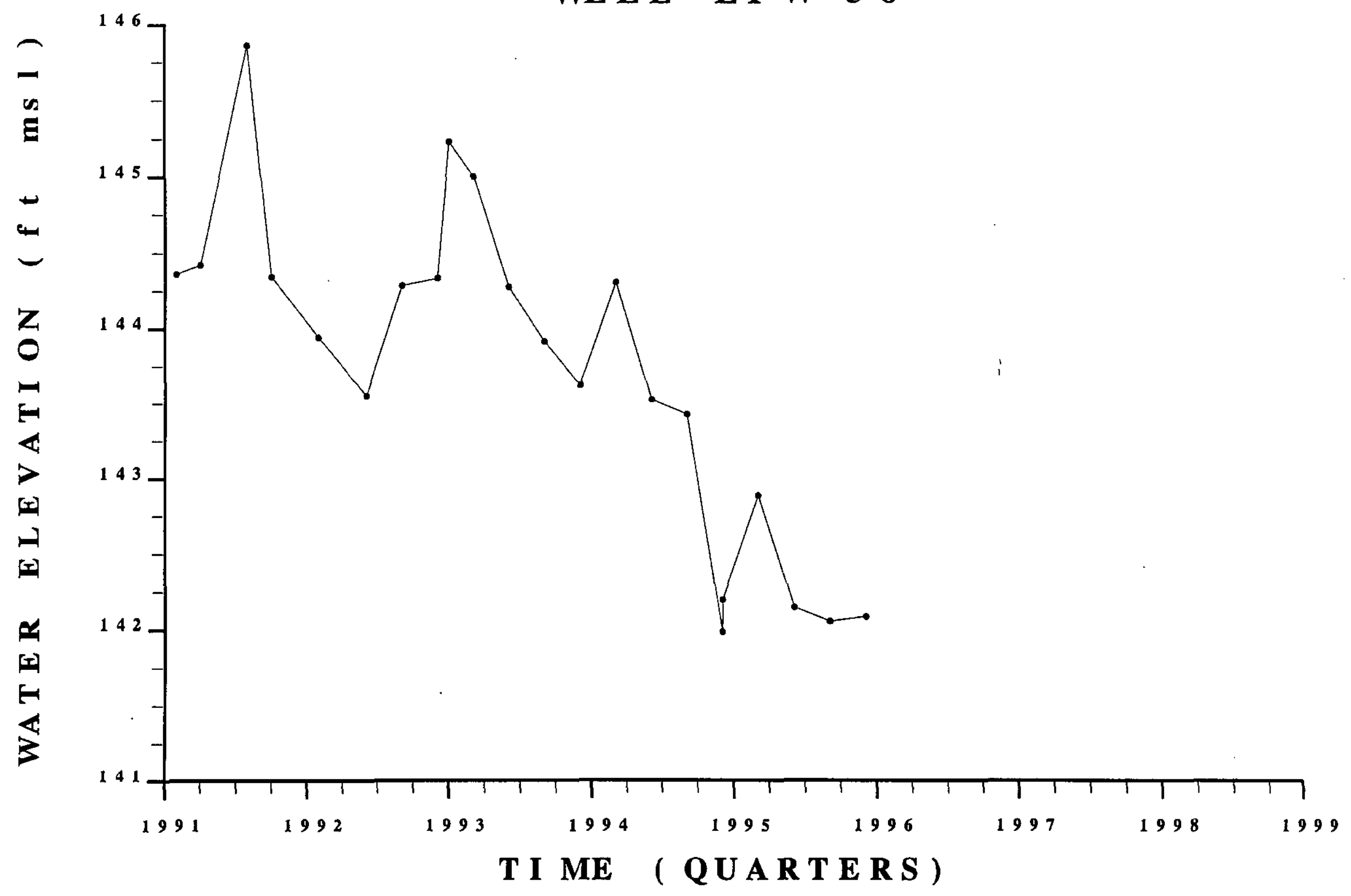




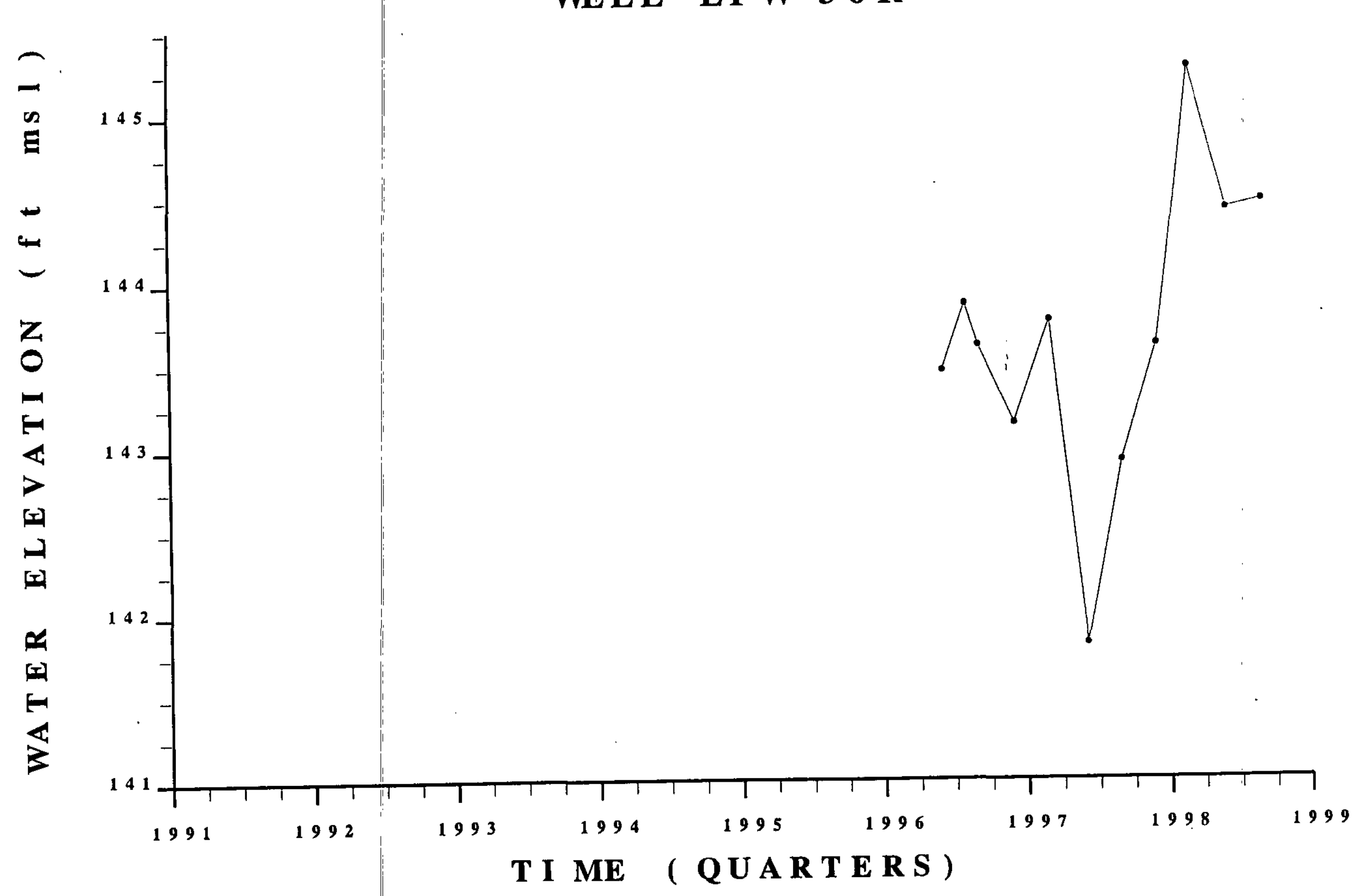

Sanitarv LandFi

H Y D R O G RA P H WELL LFW 36 R
WS R C - T R - $99-0 \begin{array}{lllll}0 & 0 & 1 & 1\end{array}$

Unc lass ified 


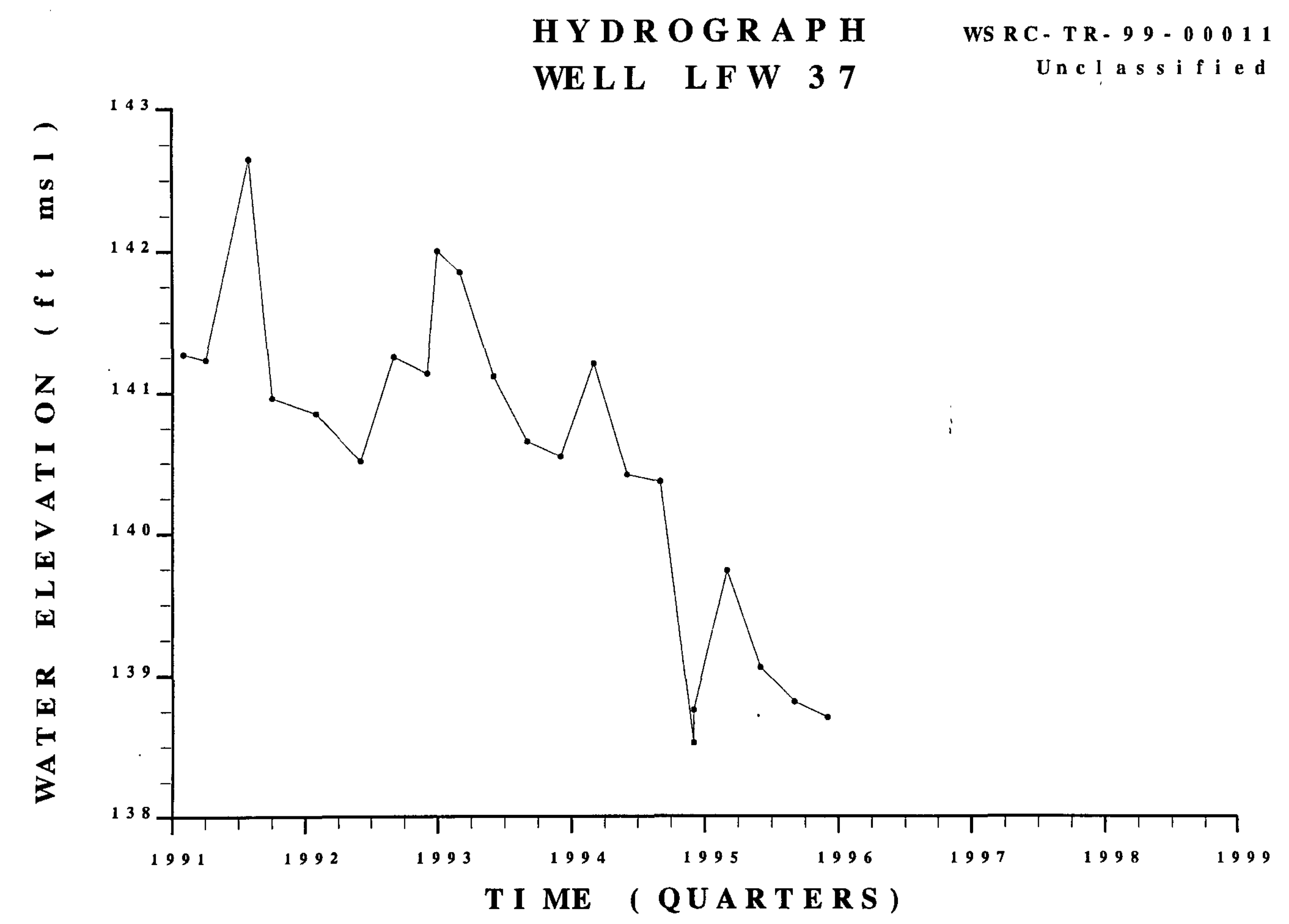

S a n i t a r v Land f i l l l

Fourth Ouarter. 1998 \& 1998 Summarv 


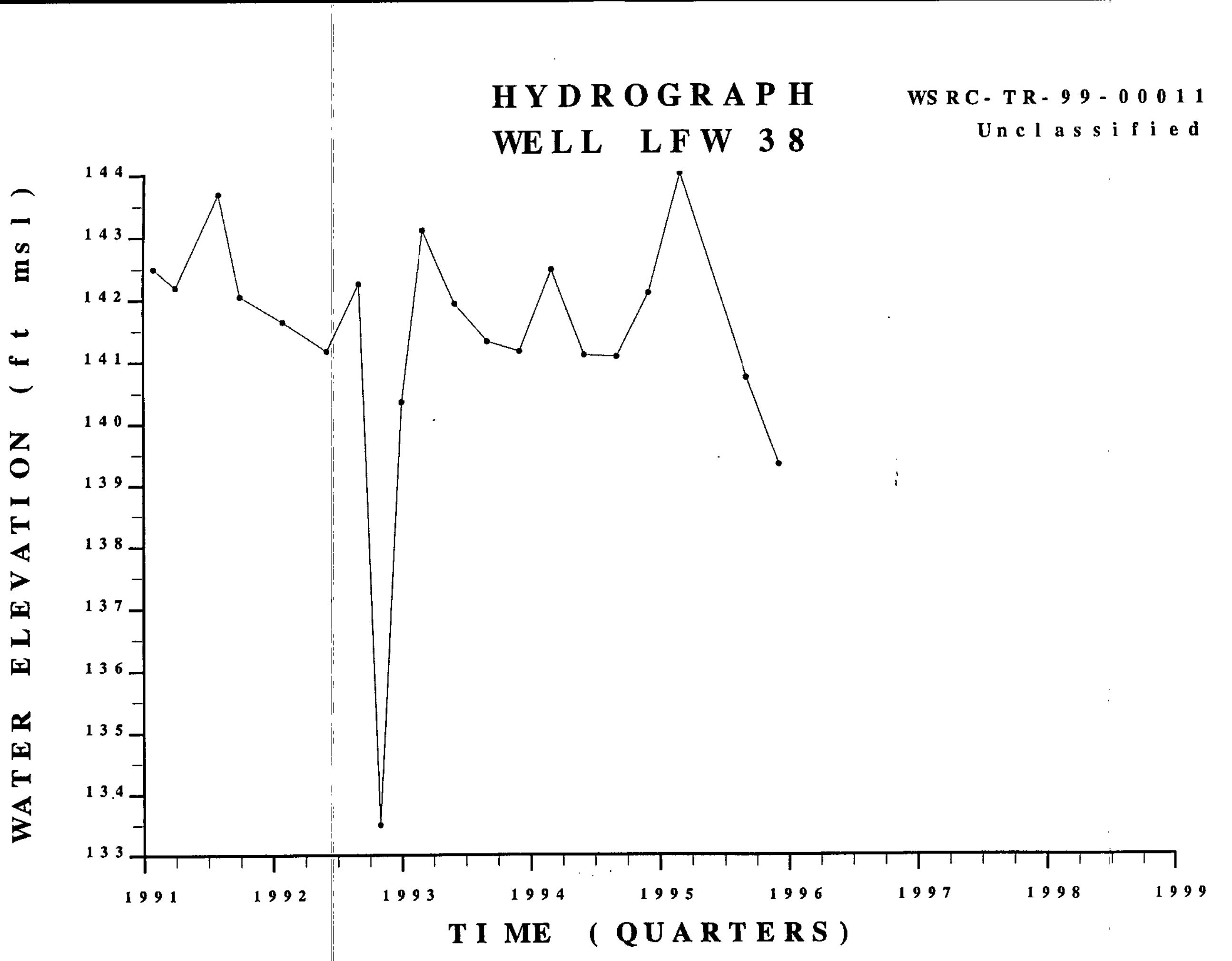




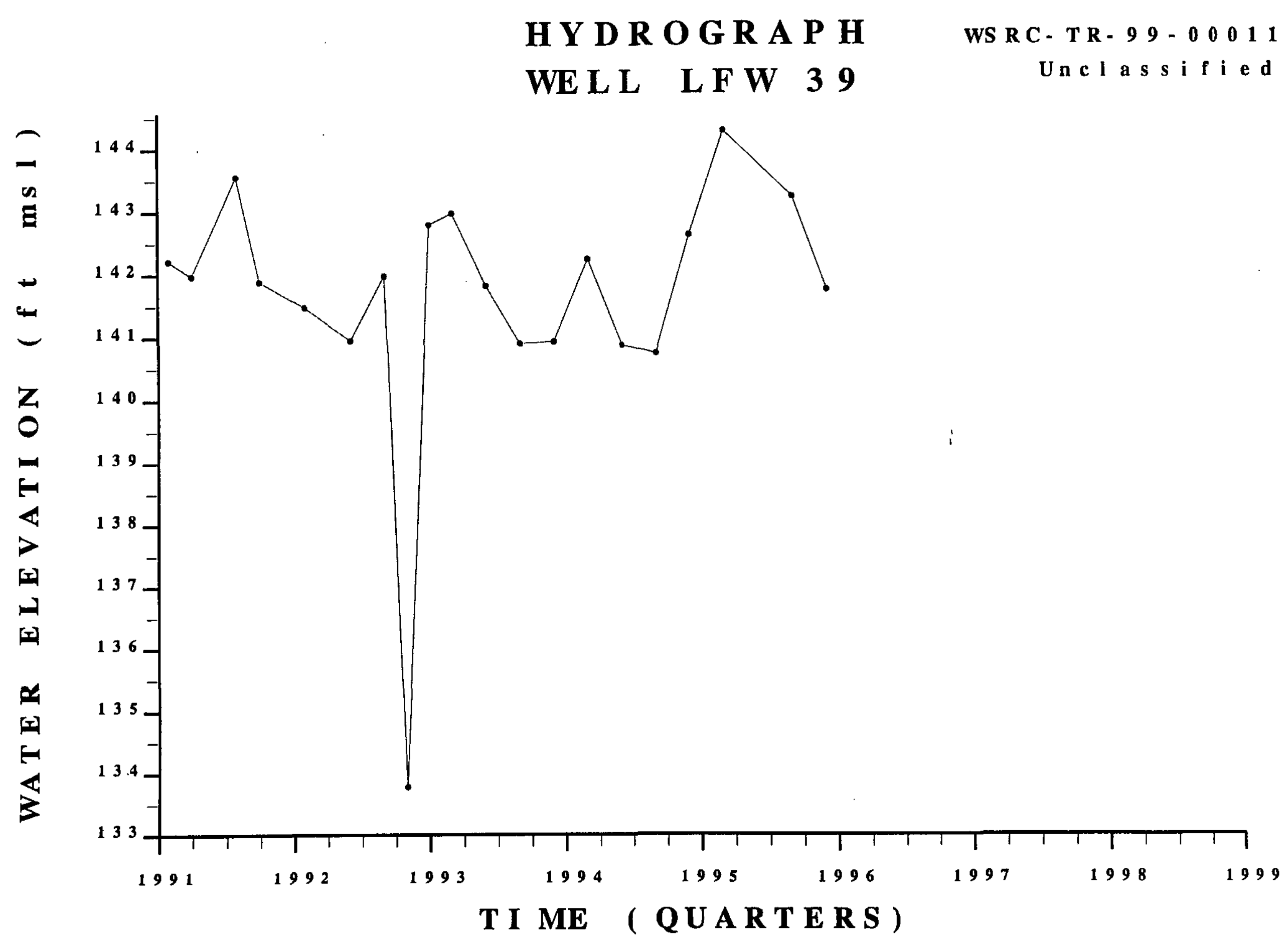


H Y D R O G R A P H WE L L L F W 40
WS R C - T R - $99-0$ - 000011 Unc l a s s i f i e d

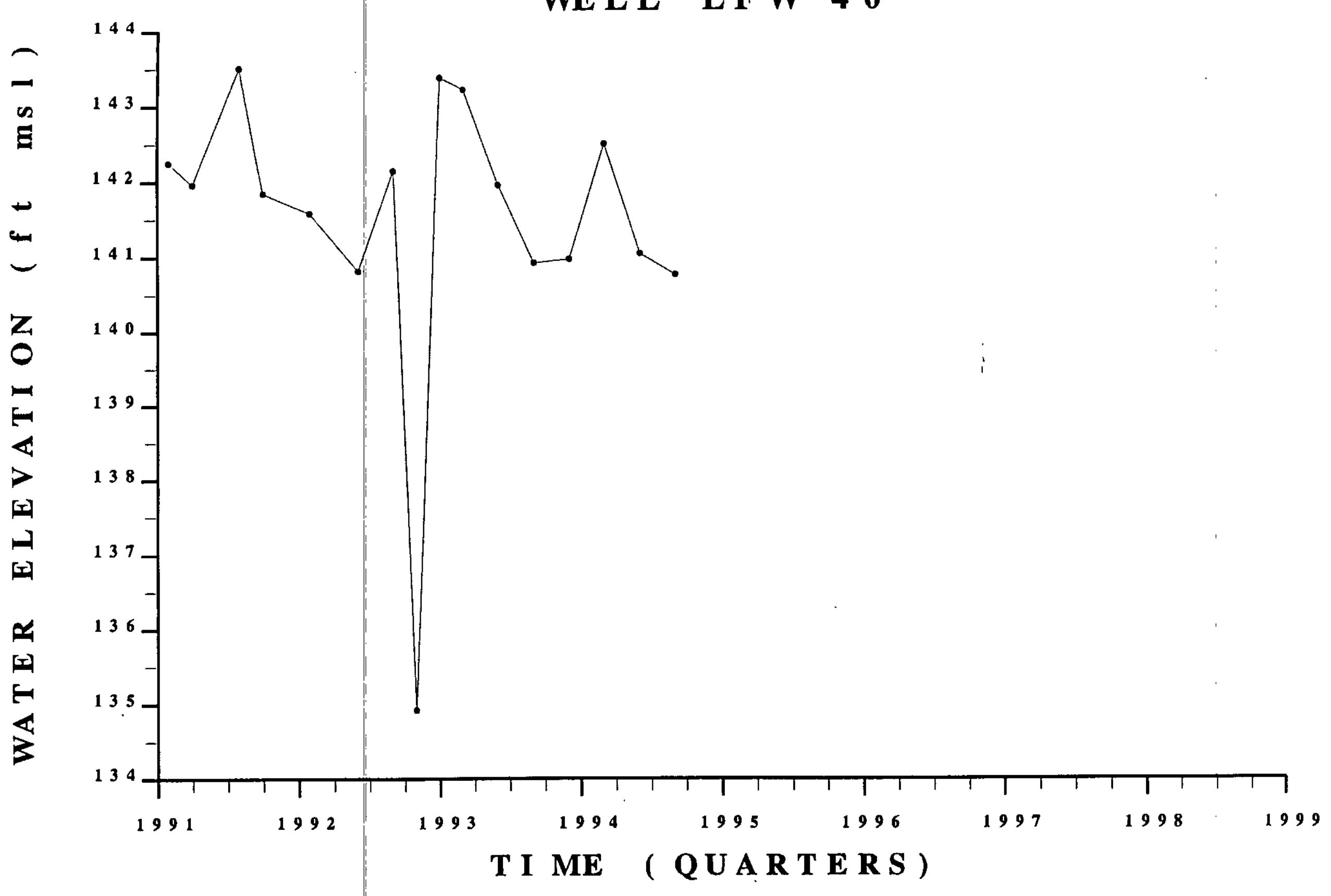


H Y D R O G R A P H WE L L L F W 41
WS R C - T R-99-0 0011 Unc l a s s i f i e d

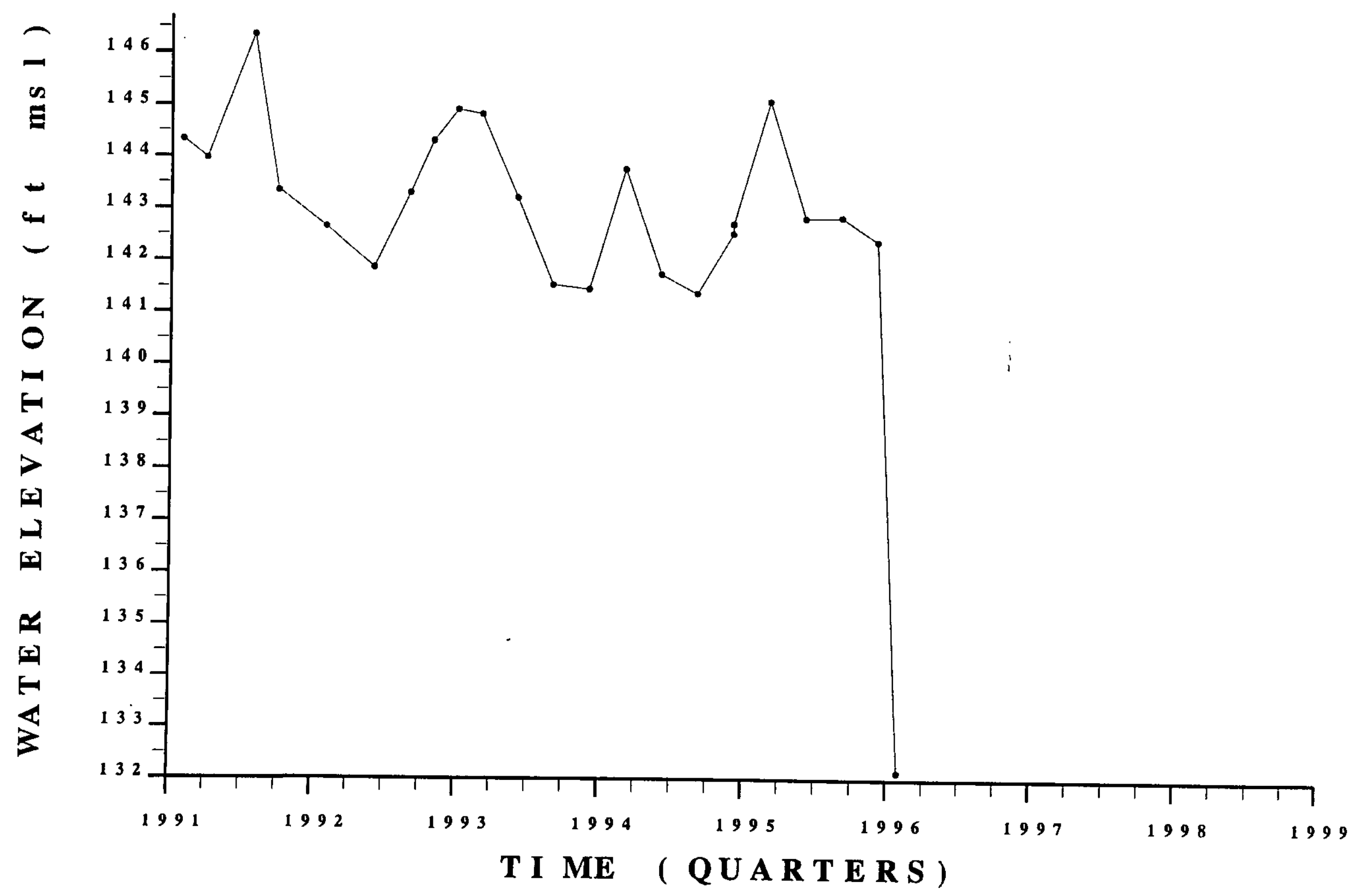

Sa n i a r v Landfi I I

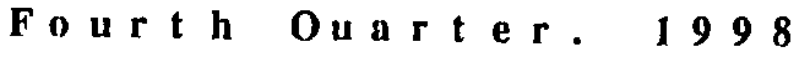
$\& \quad 1998$ 
2

H Y D R O G R A P H WELL LFW 41 R
WS R C - T R - $99-0$ - 00011

Unclass if i ed

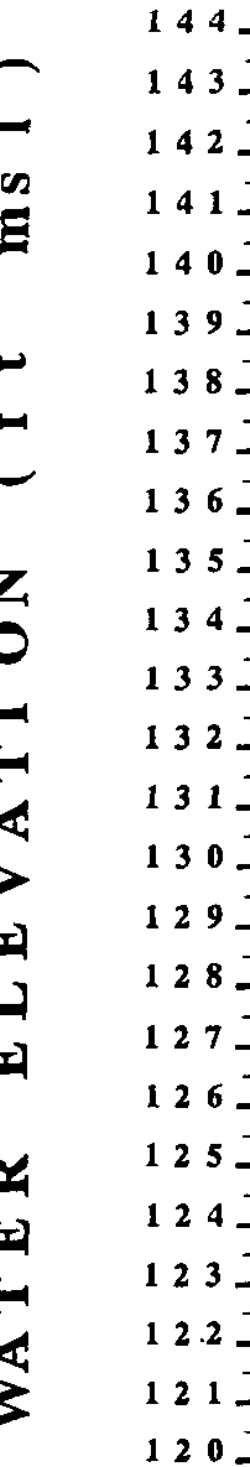

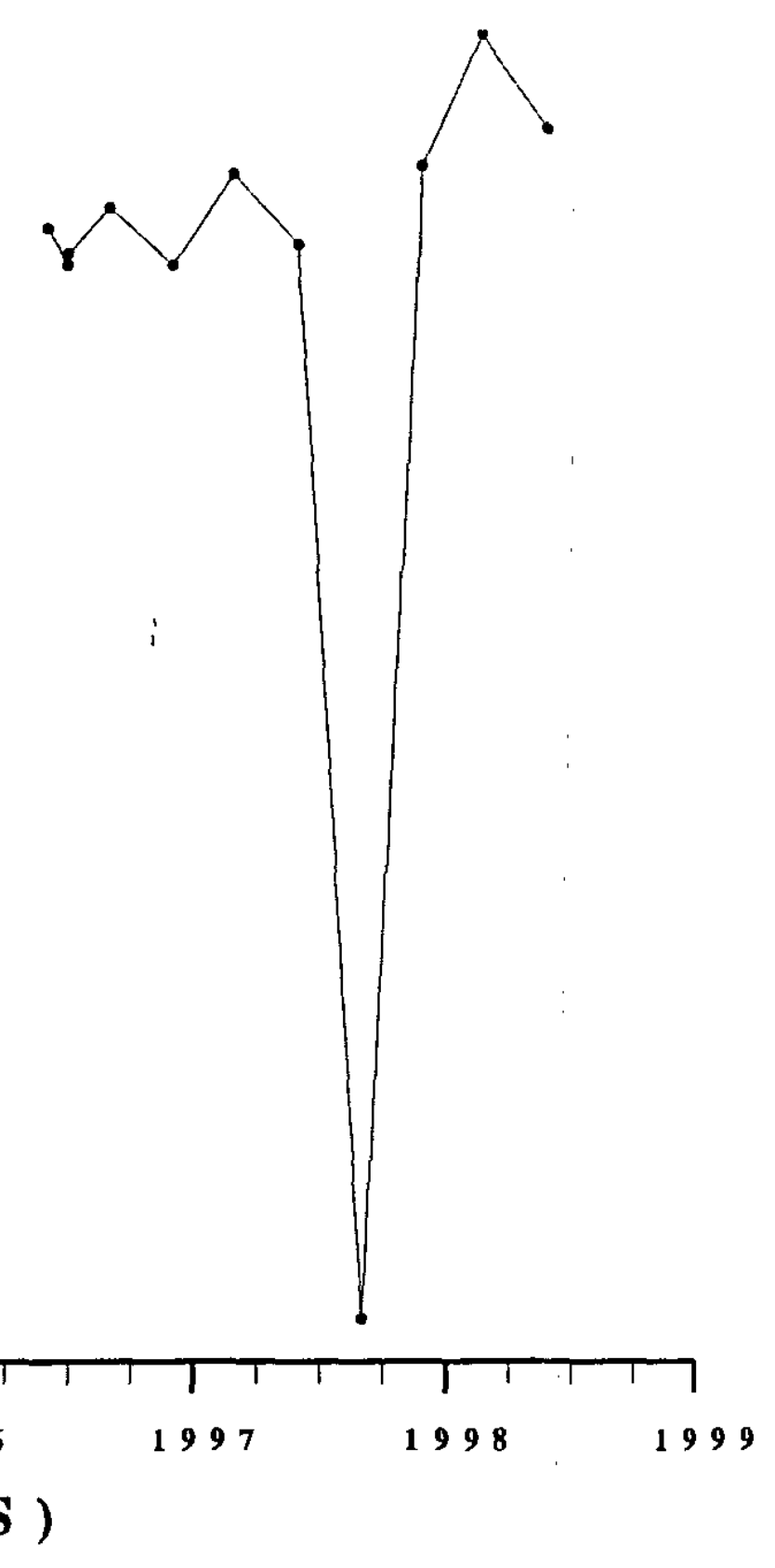

T I ME ( Q UART TRS ) 
H Y D R O G R A P H

WE L L L F W 42
WS RC-TR- $99-000011$

Unc I a s s if i ed

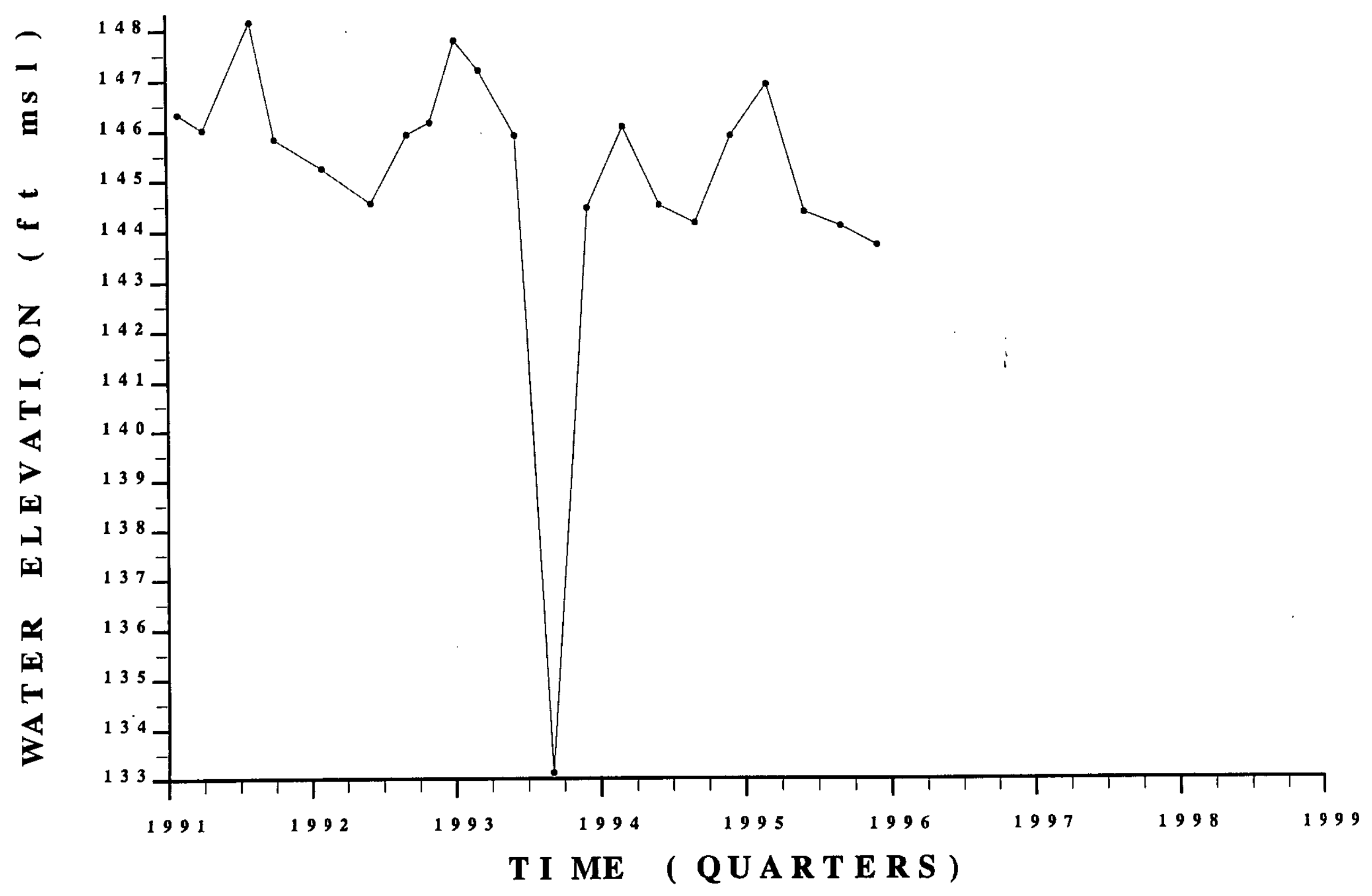

San i t a r v Land f i l l

Fourt h Ouarter.

\& 1998

S u m ma r v 


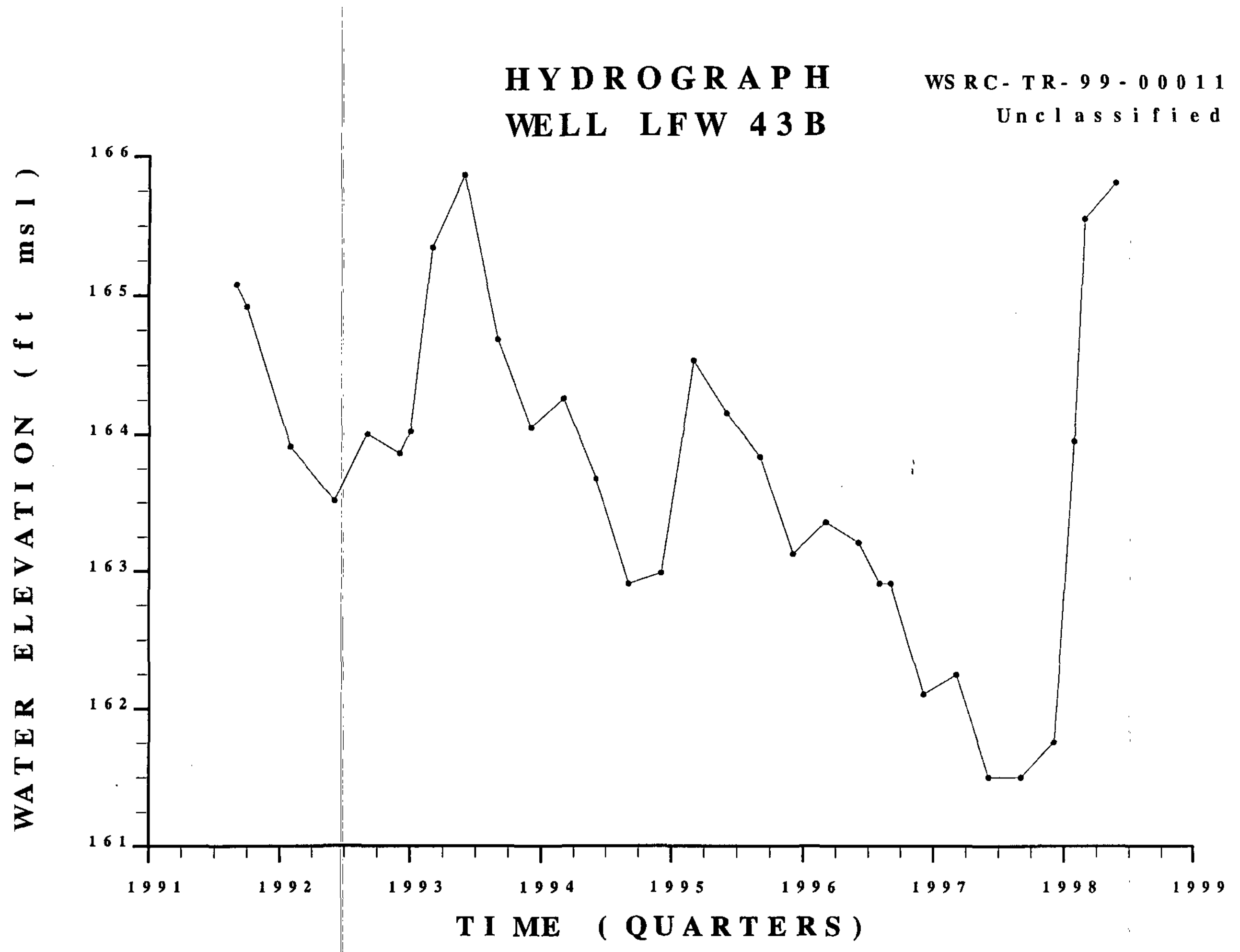

G 
H Y D R O G R A P H

WELL LFW $43 \mathrm{C}$
WS R C - T R - $99-00011$ Unc l a s s i i ed

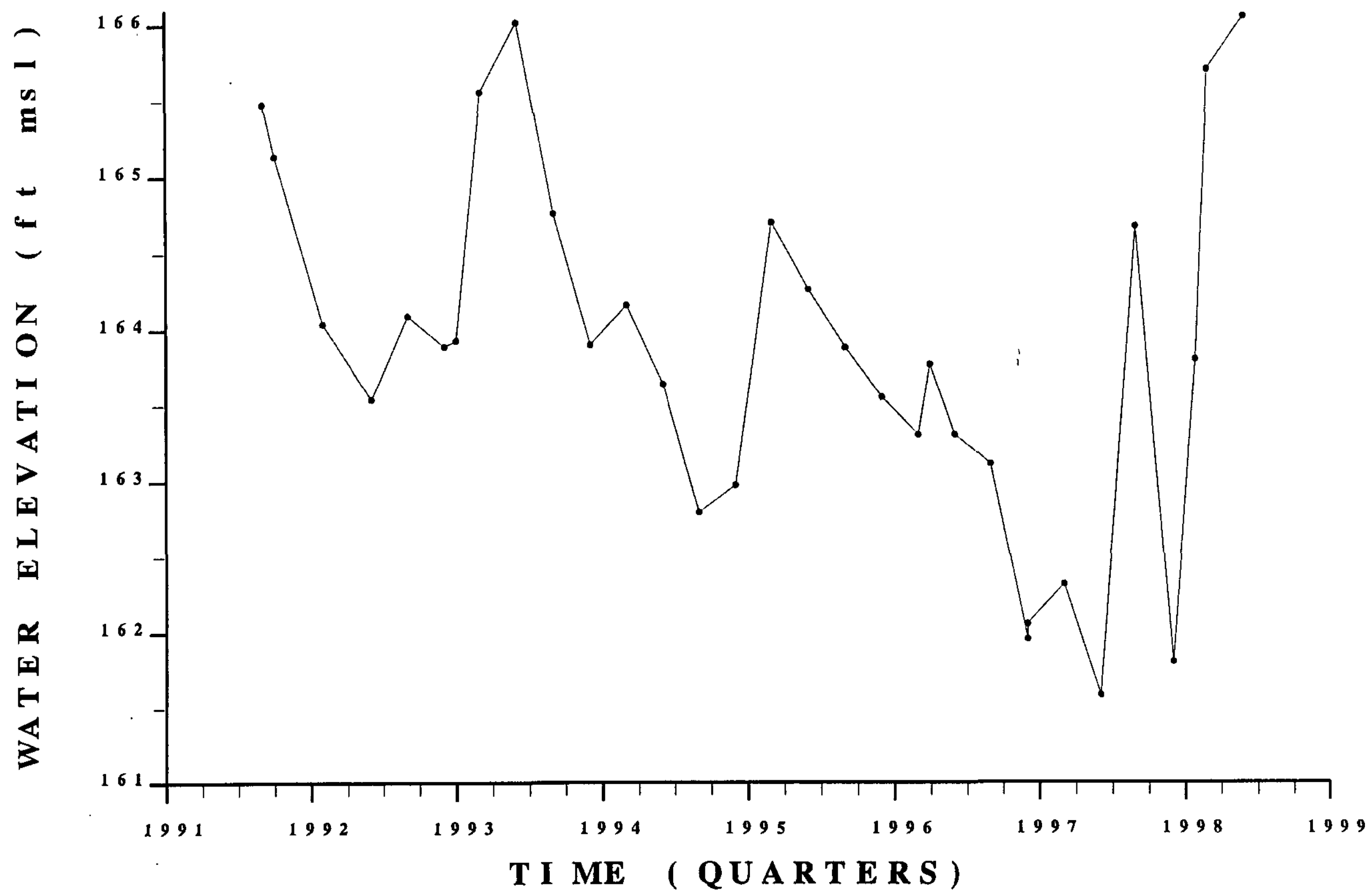

S a n i t a r v La n d f i 1 l

Fourt h Ouarter. $1998 \quad \& \quad 1998$ Summar 
H Y D R O G R A P H

WELL LFW 43 D
WS R C - T R - $99-00011$ Unclassified

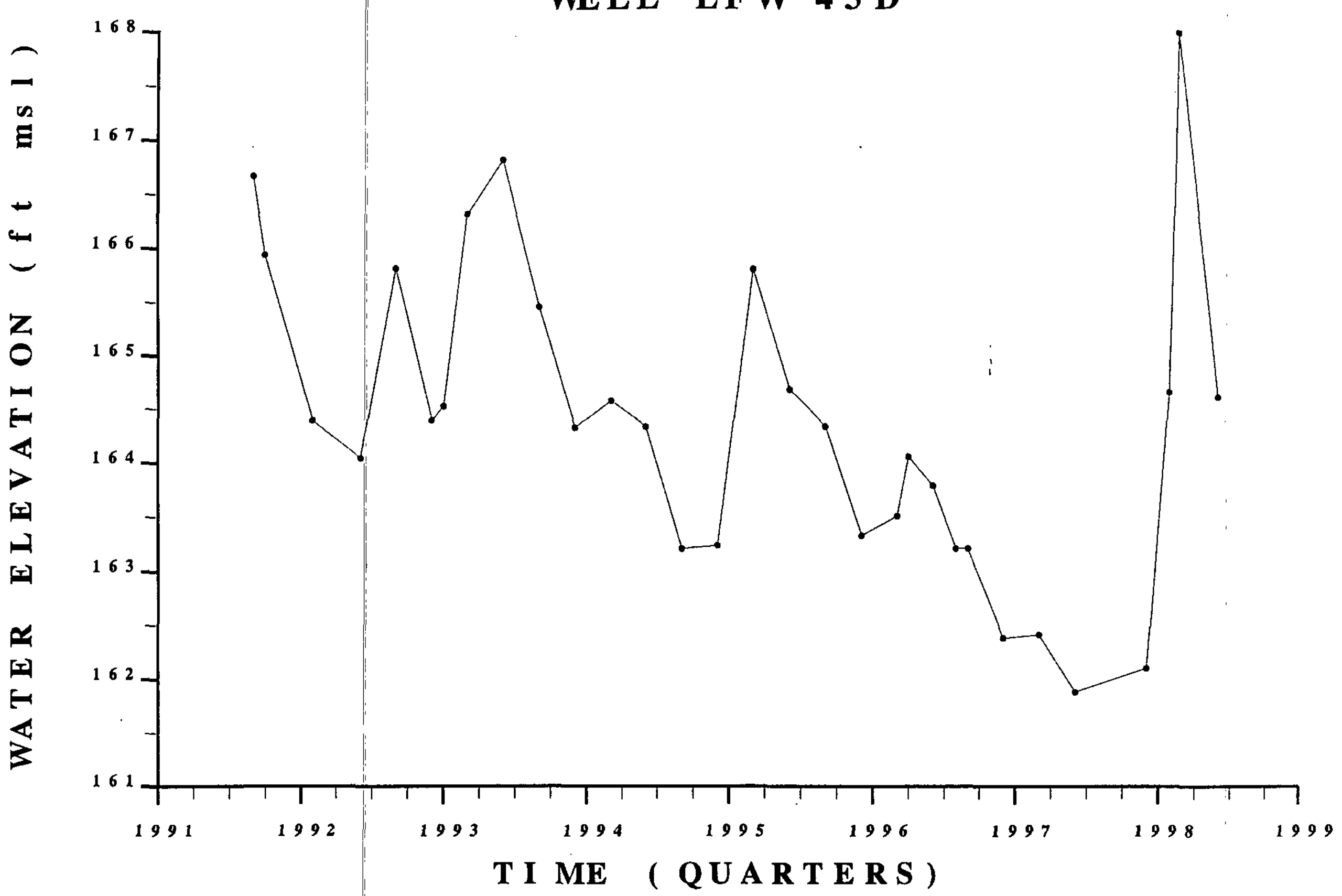

Sa nTa a 
HY D R O G R A P H WELL LFW 44 D

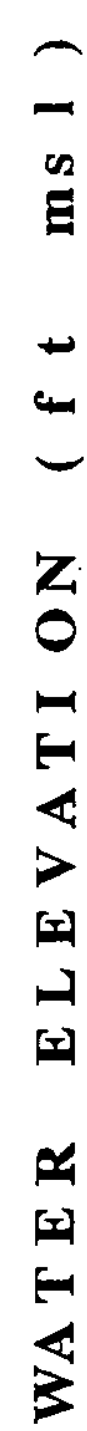

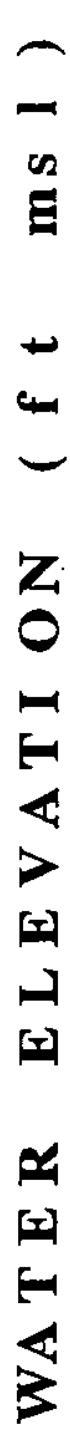

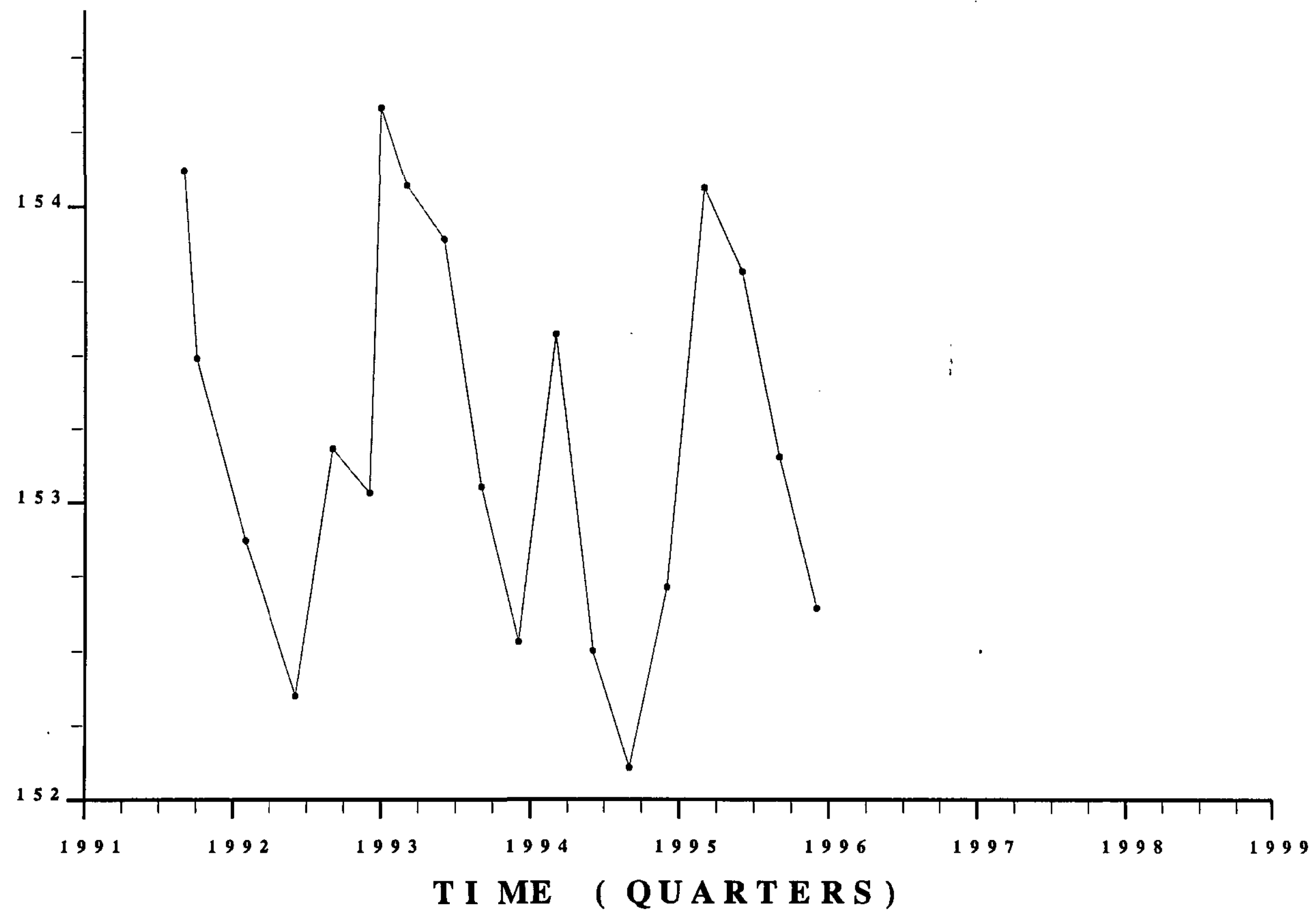




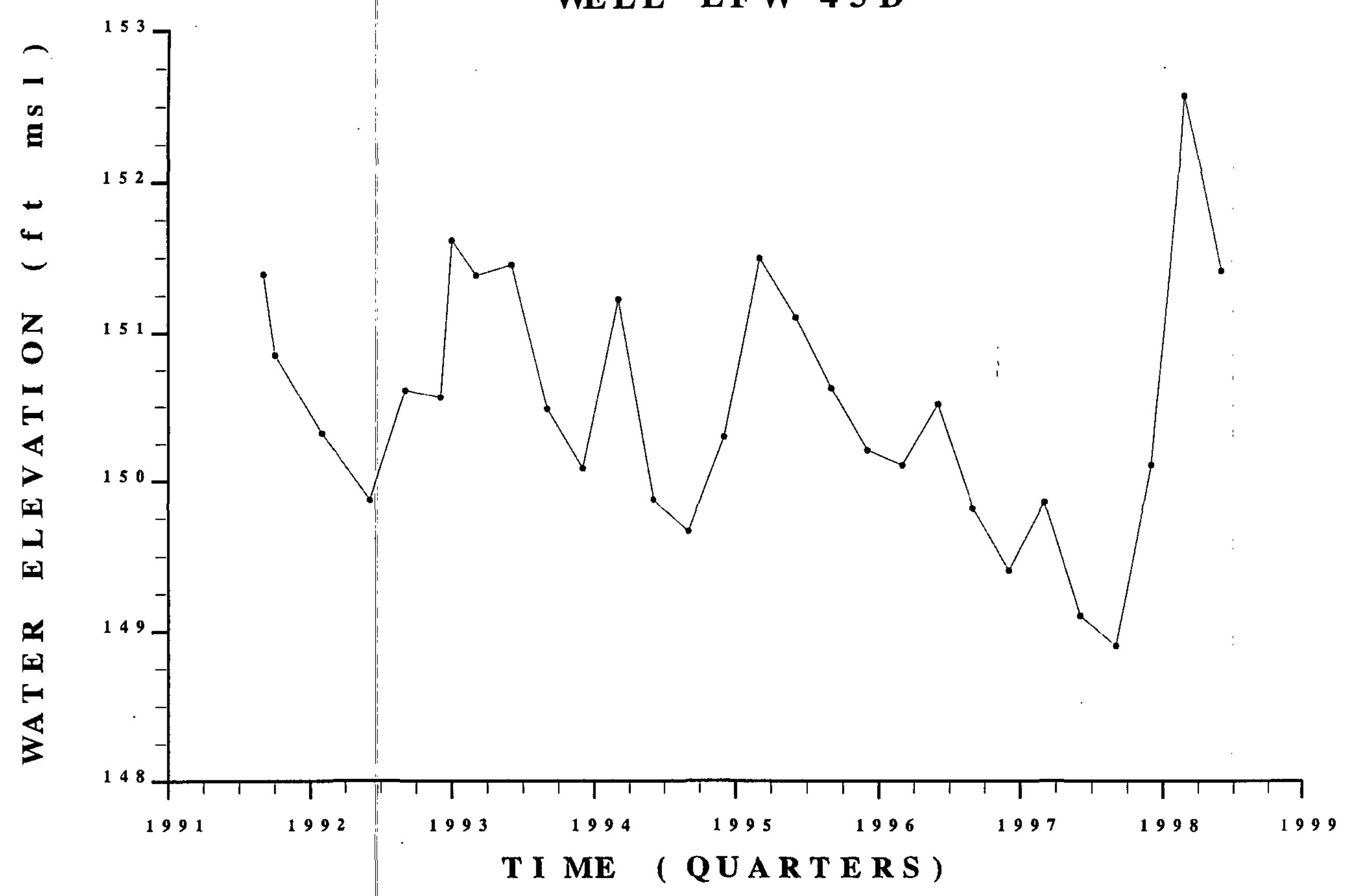

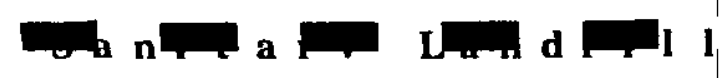

H Y D R O G R A P H WELL LFW 45 D
WS R C-TR- $99-00011$ Unc l a s s i fied 
HY D R O G R A P H WELL LF W 46 D

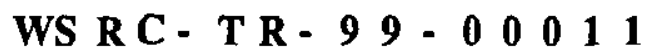
Unc l a s s i f i ed

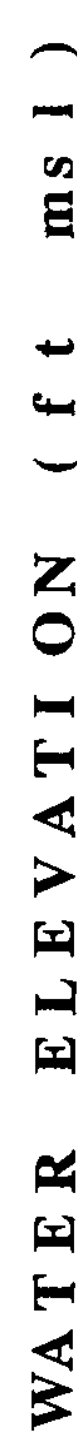

$\bar{a}$<smiles>CCC</smiles>

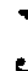

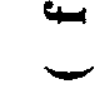

$z$

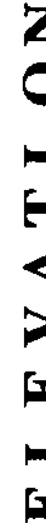

4

되

3

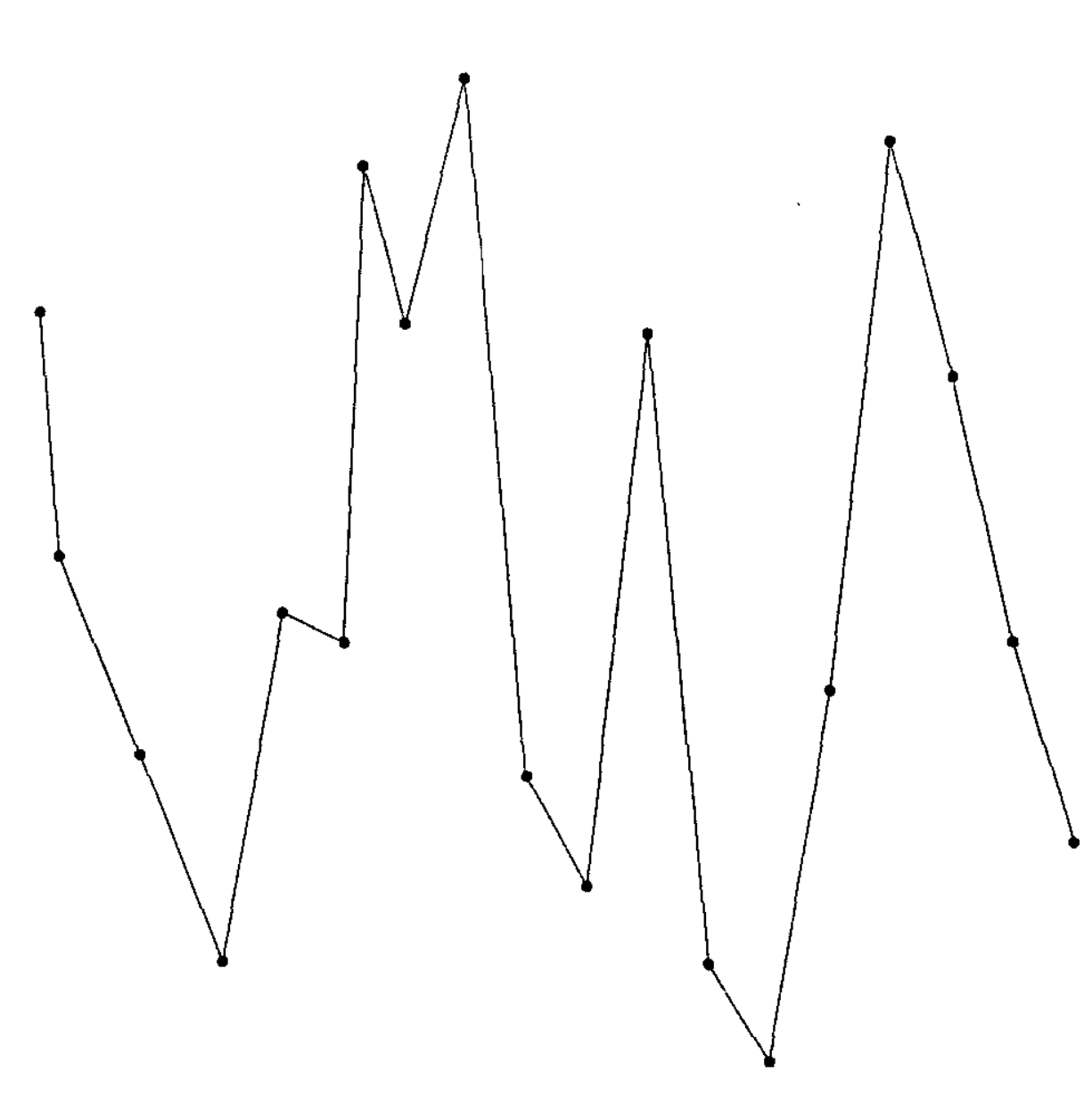

148

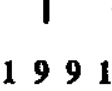

1993

T I ME ( Q UAR T E R S )

Sanitar $\mathbf{l}$ Landfill

Fourt h Ouarter.

1998

\& 1998

$S u m m a r v$ 


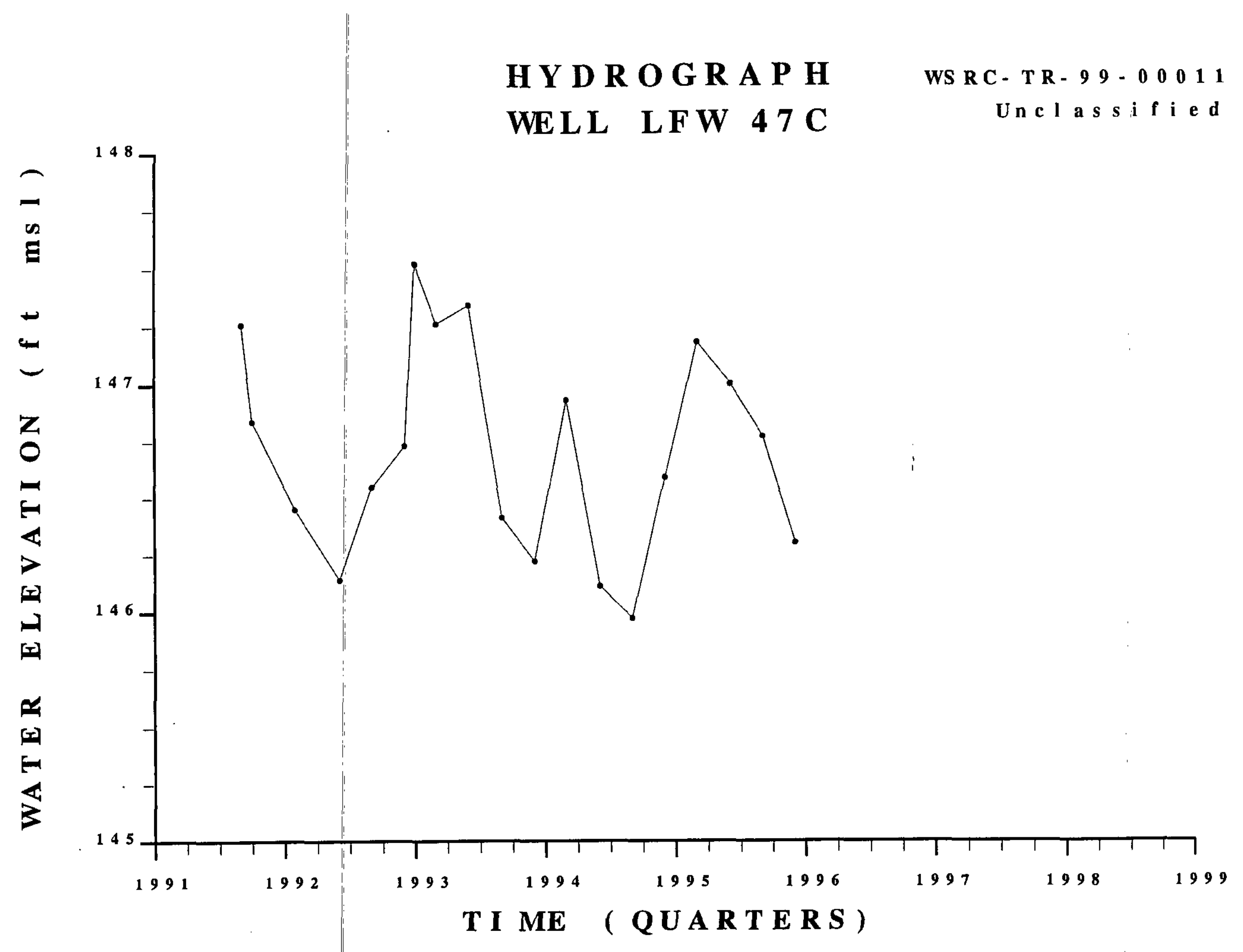

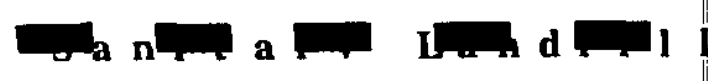


H Y D R O G R A P H

WELL LFW 47 D
WS R C - T R - $99-000011$

Unc l a s s i f i e d

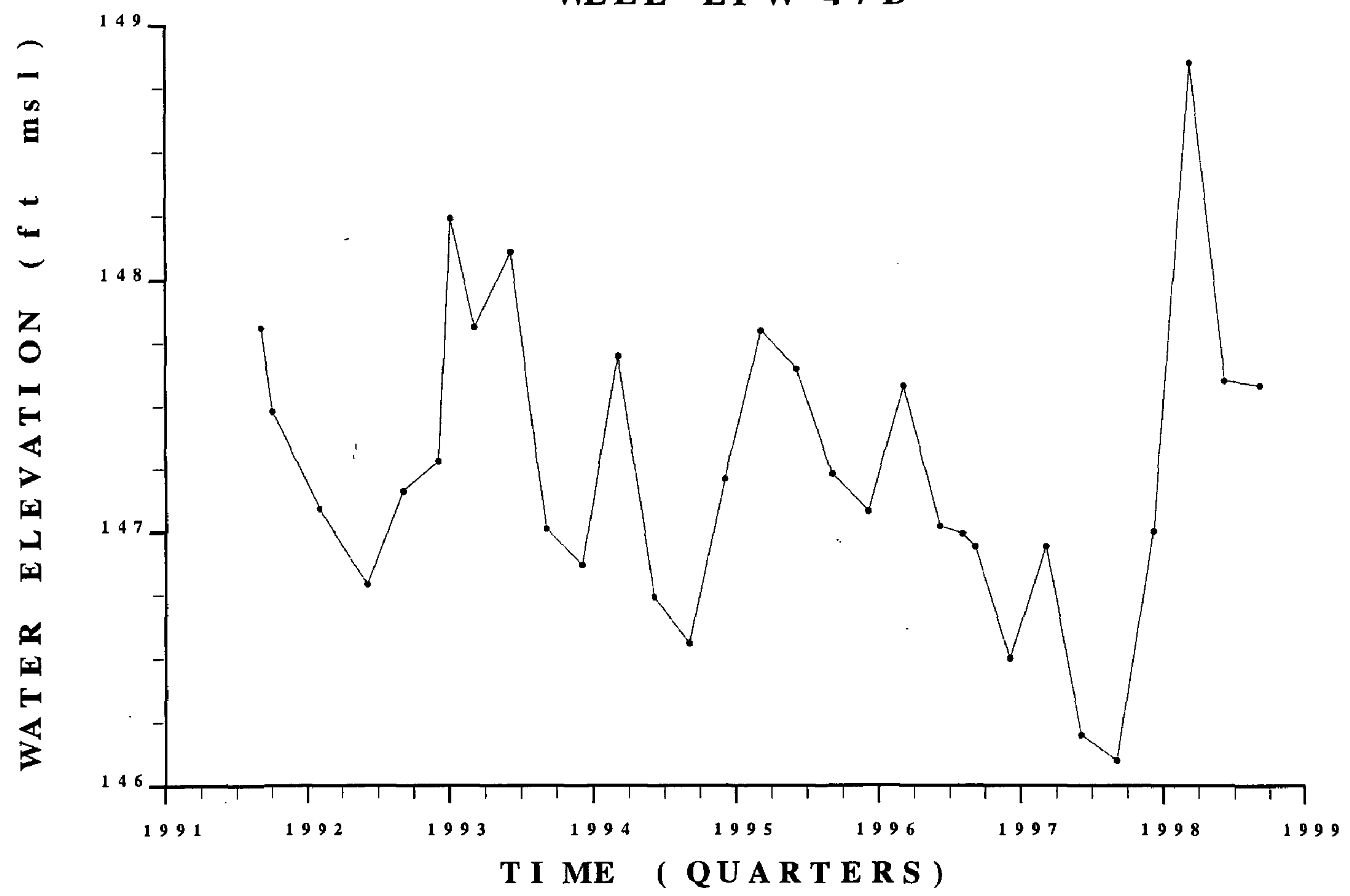

S a n i t a r v L a nd f i l I

Fourth Ou a r t r

1998

\& 1998

S u mar v 


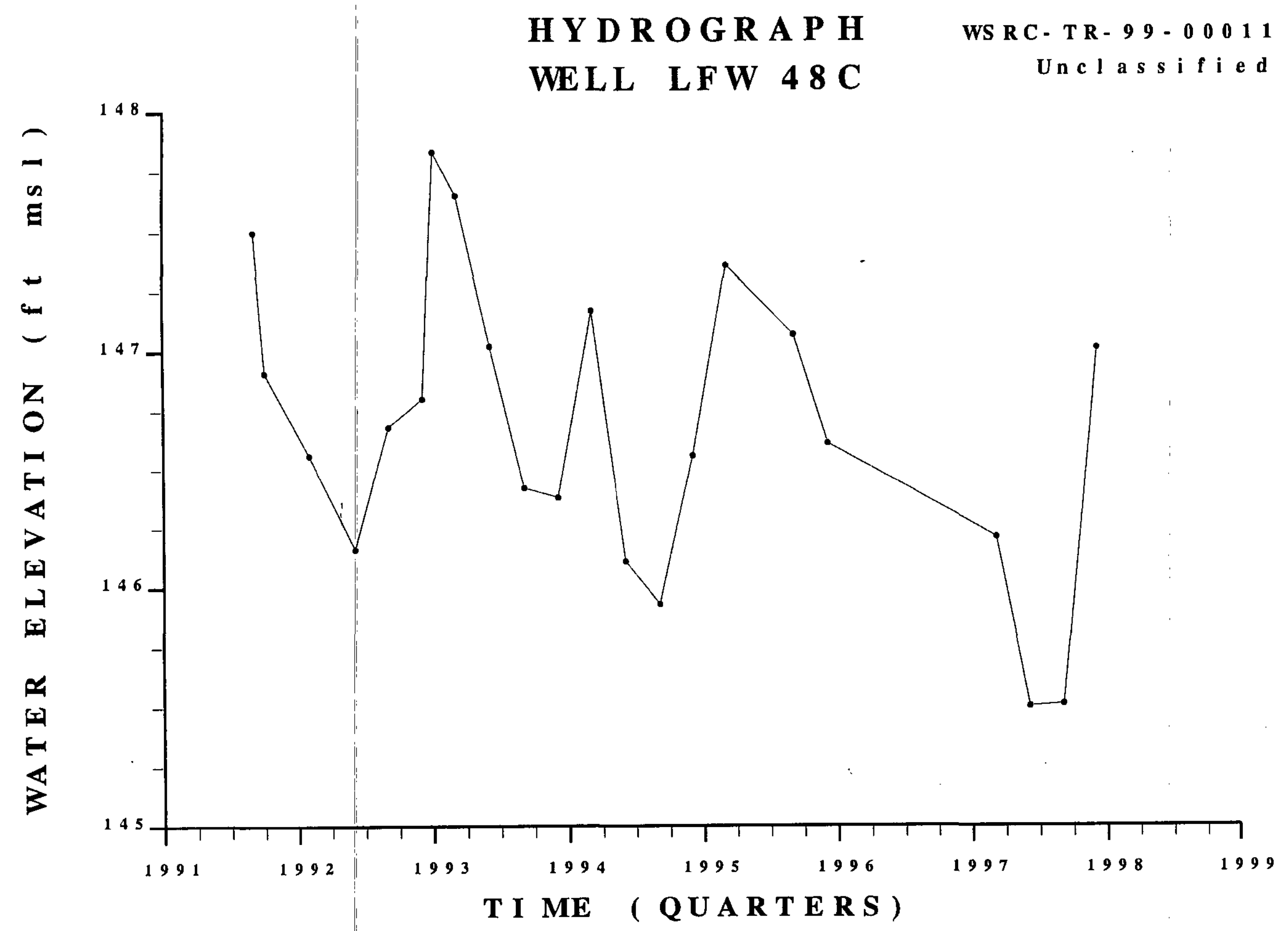

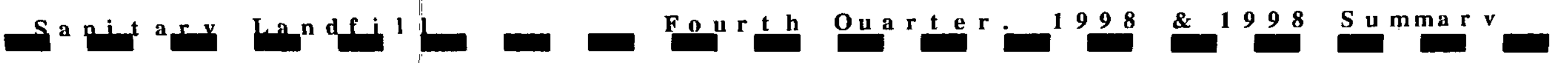


H Y D R O G R A P H

\section{WELL LFW 48 D}

WS R C - TR - 99-0 0011

Unc I a s s i f i e d

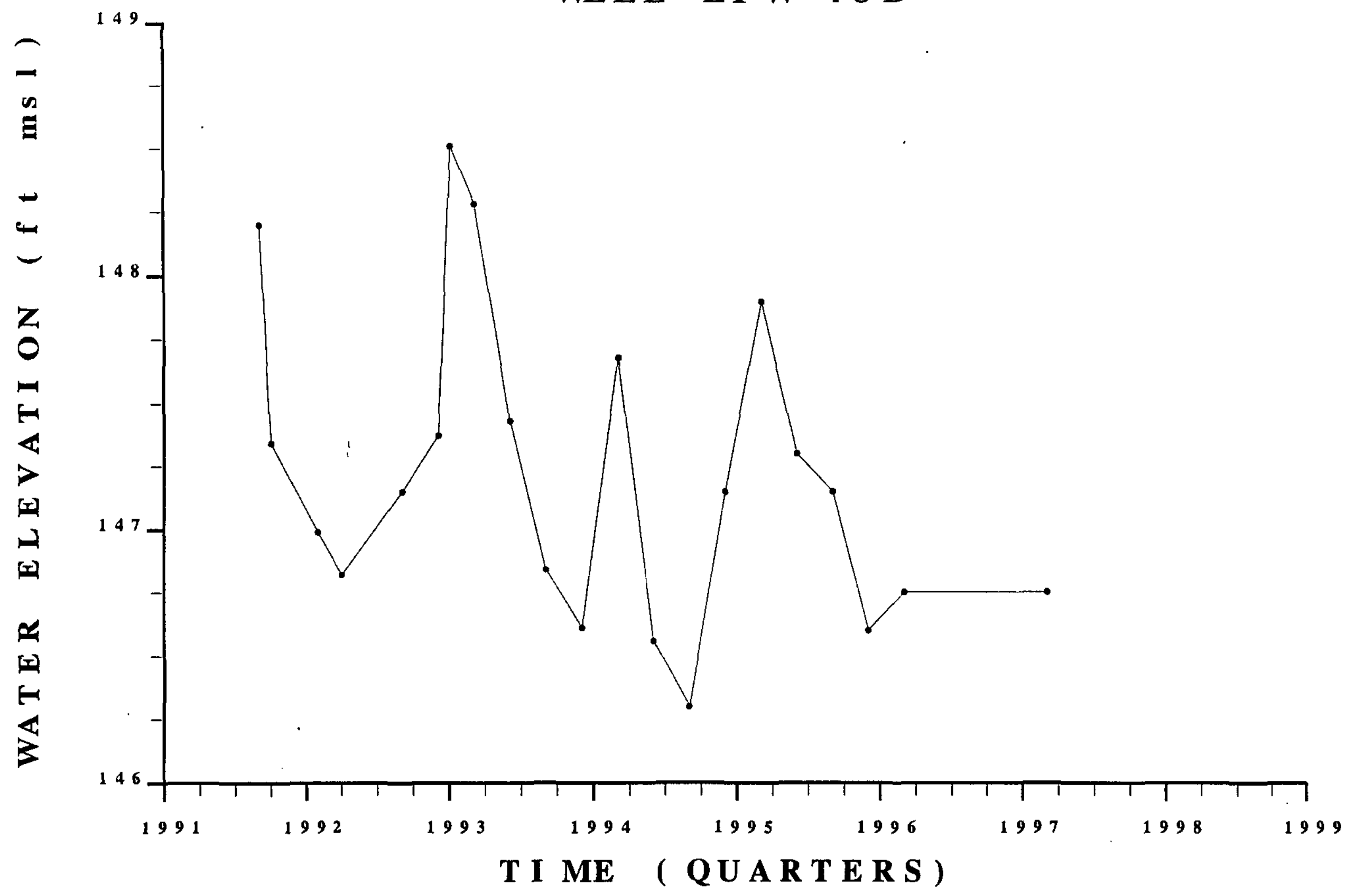

S a n i t a r v L a n d f i l l

Fourth Ouarter.

\& 1998

S u mmar v 
H Y D R O G R A P H

WELL LFW 55 C
WS R C - T R - $99-00011$

Unc l a s s i f i e d

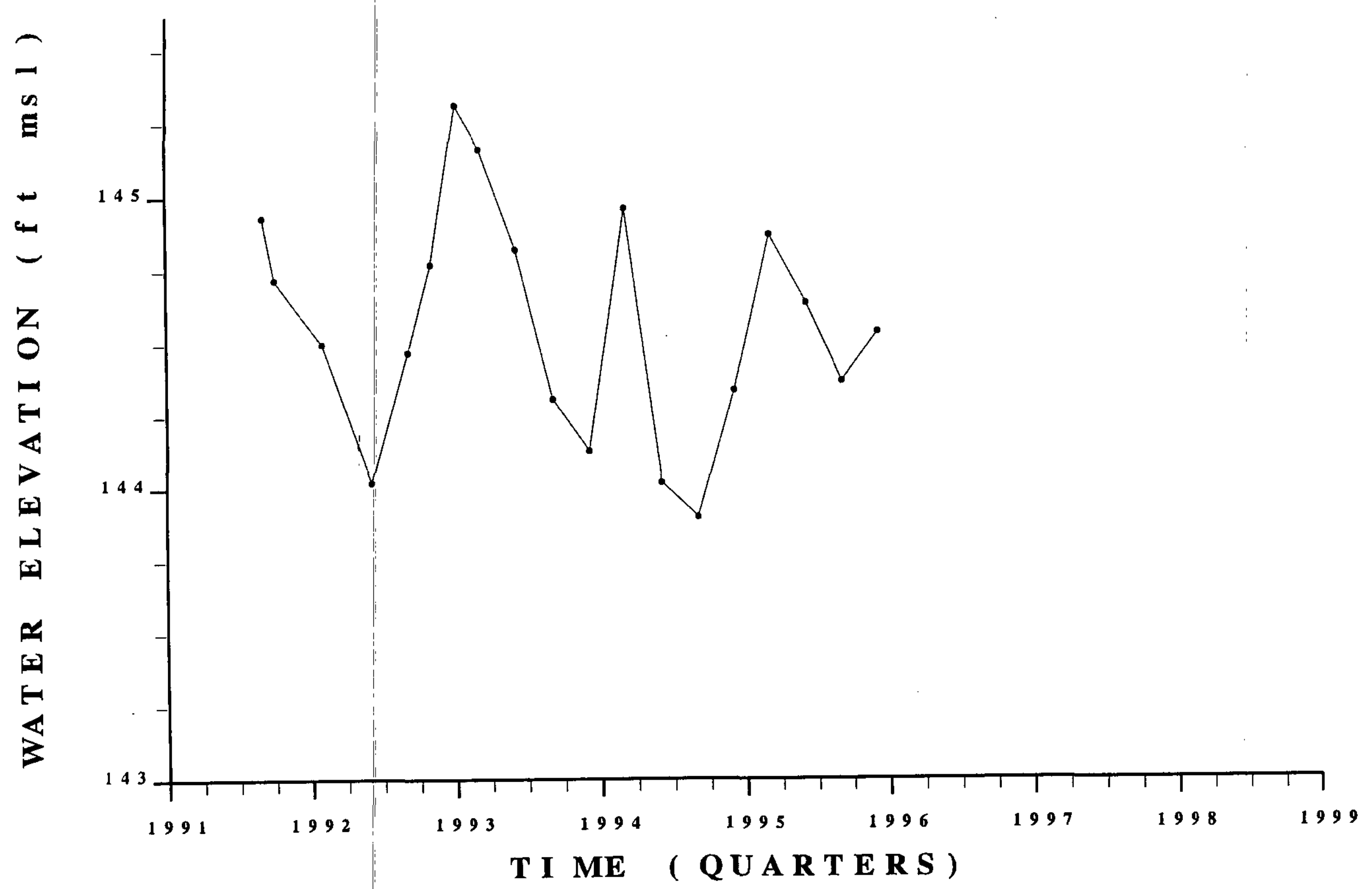

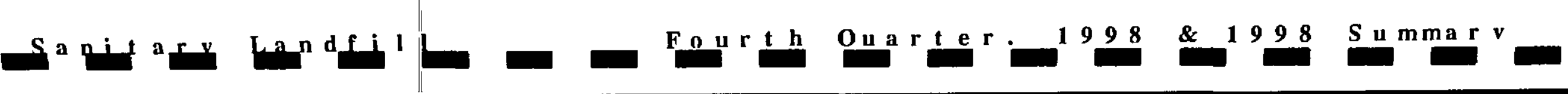


H Y D R O G R A P H WELL LFW 55 D
WS R C - T R - $99-0$ - 00011 Unc l a s s i f i e d

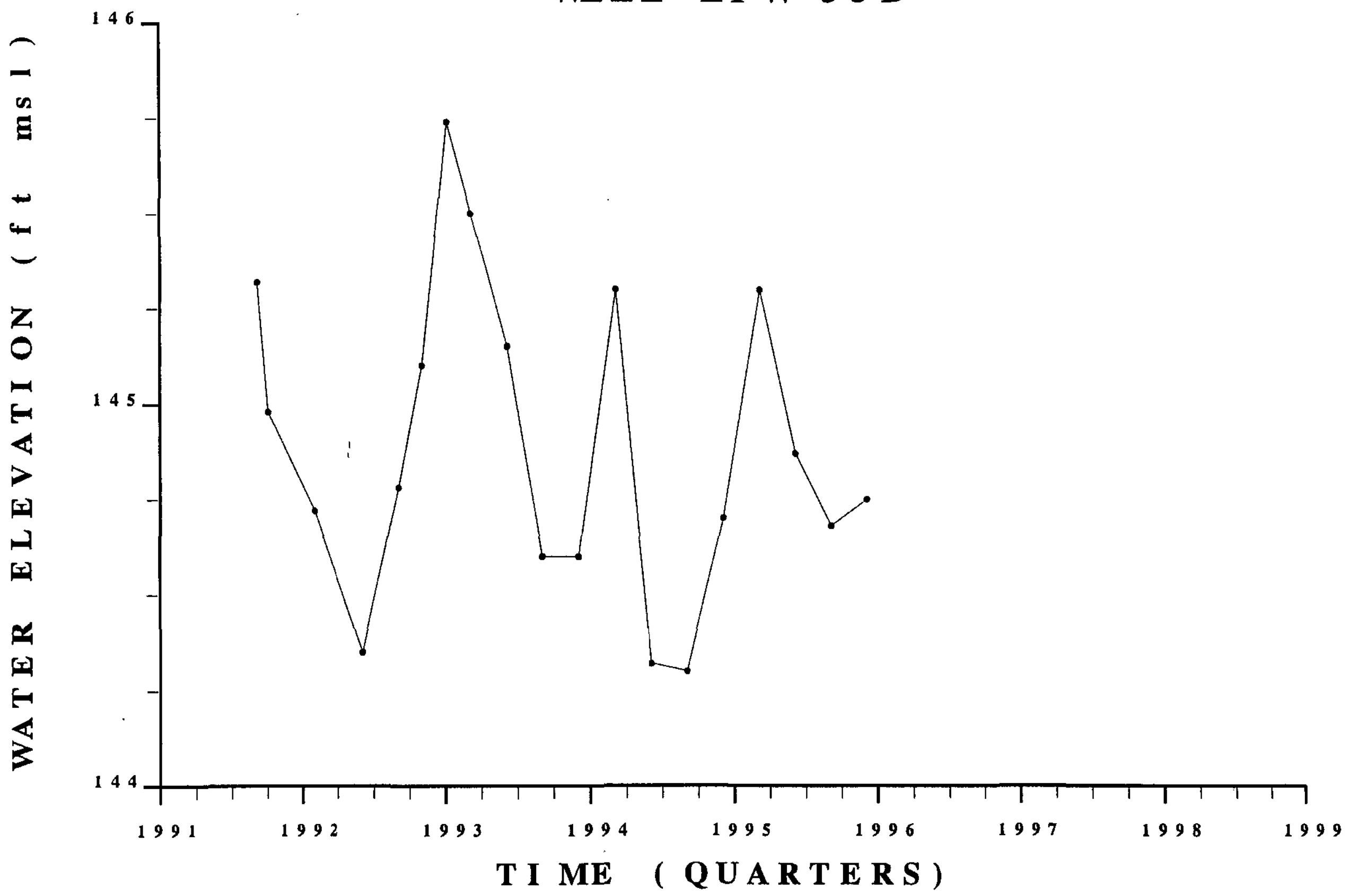

S a n i t a r v La nd f i l l

Fourth Ouarter. 1998 \& 1998 Summar 


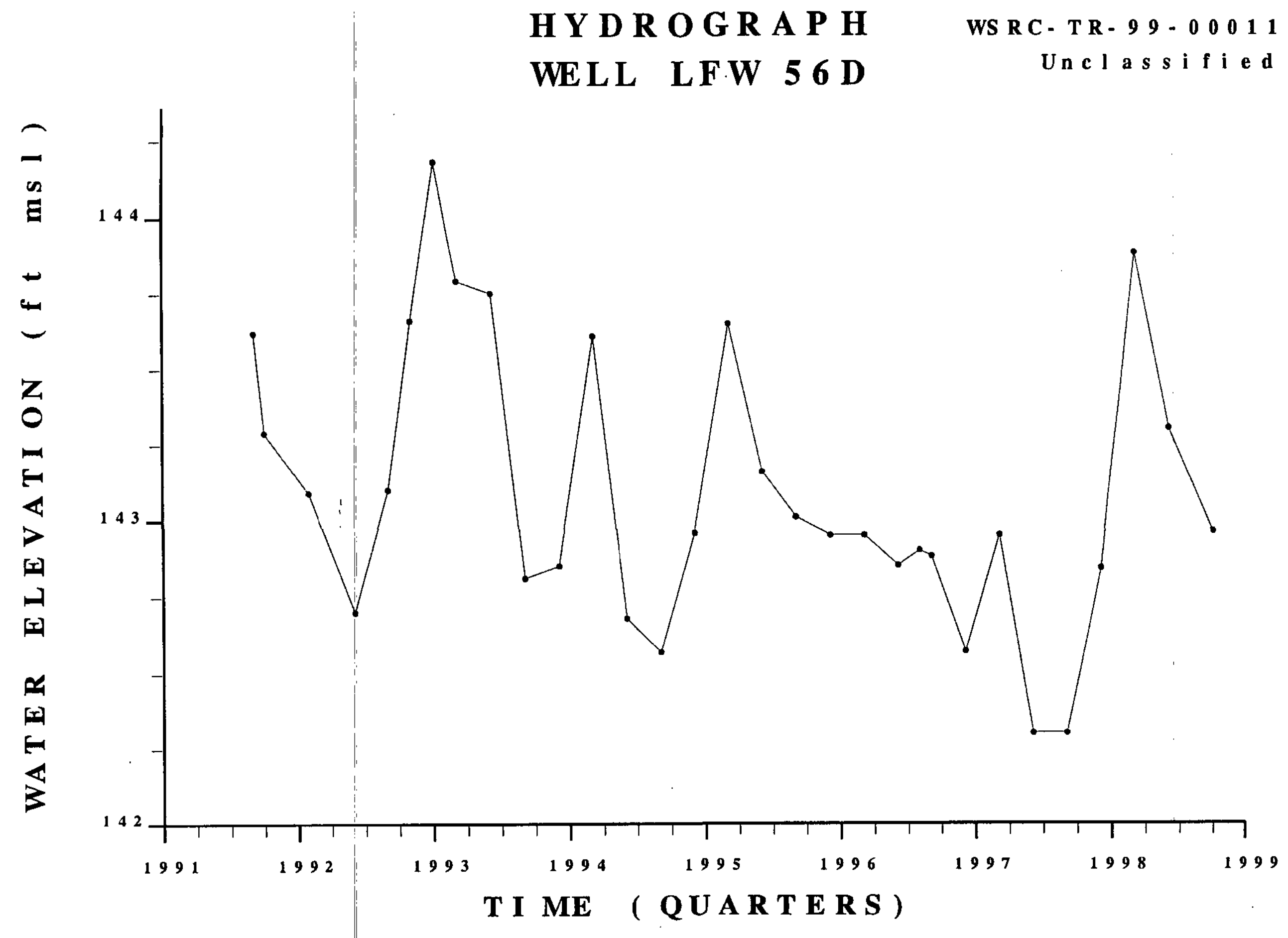

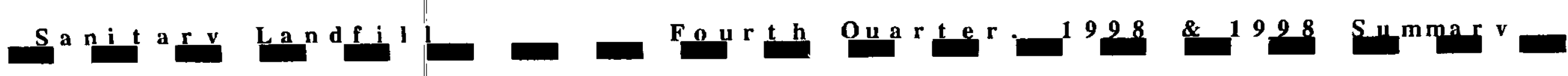


H Y D R O G R A P H

WELL L F W 57 B
WS R C - T R - $99-00011$

Unc l a s s i f i ed

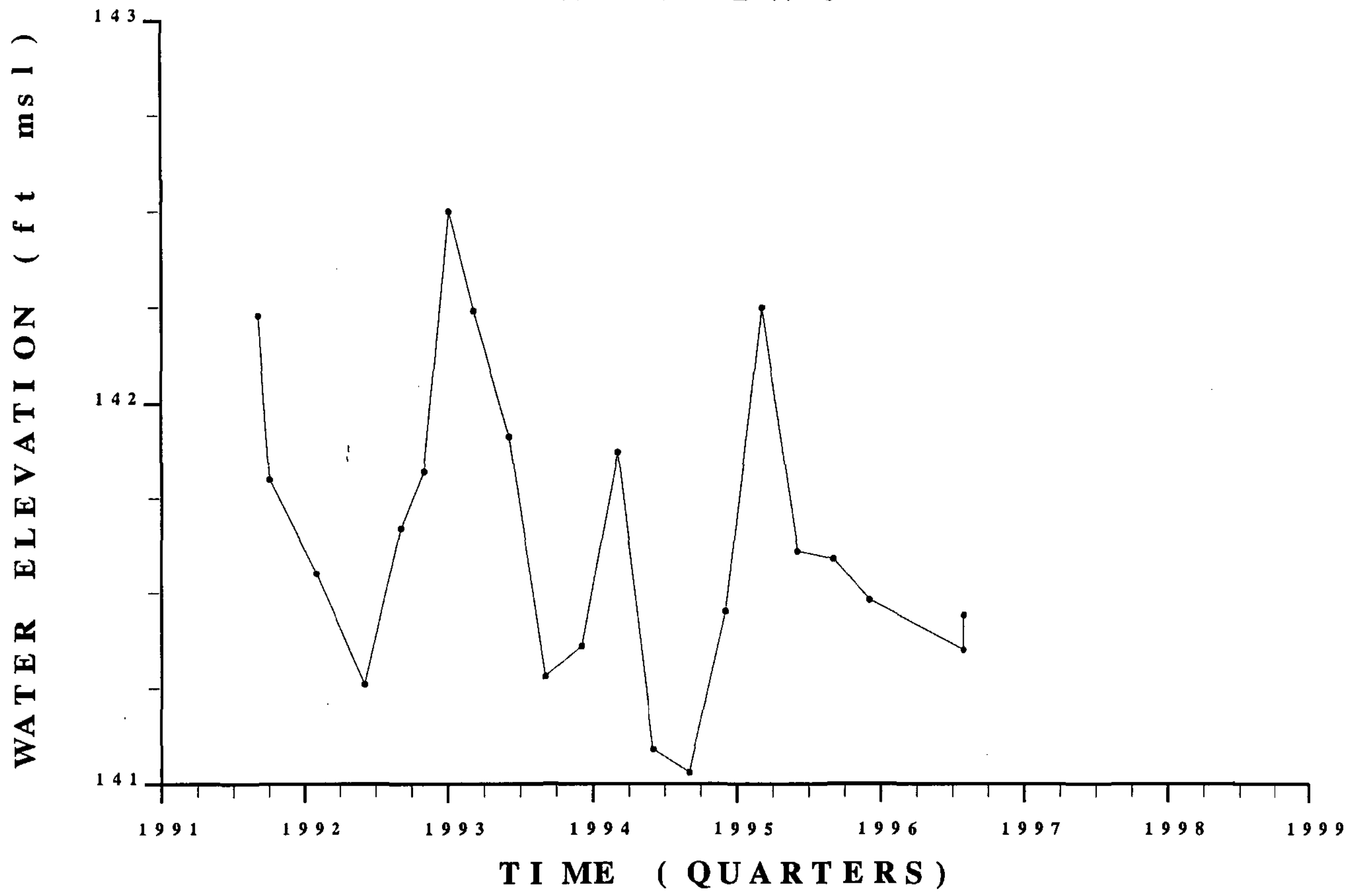

Sanitar $\mathrm{v}$ Landfill

Fourth ouarter.

\& 1998 S u mar v 


\section{HY D R O G R A P H}

WELL LFW 57 C
WS R C - T R - $99-00011$ Unc l a s s i f i ed

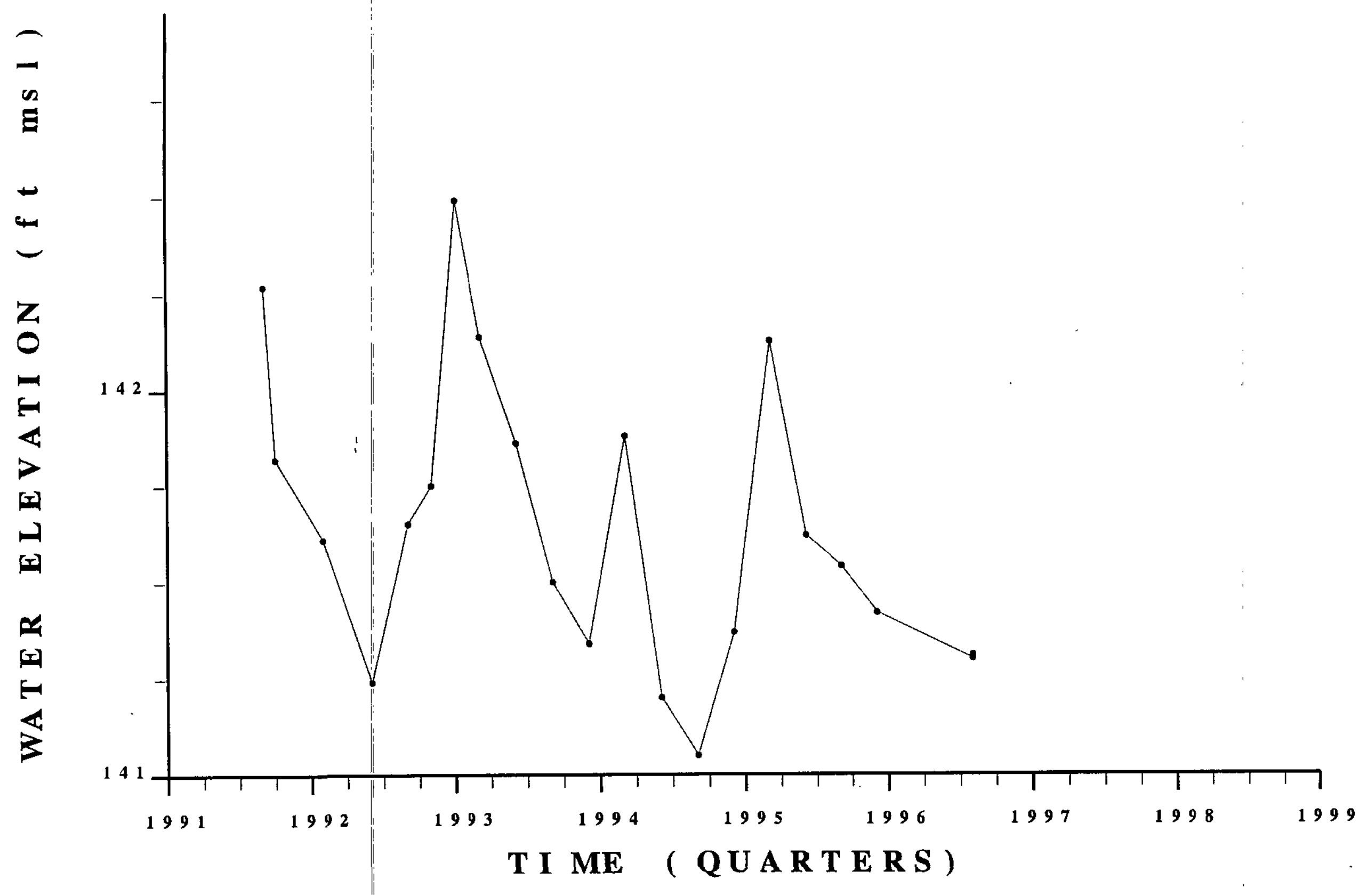

Sa n i t a r v La nd f i l l

Fourth Ouarter.

1998

\& 1998

S u m ma r v 
HYDR O G R P H WELL LFW 57 D
WS R C - T R - $99-00011$ Unc l a s s i f i ed

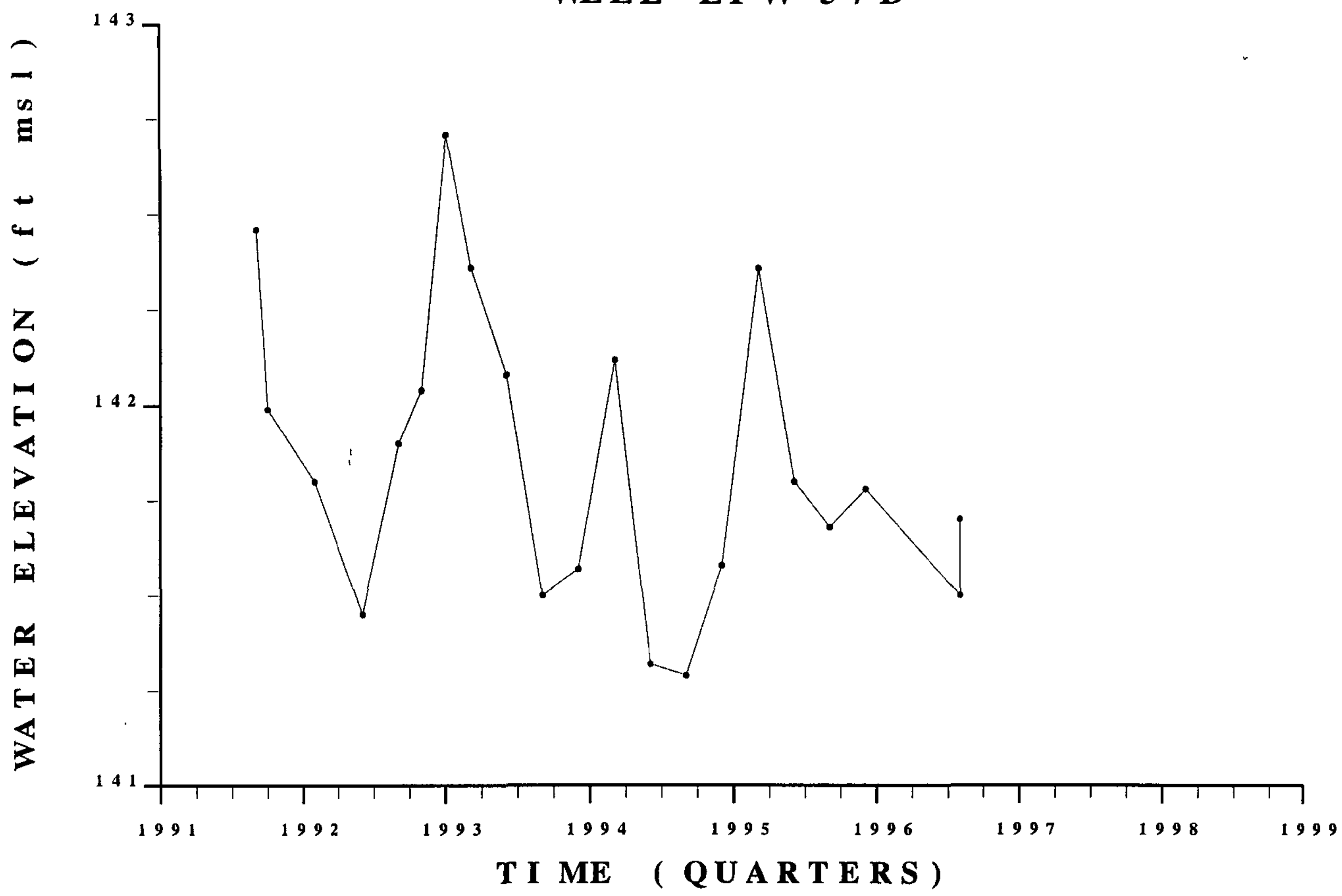




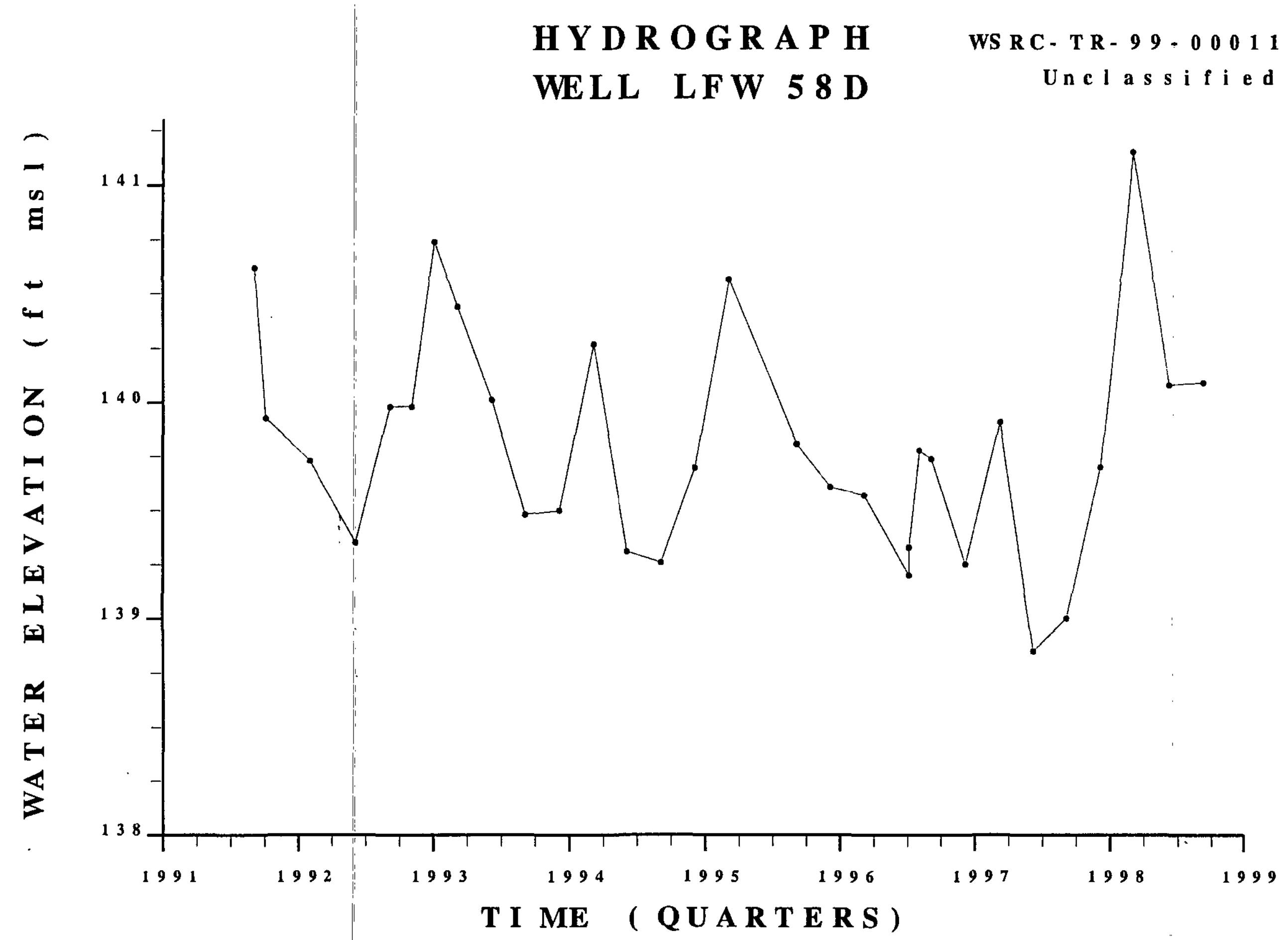

S a n i t a.r v L a n d f i l l Fourt h Ou a r t e r. 

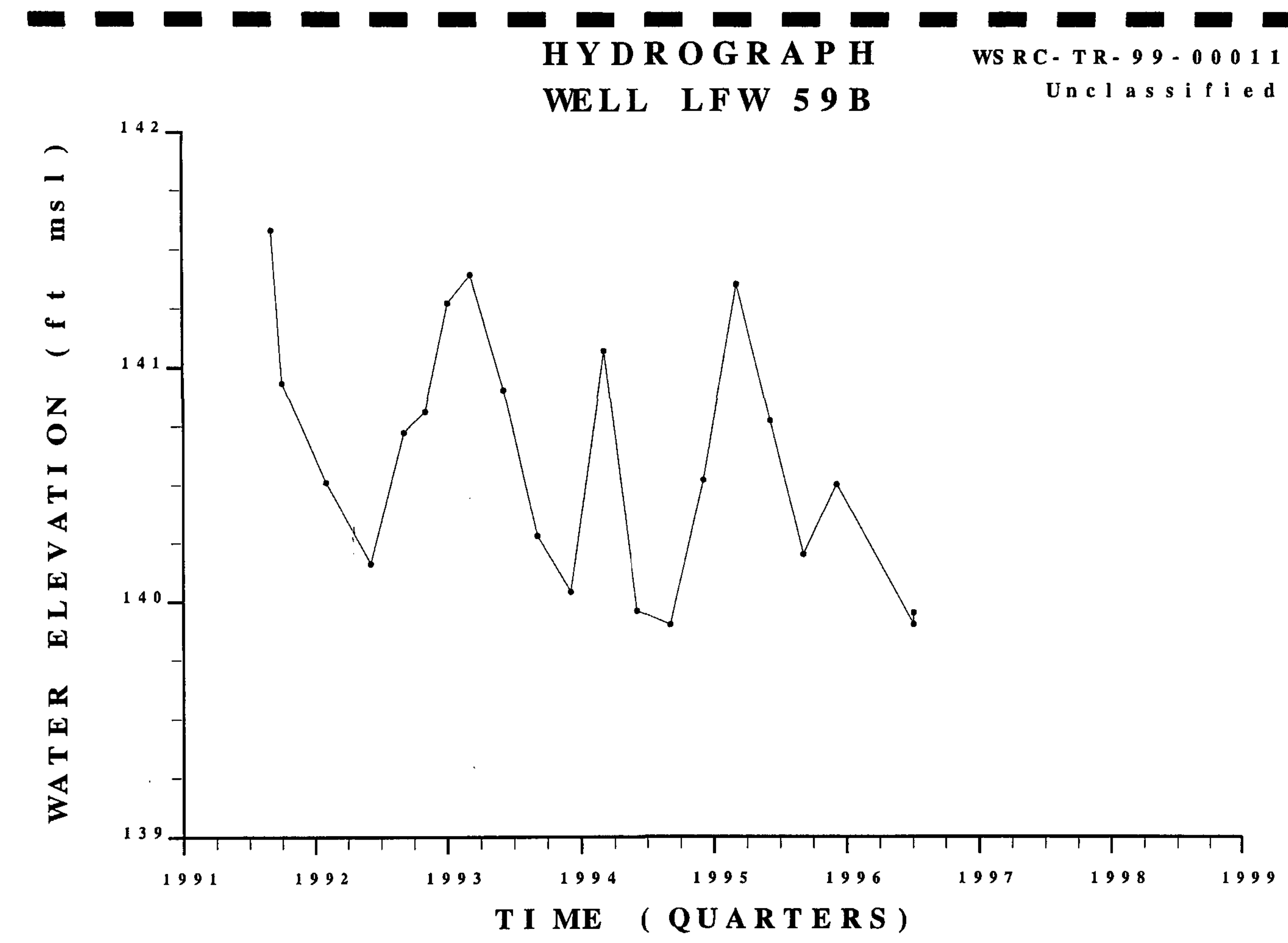

S a n i t a r v La ndf i l I

Fourth Ouater.

$\& 1998$ S u mmar 
H Y D R O G R A P H WELL LFW 59 C
WS R C - T R - $99-00011$ Unc l a s s i f i e d

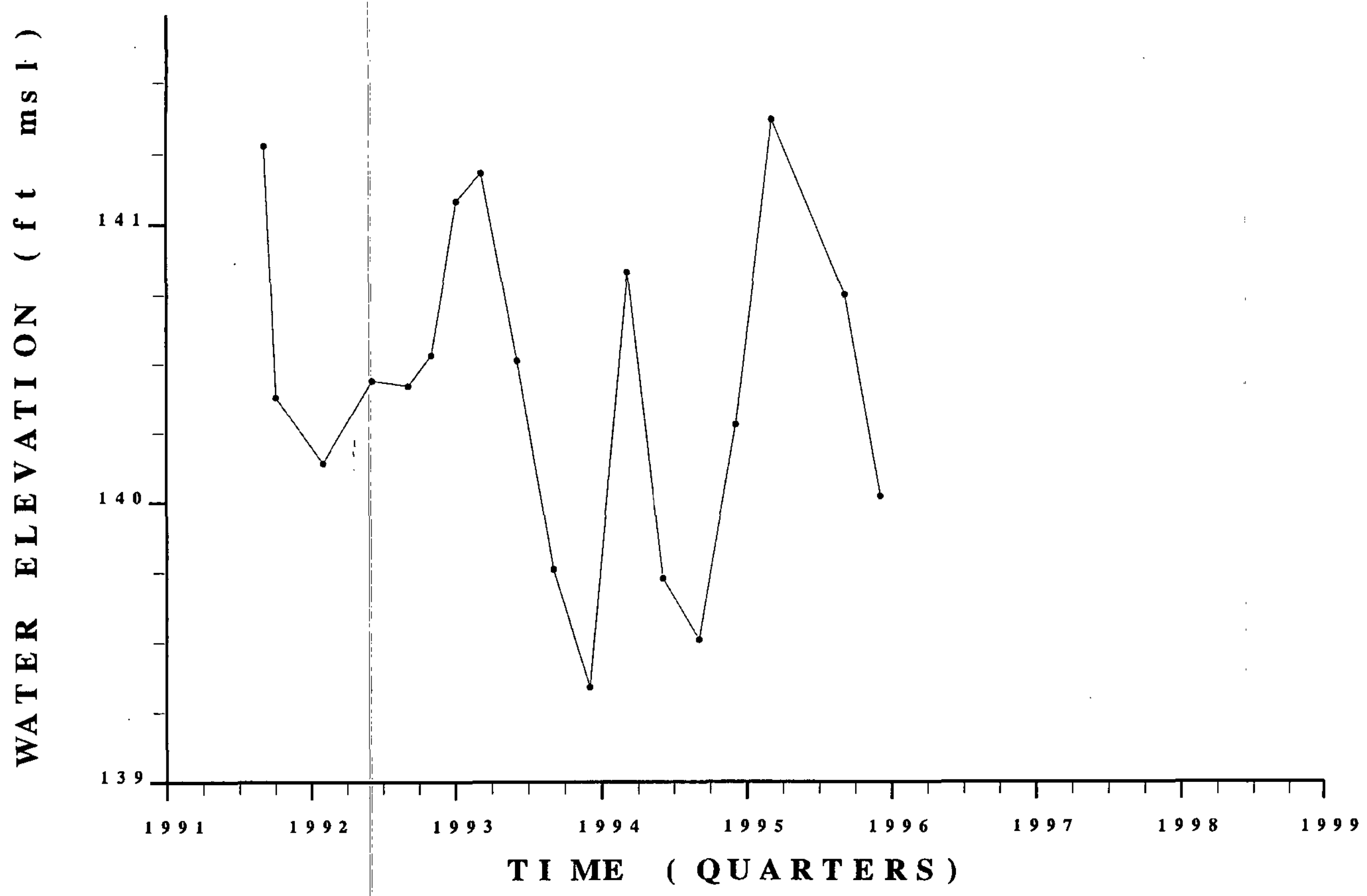

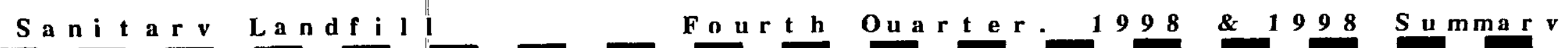


H Y D R O G R A P H WELL LFW 59 D
WS R C - T R-9 $9-00011$

Unc l as si f i e d

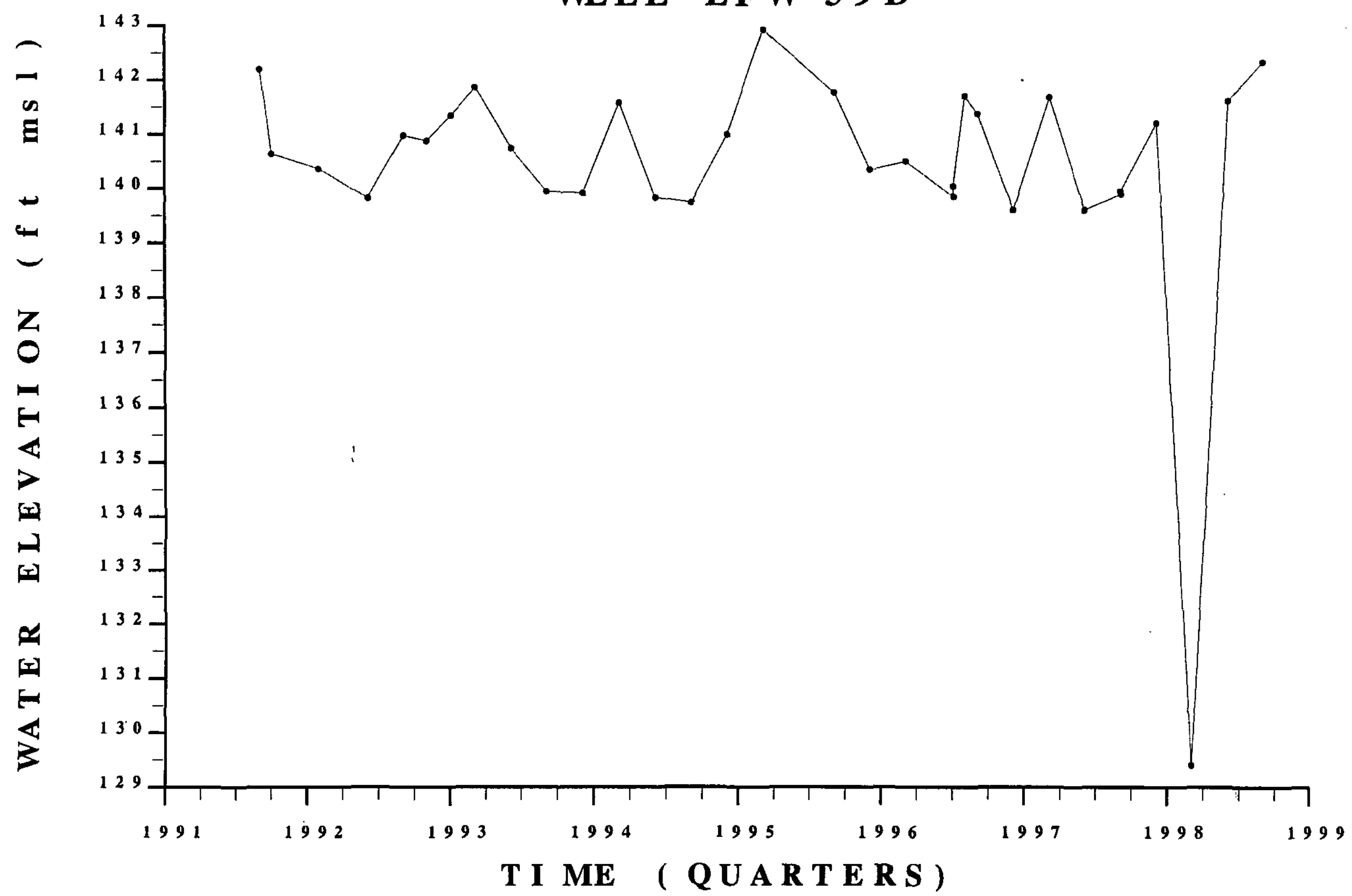

S a n i t a r v La n d f i l l

Fourth ouarter.

1998

\& 1998 S u mmar v 


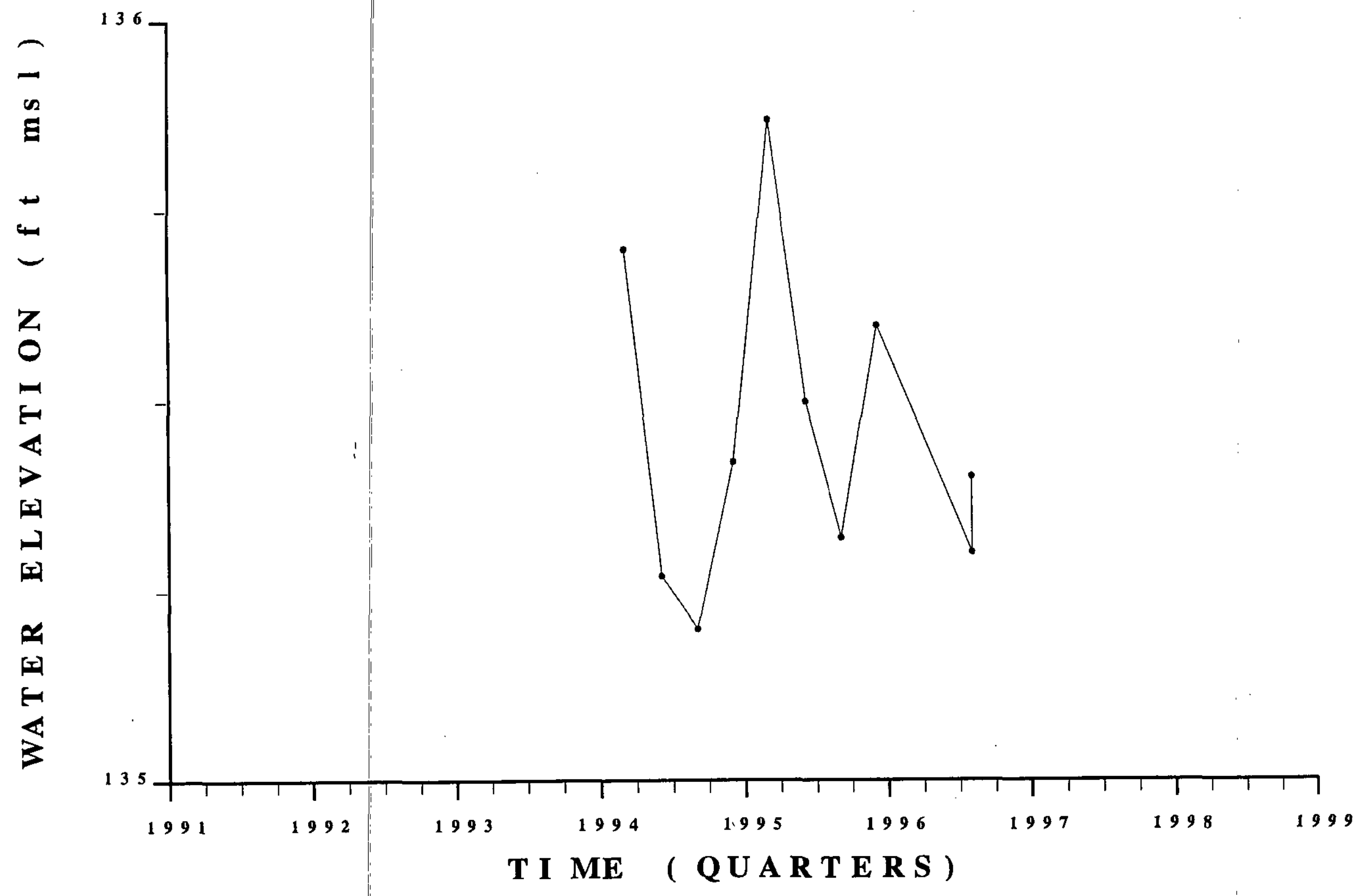

H Y D R O G R A P H WELL LFW 60 B
WS R C - T R - $990-000011$ Unc las s i f i ed 
H Y D R O G R A P H WELL LFW 60 C
WS RC-TR- $99-000011$

Unc l a s s i f i ed

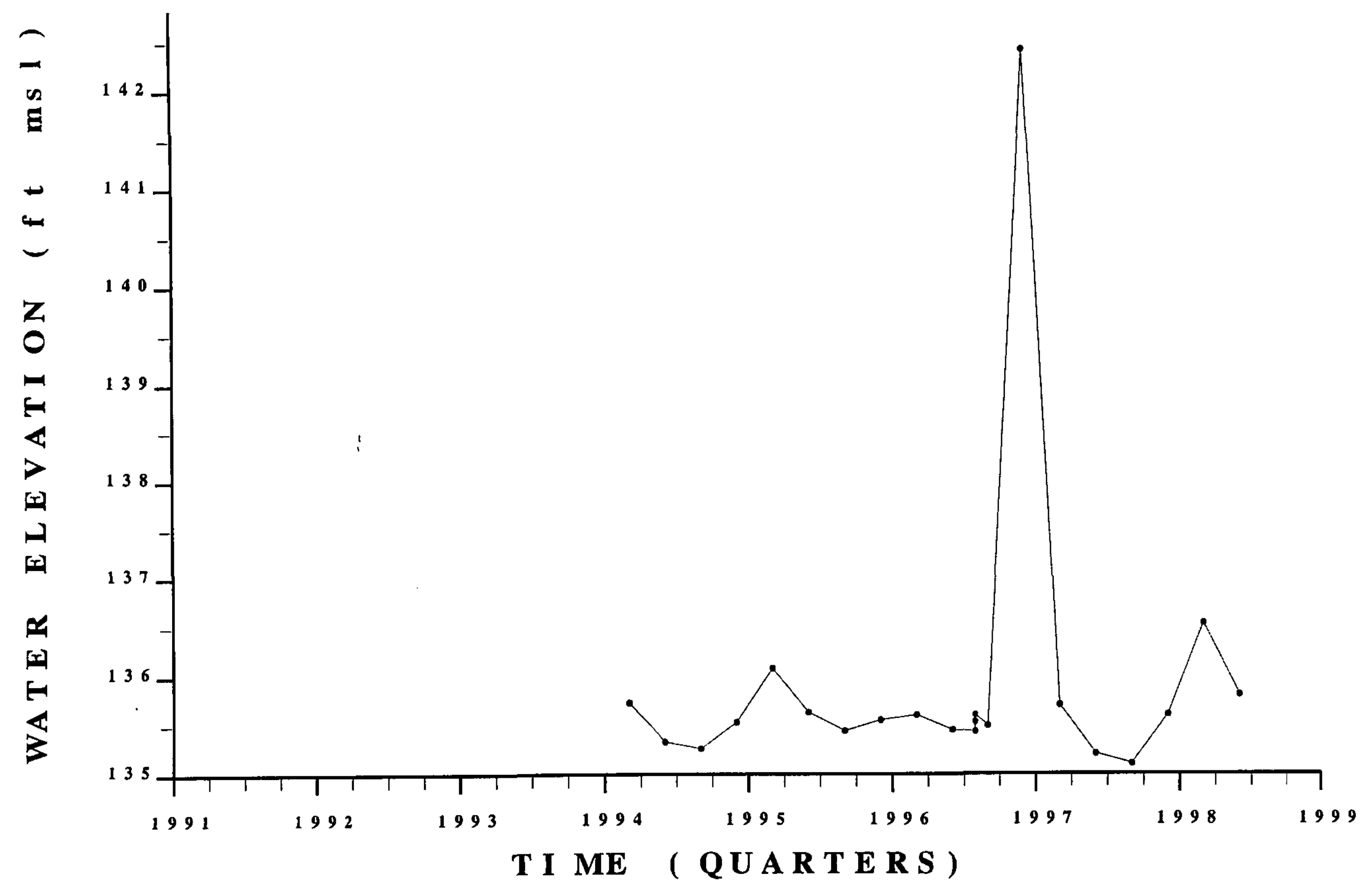

$S$ ani tar $r$ Landf i I I

Fourth Ouarter.

1998

\& 1998

S u mma r v 
H Y D R O G R A P H WELL LFW 60 D
WS RC- TR - $99-00011$ Unc las $s$ if i ed

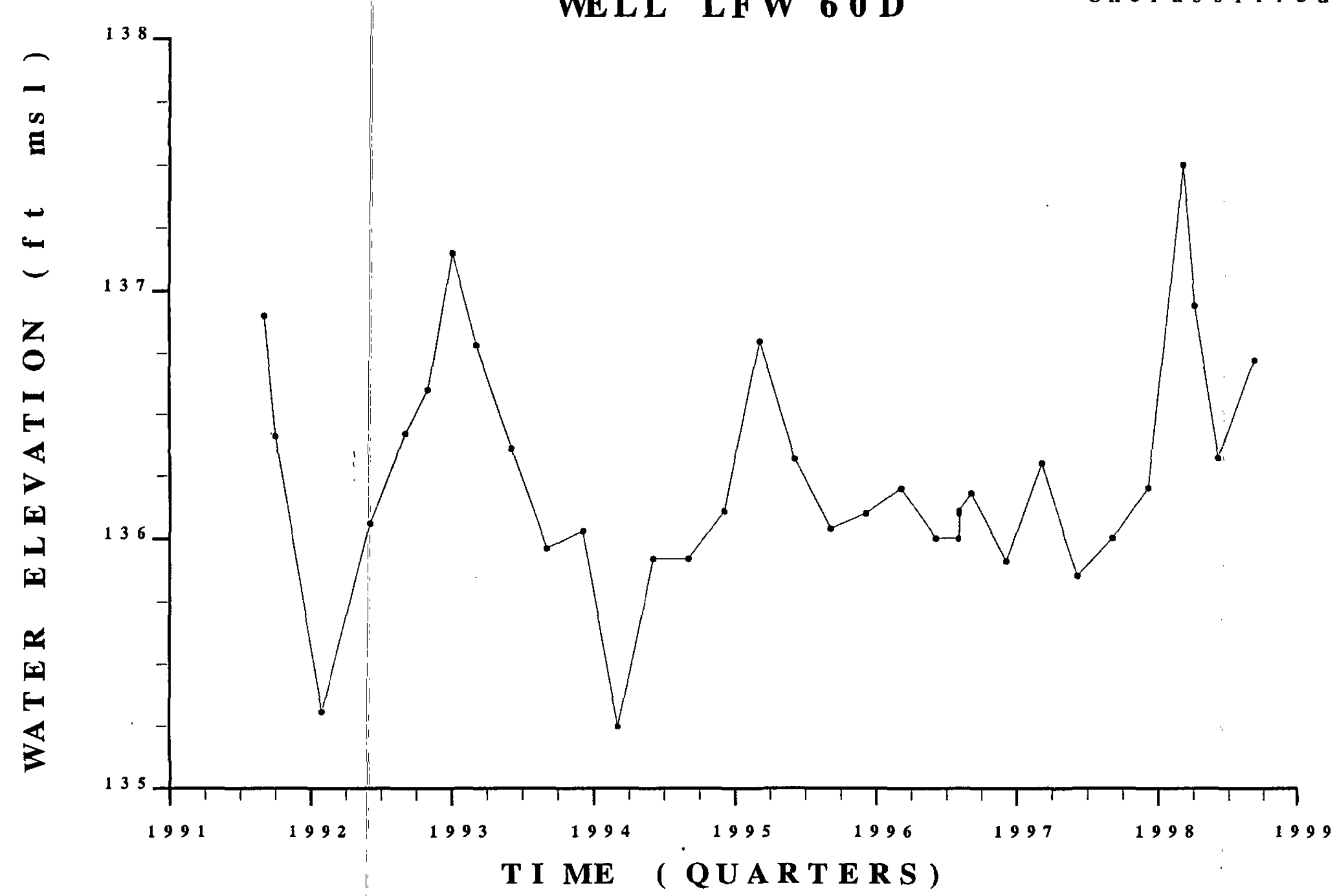


H Y D R O G R A P H WELL LFW $61 \mathrm{C}$
WS R C - T R- $99-00011$ Unc l a s s i f i e d

20
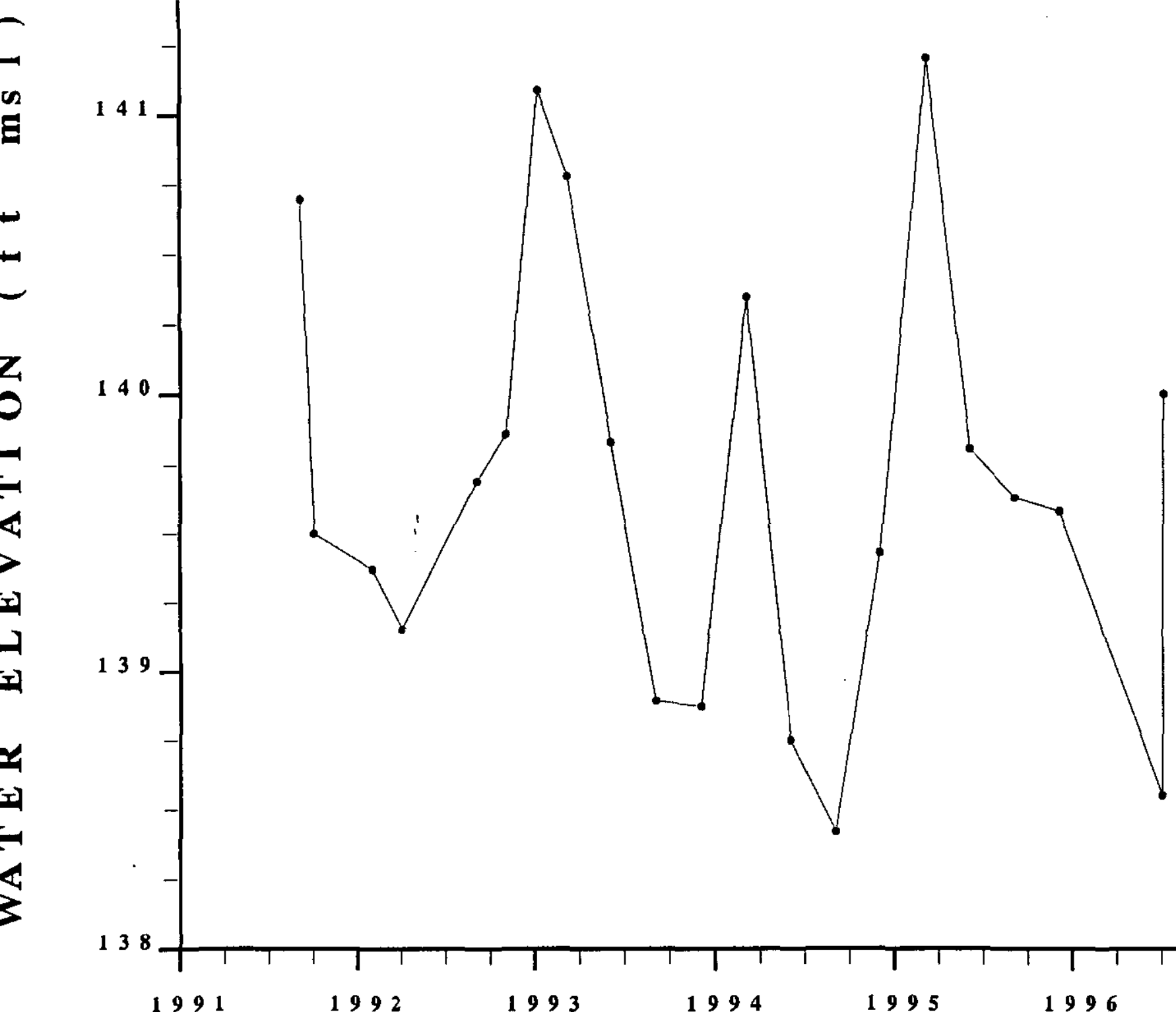

1991

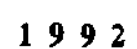

T I ME

( QUART E R S ) 
H Y D R O G R A P H WELL LFW 61 D
WS R C - T R - 99-00011 Unc l a s s i f i ed




H Y D R O G R A P H WELL LFW 62 B
WS R C - T R - $99-00011$ Unc l as s i f i ed

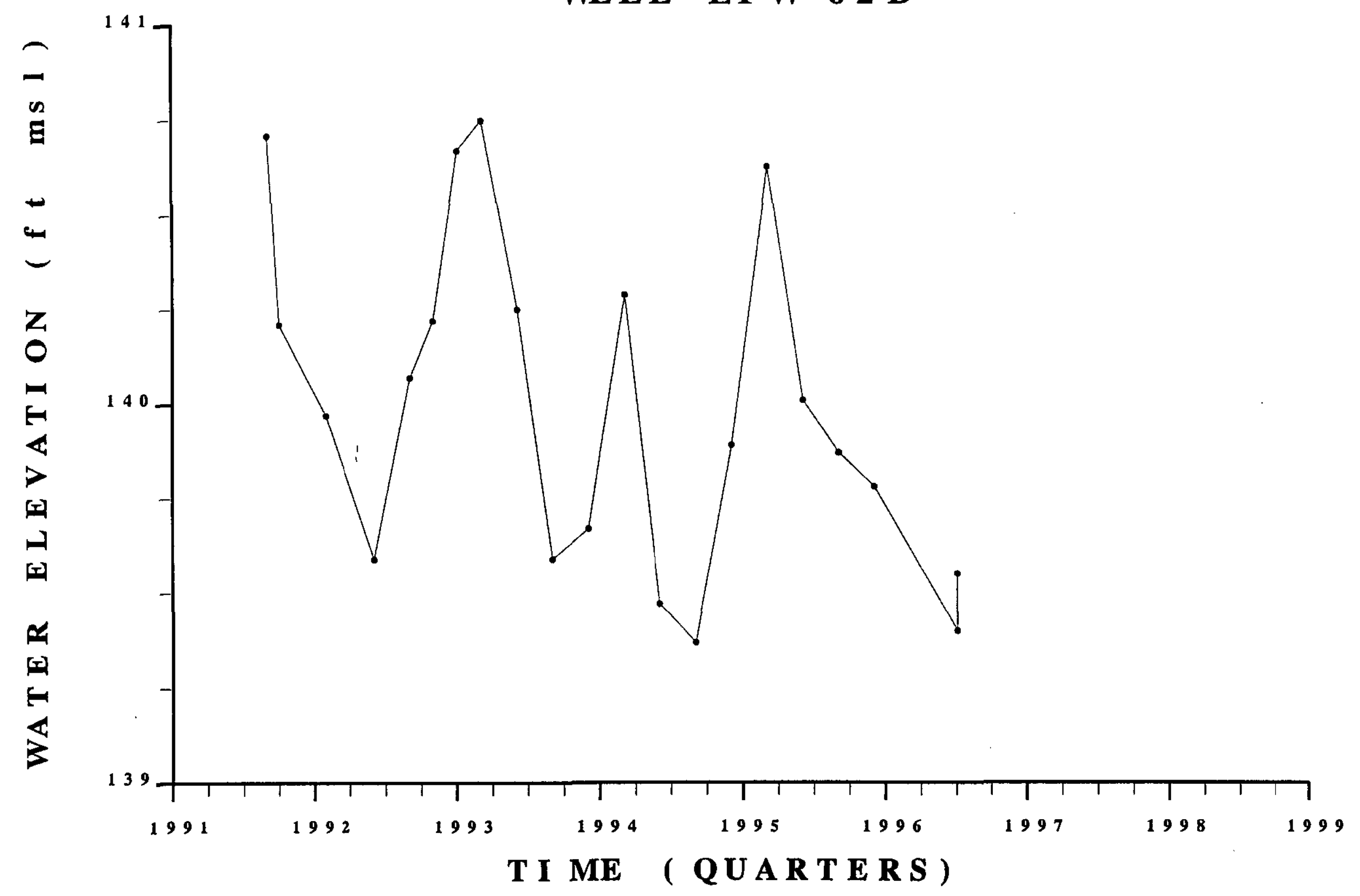

S a n i t a $\mathbf{r} \quad \mathbf{L}$ a n d f i l l Fourthouarer. 1998 $\& \quad 1998$ S umma r v 


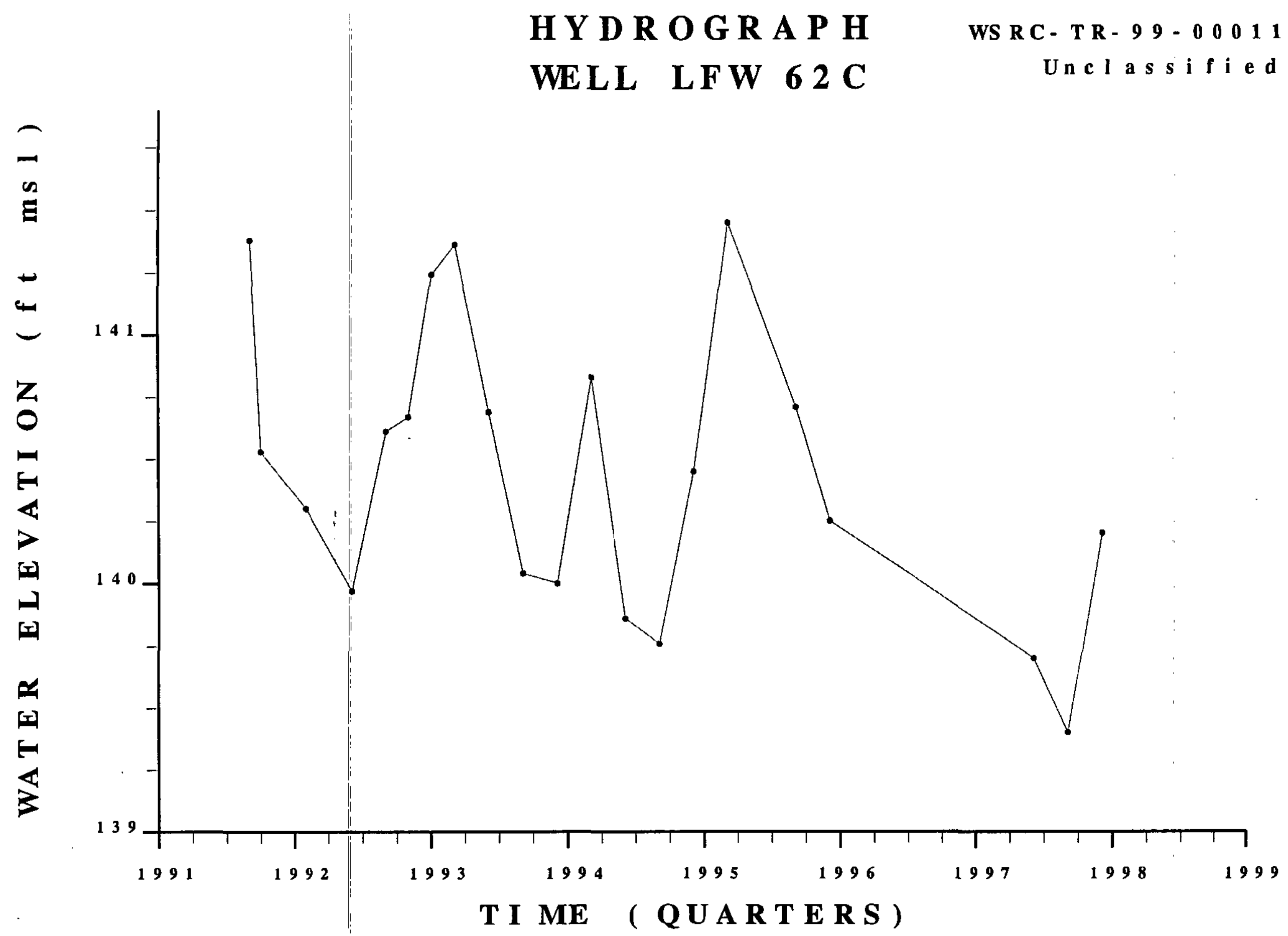



WELL LFW 62 D

Unc l a s s i fied

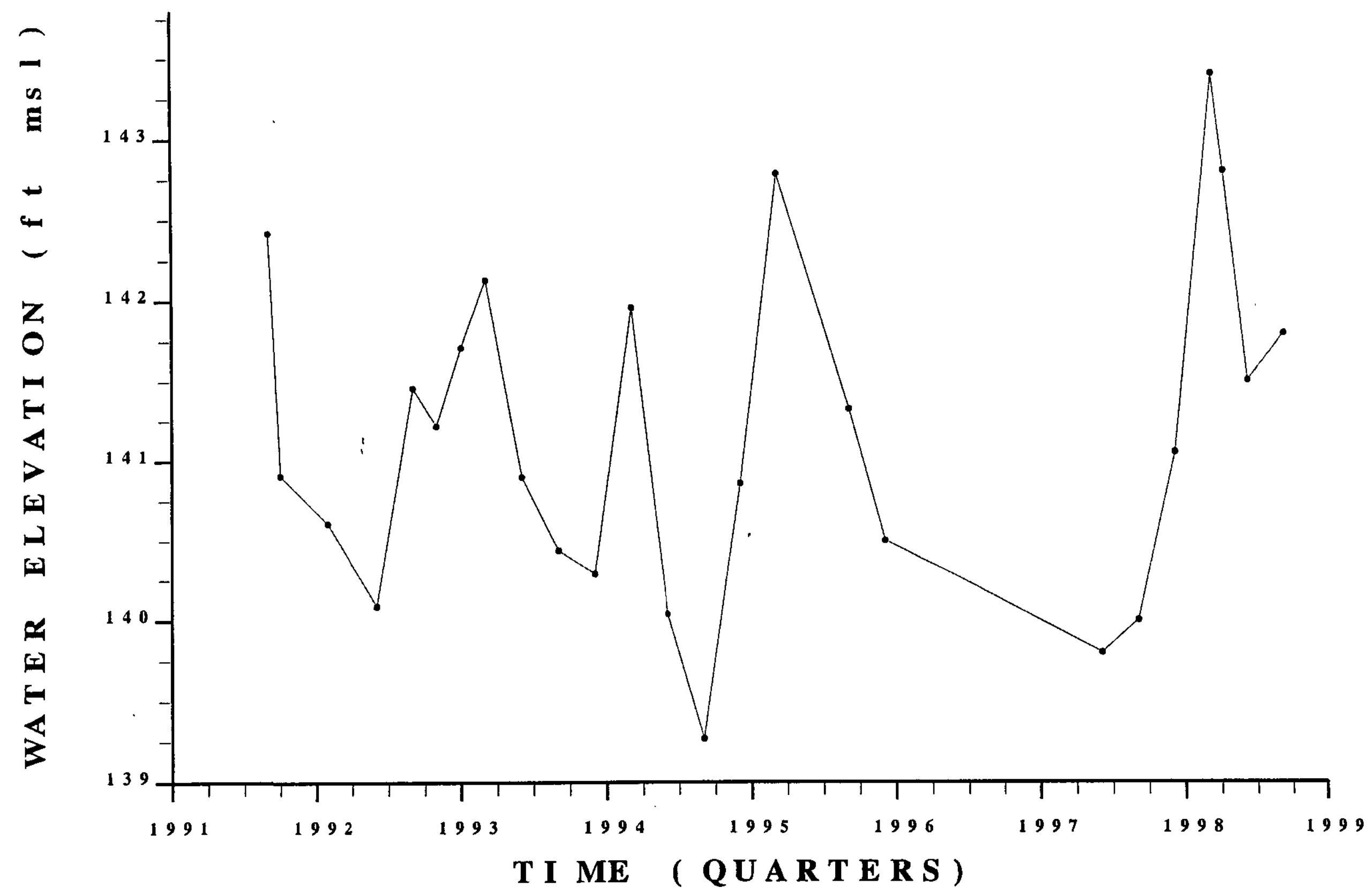




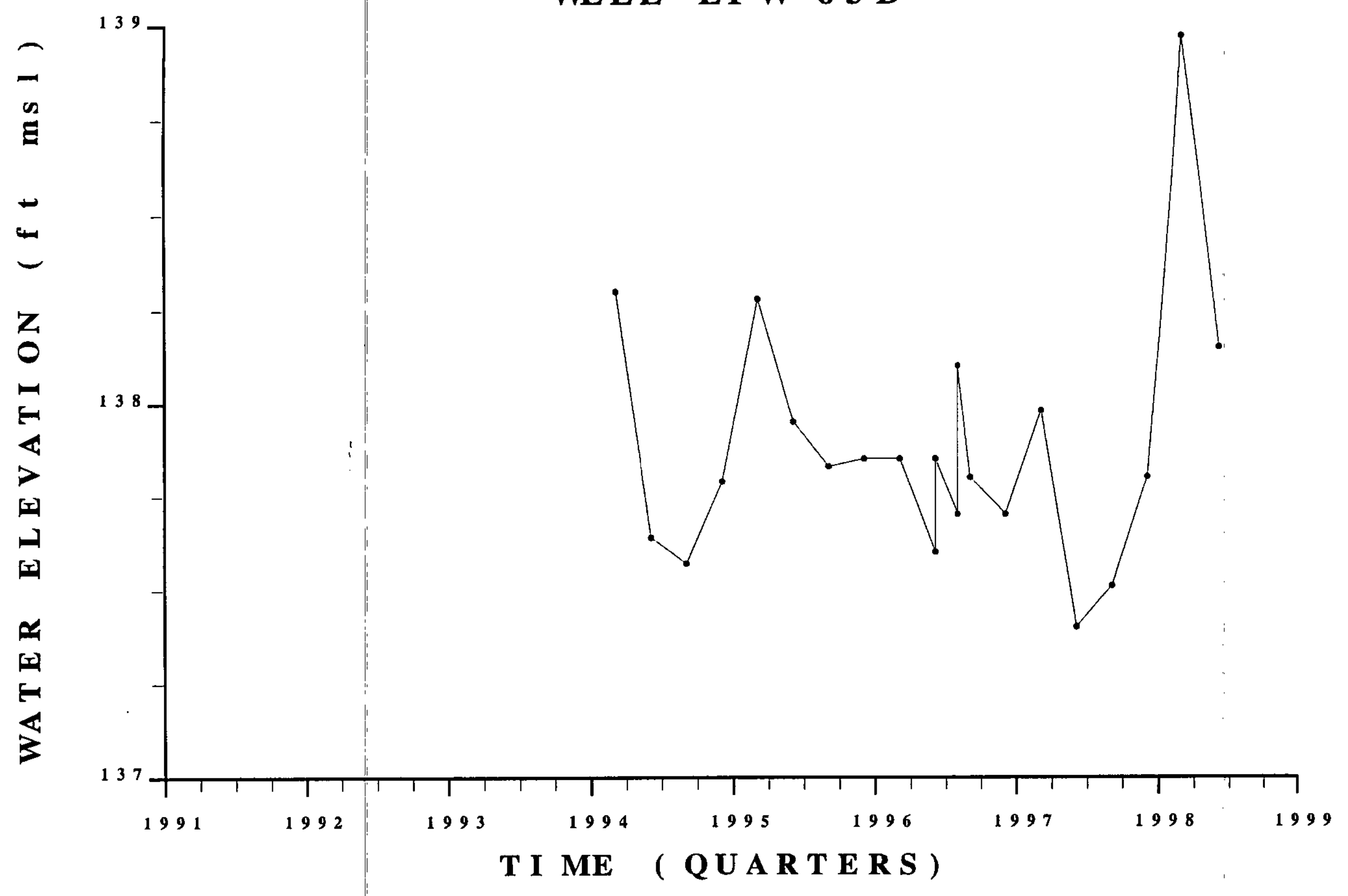

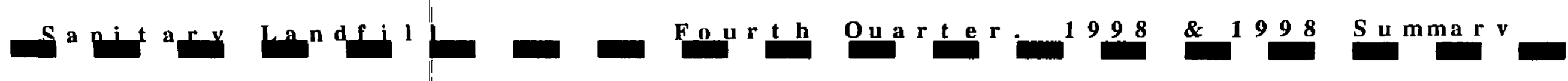




\section{H Y D R O G R A P H}

WELL LFW $63 \mathrm{C}$

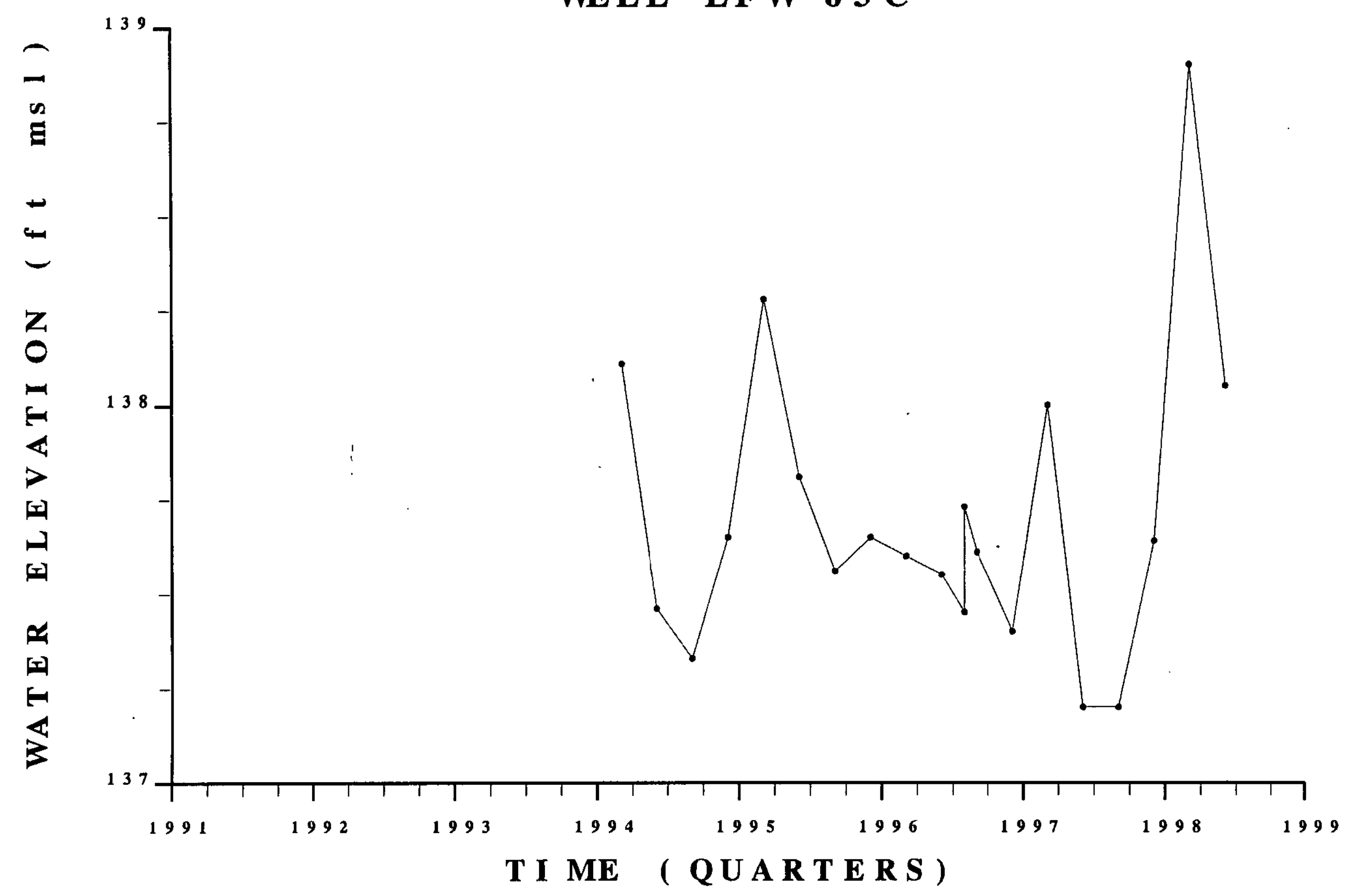

S a n i t a r v Landf i l l

Fourth Ouarter. 1998

\& 1998 Summar 


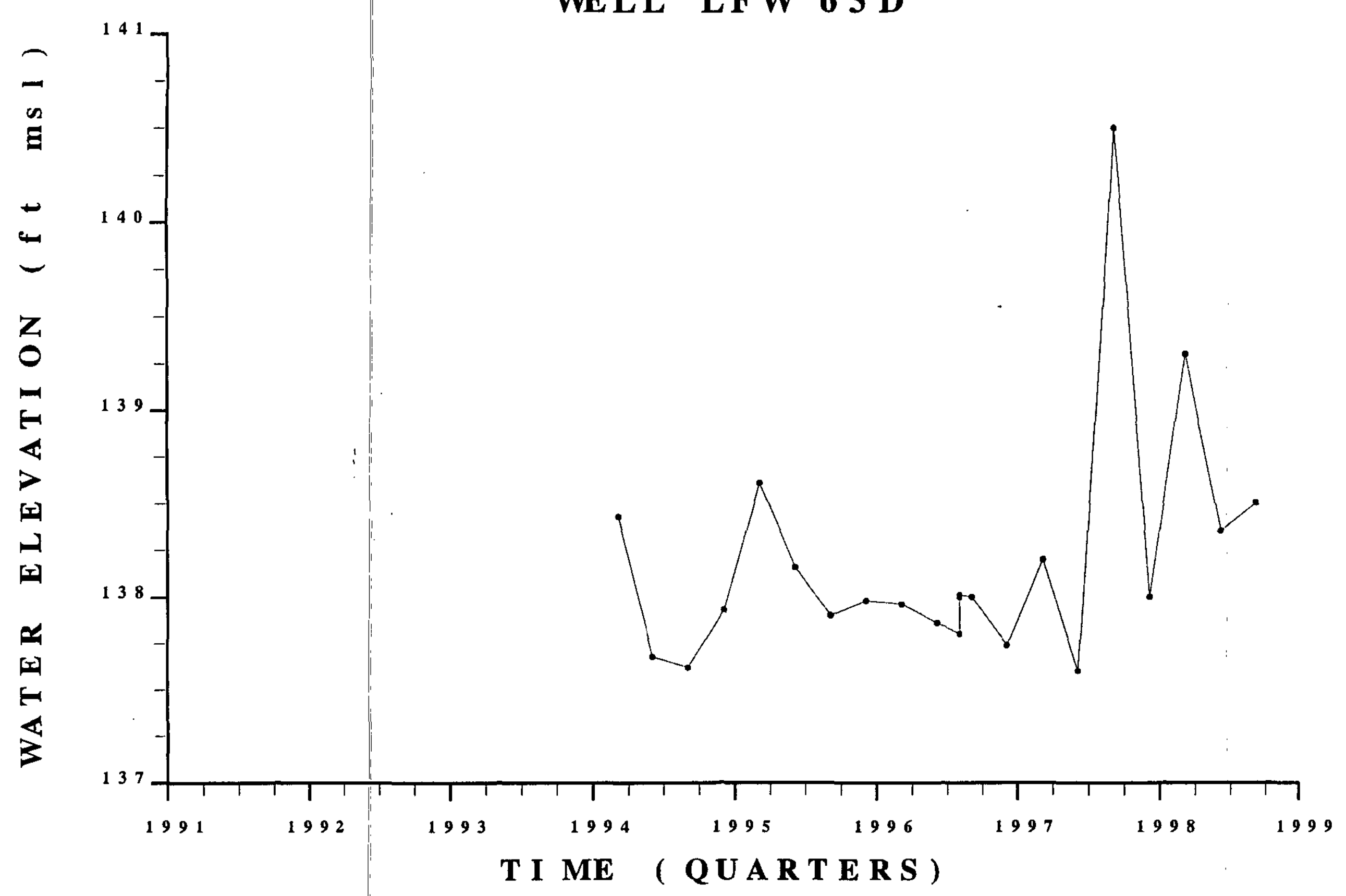

H Y D R O G R A P H

WELL LFW 63 D
WS R C - T R-99-00011

Unc l a s s i f i ed 
HY D R O G R A P H WELL LFW 64 B
WS R $\dot{C}-T R-99-00011$ Unc l a s s i f i ed

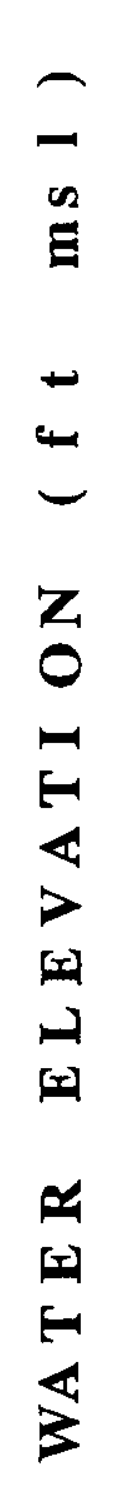

138

${ }^{138} 7$



137

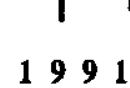

1992

1993

1994

1996

1997

1998

T I ME ( Q U A R T ER S ) 







\section{H Y D R O G R A P H}

WELL LFW 64 D
WS R C - T R- $99-00011$

Unclassif i ed

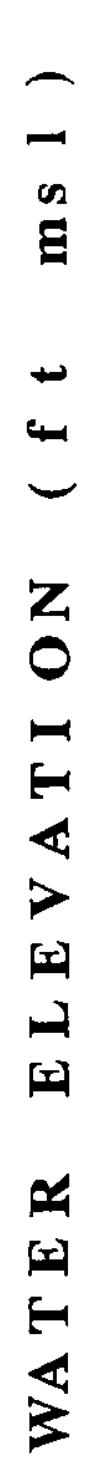
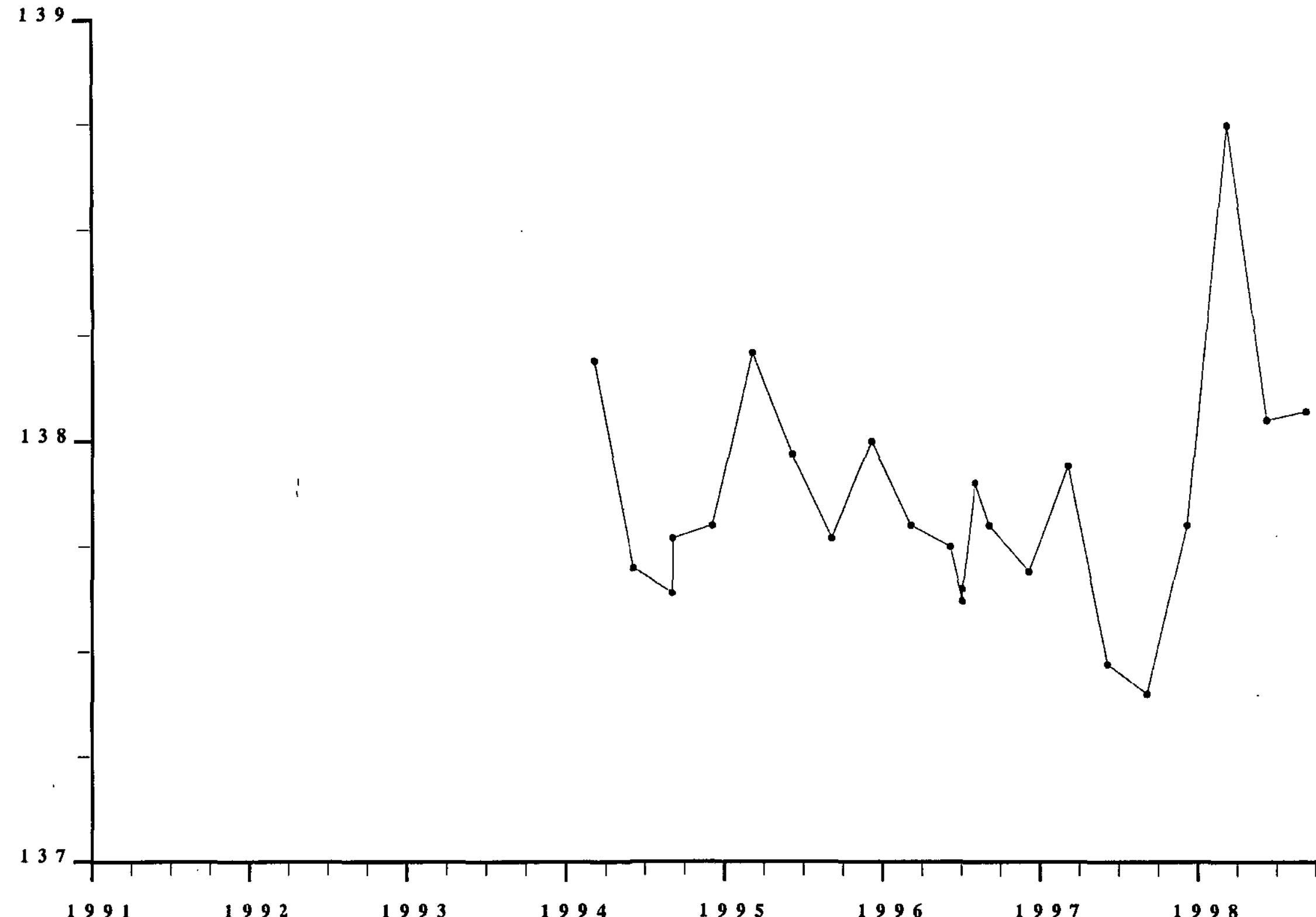

T I ME ( QUART E R S ) 


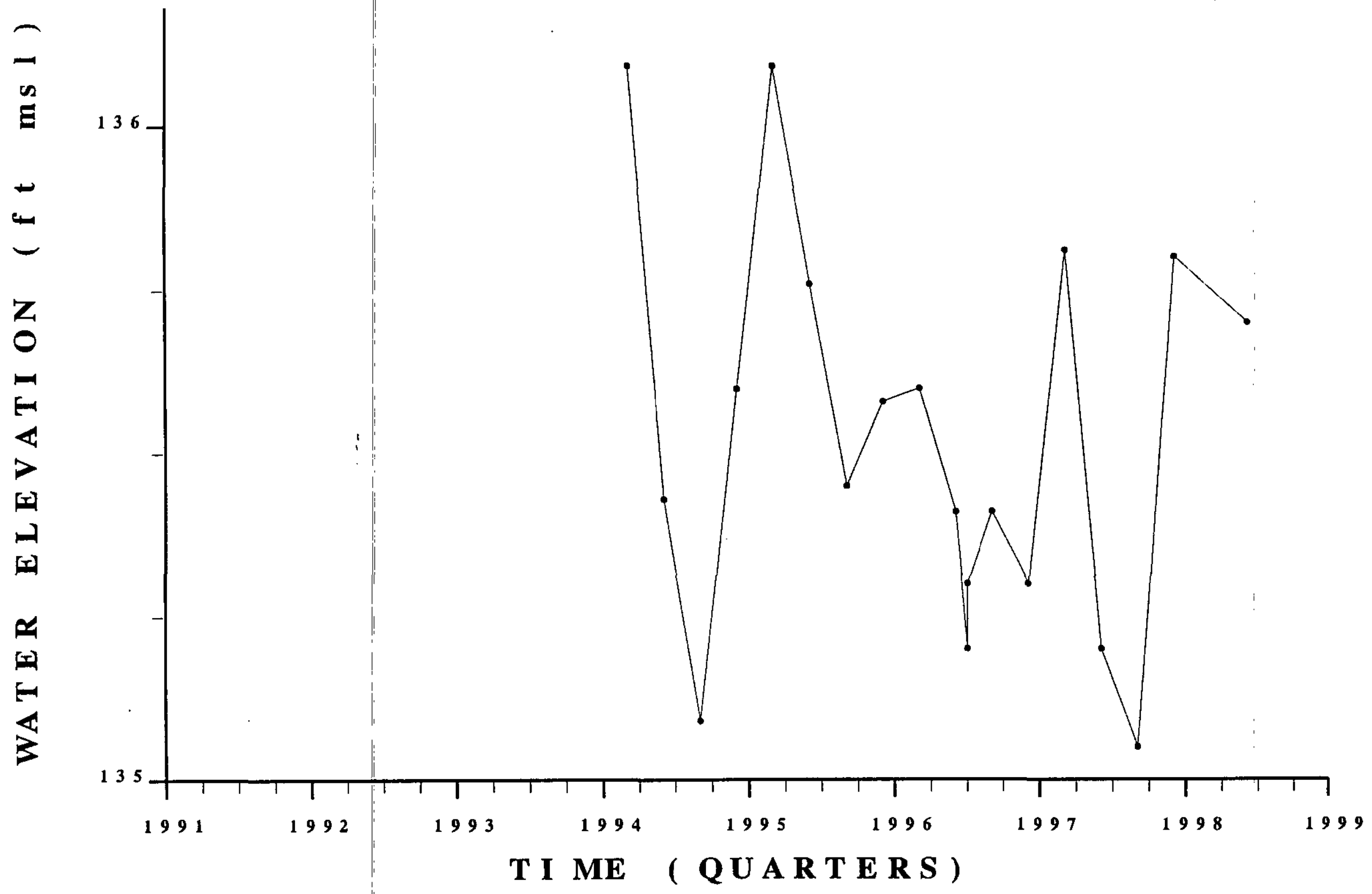

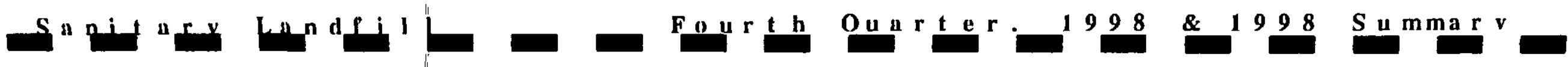


H Y D R O G R A P H WELL LFW $65 \mathrm{C}$
WS R C - TR - $99-00011$ Unc l a s s i fied

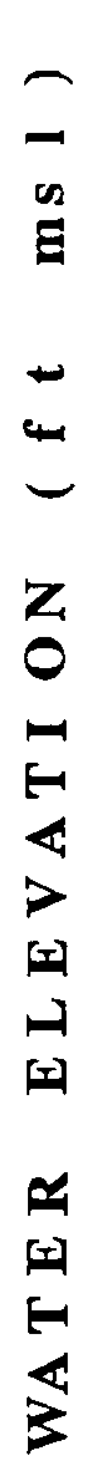

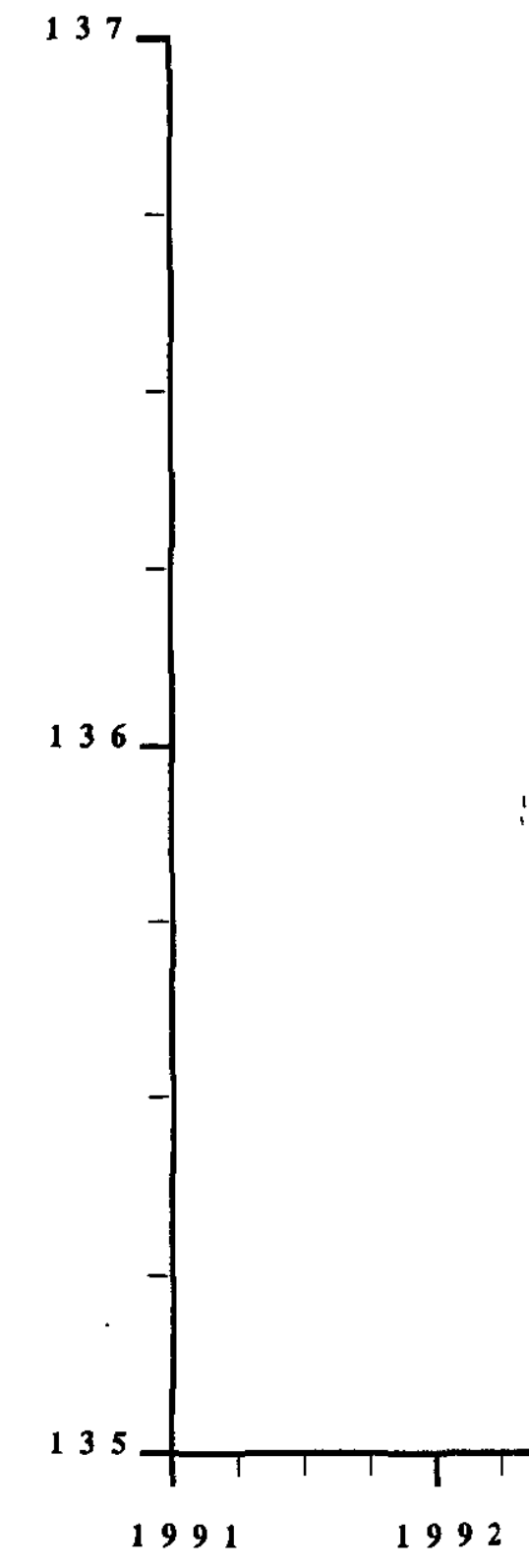

135

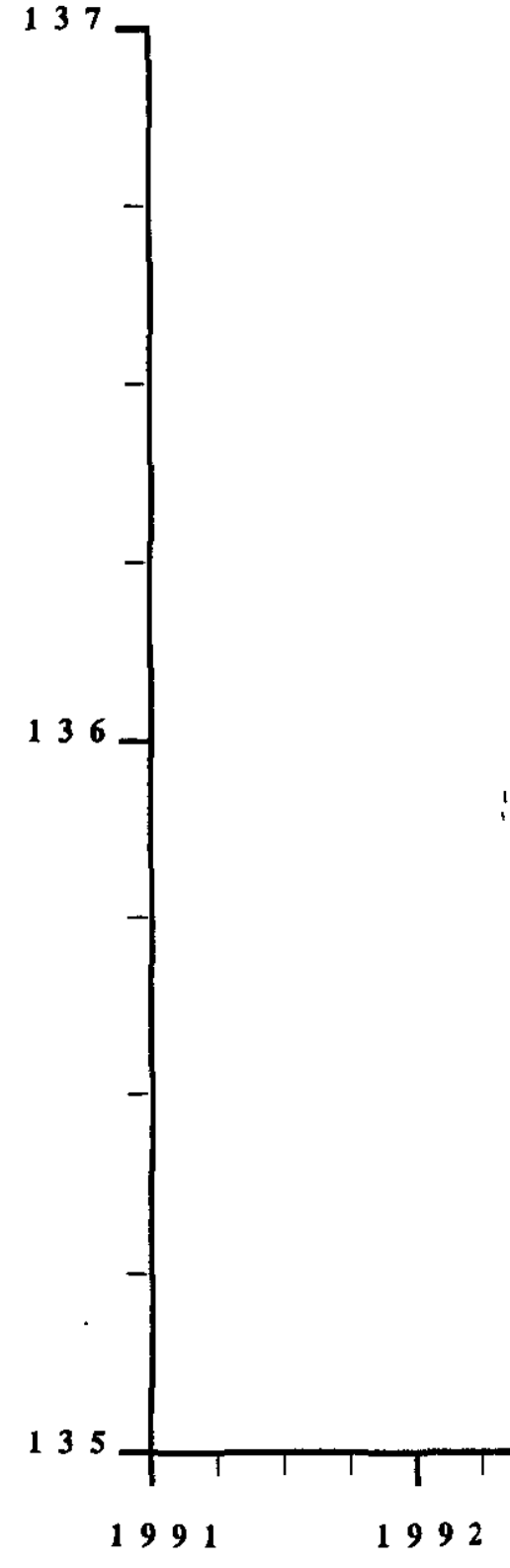

1991

1992 


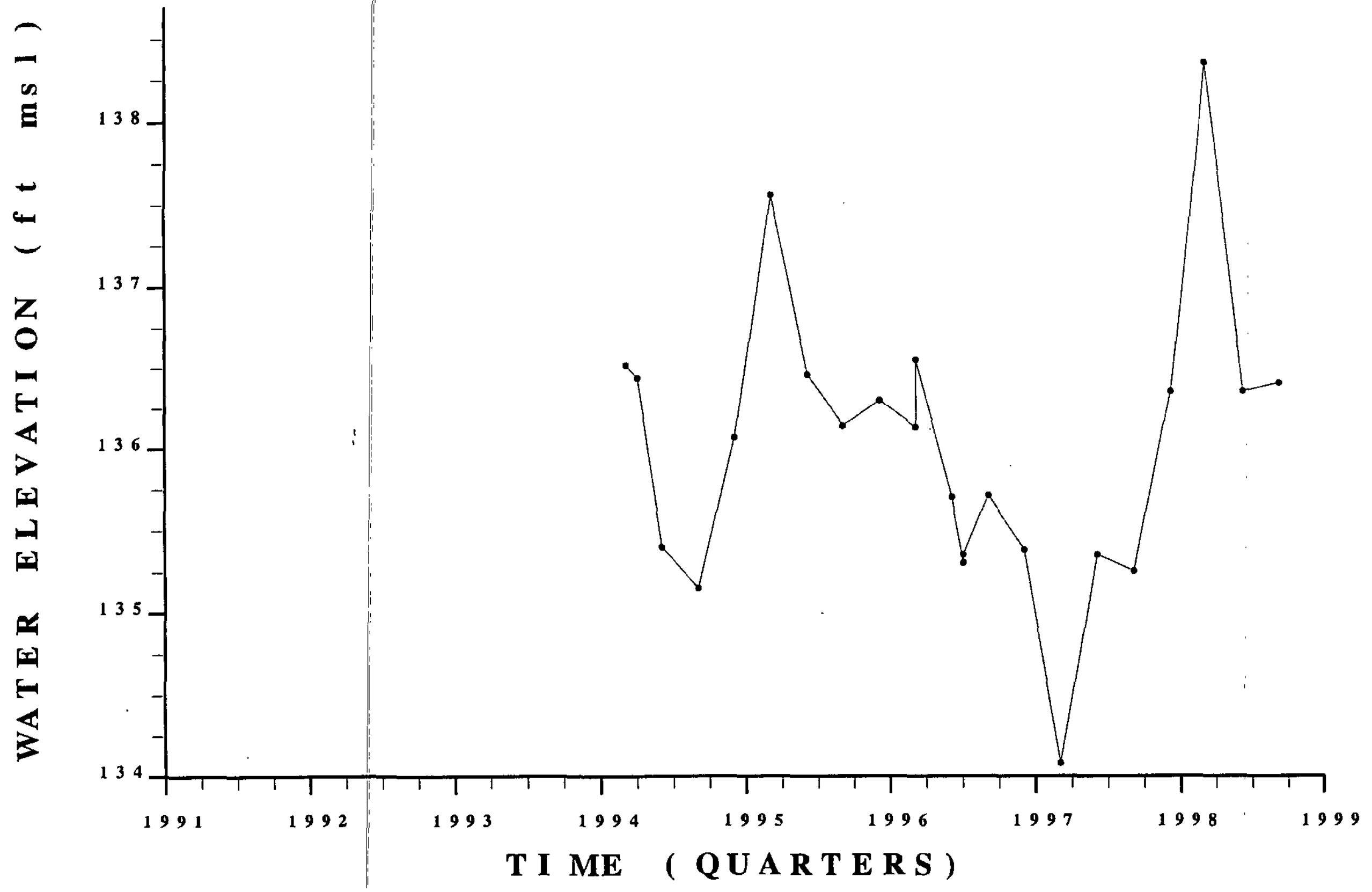

H Y D R O G R A P H WELL LFW 65 D
WS R C - T R - $99-000011$

Unc l as s if i ed

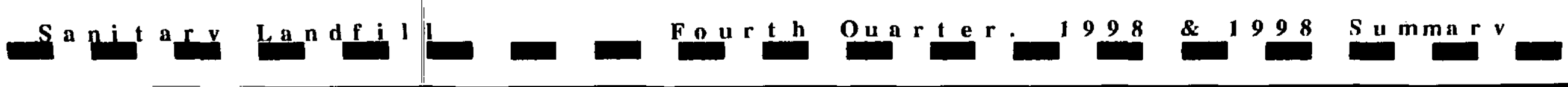




\section{H Y D R O G R A P H}

WELL LFW 66 B

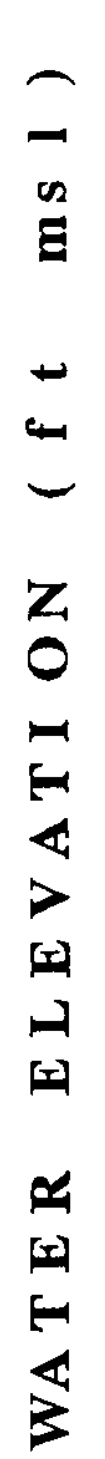

139



1991

T I ME

( Q U A R T E R ) 


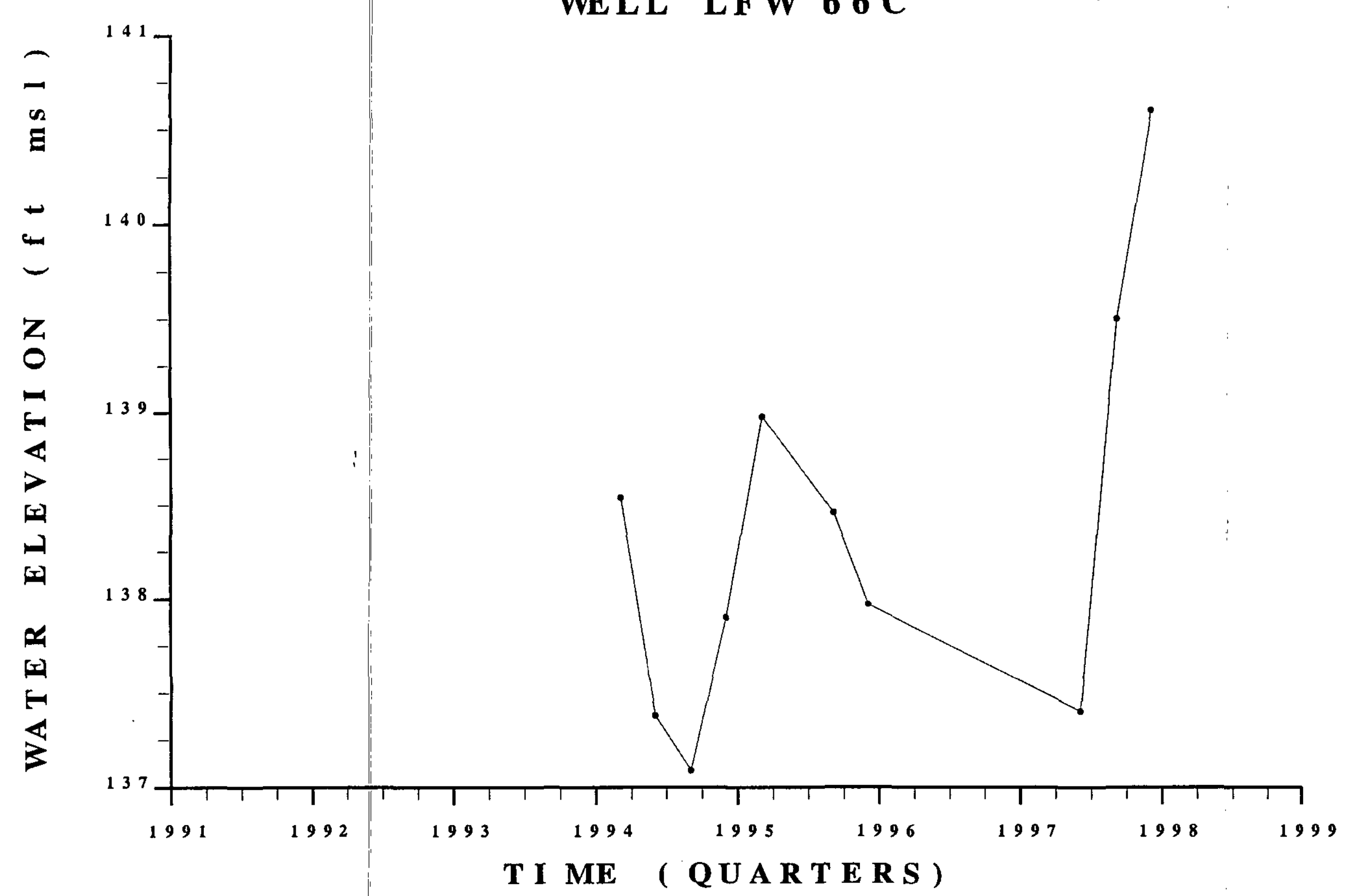




\section{H Y D R O G R A P H} WELL LFW 66 D
WS R C - T R - 99-00011 Unc l a s s i fi ed

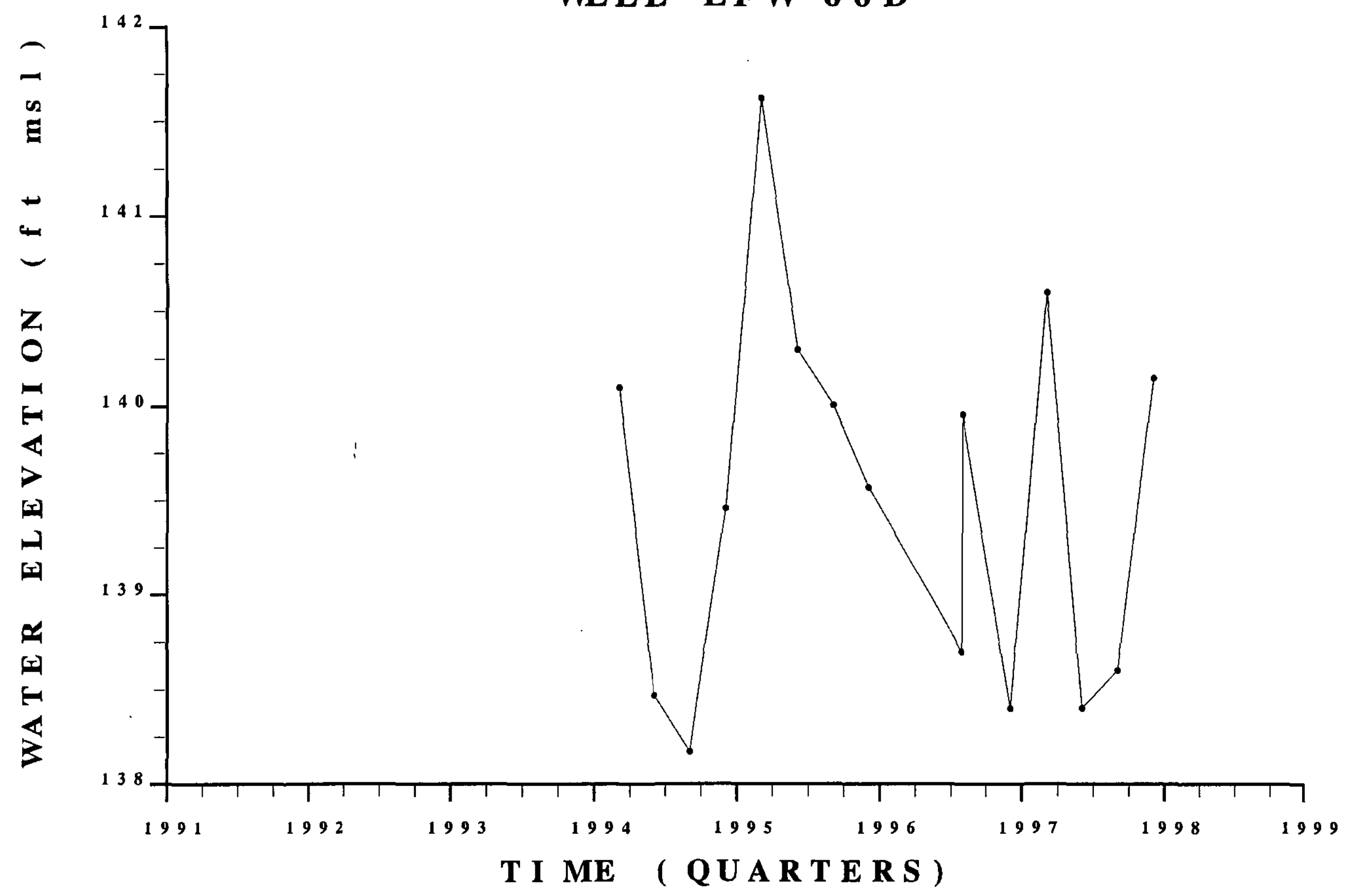

Sanitarv Landfil I

Fourth ouarter.

\& 1998

S u mma $r$ v 


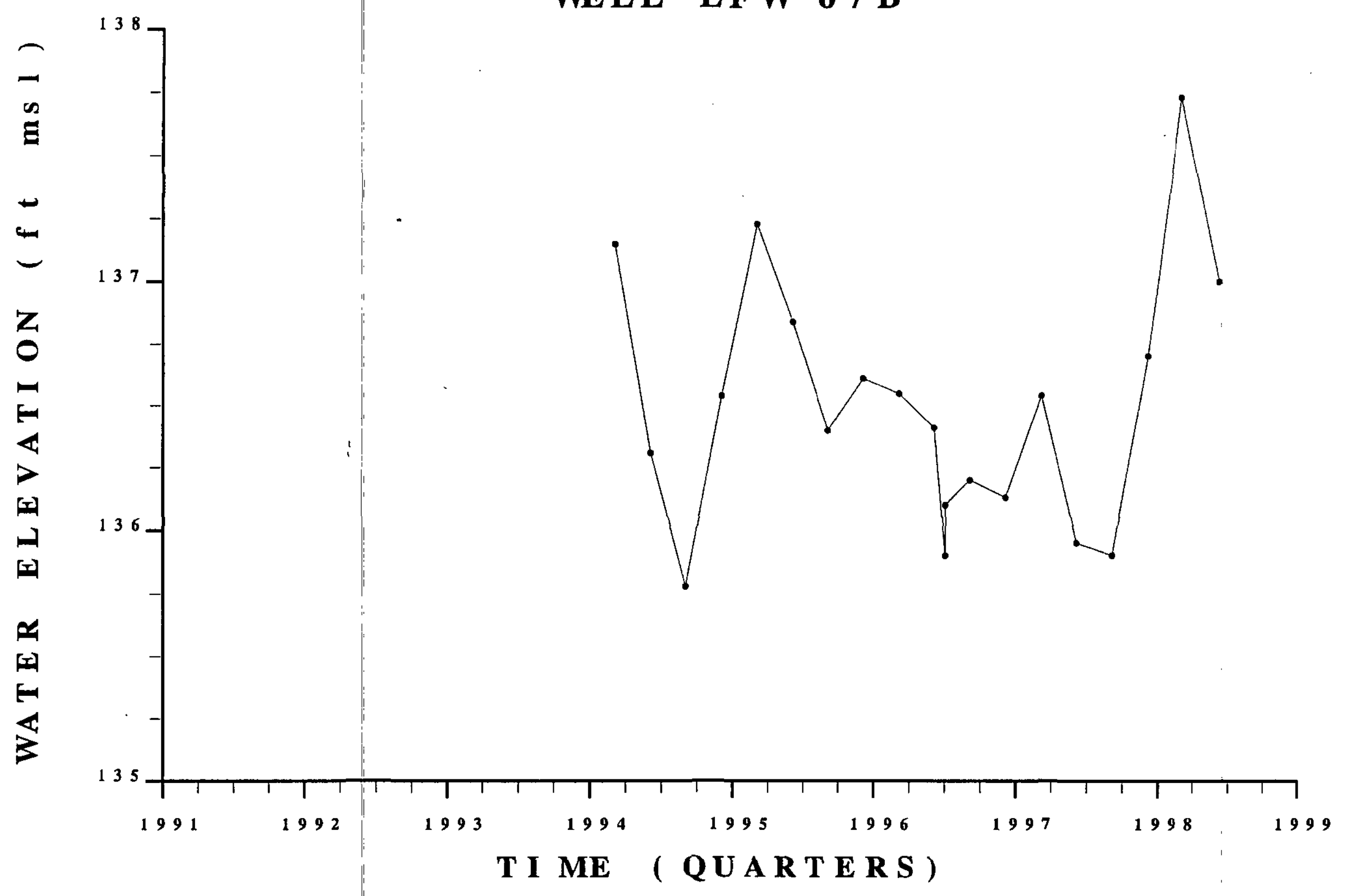

H Y D R O G R A P H WELL LFW 67 B
WS R C-T R- $99-000011$ Unc I a s s if i ed

Sanitarv Landfill 
H Y D R O G R A P H

WELL LFW $67 \mathrm{C}$
WS R C - T R - 9 9-

Unc lassified



138

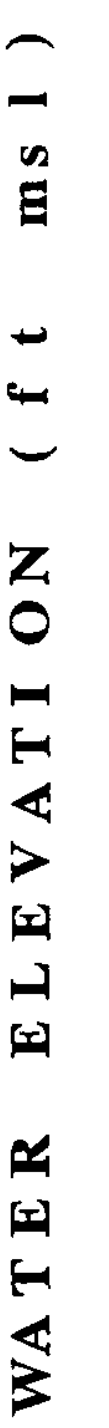

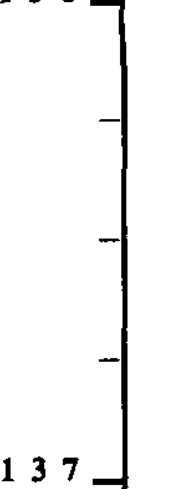

137

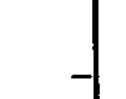

$136-$

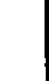

135

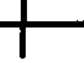

1991

1996

1997

1998

T I ME ( QUART ERS ) 


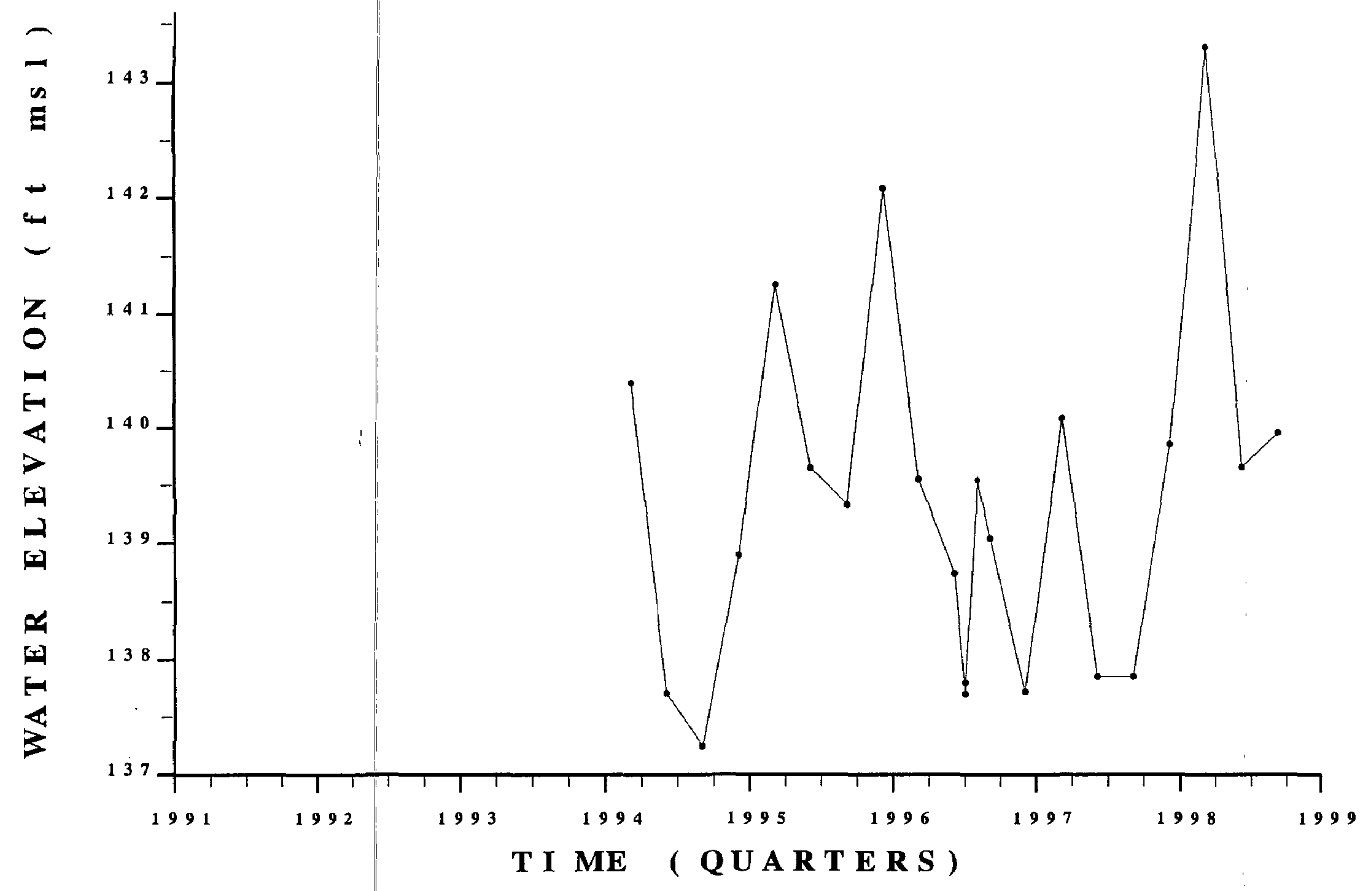

Sanitar v Landfil

Fourth Ouarter.

1998

\& 1998 S 
H Y D R O G R A P H WELL LFW 68 B
WS R C - T R - $99-00011$ Unclass i f i ed

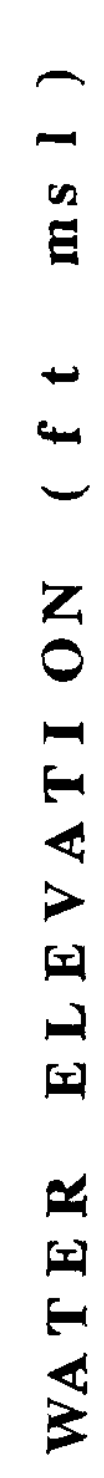

139

4

137

T I ME ( QUA R T E R ) 




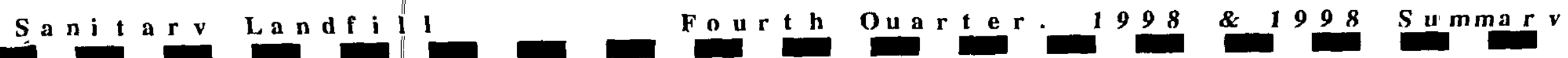


H Y D R O G R A P H WELL LFW 68 D
WS R C - T R - 99- 00011

Unc l a s s if i ed








H Y D R O G R A P H WELL LFW 69 B
WS R C - T R - $99-00011$ Unc l a s s i f i e d

\footnotetext{
Sanitarv Landfil Fourthiouarter.1998\& 1998 Summar
} 


\section{WELL LFW 69 C}

WS R C - TR- $99-00011$
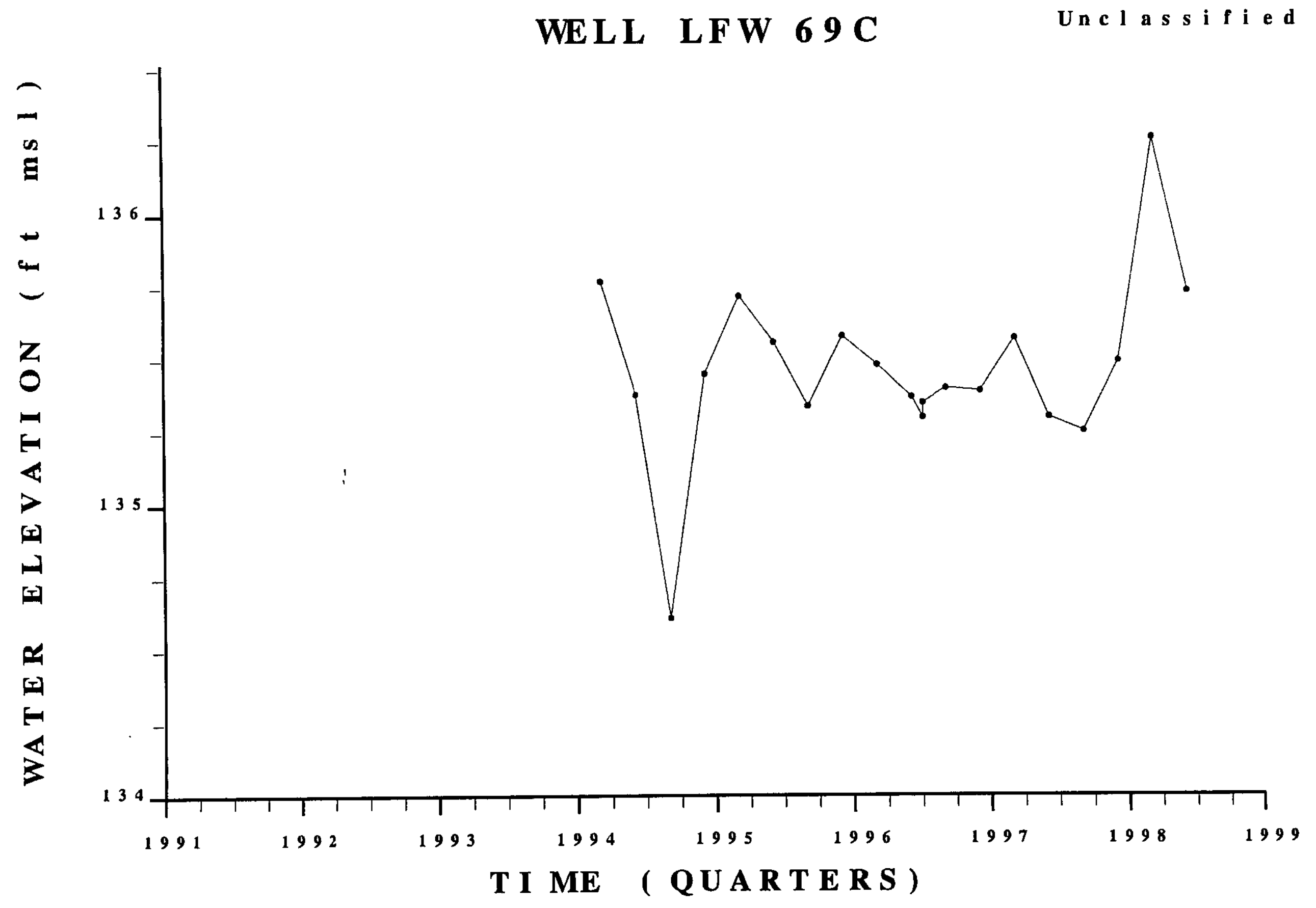

S a n i t a r v landf i l l

Fourth ouarter. 1998 \& 1998 Summar 


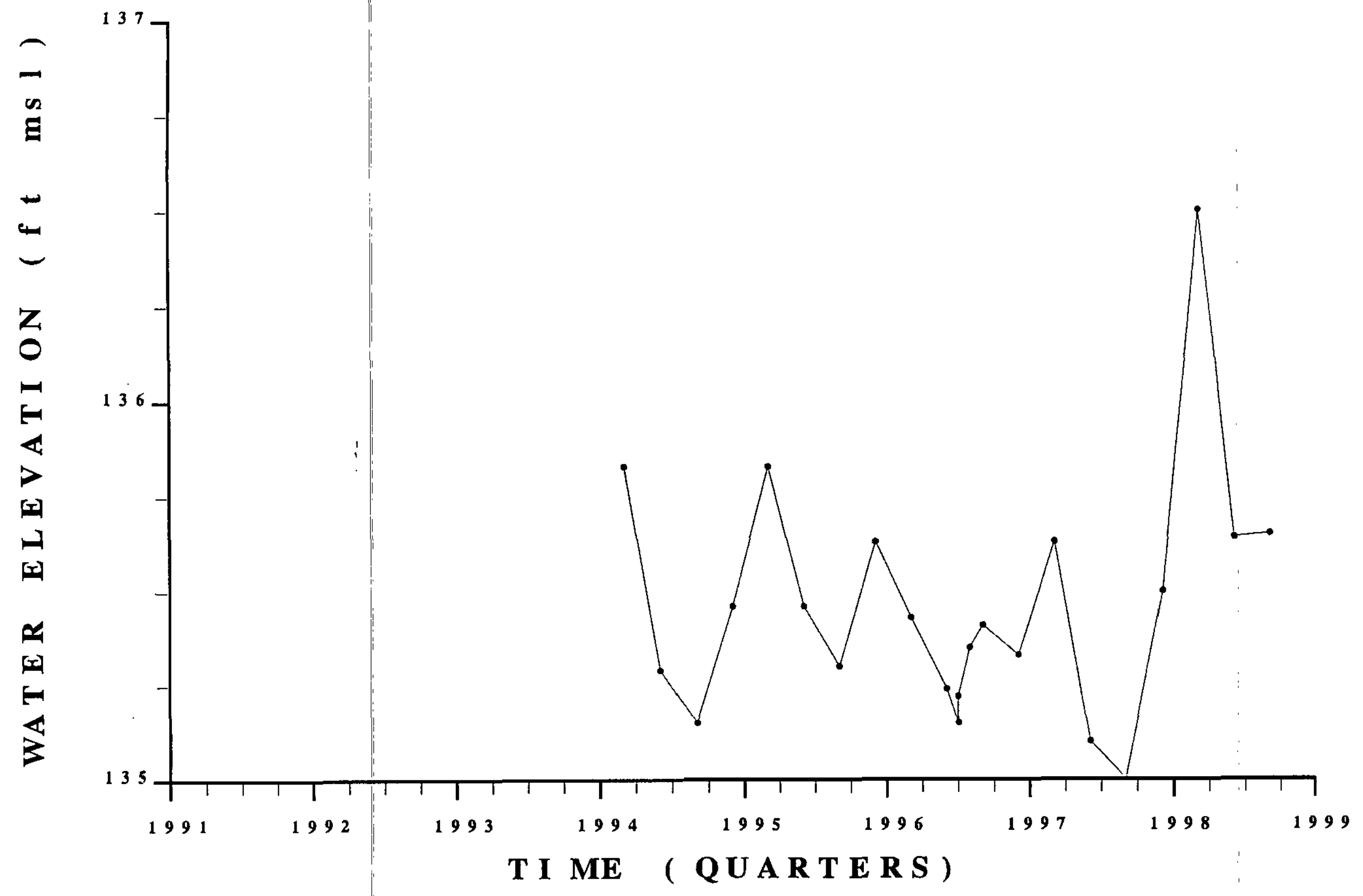

Sa n i t a r v La n d f i i i

Fourth Ou r ter. 1998 \& 1998 Summarv 
H Y D R O G R A P H WELL LFW 70 B
WS R C - T R - $99-0 \begin{array}{lllll}0 & 0 & 0 & 1 & 1\end{array}$ Unc l a s s i f i ed

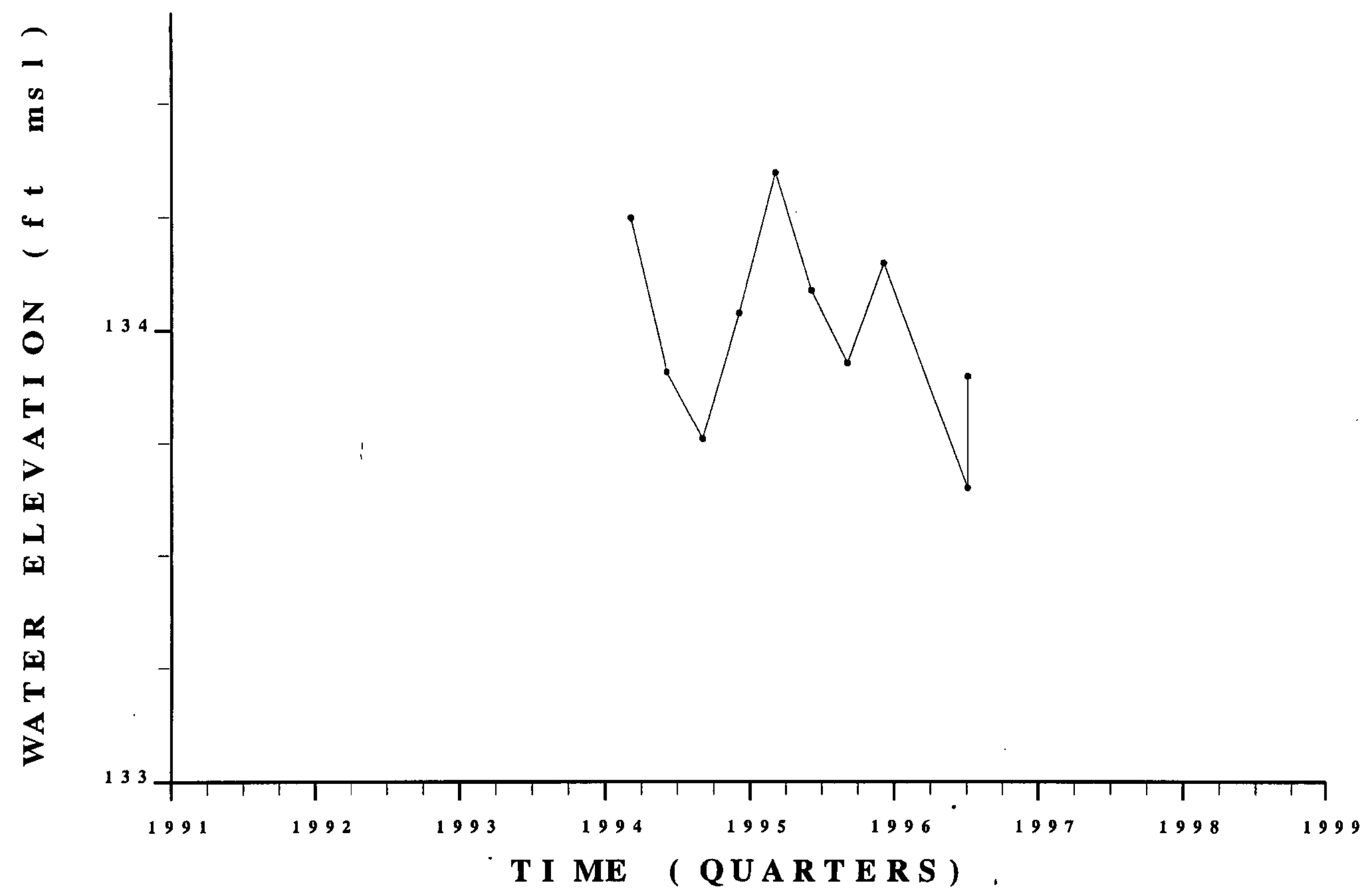

S a n i t a r v L a n d f i I I

$\begin{array}{llllll}998 & \& & 1998 & \text { S } & & \end{array}$ 


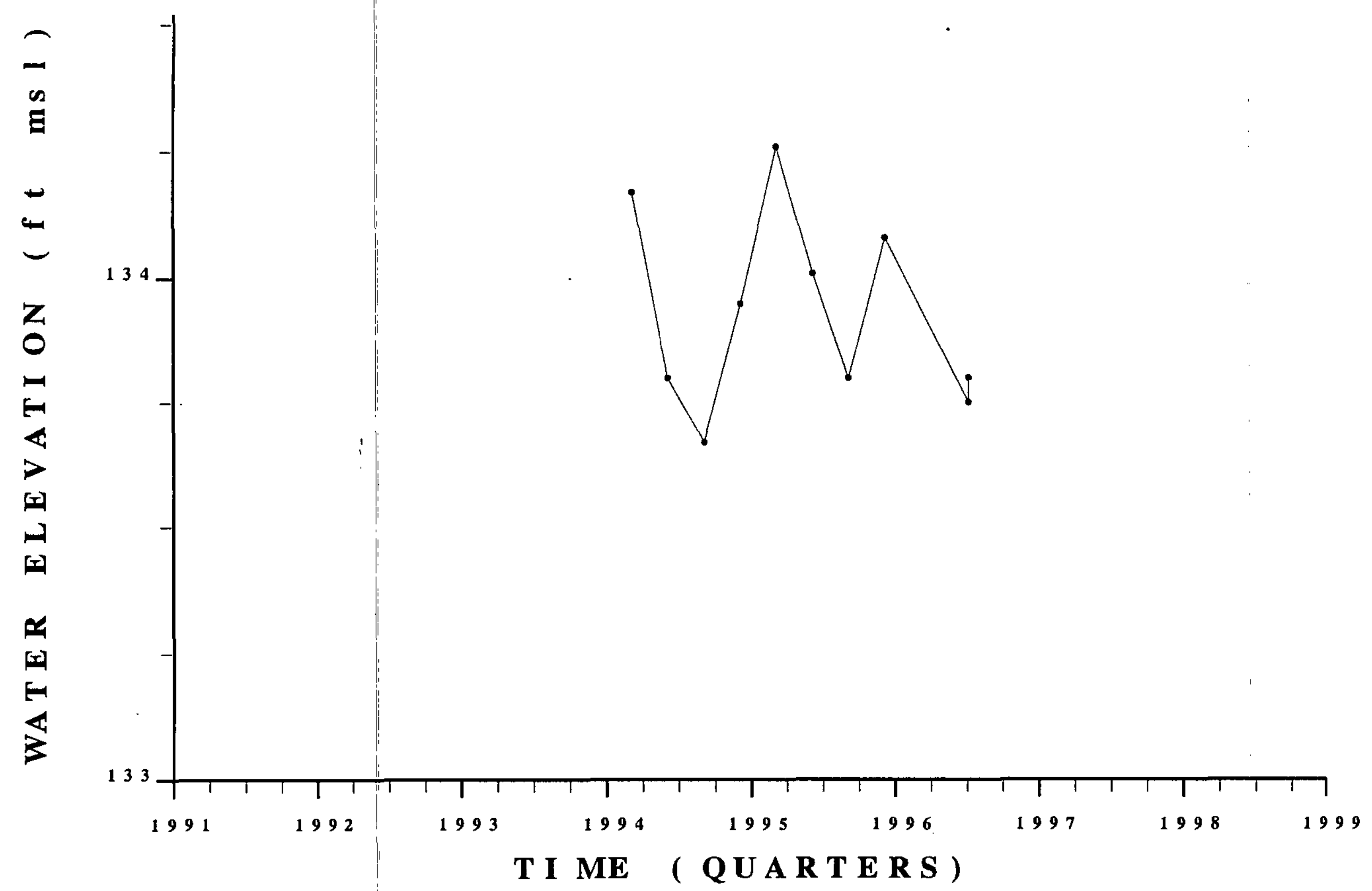


HY D R O GRA P H WELL LFW 70 D
WS R C - T R - 99-00011 Unc l as s i f i ed

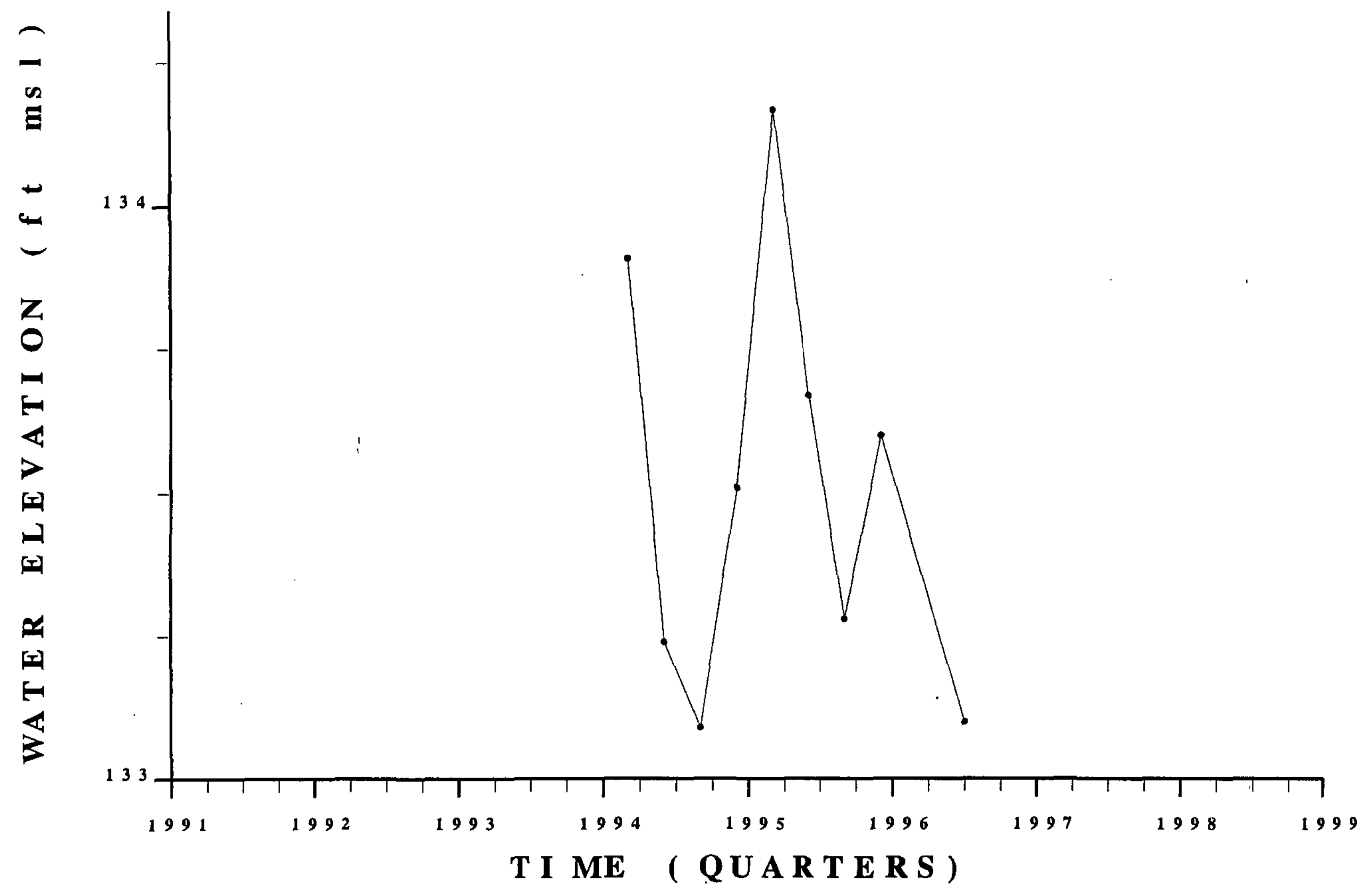




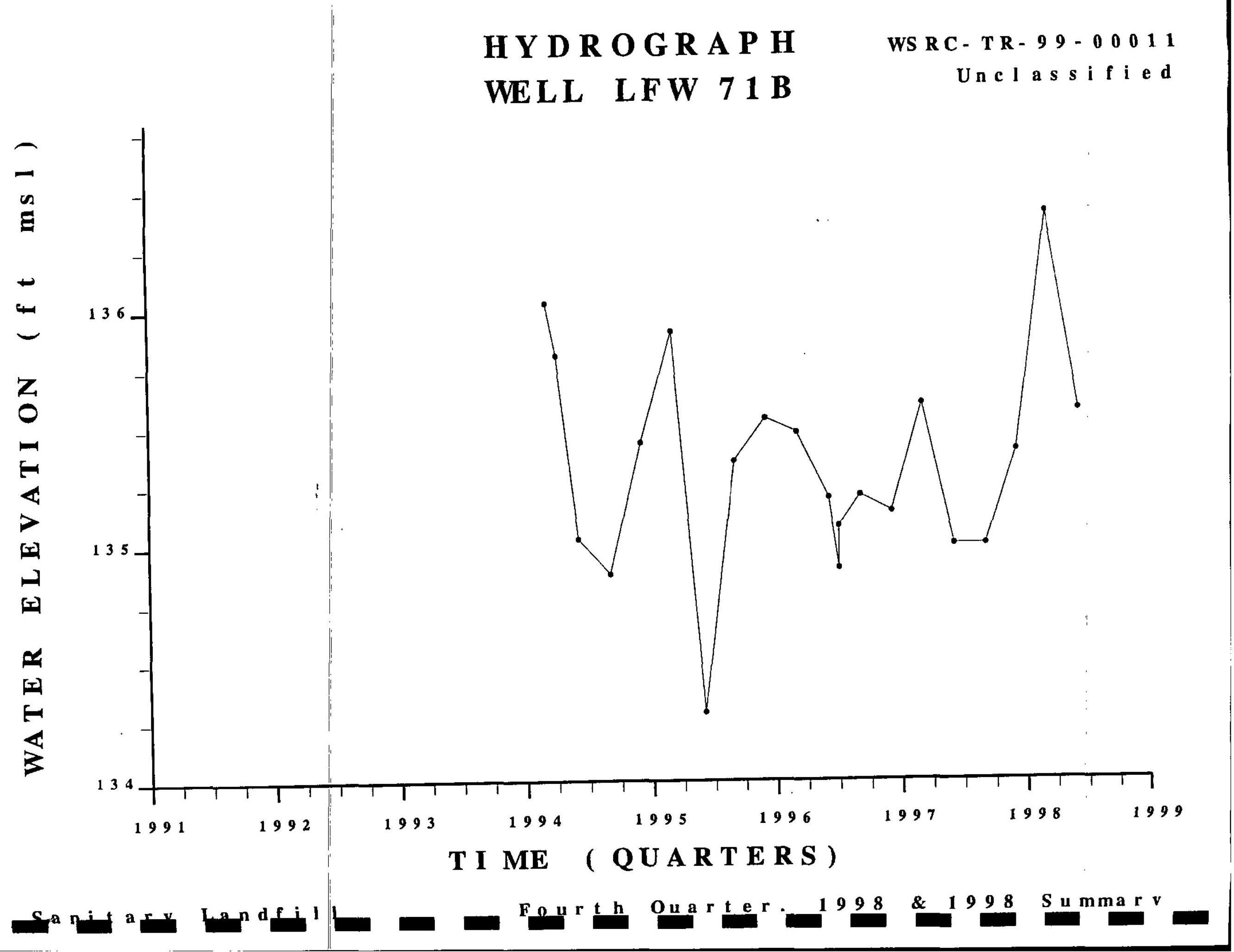









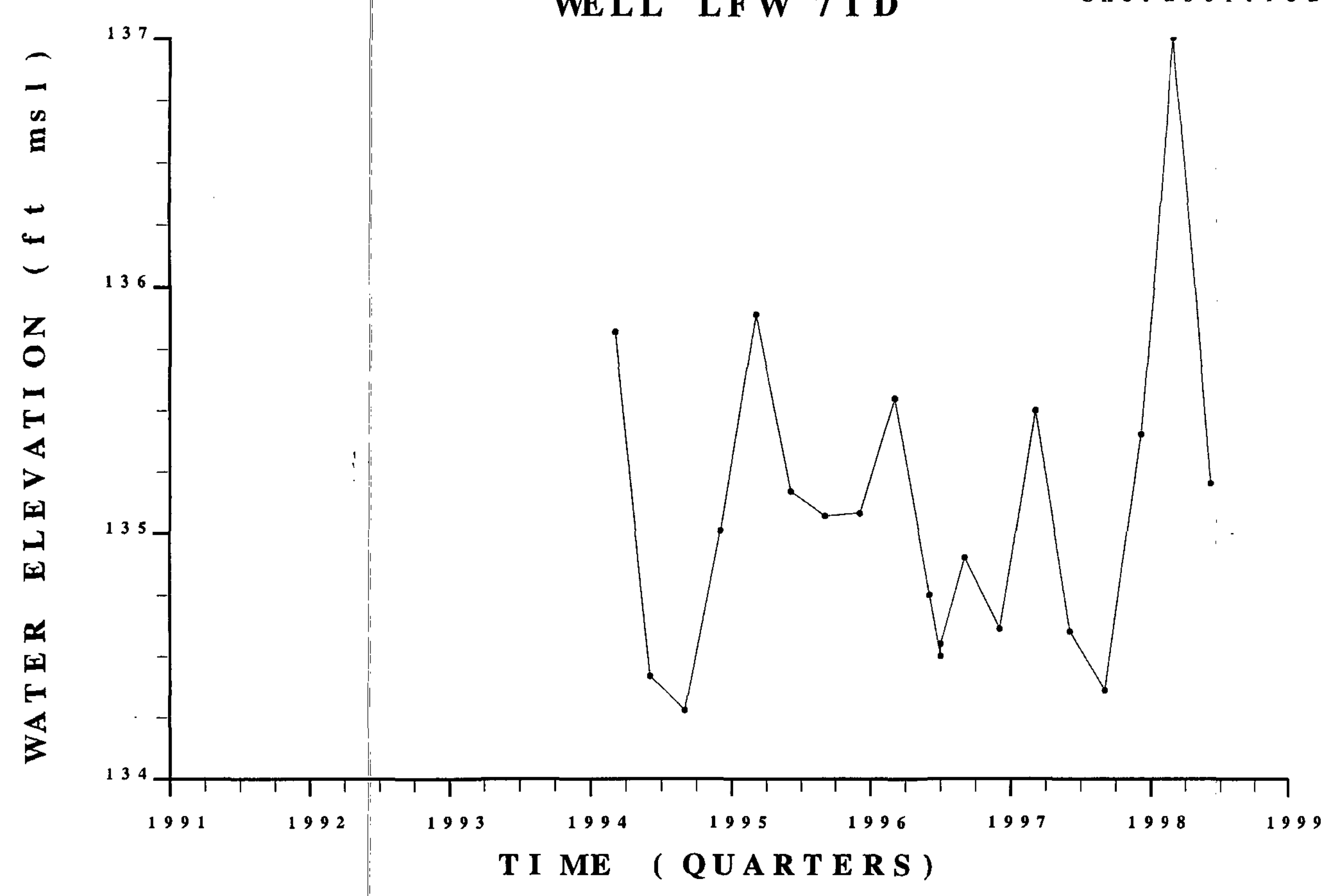

S a n i t a r v La nd f i l l

Fourth Ouarter.

1998

$\& \quad 1998 \quad$ S umma 
HYDR O GRA P H

WELL LFW 72 B
WS R C - TR- $99-00011$

Unc las s i fied



Sani t a r v landfill

Fourth ou a r t e r.

$1998 \quad \& \quad 1998$ Summar 


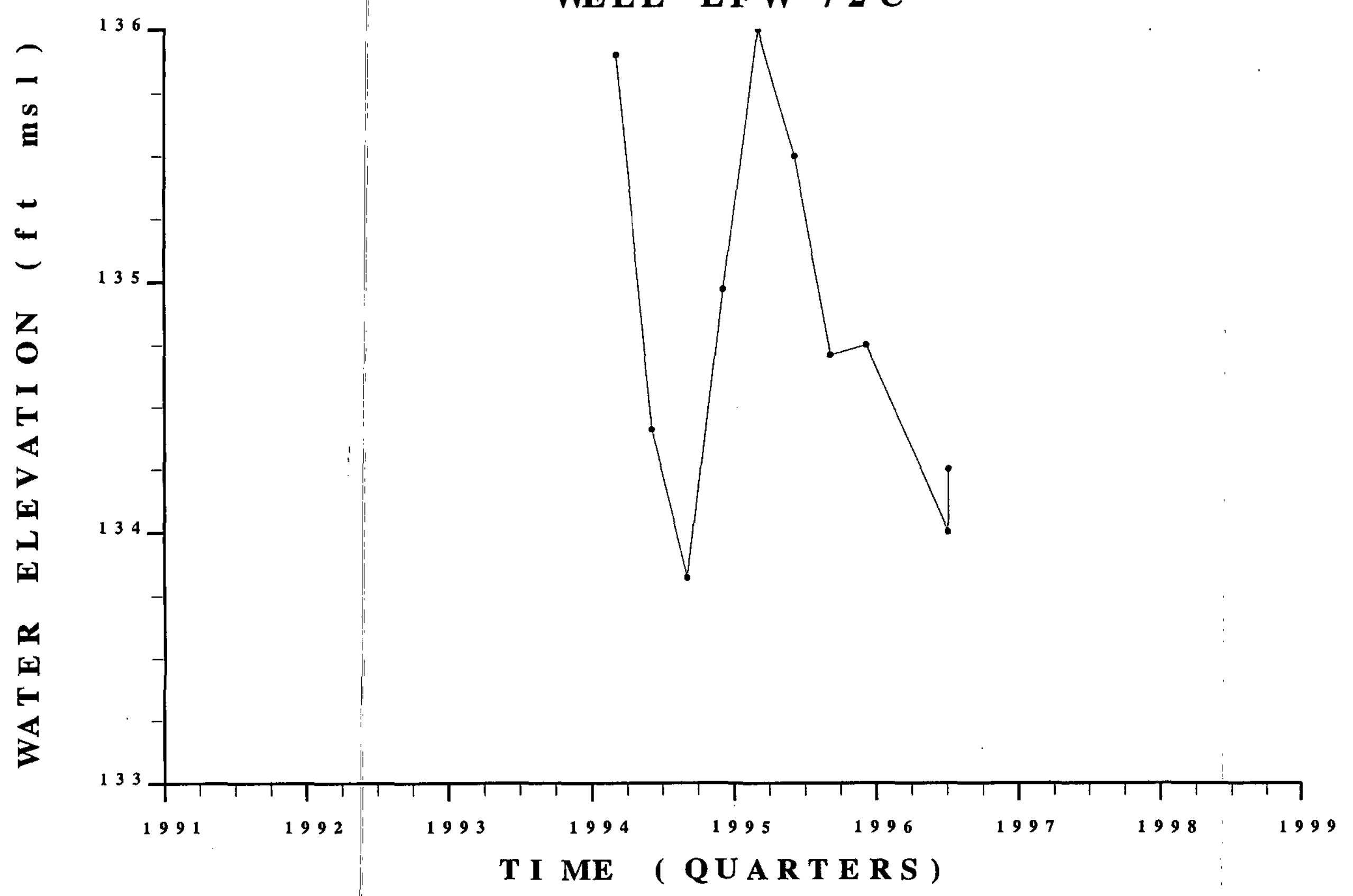

H Y D R O G R A P H WELL LFW 72 C
WS R C - T R - $99-000011$ Unclas s i f i ed

Sanitary Landfil D Fourth Ouarter.g 
H Y D R O G R A P

WELL LFW 72 D
WS R C - T R- $99-00011$

Unc l a s s i f i e d

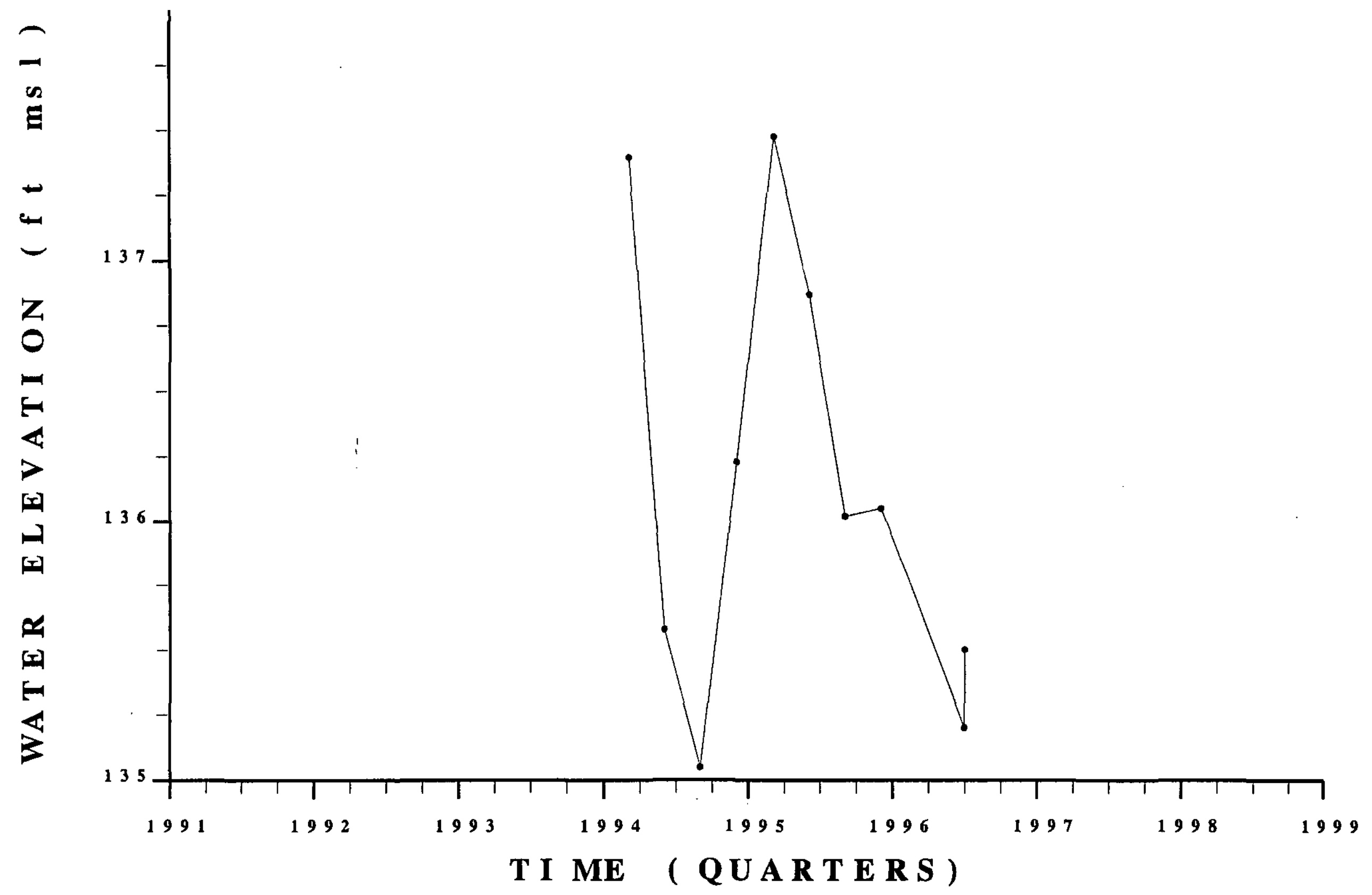

S a n i a $r$ v la nd f i l l

Fourth Ouarter.

1998

\& 1998

S u m ma r 
HY D R O G R A P H WELL LFW $74 \mathrm{C}$
WS R C - T R - 99-0 00011

Unclass i f i ed

198
197
196
195
19

$194=$

E 193 $\quad 192 \exists$

191

$190 \exists$

$189=$

$188=$

187
186
1185

$185 \exists$

$184=$

Z $\quad 183=$

○ $181=$

180

1179

178
177

$4 \quad 177$

$\quad 175=$

174
173
177

$172=$

$171 \exists$

다 $\quad 170$ 근

$168=$

167
169

() $\quad 165$ ב

$164 \mathrm{Z}$

$163 \exists$

$16 \cdot 2=$

1619

159

1991

992

T I ME ( QUART ERS )



Fourth Ougrter. 1998 \& 1998 Summarv 


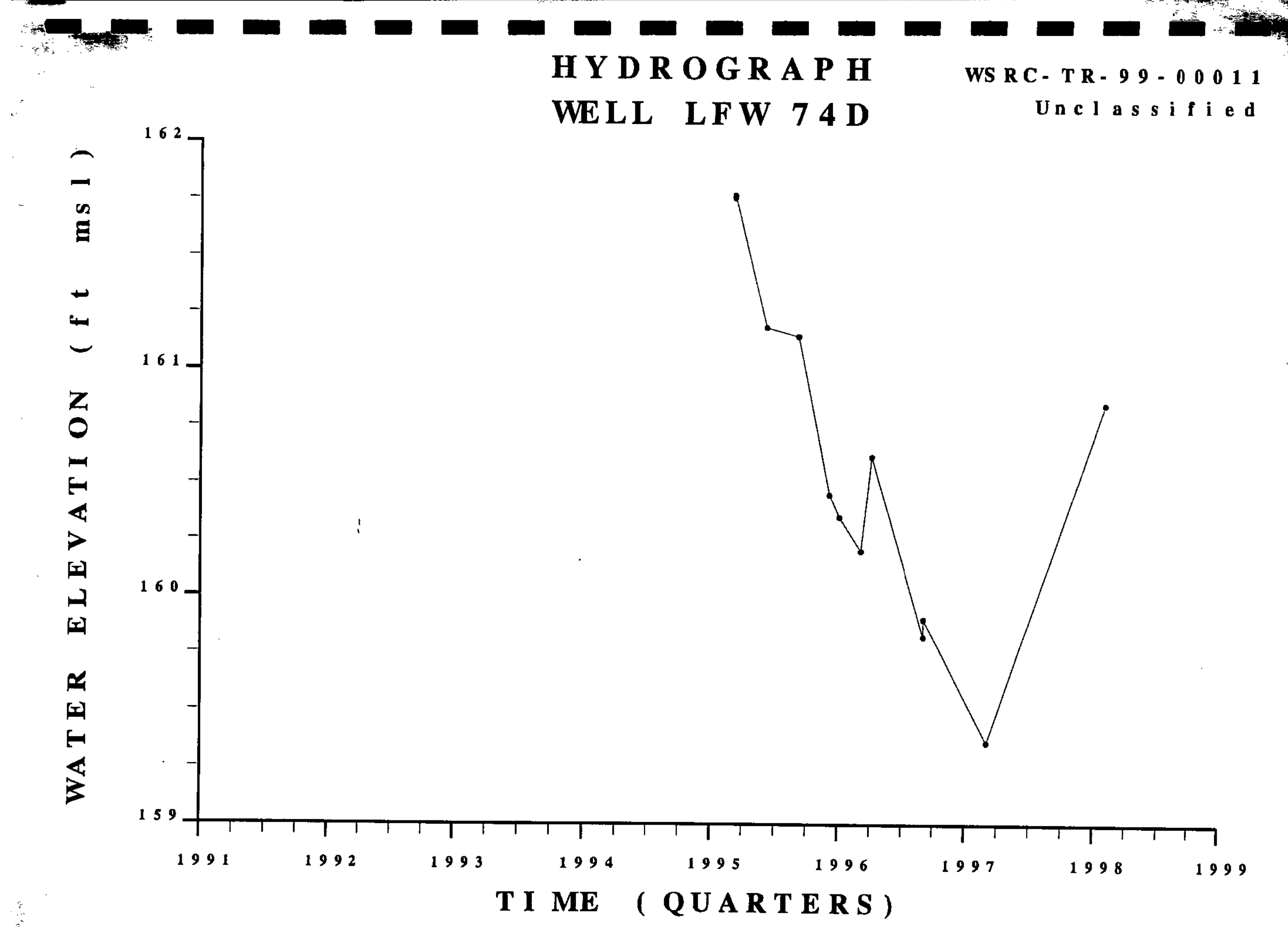


H Y D R O G R A P H WELL LFW $75 \mathrm{C}$
WS RC-TR- $99-00011$ Unc l a s $s$ i f i ed

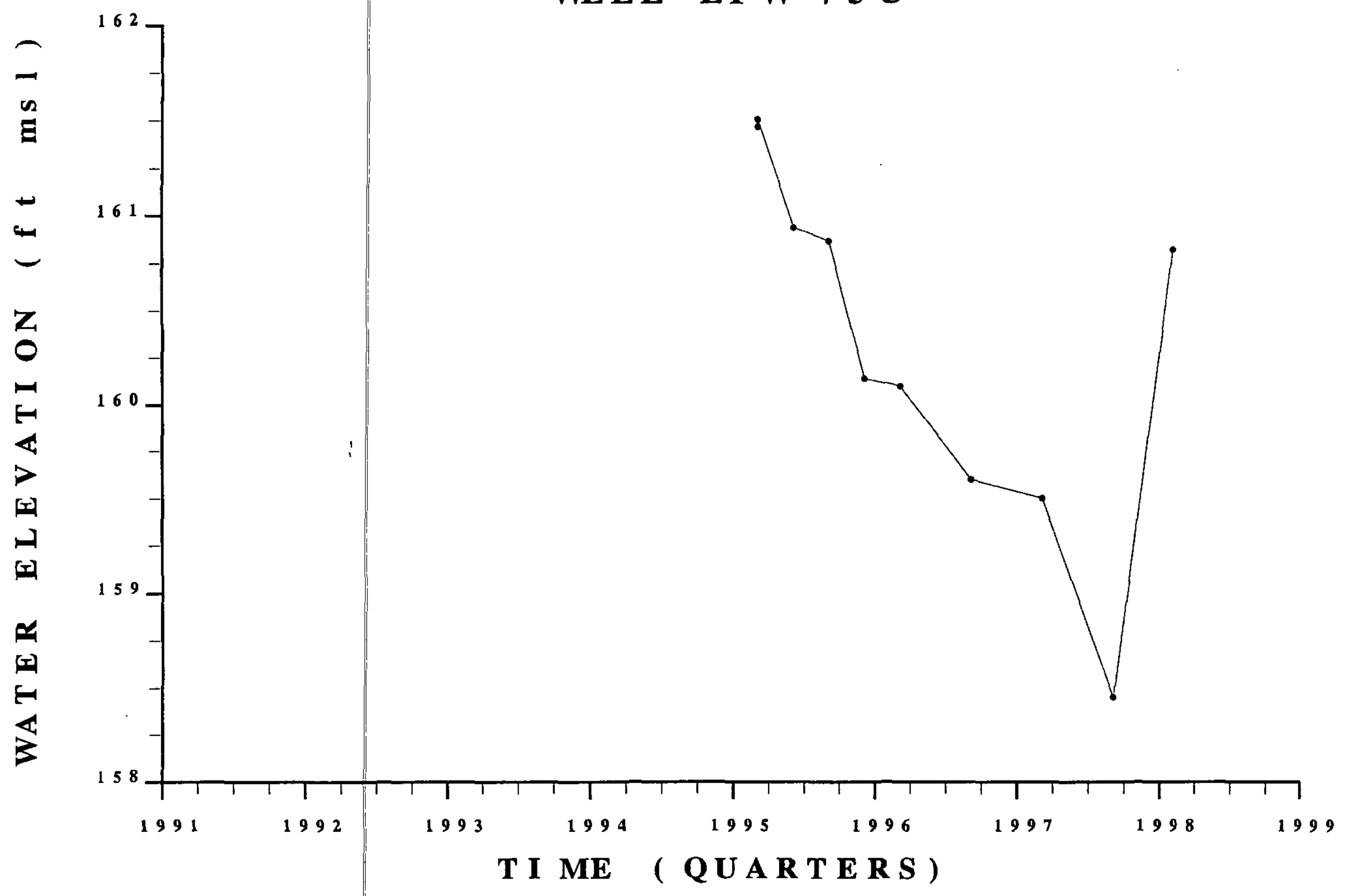

Sanitary Landfil 
H Y D R O G R A P H WELL LFW 75 D
WS R C - T R - $99-00011$

Unc l a s s i f i e d



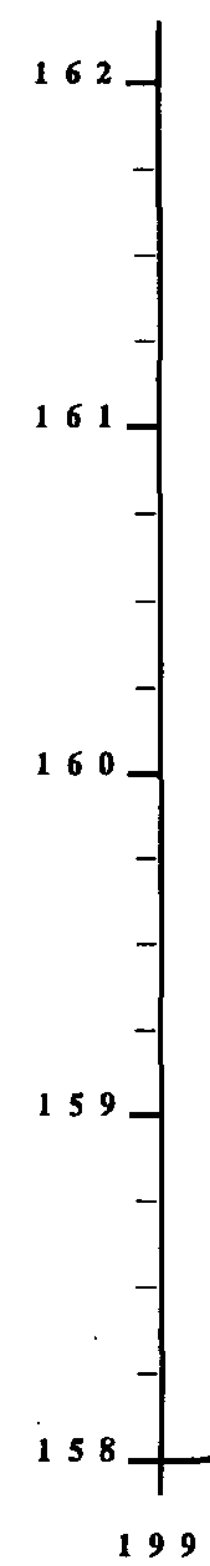

1991

1992

1993

994

1995

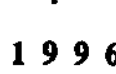

1997

1998

1999

T I ME ( QUARTER S ) 
HY D R O G R A P H WE L L L F W 76
WS R C - T R- $99-000011$ Unc l a s s i f i ed

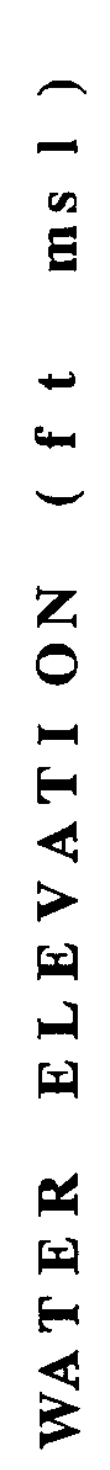

1597

158

157

1991

1993

1994

1995

1996

1997

1999

T I ME ( QUART ER S ) 
HY D R O G R A P H $\begin{array}{lll}\text { WE L L } & \text { L F W } 77\end{array}$
WS RC- T R- $99-00011$

Unc l a s s i f i ed

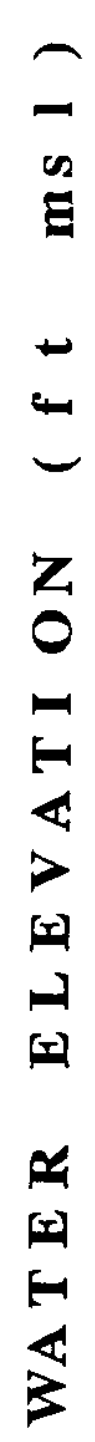

161

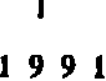

1992

1993

1995

1996

1997

( Q U A R T E R S )

S a n i t a r v L a n d f i l l

Fourth Ou a $\mathbf{t}$ e $r$.

1998

\& 1998 S mmar v 


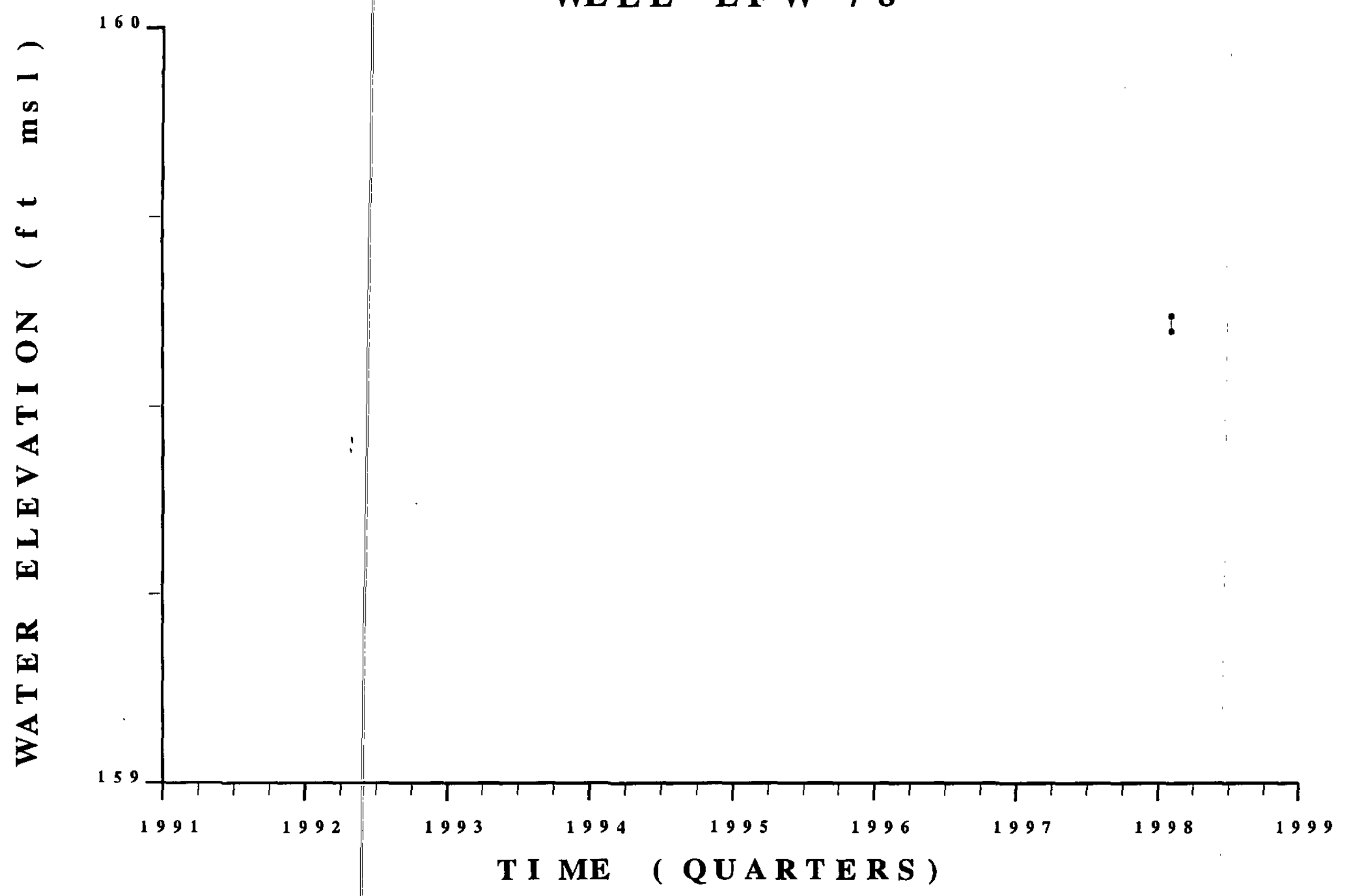

WS R C - T R - $99-000011$ Unc l a s $s$ i f i d 